\title{
BUS REAL-TIME ARRIVAL PREDICTION USING STATISTICAL PATTERN RECOGNITION TECHNIQUE
}

By

Nam Hoai Vu, M.Sc., (2000)

Hanoi University of Civil Engineering, Vietnam

A thesis submitted to the Faculty of Graduate Studies and Research in partial fulfillment of the requirements for the degree of

Doctor of Philosophy

Department of Civil and Environmental Engineering

Carleton University

Ottawa, Ontario, Canada

(C) December 2006

Nam Hoai $\mathrm{Vu}$

The Doctor of Philosophy in Civil Engineering is a joint program with the University of Ottawa, administrated by the Ottawa-Carleton Institute for Civil Engineering 


$\begin{array}{ll}\begin{array}{l}\text { Library and } \\ \text { Archives Canada }\end{array} & \begin{array}{l}\text { Bibliothèque et } \\ \text { Archives Canada }\end{array} \\ \begin{array}{l}\text { Published Heritage } \\ \text { Branch }\end{array} & \begin{array}{l}\text { Direction du } \\ \text { Patrimoine de l'édition }\end{array} \\ \begin{array}{l}\text { 395 Wellington Street } \\ \text { Ottawa ON K1A ON4 }\end{array} & \begin{array}{l}\text { 395, rue Wellington } \\ \text { Ottawa ON K1A ON4 } \\ \text { Canada }\end{array}\end{array}$

Your file Votre référence ISBN: 978-0-494-23303-0 Our file Notre référence ISBN: 978-0-494-23303-0

NOTICE:

The author has granted a nonexclusive license allowing Library and Archives Canada to reproduce, publish, archive, preserve, conserve, communicate to the public by telecommunication or on the Internet, loan, distribute and sell theses worldwide, for commercial or noncommercial purposes, in microform, paper, electronic and/or any other formats.

The author retains copyright ownership and moral rights in this thesis. Neither the thesis nor substantial extracts from it may be printed or otherwise reproduced without the author's permission.
AVIS:

L'auteur a accordé une licence non exclusive permettant à la Bibliothèque et Archives Canada de reproduire, publier, archiver, sauvegarder, conserver, transmettre au public par télécommunication ou par l'Internet, prêter, distribuer et vendre des thèses partout dans le monde, à des fins commerciales ou autres, sur support microforme, papier, électronique et/ou autres formats.

L'auteur conserve la propriété du droit d'auteur et des droits moraux qui protège cette thèse. $\mathrm{Ni}$ la thèse ni des extraits substantiels de celle-ci ne doivent être imprimés ou autrement reproduits sans son autorisation.
In compliance with the Canadian

Privacy Act some supporting forms may have been removed from this thesis.

While these forms may be included in the document page count, their removal does not represent any loss of content from the thesis.
Conformément à la loi canadienne sur la protection de la vie privée, quelques formulaires secondaires ont été enlevés de cette thèse.

Bien que ces formulaires aient inclus dans la pagination, il n'y aura aucun contenu manquant.

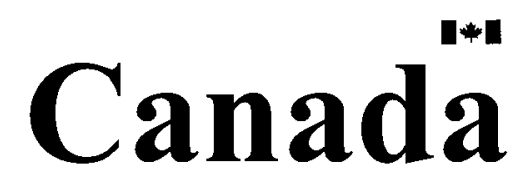




\section{Abstract}

Given the realization that real-time bus arrival information is viewed positively by passengers of public transit, many bus transit agencies of various sizes are developing Real-time Bus Arrival Information System (RETBAIS) following the implementation of Automatic Vehicle Location (AVL) and Automatic Passenger Counter (APC) systems. This research focuses on one important element of the RETBAIS, the real-time prediction model. Data required for the research were retrieved from the APC and AVL systems of the City of Ottawa/OC Transpo. The developed model has two main modules: Running Time Prediction Module (RTM) and Dwell Time Prediction Module (DTM).

The RTM is based on the statistical pattern recognition methodology. Given a pattern defining bus running time being predicted, the trained RTM automatically searches through the historical patterns which are the most similar to the new pattern and based on that, the prediction of a bus running time is made. The RTM was tested with different data sets of various bus running time situations. It was found that it worked well as indicated by the average relative prediction error of as low as $5 \%$ for the Transitway route and about $7 \%$ for the mixed-traffic bus route. Moreover, this module performed in a consistent manner even when unusual bus operational scenarios were used.

The DTM has four sub-modules. The first two sub-modules are also based on a recognition technique for predicting separately the number of passengers boarding and alighting. The third sub-module is used to examine the relationship between actual dwell times and various explanatory variables. The last one is based on the fact that passengers 
choose the most convenient door for boarding and alighting. Having tested with datasets, the DTM proved that it can predict passenger activities with satisfactory accuracy without any specific prior assumptions on the complicated relationship between dwell time and the influencing factors.

When the constituent modules are integrated, the whole model can predict bus arrival times at every downstream stop. The prediction accuracy increased with new data availability. The average relative prediction error varied from 3 to $8 \%$.

In order to provide bus dispatchers with tools for managing bus fleet, two methods to detect bus on-time performance and bus bunching were developed. By using these tools, a bus dispatcher can easily know ahead of time if the bus is on-time, late, early, or bunching is likely to occur.

By offering fast, accurate and reliable predictions, it is contended that the developed real-time prediction model will enhance the bus arrival information system and therefore will be a contribution to public transportation operation. 


\section{Acknowledgments}

I would like to express my deepest gratitude to Prof. Ata. M. Khan for encouragement, patience, support and invaluable scientific guidance in supervising this thesis.

I am grateful to the staffs of the OC Transpo (Ottawa, Canada) for several discussions, comments and data provisions on the various aspects of this thesis. Special thanks to Mr. Joe Koffman, Mr. Brian Barclay and Ms. Sylvie Paquette. I would like to say thank you to Mr. Kean Lew and Mrs. Stephen Hotard (PTV America Inc.) for helping me to use the VISSIM software.

I am greatly thankful to Prof. Yasser Hassan, Prof. William Johnson, and Prof. Steven Prus for valuable suggestions on this thesis research.

Financial support by the Vietnamese Government is gratefully acknowledged.

I want to thank my friends; Mr. Phung Viet Anh who was always willing to help me during difficulties; Mr. Jarbar Siddique for interesting conversations in the common favorite area of bus transit; Ms. Sandra Majkik who shared data with me.

I am so indebted to my wife Mrs. Huyen $\mathrm{Vu}$ and to my son $\mathrm{Hieu} \mathrm{Vu}$ for continuous encouragements, patience, sacrifice and their love. I love you both.

Four years of living and studying in this country tattooed in my mind about a beautiful country with clement people. Thank you Canada!

Nam Hoai $\mathrm{Vu}$ 


\section{TABLE OF CONTENTS}

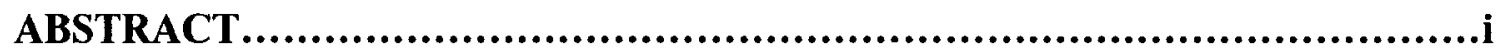

ACKNOWLEDGMENTS...............................................................ii

TABLE OF CONTENTS.................................................................iv

LIST OF TABLES........................................................................

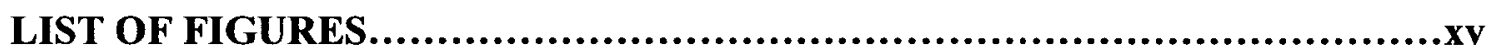

ABBREVIATIONS......................................................................xviii

\section{CHAPTER 1: INTRODUCTION}

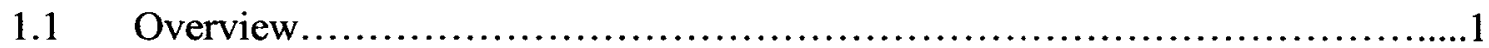

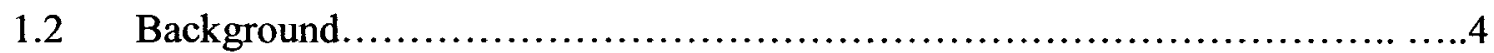

1.3 Problem Statement....................................................6

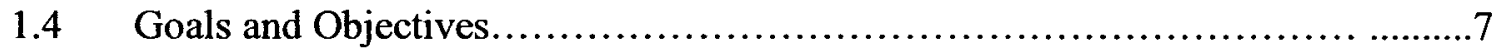

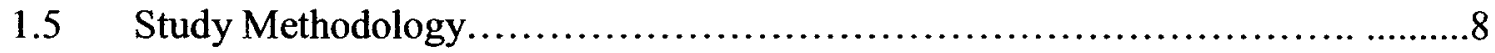

1.6 Thesis Document and Organization...................................... 11

\section{CHAPTER 2: LITERATURE REVIEW}

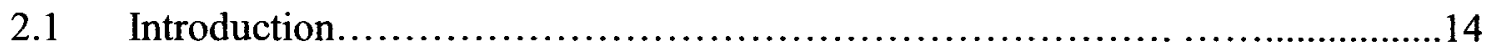

2.2 Real-Time Bus Arrival Information System: Current State of Development...14

2.2.1 AVL System and APC System.................................. 15

2.2.1.1 Automatic Vehicle Location System ......................15

2.2.1.2 Automatic Passenger Counting System ....................19

2.2.1.3 Uses of Retrieved AVL-APC data in RETBAIS.............21

2.2.2 Bus Running Time Prediction Algorithms.............................25

2.2.2.1 Blacksburg (Virginia) Prediction Algorithms................25

2.2.2.2 Portland (Oregon) and King County Metro, Seattle

(Washington) Prediction Algorithm.........................26 
2.2.2.3 Texas Transportation Institute Algorithms.................28

2.2.2.4 New Jersey University Algorithm......................28

2.2.2.5 University of Toronto Algorithm..........................29

2.2.2.6 Artificial Neural Networks (ANN)-Based Algorithms........30

2.2.2.7 Classical Statistical Regression Models.....................31

2.2.2.8 Other Prediction Models......................................33

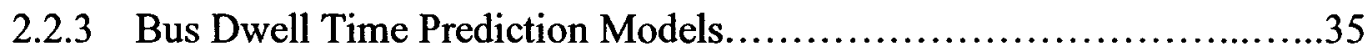

2.2.4 Media and Communication System.................................38

2.2.4.1 Information Dissemination Media........................39

2.2.4.2 Communication System................................40

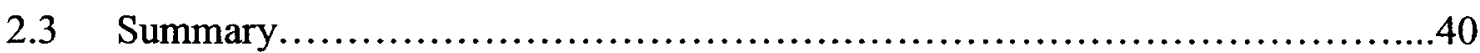

\section{CHAPTER 3: MECHANISM OF THE PROPOSED MODEL AND AVL -APC DATA COLLECTION}

3.1 Introduction...................................................................42

3.2 Component of Bus Trip Time and Influencing Factors........................42

3.2.1 Actual Moving Time..........................................44

3.2.2 Dwell Time.........................................................45

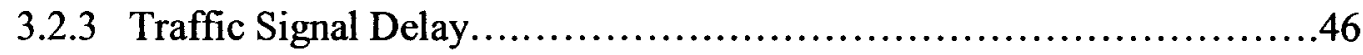

3.2.4 General Delay................................................46

3.2.5 Recovery Time................................................48

3.3 Proposed Structures and Components........................................48

3.3.1 General Discussion.............................................48

3.3.2 Assumptions...............................................50

3.3.3 Structure and the Building Modules of the Proposed Model...............51

3.3.4 Mechanism of the Proposed Model................................53

3.4 APC and AVL Data Collection............................................59

3.4.1 Bus Route Selection.......................................60

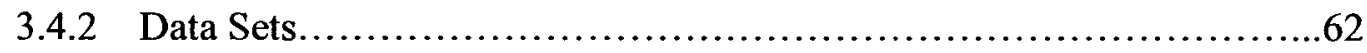


3.4.3 APC and AVL Data Description.....................................64

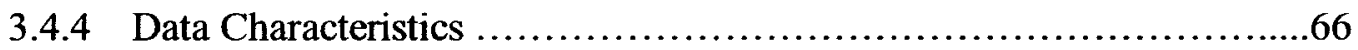

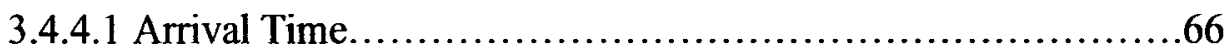

3.4.4.2 Dwell Time and Passenger Activities...........................76

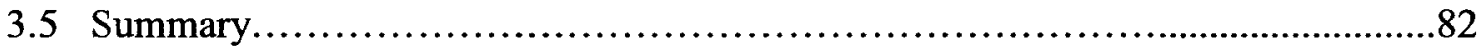

\section{CHAPTER 4: BUS RUNNING TIME PREDICTION MODULE}

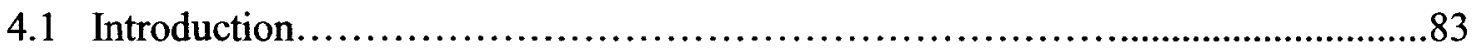

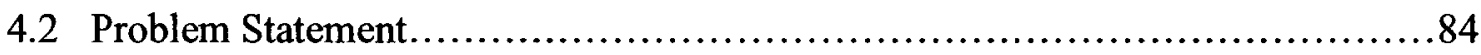

4.3 Nonparametric Regression Methods in Statistical Pattern Recognition..............85

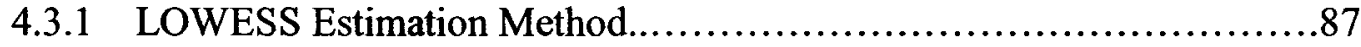

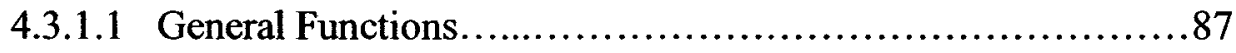

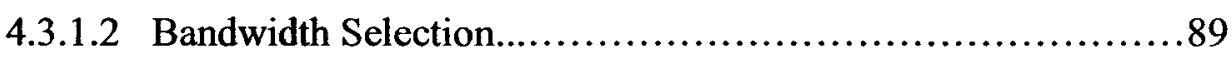

4.3.1.3 Weighting Kernel Function Selection.............................93

4.3.14 Degree of Polynomial Regression..............................93

4.4 Modelling Bus Running Time Prediction by using LOWESS Method and

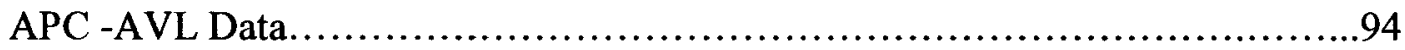

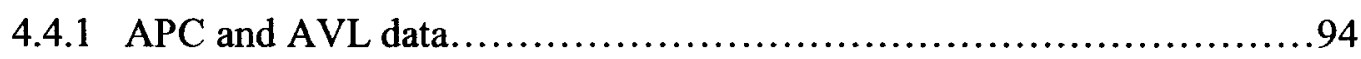

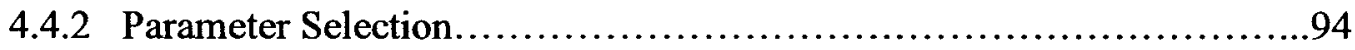

4.4.3 Pattern Selection.....................................................95

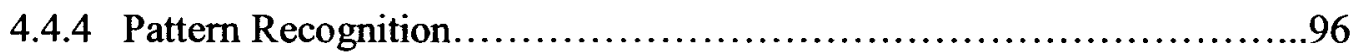

4.4.4.1 Euclidean Distance Calculation..................................96

4.4.4.2 Optimal Bandwidth by Leave-one-out Method....................96

4.4.4.3 Recognition of the Neighbours... .............................100

4.4.5 Prediction........................................................... 100

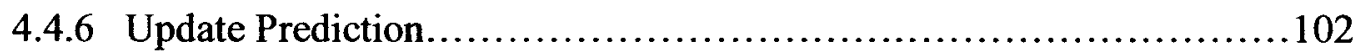

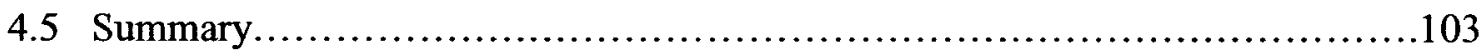




\section{CHAPTER 5: BUS DWELL TIME PREDICTION MODULE}

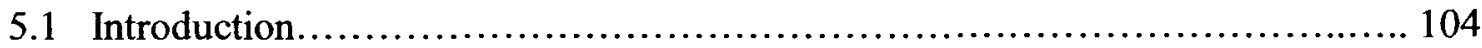

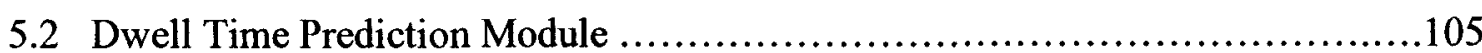

5.2.1 Discussions on Previous Works............................................... 105

5.2.2 Dwell Time Prediction Module (DTM) ...............................108

5.2.3 Real-Time Boarding Passenger Prediction Sub-module.............................110

5.2.3.1 Parameter Selection..........................................110

5.2.3.2 Pattern Selection...................................................110

5.2.3.3 Pattern Recognition...............................................................114

5.2.3.4 Prediction..................................................... 114

5.2.3.5 Update Prediction.........................................115

5.2.4 Real-time Alighting Passenger Prediction Sub-module..........................115

5.2.4.1 Parameter Selections..........................................115

5.2.4.2 Pattern Selection............................................116

5.2.4.3 Pattern Recognition.........................................116

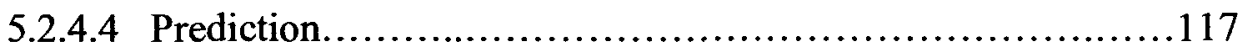

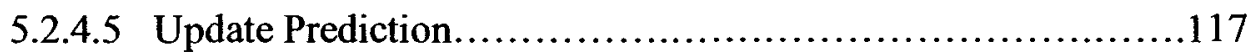

5.2.5 Regression Sub-Module ............................................ 118

5.2.5.1 Variables Selection and Preparation.............................118

5.2.5.2 Regression Functions...................................................120

5.2.6 Busiest Door Prediction Sub-Module..................................................... 127

5.2.6.1 Rigid-Body Bus..............................................

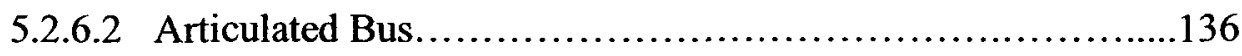

5.2.7 Method A vs. Method B and the Selection..............................142

5.2.7.1 Method A..................................................143

5.2.7.2 Method B....................................................143

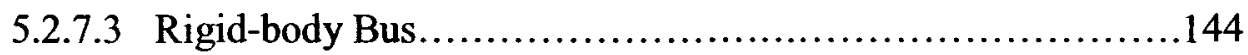

5.2.7.4 Articulated Bus............................................144

5.2.7.5 Accuracy Performance of the Two Methods...................146

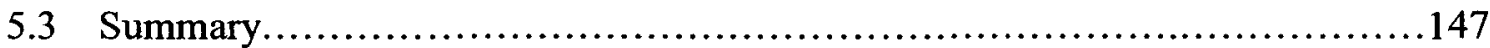

vii 


\section{CHAPTER 6: MODEL PERFORMANCE}

6.1 Introduction. 149

6.2 Computer Program Development......................................... 150

6.3 Simulation of Bus Operation Scenarios............................................. 150

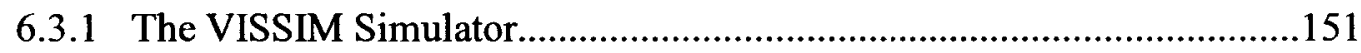

6.3.2 Transit Route Coding in VISSIM....................................153

6.3.3 Calibration and Validation....................................... 155

6.3.4 Bus Operation Scenarios, Micro-simulation Runs, and the VISSIM Outputs........................................................157

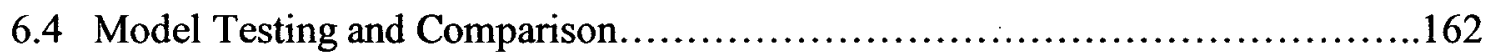

6.4.1 Evaluation Criteria for Prediction Performance.....................................162

6.4 .2 Reference Predictors............................................ 163

6.4.2.1 The Naïve Model........................................164

6.4.2.2 The Kalman Filter- Based Model..............................164

6.4.3 Testing the Developed Model and the Reference Predictors.............166

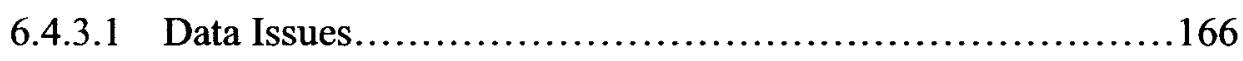

6.4.3.2 Running Time Prediction Performance......................... 167

6.4.3.3 Boarding Passenger Prediction Performance................. 176

6.4.3.4 Alighting Passenger Prediction Performance.................178

6.4.3.5 Prediction Performance with Actual Data......................180

6.4.3.6 Tukey Test for Performance Comparison.....................184

6.4.3.7 Bus Arrival Time Prediction Performance.................... 186

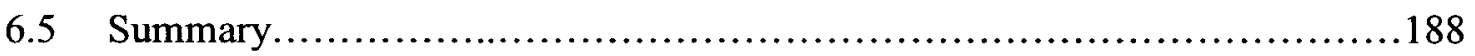

\section{CHAPTER 7: REAL-TIME PREDICTION INTERVAL, ON-LINE SCHEDULE ADHERENCE EVALUATION AND BUS BUNCHING DETECTION}

7.1 Introduction. 190

7.2 Real-time Prediction Interval........................................... 190

7.3 On-line Schedule Adherence Evaluation. .194 


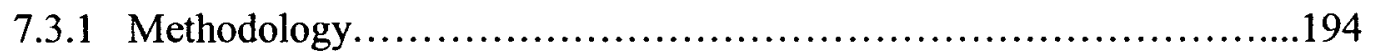

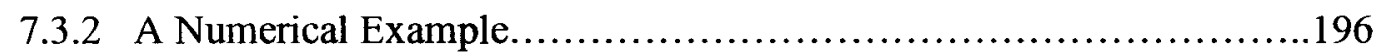

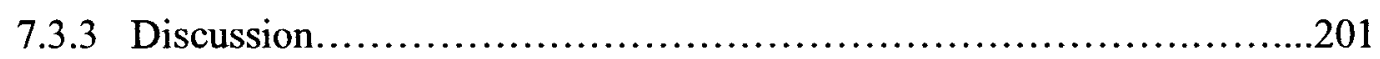

7.4 Real-time Bus Bunching Detection Method....................................203

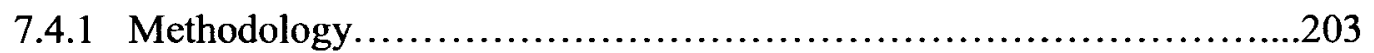

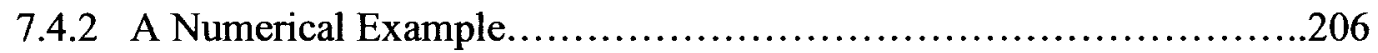

7.5 Further Possible Applications of the Developed Model............................207

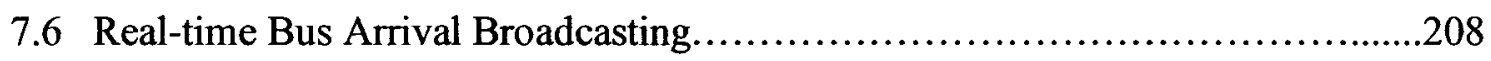

7.7 Level of Spatial and Temporal Detail of Data for the Developed Model.............209

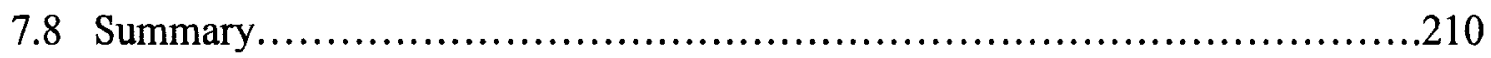

\section{CHAPTER 8: CONCLUSIONS AND RECOMMENDATIONS}

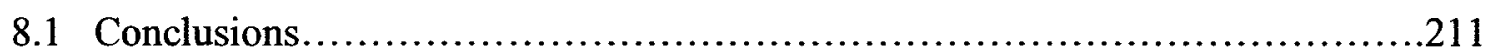

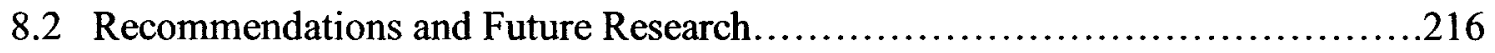

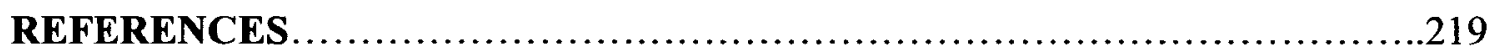

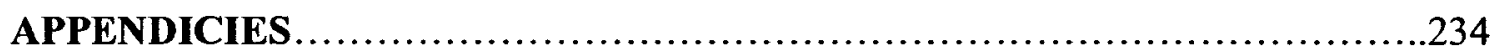

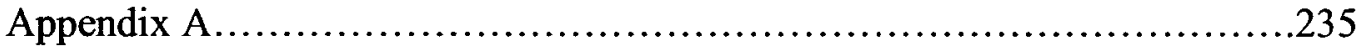

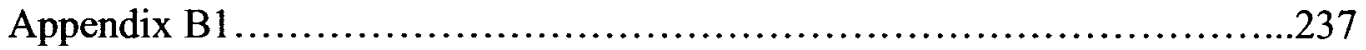

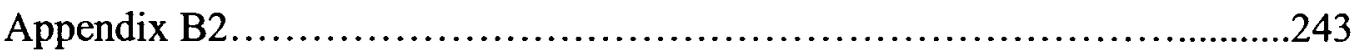

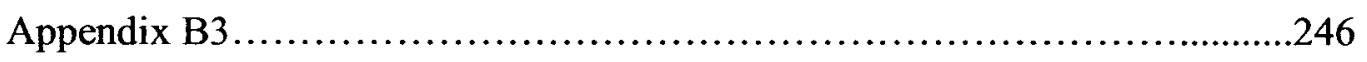

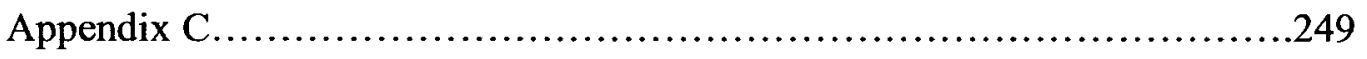

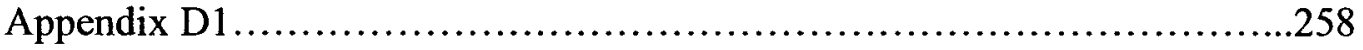

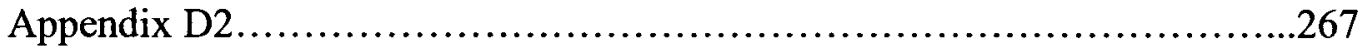

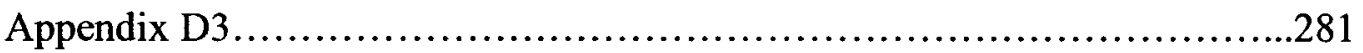

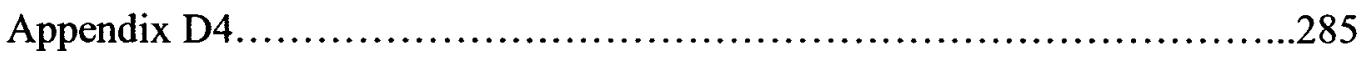

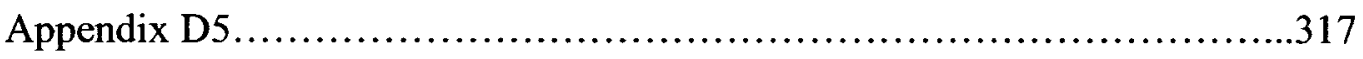

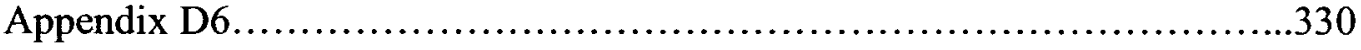

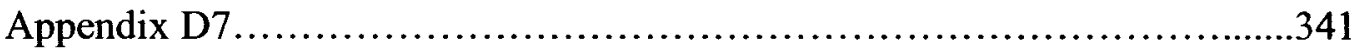

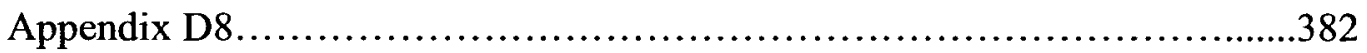


Appendix D9 


\section{LIST OF TABLES}

Table 2.1 Transit Agencies with Real-time Bus Arrival Information System........23

Table 3.1 Factors that influence Bus Running Time and Delay....................43

Table 3.2 Distance between stops BLAIR and LEBRETON. Bus Route 95 (Orleans-Nepean South)

Table 3.3 Distance between stops GREENBORO and RIDEAU. Bus Route 1

(South Keys - Ottawa Rockliffe)...................................62

Table 3.4 Data Sets for Bus Route 95 and Bus Route 1.....................................63

Table 3.5 Data Collectable by APC and AVL Systems.........................65

Table 3.6 Means and Standard Deviations of Bus on-time Performance in the Winter Bus Route 1 (Greensboro- Rideau)

Table 3.7 Means and Standard Deviations of Bus on-time Performance in the Summer Bus Route 1(Greensboro-Rideau) .....................68

Table 3.8 Means and Standard Deviations of Bus on-time Performance in the Winter. Bus Route 95(Blair- Lebreton).........................68

Table 3.9 Means and Standard Deviations of Bus on-time Performance in the Summer. Bus Route 95(Blair-Lebreton).

Table 3.10 Two-sample t statistics for Winter and Summer Time.

Route $95-$ Morning period ( 8 a.m. to 10 a.m.)......................70

Table 3.11 Two-sample $t$ statistics for Winter and Summer Time.

Route 95 - Noon period (12 a.m. to 2 p.m.)......................... 70

Table 3.12 Two-sample t statistics for Winter and Summer Time

Route 95- Afternoon period (4 p.m. to 6 p.m.)........................71

Table 3.13 Two-sample $t$ statistics for Winter and Summer Time Route 1- Morning period ( 8 a.m. to 10 a.m.).................... 72

Table 3.14 Two-sample $t$ statistics for Winter and Summer Time

Route 1- Noon period (12 a.m. to 2 p.m.). .72 
Table 3.15 Two-sample $t$ statistics for winter and summer time

Route 1- Afternoon period (4 p.m. to 6 p.m.)....................... 72

Table 3.16 Two-sample $t$ statistics for Different Time Periods of Day.

Route $95-$ the winter time (the WINTER data set)....................73

Table 3.17 Two-sample $t$ statistics for Different Time Periods of Day.

Route 95 - the summer time (the SUMMER data set)..................74

Table 3.18 Two-sample t statistics for Different Time Periods of Day.

Route 1 - the winter time (the WINTER data set) ......................74

Table 3.19 Two-sample t statistics for Different Time Periods of Day.

Route 1- the summer time (the SUMMER data set)....................75

Table 3.20 Descriptive Statistics of Passenger Activities.

Route 1 - time period (8 a.m. to 10 a.m.)............................ 79

Table 3.21 Descriptive Statistics of Passenger Activities.

Route 1- time period (12 a.m. to 2 p.m.)............................ 80

Table 3.22 Descriptive Statistics of Passenger Activities

Route 1 - time period (4 p.m. to 6 p.m.)............................. 80

Table 3.23 Descriptive Statistics of Passenger Activities

Route 95 - time period ( 8 a.m. to 10 a.m.).......................... 80

Table 3.24 Descriptive Statistics of Passenger Activities.

Route 95- time period (12 a.m. to 2 a.m.)............................. 81

Table 3.25 Descriptive Statistics of Passenger Activities.

Route 95 - time period (4 p.m. to 6 p.m.).............................. 81

Table 4.1 Common Kernel Functions for Univariate Data..........................93

Table 5.1 Variable Selections for RESM......................................119

Table 5.2 Coefficients of the Best Regression...................................123

Table 5.3 Model Summary for the Best Regression...............................124

Table 5.4 Proposed Non-linear Regression Models........................................126

Table 5.5 Validated Dataset for Logistic Regressions.............................132

Table 5.6 Best Binary Logistic Regression Equations...........................133 
Table 5.7 Model Summary of Type B-L.1 ............................... 133

Table 5.8 Prediction Performance of Type BL-1................................. 134

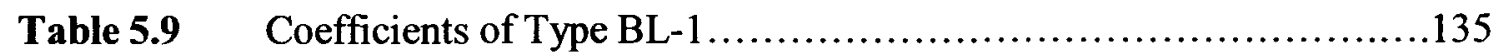

Table 5.10 The Best Multinomial Logistic Regression Equations...................139

Table 5.11 Likelihood Ratios of Type B-ML.3................................140

Table 5.12 Parameters of Type B-ML.3 ................................................ 141

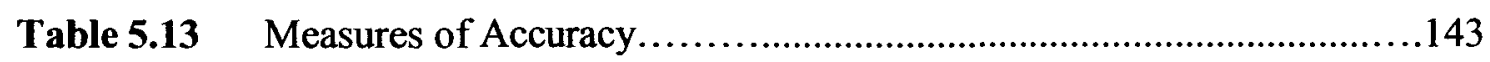

Table 5.14 Average Service Time Selection(s/p).............................144

Table 5.15 Example Output of Dwell time Prediction of

Method A and Method B Applied for Rigid-body Bus................145

Table 5.16 Example Output of Dwell time Prediction of

Method A and Method B Applied for Articulated Bus...............146

Table 5.17 Mean Absolute Prediction Error of Method A vs. Method B.............147

Table 6.1 Differences between VISSIM( version 4.1) and CORSIM (TSIS 5.1)

for public transit simulation..................................... 152

Table 6.2 Kinetic Characteristics of the OC bus................................ 153

Table 6.3 VISSIM Calibrated Parameters.................................... 157

Table 6.4 Dimensionality and Required Sample Size.......................... 159

Table 6.5 Scenario Design of Simulation.................................... 160

Table 6.6 Accuracy Criteria for Model Testing............................. 163

Table 6.7 Cross-validation MAPE, MRE, and RMSE ........................ 169

Table 6.8 Route 1- Running Time Prediction with Overall Situations:

MAPE, MRE, and RMSE ......................................171

Table 6.9 Studentized Range of Test Sets.............................. 185

Table 6.10 Result of Tukey's Procedure at Significance Level $=0.05 \ldots \ldots \ldots \ldots 185$

xiii 
Table 6.11 Holmwood Stop: Bus Arrival Time Prediction Performance of the 3 predictors.

Table 6.12 Gladstone Stop: Bus Arrival Time Prediction Performance of the 3 predictors.

Table 6.13 Rideau Stop: Bus Arrival time prediction performance of the 3 predictors.

Table 6.14 Numerical Examples of Real-time Bus Arrival Prediction Using Developed Models for 3 full trips (seconds).

Table 7.1 Real-time Prediction Interval for Bus Running Time of Route 1 (Time in second).

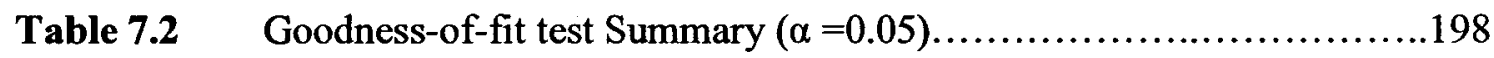

Table 7.3 Parameters of the Two Consecutive Buses..............................206

Table 7.4 Level of Spatial and Temporal Details for Data Capture................209 


\section{LIST OF FIGURES}

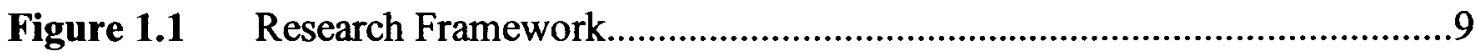

Figure 2.1 Signpost-based Automatic Vehicle Location.........................18

Figure 2.2 GPS-base Automatic Vehicle Location........................... 18

Figure 2.3 The APC System Mounted on a Bus................................19

Figure 2.4 AVL and APC Systems on a Bus................................21

Figure 3.1 Bus Trip Time Components....................................43

Figure 3.2 A Simplified Sketch of Bus Operation...............................51

Figure 3.3 Process of Real-time Prediction.....................................54

Figure 3.4A Bus Route 95 (Blair- Lebereton)..............................61

Figure 3.4B Bus Route 1 (South Keys-Rideau)..............................62

Figure 3.5 Structures of APC Data at pointcheck...............................64

Figure 3.6 Structure of APC Data on Passenger Activity.................................66

Figure 3.7 Dwell time Histogram. Route 1- Time period (8 a.m. to10 a.m.)........ 76

Figure 3.8 Dwell time Histogram. Route 1- Time period (12 a.m. to 2 p.m.).......77

Figure 3.9 Dwell time Histogram. Route 1- Time period (4 p.m. to 6 p.m.)........77

Figure 3.10 Dwell time Histogram. Route 1- Time period (4 p. m. to 6 p.m.).........77

Figure 3.11 Dwell time Histogram. Route 95 - Time period (8 a.m. to 10 a.m.).....78

Figure 3.12 Dwell time Histogram. Route 95 - Time period (12 a.m. to 2 p.m.)......78

Figure 4.1 Bandwidth Selection and NP Regression...........................90

Figure 4.2 Constant Bandwidth Problem....................................................... 90

Figure 4.3 N-Nearest Type of Bandwidth..................................

Figure 4.4 Automatic Process of Searching Optimal Number of Neighbours........97 
Figure 5.1 Dwell time Prediction Module. Method A.......................... 109

Figure 5.2 Dwell time Prediction Module. Method B...........................109

Figure 5.3 Boxplot of Dwell time. Bus route 1

(All seasons and all time dataset) ................................121

Figure 5.4 Boxplot of validated dwell time. Bus route 95

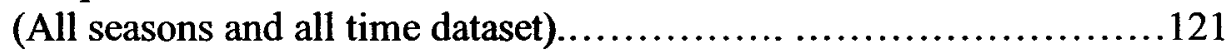

Figure 6.1 Structure and Constituent Functions of the Computer Program........ 150

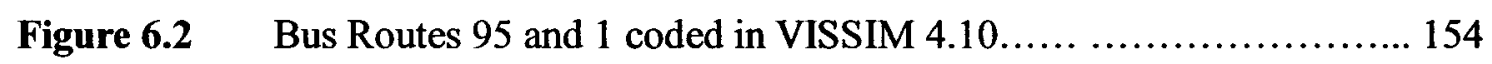

Figure 6.3 Vehicle Record Configuration Tool in the VISSIM..........................155

Figure 6.4 Route 95- Running Time Prediction: MAPE and MRE......................169

Figure 6.5 Route 95- Running Time Prediction: RMSE...................................170

Figure 6.6 Route 1- Running Time Prediction with Overall Situation:

MAPE and MRE

Figure 6.7 Route 1- Running Time Prediction with Overall Situation:

RMSE.

Figure 6.8 Route1-The Differences of MAPE Between Overall and

Slowdown zone Situations.

Figure 6.9 Route1-The difference of RME Between Overall and

Slowdown zone Situations.

Figure 6.10 Route1-The Difference of RMSE Between Overall and

Slowdown zone Situations

Figure 6.11 Nearest Neighbour and Cross-Validation Least-square Error (CR)

for Bus Running Time Prediction. Case 43-2200, Route 1.............175

Figure 6.12 Nearest Neighbour and Cross-Validation Least-square Error (CR)

for Bus Running Time Prediction. Case 54-2200, Route 95............175

Figure 6.13 Route 95- Boarding Passenger Prediction: MAPE and MRE............177

Figure 6.14 Route 95- Boarding Passenger Prediction: RMSE.....................177

Figure 6.15 Nearest Neighbour and Cross-Validation Least-square Error (CR)

xvi 
for boarding passenger prediction. Case $24-1200$, Route $95 \ldots \ldots \ldots 178$

Figure 6.16 Route 95- Alighting Passenger Prediction: MAPE and MRE..........179

Figure 6.17 Route 95- Alighting Passenger Prediction: RMSE..................179

Figure 6.18 Nearest Neighbour and Cross-Validation Least-square Error (CR) for Alighting passenger Prediction. Case 20-1200, Route 95..........180

Figure 6.19 Route 95-Running Time Prediction with Actual Data:

MAPE and MRE...............................................181

Figure 6.20 Route 95-Running Time Prediction with Actual data: RMSE......... 181

Figure 6.21 Route 1- Running Time Prediction with Actual Data:

MAPE and MRE....................................................181

Figure 6.22 Route 1-Running Time Prediction with Actual Data: RMSE...........182

Figure 6.23 Route 95- Boarding Passenger Prediction with Actual Data

MAPE and MRE.

Figure 6.24 Route 95- Boarding Passenger Prediction with Actual Data: RMSE....183

Figure 6.25 Route 95- Alighting Passenger Prediction with Actual Data

MAPE and MRE

Figure 6.26 Route 95- Alighting Passenger Prediction with Actual Data: RMSE...183

Figure 7.1 Fitted Density for OAP at Gladstone Stop-Route1 .................200

Figure 7.2 On-time Limits and Predictions Interval.........................201

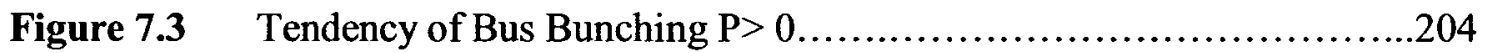

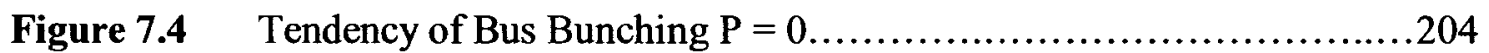

xvii 


\section{ABBREVIATIONS}

\begin{tabular}{|c|c|}
\hline ANN & Artificial Neural Networks \\
\hline $\mathrm{APC}$ & Automatic Passenger Counter \\
\hline APTS & Advanced Public Transportation System \\
\hline AVL & Automatic Vehicle Location \\
\hline ATIS & Advanced Traveler Information System \\
\hline BDSM & Busiest-door Prediction Sub-Module \\
\hline CBD & Central Business District \\
\hline CORSIM & Corridor Simulation \\
\hline $\mathrm{CV}$ & Cross-Validation \\
\hline CR & Cross-validation Least-square Error \\
\hline DGPS & Differential Global Positioning System \\
\hline DMS & Dynamic Message Sign \\
\hline DR & Dead Reckoning \\
\hline DTM & Dwell Time Prediction Module \\
\hline FTA & Federal Transit Administration \\
\hline GPS & Global Positioning System \\
\hline GIS & Geographic Information System \\
\hline OAP & Off-line Adherence Performance \\
\hline OC Transpo & Ottawa-Carleton Regional Transit Commission \\
\hline OLS & Ordinary Least Square \\
\hline PBSM & Punctuality and Bunching Prediction Sub-Module \\
\hline PDA & Personal Digital Assistant \\
\hline
\end{tabular}

xviii 
ITS

LORAN-C

LOWESS

MAPE

NP

RASM

RAP

RBSM

RMSE

RETBAIS

RESM

RTM

SPS

SO

SP Recognition

Tri-Met

VISSIM
Intelligent Transportation System

C configuration of Long Range Navigation

Locally Weighted Scatter Plot Smoothing

Mean Absolute Prediction Error

Non-Parametric Regression

Real-time Alighting Passenger Prediction Sub-Module

Real-time Adherence Performance

Real-time Boarding Passenger Prediction Sub-Module

Root Mean Square Error

Real-Time Bus Arrival Information System

Regression Sub-Module

Running time Prediction Module

Standard Positioning System

Signpost and Odometer

Statistical Pattern Recognition

Tri-County Metropolitan Transportation District of Oregon

Verkerhr In Stadten-SIMulation 


\section{Chapter 1}

\section{INTRODUCTION}

\subsection{Overview}

Many cities around the world are struggling with transportation problems. Managing congestion, reducing air and noise pollution, minimizing fuel consumption, maintaining adequate supply to serve an increasing demand for travel, and preserving community quality of life, are always the challenges for the cities to face. New or widened highways are no longer the preferred solutions of today due to fiscal constraints, conservation of land and petroleum fuels, and environmental quality objectives.

To solve the transportation problems, the development of a convenient and modern public transportation network in the city is usually considered as a first priority solution by the city authorities. Enhancing customer satisfaction by improving service quality will be a key to the success in retaining existing riders and diverting users of private automobile to the public transportation system. In America, if all people who are riding transit to work drove instead, their cars would be as long as 23,000 miles line of traffic, long enough to circle the earth (FTA 1996 Report).

Innovations in communication networks, navigation systems, Global Positioning System (GPS), Geographic Information System (GIS), sensor technologies, the Internet, and computer software, are contributing to the enhancement of the public transportation 
system. At present, the Intelligent Transportation Systems (ITS) in various countries are the vivid examples of the successes in applying new technologies in the transportation field.

The advanced public transportation system (APTS) is one of the main components in the ITS architecture in many countries. The APTS implemented by GPS, GIS, Automatic Vehicle Location System (AVL), and Automatic Passenger Counter System (APC), enables transit agencies to capture the real-time information including vehicle location, speed, operational status, and passenger occupancy. Real-time information is a valuable and reliable input for transit passenger information systems to assist transit providers in planning, operation and management.

Although various transit agencies have implemented new technologies to collect real-time information about their en-route bus fleets, they have been facing difficulties in disseminating reliable bus arrival information to the customer. Traffic conditions reflect stochastic processes. Therefore, they influence a bus adherence to schedule in a random way. It is a challenge for transit agencies to know the arrival time ahead of time. Only the real-time predictions based on real-time information are capable of attracting customers to the public transit and the use of the predicted arrival information can potentially influence the daily activities of travellers.

The APC systems have been deployed in some transit agencies in North America (the United States and Canada) since the 1980s. The time when the first AVL system was launched was even earlier. In 1969, Chicago (USA), implemented an AVL system and Toronto, (Canada) installed one in 1972. The names of AVL and APC systems explain their functions. The AVL system provides dynamically the location, speed, and 
operational status of a remote bus while an APC system is able to transmit the information about passenger boarding and alighting at stops, running time between stops, dwell times, and several service characteristics to the control center. The AVL and APC systems provide much data which can potentially serve as inputs for the development of bus arrival prediction models.

When the first AVL system was initiated in Chicago, a 10-year vision of using AVL systems to improve the schedule performance, was described optimistically by Klopfenstein, (1986, p.3). Since that time, indeed, many transit agencies still do not operate AVL and APC systems. As of 2000, only 88 out of 548 transit agencies in the United States were operating AVL system and 145 others were planning AVL systems (Schweiger, 2003). A small fraction of the 88 agencies are providing real-time arrival information to the customer via Advanced Traveller Information System (ATIS) accessed by passengers at home, work places, and bus stations via the World Wide Web (WWW) Internet system or wireless devices such as internet capable cell phones, Palm Pilots, and Personal Digital Assistants (PDAs). The reason behind the delay is not only that an AVL system is still a significant investment but also the agencies are waiting for the most preferable real-time information system that is capable of providing bus real-time arrival information, workable with the existing technologies and infrastructure, and suitable for their fiscal conditions.

The number of transit agencies using APC systems was even fewer. A survey conducted by Daniel (1998) showed that out of the 27 US transit agencies in the survey, only five agencies use the APC system. Almost all of these agencies used pencil and manual check for different purposes. The Ottawa-Carleton Regional Transit Commission 
(OC Transpo), an 827-bus system serving the capital city of Ottawa (Canada), is a pioneer and long-time user of the APC system. Over the years, data collected from the APC system and the signpost-based AVL system are serving the OC Transpo in terms of increasing the quality of service, monitoring the average loads and passenger activities, and maintaining schedule adherence. However, like many other transit agencies, the OC Transpo has not yet deployed a real-time bus arrival information system or RETBAIS for short.

Clearly, there is a need to develop models that can utilize the huge and valuable AVL-APC database for predicting bus arrival times in order to improve the quality of the arrival information disseminated to passengers.

\subsection{Background}

Transit agencies provide bus information to passengers in different ways. These vary from traditional methods such as printed schedules to modern methods such as arrival times on an electronic sign, also known as Dynamic Message Sign (DMS) located on a bus stop. There are three kinds of information that a transit passenger wants to know: the pre-trip information, the en-route information and the in-vehicle information.

Pre-trip information is static information including schedules, maps, destinations, fares, and policies. This information helps passengers in planning trips. It is updated periodically for informing passengers about every change in schedule time of bus routes, the new routes, or the new policies and fares, except the actual bus operational situations such as delay, late or early buses. To know the scheduled arrivals, a passenger can use a free brochure showing the bus map and schedule of the bus. Also, the passengers can 
access the transit agency's website to find the schedules if they are provided. Calling the transit representatives is also a method for passengers to know bus arrivals. By simply providing the bus station number, a passenger can get the scheduled times via telephone.

From the passenger's point of view, there is no certainty that the expected bus will arrive on schedule. Hence, if transit agencies cannot provide passengers actual arrivals or in other words, the en-route information, the waiting passengers can only depend on their own waiting time experiences. Therefore, they will tend to arrive to the stop a few minutes earlier than the schedule to avoid missing the bus. If the bus arrives late, this increases the passengers' waiting time. Even if the bus arrives on time, the waiting time could be perceived to be long. On the other hand, when the bus comes to the stop earlier than scheduled, passengers can miss the bus even though they come to the stop on time. In this case, the waiting time is now not a few minutes but as long as an headway. Moreover, if passengers do not know that they missed the last bus, they may experience anxiety. For on-board passengers, in-vehicle arrival information to the destinations can help them to have smooth connections with other inter-modal transit services.

Obviously, the uncertainty of bus arrival time increases passenger's waiting time and anxiety, deteriorates schedule adherence, and reduces the smooth transition among inter-modal transfer. Thus, passenger dissatisfaction is unavoidable if transit agencies still provide their customers only the static information. To satisfy customers, transit agencies should provide their passengers not only static information (e.g., pre-trip information) but also en-route and in-vehicle arrival information. Interestingly, some transit agencies that provide real-time information to passengers recognized that diversion to private 
automobile was low even when bus arrivals varied but passengers knew this information ahead of time (Schweiger et al., 2003). Therefore, providing passengers real-time arrival information is necessary and this will encourage passengers to use the transit system.

\subsection{Problem Statement}

Surveys carried out by some transit agencies revealed that the most important factor influencing bus riders' decision to ride a bus, surprisingly, is not the bus on-time performance but the real-time information about the bus of interest. On-time performance only comes as the second important factor. This shows that real-time information is playing an important role in customer's perception of transit service quality (Zhong et al., 2003).

Passengers' demand for real-time information on bus arrivals is pressing transit agencies to meet that demand in order to increase or at least, to keep their customers. Having recognized the importance of this task, various agencies in European countries, in North America (i.e., the United States, Canada), and Asian countries (e.g., Japan, Taiwan, and Korea) have been installing AVL-APC systems to assist their bus fleets as well as to develop a suitable RETBAIS. However, most of these agencies are using AVL-APC data mainly for off-line performance studies. This practice continues in spite of the fact that much more is to be gained in on-line applications such as predicting bus arrivals, predicting passengers boarding or alighting at stops, and proactive dispatch programs.

The use of APC data and AVL data is also different. While APC data downloaded daily or weekly from on-board microcomputers have been used successfully for years in the off-line analyses, for instance, passenger loads, on-time performances, etc., the AVL 
data have not yet been used adequately for neither off-line nor on-line services, except for incident response by many agencies. The reason behind the failure in using useful AVL data is that the transit agencies did not recognize the potential benefits achievable from useful AVL data. Therefore, their AVL systems were not used to match and track vehicles, and AVL and the APC systems have been used as stand-alone systems. This practice limits the on-line applications based on archived AVL-APC data (Furth et al., 2003, p.6).

The purpose of this research is to develop a methodological framework and the constituent model for advancing dynamic predictions and thus to support the real-time bus arrival information system. By using the AVL-APC data intensively, the model will be suitable for transit agencies whose bus fleets are equipped with AVL and APC systems.

\subsection{Goals and Objectives}

This research is intended to enhance the interests of transit passengers as well as transit service providers. The study aims at increasing the quality of actual arrival information (e.g., en-route and in-vehicle information) to be provided to bus transit passengers. Also, this research is intended to support bus transit providers in term of realtime control and management. For achieving the first goal, a real-time prediction model for bus arrivals was developed. To attain the second goal, the applications of the model were further developed for bus bunching and punctuality prediction in order to help dispatcher in managing the fleet.

Specific objectives of this research are: 
1. To define a research framework and constituent modules for the development of methods to support bus real-time arrival prediction.

2. To develop modules and integrate the modules to create a dynamic model that can predict real-time bus arrivals and dwell times at every stop.

3. To evaluate the accuracy of each module and the integrated set of modules.

4. To develop bunching and punctuality prediction algorithms that can help bus dispatchers in controlling the fleet under real-time situations.

5. To propose the architecture of a-real-time bus arrival system based on the developed model.

\subsection{Study Methodology}

The overall research framework is shown in Figure 1.1.

The problem definition step covered the general lack of real-time information on bus arrivals and dwell time. As noted in the previous section, the users of bus transit are placing much importance on this type of information. However, for a number of reasons, a very high proportion of public transit agencies do not provide such information. Although a number of transit agencies have taken steps to implement AVL and APC systems, they have not taken the next step to make use of the data to develop a RETBAIS. Also in this step, the issues of the existing bus arrival prediction algorithms were noted. Consequently, the problem definition step dealt with the characterization of the problem to be solved. Here, broad research goals and specific objectives were defined. 


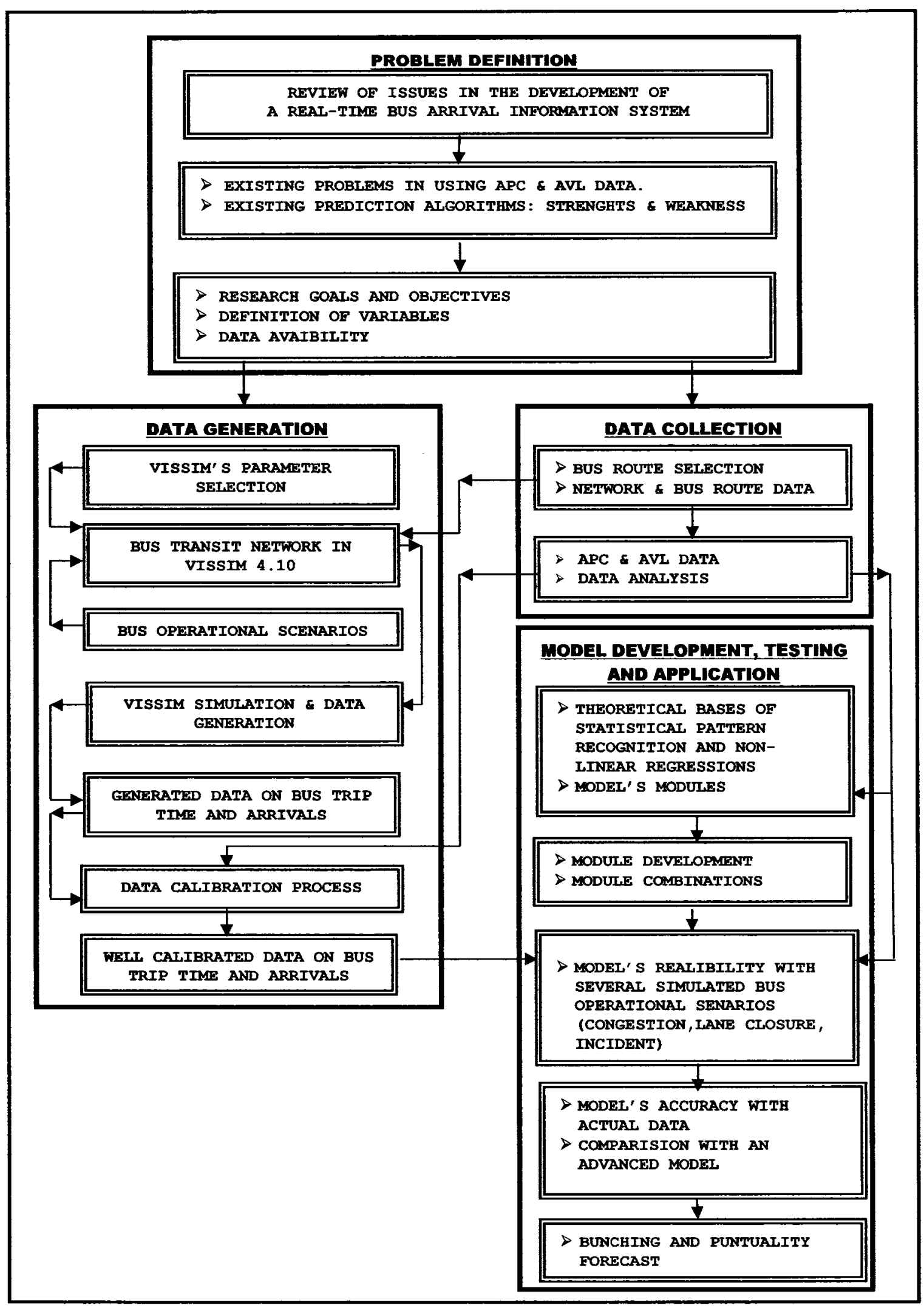

Figure 1.1: Research Framework 
In order to fulfill the research goals and objectives, further steps were accomplished. Two next steps were the data collection and the data generation. These are carried out side-by-side.

In the data collection step, the APC and AVL data collected from the OC Transpo, accompanied with other data of the transit network, were used for two purposes. On one hand, these data were mined directly for the model development and testing steps. On the other hand, they were used as the inputs for the data generation step.

The purpose of data generation step was to generate data, given the assumptions of different bus operational scenarios which are difficult to study in the form of field experiments (e.g., congestion, incidents, etc.). The VISSIM 4.10 traffic software was used for these simulation purposes. In order to make the simulated bus operations in the transit network as similar to the actual operations as possible, a calibration procedure was developed. After the calibration procedure was completed, the generated data were then used for testing the model under each scenario.

The next step involved model development and model testing procedures where the variables were defined and theoretical aspects were researched. Based on the AVL and APC data and the use of statistical pattern recognition technique and nonlinear regression methodologies, a model consisting of three modules and four sub-modules was developed. This developed model has the capability to make real-time bus arrival time and dwell time predictions. After the model was tested in terms of accuracy and reliability by using simulated data obtained from computer simulations of several bus operational scenarios, the model was also tested with real-world data. At the end of this 
step, the developed model's prediction performance on bus running time, bus dwell time and passenger activities were assessed.

Finally, for bunching detection, punctuality study, and dispatcher help, the developed model was expanded by using predicted bus running times and dwell times.

\subsection{Thesis Document and Organization}

The dissertation consists of eight chapters.

Chapter 1 provides an introduction to the subject of this research, research objectives, and the research study framework.

Chapter 2 is devoted to the literature review including current applications and the potential prediction algorithms for bus running time and dwell time.

Chapter 3 presents a broad view of real-time bus arrival prediction model developed in this thesis research. This model includes three linked modules: Running Time Prediction Module (RTM), Dwell Time Prediction Module (DTM), Punctuality and Bunching Prediction Module (PBM). The DTM is composed of four sub-modules,

including: Real-Time Boarding Passenger Prediction (RBSM), Real-time Alighting Passenger Prediction (RASM), Regression sub-module (RESM), and Busiest Door Prediction (BDSM).

Chapter 4 covers the first module, namely the RTM. In this chapter, a pattern recognition process based on a non-parametric statistical technique is presented which can predict running times on every downstream link of an en-route bus. The module's refined procedures are also presented in this chapter. 
Chapter 5 commences with some discussions on previous dwell time prediction algorithms, which have been described in the literature. Next, a framework for dwell time module and its four sub-modules are presented, followed by the solutions and procedures for the first two sub-modules. The text describes how several deficiencies of previous dwell time prediction models reported by various studies are overcome. This chapter also covers data collected by the OC Transpo on dwell time and the number of boarders, alighters and other variables such as on-time performance, bus location, bus types, and time of day. Several statistical assumptions on their relationships are examined in order to find the best statistical model for estimating dwell time.

Chapter 6 presents the method for the estimation of the developed model. First, the procedure to simulate several bus operational scenarios by using VISSIM software is introduced. Second, for comparison purposes, two reference models for bus arrival time prediction are described. One of these models can be currently considered as an advanced model and the other as the simplest model. Third, the developed model and the two reference models are compared in term of bus running time, passenger activities, and bus dwell time predictions given different bus operational scenarios and a variety of data. The comparisons are based on the Tukey's test procedure in order to provide sound conclusions. By using the evaluation system, models' accuracy and reliability are assessed.

Chapter 7 covers the methodologies for the on-line detection of bus on-time performance and bus bunching. For this purpose, a method to estimate real-time prediction interval is developed given a user-defined level of significance. On the basis of probability theory and the historical bus arrival time distribution, two methods are 
developed, one for on-line bus on-time performance evaluation and the other for on-line bus bunching detection. These provide the probability that such events may occur. Finally, the discussions on announcing of bus arrival times as well as spatial aspect and details of the database for the developed model are discussed.

Chapter 8 presents conclusions and recommendations. The contributions of this thesis research are highlighted. Recommendations are provided that include future research on different aspects of data, mathematical algorithms, and some limitations of the developed model. 


\section{Chapter 2}

\section{LITERATURE REVIEW}

\subsection{Introduction}

The application of AVL and APC systems in bus transit is becoming popular in North America and Europe. This enables the agencies to develop a RETBAIS. Based on the review of a number of publications (i.e., syntheses, reports, and articles), this chapter examines the current practices, the experiences, the benefits and problems. Also, the prediction algorithms of bus arrival time and dwell time used by transit agencies around the world are reviewed.

\subsection{Real-Time Bus Arrival Information System: Current State of Development}

The deployment of the AVL and APC systems in many transit agencies has a long history dating back to the early 1970 s. However, this became widespread through the United States and Europe only since the late 1980s and 1990s for the purpose of increasing operational efficiency. After years of using AVL and APC systems, transit agencies realized that these systems not only can increase the operational efficiency but also provide passenger real-time information which is bringing substantial benefits to the transit providers. 
The fundamental elements for deploying a RETBAIS are: the technical characteristics of the underlying AVL system, the real-time prediction models, type of media to distribute the real-time information, related costs of the system, customer and the media reaction to the system, and institutional issues with the system (Schweiger et al., 2003, p.2). In the field of interest, the literature review focuses only on the first three elements. Each element is discussed in a corresponding part, namely: the AVL and APC systems, arrival and dwell time prediction algorithms, and media and communication systems.

\subsubsection{AVL System and APC System}

\subsubsection{Automatic Vehicle Location System}

Automatic Vehicle Location (AVL) system is the backbone technology for a RETBAIS. The AVL system tracks the dynamic location of a remote bus and transmits the data to the control center. The transmitted locations of the bus along with other data such as speed, acceleration, or historical data collected from APC systems and other diagnostic systems on the bus, are used to predict the new location of the bus. As a result, the arrival time at a selected stop is predicted. Different agencies are using different technologies that belong to the four AVL categories. These, in the increasing level of advanced information technology, are presented below.

- Dead-Reckoning (DR)-based AVL system: This is the oldest and simplest navigation technology. The devices frequently used are odometers and 
compasses. Data recorded from instruments must be transmitted by radio to the central control for processing, analysis, and display. In a DR-based AVL system, a bus is equipped with a heading sensor known as an adaptation of a magnetic compass, wheel odometers, an information processor, and radio equipment. The readings of heading sensor and the readings of wheel odometers are processed by the on-board information processor and converted into modulated waves that are compatible with the bandwidth requirements, workable with both receivers and antennas, and transmittable to the control center. At the control center, the waves received from a bus then will be decoded for tracking and displaying the location of the bus.

- Radio Navigation or LORAN C-based AVL system: The C configuration of the Long Range Navigation (LORAN-C) system is the triangulation with three or more land-based transmitting stations. The instruments of a bus using LORAN-C are: a LORAN-C receiver, antenna, a data processor, and land mobile transceivers. Location is delivered based on the distances from the bus to a pair of fixed radio stations' locations. No new LORAN C-based AVL system is anticipated in the future (Okunieff, 1997).

- Signpost and Odometer (SO)-based AVL system: The system, known as radio frequency signposts, or signpost for short (both active and passive) uses radio beacon mounted on the top of the facilities. In a passive signpost system, each beacon mounted on the pole or structure above the height of the bus has a unique ID. The beacon sends a low power coded signal in order to save 
electricity. When a bus passes a signpost, the specialized receivers attached on the bus receive and decode the transmission from the signpost to recognize the signpost's ID. The location of the bus then is determined based on the last beacon ID the bus detected and the distances travelled. Figure 2.1 represents a passive SO-based AVL system. In contrast with passive system, each bus in the active system has a unique ID on a radio channel designated solely for automatic vehicle locating use. The vehicle transmission is continuous and at very low power. Therefore, when the bus crosses a signpost pole equipped with a receiver, its location is relayed and transmitted by that signpost to the control center. This alternative transmits information via underground wire system so the bus does not require a wireless transfer of data to the control center. Details on SO-AVL system can be found in Skomal (1981) and Okunieff (1997).

- Global Positioning System (GPS) - based AVL system: Development of work on GPS commenced in 1973 for the sake of supporting the positioning requirements of military operations. As of 2000, the full constellation of 24 satellites was launched to ensure that there will always be at least four satellites at all sites on the globe. Each GPS satellite transmits a unique navigation signal on two L-band frequencies, the $\mathrm{L} 1$ frequency $1575.42 \mathrm{MHz}$ and the $\mathrm{L} 2$ frequency 1227.60 MHz. Both bands carry the navigation message and Standard Positioning System (SPS). A bus equipped with GPS antenna and GPS receiver connected to an on-board microcomputer receives GPS signals, determines its location and sends the location data to the control center via wireless communication system. Figure 2.2 depicts a GPS-based AVL system for bus transit 


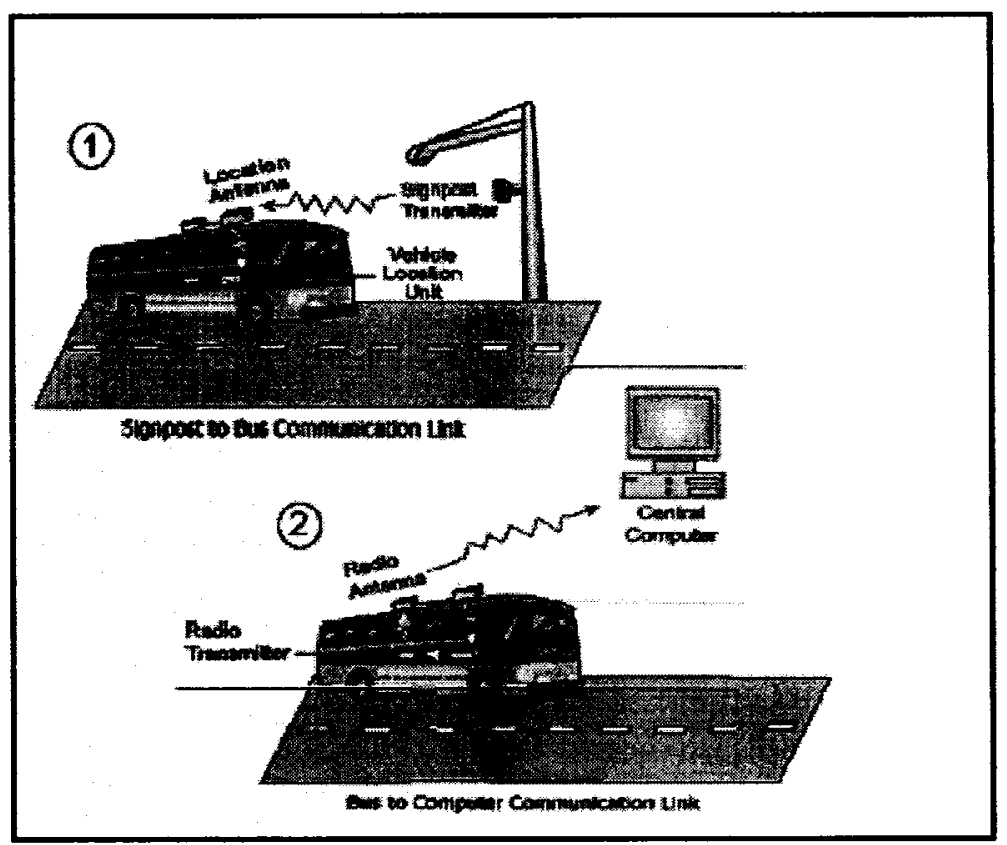

Figure 2.1: Signpost- based Automatic Vehicle Location (Source: http:// www. calccit.org/itsdecision)

1- The location antenna mounted on the bus receives the signpost signals and transmits them to the on-board Vehicle Location Unit (VLU). The VLU then recognizes the signpost's ID.

2- The location of the bus based on the signpost's ID then is transmitted to the control center via radio system.

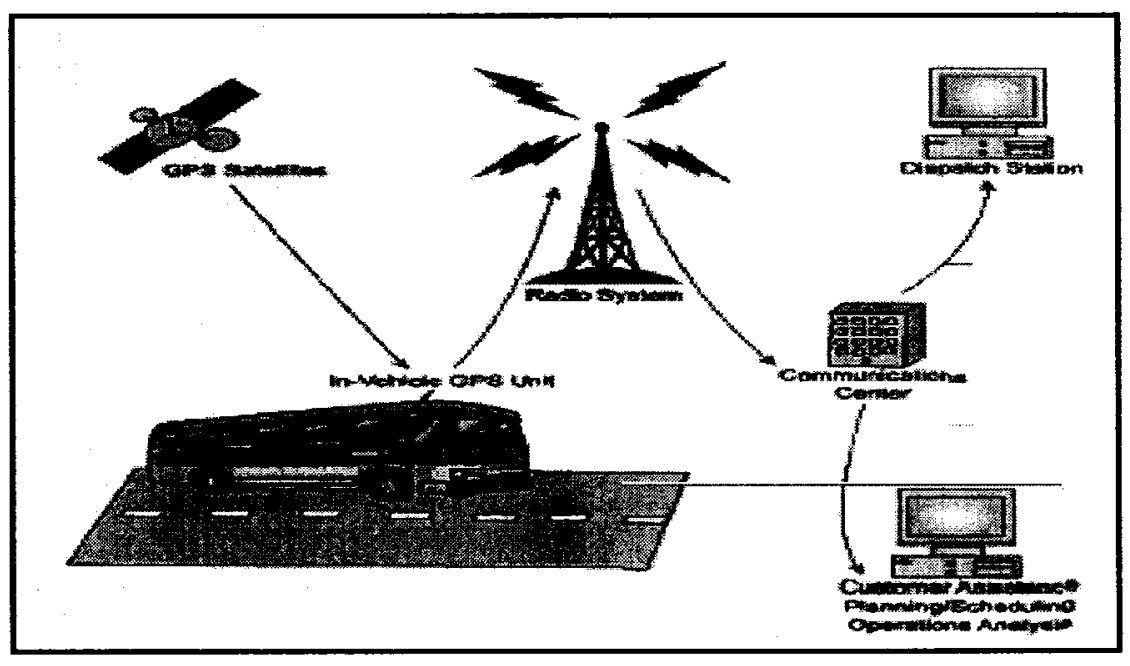

Figure 2.2: GPS-based Automatic Vehicle Location (Source: http:// www. calccit.org/itsdecision) 
- Differential GPS (DGPS)-based AVL system: In order to enhance the accuracy, DGPS requires that one of the receivers to be located as a base location. From the known location of the base, the bias errors due to the estimated position of the orbiting satellites are calculated. A bus on the DGPS based AVL system, like other GPS-based AVL systems, has a GPS antenna, GPS receivers, and an on-board microcomputer. The required additional unit is a radio link that receives correction from differential reference station.

\subsubsection{Automatic Passenger Counting System}

Automatic Passenger Counting System or APC system is an automatic means for counting boarding and alighting passengers as well as the time that the bus is at a specific location. Figure 2.3 depicts the components of an APC system mounted on a bus.

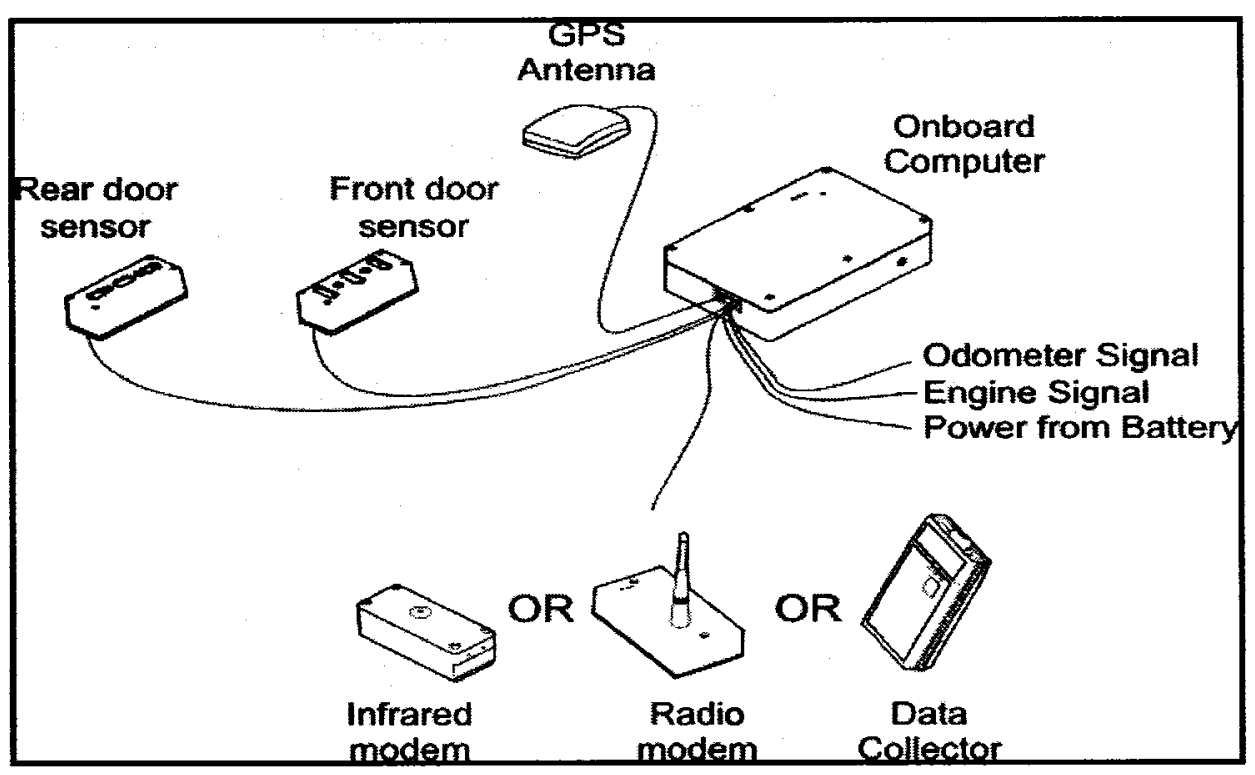

Figure 2.3: The APC system mounted on a bus (Source: http://www.infodev.ca) 
The heart of an APC system is the on-board microcomputer connected to the counting sensors attached on the door areas of a bus. Each time a passenger gets on or off the bus, the sensors will record the passenger activities and store data in the on-board microcomputer. There are two counting technologies applied in the APC system: infrared beam sensors attached on the door areas, and treadle mats mounted at the step levels. Based on the order of the broken beam in the case of infrared beam sensor or the order of stepping on the mat in the case of treadle mats, the APC system can differentiate if a passenger is getting off or alighting on the bus. The data stored in the onboard computer can be transmitted manually with data collector. To transfer data automatically, infrared or radio modems are needed. In case of using infrared devices, when the bus stops (usually at the garage or bus stop), two infrared links, one on the bus and the second on the bus stop or garage area, download data from onboard computer automatically. If the bus is equipped with a radio modem, data stored on the on-board computer can be copied automatically at the distances of up to $500 \mathrm{ft}$ between the radio transceivers. Time to download a full day data is only about 20-30 seconds (http://www.infodev.ca).

The APC system can work as a stand-alone system using one of the four AVL categories mentioned above to match passenger activities to predetermined bus stops. Figure 2.4 illustrates the integration of the APC and AVL systems. Such system can measure over 20 variables including passenger activities (boarding and alighting), bus locations (GPS coordinates, direction, bus stop location), and operational activities (speed, running time, arrival and departure time, dwell time, stop distance, access time, idle time, passenger load, etc). Moreover, an APC system integrated with the AVL system and the radio systems can provide real-time data for real-time dispatching and 
arrival data dissemination purposes (John, 1999). Therefore, the APC system has great potential as valuable source of data for either on-line or off-line applications in RETBAIS.

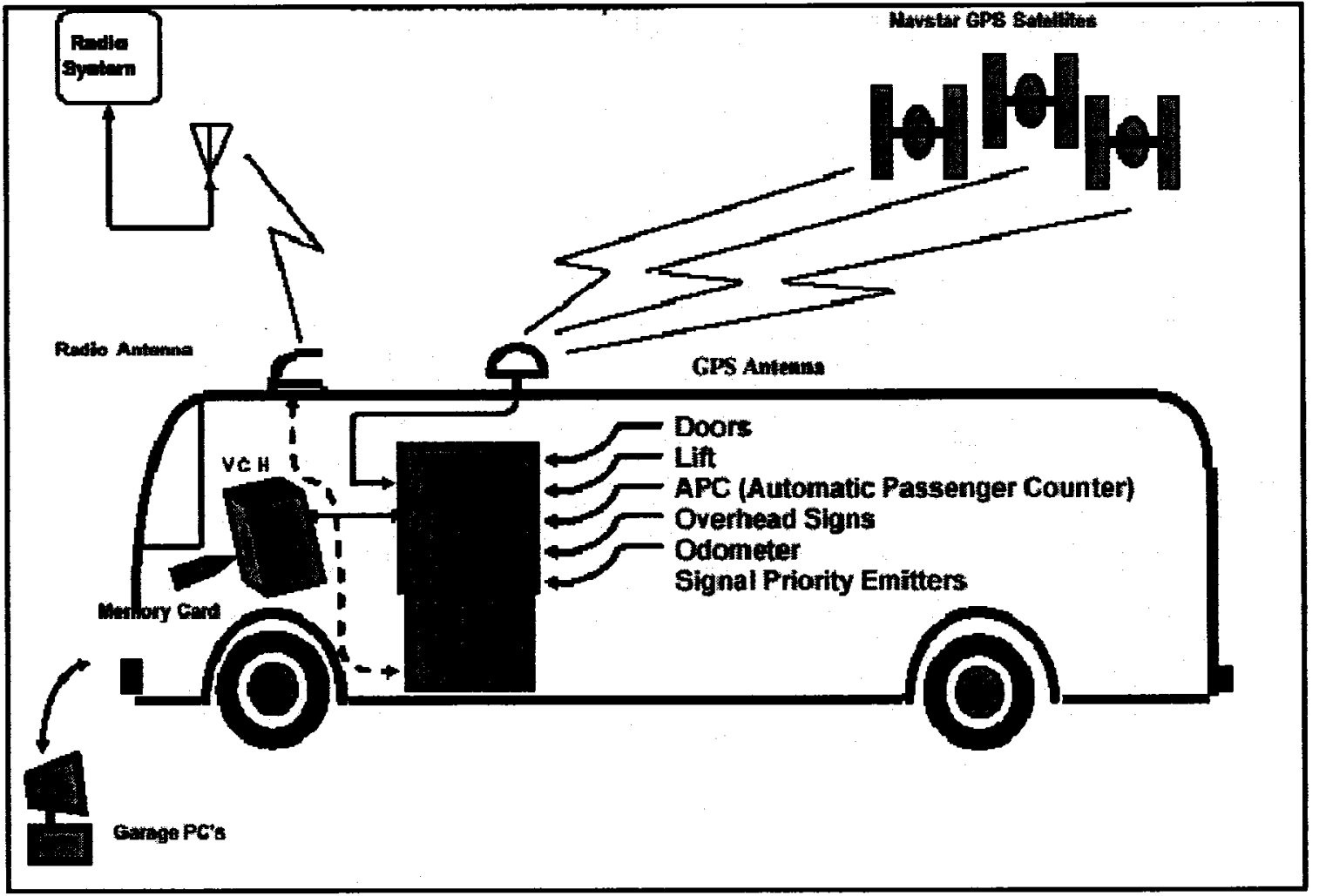

Figure 2.4: AVL and APC Systems on a Bus (Source: Furth et al., 2003)

\subsubsection{Uses of Retrieved AVL-APC data in RETBAIS}

Transit agencies use collected AVL and APC data for two main purposes: off-line performance analyses and on-line applications. While many agencies have used the collected data for off-line studies, a few agencies use these data for on-line applications, such as providing passengers en-route and in-vehicle information. The degree of using 
these data differs from one agency to another. The APC data were usually used in off-line estimates while the AVL data were used for incident response, safety, and recently for real-time location tracking. However, many agencies failed in using the AVL system for both on-line and off-line estimations (Furth et al., 2003). Very few transit agencies (for instant: Calgary Transit, Canada) considered using AVL data for off-line analysis as an objective. While the APC system can collect and store many valuable variables useful for on-line analysis, they have not been used for the real-time applications because they were not integrated with the AVL and radio systems. In contrast, the AVL system, which is considered a more "advanced" technology in real-time application, is not programmed to store data. Consequently, it has been used only as a means for incident response (Furth et al., 2003). Ironically, many agencies did have AVL system but still relied on manual check to measure running time and on-time performance whereas other agencies that had APC systems but did not integrate them with AVL systems and radio system still could not provide passengers real-time arrival information.

Some successes in North America, European countries, and Asian countries in implementing real-time bus arrival system are listed in Table 2.1. Some typical applications are detailed in terms of technology and scope.

\section{- NextBus System, United States}

The Nextbus System is well-known, serving 28 agencies over 13 states in the United States. Based on the GPS-based AVL system, satellites and wireless communication, the Nextbus System is capable of predicting real-time arrivals for buses at all bus stops. This information is updated every minute and provided to passengers in 
various ways. At bus stops, real-time arrivals are announced via dynamic message signs (DMSs).

Table 2.1: Transit Agencies with Real-time Bus Arrival Information System

\begin{tabular}{|c|c|c|c|}
\hline Agency Location & AVL system & Description & Website address \\
\hline Nextbus & GPS & $\begin{array}{l}\text { Real-time arrivals via www, palm pilot, } \\
\text { DMS, and internet capable cell phone. }\end{array}$ & www.nextbus.com \\
\hline $\begin{array}{l}\text { Cape cod, RTA, } \\
\text { Dennis, MA }\end{array}$ & GPS & Real-time bus's location via Internet & www.capcodtransit.org \\
\hline $\begin{array}{l}\text { Los Angeles, } \\
\text { LADOT, } \\
\text { LACMTA }\end{array}$ & Signpost & $\begin{array}{l}\text { Real-time arrivals via DMS at Metro } \\
\text { rapid bus stops. }\end{array}$ & www.ladottransit.com \\
\hline $\begin{array}{l}\text { San Louis Obispo } \\
\text { Transit, CA }\end{array}$ & GPS & Real-time bus arrivals at major bus stop & \\
\hline Denver RTD & GPS & $\begin{array}{l}\text { Real-time bus arrival via telephone and } \\
\text { mobile applications }\end{array}$ & www. rtd-denver.com \\
\hline $\begin{array}{l}\text { Seattle, King } \\
\text { County Metro }\end{array}$ & Signpost & $\begin{array}{l}\text { Busview and Mybus programs to } \\
\text { provide real-time information and } \\
\text { others via Internet and mobile phone }\end{array}$ & http://mybus.org \\
\hline $\begin{array}{l}\text { Ohio State } \\
\text { University }\end{array}$ & GPS & $\begin{array}{l}\text { Real-time arrivals via website and } \\
\text { message sign at stops }\end{array}$ & \\
\hline $\begin{array}{c}\text { Tri-Met } \\
\text { ( Portland, OR) }\end{array}$ & GPS & $\begin{array}{l}\text { Real-time arrivals via DMSs and real- } \\
\text { time schedule via website. }\end{array}$ & http://www.trimet.org \\
\hline $\begin{array}{c}\text { Metro transit, } \\
\text { Halifax, Canada } \\
\end{array}$ & GPS & Real-time arrival via telephone & $\begin{array}{l}\text { www.halifax.ca/metrot } \\
\text { ransit/gotime.html }\end{array}$ \\
\hline $\begin{array}{c}\text { TranLink, } \\
\text { Vancouver, } \\
\text { Canada }\end{array}$ & GPS & Real-time arrival on 98-B lines. & www.tranlink.bc.ca \\
\hline $\begin{array}{l}\text { London, United } \\
\text { Kingdom }\end{array}$ & Signpost & $\begin{array}{l}\text { Countdown system provides real-time } \\
\text { bus arrivals at stops via message signs }\end{array}$ & $\frac{\underline{\text { http://www.tfl.gov.uk/ }}}{\text { buses }}$ \\
\hline Helsinki, Finland & $\begin{array}{l}\text { DGPS and } \\
\text { Signpost }\end{array}$ & $\begin{array}{l}\text { PROMISE, provides real-time arrival } \\
\text { via internet and wireless terminals }\end{array}$ & \\
\hline $\begin{array}{l}\text { Magdeburg, } \\
\text { Germany }\end{array}$ & GPS & $\begin{array}{l}\text { PIEPSER, provides passengers the } \\
\text { delay information that may occur. }\end{array}$ & \\
\hline $\begin{array}{l}\text { Brussels, } \\
\text { Belgium }\end{array}$ & GPS & $\begin{array}{l}\text { Phoebus: Real-time arrivals at stop and } \\
\text { Internet }\end{array}$ & $\begin{array}{c}\text { www.stib.irisnet.be/FR } \\
\text { /3600F.html }\end{array}$ \\
\hline Paris, France & GPS & $\begin{array}{l}\text { SNCF -Real-time arrival via media, } \\
\text { internet. }\end{array}$ & \\
\hline $\begin{array}{l}\text { Kaohsiung, } \\
\text { Taiwan }\end{array}$ & GPS & Real-time arrival via Internet & www.mybus.com.tw \\
\hline $\begin{array}{l}\text { Taichung, } \\
\text { Taiwan }\end{array}$ & GPS & Real-time arrival via Internet & www.mybus.com.tw \\
\hline
\end{tabular}

The arrival information is also posted on the internet at http:// www.nextbus.com.

Passengers can access the website, select the bus route, direction and stop in order to 
know the dynamic arrival information. Passengers with Palm Pilot and internet capable cell phones can also get bus arrival information at anytime and anywhere.

\section{- Countdown System, London, United Kingdom:}

Transport for London or London Bus services Limited has a very large number of buses, a total of 5700 buses. The information system, called Countdown, was first piloted in 1992 in an endeavour to provide real-time arrivals for passengers. In 1996, a full scaled AVL system and Countdown Program were approved with an approximately 27 million dollars of budget. To date, 2000 countdown signs have been installed, and 2000 others are expected to be installed by the following years, covering 25 percent of the stops and serving over 60 percent of all passenger journeys. All 5700 buses in the system were equipped with signpost-based AVL system. The buses are localized by signposts in the 5000 signpost-network covering the service area. Each bus location is polled every 30 seconds. The predicted arrivals are disseminated to passengers via internet and DMSs. The information in the forms of audio and visual are in the plan. The Countdown system is well received by passengers (Source: http://www.tfl.gov.uk/buses)

\section{- Tri-County Metropolitan Transportation District of Oregon (Tri-Met)}

Tri-Met can be considered as the pioneer among over 30 agencies in the United States for using collected data from APC and AVL systems for both on-line arrival predictions and off-line performance studies. One of the keys to the success of Tri-Met after years of using the systems was the development of the Bus Dispatching System 
(BDS). Another key was that the AVL-APC interface provided the local referencing of the APC data. Hence, it increased the utilization of data resources for different purposes (Furth et al., 2003). At present, Tri-Met has a real-time bus arrival system called Tri-Met Transit Tracker system that provides real-time arrival information for selected bus routes. Passengers can access the website at http://trimet.org to get bus arrival information. The Transit Tracker project is aimed at deploying initially 250 bus stops that announce realtime arrivals to passengers. The expected rate of implementation is 50 stops per year.

\subsubsection{Bus Running time Prediction Algorithms}

Different arrival prediction models and algorithms have been developed by various transit agencies and universities. The scale and the scope of these models vary, depending upon data needs. While some models solely use data collected from the GPSbased AVL system, others use data collected from the APC systems. Several models require more data than others and additional technologies are used to collect such data (e.g., road conditions, traffic conditions). This subsection describes non-proprietary algorithms and models that have been documented and published.

\subsubsection{Blacksburg (Virginia) Prediction Algorithms}

Four algorithms were developed by Lin and Zen, (1999) to predict bus arrivals. According to the authors, first, the location of the buses and stops are tracked and relayed by satellites and GPS-based AVL system installed on the buses. To match the locations of a bus to the locations of the stops, especially on the loop routes where some stops have 
the same coordinates (e.g., longitude and latitude) but in different distances, a matching module was proposed. Second, four different algorithms were developed based on different data needs: GPS bus location data, GPS bus location data + bus schedule table, GPS bus location + bus schedule table + delay, and GPS bus location + bus schedule table + delay + time check point. In the first two algorithms, the running time of a bus is predicted based on a simple interpolation from the bus schedule table, the scheduled travel time between two major stops, the ratio of the distance between two major stops and the distance between any link pair between these two stops (e.g., from the tracked GPS bus location to a stop or link). Having observed that dwell time at time check stop was the major contributor to schedule adherence, the authors developed the last two algorithms to take delays and dwell time into consideration. Delays in these algorithms are calculated given the assumption that the lateness or earliness at an upstream stop is perceived by bus drivers. These delays may be diminished because drivers tend to adjust the speed to maintain on-time arrivals at check stops.

\subsubsection{Portland (Oregon) and King County Metro, Seattle (Washington) Prediction Algorithms}

The models created by Dailey et al., (2001) and Cathey et al., (2003) have been applied on Mybus System in King County Metro (Seattle), and Tri-Met (Portland). The model includes three components: the Tracker, the Filter, and the Predictor. According to Cathey et al., (2003), the mechanism behind this model can be summarized as follows.

- Tracker: When a request is received regarding the arrival time of a bus at a bus stop, the actual location of that bus is relayed by GPS-based AVL system. To match this location to a particular trip of a bus route, an 
elaborate tracking methodology is used. The core of this methodology is that the original GPS data on bus locations (e.g., longitude, latitude, and time) are converted into distances from these locations to an underlying pattern, called distance into pattern. Therefore, the bus locations are stored and updated easily. This eliminates the ambiguities of differentiating bus directions when a vehicle is operating back and forth on a block over the same roadway.

- Filter: A Kalman filter is applied to estimate the bus dynamic state. The state vector $X$ includes three elements: distance into pattern, speed, and acceleration. At time step $(k-1)$, before the AVL system on the bus sends the new data, the prior bus state at time step $k$ is projected. This estimation is based on the current state at time step $(k-1)$ and a transition matrix. When new AVL data are available at time step $k$, the optimal estimation of the new state (or the posterior projected state) of the bus is calculated based on the prior state and a correction. The correction is an optimal weighting of values done by multiplying a Kalman gain with the difference between the actual measured value of distance into pattern and the best prediction of its value before it is actually measured. These values then will be used to predict the next state of the bus at time step $k+I$ and the procedure is applied until the bus actually arrives at the stop.

- Predictor: Locations of the bus are updated frequently at a predetermined time interval such as at every time point or constant time intervals. 
The arrival is predicted synchronously at each time interval when the new AVL data of the bus become available.

\subsubsection{Texas Transportation Institute Algorithms}

The TransLink Lab at the Texas Transportation Institute developed two algorithms to predict bus arrival: 1-minute zone algorithm, and distance-based model. In the former algorithm, the bus route map is divided into small 1-minute zones based on the historical travel time data. When a current location of bus is detected, the travel time from that location to the predetermined destination is then calculated by counting the number of zones between these pairs. In the later algorithm, the variation in running speeds in various time period of a day (e.g., the class time), and the distance to destination are modelled to reflex the bus travel time (Chien et al., 2004).

\subsubsection{New Jersey University Algorithm}

Chen et al., (2004) created a model to predict bus arrival on the New Jersey Transit bus route 39 . Unlike the previous models discussed above, this model uses data collected from APC system intensively. Having argued that the artificial neural network (ANN) cannot be practically used in real-time bus arrival prediction due to long training time, the authors developed a combination of an ANN and a Kalman filter. First, data collected from APC data and other data sources (e.g., historical running times, distances between stops, weather conditions) were used as the inputs to train the ANN. The training back propagation algorithm and several hidden layers were examined. The well-trained 
ANN then was used for initial predictions of running times between stops, given the road conditions and traffic conditions. To capture the real-time positions of the bus, a Kalman filter-based method was also developed. The values predicted from ANN are used as the initial states of the bus at the first stop. Each time the bus arrives at the next stop, the new location, the actual running time, and the actual arrival time are relayed by AVL and APC systems, and then a Kalman gain loop uses these data ( e.g., the most updated data) for updating the new states of the bus. Therefore, the predictions are adjusted with new state and updated data.

\subsubsection{University of Toronto Algorithms}

Farhan (2002), Shalaby and Farhan (2004), developed models to predict running times and dwell times for bus route 5 in Toronto (Canada). Two Kalman filter procedures, which were first proposed by Reihoundt et al., (1997) for predicting running time and dwell time, have been developed in detail (Shalaby et al. 2004, p. 48). The predictions are made at every timepoint. To predict running time of a current bus on a link, the average historical running time (e.g., 3 days in the past) and the current running time of the previous bus on that link, are used as the initial state of the current bus. Each time the bus comes to a next stop, the state is updated by a Kalman gain and the predictions are adjusted with the most updated data collected from the AVL and APC systems. The same procedure is applied for predicting arrival rate of boarding passengers at every stop. The predicted arrival rate is assumed to be a uniform distribution, so dwell time is the product of that rate and the headway. The model outperformed in arrival 
prediction in comparison with other models (e.g., the ANN-based model and the average based- model).

\subsubsection{Artificial Neural Network (ANN)-based Algorithms}

Several ANN- based models applied in the bus transit field can be found in Chien et al., (2002) and Kalaputapu et al., (1995). Unlike the prediction models discussed so far, which require only AVL data or combined AVL-APC data, these models need different data sources collected from field measurements or simulated from simulators.

Chien et al., (2002) proposed two ANN-based models to predict arrivals: linkbased model, and stop-based model. In the first model, a segment bounded by two bus stops was considered as a series of traversed links. One bus route may have several segments. On each link, the following data were required: volume, speed, delay, average link queue time, passenger demand at stops, and bus travel distance on a link. The ANNs with the variable listed above were trained by the back propagation algorithm. During the training step, several parameters have been examined such as number of hidden layers, learning rate. If there are $M$ links on a segment, $A N N$ will be applied $M$ times to predict bus running time on each link. Therefore, the arrival time at stop $i+1$ is the sum of the departure time at stop $i$ and the total predicted travel times of all $M$ links of that segment. Unlike the first model, the second model integrated the traversed links into one link. Therefore, each segment between a pair of two stops is a link. The data that were used to train the ANN are integrated as the means and standard deviations of speeds, volumes, and delays. This model is simpler than the first one because the trained ANN is used only one time to predict running time on the link. However, it also required more variables 
than that of the link-based model to train the ANNs. There were 9 variables in this model: distance between stops, mean and standard deviation of volumes, mean and standard deviation of link speeds, mean and standard deviation of delays, number of intersections traversed between a pair of stops, passenger demand at stop. On the basis of mean absolute predictor error, both models showed good predictions when they were applied to bus route 39, New Jersey Transit.

\subsubsection{Classical Statistical Regression Models}

These models based on the classical statistic regressions vary from simple multiple linear regressions to non-linear regressions. Besides the data that can be collected by the APC or AVL systems (for example, the stop distances, average running times on the links, the number of passengers getting on or off the bus, the average speeds of bus on links), more data were required such as average delay on the link, traffic volume, densities, the number of left turning vehicles, type of vehicle, traffic signal timing, etc. The models treated bus running time or bus delay as dependent variable and other variables as independent ones.

Alfa et al., (1988) proposed three statistical models to investigate the bus running time for the transit network in the city of Winnipeg, Canada. Many variables were collected and 11 of them were carefully selected in order to get the most influencing variables. After removing the variables which had small correlations with the response variable, only four variables were retained: number of bus stops, number of stop signs, number of traffic lights, and length of the segment. These variables then were used to developed three models, one is multiple linear regression and two others are non-linear 
regression models. The second model was suitable for extrapolation of bus running time while the first model was preferable for interpolation.

Abdelfattah and Khan, (1998) developed six statistical models to capture the variations in bus transit delays under several bus operational scenarios: normal road condition (model 1), bus route in business district area (model 2), bus route including high proportion of heavy vehicles (models 3,4 ), and one-lane blocked route (models 5 \& 6). The data for building these models were measured in the field (the OC Transpo bus route 2, Ottawa, Canada) and the simulated data obtained from TRAF-NETSIM software. Thirteen variables were considered as independent variables and bus delay was used as the dependent variable. To retain the most influencing variable, several stepwise regression procedures in the SPSS software were applied. The variables with the highest influence on bus delays were: densities of left turn and through vehicles, link length, bus efficiency ratio, number of stations, number of stops, and heavy vehicle traffic density per link. The first model was linear multiple regression type and the others were all nonlinear models.

Patnaik et al., (2004) proposed a statistical regression based model using APC data. The APC database was split into two parts: 80 percent database was used for developing multiple regression models and the remaining for testing the developed models. Eleven variables collected from the APC system were used as the inputs and two models were developed using the stepwise regression method. 


\subsubsection{Other Prediction Models}

Other prediction models applied for bus arrival were found in the literature and discussed briefly as follows:

- Simulation model: Chien et al., (2000) proposed a model to predict bus arrival based on simulation. Although the enhancements developed by the authors for CORSIM (CORridor SIMulation) software were intended to increase the capability of CORSIM to capture the waiting passenger, these also were used to estimate stop-to-stop travel time. Based on the proposed model, bus schedule of each bus route was specified by users and used as input. Then the simulator generated the buses on the network based on input schedule. Therefore, given simulated road conditions, passengers, and actual bus schedule, the enhanced CORSIM could be used to predict arrival times.

- Non-real-time $A V L$ data model: Having recognized that historical AVL data are capable of providing estimated bus running time, Horbury (1999) proposed some models to estimate moving time, bus speed and dwell time. The signpost-based AVL system data collected from route 18 in London (England) were decomposed into moving time and dwell time at stop. Moving time for a trip from the origin to the destination was calculated by subtracting the total travel time and the dwell times at stops. To calculate the dwell time, boarding and alighting time models were constructed from the survey data. These models have simple linear regression forms. As a result, the moving times can be obtained. Because 
the models only used the AVL historical data, it is suitable for off-line estimations.

Bus stop-to-stop line model: Son et al., (2004) developed a real-time prediction model to project both the travel times from a bus stop to a stop line at an intersection, and from stop line to a downstream bus stop. The arrival time at the first stop and the location of the bus were recorded by the AVL system mounted on the bus ITS devices. Two Kalman filters were used to predict these travel times. The predicted arrival time at the second stop is the sum of the two predicted travel times plus the waiting times at the signal.

GPS map-based model: Weigang et al., (2004) developed models for estimating bus arrival time and bus speeds based on the GIS map. The mechanism behind the models is that the bus route is divided into a number of short, straight line sub-routes. The location of bus relayed by GPS system then is matched to find the bus position in the GIS map and an algorithm was developed to make an accurate prediction for the distance from the tracked bus position. If bus speed information is available, then the running time is simply projected by dividing the distance by the speed. 


\subsubsection{Bus Dwell time Prediction Models}

Most of the models developed in the past considered dwell time as a delay and explored the influencing factors on dwell time. Prior to the applications of the APC system to bus transit, because of high cost and labor-power in data collection, studies at that time focused mostly on the relationship between dwell time and the number of passengers boarding and alighting which were counted manually at point checks.

Back in 1974, Kraft and Berger found that the time for serving one boarding passenger is about 4.5 seconds and dwell time for boarding is 2 seconds plus time for serving M boarding passengers (Kraft et al., 1974).

Having studied dwell time and passenger activities in Lafayette, Indiana (USA), Guenther et al., (1983) generated some conclusions about dwell time:

The number of passengers boarding and alighting at each stop follows Poisson distribution only when they are relatively low.

- Negative binomial distribution is acceptable as a descriptor of boarding and alighting passengers under all volume conditions.

The assumption that dwell time per passenger is independent of the number of passengers boarding and alighting may be erroneous.

The relationship between average dwell time per passenger and the total number of boarding and alighting passengers at a bus stop was found as $\varepsilon=5.0-1.2[\ln (z)]$

Where: $z$ is the number of boarders and alighters at a stop 
Another model developed for bus dwell time estimation can be found in the works of Senevirate (1988). The author found that the Gamma distribution fits data of boarding and alighting time distribution better than the Normal and the log-normal distributions did.

In the early 1990s, the equations relating dwell time to the number of passengers as well as other data collected by the APC and AVL systems such as, fare box type, number of vehicle's doors, lifting operations, bus schedule adherence performances, bus locations, and bus speed were first developed.

Marshall et al., (1990) developed six regression models (four linear and two exponential regressions) to relate dwell time to the number of passengers, the fare collection methods (coins, bill) and the bus induced-delay. The authors concluded that exponential regressions were suitable and did not create negative dwell time when no boarding and alighting passenger are recorded.

Levine et al., (1994) considered dwell time to consist of five components (boarding and alighting time, waiting time for other passenger activities, waiting time by buses that advanced ahead of schedule, waiting time for re-entry to traffic stream, and time for attending equipment). The Levine's models were mainly devoted to low-floorbus boarding time. The findings showed that a low-floor bus saves on the average 17.5 sec per full bus per stop, $0.16 \mathrm{sec}$ per passenger with unimpeded movement and $5.78 \mathrm{sec}$ per passenger using mobility aids.

The Highway Capacity Manual (HCM) 2000 suggested a formula for the calculation of dwell time as follows

$$
D=\left(P_{a}\right)\left(t_{a}\right)+\left(P_{b}\right)\left(t_{b}\right)+t_{o p}
$$


where: $\quad D=$ dwell time in seconds

$P_{a}=$ Number of alighting passengers through the busiest door

$t_{a}=$ Passenger alighting time per passenger

$P_{b}=$ Number of boarding passengers through the busiest door

$t_{b}=$ Passenger boarding time per passenger

$t_{o p}=$ Door opening and closing time

Rajbhandari et al., (2003) used statistical regressions to generate four models for dwell time as a function of the number of boarding and alighting passengers, and the number of standees. Like other authors, Rajbhandari found that the dwell time has a nonlinear relation with the number of passengers as shown below

$$
D T=a+b(\text { Total })+c(\text { Total })(S)
$$

where: $a, b, c=$ are regression parameters

Total $=$ Number of boarding and alighting passengers

$\mathrm{S}=$ Number of standees

$\mathrm{DT}=$ Dwell time.

Dueker et al., (2004) collected data by using the APC system in TriMet transit system for developing multiple regression models. The determinants of dwell time include passenger activity, lift operation and other effects.

Bertini and Geneidy, (2004) also used data from TriMet to model the dwell time. The data were 452 stop samples of 14 trips of bus route 14 . The authors found that dwell time and passenger activities are related as follows: 


$$
\text { Dwell time }(s)=5.8+0.85 N_{a}+3.6 N_{b}
$$

Where: $N_{a}, N_{b}$ is the number of boarding and alighting passengers, respectively

Shalaby et al., (2004) proposed a Kalman filter to predict the passenger arrival rate based on the historical data of the number of passengers arriving at a stop. Once the passenger arrival rate has been predicted, it is assumed to be constant during the headway. Thus, the predicted number of boarding passengers is the product of the headway and the predicted passenger arrival rate. Dwell time is then calculated by multiplying the predicted boarding passengers and an average serving time per boarder.

Fang and Min (2005) generated a model to estimate the dwell time. First, the author surveyed the distribution of passengers boarding and alighting times via front door and the rear doors. Second, they developed several probabilistic distributions (e.g., lognormal, Gamma, and Weibull) to fit the frequency distribution. The probability of the service time duration for both alighting and boarding is predicted by using Monte-Carlo simulation.

\subsubsection{Media and Communication System}

Media and communication system play an important role in the success of a RETBAIS. Real-time arrivals will be useless if they cannot be disseminated immediately to passengers. A bus fleet well-equipped with APC and AVL systems will not work efficiently if the data collected cannot be transmitted sufficiently and dynamically to the control center. This subject is discussed in two parts: information dissemination media for transit users and communication system for transit providers. 


\subsubsection{Information Dissemination Media}

Printed medium is the simplest way transit agencies provide information to passengers. Besides this traditional method, passengers can access the telephone system to get traveller information. The innovations in information technologies now enable transit agencies to have various media to distribute information to passengers. Currently, passengers can get transit information from cell phones, PDAs, pagers, Palm Pilots, internet capable mobile phones, kiosks, video monitors, DMSs, and the Internet.

The DMSs and video monitors are prevalent at bus stops. The DMSs and Video monitors are non-interactive displays where passengers can get only en-route or invehicle information if such devices are installed in the buses. The information shown on the electronic signs usually includes: time and date, route number and destination, waiting time (countdown or time range), and service interruption. Kiosk, internet capable cell phones, and the Internet allow passengers to get information actively. Therefore, passengers can get all kind of information they need (pre-trip information, en-route and in-vehicle information).

Providing information via Palm pilots, pagers, PDAs and cell phones is a preferable method that transit agencies aim at because such devices are personal and wireless. Therefore, they enable passengers to get real-time information anytime and anywhere at low cost.

Some current deployments of dissemination media are listed in Table 2.1. (Further details can be seen in TCRP Report 92, 2003). 


\subsubsection{Communication System}

Most of agencies use radio system to transmit data. As the demand for real-time information is increasing, the requirements for data transmission are on the rise. This can cause "overcrowding of spectrum" placed on the available radio channels dedicated for the AVL system. Therefore, the agencies are looking for the alternative solutions such as using "spread spectrum", narrowing radio communication band, renting commercial radio service, using infrared or high capacity frequency link (John, 1999).

\subsection{Summary}

In many transit agencies, AVL and APC have long been used for increasing operational efficiency. However, it is in recent years that the agencies have recognized the benefits of applying AVL, APC and other advanced technologies for the provision of passenger real-time information.

The use of collected APC data for off-line analysis has been the practice in transit agencies, but the AVL system was not considered as an objective. Many agencies installed AVL systems for their bus fleet, but cannot provide real-time arrival information to passengers. They use the AVL system only for incident response while they use manual check for on-time performance estimation and running time analysis. The APC systems have been used for off-line studies only in spite of the fact that these can provide valuable data for on-line applications. 
Bus arrival prediction models reported in literature very seldom use the collected APC data for real-time prediction while it is known that an integration of APC and AVL system with radio systems can meet the real-time information requirements. Almost all of the prediction models are based on the GPS-based AVL data and other diverse sources of data.

None of the arrival prediction models based on AVL data predict dwell times and include them in the total predicted trip time. This may cause inaccurate predictions of arrival for downstream stops when the bus serves crowded upstream stops. 


\section{Chapter 3}

\section{MECHANISM OF THE PROPOSED MODEL AND AVL-APC DATA COLLECTION}

\subsection{Introduction}

Following the examination of the previous works involved in real-time bus running time and dwell predictions, this chapter presents the framework of the developed model for real-time bus arrival predictions and its building blocks. This chapter is organized as follows: First, the components of a bus trip time are investigated in order to emphasize the roles of running time and dwell time in bus trip time analyses and predictions. Second, various modules are described and their integration into the complete model is covered. Last, the study design including the process of APC and AVL data collection and data descriptions are discussed.

\subsection{The Components of Bus Trip Time and Influencing Factors}

To date, many models and algorithms have been developed to investigate the factors that influence bus running time and delay. The most influencing factors found in studies are shown in Table 3.1. The identified factors influence different components of the bus trip time. Therefore, in analyzing and estimating accurately the components of the 
bus trip time, causal factors should be studied, given that their effects will ultimately influence actual bus operation on the route.

Table 3.1: Factors that influence Bus Running Time and Delay

\begin{tabular}{|l|l|}
\hline \multicolumn{1}{|c|}{ Studies } & \multicolumn{1}{c|}{ Factors } \\
\hline Afdail et al., (1988) & - Number of bus stops \\
Abdelfattah and Khan (1998) & - Link length \\
& - Number of traffic lights \\
& - Densities, left turning and through \\
& vehicles \\
& mixed-traffic stream \\
\hline Abkowitz and Engelstein (1984) & - The number of passengers boarding \\
& or alighting at stops. \\
\hline Senevirante (1988) & - Dwell time \\
Patnaik et al., (2004) & - Service characteristics \\
\hline McKnight et al.,(2003) & - Traffic congestion \\
\hline
\end{tabular}

According to Maloney and Boyle (1999), a bus trip time includes the following components: (1) actual moving times between stops; (2) dwell time at stops; (3) traffic signal delay; (4) general traffic delays, and (5) recovery time at the end of each trip.

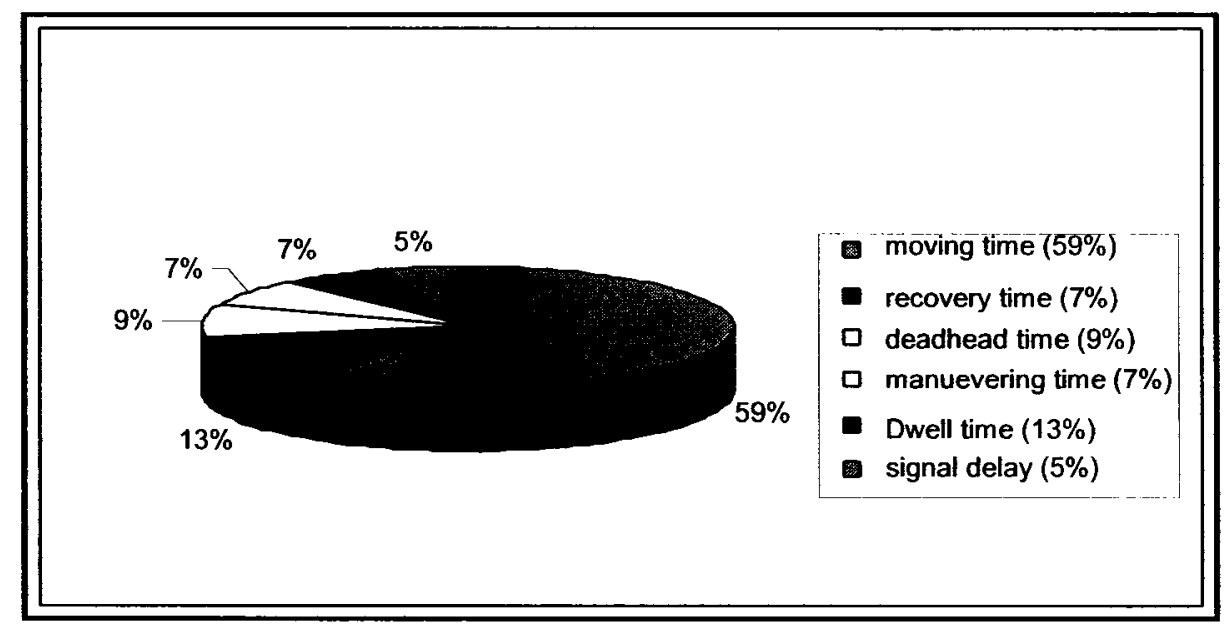

Figure 3.1: Bus Trip Time Components (Modified from Manoley et al., 1999) 
Maloney and Boyle (1999) showed that actual moving time between stops is the primary component accounting for overall 59 percent of total trip time, followed by recovery time $(13 \%)$, deadhead time $(9 \%)$, time maneuvering to access and leave the stop (7\%), dwell time (7\%), and signal delay (5\%) (see Figure 3.1). The following paragraphs briefly discuss each component of the bus trip time.

\subsubsection{Actual Moving Time}

Actual moving time of a bus is the time that the bus is in motion. It is heavily influenced by three main factors: schedule, distance between stops, and speed of the bus.

A short schedule will force bus drivers to speed up whenever possible and rush to the stops. Therefore, a short schedule may shorten the moving time of the bus in some cases, but may raise safety concerns on the road and put pressure on the driver and the dispatcher to keep the schedule adherence. In contrast, a long schedule may lead the bus to come to the stops earlier than scheduled time. If the arrivals are consistently early, the moving times may be increased intentionally by operators (i.e., to slow down the bus). As a result, passengers will spend more time in underutilized buses (Senevirante, 1990).

The spaces between bus stops also influence moving time. A short distance does not permit the attainable cruise speed of buses since the available distance between stops is used for deceleration and acceleration operations. On the other hand, Levison (1983) pointed out that the fewer the bus stops, the greater the number of passengers waiting at downstream stop. Therefore, elimination of some stops will enhance average speed but can increase access time for passengers. However, Levison found that on the basis of 
statistical analysis, reducing the number of stops did not influence the average speed to a high degree given the fact of traffic signals.

The speed of the bus is an important factor that influences the bus movement time. However, speed is affected by many factors. Traffic densities and congestions are the major causes of low speeds of buses running in mixed traffic bus routes in the downtown area. For instant, the kilometers travelled by a bus in one hour decreases significantly from $22.5 \mathrm{~km} / \mathrm{h}$ in suburban area to $16 \mathrm{~km} / \mathrm{h}$ in the city, and $8 \mathrm{~km} / \mathrm{h}$ in the central business district (CBD) (Levinson, 1983). The speed of bus during the rush hour periods was 1.4 to 1.6 times as slow as that of the car (Levinson, 1983) or equal to $42-59$ percent of car's speeds (McKnight et al., 1997). In a manner similar to the effect of the number of bus stops, the number of traffic signals also reduces the speed of the buses when they approach and leave the traffic signals.

\subsubsection{Dwell Time}

Dwell time is the time required for the bus at stop to serve at the busiest door plus time for closing and opening door (HCM 2000). Major factors that affect dwell time found by many studies are the number of boarding and alighting passengers, bus stop spacing, fare collection method, type of bus, and type of on-board circulation. However, the relationship between dwell time and the number of boarding and alighting passengers was considered by many researchers to be sufficient for their purposes. Many models have been developed to investigate this relationship. They vary from simple descriptive statistical models (Bertini et al., 2004; Rajat et al., 2003; Lin and Wilson, 1992), to probabilistic models such as Poisson model (Chien et al., 2000), Gamma model 
(Senevirante, 1988), natural logarithm model (Guenthner et al., 1983), and exponential model (Leo et al., 1990). The previous works on dwell time shows another trend, namely to project dwell time based on the relationships between dwell time and the bus service characteristics. The models belonging to this trend can be found in following publications (Kraft et al., 1974; Kostopolous et al., 1985; Dueker et al., 2004). These models are the multiple regressions where dwell time is the dependent variable and types of fare collection, number of doors, and on time performance are used as independent variables.

\subsubsection{Traffic Signal Delay}

Traffic signal delay accounts for a large proportion (i.e., up to $32-45$ percent) of total delay (Levison, 1986). Most of this delay is due to waiting and queuing at signalized intersections. In the Highway Capacity Manual 2000 (TRB 2000), a bus in a traffic flow is converted into equivalent passenger car units so that an average delay per queuing vehicle is calculated. This value can be considered as the delay of a bus at a signalized intersection.

\subsubsection{General Delay}

General delay refers to delays caused by traffic congestion, crowded bus berths, and lane changing maneuvers. It is fair to say that bus travel time delay is not only caused by ordinary vehicles sharing the lane with buses but also by the buses themselves. Slow and large buses in heavy traffic stream, like other heavy vehicles, cause car drivers to avoid travelling in the same lane and consequently result in intricate lane changing 
maneuvers. Hence, this characteristic of traffic flow increases delay. According to Ubitran et al., (1986), traffic congestion has a severe adverse impact on bus travel time. So far, very few studies have been found in the literature that examined the direct impacts of congestion on bus travel time. Currently, MacKnigh et al., (2003) developed models to quantify the impacts of congestion on bus travel time in their studies of 39 bus routes in Northern New Jersey (USA). The following is such a model:

$$
d B T T_{r_{-} f f}=0.73\left(T T T_{r_{-} a}-T T T_{r_{-} f f}\right)
$$

Where:

$d B T T_{r_{f f}}=$ Change in travel time rate (minutes per mile) due to congestion for buses on route

$T T T_{r_{-} a}=$ Travel time rate for traffic under existing conditions

$T T T_{r_{-f f}}=$ Travel time rate for traffic under free flow conditions

$0.73=$ Statistical parameter resulted from calibration study

To quickly estimate the actual travel time rate, travel time rate indices (TRIs), which are the extra amounts of time (in terms of minutes/mile) for travel over a link, were used.

$$
T R I_{s}=T T T_{r_{-} a} / T T T_{r_{-f f}} \text { or } T T T_{r_{-} a}=T R I s . T T T_{r_{f f f}}
$$

From Equations 3.1 \& 3.2, bus travel time increment due to congestion is as below.

$$
d B T T_{r_{-} f}=0.73 T T T_{r_{-f f}}\left(T R I_{s}-1\right)
$$




\subsubsection{Recovery Time}

Recovery time is the required time for layover of a bus driver at the end of each trip. The length of recovery time is based on the labor agreement with bus drivers and it is usually equal to at least 10 percent of a round trip time (Pile et al., 1998).

Obviously, if buses are running on the exclusive bus route or on the Transitway, many factors influencing the travel time components under mixed operations are eliminated. In contrast, if buses are running in the mixed-traffic bus lanes where other vehicles share the same lane with buses, the buses are vulnerable to delays caused by the many frictions such as traffic volume, incidents, curb parking operations, etc. For this reason, predicting the influences on the components of a bus trip in mixed traffic bus route will continue to be a challenge. For information, it should be noted that mixedtraffic bus operation accounts for almost 99 percent of total bus route distances in North America (Kittelson and Associates Inc., 2003).

\subsection{Proposed Model Structures and Components}

\subsubsection{General Discussion}

As discussed above, bus travel time is complex and is influenced by many stochastic factors. Hence, to predict bus travel time, one should consider all bus trip time components. The recovery time component is an exception which does not influence the accuracy of bus arrival time predictions. Other identified components play a role in the success of a bus arrival prediction model. If any of these components is not adequately 
modelled, one may get poor predictions of bus arrivals, especially when the bus operates in the mixed traffic route during rush hours.

The models reported in literature can be divided into three groups. In the first group are the models which aggregate all components (e.g., actual moving time, general delays, traffic signal delays, and dwell times) into one variable called running time and predict its value. These models can be found in the works of Chen et al. (2004), Chien et al. (2002), Abdelfattah et al. (1998), and Kalaputapu et al. (1995). The second group includes the models that integrate moving time, general delays and either traffic signal or dwell time into the running time. The bus arrival prediction is the sum of that running time and estimated traffic signal delays or dwell time at stops. Please refer to the works of Shalaby et al. (2004), Son et al. (2004), Lin and Zen (1999), and Horbury (1999) for further details of these models. The last group includes the models that take into account only one main component of bus travel time, the actual moving time. These models are based only on the data of the actual location of the bus provided by the GPS-based AVL system and make the prediction of a new location of the bus in the next time step. Some of these models can be found in the publications of Dailey et al. (2001), Cathey et al. (2003), and Weigang et al. (2004).

Each group has drawbacks and advantages. The models in the first group do not require as elaborate data as required by the second group. However, because of the aggregation, these models cannot capture the effects of one component on another. For example, a long moving time can cause a crowded downstream stop(s) and this, in turn, results in a long dwell time contributing to a late arrival at the following stops. On the contrary, the models in the second group which separate dwell time as an independent 
component can differentiate the effects. Consequently, they are more flexible than the first ones. Because of their flexibility, such models may be applicable for both arrival time prediction and dynamic bus control actions. The models in the last group were advanced for tracking the dynamic bus locations. However, based only on the locations of the bus and ignoring dwell time and other components of a bus trip time, the accuracy of these models is good for an arrival prediction of one stop ahead but it may deteriorate at the downstream stops after that stop.

The model proposed in this study takes into account the advantages of different models aforementioned. In order to use data collectable by APC and AVL systems, moving times, traffic signal delays and general delays are integrated into running times while dwell time is treated separately. The proposed model has three modules. The first module is for predicting the running time, the second module is for predicting dwell time, and the last module is for model application. By separating dwell time and running time prediction, the proposed model can capture the effects of running time on dwell time and vice versa. Also, basing on the latest updates of the dynamic locations of the bus, each module will adjust its predictions at every time step. Therefore, the whole model can be applied for real-time predictions.

\subsubsection{Assumptions}

The following assumptions are made in this research study:

- A bus route under consideration is composed of links and bus stops/timepoints where a bus link is a route section whose two ends are two bus stops or timepoints.

- All buses in the bus route are equipped with APC, AVL and radio systems. 
- Each time a bus arrives at and departs from a stop, the following data are recorded and transmitted dynamically to the control center: location of the bus at this stop, the bus trip ID, the times the bus arrives and departs the stop, the number of passengers boarding and alighting at the stop, and the running time the bus travelled on the previous link.

Figure 3.2 presents a simplified sketch of bus operation on a route.

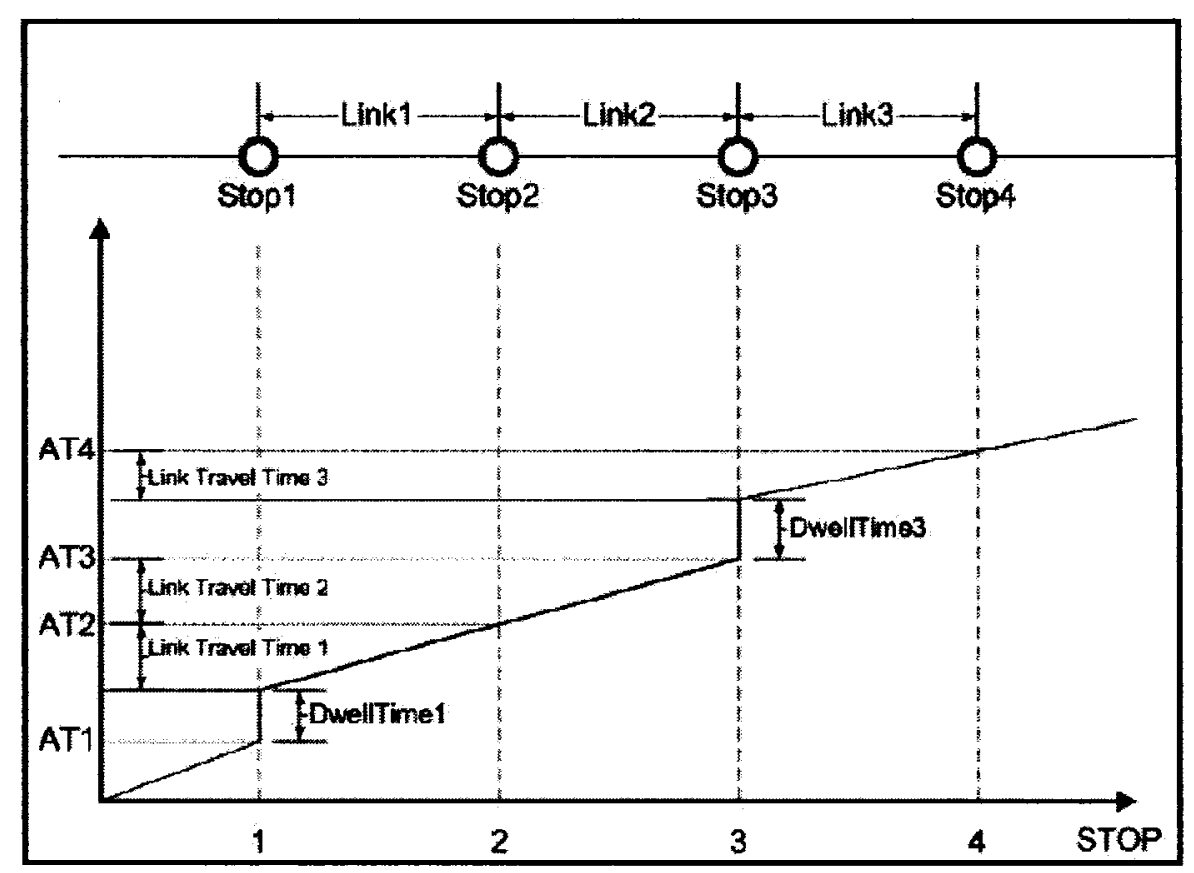

Figure 3.2: A Simplified Sketch of Bus Operation on a route

\subsubsection{Structure and the Building Modules of the Proposed Model}

As mentioned above, to take advantages of the previous models, the proposed model for bus arrival time prediction in this study is the combination of the three modules. The first module predicts running times and is therefore called Running Time 
prediction Module (RTM). The second module predicts dwell times, and it is called Dwell Time prediction Module (DTM). The last module predicts bus punctuality and bunching, and it is called Punctuality and Bunching prediction Module (PBM).

Running times in a bus link are predicted by the first module (RTM) in the following manner. Given a current running time situation, the historical data of running times of each link are used as a basis to predict running times. Each time the bus comes to a stop or when the data collected by APC and AVL become available, the most updated data are used to predict new bus running times. These data are also stored to update historical data. The running time prediction module is described in details in chapter 4 and 6.

Dwell time and passenger occupancy prediction module (DTM) is based on the passenger activities recorded at the stops (i.e., both historical data and the current data). The proposed dwell time prediction module is presented in chapter 5 and 6 .

The punctuality and bunching prediction module (PBM) is described in chapter 7 . In this module, a method to estimate prediction interval is to be examined. Based on this method and by using probability theory, two algorithms are developed for bus bunching and bus on-time detection.

In general, to predict bus arrival/departure time at stop $i+1$, given the recorded departure time of the bus at previous stop (i) denoted as $D p_{\text {actual, }}$, , the first two modules (i.e. RTM and DTM) are connected together by two simple general equations:

$$
\begin{gathered}
A r_{i+1}=D p_{\text {actual, } i}+r_{i_{-} i+1} \\
D p_{i+1}=A r_{i+1}+d w e l l_{i+1}
\end{gathered}
$$

Where: 
$A r_{i+1}$ is the predicted arrival time at stop $i+1$

$D p_{i+1}$ is the predicted departure time at stop $i+1$

$D p_{a c t u a l, i}$ is the actual departure time of the bus at stop $i$

$r_{i_{-} i+1}=$ predicted running time the bus will spend on the link between two stops, obtained by RTM.

$d w e l l_{i+1}=$ predicted dwell time of the bus at stop $i+1$, obtained by DTM

Once the arrival prediction has been made for a bus stop, this value will be compared to the scheduled arrival time that the bus is supposed to be at that stop. This predicted time difference will indicate if the bus is likely to be early, on-time, or late. Based on this information and other predicted variables, the PBM will provide the best suggestions to bus dispatcher in support of decision making.

\subsubsection{The Mechanism of The Proposed Model}

Figure 3.3 shows the process of real-time prediction of the proposed model. For detailed descriptions, let us assume that the bus route under consideration has $n$ bus stops denoted as $S T O P_{i}$ (where $\mathrm{i}=1$ to $n$ ) and bus $k$ has just arrived at bus stop $S T O P_{i}$. We call the actual time that bus arrived at stop $i$ as $A r_{\text {actual, } i}$ and the actual time the bus left the

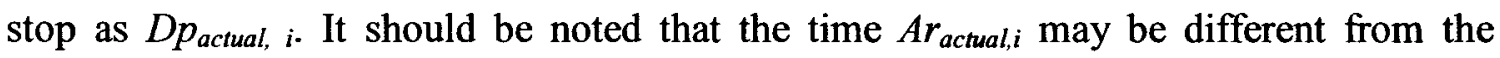
scheduled time $S c_{i}$ of bus $k$ at stop $i$ due to travel time variations. Let us denote $A r_{j}$ and $D p_{j} \quad$ as the predicted arrival time and departure time of bus $k$ at a downstream stop $j$ (where $j=i+1$ to $n$ ). 


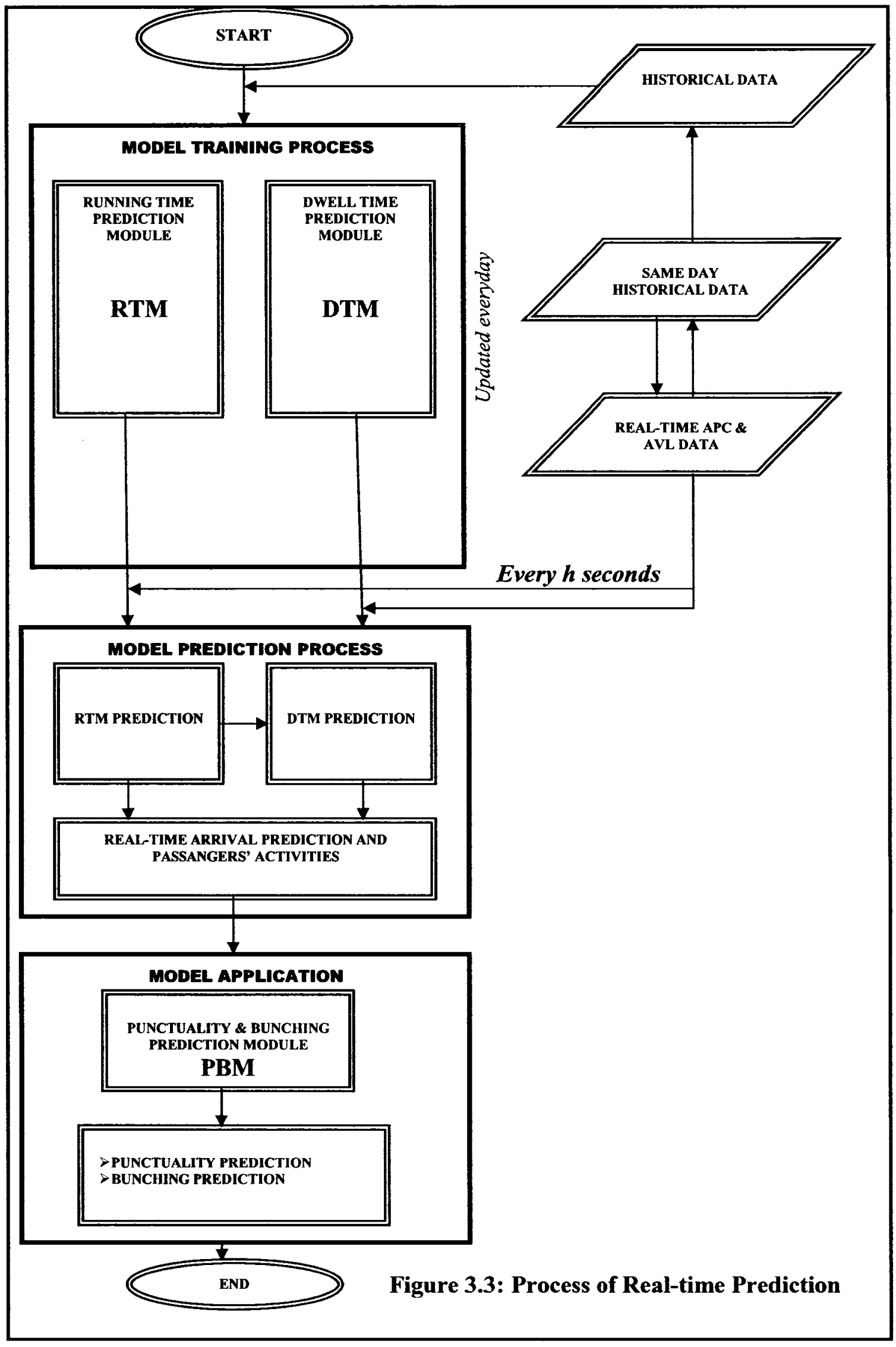


To predict the arrival times of bus $k$ at downstream stops (e.g., $i+l$, $i+2 \ldots \mathrm{n})$, the following steps are applied.

\section{Initial Arrival Predictions:}

- Running time module will predict running times of bus $k$ on all downstream links (i.e., $\operatorname{LINK}_{i_{-} i+1}, \operatorname{LINK}_{i+1_{-} i+2} \ldots L I N K_{n-I_{-} n}$ ). The predicted running times are coded as $r_{i_{-} i+1}, r_{i+I_{-} i+2} \ldots r_{n-I_{-} n}$.

- Dwell time module will predict dwell times of bus $k$ at all downstream

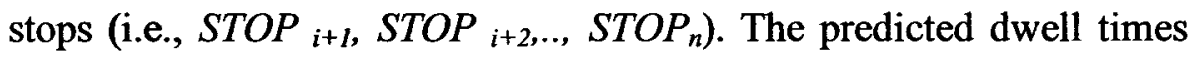
are denoted as: $\quad d$ well $_{i+1}, d$ well $_{i+2} \ldots, d$ well $_{n}$.

- Because the actual departure time at stop $i$ was recorded ( $\left.D p_{\text {actual, }}\right)$, the predicted arrival of bus $k$ at the next stop $(i+1)$ is

$$
A r_{i+1}=D p_{a c t u a l, i}+r_{i-i+1}
$$

- The predicted arrival time of bus $k$ at stop $i+2$ then will be calculated

$$
\begin{aligned}
& A r_{i+2}=D p_{i+1}+r_{i+1} i+2 . \\
& D p_{i+1}=A r_{i+1}+d w e l_{i+I} .
\end{aligned}
$$

Substitute $D p_{i+1}$ of Equation 3.8 into Equation 3.7, so we have:

$$
A r_{i+2}=A r_{i+1}+d w e l l_{i+1}+r_{i+1}{ }^{i+2}
$$

Substitute $A r_{i+l}$ of Equation 3.6 into Equation 3.9, so we have:

$$
A r_{i+2}=D p_{a c t u a l, i}+r_{i_{-} i+1}+d \text { well }_{i+1}+r_{i+I_{-} i+2}
$$

- The same mathematical operation is applied for all other stops. For example, the predicted arrival time of bus $k$ at stop $i+3$ will be calculated as: 


$$
\begin{aligned}
& A r_{i+3}=D p_{i+2}+r_{i+2 \_}{ }_{-i+3} \\
& D p_{i+2}=A r_{i+2}+d w_{e l l} i_{i+2}
\end{aligned}
$$

Substitute $D p_{i+2}$ of Equation 3.12 into Equation 3.11. We have:

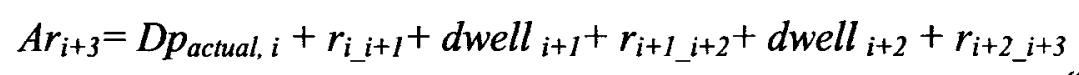

Hence, we have initial predicted arrivals of bus $k$ at all downstream stops even when bus $k$ is currently at an upstream stop.

\section{Update Arrival Predictions}

The predicted travel time to each down stream stop will be updated when the most recent bus arrival information becomes available at every $h$ seconds. For example, as the bus departs from stop 1, the running time prediction module, together with dwell time prediction module, predicts arrival times from first stop to all downstream stops in the fashion as mentioned above. By the time the bus arrives at stop 2, new updated arrival information, running times and passenger activities of the buses on the bus route have already been collected by the AVL and APC systems. These data then will be used by the two modules to make new predictions of running times and dwell times. As a result, the arrival predictions will be updated.

In general, when bus $k$ comes to the next stop (e.g., stop $i+1$ ), the new

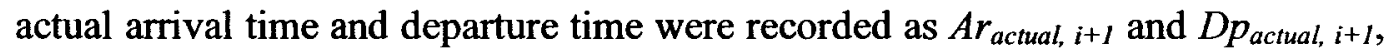
respectively. New updated arrival predictions then will be calculated at time step $D p_{a c t u a l, ~}^{i+1}$ as follows: 
- Running time module will use updated information to predict new running times of bus $k$ on all downstream links after stop $i+1$ (e.g., $\left.\operatorname{LINK}_{i+1 \_i+2,} \operatorname{LINK}_{i+2 \_i+3} \ldots, L I N K_{n-1 \_n}\right)$. The new predicted running times are coded as $r^{(l)}{ }_{i+1} i+2, r^{(l)}{ }_{i+2}{ }_{i+3} \ldots, r_{n-1}^{(l)} n$. The superscripts attached with each variables indicates the number of times the predicted running times have been updated. In this case, the running times have been updated for the first time.

- Dwell time module will use the most updated information to predict dwell times of bus k at all downstream stops (e.g., STOP ${ }_{i+2}, S T O P_{i+3} \ldots, S T O P_{n}$ ). The new predicted dwell times are denoted as: $\quad d w e l l{ }_{i+2}^{(l)}, \ldots, d w e l l^{(l)}{ }_{n}$. Like running time variables, the superscripts indicate the number of times that dwell times have been updated.

- New updated arrival prediction at stop $(i+2)$ will be calculated as follows $A r^{(l)}{ }_{i+2}=D p_{\text {actual }, i+1}+r^{(1)}{ }_{i+1} i+2$

The same mathematical operation will be applied to update the arrival predictions for all downstream stops. For example, the updated arrival time of bus $k$ at stop $i+3$ will be as follows:

$$
\begin{aligned}
& A r^{(l)}{ }_{i+3}=D p^{(l)}{ }_{i+2}+r^{(l)}{ }_{i+2}{ }_{-}{ }^{i+3} \\
& D p^{(l)}{ }_{i+2}=A r^{(l)}{ }_{i+2}+d \text { ell }^{(l)}{ }_{i+2}
\end{aligned}
$$

Substituting Equations 3.14 and 3.16 into Equation 3.15 gives

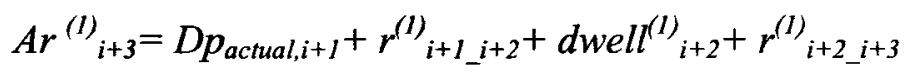


- The same procedures will be applied every time the bus actually comes to a downstream stop (e.g., stop $i+2, i+3 \ldots, n$ ). For example, the prediction on arrival time of bus $k$ at stop $(i+3)$ is updated when bus $k$ arrives at next stop (i.e. stop $i+2$ ) as shown below.

$$
A r^{(2)}{ }_{i+3}=D p_{\text {actual, } i+2}+r^{(2)}{ }_{i+2} i+3
$$

If we compare the first updated value of predicted arrival time at stop $(i+3)$ (Eq. 3.17), and that of the initial prediction (Eq.3.13), we can see that the new value is updated based on the two most recent updated information. First, it is based on the actual departure time recorded at new stop $D p_{\text {actual },+1 .}$. Therefore, the model can capture the adverse effects of an incident that may cause a late departure at stop $(i+1)$ when the bus was running in the $L I N K_{i-i+l}$. Second, it is based on the first time updated running times

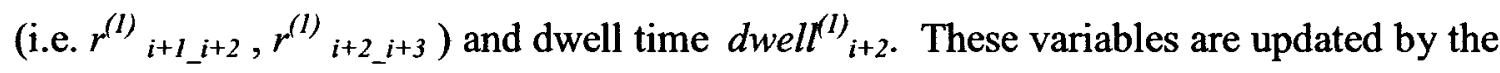
RTM and DTM, given the newest data collected from the APC and AVL systems. Once again, if we compare the first updated arrival time at stop $(i+3)$ in Equation 3.17 and the second updated one in Equation 3.18, we can see the second updated arrival based on the new actual departure time at stop $D p_{a c t u a l, i+2}$ and the second updated running time $r^{(2)}{ }_{i+2}{ }^{i+3}$. Hence, the model can capture the dynamic changes in bus travel times.

\section{Interpolate Arrival times at Skipped Stops}

Following the mechanism presented above we can see that, at each time step, or in other words, at each time the bus left an upstream stop, say $D p_{a c t u a l, i}$, the predictions of arrivals of this bus at all following stops will be made based on the actual recorded time 
$D p_{a c t u a l, i}$, the predicted running times $r_{j}$ and the predicted dwell times $d w e l l_{j}(j=i+1$ to n). A question can be asked as to how the proposed model predicts the arrivals at downstream stops when the bus comes to an upstream stop without stopping due to serving requirements? In practice, if the bus does not stop at a bus stop, neither location of the bus stop nor passenger activities at this stop are recorded. In this case, dwell time is definitely zero but the arrival time is not known. In case of a bus equipped with GPS-based AVL system, the interpolated time that the bus approaches the stop can be considered as the GPS time recorded when the bus was closest to this stop. However, if the bus did not have a GPS-based AVL system, (e.g., the bus has another AVL technology such as S/O-based AVL or DR-based AVL), the arrival time of the bus can be estimated as follows:

- Assume three bus stops A, B, and C and that bus $k$ passed stop B without stopping.

- The previous buses had the running times in $L I N K_{A_{-} B}$ are $r(k-d)$ (where $d=1,2$..). Approximately, the interpolated arrival of bus $\mathrm{k}$ will be

$\operatorname{Ar}(k)_{\text {interpolated, } B}=D p(k)_{\text {actual, } A}+r(k)_{\text {Average } A_{-} B}$

Where: $r(k)_{A \text { verage } A_{-} B}$ is the average of the same-day historical running times of the previous buses running on the $\operatorname{LINK}_{A_{-} B}$ in one hour period.

\subsection{APC and AVL Data Collection}

Based on how the proposed model works (i.e. its mechanism) and its building elements, a study design containing the processes of data collection and data reduction was developed. It includes: data collection, data description, and data reduction. 


\subsubsection{Bus Route Selection}

In order to develop a model capable of predicting real-time bus arrival under different operational scenarios such as buses operating during off-peak and peak-hour, on mixed traffic route or on exclusive bus lane, the selected bus routes have to cover these situations. In this study, data retrieved from APC and AVL systems of the OC Transpo were used. Two bus routes were selected as the study subjects; route 95 (Orleans-Fallow Field) and bus route 1 (South Keys-Ottawa Rockcliffe). The reasons behind the selections are:

- Bus route 95 is one of the three major bus routes in Ottawa (i.e. route 95 , route 96 and route 97 ) and it operates 22 hours a day from 4:00 a.m. to 2:30 a.m. Almost all of the buses $(95 \%)$ operating on this route are articulated buses.

- Almost all of the segments of route 95 are on the Transitway and the others are designated as exclusive bus lanes located on highway 417, Albert and Salter streets.

- Route 1 is a mixed-traffic bus route located almost entirely on Bank Street, an important radial road in the city of Ottawa.

- Both bus routes cross the central business district (CBD) of Ottawa.

Bus route 95 has 63 stops. It connects Orleans to South Nepean and is 31.4 kilometers in length. In this study, the route segment shown in Figure 3.4A from Blair to Lebreton station was selected because it contains both Transitway and exclusive bus lanes. This segment is $10.26 \mathrm{~km}$ long; it includes 26 stops and the average distance between two stops is $0.39 \mathrm{~km}$. Out of 26 stops in this segment, seven stops were chosen and their data are collected and tabulated in Table 3.2. The schedule headways during 
weekday vary from 3 to 5 minutes in peak-hour to 15 minutes after 10 p.m., and 30 minutes after midnight.

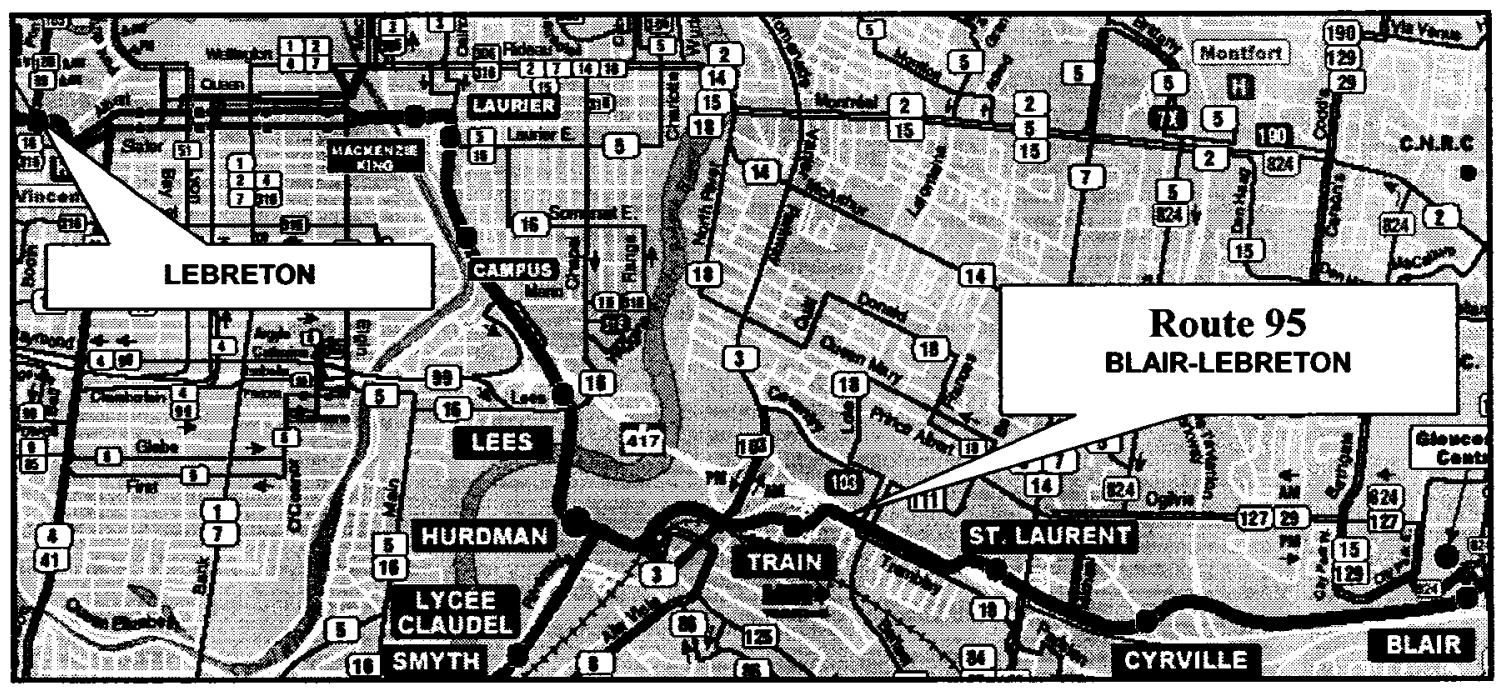

Figure 3.4A: Bus Route 95 (Blair-Lebereton)

Table 3.2: Distance between stops BLAIR and LEBRETON. Bus Route 95 (Orleans-Nepean South)

\begin{tabular}{|l|l|c|c|}
\hline Stop ID & STOP DESCRIPTION & $\begin{array}{c}\text { Distance between stops } \\
(\mathbf{k m})\end{array}$ & $\begin{array}{c}\text { Accumulated } \\
\text { Distances (km) }\end{array}$ \\
\hline EE915 & BLAIR 2B & 0.00 & 0.00 \\
\hline EB 905 & ST LAURENT 2B & 2.79 & 2.79 \\
\hline AE900 & TRAIN 2A & 1.19 & 3.98 \\
\hline CE940 & LEES 2B & 2.16 & 6.14 \\
\hline CD910 & MACKEZINE KING 2A* & 1.87 & 8.01 \\
\hline CA920 & ALBERT -KENT* & 1.19 & 9.2 \\
\hline CJ 900 & LEBRETON* & 1.06 & 10.26 \\
\hline
\end{tabular}

Note: * bus stop is in CBD area. Please see Appendix $\mathbf{A}$ for the map of route 95

Bus route 1 has 65 bus stops lying between Ottawa Rockliffe and South Keys. It is a mixed-traffic bus route because it is designated mainly on Bank, Wellington, Rideau, St. Patrick and Beachwood streets. 


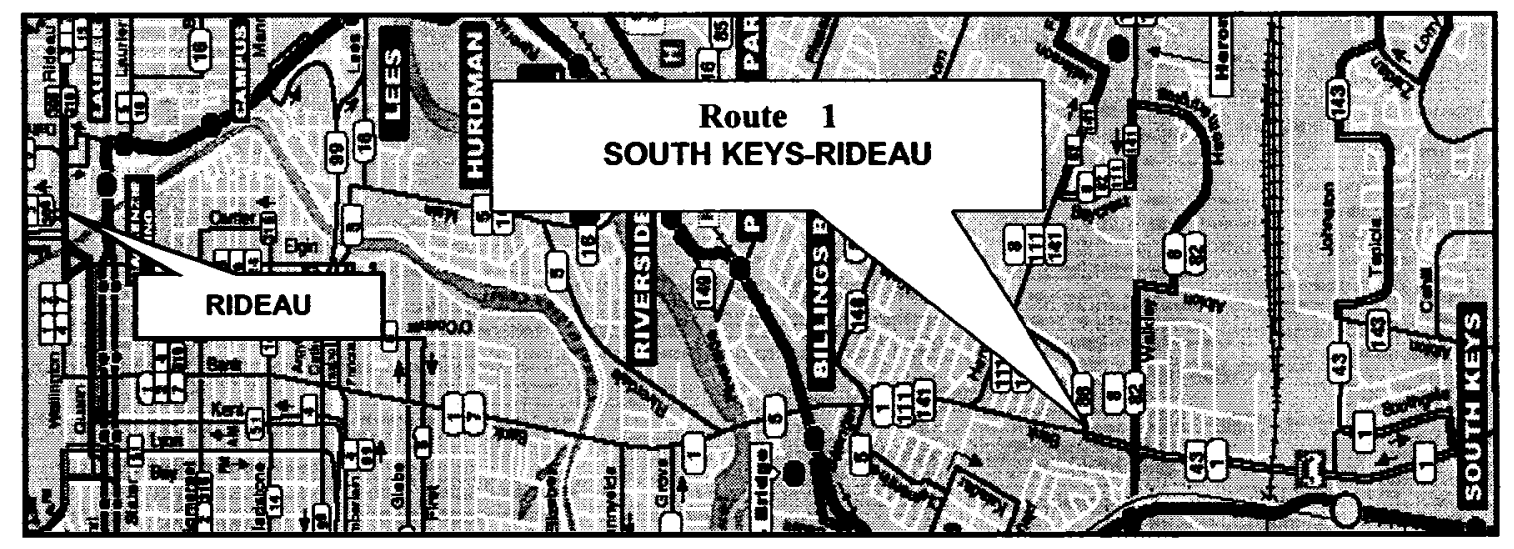

Figure 3.4B: Bus route 1 (South Keys-Rideau)

Table 3.3: Distance between stops GREENSBORO and RIDEAU. Bus Route 1 (South Keys - Ottawa Rockliffe)

\begin{tabular}{|c|c|c|c|}
\hline Stop ID & $\begin{array}{c}\text { STOP } \\
\text { DESCRIPTION }\end{array}$ & $\begin{array}{c}\text { Distance between } \\
\text { stops }(\mathrm{km})\end{array}$ & $\begin{array}{c}\text { Accumulated } \\
\text { Distances }(\mathrm{km})\end{array}$ \\
\hline RF900 & GREENSBORO 1A & 0.00 & 0.00 \\
\hline RF075 & BANK-JOHNSTON NS & 3.19 & 3.19 \\
\hline RA190 & BANK- WALKLEY & 1.19 & 4.38 \\
\hline RA945 & BILLINGS BRIDGE 4C & 2.70 & 7.08 \\
\hline CH080 & BANK -HOLMWOOD* & 2.12 & 9.20 \\
\hline CF630 & BANK- GLADSTONE* & 1.55 & 10.75 \\
\hline CD 920 & RIDEAU 3A* & 2.1 & 12.85 \\
\hline
\end{tabular}

Note: ${ }^{*}$ bus stop is in CBD area. Please see Appendix $A$ for the map of route 1

In this study, the segment connecting Greensboro stop and Rideau stop as shown in Figure 3.4B, is selected and seven bus stops were considered as timepoints (see Table 3.3). The schedule of bus route 1 varies depending upon the time of day, from 12 minutes in peak-hour period to 30 minutes after 7 p.m.

\subsubsection{Data Sets}

Data from the APC-AVL systems available from the OC Transpo for each selected stop were clustered by time periods in a day and seasons in a year. In this study, 
only weekday data were of interest. Weekday peak period data are considered as the ones that record bus arrivals at stops from 8 a.m. to 10 a.m., 12:00 noon to 2 p.m., from 4 p.m. to 6 p.m. in the afternoon. Weekday evening is considered as the time of bus arrivals after 7 p.m. However, this study did not consider weekday evening.

Data were also clustered by season in order to capture the various passenger activities and on-time bus performance in different seasons. Four seasonal data sets, namely SEASON1 (January $1^{\text {st }}$ to April $30^{\text {th }}$ ), SEASON2 (May $1^{\text {st }}$ to $31^{\text {st }}$ Jun), SEASON3 (July $1^{\text {st }}$ to August $30^{\text {th }}$ ), and SEASON4 (September $1^{\text {st }}$ to December $30^{\text {th }}$ ) were also clustered. The data for bus route 95 and bus route 1 were collected for one year, covering from Season 4 of 2004 to Season 4 of 2005. The number of observations during peak-periods in each sample is shown in Table 3.4. Each observation noted in Table 3.4 contains a set of information, including stop ID, route and route direction, time period and date, actual and schedule arrival, the number of passengers boarding and alighting at a stop, the number of passengers on-board when the bus leaves the stop or passes it. These data are synthesized in the tabular form called APC point check as shown in Figure 3.5.

Table 3.4: Data Sets for Bus Route 95 and Bus Route 1

\begin{tabular}{|c|c|c|c|c|c|c|c|c|c|c|c|c|}
\hline \multirow{2}{*}{ Route } & \multicolumn{3}{|c|}{ SEASON 1 } & \multicolumn{3}{c|}{ SEASON 2 } & \multicolumn{3}{c|}{ SEASON 3 } & \multicolumn{3}{c|}{ SEASON 4 } \\
\cline { 2 - 12 } & $\begin{array}{c}\mathbf{8 - 1 0} \\
\text { a.m. }\end{array}$ & $\begin{array}{c}\mathbf{1 2 - 2} \\
\text { p.m }\end{array}$ & $\begin{array}{c}\text { 4- } \\
\text { 6p.m. }\end{array}$ & $\begin{array}{c}\mathbf{8 - 1 0} \\
\text { a.m. }\end{array}$ & $\begin{array}{c}\mathbf{1 2 - 2} \\
\text { p.m. }\end{array}$ & $\begin{array}{c}\text { 4- } \\
\text { 6p.m. }\end{array}$ & $\begin{array}{c}\mathbf{8 - 1 0} \\
\text { a.m. }\end{array}$ & $\begin{array}{c}\text { 12-2 } \\
\text { p.m. }\end{array}$ & $\begin{array}{c}\text { 4- } \\
\text { 6p.m }\end{array}$ & $\begin{array}{c}\mathbf{8 - 1 0} \\
\text { a.m. }\end{array}$ & $\begin{array}{c}\text { 12-2 } \\
\text { p.m. }\end{array}$ & $\begin{array}{c}\text { 4- } \\
\text { 6p.m. }\end{array}$ \\
\hline $\mathbf{9 5}$ & $\mathbf{4 7 2}$ & 998 & 1220 & $\mathbf{8 9 0}$ & 744 & 931 & 849 & 494 & 700 & 592 & 450 & 600 \\
\hline $\mathbf{1}$ & 513 & 335 & 189 & 291 & 203 & 113 & 297 & 210 & 130 & 389 & 312 & 150 \\
\hline
\end{tabular}




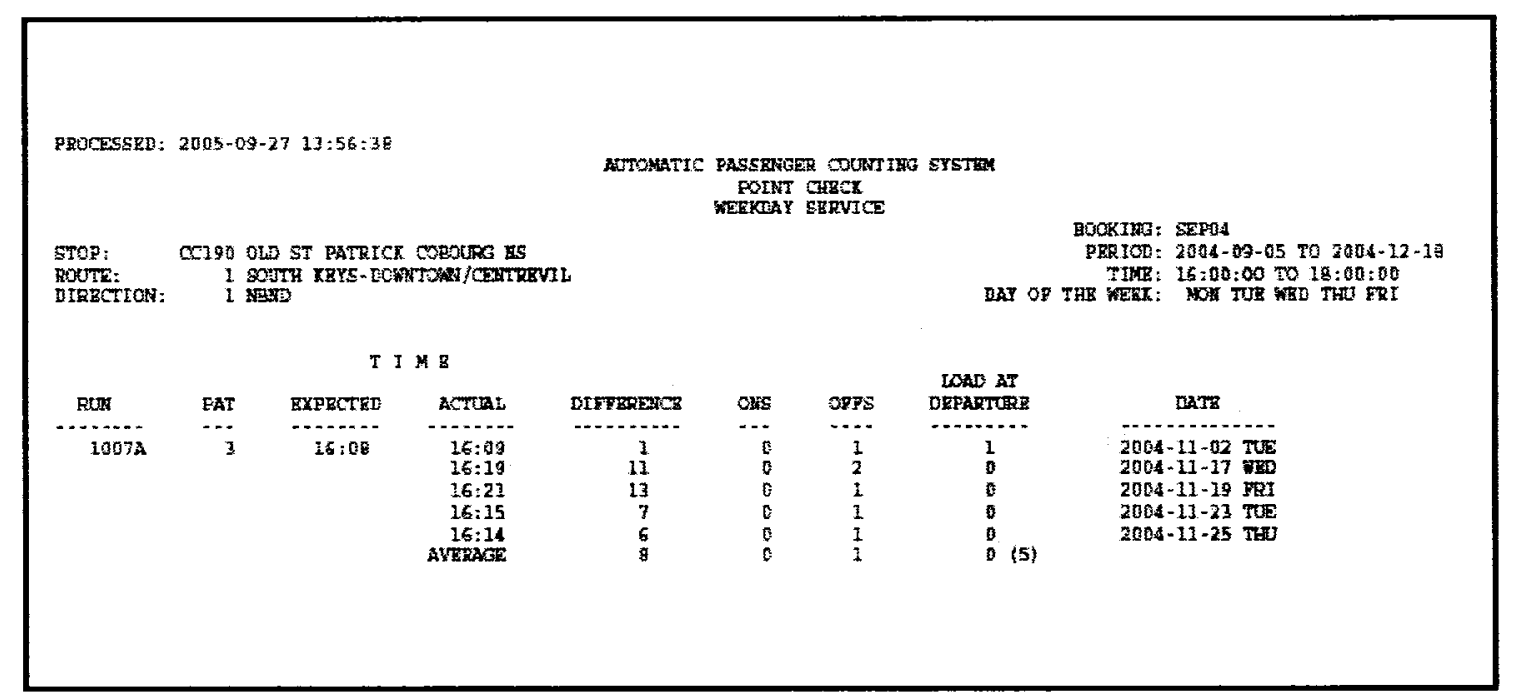

Figure 3.5: Structure of APC Data at pointcheck

\subsubsection{APC and AVL Data Description}

The data sets used in this study were collected from the APC system mounted on the buses. The OC Transpo has been equipping APC system on about 10 percent of their bus fleet. These units are circulated among all bus routes routinely to get the operational data of all bus routes for analysis purposes. At present, the location of a bus is identified by the odometers and signpost-based AVL system. The GPS-based AVL system has been deployed in the OC Transpo and was expected to be complete at the end of 2005 . The APC system can collect many data items automatically, and at the same time. Table 3.5 presents the descriptions of data collectable by APC and AVL systems of the OC Transpo. 
Table 3.5: Data Collectable by APC and AVL systems

\begin{tabular}{|c|c|}
\hline Collectable Data & Description \\
\hline SCHED DEP TIME & Scheduled departure time at specified section \\
\hline DATE & The date on which the observation occurs \\
\hline TIME OF DAY & Time period of bus operation \\
\hline DOOR OPENING TIME & Bus door opening time \\
\hline DOOR CLOSING TIME & Bus door closing time \\
\hline EXCESS_TIME & $\begin{array}{l}\text { The portion of time spent at a stop other than servicing passengers; that } \\
\text { is , the stop time at a stop excluding the dwell time }\end{array}$ \\
\hline ATC_DEP_TIME & Actual departure time \\
\hline MOVING_TIME & $\begin{array}{l}\text { Time spent Moving Between Stops; The proportion of time between } \\
\text { departing the first stop and the arrival at the second stop-excluding any } \\
\text { idle times, stop times, and stop-and-go times. }\end{array}$ \\
\hline STOPGO_TIME & $\begin{array}{l}\text { The portion of the running time spent in a stop and go situation (stop } \\
\text { time less than } 10 \text { seconds). }\end{array}$ \\
\hline IDLE TIME & The portion of the running time spent in an idle situation. \\
\hline DWELL TIME & The portion of running time spent in servicing passengers \\
\hline EXPECTED TIME & The scheduled time at the stop, as obtained from the itinerary \\
\hline ACTUAL TIME & The actual time that observed trip passed the stop. \\
\hline ONS 1, ONS 2, ONS 3 & $\begin{array}{l}\text { The number of boarding passengers at the bus stop used front door, the } \\
\text { first rear door, and the second rear door. }\end{array}$ \\
\hline OFFS 1, OFFS 2, OFF3 & $\begin{array}{l}\text { The number of alighting passengers the bus stop at the stop used front } \\
\text { door, the first rear door, and the second rear door. }\end{array}$ \\
\hline LOAD AT DEPARTURE & The number of passengers on the bus as the bus leaves or passes the stop \\
\hline STOP DISTANCE & Travel distance between two consecutive stops \\
\hline STATTTIME & $\begin{array}{l}\text { Stationary time: The time the bus not in motion; vary from } 10 \text { to } 45 \\
\text { seconds }\end{array}$ \\
\hline TRIP ID & Unique index associated with a trip \\
\hline STOPID & Unique index associated with a bus stop \\
\hline TRIP STATUS & Start or end a trip \\
\hline MATCH & Unique index associated with a pattern in each pick data \\
\hline$L A T$ & GPS Latitude* (As of August 2005) \\
\hline$L O N$ & GPS Longitude* (As of August 2005$)$ \\
\hline
\end{tabular}

Note: * Not available

Another type of data that records the passenger activity at bus doors is also collected by the OC Transpo. Figure 3.6 presents an example of such data. There are 6780 and 2800 recorded observations for bus route 95 and bus route 1 for all selected stops, respectively. 


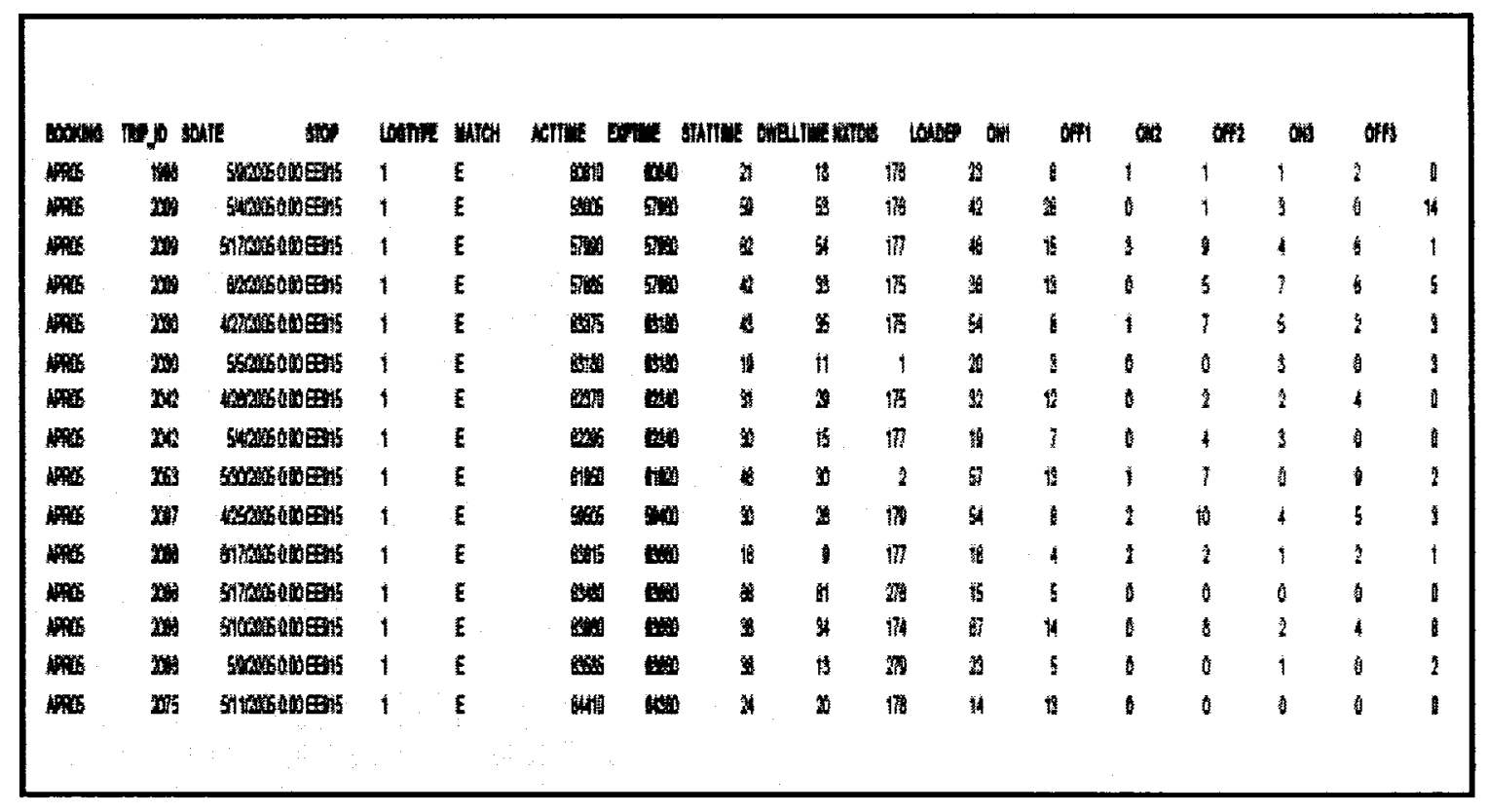

Figure 3.6: Structure of APC Data on Passenger Activity

\subsubsection{Data Characteristics}

Before developing the model, it is necessary to analyze the acquired data, so that we can obtain the general characteristics of arrival times and dwell times at each bus stop in different period of time. In order to do that, descriptive statistics were applied.

\subsubsection{Arrival time}

Arrival time was analyzed in term of bus on-time performance, measuring the differences between the schedule and actual arrival. The data were collected at each bus stop and clustered by time of day and seasonal time (e.g., SEASON 1, SEASON 2, SEASON 3, and SEASON 4). A positive value of difference indicates the bus was behind the schedule, or lateness, while a negative one represents earliness. 
Two periods of time in the year 2005 were chosen to consider the effects of weather on bus on-time performance. The WINTER data set (Jan $3^{\text {rd }}$ to Apr $23^{\text {rd }}, 2005$ ) represents winter conditions and the SUMMER data set (Jun $26^{\text {th }}$ to September $4^{\text {th }}, 2005$ ) accounts for summer conditions. The means and standard deviations of lateness and earliness of buses on bus route 1 in the two seasons are shown in Tables 3.6 and 3.7 while that of bus route 95 are shown in Tables 3.8 and 3.9 .

Table 3.6: Means and Standard Deviations of Bus on-time Performance in the winter. Bus Route 1 (Greensboro - Rideau)

\begin{tabular}{|c|c|c|c|c|}
\hline Time Period & Stop Name & Mean (minute) & $\begin{array}{l}\text { Standard } \\
\text { Deviation } \\
\text { (Minute) }\end{array}$ & $\begin{array}{l}\text { Number of } \\
\text { observations }\end{array}$ \\
\hline \multirow{7}{*}{$\begin{array}{l}8 \text { a.m.-10 a.m. } \\
\text { (WINTER) }\end{array}$} & GREENSBORO 1A & -0.02 & 1.38 & 51 \\
\hline & BANK-JOHNSTON NS & -0.88 & 1.48 & 49 \\
\hline & BANK- WALKLEY & -1.59 & 1.47 & 56 \\
\hline & BILLINGS BRIDGE 4C & 0.06 & 1.90 & 67 \\
\hline & BANK -HOLMWOOD & 2.02 & 2.40 & 78 \\
\hline & BANK- GLADSTONE & 4.71 & 2.55 & 81 \\
\hline & RIDEAU 3A & 3.17 & 3.43 & 53 \\
\hline \multirow{7}{*}{$\begin{array}{l}12 \text { noon -2 p.m. } \\
\text { (WINTER) }\end{array}$} & GREENSBORO 1A & 1.07 & 2.15 & 45 \\
\hline & BANK-JOHNSTON NS & -0.63 & 2.66 & 43 \\
\hline & BANK-WALKLEY & -0.64 & 3.02 & 42 \\
\hline & BILLINGS BRIDGE 4C & 1.05 & 2.71 & 42 \\
\hline & BANK -HOLMWOOD & 1.63 & 3.11 & 40 \\
\hline & BANK- GLADSTONE & 4.65 & 3.72 & 40 \\
\hline & RIDEAU 3A & 4.87 & 3.84 & 49 \\
\hline \multirow{7}{*}{$\begin{array}{l}4 \text { p.m. - } 6 \text { p.m. } \\
\text { (WINTER) }\end{array}$} & GREENSBORO 1A & 0.67 & 1.39 & 20 \\
\hline & BANK-JOHNSTON NS & -0.87 & 3.03 & 23 \\
\hline & BANK- WALKLEY & -0.83 & 2.93 & 24 \\
\hline & BILLINGS BRIDGE 4C & 0.54 & 3.16 & 24 \\
\hline & BANK -HOLMWOOD & 2.42 & 3.73 & 24 \\
\hline & BANK- GLADSTONE & 4.52 & 3.80 & 23 \\
\hline & RIDEAU 3A & 4.17 & 4.61 & 28 \\
\hline
\end{tabular}


Table 3.7: Means and Standard Deviations of Bus on-time Performance in the Summer. Bus Route 1 (Greensboro - Rideau)

\begin{tabular}{|c|c|c|c|c|}
\hline Time Period & Stop Name & Mean (minute) & $\begin{array}{l}\text { Standard } \\
\text { Deviation } \\
\text { (Minute) }\end{array}$ & $\begin{array}{l}\text { Number of } \\
\text { observations }\end{array}$ \\
\hline \multirow{7}{*}{$\begin{array}{l}8 \text { a.m.-10 a.m. } \\
\text { (SUMMER) }\end{array}$} & GREENSBORO 1A & 0.66 & 1.21 & 32 \\
\hline & BANK-JOHNSTON NS & -0.91 & 1.81 & 33 \\
\hline & BANK- WALKLEY & -1.88 & 1.95 & 34 \\
\hline & BILLINGS BRIDGE 4C & -0.27 & 1.99 & 49 \\
\hline & BANK -HOLMWOOD & 2.17 & 2.73 & 48 \\
\hline & BANK- GLADSTONE & 5.23 & 3.39 & 47 \\
\hline & RIDEAU 3A & 3.55 & 3.73 & 45 \\
\hline \multirow{7}{*}{$\begin{array}{l}12 \text { noon -2p.m. } \\
\text { (SUMMER) }\end{array}$} & GREENSBORO 1A & 0.96 & 2.33 & 28 \\
\hline & BANK-JOHNSTON NS & -0.41 & 3.07 & 27 \\
\hline & BANK- WALKLEY & -0.11 & 3.39 & 27 \\
\hline & BILLINGS BRIDGE 4C & 1.26 & 3.38 & 27 \\
\hline & BANK -HOLMWOOD & 1.48 & 2.67 & 27 \\
\hline & BANK- GLADSTONE & 5.30 & 3.39 & 27 \\
\hline & RIDEAU 3A & 3.55 & 4.82 & 27 \\
\hline \multirow{7}{*}{$\begin{array}{l}4 \text { p.m.-6p.m. } \\
\text { (SUMMER) }\end{array}$} & GREENSBORO 1A & & & \\
\hline & BANK-JOHNSTON NS & -0.60 & 1.82 & 21 \\
\hline & BANK- WALKLEY & -0.5 & 1.93 & 20 \\
\hline & BILLINGS BRIDGE 4C & 0.95 & 1.68 & 19 \\
\hline & BANK -HOLMWOOD & 1.29 & 2.03 & 18 \\
\hline & BANK- GLADSTONE & 3.65 & 2.17 & 18 \\
\hline & RIDEAU 3A & 0.94 & 2.01 & 33 \\
\hline
\end{tabular}

Table 3.8: Means and Standard Deviations of Bus on-time Performance in the Winter. Bus Route 95 (Blair - Lebreton)

\begin{tabular}{|c|c|c|c|c|}
\hline Time period & Stop Name & Mean (minute) & $\begin{array}{r}\text { Standard } \\
\text { Deviation } \\
\text { (minute) }\end{array}$ & $\begin{array}{c}\text { Number of } \\
\text { Observations }\end{array}$ \\
\hline \multirow{5}{*}{$\begin{array}{l}\text { 8a.m.-10 a.m. } \\
\text { (WINTER) }\end{array}$} & ST LAURENT 2B & 0.55 & 2.65 & 126 \\
\hline & TRAIN 2A & 0.79 & 2.34 & 122 \\
\hline & LEES 2B & 1.10 & 2.31 & 126 \\
\hline & ALBERT -KENT & 2.36 & 2.78 & 129 \\
\hline & LEBRETON & 0.95 & 3.04 & 134 \\
\hline \multirow{4}{*}{$\begin{array}{c}12 \text { noon-2 } \\
\text { p.m. } \\
\text { (WINTER) }\end{array}$} & BLAIR 2B & -0.59 & 1.66 & 111 \\
\hline & ST LAURENT 2B & -0.27 & 1.70 & 111 \\
\hline & TRAIN 2A & -0.03 & 2.63 & 107 \\
\hline & LEBRETON & -0.40 & 2.12 & 110 \\
\hline \multirow{7}{*}{$\begin{array}{l}4 \text { p.m.-6 p.m. } \\
\text { (WINTER) }\end{array}$} & BLAIR 2B & -0.11 & 1.72 & 138 \\
\hline & ST LAURENT 2B & 0.40 & 1.81 & 138 \\
\hline & TRAIN 2A & 0.94 & 1.94 & 134 \\
\hline & LEES 2B & 0.97 & 2.04 & 143 \\
\hline & MACKEZINE KING 2A & 2.76 & 2.88 & 138 \\
\hline & ALBERT -KENT & 3.20 & 2.50 & 143 \\
\hline & LEBRETON & 0.59 & 2.66 & 143 \\
\hline
\end{tabular}


Table 3.9: Means and Standard Deviations of Bus on-time Performance in the Summer. Bus route 95 (Blair - Lebreton)

\begin{tabular}{|c|c|c|c|c|}
\hline Time period & Stop Name & $\begin{array}{c}\text { Mean } \\
\text { ( Minute) }\end{array}$ & $\begin{array}{l}\text { Standard } \\
\text { Deviation } \\
\text { (Minute) }\end{array}$ & $\begin{array}{c}\text { Number of } \\
\text { Observations }\end{array}$ \\
\hline \multirow{7}{*}{$\begin{array}{l}8 \text { a.m.-10 a.m. } \\
\text { (WINTER) }\end{array}$} & BLAIR 2B & 0.17 & 0.162 & 98 \\
\hline & ST LAURENT 2B & 0.47 & 1.68 & 95 \\
\hline & TRAIN 2A & 0.78 & 1.67 & 98 \\
\hline & LEES 2B & 0.85 & 1.80 & 99 \\
\hline & MACKEZINE KING 2A & 1.71 & 1.87 & 103 \\
\hline & ALBERT -KENT & 2.28 & 2.16 & 102 \\
\hline & LEBRETON & 0.61 & 2.43 & 103 \\
\hline \multirow{7}{*}{$\begin{array}{l}12 \text { noon -2p.m. } \\
\text { (WINTER) }\end{array}$} & BLAIR 2B & -0.19 & 1.64 & 59 \\
\hline & ST LAURENT 2B & 0.43 & 1.70 & 61 \\
\hline & TRAIN 2A & 0.97 & 1.67 & 61 \\
\hline & LEES 2B & 0.62 & 1.55 & 63 \\
\hline & MACKEZINE KING 2A & 1.43 & 1.65 & 61 \\
\hline & ALBERT -KENT & 2.08 & 2.11 & 64 \\
\hline & LEBRETON & 0.63 & 2.52 & 60 \\
\hline \multirow{7}{*}{$\begin{array}{l}4 \text { p.m-6 p.m. } \\
\text { (WINTER) }\end{array}$} & BLAIR 2B & 0.67 & 1.97 & 80 \\
\hline & ST LAURENT 2B & 1.27 & 1.96 & 83 \\
\hline & TRAIN 2A & 1.78 & 2.09 & 81 \\
\hline & LEES 2B & 1.88 & 2.27 & 79 \\
\hline & MACKEZINE KING 2A & 3.26 & 2.57 & 79 \\
\hline & ALBERT -KENT & 3.63 & 2.88 & 80 \\
\hline & LEBRETON & 0.90 & 2.87 & 84 \\
\hline
\end{tabular}

From the tables, we can see that, in the same time period, the lateness of the stops located in the CBD area are always longer than that of the stops located outside the CBD. For route 1 , the means of times decrease in the afternoon both in the winter and summer, implying that the adherence to the schedule is better in the afternoon. In contrast, for route 95 , the better adherence to the schedule is found in the middle of the day.

Further analyses were carried out in the form of two-sample-t test technique. The means of bus on-time performances at the same bus stop were compared in terms of different time periods (e.g., 8 a.m. to 10 a.m., 12 noon to 2 p.m., and 4 p.m. to 6 p.m.) and various seasons (e.g., the winter and the summer). The results of the tests for bus 
route 95 are shown in the Tables 3.10 to 3.12 , and those of bus route 1 are presented in

Tables 3.13 to 3.15 .

Table 3.10: Two-sample $t$ statistics for Winter and Summer Time Route 95- Morning period (8 a.m. to 10 a.m.)

\begin{tabular}{|l|c|c|c|}
\hline \multirow{2}{*}{\begin{tabular}{l} 
Ho: $m_{l}=m_{2}$ \\
\multicolumn{1}{|c|}{ STOP $m_{l} \neq m_{2}$}
\end{tabular}} & \multicolumn{3}{|c|}{ Two-sample t values ( 2 tail-test) } \\
\cline { 2 - 4 } & $t_{l}$ & $d f_{I}$ & $T_{I}$ \\
\hline BLAIR 2B & $\underline{0.721}$ & 98 & 1.984 \\
\hline ST LAURENT 2B & $\underline{0.268}$ & 95 & 1.984 \\
\hline TRAIN 2A & $\underline{0.037}$ & 98 & 1.984 \\
\hline LEES 2B & $\underline{0.912}$ & 99 & 1.984 \\
\hline MACKEZINE KING & $\underline{1.312}$ & 103 & 1.984 \\
\hline ALBERT -KENT & $\underline{0.307}$ & 102 & 1.984 \\
\hline LEBRETON & $\underline{0.957}$ & 103 & 1.984 \\
\hline
\end{tabular}

Notes for tables (3.10) to (3.12)

$t_{l}=\mathrm{t}$ values resulted from the comparisons the means measured in the winter time to the mean measured in the summer time, given the same time period of day.

$d f 1=$ Degree of freedom corresponding with $t_{l}$

$T 1 \quad=$ Critical $\mathrm{T}$ values with 0.05 level of significance corresponding with $d f 1$.

Underlined values: Not significant at 0.05 level or cannot reject $\boldsymbol{H o} .(\boldsymbol{t}<\boldsymbol{T})$

Table 3.11: Two-sample $t$ statistics for winter and summer time Route 95- Noon period (12 a.m. to 2 p.m.)

\begin{tabular}{|l|c|c|c|}
\hline \multirow{2}{*}{$\begin{array}{l}H o: \\
\text { Sa: } m_{l}=m_{2} \neq m_{2}\end{array}$} & \multicolumn{2}{|c|}{ Two-sample t values (2 tail-test) } \\
\cline { 2 - 4 } & $t_{l}$ & $d f_{l}$ & $T_{l}$ \\
\hline BLAIR 2B & $\underline{2.507}$ & 59 & 2.001 \\
\hline ST LAURENT 2B & 2.684 & 61 & 2.000 \\
\hline TRAIN 2A & 3.726 & 63 & 2.000 \\
\hline LEES 2B & 1.749 & 61 & 2.000 \\
\hline MACKEZINE KING & 2.779 & 63 & 2.000 \\
\hline ALBERT -KENT & 3.482 & 61 & 2.000 \\
\hline LEBRETON & 2.689 & 64 & 2.000 \\
\hline
\end{tabular}


Table 3.12: Two-sample $t$ statistics for Winter and Summer Time Route 95- Afternoon period (4 p.m. to 6 p.m.)

\begin{tabular}{|c|c|c|c|}
\hline \multicolumn{4}{|l|}{$\begin{array}{l}\boldsymbol{H o}: m_{I}=m_{2} \\
\boldsymbol{H a}: m_{l} \neq m_{2}\end{array}$} \\
\hline \multirow{2}{*}{ STOP } & \multicolumn{3}{|c|}{ Two-sample t values ( 2 tail-test) } \\
\hline & $t_{I}$ & $d f_{1}$ & $T_{1}$ \\
\hline BLAIR 2B & 2.949 & 80 & 1.990 \\
\hline ST LAURENT 2B & 3.287 & 83 & 1.990 \\
\hline TRAIN 2A & 2.933 & 81 & 1.990 \\
\hline LEES 2B & 2.963 & 79 & 1.990 \\
\hline MACKEZINE KING & 1.319 & 79 & 1.990 \\
\hline ALBERT -KENT & 1.120 & 80 & 1.990 \\
\hline LEBRETON & $\overline{0.816}$ & 84 & 1.990 \\
\hline
\end{tabular}

For route 95 (i.e. the Transitway bus route), as shown in Tables 3.10 to 3.12 , almost all of the means based on collected data at bus stops during the noon period (12 noon to 2 p.m.) and during the afternoon period ( 4 p.m. to 6 p.m.) are statistically significant in terms of difference between winter and summer at 0.05 level of significance. This means that the buses of route 95 adhered to the schedule differently depending upon seasons during such time periods. However, for the morning period (8 a.m. to 10 a.m.), such differences were not found. In other words, during the morning period, there is no difference in bus on-time performance between the winter and the summer.

Also, for bus route 1 (i.e. mixed traffic bus route), the test results in Tables 3.13 to 3.15 show that at 0.05 significance level, the null hypothesis (i.e., the means measured in the winter time are the same as the means recorded in the summer time) cannot be rejected, for any time period of day. These imply that there are no statistical differences in bus adherence to schedule between the winter and the summer time for the mixed-bus route. 
Table 3.13: Two-sample $t$ statistics for Winter and Summer Time Route 1- Morning period (from 8 a.m. to 10 a.m.)

\begin{tabular}{|c|c|c|c|}
\hline \multicolumn{4}{|l|}{$\begin{array}{l}H o: m_{l}=m_{2} \\
\text { Ha: } m_{l} \neq m_{2}\end{array}$} \\
\hline \multirow{2}{*}{ STOP } & \multicolumn{3}{|c|}{ Two-sample t values ( 2 tail-test) } \\
\hline & $t_{I}$ & $d f_{1}$ & $T_{1}$ \\
\hline GREENSBORO 1A & 2.359 & 32 & 2.038 \\
\hline BANK-JOHNSTON NS & 0.079 & 33 & 2.036 \\
\hline BANK- WALKLEY & 0.748 & 34 & 2.034 \\
\hline BILLINGS BRIDGE & 1.978 & 49 & 2.009 \\
\hline BANK -HOLMWOOD & 1.327 & 48 & 2.011 \\
\hline BANK- GLADSTONE & $\underline{1.813}$ & 47 & 2.013 \\
\hline RIDEAU 3A & 0.521 & 45 & 2.015 \\
\hline
\end{tabular}

Notes for tables (3.13) to (3.15)

$t_{I} \quad=t$ value that is resulted from the comparisons the means measured in the winter time to the mean measured in the summer time, given the same time period of day.

$d f_{1} \quad=$ Degree of freedom corresponding with $t_{1}$

$T 1 \quad=$ Critical $\mathrm{T}$ values with 0.05 level of significance corresponding with $d f 1$.

Underlined values: Not significant at 0.05 level or cannot reject $\boldsymbol{H o} .(t<T)$

Table 3.14: Two-sample $t$ statistics for winter and summer time Route 1- Noon period (12 a.m. to 2 p.m.)

\begin{tabular}{|l|c|c|c|}
\hline \multirow{2}{*}{$\begin{array}{l}\text { Ho: } m_{1}=m_{2} \\
\text { Ha: } m_{1} \neq m_{2}\end{array}$} & \multicolumn{3}{|c|}{ Two-sample t values ( 2 tail-test) } \\
\hline \multirow{2}{*}{ STOP } & $t_{1}$ & $d f_{1}$ & $T_{1}$ \\
\cline { 2 - 4 } & $\underline{0.202}$ & 28 & 2.048 \\
\hline GREENSBORO 1A & $\underline{0.307}$ & 27 & 2.052 \\
\hline BANK-JOHNSTON NS & $\underline{0.661}$ & 27 & 2.052 \\
\hline BANK- WALKLEY & $\underline{0.272}$ & 27 & 2.052 \\
\hline BILLINGS BRIDGE & $\underline{0.211}$ & 27 & 2.052 \\
\hline BANK-HOLMWOOD & $\underline{0.740}$ & 27 & 2.052 \\
\hline BANK- GLADSTONE & $\underline{1.225}$ & 27 & 2.052 \\
\hline RIDEAU 3A & & & \\
\hline
\end{tabular}

Table 3.15: Two-sample $t$ statistics for winter and summer time Route 1- Afternoon period (4 p.m. to 6 p.m.)

\begin{tabular}{|c|c|c|c|}
\hline $\begin{array}{l}\text { Ho: } m_{1}=m_{2} \\
H a: m_{I} \neq m_{2}\end{array}$ & \multirow{2}{*}{\multicolumn{3}{|c|}{ Two-sample t values ( 2 tail-test) }} \\
\hline \multirow{2}{*}{ STOP } & & & \\
\hline & $t_{1}$ & $d f_{I}$ & $T_{1}$ \\
\hline \multicolumn{4}{|l|}{ GREENSBORO 1A } \\
\hline BANK-JOHNSTON NS & 0.356 & 21 & 2.080 \\
\hline BANK- WALKLEY & 0.444 & 20 & 2.086 \\
\hline BILLINGS BRIDGE & $\underline{0542}$ & 19 & 2.093 \\
\hline BANK -HOLMWOOD & 1.257 & 18 & 2.101 \\
\hline BANK- GLADSTONE & $\overline{0.929}$ & 18 & 2.101 \\
\hline RIDEAU 3A & $\overline{3.440}$ & 28 & 2.048 \\
\hline
\end{tabular}


In order to test if the on-time performance of the buses varies depending upon the different time periods of the same day for a given season, the means of on-time performance collected at bus stop during each time period were also compared by the same technique described above. The test results are shown in Tables 3.16 and 3.17 for bus route 95 , and Tables 3.18 and 3.19 for bus route 1 .

Table 3.16: Two-sample t statistics in Different Time Periods of Day Route 95- The winter time (the WINTER data set)

\begin{tabular}{|l|c|c|c|c|c|c|}
\hline Ho: $m_{1}=m_{2}, H a: m_{l} \neq m_{2} ; H^{\prime}: m_{1}=m_{3}, H a^{\prime}: m_{l} \neq m_{3}$ \\
\cline { 2 - 7 } STOP & \multicolumn{7}{|c|}{ Two-sample t values ( 2 tail-test) } \\
& $t_{2}$ & $d f_{2}$ & $T_{2}$ & $t_{3}$ & $d f_{3}$ & $T_{3}$ \\
\hline BLAIR 2B & 3.014 & 110 & 1.984 & $\underline{1.533}$ & 119 & 1.984 \\
\hline ST LAURENT 2B & 2.867 & 110 & 1.984 & $\underline{0.532}$ & 126 & 1.984 \\
\hline TRAIN 2A & 2.477 & 107 & 1.984 & $\underline{0.555}$ & 122 & 1.984 \\
\hline LEES 2B & 4.926 & 119 & 1.984 & $\underline{0.486}$ & 126 & 1.984 \\
\hline MACKEZINE & 4.628 & 115 & 1.984 & $\underline{1.950}$ & 128 & 1.984 \\
\hline ALBERT -KENT & 4.941 & 138 & 1.984 & 2.609 & 129 & 1.984 \\
\hline LEBRETON & 4.074 & 110 & 1.984 & $\underline{1.046}$ & 134 & 1.984 \\
\hline
\end{tabular}

Notes for tables (3.16) \& (3.17):

Underlined values: Not significant at 0.05 level or cannot reject $H o$ or $H_{0} \cdot(t<T)$

$t_{2}, t_{3}=$ Values that are resulted from the comparisons between the means measured the morning period (8 A.M. to 10 A.M.), the means measured in noon period (12 A. M. to 2 P.M.), and those of afternoon period (4 P.M. to 6 P.M.), respectively.

$d f 2, d f 3=$ Degree of freedom corresponding with $t_{2}, t_{3}$

$T_{2}, T_{3}=$ Critical $\mathrm{T}$ values with 95 percent degree of confidence corresponding with $d f_{2}, d f_{3}$.

For bus route 95 , the test results show clear differences between the means of on-time performance measured in different time periods of day. As shown in Table 3.16, during the winter time, there are statistically significant differences of bus on-time performance between morning and noon time period. For the summer time, the statistical differences of the means are found between morning and afternoon period (Table 3.17). For bus route 1 , the differences between the means are not as clear as those of bus route 
Table 3.19: Two-sample t statistics in Different Time Periods of Day Route 1- The summer time (the SUMMER data set)

\begin{tabular}{|l|c|c|c|c|c|c|}
\hline Ho: $m_{l}=m_{2}, H a: m_{l} \neq m_{2} ; H^{\prime}: m_{1}=m_{3}, H a: m_{l} \neq m_{3}$ \\
\cline { 2 - 7 } & \multicolumn{7}{|c|}{ Two-sample t values ( 2 tail-test) } \\
\cline { 2 - 7 } & $t_{2}$ & $d f_{2}$ & $T_{2}$ & $t_{3}$ & $d f_{3}$ & $T_{3}$ \\
\hline GREENSBORO 1A & 0.613 & 28 & 2.048 & & & \\
\hline BANK-JOHNSTON NS & $\underline{0.747}$ & 27 & 2.052 & $\underline{0.611}$ & 21 & 2.080 \\
\hline BANK-WALKLEY & 2.414 & 27 & 2.052 & 2.528 & 20 & 2.086 \\
\hline BILLINGS BRIDGE & 2.155 & 27 & 2.052 & 2.547 & 19 & 2.093 \\
\hline BANK-HOLMWOOD & $\underline{1.066}$ & 27 & 2.052 & $\underline{1.419}$ & 18 & 2.101 \\
\hline BANK-GLADSTONE & $\underline{0.086}$ & 27 & 2.052 & 2.209 & 18 & 2.101 \\
\hline RIDEAU 3A & $\underline{0.00}$ & 27 & 2.052 & 2.633 & 28 & 2.048 \\
\hline
\end{tabular}

In this subsection, the data on arrival time of the bus were investigated thoroughly. Based on the results of two-sample-t tests, some conclusions on the bus arrival times of the two bus routes can be drawn.

- For mixed traffic bus route (i.e. bus route 1), the statistical differences of bus on time performance recorded at stops during winter and summer time are not found for all three time periods of the day (i.e. morning, noon, and afternoon). This implies that the winter does not have much influence on punctuality of the buses on a mixed bus route. They were always behind schedule. However, for Transitway (i.e. bus route 95), such differences were found for middle of the day and the afternoon.

- For the same season and for the same day, bus on-time performance was different, depending upon time periods and type of bus routes. For bus route 95, these differences were very clear. At almost all the selected stops of this bus route, the differences can be found between morning time and noon time during the winter time and between morning and afternoon time in the summer time. In the winter time, buses tend to be on-time in the noon time. During summer time, buses tend to arrive to stop later in 
the afternoon. For bus route 1 , the differences were not clear. On the average, buses were behind schedule by the same amount.

\subsubsection{Dwell Time and Passenger Activities}

According to the OC Transpo, dwell time is the time counted from door opening time to the moment when the last passenger activity is finished. In order to eliminate the abnormal dwell time that could have bad effects on the results of the analysis, the study deleted dwell times of over 180 seconds ( 3 minutes). According to OC Transpo, these are regarded as "unusual events" and therefore these data should not be included in the analysis. Figures 3.7 to 3.12 present the histograms of dwell times based on one year's observations of each time period of day for each bus route.

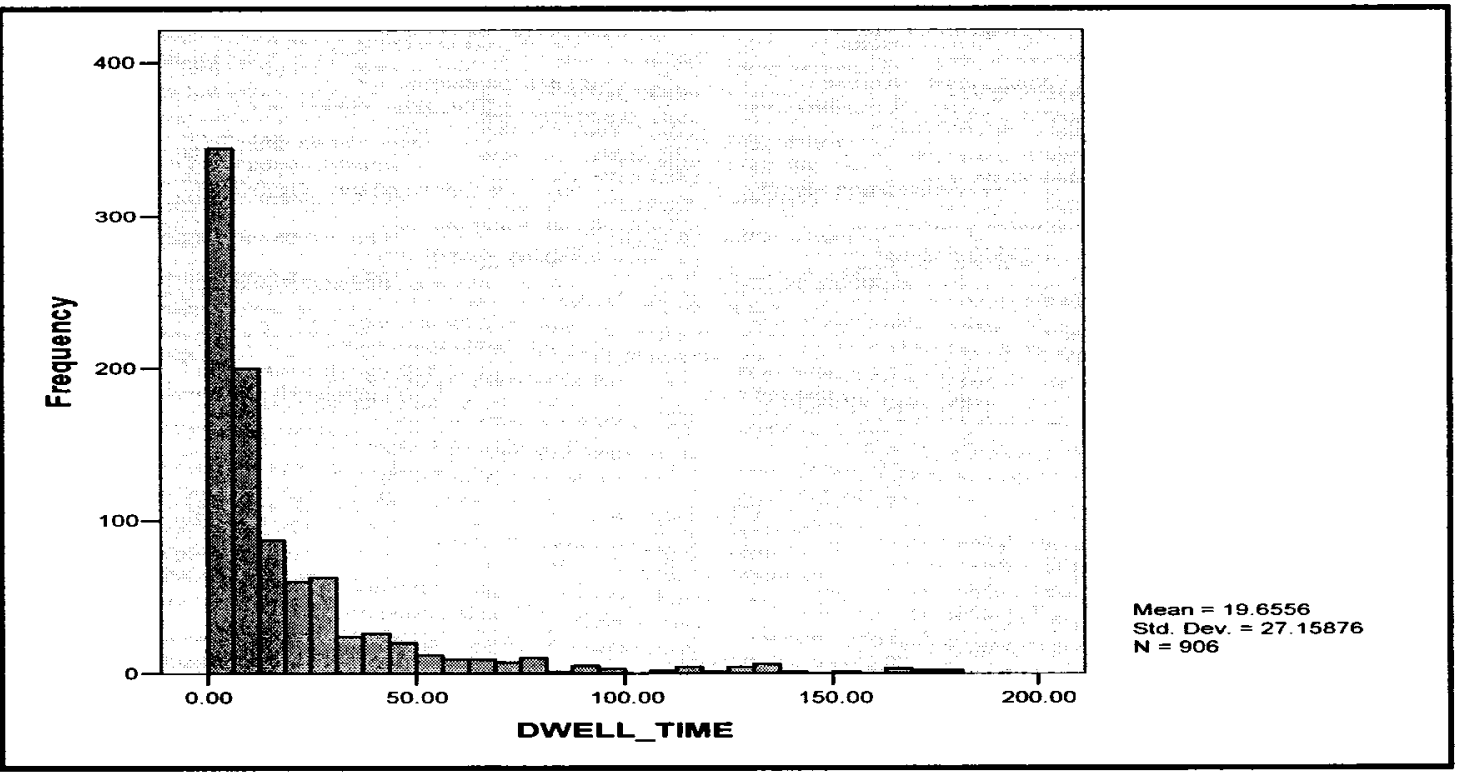

Figure 3.7: Dwell time Histogram for Route 1-Time period (8 a.m. to 10 a.m.) 


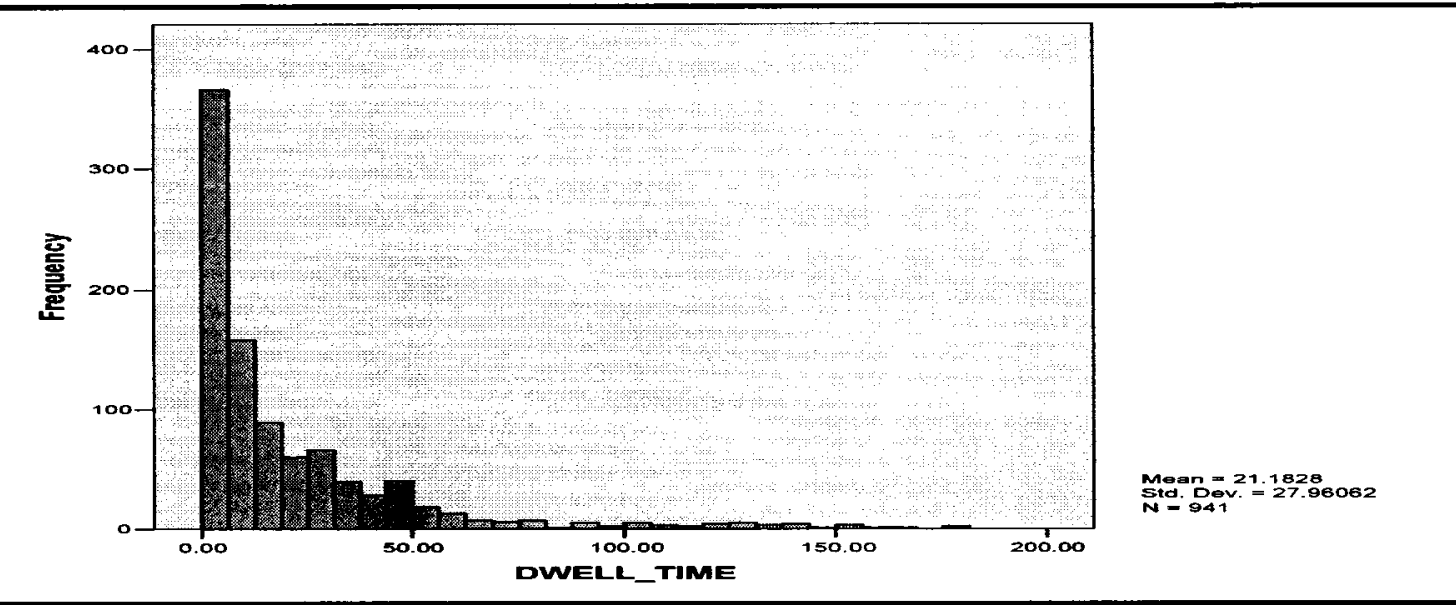

Figure 3.8: Dwell time Histogram for Route 1- Time period (12 a.m. to 2 p.m.)

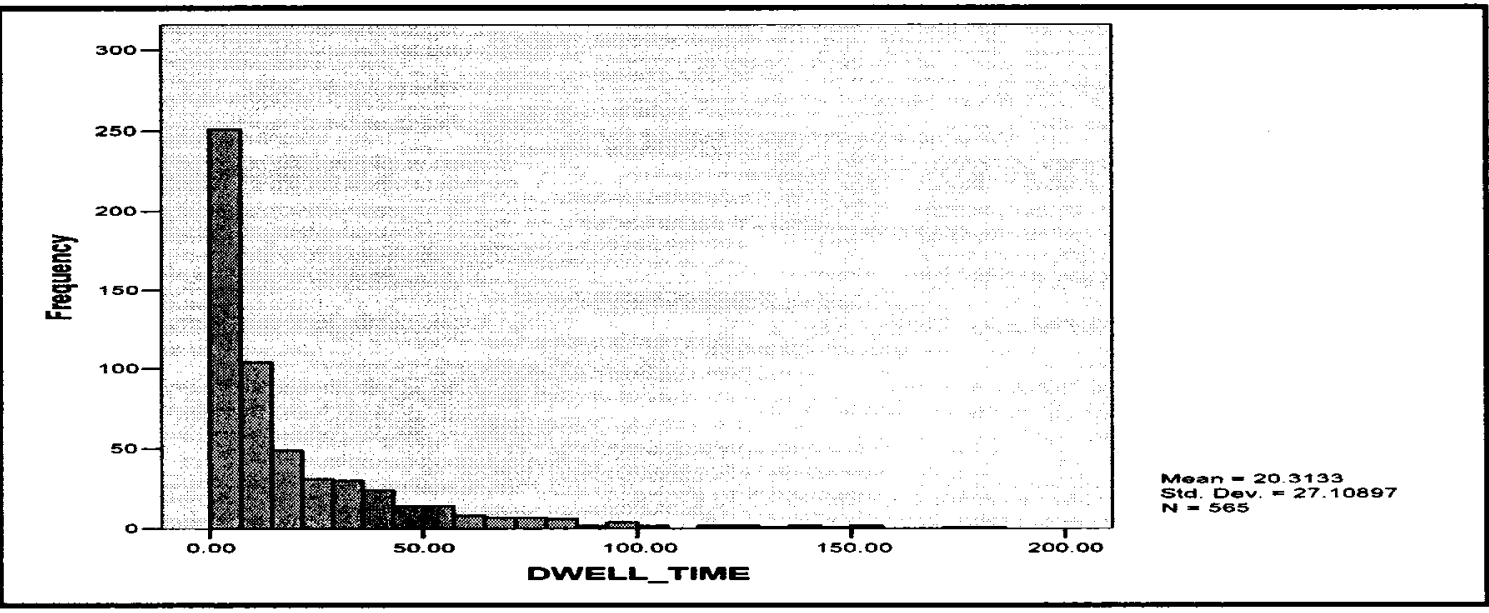

Figure 3.9: Dwell time Histogram for Route 1- Time period (4 p.m. to 6 p.m.)

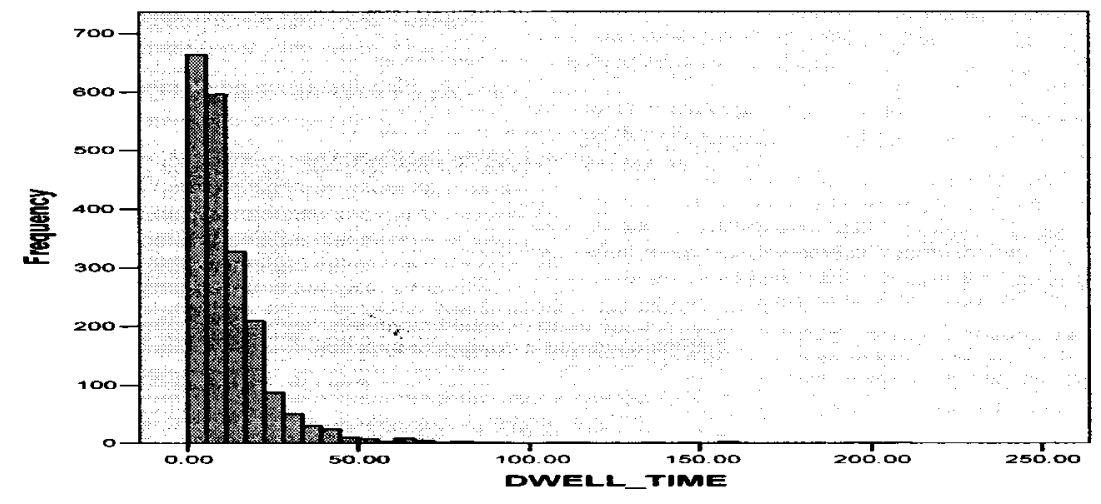

Figure 3.10: Dwell time Histogram for Route 95- Time period (8 a.m. to 10 a.m.) 

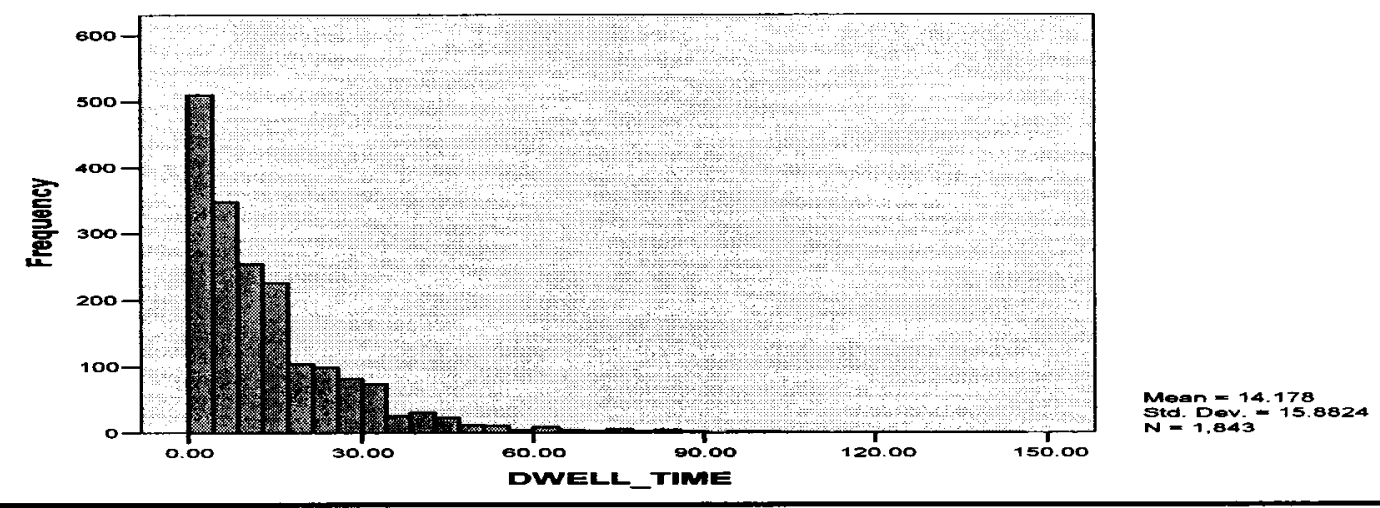

Figure 3.11: Dwell time Histogram for Route 95- Time period (12 a.m. to 2 p.m.)

As shown in the histograms, route 95 has smaller means and standard deviations than that of route 1 . As described previously, these differences reflect type of bus used and route characteristics. This means that its dwell time per stop are smaller and more stable than that of bus route 1 . The average dwell time per stop tends to be longer in the noon and afternoon compared to that of morning in both types of bus routes. It varies in the range of 12 to 17 seconds for route 95 and 19 to 21 seconds for route 1.

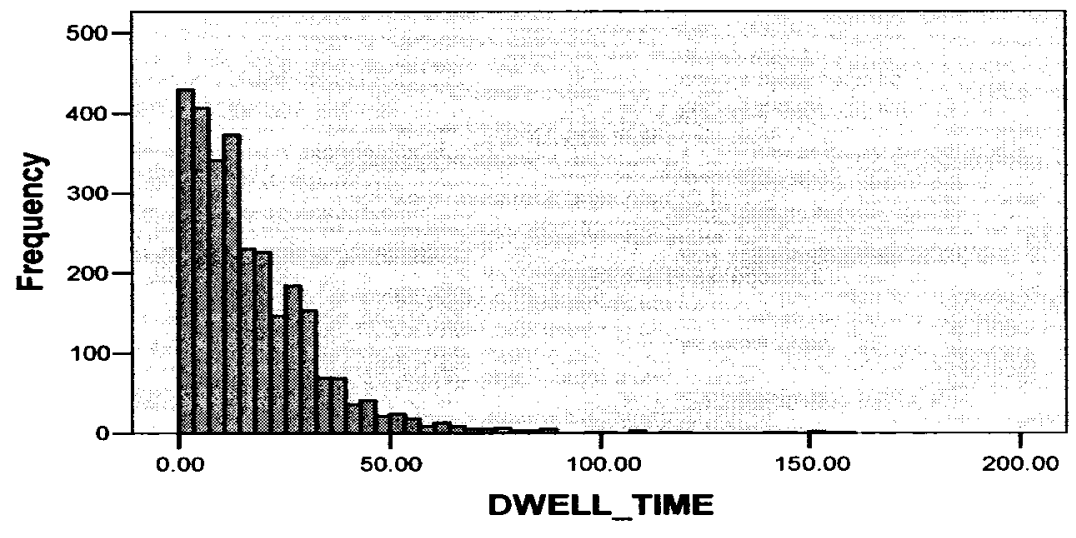

Figure 3.12: Dwell time Histogram for Route 95-Time period (4p.m. to 6 p.m.) 
Passenger activity is composed of the passenger movements through the bus doors. Most of the buses in route 95 are low-floor articulated buses with 3 doors while the buses running on route 1 are mostly rigid-body buses. Boarding passengers are required to board at front door while alighting passengers are allowed to get off the bus at all doors. However, boarding passengers can also get on an articulated bus at the rear doors in the peak-hours.

Tables 3.20 to 3.25 present the descriptive statistics of passenger activities on each bus route. As shown in the tables, the number of passengers using front door for boarding is about 2.5 times larger than that of using rear doors on average. By comparing the variables $O F F 1$ ( the number of alighting passengers using front door) in route 1 and route 95 , we can see that the ratios between the number of passengers using rear doors and the front door for alighting are about 1.2 to 1.5 for route 1 , but about 5.8 to 6.3 for route 95 . Obviously, passengers in rigid body buses tend to use front door for alighting more frequently compared to that of articulated buses.

Table 3.20: Descriptive Statistics of Passenger Activities. Route 1- Time period: (8 a.m. to 10 a.m.)

\begin{tabular}{|l|c|c|c|c|c|}
\hline Variable & $\mathbf{N}$ & Minimum & Maximum & Mean & Std. Deviation \\
\hline ON_1 & 1056 & .00 & 54.00 & 4.3163 & 6.62927 \\
\hline OFF_1 & 1056 & .00 & 19.00 & .9489 & 1.89468 \\
\hline OFF_2 & 1056 & .00 & 19.00 & .1913 & 1.12758 \\
\hline OFF_3 & 1056 & .00 & 37.00 & 1.5426 & 3.72237 \\
\hline TOTAL_ON & 1056 & .00 & 54.00 & 4.3920 & 6.72462 \\
\hline TOTAL_OFF & 1056 & .00 & 55.00 & 3.0123 & 5.66329 \\
\hline LOAD_ARR & 1056 & .00 & 71.00 & 19.2983 & 16.29168 \\
\hline TOTAL_PAS & 1056 & .00 & 57.00 & 7.5616 & 9.02708 \\
\hline LOAD_DEP & 1056 & .00 & 65.00 & 19.5019 & 16.34428 \\
\hline
\end{tabular}

Note: Variables have been defined in Table 3.5 . 
Table 3.21: Descriptive Statistics of Passenger Activities.

Route 1- Time period: (12 a.m. to 2 p.m.)

\begin{tabular}{|l|c|c|c|c|c|}
\hline Variable & $\mathbf{N}$ & Minimum & Maximum & Mean & $\begin{array}{c}\text { Std. } \\
\text { Deviation }\end{array}$ \\
\hline ON_1 & 1072 & .00 & 29.00 & 4.0606 & 5.31589 \\
\hline OFF_1 & 1072 & .00 & 19.00 & 1.3302 & 2.48977 \\
\hline OFF_2 & 1072 & .00 & 16.00 & .2407 & 1.41945 \\
\hline OFF_3 & 1072 & .00 & 27.00 & 1.7043 & 3.69738 \\
\hline TOTAL_ON & 1072 & .00 & 29.00 & 4.0886 & 5.31760 \\
\hline TOTAL_OFF & 1072 & .00 & 37.00 & 3.2752 & 5.99532 \\
\hline LOAD_ARR & 1072 & .00 & 56.00 & 15.3330 & 13.56143 \\
\hline LOAD_DEP & 1072 & .00 & 56.00 & 16.1465 & 13.76907 \\
\hline TOTAL_PAS & 1072 & .00 & 44.00 & 7.3638 & 8.80063 \\
\hline
\end{tabular}

Table 3.22: Descriptive Statistics of Passenger Activities. Route 1- Time period: (4 p.m. to 6 p.m.)

\begin{tabular}{|l|c|c|c|c|c|}
\hline Variable & Observations & Minimum & Maximum & Mean & $\begin{array}{c}\text { Std. } \\
\text { Deviation }\end{array}$ \\
\hline ON_1 & 648 & .00 & 33.00 & 4.5077 & 6.21833 \\
\hline OFF_1 & 648 & .00 & 14.00 & 1.4105 & 2.46672 \\
\hline OFF_2 & 648 & .00 & 17.00 & .1867 & 1.35414 \\
\hline OFF_3 & 648 & .00 & 19.00 & 1.7160 & 3.27176 \\
\hline TOTAL_ON & 648 & .00 & 33.00 & 4.5556 & 6.22082 \\
\hline TOTAL_OFF & 648 & .00 & 29.00 & 3.3133 & 5.48954 \\
\hline LOAD_ARR & 648 & .00 & 59.00 & 14.7469 & 13.32245 \\
\hline TOTAL_PAS & 648 & .00 & 50.00 & 7.8688 & 9.29774 \\
\hline LOAD_DEP & 648 & .00 & 58.00 & 15.9892 & 13.47623 \\
\hline
\end{tabular}

Table 3.23: Descriptive Statistics of Passenger Activities. Route 95- Time period (8 a.m. to 10 a.m.)

\begin{tabular}{|l|c|c|c|c|c|}
\hline Variables & Observations & Minimum & Maximum & Mean & Std. Deviation \\
\hline ON_1 & 2039 & .00 & 21.00 & 2.7533 & 2.94677 \\
\hline ON_2 & 2039 & .00 & 15.00 & .8524 & 1.74539 \\
\hline ON_3 & 2039 & .00 & 15.00 & .8818 & 1.76050 \\
\hline OFF_1 & 2039 & .00 & 21.00 & .7396 & 1.59007 \\
\hline OFF_2 & 2039 & .00 & 27.00 & 2.1918 & 2.98886 \\
\hline OFF_3 & 2039 & .00 & 40.00 & 2.4998 & 3.38369 \\
\hline TOTAL_ON & 2039 & .00 & 37.00 & 4.4875 & 5.33277 \\
\hline TOTAL_OFF & 2039 & .00 & 79.00 & 5.4311 & 7.14610 \\
\hline LOAD_DEP & 2039 & .00 & 97.00 & 30.2325 & 20.35251 \\
\hline LOAD_ARR & 2039 & .00 & 96.00 & 31.1761 & 17.77509 \\
\hline
\end{tabular}


Table 3.24: Descriptive Statistics of Passenger Activities. Route 95- Time period (12 a.m. to 2 p.m.)

\begin{tabular}{|l|c|c|c|c|c|}
\hline Variables & $\mathrm{N}$ & Minimum & Maximum & Mean & Std. Deviation \\
\hline ON_1 & 1843 & .00 & 23.00 & 3.6316 & 3.76717 \\
\hline ON_2 & 1843 & .00 & 17.00 & 1.3820 & 2.37809 \\
\hline ON_3 & 1843 & .00 & 15.00 & 1.3006 & 2.32106 \\
\hline OFF_1 & 1843 & .00 & 15.00 & .9078 & 1.58890 \\
\hline OFF_2 & 1843 & .00 & 23.00 & 2.6088 & 3.33336 \\
\hline OFF_3 & 1843 & .00 & 22.00 & 3.0222 & 3.96141 \\
\hline TOTAL_ON & 1843 & .00 & 41.00 & 6.3142 & 7.11558 \\
\hline TOTAL_OFF & 1843 & .00 & 48.00 & 6.5388 & 7.96463 \\
\hline LOAD_ARR & 1843 & .00 & 88.00 & 29.2702 & 14.47510 \\
\hline LOAD_DEP & 1843 & .00 & 98.00 & 29.0456 & 16.98444 \\
\hline
\end{tabular}

Table 3.25: Descriptive Statistics of Passenger Activities. Route 95- Time period (4 p.m. to 6 p.m.)

\begin{tabular}{|l|c|c|c|c|c|}
\hline Variables & $\mathrm{N}$ & Minimum & Maximum & Mean & Std. Deviation \\
\hline ON_1 & 2839 & .00 & 33.00 & 4.0775 & 4.18456 \\
\hline ON_2 & 2839 & .00 & 19.00 & 1.7263 & 2.66858 \\
\hline ON_3 & 2839 & .00 & 26.00 & 1.8859 & 2.93571 \\
\hline OFF_1 & 2839 & .00 & 17.00 & 1.0398 & 1.93940 \\
\hline OFF_2 & 2839 & .00 & 26.00 & 2.9362 & 4.11590 \\
\hline OFF_3 & 2839 & .00 & 36.00 & 3.6404 & 5.09293 \\
\hline TOTAL_ON & 2839 & .00 & 52.00 & 7.6897 & 8.05438 \\
\hline TOTAL_OFF & 2839 & .00 & 68.00 & 7.6164 & 10.34543 \\
\hline LOAD_ARR & 2839 & .00 & 104.00 & 39.6840 & 19.96143 \\
\hline LOAD_DEP & 2839 & .00 & 106.00 & 39.7573 & 21.18925 \\
\hline
\end{tabular}

The average load factors ( the number of passengers in a transit vehicle prior to stopping divided by vehicle capacity) of both bus routes are quite low at about $35 \%$ and $52 \%$ for route 1 and route 95 , respectively. On the average, there are 4 boarding passengers and 3 alighting passengers for each stop of route 1. The corresponding numbers for route 95 are 6 and 7 for each stop. 


\subsection{Summary}

In this chapter, based on the observations of a bus travel time and its components, the framework of the proposed model for bus arrival prediction and its modules were presented. Because moving time accounts for about 60 percent of total travel time and it is vulnerable to delays caused by many unforeseen stochastic traffic-related factors, it is difficult to examine the effect of each factor on moving time separately. Therefore, the proposed model aggregated moving time and relevant delays into running time. By doing so, running time can be obtained from the departure time and the arrival time recorded by the APC and AVL systems between two consecutive bus stops. Dwell time is predicted separately from running time so that the model can capture the effect of long running times to long dwell time at bus stops, which may cause the bus to be behind the schedule at the stops thereafter.

The mechanism of the whole model with its three constituent modules was presented in order to explain how the model predicts real-time bus arrivals. Before going to develop the prediction model, the APC and AVL data were collected from the OC Transpo and processed. To understand the preliminary operational characteristics of the two bus routes, namely route 95 and 1 , which were selected for modelling purposes, several descriptive statistics were discussed. In the next chapter, the first module of the model will be described. 


\section{Chapter 4}

\section{BUS RUNNING TIME PREDICTION MODULE}

\subsection{Introduction}

Observations on bus travel time components presented in the previous chapter show that running time is the key component in overall terms. Hence, if a model makes poor predictions of bus running time, this will cause the failure of the overall model itself. For this reason, almost all bus arrival prediction models and algorithms reported in literature paid most attention to running time prediction. Also, almost all of these models tried to incorporate most influencing factors in their predictions. In theory, on the basis of all influencing factors, it should be possible to make better predictions. In practice, however, this has been a difficult task because of the data availability and technical constrains.

Data availability is of most concern when a researcher attempts to develop a prediction model, given that the quality and quantity of data influences model development in a major way. Data can be divided into static data and dynamic data. The static data such as number of stops, number of intersections, link length, curve radius and so on, of course, are the simplest data that can be collected by common measurements. However dynamic data, whose characteristics vary over time such as densities, volumes and speeds of traffic stream, proportion of left turn and heavy vehicles, are not always 
available and collectable. Although, from a technical perspective, sensors embedded on the bus routes or the traffic cameras can capture these dynamic data; practically, not all the bus routes have sensors and cameras or even if this was the case, not all these data can be transmitted dynamically. This eliminates the applications of the models based on the above data to make real-time predictions where up-to-minute input data are the vital requirements.

To eliminate the difficulties of data availability, an innovative algorithm for bus running time prediction is advanced in this research in order to develop a real-time bus arrival prediction model that is based on the available APC and AVL data. The algorithm is based on a statistical pattern recognition technique where the bus operational state in the past that is most similar to a current bus state reported by the APC-AVL systems is recognized and used to predict the running time of the bus. The methodology to apply the technique to bus running time predictions and the detailed procedure are presented in the following sections.

\subsection{Problem Statement}

Running time is a stochastic process influenced by many stochastic factors. Hence, capturing the distribution of running times with these factors by using specific probabilistic formulations is difficult. No publication reporting an examination of such distributions has been found in the published literature.

The duration of time that a bus has just spent from one stop to another, by itself, reflects the sum of influences including traffic conditions. For example, if the bus was unusually behind the schedule, it could be due to an incident while the bus was running 
on the link. If the bus was late in the usual manner, this could be due to traffic surges during rush hours. If the bus was earlier than schedule time, it could be due to travel in light traffic. Intuitively, the buses running on the same link and in short headways under similar traffic conditions will tend to have similar running situations on that link. In other words, running times of buses observed around a specific time in the past, say $t$, may contain information about the running time a bus will spend at time $t$ in the future. This standpoint stimulates the applications of pattern recognition to bus running time predictions.

A pattern can be seen as a set of measurements or observations represented in a vector or matrix notation (Schalkoff, 1992, p.6). According to this definition, running times of the buses, together with other traffic data, around a specific departure time of a bus under consideration, can be considered as a pattern for the feature of interest (i.e. running time) of this bus at the time in question. In this research, the statistical pattern recognition technique was chosen to develop a model for predicting bus running times. Specifically, a nonparametric regression technique is proposed for the recognition of the bus running situations in the past that exhibit the closest similarity to the current bus running situation. These, the local neighbours, then will be used to estimate the running times of the buses.

\subsection{Nonparametric Regression Method in Statistical Pattern Recognition}

Nonparametric regression (NP) methods, or kernel-based methods, are the new developments in statistical pattern (SP) recognition (Schalkoff, 1992). They stem from curve smoothing techniques and non-linear time series methods defined in the 1970 s. 
The term nonparametric was used to differentiate these methods from the parameter regression ones where the data were used to build a series of equations with parameters resulting from parameter fittings. Various prior relations among data variables have been defined such as linear, logistic, log-linear, and so on, depending upon the data.

The nonparametric regression methods, in contrast, do not need any specific prior relation among data. Hence, there are no parameter estimates in NP regression. Instead, to forecast, the NP regression retains the data and searches through them for past similar cases. This strength makes NP regression to be a powerful method due to its flexible adaptations in a wide variety of situations.

Another advantage of the NP regression over the traditional parametric regression is that because of no parameter estimates, NP regression can adapt easily with new data added to the model while parameter regression method needs expensive parameter re-computations. This is an especially important characteristic of NP for realtime applications to predictions where new data are added to the model continuously.

Like artificial neural network (ANN) models, the NP regression models can be trained from actual data; but unlike an ANN model which is considered as a "black-box" method, the NP regression provides statistical inference and confidence interval. There are three popular types of nonparametric regression (Andersen, 2004).

- Locally Weighted Scatter plot Smoothing (LOWESS): Model fits data by local polynomial regressions and join them together. This type is most widely used in many application fields.

- Smoothing Splines: Models partition the data and fit separate piecewise regression to each section, smoothing them together where they join. 
- Generalized additive models: The extensions of the types above to specific situations such multiple and multivariate regression, and to non-quantitative dependent variables.

In this study, the use of LOWESS method is proposed as the basis for model development. The choice of this method over other methods such as spline regression is based on the task at hand. Our interest is with pointwise estimates and not with the continuous regression curve. The details of LOWESS method are discussed below.

\subsubsection{LOWESS Estimation Method}

\subsubsection{The General Functions}

LOWESS method was first introduced by Cleveland (1979) and further developed for multivariate models (Cleveland et al., 1988). In the 1979 publication, Cleveland proposed the model for bivariate data $\left(X_{1}, Y_{1}\right), \ldots,\left(X_{n}, Y_{n}\right)$ which are the independent and identically distributed (i.d.d) samples drawn from population $(X, Y)$. Given a value defined by $x_{o}$, the estimated value of $Y \mid X=x_{o}$ is calculated by the regression function $m(x)$. Cleveland supposed that function $m(x)$ can be expanded by Taylor series expansion in a neighbourhood of $x_{o}$ as follows:

$$
m(x) \approx m\left(x_{0}\right)+m^{\prime}\left(x_{0}\right)\left(x-x_{0}\right)+\frac{m^{\prime \prime}}{2 !}\left(x-x_{0}\right)^{2}+\ldots+\frac{m^{(p)}}{p !}\left(x-x_{0}\right)^{p}
$$

This polynomial is fitted locally by a weighted least squares regression minimization (Fan et al., 1996). 


$$
\begin{aligned}
& \sum_{i=1}^{n}\left\{Y_{i}-\beta_{0}-\sum_{j=0}^{p} \beta_{j}\left(X_{i}-x_{0}\right)\right\}^{j} K_{h}\left(X_{i}-x\right) \\
& K_{h}(.)=K(. / h) / h
\end{aligned}
$$

Where: $\quad h=$ bandwidth controlling the size of the local neighbourhood $K_{h}$ (.) is a Kernel function assigning weights to each datum point.

The solution of Equation 4.2 gives a series of $\stackrel{\Lambda}{\beta}_{j}, j=0, \ldots, p$.

Obviously, from Taylor expansion, we have

$$
\hat{\beta}_{j}=\frac{{\stackrel{\Lambda}{m_{j}}\left(x_{0}\right)}_{j !}}{j !}
$$

where: $\hat{m}_{j}\left(x_{0}\right)$ is an estimator of $m^{(j)}\left(x_{0}\right)$

As $\hat{m}_{j}\left(x_{0}\right)$ is defined from Equation $4.4, m^{(j)}\left(x_{0}\right)$ is also defined to estimate the entire function $m^{(j)}($.

In the matrix notation, Equation 4.2 can be denoted as

$$
\underset{\beta}{\operatorname{Min}}(\boldsymbol{y}-\boldsymbol{X} \beta)^{T} \boldsymbol{W}(\boldsymbol{y}-\boldsymbol{X} \beta)
$$

where:

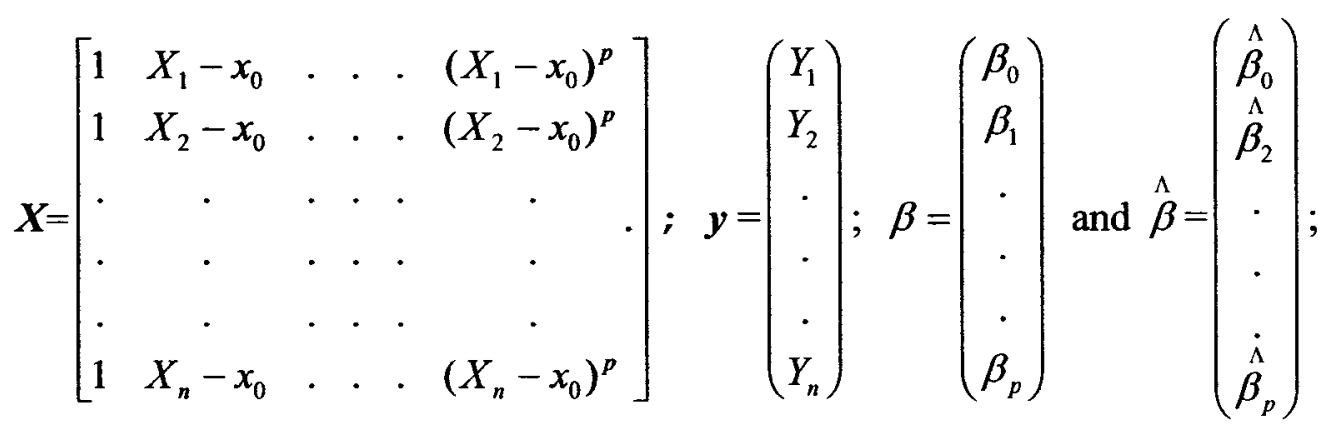


$W$ is the $n \times n$ diagonal matrix of weights

$$
W=\operatorname{diag}\left\{K_{h}\left(X_{i}-x_{0}\right)\right\}
$$

The solution of Equation 4.5 can be written as:

$$
\stackrel{\Lambda}{\beta}=\left(\boldsymbol{X}^{T} \boldsymbol{W X}\right)^{-1} \boldsymbol{X}^{T} \boldsymbol{W} \boldsymbol{y}
$$

where: $\boldsymbol{X}^{T}$ is the transpose matrix of matrix $\mathbf{X}$

Once $\hat{\beta}$ is defined, the estimation of $Y$ given $X=x_{o}$ is easily defined, or a prediction is made.

From the general equations, we can see that there is a series of issues that need to be discussed. They are: the bandwidth parameter (or smoothing parameter), the type of Kernel function, the degree of polynomial of the regression function.

\subsubsection{Bandwidth Selection}

Bandwidth plays an important role in the accuracy of the estimation made by LOEWSS. Bandwidth selection is the process of recognizing suitable neighbours around $x_{o}$, or the focal point (Andersen, 2004). In ST Recognition, the recognition of a point belonging to a neighbourhood of a focal point is usually based on the Euclidian distance from the focal point to that point, and a selected bandwidth. In essence, to find an optimal bandwidth is to balance the trade-off between variance and bias (Fan et al., 1996). A too large bandwidth can create a large modelling bias. In contrast, a very small bandwidth can cause a noisy estimate or large variance. Figure 4.1 depicts the effects of bandwidth on NP regression. 
Several methods have been developed to find an optimal bandwidth given a dataset. Generally speaking, there are two kinds of bandwidth selection: constant bandwidth and variable bandwidth. Constant bandwidth regulates a constant range of $x$ given a focal point while variable bandwidth changes upon the location of the focal point. Constant bandwidth is simple to apply but has a drawback of no neighbour recognition.

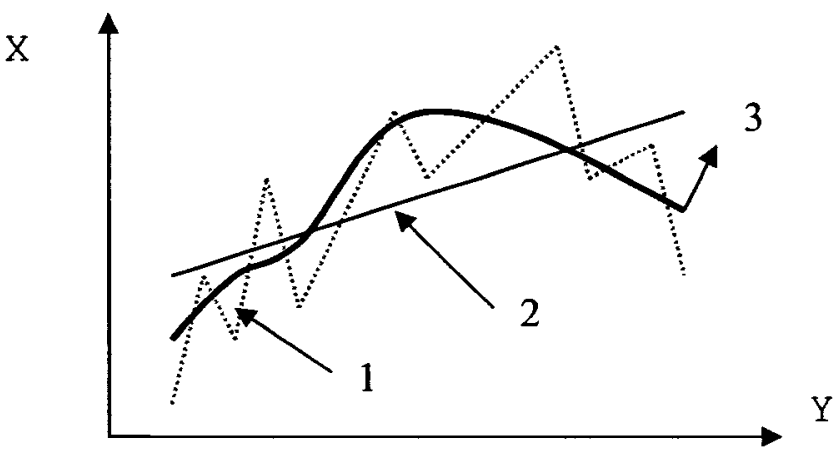

Figure 4.1: Bandwidth Selection and NP Regression

(Source: Modified from Fan et al., 1996, p.8)

Note: (1) Too small bandwidth ( $h=0$ ) causes noisy estimation and large modelling variance, (2) Too large bandwidth $(h=\infty)$ causes modelling bias, (3)As $h$ runs from 0 to $\infty$, the resulting estimate runs from the most complicated curve to the simplest curve.

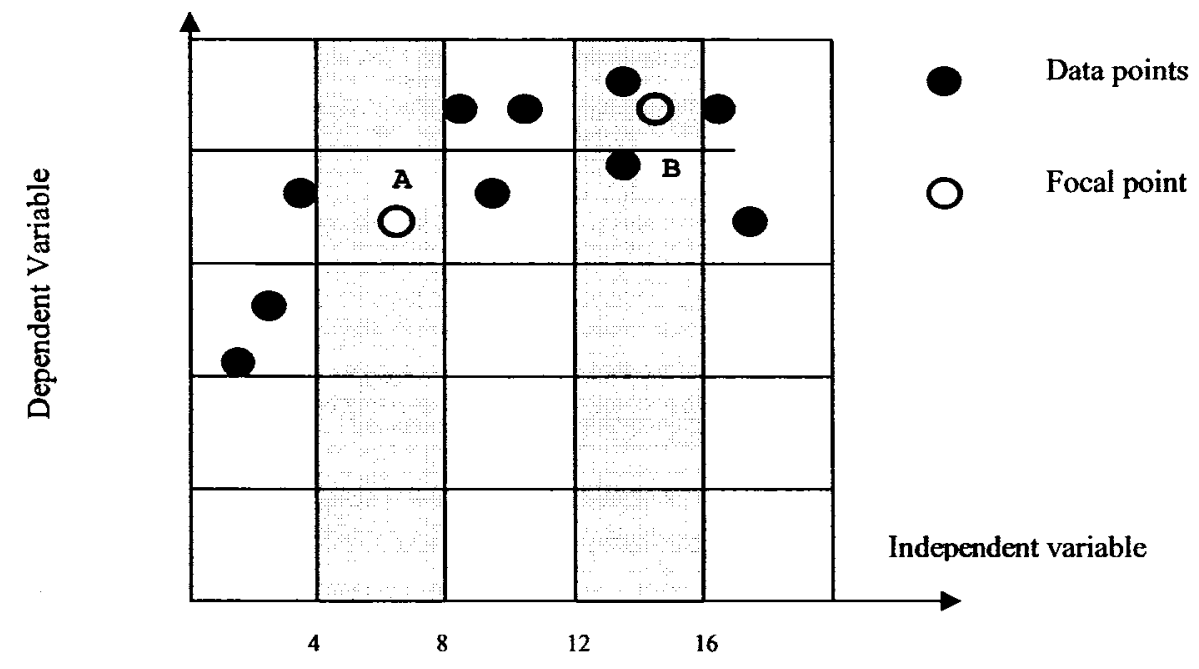

Figure 4.2: Constant bandwidth Problem

Note: If constant bandwidth is 4 units, no neighbour is found around focal $A$ whereas focal $B$ has 2 neighbours 


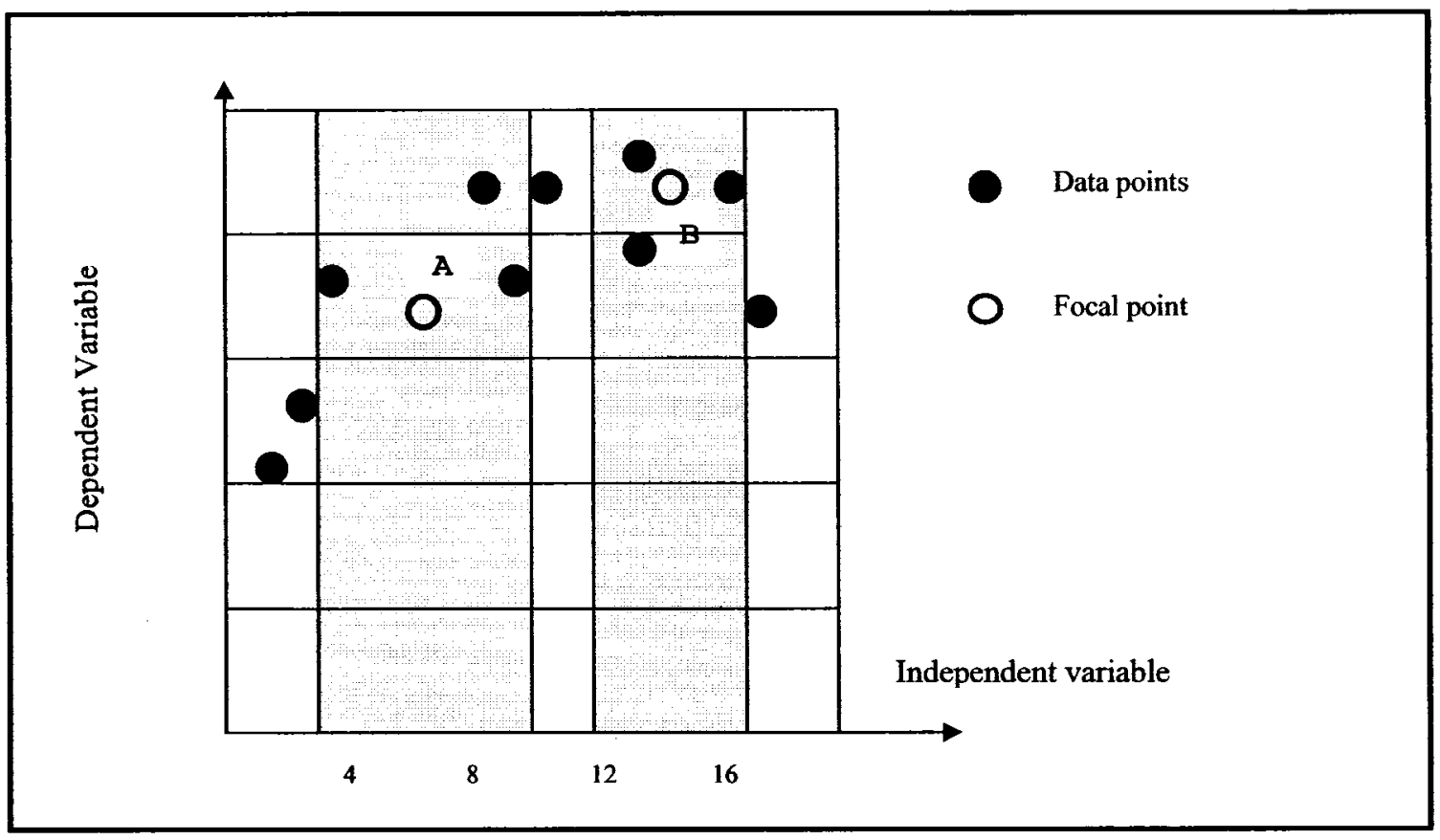

Figure 4.3: N-Nearest type of bandwidth

Note: If $N$ nearest neighbours are 3, focal $A$ always has 3 neighbours and so does the focal $B$

Therefore, the $N$-nearest neighbour type of bandwidth, where $N$ neighbours nearest to a given focal are always recognized, is more common. The difference between the two methods is shown in Figures $4.2 \& 4.3$.

In a study published in 1979 , Cleveland proposed to use the distance from the $\mathrm{r}^{\text {th }}$ nearest neighbour of $x_{0}$ as the defined bandwidth. However, the author did not discuss the value of $r$ that should be chosen. Instead, Cleveland suggested that "Instead of thinking in terms of $q$, the number of points in the neighbourhood, we think in terms of $f=q / n$, the fraction of points in the neighbourhood". Because the $f$ values were not suggested, so $f$ was chosen without a certainty that the bandwidth is optimal.

Another method to find optimal bandwidth is cross-validation (Hardle, 1990). The main feature of this method is the use of two samples drawn from the same 
population. The first sample, called the screening sample contains $n_{c}$ data points used for building a regression model. The model then is applied to the predictors for the second sample called the validation sample with $n_{v}$ data points. There are different ways to split $n_{c}$ and $n_{v}$ from population of $\mathrm{n}$ to built up different models. Out of those models, there will be several models offering the best average predictive ability. When sample size is small and the time for computing bandwidth is limited, the simplest cross-validation with $n_{v}=l$ is preferable. This method can also be called the leave-one- out method.

In the leave-one-out method presented by Hardle (1990), for each given $\left(x_{i}, y_{i}\right)$, $(n-1)$ remaining points after removing $\left(x_{i}, y_{i}\right)$ from the sample are used to build regression function $m(x)$ and to predict value of $\hat{y}_{i}=\hat{m}_{h, i}\left(x_{i}\right)$, given $x_{i}$ and a bandwidth $h$. The values of $h$ then are changed to find the optimal bandwidth $\stackrel{\Lambda}{h}$ satisfying the equations (Hardle, 1990, pp.148-153)

$$
\stackrel{\Lambda}{h}=\underset{h}{\arg \min }[C V(h)]
$$

Where CV (h) is cross-validation function

$$
C V(h)=n^{-1} \sum_{j=1}^{n}\left[\begin{array}{l}
\Lambda \\
y_{j}
\end{array}-y_{i}^{(h)}\right]^{2}
$$

Other methods to find the optimal bandwidth are: plug-in-out method (details can be seen in Hardle 1990; Fan et al., 1996; and Loader, 1999), automatic bandwidth and order selection (William 1995; Zhang 2000). These methods are based on the trade-off between variance and bias to find optimal bandwidth automatically. However, these methods are very complicated and difficult to apply in practice. 


\subsubsection{Weighting Kernel Function Selection}

The goal of Kernel function is to assign suitable weights in response to each neighbour $x_{i}$ of a focal $x_{0}$. The kernel function is a continuous and symmetric function which integrates to 1 .

$$
\int K(u) d u=1
$$

There are several possible kernel functions that can be applied for estimation. According to Loader (1999), the selection of Kernel function has small influence on the trade-off between variance and bias of the estimation.

Table 4.1: Common Kernel Functions for Univriate data

\begin{tabular}{|c|c|}
\hline Kernel Function & Analytic Form, K(x) \\
\hline Rectangular & $1 / 2$ for $|u| \leq 1,0$ otherwise \\
\hline Triangular & $1-|u|$ for $|u|<1,0$ otherwise \\
\hline Biweight & $\frac{15}{16}\left(1-u^{2}\right)^{2}$ for $|u|<1,0$ otherwise \\
\hline Tri-cube weight & $\left(1-|u|^{3}\right)^{3}$ for $|u|<1,0$ otherwise \\
\hline Normal & $\frac{1}{\sqrt{2 \pi}} \exp \left(-u^{2} / 2\right)$ \\
\hline Epanechnikov & $\frac{3}{4}\left(1-u^{2} / 5\right) / \sqrt{5}$ for $\left.|u|<\sqrt{5},\right)$, ) o otherwise \\
\hline
\end{tabular}

Note: $u=\frac{x_{i}-x_{0}}{h} ; h=$ bandwidth. Source: Webb Andrew (2002), p.109.

\subsubsection{Degree of Polynomial Regression}

The selection of degree of polynomial is the balance between computational ease and the need to reproduce patterns in data. Cleveland (1979) suggested using linear regression for adequate smoothing and computational ease. 


\subsection{Modelling Bus Running Time Prediction by Using LOWESS Method and APC - AVL Data}

Before applying LOWESS method and AVL and APC data for modelling bus running time prediction, it is necessary to determine the parameters for the LOWESS method and the APC-AVL data that will be used in the model.

\subsubsection{APC and AVL Data}

As the purpose of this research is to develop a model capable of predicting running time by using APC and AVL data, it is necessary to discuss the data being used in the model. Data collected by the APC and the AVL systems were shown in Table 3.5. Actual running times from one stop to another stop can be obtained in two ways: subtracting the arrival time of the bus at the stop and the actual departure time of that bus at the stop right before; or summation of the moving time, the stationary and the stopand-go time of the bus. These running times are used in modelling.

\subsubsection{Parameter Selection}

The role of each parameter in LOWESS method has been presented in the previous parts of this chapter. To apply this method for modelling real-time bus running time predictions, the following parameters and assumptions are used. 
- $\quad$ Bandwidth selection method: The leave-one-out method is chosen because of its ease in computation while it can maintain an automatic optimal bandwidth. Hence, it is suitable for real-time estimations.

- Kernel function selection: The Tri-cube weight function is selected.

- Degree of polynomial regression function: The linear regression is proposed.

\subsubsection{Pattern Selection}

Assume that bus $k$ leaves stop $i$ at time $D p(k)_{\text {actual, } i}$ We have to predict running time the bus will spend to arrive at the next stop $i+1, r_{0}(k)$. By that time $D p(k)_{a c t u a l, i}$, the running times of the previous buses ( e.g., $k-1, k-2, \ldots, k-d$ ) in the link bounded by stop $i$ and stop $(i+1)$ have been collected. Let us denote these running times as $r_{0}(k-1), r_{0}(k-$ 2), ..., $r_{0}(k-d)$. Now, the running time being predicted $r_{0}(k)$ is presented by pattern $\boldsymbol{R}_{0}$

$$
\boldsymbol{R}_{0}=\left[r_{0}(k-1), r_{0}(k-2), \ldots, r_{0}(k-d)\right]^{\mathrm{T}}
$$

Where:

$d=$ the number of previous buses that should be taken into account in making the running prediction of the current bus.

Also in the past, each running time experienced by bus $k$ in the link $(i+i+1)$ is also presented by its own pattern whose elements are defined similarly above. So we have $\boldsymbol{R}_{l}$, $\boldsymbol{R}_{2}, \ldots, \boldsymbol{R}_{m}$ patterns, representing the corresponding running times of bus $k$ as recorded in the past $r_{1}(k), r_{2}(k), \ldots, r_{m}(k)$.

$$
\begin{aligned}
\boldsymbol{R}_{j} & =\left[r_{j}(k-1), r_{j}(k-2), \ldots, r_{j}(k-d)\right]^{\mathrm{T}} \\
(m & =\text { number of observations collected in the past, } j=1 \text { to } m) .
\end{aligned}
$$


To predict running time of bus $k$ at current time with a given pattern $\boldsymbol{R}_{0}$, the following steps are developed to recognize the running times of bus $k$ in the past $r_{j}(k)$ which have the most similar patterns to pattern $\boldsymbol{R}_{0}$.

\subsubsection{Pattern Recognition}

The common way that is applied in SP recognition so as to recognize and classify features in the same group, is based on the Euclidian Distances and a selected bandwidth. Therefore, this step includes two sub-steps: calculate the Euclidian distances from pattern $\boldsymbol{R}_{0}$ to the other patterns $\boldsymbol{R}_{1}, \boldsymbol{R}_{2}, \ldots, \boldsymbol{R}_{m}$, and recognize the neighbourhood.

\subsubsection{Euclidian Distance Calculation}

The Euclidian distance between patterns $R o$ and $R j(j=1$ to $m$ ) can be calculated as follows:

$$
u_{j}=\sqrt{\left[r_{j}(k-1)-r_{0}(k-1)\right]^{2}+\left[r_{j}(k-2)-r_{0}(k-2)\right]^{2}+\ldots+\left[r_{j}(k-d)-r_{0}(k-d)\right]^{2}}
$$

\subsubsection{Finding Optimal Bandwidth by Leave-One-Out Method}

A running time recorded in the past $r_{j}(k)$ will be recognized as the neighbour of the one being predicted $r_{0}(k)$, called the focal point, when its Euclidian distance to the focal point is smaller than a regulated bandwidth. 


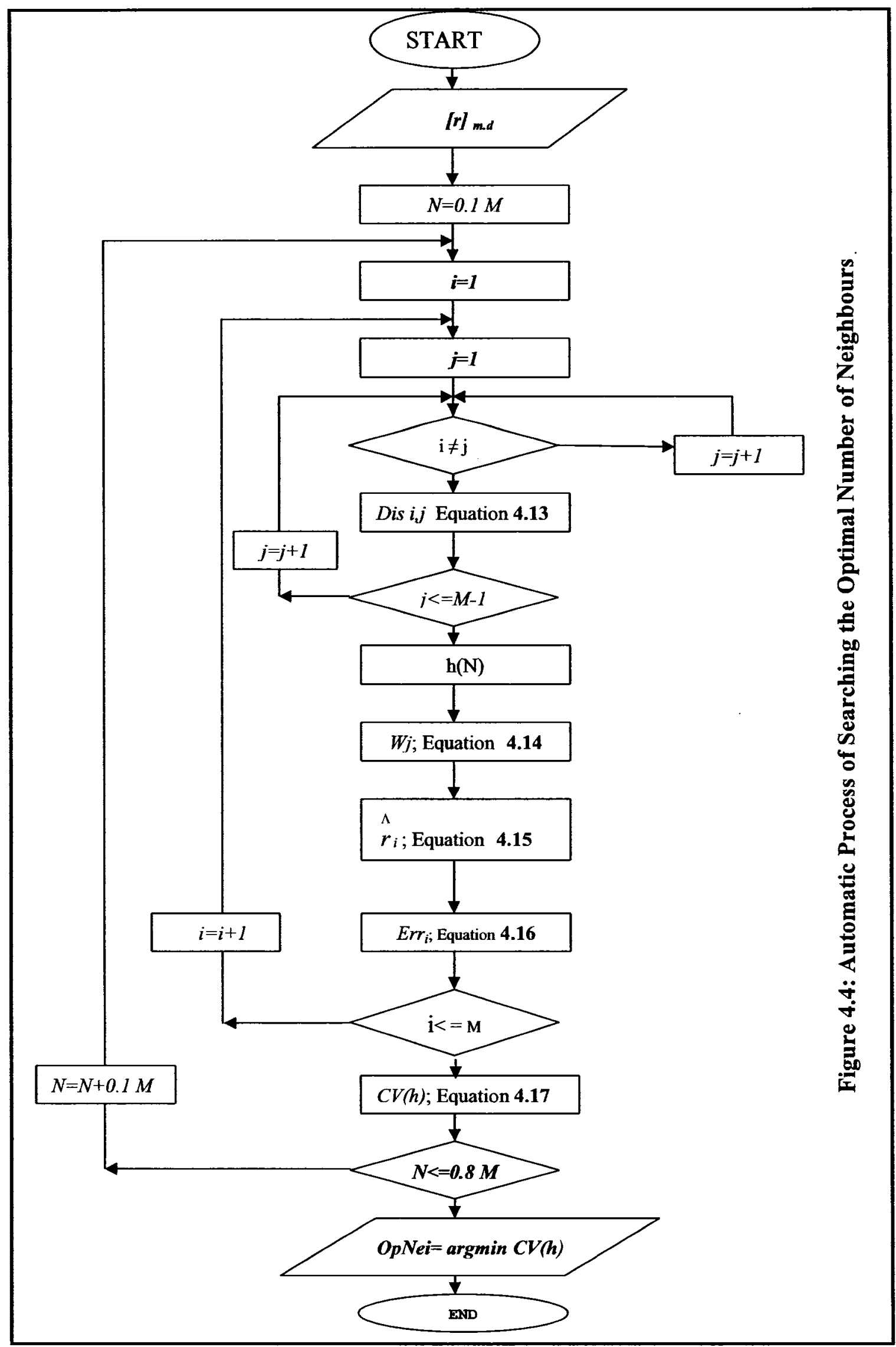


The distances have been defined by Equation 4.12 for all running times. Now we define the bandwidth by using leave-one-out technique presented by Hardle (1990).

We have $m$ observations of running times $r_{j}(k)$ from the past and the corresponding pattern for each, $\boldsymbol{R}_{j}(k)$. Following the algorithm suggested by Hardle, and applying Nadaraya-Watson kernel (shown on the next page) estimation for the sake of shortening the time to find the optimal bandwidth, the following steps shown in Figure 4.4 are pursued.

Regulate the initial $N$-nearest neighbours as $0.1 \mathrm{~m}$ (for example, if $\mathrm{m}=100$ observations, so $\mathrm{N}=10$, meaning that 10 neighbours will be around a focal point) and the increment for each step is $0.1 \mathrm{~m}$.

FOR $N=0.1 \mathrm{~m}, N=N+0.1 \mathrm{~m}$ TO $M=0.8 \mathrm{~m}$ DO (LOOP1) FOR $i=1$ to $M$ do (LOOP 2)

1. Consider the running time $r_{i}(k)$ as the focal point, $(i=1$ to $m)$. It should be noted that it is represented by a pattern namely $\boldsymbol{R}_{i}(k)=\left[r_{i}(k-1), r_{i}(k-2), \ldots, r_{i}(k-d)\right]^{\mathrm{T}}$.

2. Use (m-1) remaining running times to build a $N$-nearest neighbour regression to predict the value of $r_{i}(k)$, as follows:

- Calculate the Euclidean distances from the remaining running times to the focal $r_{i}(k)$ as:

- Calculate the Euclidian distance

$$
\begin{aligned}
& d i s_{j}=\sqrt{\left[r_{j}(k-1)-r_{i}(k-1)\right]^{2}+\left[r_{j}(k-2)-r_{i}(k-2)\right]^{2}+\ldots+\left[r_{j}(k-d)-r_{i}(k-d)\right]^{2}} \\
& \quad \text { with }(i \neq j)
\end{aligned}
$$

- Arrange the $d_{i s}$ in the increasing order 
- Select the $N^{\text {th }}$ smallest of the arrangement above. The bandwidth is the distance from the focal to the $N^{\text {th }}$ nearest neighbour. Let us call that value as $h(N)$.

- Calculate the weight by applying tri-cube weight Kernel function:

$$
\begin{aligned}
& W(j)=\frac{70}{81}\left(1-\left|\frac{\text { dis }_{j}}{h(N)}\right|^{3}\right)^{3} \cdot \mathrm{I}_{[0,1]} \\
& \text { Where: } \mathrm{I}_{[0,1]}=1 \text { if } 0 \leq \mid \text { dis }_{j} / h(N) \mid<1 \text { otherwise } \mathrm{I}_{[0,1]}=0
\end{aligned}
$$

- For the sake of computational ease, the predicted value of $r_{i}(k)$ made at $h(N)$ is computed by the Nadaraya-Watson kernel estimation.

$$
{ }_{r_{i}}(k)=\frac{\sum_{j=1, j \neq i}^{m-1} W_{j} r_{j}(k)}{\sum_{j=1, j \neq i}^{m-1} W_{j}}
$$

- Calculate the prediction error made at $h_{(N)}$ as :

$$
\operatorname{Err}_{i}(k)=\left[\hat{r}_{i}(k)-r_{i}(k)\right]^{2}
$$

\section{END LOOP 2}

3. Calculate the Cross-validation function

$$
C V(h)=\frac{\sum_{i=1}^{m} \operatorname{Err}_{i}(k)}{m}
$$

\section{END LOOP 1}

Determine the optimal number of neighbours

$$
O p N e i=\underset{h}{\arg } \operatorname{MIN}\{C V(h)\}
$$




$$
H=d i s_{O p N e i}
$$

Where: dis OpNei $_{\text {is }}$ the Euclidian distance from the focal point to the smallest Opnei $i^{\text {th }}$ neighbour.

\subsubsection{Recognition of the Neighbours}

The Euclidian distances (Eq. 4.12) and the optimal band width $H$ (Eq. 4.18B) have been defined. Hence, given focal point $r_{0}(k)$ and its pattern $\boldsymbol{R}_{0}$ ( see Eq.4.11A), a point $r_{j}(k)$ will be recognized as the neighbour of $r_{0}(k)$ by applying Tri-cube weight kernel function as follows:

Calculate the weight $K_{j}=\frac{70}{81}\left(1-\left|\frac{u_{j}}{H}\right|^{3}\right)^{3} I_{[0,1]}$

Where: $I_{[0, I]}=1$ if $0 \leq\left|u_{j} / H\right|<1$ otherwise $I_{[0,1]}=0 ;(j=1$ to $m)$

- From Equation 4.19, we can see that if $r_{j}(k)$ has a distance larger than the bandwidth $\mathrm{H}$, the weight assigned for that point is zero, meaning that $r_{j}(k)$ does not belong to the neighbourhood of focal $r_{0}(k)$. Otherwise, it is a neighbour of $r_{0}(k)$. The influence of this neighbour on the focal point is taken into account by a kernel weight $K_{j}$ depending upon its location.

\subsubsection{Prediction}

When well defined, the weighted neighbours will be used as independent variables of a polynomial regression in order to estimate the dependent variable which is 
being predicted. In our model, the running time of bus $k$ being predicted is the dependent variable (i.e., $\left.r_{0}(k)\right)$. The weighted neighbours of this dependent variable are the historical running times recorded in the past (i.e., $\left.r_{j}(k)\right)$ and recognized by the steps mentioned above. Although a higher degree of polynomial regression results in better predictions, the polynomial regression in this study is proposed to be linear regression for the sake of balancing computational ease and adequate prediction (Cleveland et al., 1979).

Equations from 4.2 to 4.7 present the procedure for predicting a univariate variable by using weighted least-square regression minimization. In our case, the prediction is univariate response but its pattern is a multi-dimensional vector. Therefore, the procedure above has been further developed by applying the works of Fan et al., (1996) for multivariate regression as follows.

- Weighted least square regression function has similar form as for Equation 4.5

$$
\min (y-X \beta)^{T} W(y-X \beta)
$$

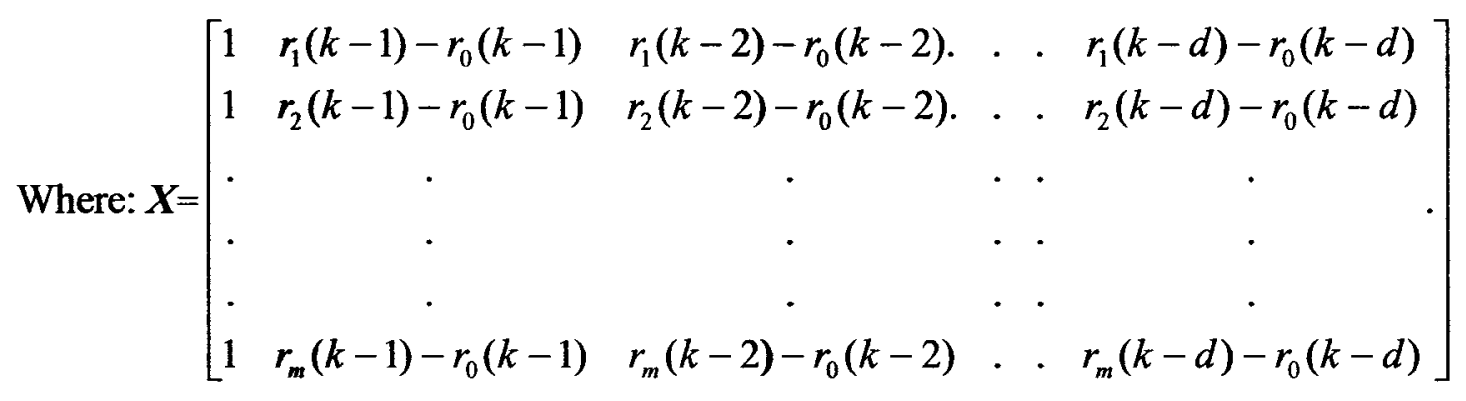

$$
\boldsymbol{y}=\left(\begin{array}{c}
r_{1}(k) \\
r_{2}(k) \\
\cdot \\
\cdot \\
\cdot \\
r_{m}(k)
\end{array}\right) ; \quad \beta=\left(\begin{array}{c}
\beta_{0} \\
\beta_{1} \\
\cdot \\
\cdot \\
\cdot \\
\beta_{d}
\end{array}\right) \quad \text { and the estimated values of beta } \hat{\beta}=\left(\begin{array}{c}
\Lambda \\
\beta_{0} \\
\beta_{1} \\
\cdot \\
\cdot \\
\dot{i} \\
\beta_{d}
\end{array}\right)
$$


$W$ is the matrix of weights defined as below

$$
\boldsymbol{W}=\left[\begin{array}{cccccc}
K_{1} & 0 & 0 & 0 & 0 & 0 \\
0 & K_{2} & 0 & . & . & . \\
. & 0 & K_{3} & 0 & . & . \\
. & . & 0 & . & 0 & . \\
. & . & . & 0 & . & 0 \\
0 & . & . & . & 0 & K_{m}
\end{array}\right]
$$

Where: $K s^{\prime}$ values are computed in Equation (4.19).

- The solution of Equation 4.20 is

$$
\stackrel{\Lambda}{\boldsymbol{\beta}}=\left(\boldsymbol{X}^{T} \boldsymbol{W} \boldsymbol{X}\right)^{-1} \boldsymbol{X}^{T} \boldsymbol{W} \boldsymbol{y}
$$

The predicted value of $r_{0}(k)$ or the running time that bus $k$ will likely spend in the $\operatorname{LINK}_{i_{-} i+1}$ is

$$
r_{0}(k)=\hat{\beta}_{0}+\sum_{j=1}^{d} \hat{\beta}_{j}, r_{0}(k-j)
$$

\subsubsection{Update Prediction}

The mechanism of how the bus arrival time prediction model updates its predictions was discussed in the previous chapter. As described earlier, when the bus arrives at the next stop, new data are collected and transmitted to the control center. These data then will be used by this module to predict running times and for all downstream links. The updating process follows the same steps above as noted from pattern recognition to prediction. Each time the bus arrives at a stop, if there are $M$ links between the current stop (i.e. the stop the bus has just arrived) and the last stop, the RTM will be applied $M$ times for predicting the running time that the bus will likely spend on each link. 


\subsection{Summary}

In this chapter, an innovative algorithm to predict running times of bus transit is developed and its details are noted. The methodology applied in this algorithm is based on the LOWESS method, a method in the family of Statistical Pattern Recognition. The reasons for choosing this method are as follows: (1) it is a powerful method because it is not based on any specific assumptions on the relationship of variables. (2) The predictions made by LOWESS method are based on the local historical database (both past and current data base) which was recorded around the time the prediction is being made, so the method can capture the surges in traffic stream during peak hours as well as the changes due to traffic incidents.

To predict running time by applying LOWESS-based method, four steps were proposed: (1) pattern selection, (2) pattern recognition, (3) prediction, and (4) updating of prediction. Of the four steps, the second step is the most elaborate. This includes a procedure from defining Euclidian distances, finding optimal bandwidth by using leaveone-out method, and recognizing the neighbours. After recognition, the weighted neighbours are used in predicting running times by applying multivariate linear regression.

The prediction performance of this module will be presented in chapter 6 . 


\section{Chapter 5}

\section{BUS DWELL TIME PREDICTION MODULE}

\subsection{Introduction}

From the transit passenger point of view, one would argue that the predictions of dwell times, departure times and the number of passengers are not what a passenger wants to know. This standpoint is true; however, these predictions contribute to the accuracy of announced arrivals that passengers are interested in. Although dwell time accounts for a smaller portion of total delay (12-26\%) compared to that of running time (48-75\%), this cannot be neglected, especially when the buses serve high density population areas (Levinson, 1983).

As opposed to the above point of view, bus transit providers have considered that data on passenger activities at each stop, and the number of on-board passengers are extremely important for analyzing ridership and the relationship between passenger loading, running times and on-time performance (Pile et al., 1998). In terms of real-time control, the predicted number of passengers boarding and alighting at a stop can help bus dispatchers to have suitable pro-active solutions once a crowded bus stop is detected in advance or the knowledge that bunching is likely to occur.

Dwell time is influenced by many factors belonging to two groups. The first group relates to passenger activities such as a number of passengers boarding and 
alighting, types of passengers (e.g., age, physical health, sex). The second group relates to bus service activities such as types of fare collection, number of doors, seat-capacity, type of bus (e.g., rigid-body bus, articulated bus, low-floor bus or high-deck bus), and service frequencies. Before the invention of the APC system, dwell time analysis was limited due to time consuming and highly labor-intensive manual counting. The APC systems, at present, are creating great potential for studies on dwell time by providing much data not only in quantity but also in quality. This also enables the applications of real-time prediction method for predicting bus passenger activities and analyzing delays.

The proposed real-time dwell time prediction module described in this chapter is one part of the entire proposed model developed for predicting bus arrivals. Specifically, predicted dwell times are the chains to link a pair of predicted running times detailed in chapter 4 . Thus, this module enables the entire model to predict arrival times and departure times of buses at all stops. This chapter is organized as follows. First, some drawbacks of previous dwell time prediction models are discussed. Second, four proposed sub-modules are then described in detail and integrated in a complete bus dwell time prediction module. Last, the summary closes the chapter.

\subsection{Bus Dwell Time Prediction Module}

\subsubsection{Discussions on the Previous Works}

A number of problems uncovered in the previous works on dwell time prediction require further discussions: 
- As shown in the literature review, it was recognized that the number of boarding and alighting passengers is the vital key to determine the dwell time. However, no discussion on how to predict these numbers is found except in the works of Farhan (2002) and Shalaby et al., (2004). To predict dwell time, the previous models require the values of the number of passenger boarding and alighting at stops. Unfortunately, these values were not available and need to be predicted as well. Therefore, it is reasonable to say that the number of predicted alighters and boarders at a stop is the root of dwell time prediction if one want to know dwell time in advance. Once the number boarding and alighting passengers has been predicted, all the previously noted models can be used to predict dwell time. Otherwise, they can be used only for off-line estimation purposes when the information on passenger activities of buses is available.

- The assumption that a long headway or a long service frequency can result in a long dwell time at downstream stops was widely accepted and used by many authors (Koutsopoulos et al., 1985; Lin et al., 1999; Ding et al., 2000; Chien et al., 2000; Fu and Liu, 2003; Shalaby and Farhan, 2004). However, the relationship between dwell time and headway was still underestimated. Instead, this assumption is quantified by a simple equation shown below:

$$
d w e l l=s f . \text { apar. } t_{b}
$$

Where:

$$
\begin{aligned}
& d w e l l=\text { Dwell time }(\mathrm{sec}) \\
& s f \quad=\text { Service frequency or headway }(\mathrm{sec})
\end{aligned}
$$


apar $=$ Average passenger arrival rate (passenger/sec) where arrival rate distribution is usually assumed as a Poisson distribution or uniform distribution;

$t_{b}=\quad$ Average boarding time per passenger ( $\left.\mathrm{sec} / \mathrm{pas}\right)$

This assumption has some drawbacks:

- First, as shown in Equation 5.1, these models assume that arrival passenger flow is uniform. This assumption may be erroneous in case of long headways where passengers tend to arrive at stops around the announced arrival times (Abkowitz et al., 1986).

- Second, based on the assumption above, one could argue that if no passenger comes to the stop during a headway time, the value of average passenger arrival rate will be zero. This will result in a zero value of dwell time, meaning that the bus will not stop at that stop because there is no boarding demand. However, there may be an alighting demand and a stop may be made with a positive dwell time. Hence, the above assumption should be modified to capture this case.

- It is the fact that boarding passengers at the front door usually wait for the last alighting passenger before getting on the bus. If the bus has two doors and some passengers get off at the front door, dwell time may be lengthened. Therefore, the passenger decision on taking front door for getting off the bus will play an important role in predicting dwell time. If the bus has more than 2 doors (e.g., articulated bus), where passengers can board in rear doors, the dwell time will be the time for serving the busiest door. However, which door 
will be the busiest door in the above cases and what is the role the passengers play in determining dwell time are still open questions.

\subsubsection{The Dwell time Prediction Module (DTM)}

The DTM proposed in this study is dynamic in a manner that it is based on the real-time predicted boarding and alighting passengers at a stop. In order to do this, two of its sub-modules, namely Real-time Boarding Passenger Prediction (RPSM) and Realtime Alighting Passenger Prediction (RASM), have been developed separately. These predictions then are used as the inputs for two other sub-modules called Regression (RESM) and Busiest door (BDSM). The RESM is based on the collected data from AVL and APC systems in the OC Transpo. In this sub-module, the relationship between dwell time and a series of variables (e.g. the number of passengers, bus type, on time performance, and so on) is examined in the form of several statistical regressions. The BDSM is based on the equation suggested by HCM 2000 (Eq. 2.2) with modifications in order to find the busiest door.

By combining the sub-modules, two methods (A\&B) are proposed in this research for predicting dwell time. Method A is the combination of BPSM, APSM, and RESM. Method B includes BPSM, APSM, and BDSM. Figures 5.1 and 5.2 depict the frameworks of the two methods. 


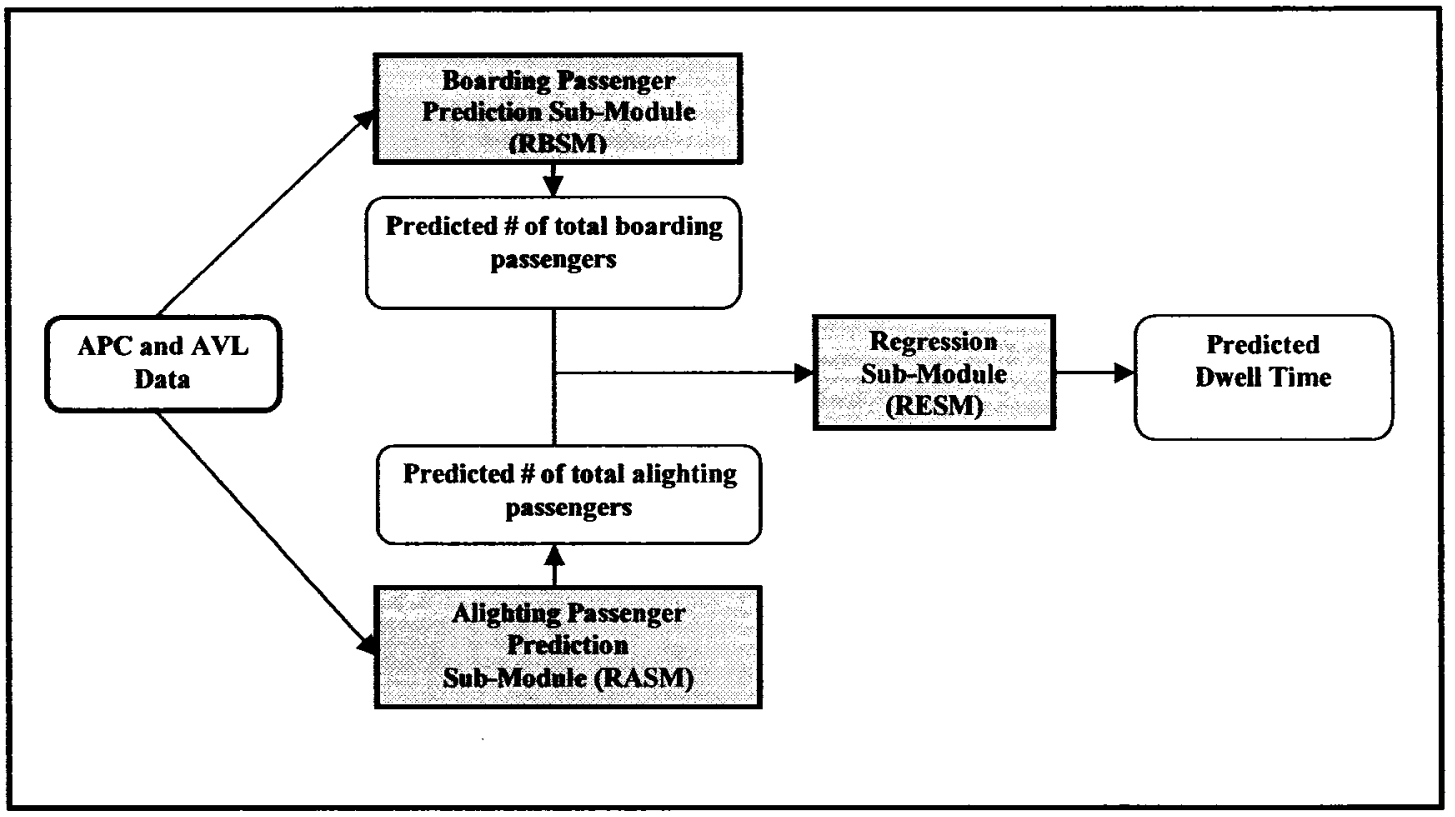

Figure 5.1: Dwell time Prediction Module. Method A

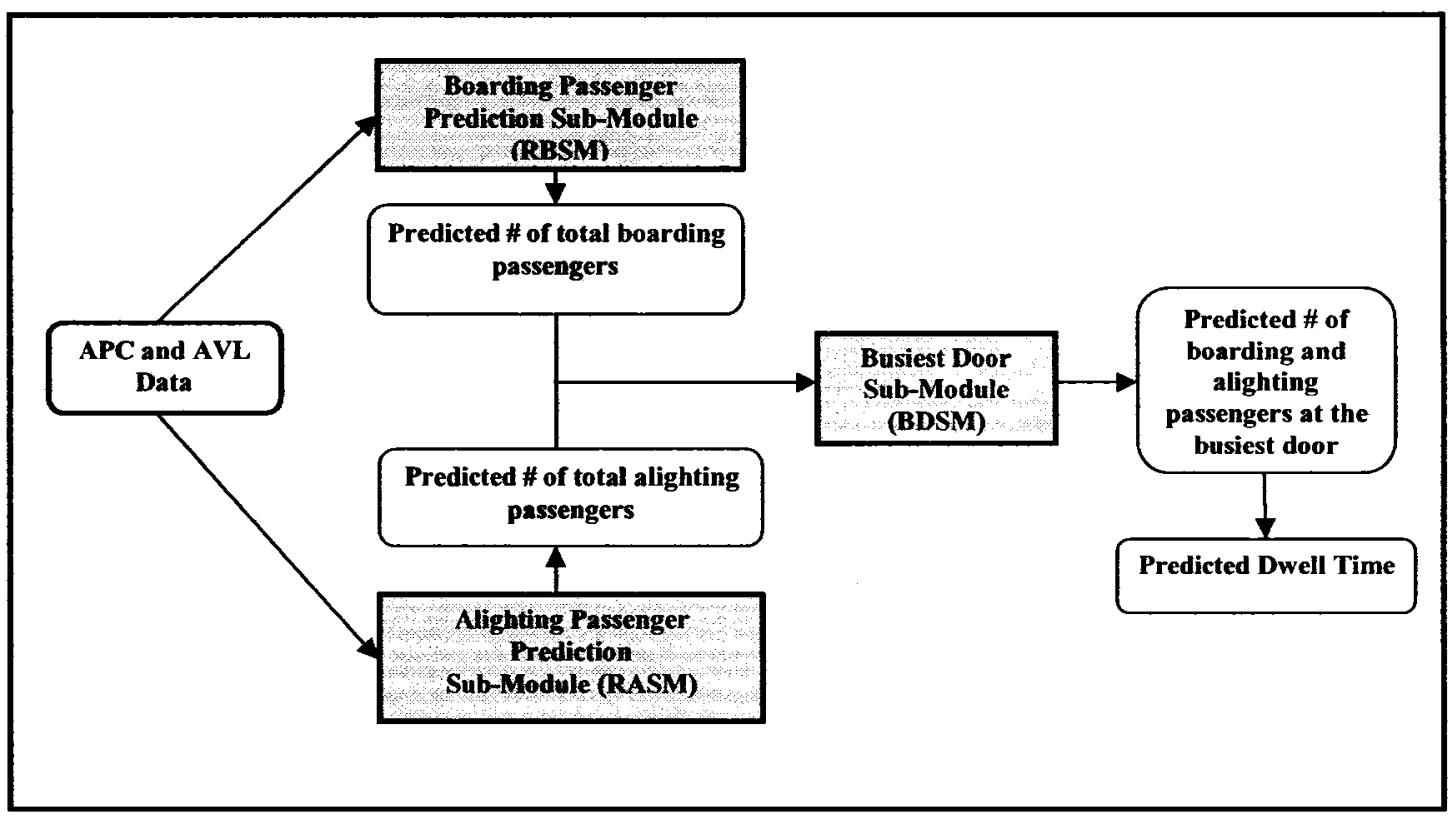

Figure 5.2: Dwell time Prediction Module. Method B

The details of each sub-module are presented separately in the following sections and the better prediction method is identified. 


\subsubsection{Real-time Boarding Passenger Prediction Sub-Module}

The LOWESS-based method developed in chapter 4 is used here to predict boarding passengers at a stop. The steps to obtain a prediction on the number of passengers boarding bus $(k)$ at stop $(i+1)$ are presented below.

\subsubsection{Parameter Selection}

The method applied to select parameters in chapter 4 is used in this chapter as follows: leave-one-out method for optimal bandwidth selection; Tri-cube weight function to recognize the neighbours, and Nadaraya-Watson kernel function for prediction.

\subsubsection{Pattern Selection}

As discussed earlier, there is an erroneous assumption on the distribution of passenger arrival flow at a stop. In this research, the author intended to capture the relationship between headway and the number of boarding passengers at a downstream stop without any prior mathematical assumption about this relationship.

Assume that bus $k$ leaves stop $i$ at time $D p(k)_{a c t u a l, i}$. We have to predict the number of passengers boarding on bus $k$ when it arrives at the next stop $i+1$. Let us denote this variable as $b_{0}(k)$. At time step $D p(k)_{a c t u a l, i}$, data on the number of boarding passengers on previous buses at stop $(i+1)$ have been collected. We denote these numbers as $b_{0}(k-1)$, $b_{0}(k-2), \ldots, b_{0}(k-d)$. 
Also at that time step, running times of the previous buses (e.g., $r_{0}(k-1)$, $\left.r_{0}(k-2), \ldots, r_{0}(k-d)\right)$ in the link bounded by stop $i$ and stop $(i+1)$ have been available. We already have the predicted running time that bus $k$ on the $L I N K_{i_{-} i+1}$. This value is $r_{0}(k)$ in Equation 4.22 (Chapter 4).

In order to take the relationship between the bus headway and the number of boarding passengers without any prior assumption on it, we consider the number of boarding passengers on the previous bus at stop $i+1$ as well as the recorded headway of this bus as the elements of a pattern defining the number of boarding passengers being predicted when bus $k$ arrives at stop $i+1$. Because the running time of bus $k$, which has been predicted, may have influence on the number of boarders being predicted, it is also included as an element of the pattern above.

Based on the above assumption, we can say that the boarding passengers being predicted $b_{0}(k)$ is presented by a pattern, namely $\left(\boldsymbol{B}_{0}\right)$, whose three elements are the number of boarding passengers recorded from the previous bus, the running time of that bus, and the predicted running time bus $k$ will spend to come to stop $i+1$.

$$
B_{0}=\left[b_{0}(k-1), r_{0}(k-1), r_{0}(k)\right]^{\mathrm{T}}
$$

Also in the past, the number of boarding passengers on bus $k$ at stop $(i+1)$ is also presented by its own pattern whose elements are defined similarly above. So we have $\boldsymbol{B}_{l}$, $\boldsymbol{B}_{2}, \ldots, \boldsymbol{B}_{m}$ patterns, representing the corresponding number of boarders of bus $k$ recorded in the past $b_{1}(k), b_{2}(k), \ldots, b_{m}(k)$.

$$
\begin{aligned}
& \boldsymbol{B}_{j}=\left[b_{j}(k-1), r_{j}(k-1), r_{j}(k)\right]^{\mathrm{T}} \\
& (m=\text { number of observations colleted in the past, } j=1 \text { to } m) .
\end{aligned}
$$


Because the patterns contain elements measured on different scales, it is necessary to standardize them. We have:

$$
S B_{j}=\left[s b_{j}(k-1), s r_{j}(k-1), s r_{j}(k)\right]^{\mathrm{T}}
$$

Where:

- $\quad \boldsymbol{S B}_{\boldsymbol{j}}(j=1$ to $m)=$ standardized pattern of $\boldsymbol{B}_{j}$

- $\quad s b_{j}(k-1)=$ standardized values of $b_{j}(k-1) ; j=1$ to $m$

$$
s b_{j}(k-1)=\frac{b_{j}(k-1)-\bar{b}(k-1)}{S b(k-1)}
$$

- $\quad \bar{b}(k-1)=$ The mean of the number of boarding passengers on bus $(k-1)$

$$
\bar{b}(k-1)=\frac{\sum_{j=1}^{m} b_{j}(k-1)}{m}
$$

- $\quad S b(k-1)=$ Standard deviation of boarding passengers on bus $(k-1)$

$$
S b(k-1)=\sqrt{\frac{\sum_{j=1}^{m}\left[b_{j}(k-1)-\bar{b}(k-1)\right]^{2}}{m-1}}
$$

- $\quad s r_{0}(k-1)=$ Standardized value of running time $r_{0}(k-1)$

$$
s r_{0}(k-1)=\frac{r_{0}(k-1)-\bar{r}(k-1)}{S r(k-1)}
$$

- $\quad s r_{j}(k-I)=$ Standardized values of running times $r_{j}(k-1) ;(j=1$ to $\mathrm{m})$ 


$$
s r_{j}(k-1)=\frac{r_{j}(k-1)-\bar{r}(k-1)}{S r(k-1)}
$$

Where:

$-r(k-1)=$ Mean of running times of bus $k-1$

$$
\bar{r}(k-1)=\frac{\sum_{j=1}^{m} r_{j}(k-1)}{m}
$$

- $S r(k-1)=$ Standard deviation of running times of bus $k-1$

$$
S r(k-1)=\sqrt{\frac{\sum_{j=1}^{m}\left[r_{j}(k-1)-\bar{r}(k-1)\right]^{2}}{m-1}}
$$

- $s r_{0}(k)=$ Standardized value of running time $r_{0}(k)$

- $s r_{j}(k)=$ Standardized values of running times $r_{j}(k)$

$$
\begin{aligned}
& s r_{0}(k)=\frac{r_{0}(k)-\bar{r}(k)}{S r(k)} \\
& s r_{j}(k)=\frac{r_{j}(k)-\bar{r}(k)}{S r(k)}
\end{aligned}
$$

Where:

- $\quad r(k)=$ Mean of running times of bus $k$ 


$$
r^{(k)}=\frac{\sum_{j=1}^{m} r_{j}(k)}{m}
$$

- $\quad S r(k)=$ Standard deviation of running times of bus $k$

$$
S r(k)=\sqrt{\frac{\sum_{j=1}^{m}\left[r_{j}(k)-\bar{r}(k)\right]^{2}}{m-1}}
$$

\subsubsection{Pattern Recognition}

Following standardization, the patterns are used to recognize the neighbours of $b_{0}(k)$ represented by pattern $\boldsymbol{B}_{0}$ (Eq.5.2). The automatic procedure to recognize the neighbours around $b_{0}(k)$ is similar to the one presented in Section 4.4 .4 of which we replace the $r_{0}(k)$ and $r_{j}(k)$ with their patterns by the focal $b_{0}(k)$ and $b_{j}(k)$ with the corresponding patterns. For the sake of reducing a lengthy expression, this procedure is not shown here.

\subsubsection{Prediction}

To predict the standardized value of boarders $s b_{0}(k)$, we use the NadarayaWatson kernel estimation function as follows.

$$
\hat{b}_{0}(k)=\frac{\sum_{j=1, j \neq i}^{\text {opNei }} K_{j} \cdot b_{j}(k)}{\sum_{j=1, j \neq i}^{\text {OpNei }} K_{j}}
$$

Where 
$O p N e i$ is the optimal number of neighbours around $b_{0}(k)$. Please see equations 4.18A and 4.18 B for the definition of $O p N e i$.

$K_{j}$ is the weight assigned for the $j^{\text {th }}$ neighbour of the defined neighbourhood. Please refer to equation 4.19 for the definition of $K_{j}$

$\hat{b}_{0}=$ The predicted value of $b_{0}(k)$

\subsubsection{Update Prediction}

The updating process follows the same steps above as noted from pattern recognition to prediction. If there are $M$ stops between the current stop (i.e. the stop the bus has just arrived) and the last stop, the DTM will be applied $M$ times for predicting the number of boarders on the bus when it arrives at each stop.

\subsubsection{Real-Time Alighting Passenger Prediction Sub-module}

LOWESS-based method is also applied to predict the number of alighting passengers at a stop. The procedure is as shown below.

\subsubsection{Parameter Selection}

The following methods are applied to select parameters in modelling alighting passenger predictions: leave-one-out method for optimal bandwidth selection; Tri-cube weight function to recognize the neighbours, and Nadaraya-Watson kernel function for prediction. 


\subsubsection{Pattern Selection}

The number of alighting passengers at a stop does not depend on the service frequency. Therefore, the pattern for the number of alighting passengers of bus $k$ that are being predicted at stop $i+1$, written as $a_{0}(k)$, is selected as the composition of only the number of alighting passengers of the two previous buses denoted as $a_{0}(k-1)$ and $a_{0}(k-2)$

$$
\boldsymbol{A}_{0}=\left[a_{0}(k-1), a_{0}(k-2)\right]^{\mathrm{T}}
$$

Where:

$a_{0}(k) \quad=$ the being predicted number of passengers alighting from bus $k$ at stop $i+1$.

$a_{0}(k-1), a_{0}(k-2)=$ the number of alighting passengers on the two previous buses $(k-1)$ and $(k-2)$ at stop $i+1$

Also in the past, each number of alighting passengers on bus $k$ at stop $(i+1)$ is presented by its own pattern with its elements defined in a manner similar to the one above. So we have the patterns $A_{1}, A_{2} \ldots A_{m}$

$A j=\left[a_{j}(k-1), a_{j}(k-2)\right]^{\mathrm{T}}$

Where: $\mathrm{j}=1$ to $\mathrm{m} ; \mathrm{m}=$ the number of observations in the past

\subsubsection{Pattern Recognition}

The procedure to recognize the neighbours around $a_{0}(k)$ is similar to the one presented in Section 4.4.4. 


\subsubsection{Prediction}

Again, we use Nadaraya-Watson kernel estimation function as follows

$$
\Lambda_{0}(k)=\frac{\sum_{j=1, j \neq i}^{o_{p N e i}} K_{j} \cdot a_{j}(k)}{\sum_{j=1, j \neq i}^{o_{p N e i}} K_{j}}
$$

Where

$O p N e i$ is the optimal number of neighbours around $a_{0}(k)$. Please see equations 4.18A and 4.18 B for definition of $O p N e i$

$K_{j}$ is the weight assigned for the $j^{\text {th }}$ neighbour of the defined neighbourhood. Please refer to equation 4.19 for the definition of $K_{j}$

$$
\hat{a_{0}}=\text { The predicted value of } a_{0}(k)
$$

\subsubsection{Update Prediction}

The updating process follows the same steps as noted above from pattern recognition to prediction. Each time the bus arrives at a stop, if there are $M$ stops between the current stop (i.e. the stop the bus has just arrived) and the last stop, the RASM will be applied $M$ times for predicting the number of de-boarders on the bus when it arrives at each stop. 


\subsubsection{Regression Sub-Module}

This sub-module is developed in order to examine the relationships between dwell time and other variables influencing it. It is a component of method A mentioned in Section 5.2.2. Data collected from APC and AVL systems of the OC Transpo are used to find these relationships.

\subsubsection{Variable Selection and Preparation}

The variables selected in the regression models are shown in Table 5.1. Because some variables were not colleted by the APC-AVL systems but can be transformed from other variables, it is necessary to prepare data before going to the regression analyses.

The variables were transformed and calculated as shown below. The explanations of variables are in Tables 3.5 and 5.1.

$$
\begin{aligned}
& L O A D \_A R R:=\left(L O A D \_D E P\right)+\left(T O T A L \_O F F S\right)-\left(T O T A L \_O N S\right) \\
& T O T A L \_O N S=\left(O N \_1\right)+\left(O N \_2\right)+\left(O N \_3\right) \\
& T O T A L_{-} O F F S=\left(O F F_{-} 1\right)+\left(O F F_{-} 2\right)+\left(O F F_{-} 3\right) \\
& P U N T=\left(A C T \_T I M E\right)-\left(E X P E C \_T I M E\right) \\
& \text { TOTAL_PASS }=\left(T O T A L \_O N S\right)+\left(T O T A L \_O F F S\right) \\
& L F=\left(L O A D \_A R R\right) / B U S \_C A P A
\end{aligned}
$$

where: BUS_CAPA is the number of designated seats of transit bus; $B U S \_C A P A$ $=40$ for rigid bus; $=65$ for articulated bus. 
Table 5.1: Variable Selections for RESM

\begin{tabular}{|c|c|c|c|c|}
\hline Variable & $\begin{array}{l}\text { Type of } \\
\text { Variable }\end{array}$ & Code & Description of Variable & Reason for Selection \\
\hline $\begin{array}{l}\text { Boarding } \\
\text { passengers }\end{array}$ & Numeric & TOTAL_ONS & $\begin{array}{l}\text { The total number of } \\
\text { passengers boarding at a stop }\end{array}$ & $\begin{array}{l}\text { It has a major influence } \\
\text { on dwell time. }\end{array}$ \\
\hline $\begin{array}{l}\text { Alighting } \\
\text { passengers }\end{array}$ & Numeric & TOTAL_OFFS & $\begin{array}{l}\text { The total number of } \\
\text { passengers alighting at a stop }\end{array}$ & $\begin{array}{c}\text { It has an influence on } \\
\text { dwell time }\end{array}$ \\
\hline $\begin{array}{l}\text { Boarding } \\
\text { and } \\
\text { alighting }\end{array}$ & Numeric & TOTAL_PASS & $\begin{array}{l}\text { Total passengers getting on } \\
\text { and off at a bus stop }\end{array}$ & $\begin{array}{l}\text { It has a major influence } \\
\text { on dwell time. }\end{array}$ \\
\hline $\begin{array}{l}\text { On- board } \\
\text { passengers } \\
\text { prior stop }\end{array}$ & Numeric & $L O A D \_A R R$ & $\begin{array}{l}\text { Number of passengers on the } \\
\text { bus before it arrives or passes } \\
\text { the stop. }\end{array}$ & $\begin{array}{l}\text { This effects the } \\
\text { circulation in the bus. } \\
\text { Therefore it will } \\
\text { influence dwell time }\end{array}$ \\
\hline $\begin{array}{l}\text { Loading } \\
\text { factor }\end{array}$ & Numeric & $L F$ & $\begin{array}{l}\text { Ratio between the number of } \\
\text { on-board passengers prior stop } \\
\text { and the capacity of the bus }\end{array}$ & $\begin{array}{l}\text { A value of LF close to } 1 \\
\text { means that the bus is } \\
\text { likely full. Hence, this } \\
\text { may cause a long dwell } \\
\text { time. }\end{array}$ \\
\hline $\begin{array}{c}\text { Alighting } \\
\text { passengers } \\
\text { at front door }\end{array}$ & Numeric & $O F F_{-} 1$ & $\begin{array}{l}\text { Number of alighting } \\
\text { passengers using front door }\end{array}$ & $\begin{array}{c}\text { If passengers alight at } \\
\text { front door, it will increase } \\
\text { dwell time. }\end{array}$ \\
\hline Punctuality & Numeric & PUNT & $\begin{array}{l}\text { Measured by lateness and } \\
\text { earliness of the bus. }\end{array}$ & $\begin{array}{c}\text { A long lateness can cause } \\
\text { a crowded downstream } \\
\text { stop resulting in a long } \\
\text { dwell time. }\end{array}$ \\
\hline Bus Type & Dummy & $B U S \_T Y P E$ & $\begin{array}{c}\text { Articulated bus or rigid bus } \\
\text { (all are low-floor buses). } \\
\text { 1=Articulated Bus; } \\
\text { 0 = Rigid bus }\end{array}$ & $\begin{array}{l}\text { Dwell time depends on } \\
\text { bus type }\end{array}$ \\
\hline Time & Dummy & TIME & $\begin{array}{c}\text { Time of day (Morning, noon, } \\
\text { after noon). } 1=\text { Morning; } 2= \\
\text { Noon; } 3=\text { afternoon }\end{array}$ & $\begin{array}{l}\text { Dwell time depends on } \\
\text { time of day }\end{array}$ \\
\hline Season & Dummy & SEASON & $\begin{array}{c}0=\text { Winter; } 1=\text { Spring; } \\
2=\text { Summer; } 3=\text { Fall }\end{array}$ & $\begin{array}{l}\text { Dwell time may be } \\
\text { different for summer and } \\
\text { winter }\end{array}$ \\
\hline $\begin{array}{l}\text { Number of } \\
\text { Doors }\end{array}$ & Numeric & DOORS & Self explanatory & $\begin{array}{l}\text { Increasing number of } \\
\text { doors reduces dwell time }\end{array}$ \\
\hline $\begin{array}{c}\text { Stop } \\
\text { location }\end{array}$ & Dummy & STOP_LOCA & $\begin{array}{c}1=\text { Stop located at CBD } \\
0=\text { Otherwise }\end{array}$ & $\begin{array}{l}\text { The location of bus stop } \\
\text { may effect dwell time }\end{array}$ \\
\hline
\end{tabular}




\subsubsection{Regression Functions}

Before going to regression analyses, dwell times being used in the regression functions are processed to delete unreliable records. One of these records is the zero values, meaning that the bus does not stop at the bus stops. Such records are not included in the model and being deleted. The other records that should be removed from the dataset are the extreme values of dwell times and outliers. However, deleting these records cannot be done without any consideration. In this study, the records of dwell time over 180 seconds are deleted without any concern because such time durations are usually the layovers. After removing these records, the remaining data set was examined to find additional records that should be deleted.

Examinations of the data show that the mean of dwell time recorded for all seasons and for all times of day for bus 95 and 1 are 15.86 and 20.40 seconds, respectively. The records, which are suggested by the SPSS package as the extreme cases and outliers, are the ones with durations of over 44 seconds in bus route 95 and 57 seconds in bus route 1. The boxplots shown in Figures 5.3 and 5.4 present the outliers and extreme cases which are signified by small circles and asterisks ${ }^{1}$, respectively. According to the SPSS, the cases with values between 1.5 and 3 box lengths from the upper or lower edge of the box are considered as outliers. The cases with values higher than 3 box lengths from both edges of the box are considered as extreme cases. The box length is the interquartile range.

The outliers and extreme cases as mentioned above (i.e. cases with recorded dwell time larger than 57 seconds for buses of route 1 and 44 seconds for bus 95) should be

\footnotetext{
${ }^{1}$ The number beside the asterisk (or circle) is the case number
} 
erased in order to improve the prediction capability of the regression models. In this study, the dwell times lower than 57 seconds are kept for both routes. The sample size of the database for regression analysis reduces from 9983 cases to 8685 cases after outlier and zero value deletions ${ }^{2}$.

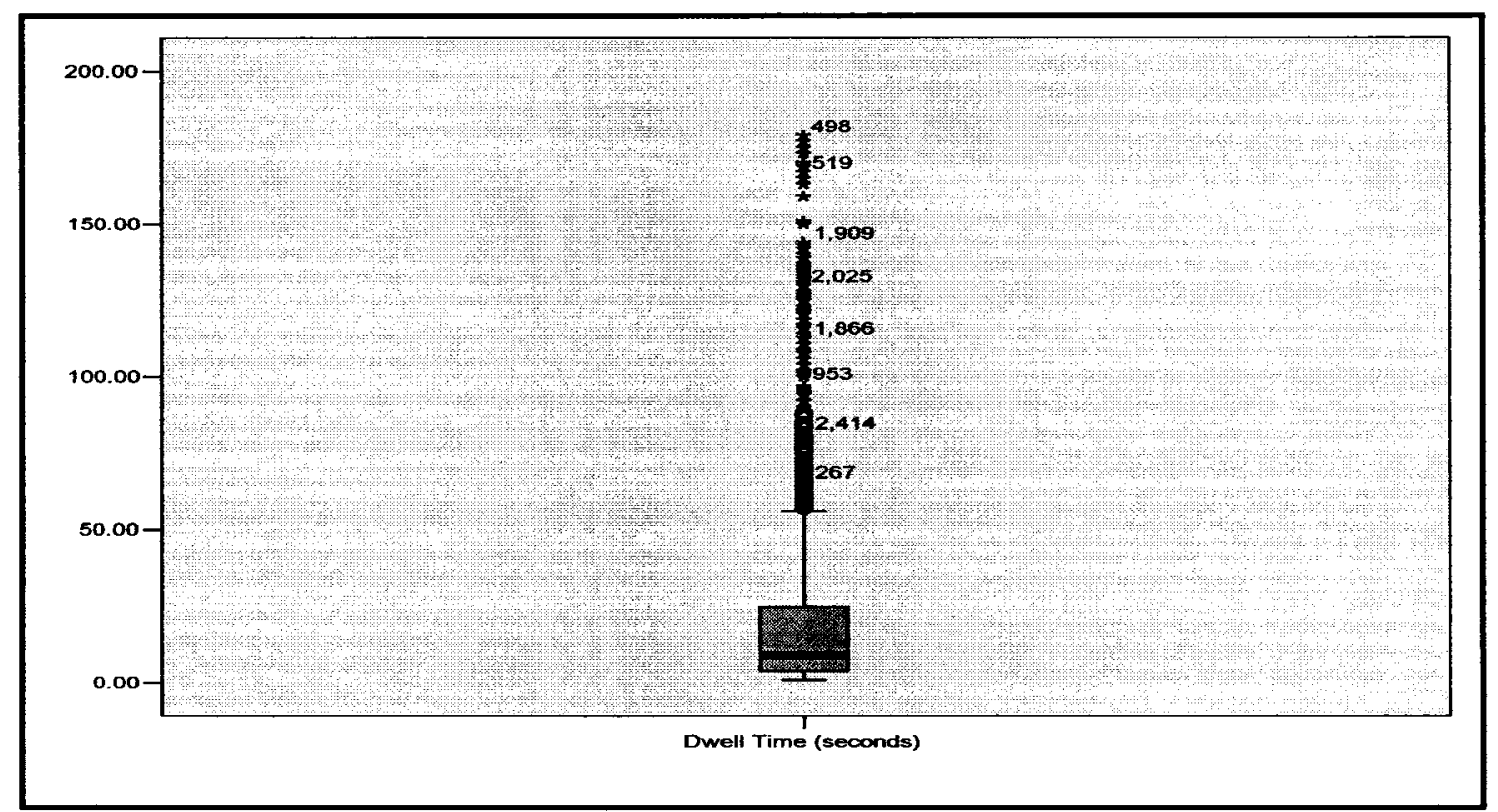

Figure 5.3: Boxplot of Dwell time. Bus route 1

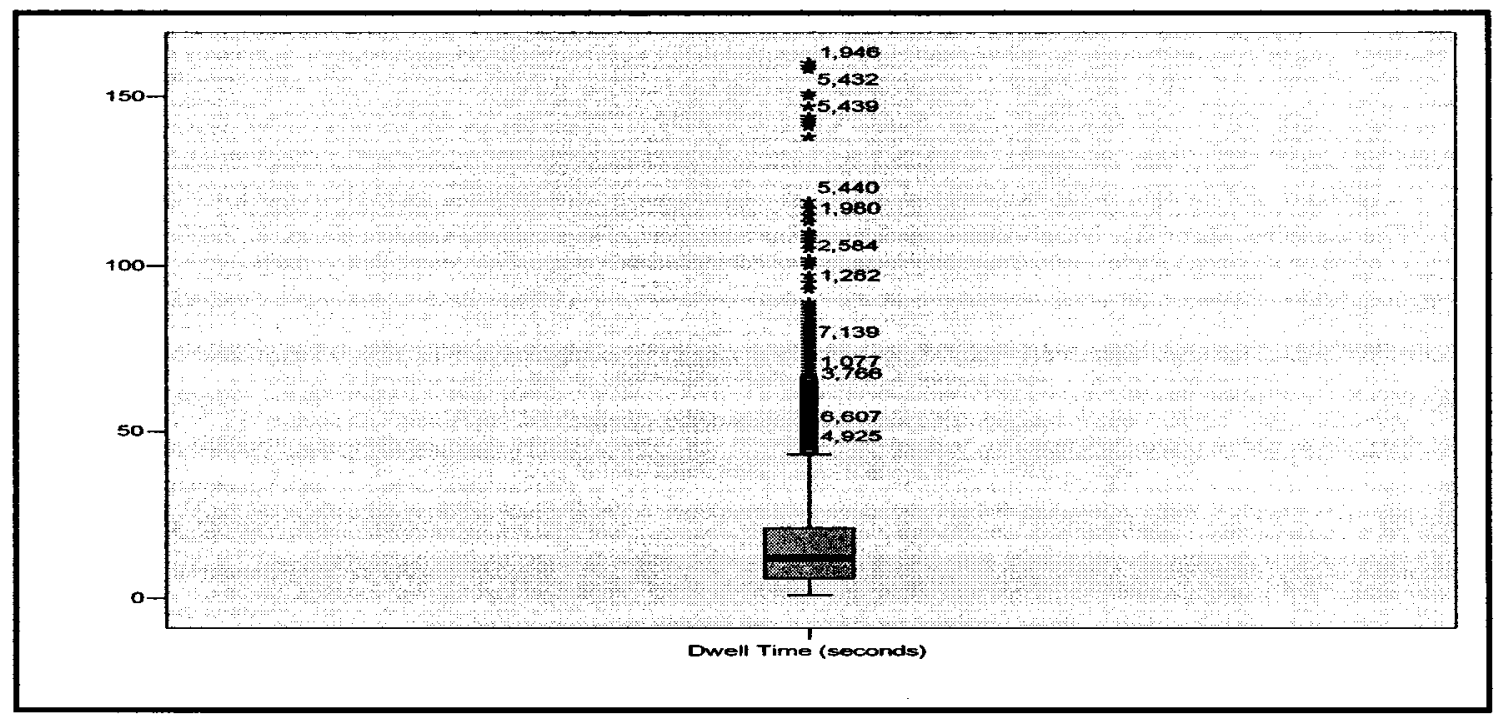

Figure 5.4: Boxplot of Dwell time. Bus route 95

\footnotetext{
${ }^{2}$ It was found that when using all data without any outlier and extreme case deletion for a pilot multipleregression, the $R^{2}$ of this equation was rather low. Please see Appendix B1 for this regression.
} 


\section{Type A-1: Simple Linear Regression}

The general regression equation is shown below.

$$
\begin{aligned}
& d w e l l=\beta_{0}+\beta_{1} \cdot\left(T O T A L_{-} O N\right)+\beta_{2} .\left(T O T A L_{-} \text {OFF }\right)+\beta_{3} .\left(T O T A L_{-} P A S S\right)+ \\
& \beta_{4} .\left(L O A D_{-} A R R\right)+\beta_{5} \cdot(L F)++\beta_{6} .(P U N T)+\beta_{7} .\left(B U S \_T Y P E\right)+\beta_{8} \cdot(T I M E)+ \\
& \beta_{9} .(\text { SEASON })+\beta_{10} .(D O O R S)+\beta_{11} .(\text { STOP_LOCA })
\end{aligned}
$$

The backward stepwise regression method is selected in order to track the effect of each variable on dwell time. As a part of this method, variables with significance values of more than 0.15 (i.e., they are statistically not significant) are not included in the model. From this perspective, one may argue that a significance level of 0.05 should be better. Of course, a selection of a low value of level of significance (i.e. high confidence level) will reduce the risk of taking an unimportant variable in the regression model. However, 0.05 is too low and often results in the deletion of the important variables from the model. Therefore, the values of $\alpha=0.15-0.2$ are suggested (Scott Menard, 2001, p. $64)$.

The SPSS (Version 13.0) package was used to develop the regression model. Eighty percent of the total cases were drawn randomly. The remaining cases were used for testing the models. The models showing the largest $R$-square, along with significant $\mathrm{t}-$ test for parameters, were selected. The results of the three runs are shown in Tables 5.2 and 5.3. 
Table 5.2: Coefficients of the Best Regression

\begin{tabular}{|c|c|c|c|c|c|c|}
\hline \multicolumn{7}{|c|}{ Coefficients a } \\
\hline & & \multicolumn{2}{|c|}{$\begin{array}{l}\text { Unstandardized } \\
\text { Coefficients }\end{array}$} & \multirow{2}{*}{$\begin{array}{c}\begin{array}{c}\text { Standardized } \\
\text { Coefficients }\end{array} \\
\text { Beta }\end{array}$} & \multirow[b]{2}{*}{$t$} & \multirow[b]{2}{*}{ Sig. } \\
\hline \multicolumn{2}{|c|}{ Model } & B & Std. Error & & & \\
\hline \multirow[t]{10}{*}{1} & (Constant) & 15.750 & 1.154 & & 13.648 & .000 \\
\hline & TOTAL_ONS & 1.220 & .016 & .673 & 75.097 & .000 \\
\hline & TOTAL_OFFS & .438 & .016 & .246 & 27.173 & .000 \\
\hline & LOAD_ARR & .072 & .029 & .112 & 2.495 & .013 \\
\hline & LF & -4.935 & 1.649 & -.125 & -2.992 & .003 \\
\hline & TIME & .113 & .119 & .008 & .953 & .341 \\
\hline & PUNT & .003 & .001 & .047 & 5.102 & .000 \\
\hline & SEASON & .097 & .088 & .009 & 1.098 & .272 \\
\hline & STOP_LOCATE & 2.125 & .217 & .089 & 9.804 & .000 \\
\hline & DOORS & -4.361 & .419 & -.159 & -10.401 & .000 \\
\hline \multirow[t]{9}{*}{2} & (Constant) & 16.020 & 1.119 & & 14.317 & .000 \\
\hline & TOTAL_ONS & 1.222 & .016 & .674 & 75.854 & .000 \\
\hline & TOTAL_OFFS & .439 & .016 & .246 & 27.183 & .000 \\
\hline & LOAD_ARR & .075 & .028 & .117 & 2.617 & .009 \\
\hline & LF & -5.086 & 1.642 & -.129 & -3.098 & .002 \\
\hline & PUNT & .003 & .001 & .047 & 5.096 & .000 \\
\hline & SEASON & .093 & .088 & .009 & 1.055 & .291 \\
\hline & STOP_LOCATE & 2.127 & .217 & .089 & 9.816 & .000 \\
\hline & DOORS & -4.385 & .419 & -.160 & -10.478 & .000 \\
\hline \multirow[t]{8}{*}{3} & (Constant) & 16.175 & 1.109 & & 14.583 & .000 \\
\hline & TOTAL_ONS & 1.222 & .016 & .674 & 75.856 & .000 \\
\hline & TOTAL_OFFS & .438 & .016 & .246 & 27.163 & .000 \\
\hline & LOAD_ARR & .074 & .028 & .116 & 2.605 & .009 \\
\hline & LF & -5.064 & 1.641 & -.128 & -3.085 & .002 \\
\hline & PUNT & .003 & .001 & .047 & 5.116 & .000 \\
\hline & STOP_LOCATE & 2.126 & .217 & .089 & 9.812 & .000 \\
\hline & DOORS & -4.397 & .418 & -.161 & -10.511 & .000 \\
\hline
\end{tabular}

$$
\begin{aligned}
& \underset{R^{2}=0.581}{d w e l l}=16.175+\underset{t=14.583}{2.126}(\text { STOP_LOCATE })+\underset{t=2.812}{0.438}(\text { TOTAL_.63 } \\
& +\underset{t=75.856}{1.222}(\text { TOTAL } \text { ONFS })+ \\
& -\underset{t=-3.085}{5.064}(L F)+\underset{t=0}{0.003}(\text { PUNT })
\end{aligned}
$$

After 3 runs, the stepwise regression method returned the best regression as shown in Table 5.2. Two out of 9 variables were removed from the model (i.e. TIME and SEASON). All parameters are statistically significant. With $\boldsymbol{R}$-square of 0.581 , the model is satisfactory.

Following Equation 5.27, if we control other variables, dwell time will increase by 2.13 seconds if bus stop is located in the CBD area compared to that of a stop outside this 
area. The number of doors has a strong influence on dwell time. If the bus has 3 doors instead of 2 doors, dwell time can decrease up to 4.34 seconds when other variables are controlled. Dwell time will increase if the bus has more boarding or alighting passengers.

Table 5.3: Model Summary for the Best Regression

\begin{tabular}{|c|c|c|c|c|}
\hline \multicolumn{5}{|c|}{ Model Summary } \\
\hline Model & $\mathbf{R}$ & R Square & $\begin{array}{l}\text { Adjusted } \\
\text { R Square } \\
\end{array}$ & $\begin{array}{l}\text { Std. Error of } \\
\text { the Estimate }\end{array}$ \\
\hline 1 & $.763^{\mathrm{a}}$ & .582 & .581 & 7.66728 \\
\hline 2 & $.763^{b}$ & .582 & .581 & 7.66722 \\
\hline 3 & $.763^{c}$ & .581 & .581 & 7.66729 \\
\hline a. & \multicolumn{4}{|c|}{$\begin{array}{l}\text { Predictors: (Constant), DOORS, SEASON, STOP } \\
\text { LOCATE, TIME, TOTAL_OFFS, LF, TOTAL_ONS, PUNT, } \\
\text { LOAD_ARR }\end{array}$} \\
\hline b. & \multicolumn{4}{|c|}{$\begin{array}{l}\text { Predictors: (Constant), DOORS, SEASON, STOP } \\
\text { LOCATE, TOTAL_OFFS, LF, TOTAL_ONS, PUNT, } \\
\text { LOAD_ARR }\end{array}$} \\
\hline c. & \multicolumn{4}{|c|}{$\begin{array}{l}\text { Predictors: (Constant), DOORS, STOP_LOCATE, } \\
\text { TOTAL_OFFS, LF, TOTAL_ONS, PUNT, LOAD_ARR }\end{array}$} \\
\hline
\end{tabular}

Typel A-2: Non-Linear Regression

Besides the multiple linear regression developed above, a series of non-linear regression types of function are also examined.

- Type A-2.1:

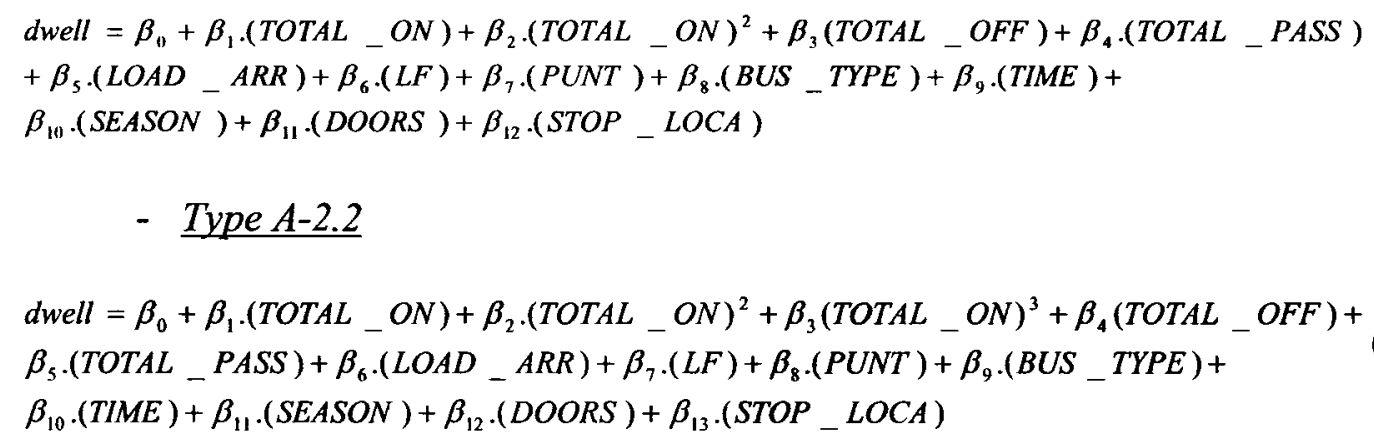

- Type A-2.3 


$$
\begin{aligned}
& d w e l l=\beta_{0}+\beta_{1} \cdot(\text { TOTAL_ON })+\beta_{2} \cdot(\text { TOTAL_ON })^{2}+\beta_{3}(\text { TOTAL_OFFS })^{2}+ \\
& \beta_{4}(\text { TOTAL_OFF })\left(T O T A L_{-} O F F\right)+\beta_{5} .\left(T O T A L_{-} P A S S\right)+\beta_{6} .\left(L O A D_{-} A R R\right)+\beta_{7} .(L F)+ \\
& \beta_{8} \cdot(P U N T)+\beta_{9} \cdot(\text { BUS_TYPE })+\beta_{10} \cdot(\text { TIME })+\beta_{11} \cdot(\text { SEASON })+\beta_{12} \cdot(D O O R S)+\beta_{13} \cdot(\text { STOP_LOCA }) \\
& \text { - Type A-2.4 (Gauss Regression) } \\
& d w e l l=b_{1} \cdot\left(1-b_{3} \exp \left(-b_{2} \cdot\left(T O T A L_{-} P A S S\right)^{2}\right)\right) \\
& \text { - Type A-2.5 (Weibull Regression) } \\
& \left.d w e l l=b_{1}-b_{2} \cdot \exp \left(-b_{3} \cdot\left(T O T A L_{-} P A S S\right)^{b_{4}}\right)\right) \\
& \text { - Type A-2.6 (Morgan-Mercer-Florin Regression) } \\
& d w e l l=\left(b_{1} b_{2}+b_{3} . T_{O T A L} P A S S^{b_{4}}\right) /\left(b_{2}+\text { TOTAL_PASS }{ }^{b_{4}}\right)
\end{aligned}
$$

The types from A-2.1 to A-2.3 are polynomial regressions in term of variables TOTAL_ONS and TOTAL_OFF whereas types from A-2.4 to A-2.6 are the common nonlinear regressions where the relationship between dwell time and TOTAL_PASS (e.g., total number of passengers getting on and off the bus) is examined. To find the best regression equation for each type, backward regression method was applied for the types from A-2.1 to A-2.3 and the Gauss-Newton method was applied for the rest. By using the SPSS version 13 , we can find the best regression equations. Their $R$-square values and parameters are presented in Table 5.4. Details can be seen in Appendix B1.

Out of the types from A-1 to A2-6, type A-2.3 has the highest $R$-square, meaning that it is the most accurate type as compared with others. As a result, the use of type A2.3 is proposed in this research to predict dwell time.

Although type A-2.6 is less accurate than type A-2.3, it is a compact one requiring only the total of passenger activities through bus doors recorded or predicted. Therefore, it should be used in case of lack of data on some variables. 
Table 5.4: Proposed Non-linear Regression Models

\begin{tabular}{|c|c|c|}
\hline Type & The best regression equations & $\begin{array}{c}R- \\
\text { square }\end{array}$ \\
\hline A-2.1 & 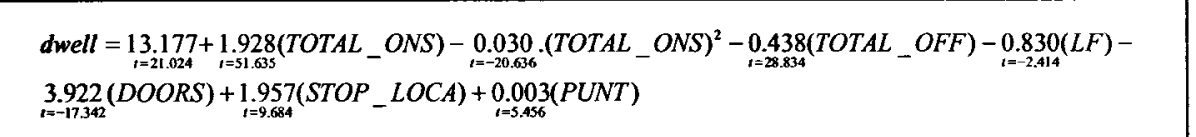 & $\mathbf{0 . 6 1 0}$ \\
\hline A-2.2 & 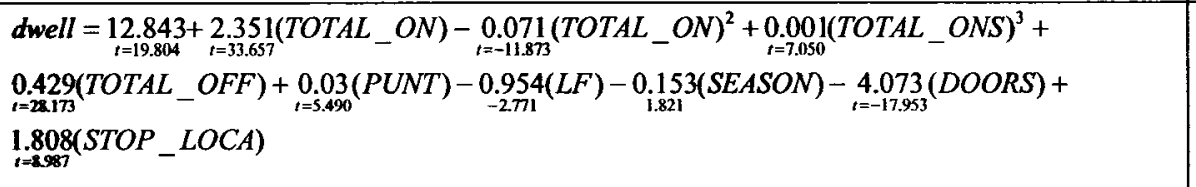 & 0.618 \\
\hline A-2.3 & 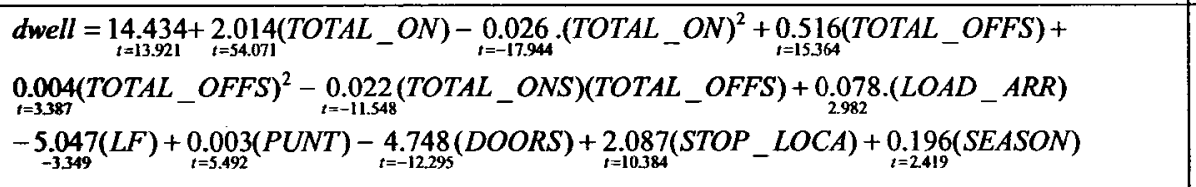 & 0.634 \\
\hline A-2.4 & $d w e l l=31.08\left(1-0.795 \exp \left(-0.346 .\left(10^{-1} . T O T A L_{-} P A S S\right)^{2}\right)\right)$ & 0.502 \\
\hline A-2.5 & $d w e l l=1184.802-91.856 \exp \left(-2.557\left(10^{-1} \text { TOTAL_PASS }\right)^{-0.003}\right)$ & 0.477 \\
\hline A-2.6 & $d w e l l=\left(-146.259+470.907\left(T O T A L_{-} P A S S\right)^{0.561}\right) /\left(97.767+\left(10^{-1} \text { TOTAL_PASS }\right)^{0.56}\right.$ & 0.528 \\
\hline
\end{tabular}

To predict dwell time, the number of boarding passengers (TOTAL_ONS) and the number of alighting passengers (TOTAL_OFFS), which can be predicted from previous sub-modules (i.e., BPSM and APSM), is used in regression A-2.3. Other related variables (i.e., $L O A D \_A R R, P U N T$, and $L F$ ) can also be determined indirectly by using Equations 5.20, 5.23 and 5.25. It should be noted that data on all variables that are used in this type are all obtainable from the APC and AVL systems mounted on the buses. 


\subsubsection{Busiest Door Prediction Sub-Module}

If we assume that all passengers board at the front door and alight at the reardoor, the dwell time can be easily predicted as the maximum of the two service times, one for boarding passengers at front door and one for alighting passengers at the rear door. However, data colleted from the APC system by the OC Transpo shows the fact that the on-board passengers alight at all doors while waiting passengers can get on the bus at the front door of a 2 door-bus and from all doors of the 3-door bus. This implies that the dwell time is actually the service time at the busiest door. This can be seen in the suggestions of the Highway Capacity Manual 2000 (Equation 2.2). However, how to find the busiest door was not discussed in literature. In this sub-module, the equation provided by the HCM (2000) is used with changes in finding the most crowded door. It should be recalled that the sub-module is a part of method $\mathrm{B}$, another method proposed in this study for predicting dwell time in real-time, given the predicted number of boarding and alighting passengers. Please refer to Fig.5.2 for details. In this research, dwell time is calculated by using Equation 5.33.

$$
d w e l l=a t_{a}+b \cdot t_{b}+t_{o p}
$$

Where: $d w e l l=$ Predicted dwell time in seconds

$a \quad=$ Predicted number of alighting passengers through the busiest door

$t_{a} \quad=$ Passenger alighting time per passenger ( follows HCM 2000)

$b \quad=\quad$ Predicted number of boarding passengers through the busiest door

$t_{b} \quad=$ Passenger boarding time per passenger (follows HCM 2000)

$t_{o p} \quad=$ Door opening and closing time ( 4 seconds) 
In order to find the busiest door for each type of bus, data are clustered upon rigid body bus and articulated bus. The methodologies to find the busiest door for each type are presented below.

\subsubsection{Rigid-Body Bus}

A rigid-body bus has two doors, one in front and the other in the rear. At a bus stop, passengers can only get on the bus from the front door while they can get off the bus at any door convenient for them. Before the bus completely opens the doors, alighting passengers tend to move to the doors for ease of exiting. Alighting passengers have priority to get off the bus first. If some passengers get off via the front door, onground passengers have to wait until the last alighter leaves the bus before getting on. This will lengthen dwell time compared to the case when all alighting passengers use the rear door and all boarding passengers use the front door. For example, assume that there are 8 alighting passengers and 3 boarding passengers. Serving time for each alighter is assumed to be 2.5 seconds and 5 seconds for each boarder. Hence, servicing time to board all 3 passengers is 15 seconds at the front door, and 20 seconds are required for 8 alighting passengers, provided that all alighters use the rear door and all boarders use the front door. The busier door is the rear door (i.e., 20 seconds). However, if 4 out of 8 alighters use the front door, the servicing time at this door will be 25 seconds and for the rear door it is only 10 seconds. The busier door now is the front door instead of the rear door as found in the first case and the extra service time is 5 seconds. Obviously, if 
passengers get off at the front door, the dwell time is increased. If we can find the busier door, we will have a better prediction of dwell time of the bus.

On-board passengers are likely to choose the most convenient door for alighting. Of course, passengers near a door will usually use that door for alighting because of a short distance. Based on the distance and the ease to move to the doors, passengers in the middle of the bus will choose a door for alighting. In general, passengers will use the door that offers them the highest convenience. Intuitively, the convenience of a door can be presented by several variables such as the number of boarding and alighting passengers, the number of standees on the bus, the stop location, time of day, season, and so on. In other words, the likelihood (or the probability) that a door is the busier door depends on an unobserved variable (i.e. the convenience that a door offers passengers at each bus stop) whose values can be quantified by a series of data retrieved from APC and AVL systems. Let us call $U$ as "convenience" function. Assume that we have to relate probability that the front door is the busier door $\left(P_{\text {front door }}\right)$ with convenience value that the front door offers alighting passengers at bus stops.

Obviously, we cannot model this probability directly as $P_{\text {front_door }}=U$, because the values of $U$ may vary outside the range 0 and 1 while the probability must lie between this range. To solve the problem, a commonly used approach in logistic regression is to relate the probability with the odds.

Let us assume that the odds front door describes the ratio of the probability that the front door is the busier door to the probability that it is not so. It is easy to convert back and forth between odds and probability by using Equations 5.34 and 5.35. As shown in the equations, probabilities range from 0 to 1 , whereas odds range from 0 to infinity. An 
odds equal to 1 indicates equal probabilities that the front door is the busier door and it is not so (i.e., 50\%). For odds greater than 1, that the front door is the busier door is more likely and vice versa.

$$
\begin{gathered}
o d d s_{\text {front_door }}=\frac{P_{\text {front_door }}}{1-P_{\text {front_door }}} \\
P_{\text {front_door }}=\frac{o d d s_{\text {front_door }}}{1+o d d s_{\text {front_door }}}
\end{gathered}
$$

Where:

$P_{\text {front } \_ \text {door }}=$ the probability the front door is the busier door

The natural logarithm of the odds that the front door is the busier door is called the Logit (front_door).

$$
\text { Logit (front_door })=\ln \left(\text { odd }_{s_{\text {front_door }}}\right)=\ln \left(\frac{P_{\text {front_door }}}{1-P_{\text {front_door }}}\right)
$$

Obviously, the variable Logit (front_door) varies from negative infinity to positive infinity. Therefore, if we model Logit (front_door) $=U$, we will have a form of logistic regression.

$$
\text { Logit (front_door) }=U
$$

We can convert Logit (front_door) back to the odds by exponentiation. From Equations 5.37 and 5.38 we have

$$
\text { Odds }_{\text {front_door }}=e^{U}
$$


Substitute the odds $s_{\text {front_door }}$ in Equation 5.38 into Equation 5.35, so the probability that the front door is the busier door can be calculated as below.

$$
P_{\text {front_door }}=P_{1}=\frac{e^{U}}{1+e^{U}}
$$

The probability that the rear door is the busier door $\left(P_{\text {rear }}\right.$ door $)$ is as shown below

$$
P_{\text {rear } r_{-} \text {door }}=P_{2}=\frac{1}{1+e^{U}}
$$

Several types of $U$ equations are proposed in the Equations from 5.41 to 5.43 . The regression coefficients of each equation are estimated through an interactive maximum likelihood method.

\section{$\underline{\text { Type B-L.I }}$}

$U=\beta_{0}+\beta_{1} \cdot\left(T O T A L_{-} O N S\right)+\beta_{2} \cdot\left(T O T A L_{-} O F F S\right)+\beta_{3} \cdot\left(L O A D_{-} A R R\right)+\beta_{4} \cdot(L F)+\beta_{6} \cdot(P U N T)+$

$\beta_{7} \cdot($ TIME $)+\beta_{8} \cdot($ SEASON $)+\beta_{9} \cdot($ STOP_LOCA $)$

\section{$\underline{\text { Type B-L.2 }}$}

$U=\beta_{0}+\beta_{1} \cdot\left(T O T A L_{-} O N\right)+\beta_{2} \cdot\left(T O T A L_{-} O N\right)^{2}+\beta_{3}\left(T O T A L_{-} O N\right)^{3}+$

$+\beta_{56} \cdot\left(L O A D_{-} A R R\right)+\beta_{6} \cdot(L F)+\beta_{7} \cdot(P U N T)+\beta_{8} \cdot(T I M E)+\beta_{9} \cdot(S E A S O N)+\beta_{110} \cdot\left(S T O P \_L O C A\right)$

\section{Type B-L.3}

$U=\beta_{0}+\beta_{1} \cdot\left(T O T A L_{-} O N\right)+\beta_{2} .\left(T O T A L_{-} O N\right)^{2}+\beta_{3}\left(T O T A L_{-} O F F S\right)^{2}+\beta_{4}\left(T O T A L_{-} O N S\right)\left(T O T A L \_O F F S\right)+$ $\beta_{5} \cdot\left(L O A D_{-} A R R\right)+\beta_{6} \cdot(L F)+\beta_{7} \cdot(P U N T)+\beta_{8} \cdot(T I M E)+\beta_{9} \cdot(S E A S O N)+\beta_{10} \cdot\left(S T O P_{-} L O C A\right)$

Now, we can apply binary logistic regression. APC data of 7 bus stops of route 1 were used to develop the model. At each stop, the sums of passengers through the front door and the rear door were calculated for each door and compared to each other for every bus trip. If the front door is the busier door then it is coded as 1 , otherwise as 0 . In this research, the dwell times longer than 180 seconds or equal to zero are not included in 
the model. Also, the cases that recorded boarding passengers at the rear door are considered as "unusual events" and erased.

The validated dataset is shown in Table 5.5. Out of the 2642 cases, there are 1740 cases recording that the busier door is the front door, accounting for 68.3 percent of the total.

The backward stepwise method was used in this research in order to delete the variables with significance level larger than 0.1 , and as the sequence, the best regression was being found. To do this, the SPSS 13 package was used to develop the model for each type of $U$ presented in Equations 5.41, 5.42 and 5.43. The overall significance was tested with Model Chi-squared as called by SPSS, which is derived from the likelihood of observing the actual data under assumption that the model that has been fitted is accurate.

The best regression function of each type from B-L.1 to B-L.3 after the backward stepwise procedure were found and tabulated in Table 5.6. Details can be seen in Appendix B2.

Table 5.5: Validated Dataset for Logistic Regressions

\begin{tabular}{|ll|r|r|r|r|}
\hline \multicolumn{7}{|c|}{ VALIDATED_BUSIER_DOOR } \\
\hline & & & & \\
\hline & Frequency & Percent & Valid Percent & \multicolumn{2}{c|}{$\begin{array}{c}\text { Cumulative } \\
\text { Percent }\end{array}$} \\
\hline Valid & 00 & 808 & 30.6 & 31.7 & 31.7 \\
& 1.00 & 1740 & 65.9 & 68.3 & 100.0 \\
& Total & 2548 & 96.4 & 100.0 & \\
Missing & System & 94 & 3.6 & & \\
Total & & 2642 & 100.0 & & \\
\hline
\end{tabular}


Table 5.6: Best Binary Logistic Regression Equations

\begin{tabular}{|c|c|c|c|c|}
\hline Type & The Best Logistic Regression Equation & $-2 L L$ & $\begin{array}{c}\text { Nagellerke } \\
\text { R } \\
\text { quare }\end{array}$ & $\begin{array}{l}\text { Overall } \\
\text { Accuracy }\end{array}$ \\
\hline B-L.1 & 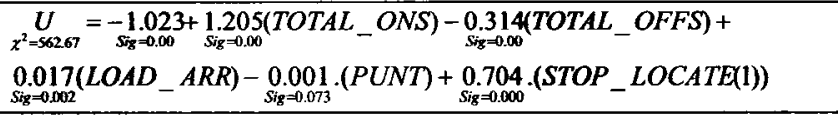 & 1804.39 & 0.586 & $86.3 \%$ \\
\hline B-L.2 & 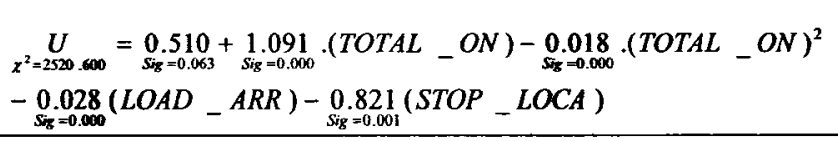 & 948.378 & 0.469 & $89 \%$ \\
\hline B-L.3 & 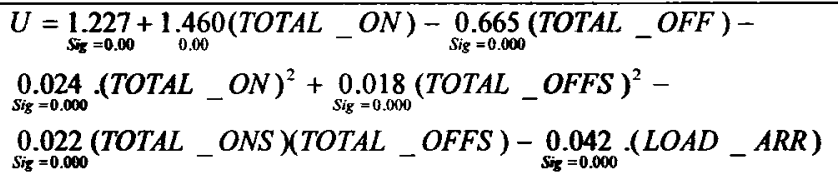 & 861.847 & 0.529 & $89 \%$ \\
\hline
\end{tabular}

As can be seen in Table 5.6, all types are quite powerful in term of prediction. To obtain further information for selection, we use the $R_{L}^{2}$, which was suggested by Menard (2001, p.27). According to him " $R_{L}^{2}$ is the most appropriate for logistic regression because it is conceptually closest to the Ordinary Least Square".

$$
R_{L}^{2}=\frac{\chi^{2}}{\chi^{2}-2 L L}
$$

Where: $\chi^{2}=$ the Chi-square of the logistic regression model.

Following Equation 5.44, the $R_{L}^{2}$ for types B-L.1, B-L.2 and B-L.3 are 0.433, 0.372 and 0.430 , respectively. Type B-L. 1 shows the highest $R_{L}^{2}$. As a result, it is used for this research.

Table 5.7: Model Summary of Type B-L1

\begin{tabular}{|c|c|c|c|}
\hline Step & $\begin{array}{c}-2 \text { Log } \\
\text { likelihood }\end{array}$ & $\begin{array}{c}\text { Cox \& Snell } \\
\text { R Square }\end{array}$ & $\begin{array}{c}\text { Nagelkerke R } \\
\text { Square }\end{array}$ \\
\hline 1 & $1804.229(a)$ & .418 & .586 \\
\hline 2 & $1804.777(a)$ & .418 & .586 \\
\hline
\end{tabular}

a Estimation terminated at iteration number 8 because parameter estimates changed by less than .001 . 
Table 5.8: Prediction Performance of Type B-L.1

\begin{tabular}{|c|c|c|c|c|c|}
\hline \multicolumn{6}{|c|}{ Classification Table ${ }^{a}$} \\
\hline \multirow{3}{*}{\multicolumn{3}{|c|}{ Observed }} & \multicolumn{3}{|c|}{ Predicted } \\
\hline & & & \multicolumn{2}{|c|}{ BUSIER DOOR } & \multirow[b]{2}{*}{$\begin{array}{c}\text { Percentage } \\
\text { Correct }\end{array}$} \\
\hline & & & $\begin{array}{l}\text { Busier door } \\
\text { is rear door }\end{array}$ & $\begin{array}{l}\text { Busier Door } \\
\text { is Front door }\end{array}$ & \\
\hline Step 1 & $\begin{array}{l}\text { BUSIER_DOOR } \\
\text { Overall Percentage }\end{array}$ & $\begin{array}{l}\text { Busier door is rear door } \\
\text { Busier Door is Front door }\end{array}$ & $\begin{array}{l}647 \\
189\end{array}$ & $\begin{array}{r}161 \\
1551\end{array}$ & $\begin{array}{l}80.1 \\
89.1 \\
86.3\end{array}$ \\
\hline Step 2 & $\begin{array}{l}\text { BUSIER_DOOR } \\
\text { Overall Percentage }\end{array}$ & $\begin{array}{l}\text { Busier door is rear door } \\
\text { Busier Door is Front door }\end{array}$ & $\begin{array}{l}647 \\
188\end{array}$ & $\begin{array}{r}161 \\
1552\end{array}$ & $\begin{array}{l}80.1 \\
89.2 \\
86.3\end{array}$ \\
\hline
\end{tabular}

a. The cut value is .500

As shown in Table 5.7, it has a pseudo $R^{2}$ statistics of $0.586\left(\right.$ Nagelkerke $\left.R^{2}\right)$ and $0.418\left(\right.$ Cox \& Snell $\left.R^{2}\right)$, which indicate a satisfactory model.

Table 5.8 presents prediction performance of type BL-1. The overall correct prediction of the model is up to 86.3 percent. Out of the 1740 cases recorded showing that the busier door is the front door, this model predicted correctly 1552 cases. This results in an accuracy of up to 89.1 percent. Also, out of the observed 708 cases where the rear door is the busier door, the model predicted correctly 647 cases, resulting in a good prediction up to $80.1 \%$ of accuracy.

Table 5.9 tabulates the coefficients of type B-L.1. The odds ratio associated with each coefficient is presented as $\operatorname{Exp}(B)$ shown in the last column of this table. The odds ratio is the number by which we would multiply the odds front_door $_{\text {(the probability divided }}$ by 1 minus the probability, as shown in Equation 5.34) for each one-unit increase in the dependent variables. An odds ratio greater than 1 indicates that the odds $s_{\text {front }}$ door 
increases when the independent variable increases and an odds ratio smaller than 1 indicate that the oddsfront_door decrease when the independent variable increases.

Table 5.9: Coefficients of Type BL-1

\begin{tabular}{|c|c|c|c|c|c|c|c|}
\hline & & $B$ & S.E. & Wald & $d f$ & Sig. & $\operatorname{Exp}(B)$ \\
\hline \multirow{13}{*}{$\begin{array}{l}\text { Step } \\
1(a)\end{array}$} & TOTAL_ONS & 1.203 & .059 & 419.818 & 1 & .000 & 3.331 \\
\hline & TOTAL_OFFS & -.314 & .021 & 217.103 & 1 & .000 & .730 \\
\hline & LOAD_ARR & .017 & .006 & 9.586 & 1 & .002 & 1.018 \\
\hline & PUNT & -.001 & .000 & 3.190 & 1 & .074 & .999 \\
\hline & SEASON & & & 7.004 & 3 & .072 & \\
\hline & SEASON(1) & -.183 & .154 & 1.416 & 1 & .234 & .833 \\
\hline & SEASON(2) & -.088 & .172 & .260 & 1 & .610 & .916 \\
\hline & SEASON(3) & .238 & .170 & 1.954 & 1 & .162 & 1.268 \\
\hline & STOP_LOCATE(1) & .704 & .177 & 15.838 & 1 & .000 & 2.023 \\
\hline & TIME & & & .548 & 2 & .760 & \\
\hline & TIME(1) & .089 & .156 & .328 & 1 & .567 & 1.093 \\
\hline & TIME(2) & .112 & .154 & .526 & 1 & .468 & 1.118 \\
\hline & Constant & -1.105 & .250 & 19.548 & 1 & .000 & .331 \\
\hline \multirow[t]{10}{*}{$\begin{array}{l}\text { Step } \\
2(a)\end{array}$} & TOTAL_ONS & 1.205 & .059 & 420.852 & 1 & .000 & 3.337 \\
\hline & TOTAL_OFFS & -.314 & .021 & 218.743 & 1 & .000 & .730 \\
\hline & LOAD_ARR & .017 & .006 & 9.620 & 1 & .002 & 1.018 \\
\hline & PUNT & -.001 & .000 & 3.213 & 1 & .073 & .999 \\
\hline & SEASON & & & 6.913 & 3 & .075 & \\
\hline & SEASON(1) & -.186 & .154 & 1.462 & 1 & .227 & .830 \\
\hline & SEASON(2) & -.096 & .171 & .317 & 1 & .573 & .908 \\
\hline & SEASON(3) & .230 & .170 & 1.840 & 1 & .175 & 1.259 \\
\hline & STOP_LOCATE(1) & .704 & .177 & 15.844 & 1 & .000 & 2.023 \\
\hline & Constant & -1.023 & .223 & 21.020 & 1 & .000 & .359 \\
\hline
\end{tabular}

a Variable(s) entered on step 1: TOTAL_ONS, TOTAL_OFFS, LOAD_ARR, PUNT, SEASON, STOP_LOCATE, TIME.

Because the odd ratios of the independent variables TOTAL_ON, LOAD_ARR and STOP_LOCATE(1) are all greater than 1, the odds front_door will increase when these variables increase. In contrast, the odds $s_{\text {front_door }}$ will decrease with the increments of the variables TOTAL_OFF and PUNT.

Specifically, while controlling other variables, if the bus has one more boarding passenger, the oddsfront_door will increase 3.33 times. This is obvious because all 
passengers have to board at the front door. Similarly, the odds front_door $_{\text {will increase } 1.02}$ with one unit loading factor increment. This result is expected because on-board passengers tend to alight via front door because of the difficulties in circulation. This odds will be doubled if the bus serves in CBD area. In contrast, if the bus has one more alighting passenger, the odds $s_{\text {front_door }} \quad$ will decrease 0.83 times. Also, the odds $s_{\text {front_door }}$ will reduce if the bus arrives at stop later.

Once the busier door has been defined, dwell time will be determined by Equations 5.45, 5.46 and 5.47.

$$
\begin{aligned}
& d w e l l=P_{1} \cdot\left(\text { TOTAL_OFFS } t_{a}+\left(\text { TOTAL_ONS } t_{b}+t_{o p} \quad \text { if } P_{l} \geq P_{2}\right.\right. \\
& d w e l l=\operatorname{Max}\left\{P_{1} .\left(T O T A L_{-} \text {OFFS }\right) t_{a}+\left(T O T A L_{-} \text {ONS }\right) . t_{b} ; P_{2} .(\text { TOTAL_OFFS }) . t_{a}\right\}+t_{o p} \\
& \quad \text { if } \quad P_{1}<P_{2} \\
& \quad P_{2}=1-P_{1}
\end{aligned}
$$

Where:

TOTAL_ONS and TOTAL_OFFS = Total the number of boarding and alighting passengers.

$t_{a}, t_{b}=$ Average service time per passenger for alighting and boarding, respectively.

$$
t_{o p}=\text { Door opening and closing time ( } 4 \text { seconds) }
$$

\subsubsection{Articulated Bus}

Unlike a rigid bus, an articulated bus has three doors and passengers can board via rear doors. To predict the busiest door out of the three doors of the bus, multinomial Logit regression is applied. 
Mathematically, the extension of the binary logistic regression, which was applied for rigid-body bus, to multinomial logistic regression is straightforward. Let us assume the second rear door is the reference category (i.e., the probability that this door is the busiest door). We call the natural logarithm of the odd (front door) (the ratio of the probability that the front door is the busiest door to the probability that the second rear door is the busiest door) is the Logit of front door, written as Logit (front_door). Similarly, we denote Logit (first_rear_door) as the natural logarithm of the ratio of the probability that the first rear door is the busiest door to the probability that second rear door is the busiest door). In mathematical notations, we have:

$$
\begin{aligned}
& \text { Logit }(\text { front_door })=\ln \frac{P_{1}}{P_{3}} \\
& \text { Logit }(\text { first_rear_door })=\ln \frac{P_{2}}{P_{3}} \\
& P_{1}+P_{2}+P_{3}=1
\end{aligned}
$$

Where:

$P_{1}, P_{2}$, and $P_{3}=$ the probabilities that the front door, the first rear door and the last rear door is the busiest door, respectively.

$$
\text { ln = natural logarithm or logarithm base } e
$$

Similar to the explanations in the case of two-door bus, we use the convenience functions namely $U_{I}$ and $U_{2}$ whose variables are collectable from APC and AVL systems, one for each door (i.e., the front door and the first rear door) relative to the second rear door (i.e., the reference category), to describe the relationship between the probabilities and the collected data. In other words, we model Logit (front_door) $=U_{I}$ and Logit 
(first_rear_door) $=U_{2}$. Therefore, based on Equations 5.48, 5.49, and 5.50 with several mathematical operations, we have the probability that the front door is the busiest door is:

$$
P_{1}=\frac{e^{U_{1}}}{e^{U_{1}}+e^{U_{2}}+1}
$$

Similarly, the probability that the first rear door is the busiest door is:

$$
P_{2}=\frac{e^{U_{2}}}{e^{U_{1}}+e^{U_{2}}+1}
$$

And, the probability that the second rear door is the busiest door is:

$$
P_{3}=\frac{1}{e^{U_{1}}+e^{U_{2}}+1}
$$

Some types of $U_{1}$ and $U_{2}$ equations are examined in order to find the best multinomial Logit regressions. The types of $U$ 's function are formulated as follows

\section{Type B-ML.I}

$U_{1}=\beta_{0}+\beta_{1-1} \cdot\left(T O T A L_{-} O N S\right)+\beta_{2-1} \cdot\left(T O T A L_{-} O F F S\right)+\beta_{3-1} \cdot\left(L O A D_{-} A R R\right)+\beta_{4-1} \cdot(L F)+$

$+\beta_{5-1} \cdot(P U N T)+\beta_{6-1} \cdot(T I M E)+\beta_{7-1} \cdot($ SEASON $)+\beta_{8-1} \cdot($ STOP_LOCA $)$

$U_{2}=\beta_{0}+\beta_{1-2} .\left(T O T A L_{-} O N S\right)+\beta_{2-2} .\left(T O T A L_{-} O F F S\right)+\beta_{3-2} .\left(L O A D_{-} A R R\right)+\beta_{4-2} .(L F)+$

$+\beta_{5-2} \cdot(P U N T)+\beta_{6-2} \cdot($ TIME $)+\beta_{7-2} \cdot($ SEASON $)+\beta_{8-2} \cdot($ STOP $-L O C A)$

\section{$\underline{\text { Type B- ML.2 }}$}

$U_{1}=\beta_{0}+\beta_{1-1} \cdot\left(T O T A L_{-} O N S\right)+\beta_{2-1} .\left(T O T A L_{-} O F F S\right)+\beta_{3-1}\left(T O T A L_{-} O N\right)^{2}+\beta_{4-1} \cdot\left(L O A D_{-} A R R\right)+$

$\beta_{5-1 \cdot} \cdot(L F)++\beta_{6-1} \cdot(P U N T)+\beta_{7-1} \cdot(T I M E)+\beta_{8-1 \cdot} \cdot(S E A S O N)+\beta_{9-1} \cdot\left(S T O P \_L O C A\right)$

$U_{2}=\beta_{0}+\beta_{1-2 \cdot} \cdot\left(T O T A L_{-} O N S\right)+\beta_{2-2 \cdot} \cdot\left(T O T A L_{-} O F F S\right)+\beta_{3-2}(\text { TOTAL_ON })^{2}+$

$\beta_{4-2 \cdot} \cdot\left(L O A D_{-} A R R\right)+\beta_{5-2 \cdot} \cdot(L F)++\beta_{6-2} \cdot(P U N T)+\beta_{7-2} \cdot(T I M E)+\beta_{8-2} \cdot(S E A S O N)+\beta_{9-2} \cdot(S T O P+L O C A)$

\section{Type B-ML.3}

$U_{1}=\beta_{0}+\beta_{1-1} \cdot($ TOTAL_ONS $)+\beta_{2-1} \cdot($ TOTAL_OFFS $)+\beta_{3-1}(\text { TOTAL_ON })^{2}+\beta_{4-1}(\text { TOTAL_OFF })^{2}$

$+\beta_{5-1}($ TOTAL_ONS $)($ TOTAL_OFFS $)+\beta_{6-1} \cdot($ LOAD _ ARR $)+\beta_{7-1} \cdot(L F)+$

$+\beta_{8-1} \cdot(P U N T)+\beta_{9-1} \cdot($ TIME $)+\beta_{10-1} \cdot($ SEASON $)+\beta_{11-1} \cdot($ STOP_LOCA $)$ 
In order to find the coefficients of the regression types above, APC data from 7 stops on the bus route 95 were used. Total number of boarding and alighting passengers at each door of the bus at each bus stop were calculated and compared with those of the other doors. If a door is the busiest door, it is coded as 1 and the remaining doors are coded as zeros. By using the multinomial logistic regression function of the SPSS 13.0 software for each type of Us named as B-ML.1, B-ML.2 and B-ML.3, we have the best Multinomial Logistic Regression for each. The results of these procedures are tabulated in Table 5.10. Please see Appendix B3 for the detail of each type.

Table 5.10: The Best Multinomial Logistic Regression Equations

\begin{tabular}{|c|c|c|c|c|}
\hline Type & The Best Logistic Regression Equation & $\underset{\text { deviance }}{\chi^{2}-}$ & $\begin{array}{c}\text { McFaden } \\
R \\
\text { square }\end{array}$ & $\begin{array}{c}\text { Overall } \\
\text { Avcuracy }\end{array}$ \\
\hline $\begin{array}{c}\text { B- } \\
\text { ML.1 }\end{array}$ & $\begin{array}{l}U_{1}=1.302+0.098 * \text { TOTAL_ONS }-0.308 * \text { TOTAL_OFFS }- \\
6.10^{-3} * \text { LOAD_ARR }-0.105 * \text { TIME }+6.7 .10^{-3} * \text { SEASON }+ \\
0.549 * \text { STOP_LOCA } \\
U_{2}=-0.133-0.001^{*} \text { TOTAL_ONS-0.023*TOTAL_OFFS+ } \\
0.004^{*} \text { LOAD_ARR-0.114*TIME-0.022*SEASON+0.057*STOP_LOCA }\end{array}$ & 10360.49 & 0.193 & 62.3 \\
\hline $\begin{array}{c}\text { B- } \\
\text { ML.2 }\end{array}$ & $\begin{array}{l}U_{1}=1.602+1.50 * \text { TOTAL_ONS }-0.30 * \text { TOTAL_OFFS }-0.003 * \text { TOTAL_ON }{ }^{2}- \\
0.013^{*} \text { LOAD_ARR }-0.116 * \text { TIME }+ \\
0.122 * \text { SEASON }+0.659 * \text { STOP_LOCA } \\
\left.U_{2}=-0.228+0.016\left(T O T A L_{-} O N S\right)-0.023 .\left(T O T A L_{-} O F F S\right)+0.003 L O A D_{-} A R R\right) \\
-0.104 .(T I M E)-0.011(S E A S O N)+0.034 .\left(S T O P_{-} L O C A\right)\end{array}$ & 8352.10 & 0.194 & 65.2 \\
\hline $\begin{array}{c}\text { B- } \\
\text { ML.3 }\end{array}$ & 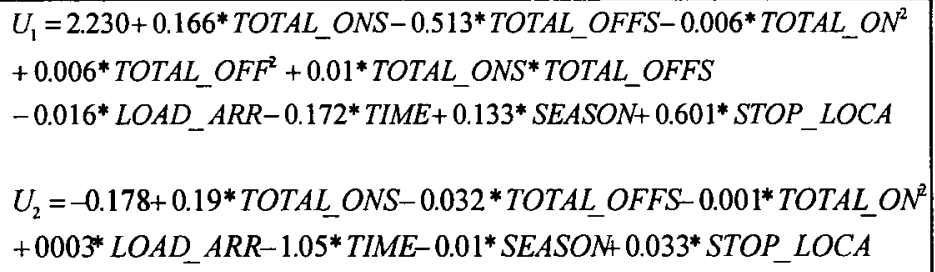 & 861.85 & 0.224 & 65.9 \\
\hline
\end{tabular}


As shown in Table 5.10, type ML-3 provides the largest $M c F a d d d e n \boldsymbol{R}_{L}^{2}$ of 0.224 and the overall accuracy of 65.9 percent. Therefore, type ML-3 is suggested for busiest door prediction of articulated bus. Also, it works fairly well, as indicated by $\chi^{2}$ of 861.847 and $M c F a d d d e n \boldsymbol{R}_{L}^{2}$ of 0.224

The overall Likelihood Ratio tests of this type are presented in Table 5.11 on the basis of SPSS application. The results show that all parameters are statistically significant, except the variable $P U N T$.

Table 5.11: Likelihood Ratios of type B-ML.3

\begin{tabular}{|c|c|c|c|c|c|c|}
\hline \multirow{2}{*}{ Effect } & \multicolumn{2}{|c|}{ Model Fitting Criteria } & \multicolumn{2}{c|}{ Likelihood Ratio Tests } \\
\cline { 2 - 7 } & $\begin{array}{c}\text { AIC of } \\
\text { Reduced } \\
\text { Model }\end{array}$ & $\begin{array}{c}\text { BIC of } \\
\text { Reduced } \\
\text { Model }\end{array}$ & $\begin{array}{c}-2 \text { Log } \\
\text { Likelihood of } \\
\text { Reduced } \\
\text { Model }\end{array}$ & Chi-Square & df & Sig. \\
\hline Intercept & 8372.224 & 8503.287 & 8332.224 & 285.443 & 2 & .000 \\
\hline LOAD_ARR & 8090.781 & 8234.950 & $8046.781(\mathrm{a})$ & .000 & 0 &. \\
\hline LF & 8090.781 & 8234.950 & $8046.781(\mathrm{a})$ & .000 & 0 &. \\
\hline PUNT & 8086.832 & 8217.895 & 8046.832 & .051 & 2 & .975 \\
\hline SEASON & 8104.913 & 8235.976 & 8064.913 & 18.132 & 2 & .000 \\
\hline STOP_LOCATE & 8143.819 & 8274.882 & 8103.819 & 57.038 & 2 & .000 \\
\hline TIME & 8099.926 & 8230.989 & 8059.926 & 13.145 & 2 & .001 \\
\hline TOTAL_ONS & 8213.854 & 8344.917 & 8173.854 & 127.073 & 2 & .000 \\
\hline TOTAL_OFFS & 9061.769 & 9192.832 & 9021.769 & 974.988 & 2 & .000 \\
\hline Total_On_squared & 8205.349 & 8336.412 & 8165.349 & 118.568 & 2 & .000 \\
\hline TotalOffPw & 8125.214 & 8256.277 & 8085.214 & 38.433 & 2 & .000 \\
\hline TOTALOn_and_OFF & 8191.669 & 8322.732 & 8151.669 & 104.888 & 2 & .000 \\
\hline
\end{tabular}

The chi-square statistic is the difference in -2 log-likelihoods between the final model and a reduced model. The reduced model is formed by omitting an effect from the final model. The null hypothesis is that all parameters of that effect are 0 .

a This reduced model is equivalent to the final model because omitting the effect does not increase the degrees of freedom.

As shown in Table 5.12, the probabilities of front door and the first rear door are both reduced when more passengers get off the buses, but the front door has a larger reduction. In contrast, these probabilities increase when more passengers get on the bus 
and the influence on the front door has a larger increment. Interestingly, location of bus stop has a quite strong influence on passenger's door choice. This can be seen shown in Logit (door_1_busiest) and Logit (door_2_busiest) of 1.824 and 1.033, if the stop is located in the CBD area.

Table 5.12: Parameters of Type B-ML.3

\begin{tabular}{|c|c|c|c|c|c|c|c|c|c|}
\hline \multicolumn{10}{|c|}{ Parameter Estimates } \\
\hline & & \multirow[b]{2}{*}{ B } & \multirow[b]{2}{*}{ Std. Error } & \multirow[b]{2}{*}{ Wald } & \multirow[b]{2}{*}{ df } & \multirow[b]{2}{*}{ Sig. } & \multirow[b]{2}{*}{$\operatorname{Exp}(B)$} & \multicolumn{2}{|c|}{$\begin{array}{l}\text { 95\% Confidence Interval for } \\
\text { Exp(B) }\end{array}$} \\
\hline \multicolumn{2}{|c|}{ Busiest_doof } & & & & & & & Lower Bound & Upper Bound \\
\hline \multirow{12}{*}{ door 2 busiest } & Intercept & -.178 & $\begin{array}{l}.179 \\
\end{array}$ & .993 & 1 & .319 & & & \\
\hline & LOAD_ARR & .003 & .003 & 1.300 & 1 & .254 & 1.003 & .998 & 1.008 \\
\hline & LF & $0^{\mathrm{b}}$ & & & 0 & & & & \\
\hline & PUNT & .000 & .000 & .008 & 1 & .930 & 1.000 & .999 & 1.001 \\
\hline & SEASON & -.010 & .039 & .061 & 1 & .806 & .990 & .918 & 1.069 \\
\hline & STOP_LOCATE & .033 & .092 & .127 & 1 & .721 & 1.033 & .863 & 1.237 \\
\hline & TIME & -.105 & .051 & 4.232 & 1 & .040 & .901 & .815 & .995 \\
\hline & TOTAL_ONS & .019 & .017 & 1.276 & 1 & .259 & 1.019 & .986 & 1.054 \\
\hline & TOTAL_OFFS & -.032 & .014 & 5.113 & 1 & .024 & .969 & .942 & .996 \\
\hline & Total_On_squared & -.001 & .001 & 1.115 & 1 & .291 & .999 & .998 & 1.000 \\
\hline & TotalOffPw & .000 & .000 & .621 & 1 & .431 & 1.000 & 1.000 & 1.001 \\
\hline & TOTALon_and_OF & .000 & .001 & .112 & 1 & .738 & 1.000 & .998 & 1.001 \\
\hline \multirow[t]{12}{*}{ Door 1 busiest } & Intercept & 2.230 & .159 & 195.865 & 1 & .000 & & & \\
\hline & LOAD_ARR & -.016 & .002 & 43.579 & 1 & .000 & .984 & .979 & .989 \\
\hline & LF & $0^{b}$ & & & 0 & & & & \\
\hline & PUNT & .000 & .000 & .022 & 1 & .882 & 1.000 & .999 & 1.001 \\
\hline & SEASON & .133 & .036 & 13.534 & 1 & .000 & 1.142 & 1.064 & 1.226 \\
\hline & STOP_LOCATE & .601 & .088 & 47.085 & 1 & .000 & 1.824 & 1.536 & 2.166 \\
\hline & TIME & -.172 & .048 & 12.841 & 1 & .000 & .842 & .767 & .925 \\
\hline & TOTAL_ONS & .166 & .016 & 102.289 & 1 & .000 & 1.181 & 1.143 & 1.219 \\
\hline & TOTAL_OFFS & -.513 & .018 & 851.036 & 1 & .000 & .599 & .578 & .620 \\
\hline & Total_On_squared & -.006 & .001 & 92.083 & 1 & .000 & .994 & .993 & .995 \\
\hline & TotalOffPw & .006 & .001 & 53.559 & 1 & .000 & 1.006 & 1.004 & 1.007 \\
\hline & TOTALon_and_OF & .010 & .001 & 72.065 & 1 & .000 & 1.010 & 1.007 & 1.012 \\
\hline
\end{tabular}

Following the best multinomial logistic regression type, we can estimate the dwell time at the busiest door, as shown in Equations 5.60 and 5.61.

$$
\begin{gathered}
d w e l l=P_{\text {Busiest_door }}(\text { TOTAL_ONS }) t_{a}+P_{\text {Busiest_door }}\left(\text { TOTAL_OFFS } t_{b}+t_{o p}\right. \\
P_{\text {Busiest_door }}=\operatorname{Max}\left(P_{1}, P_{2} \text { and } P_{3}\right)
\end{gathered}
$$


$P_{1}, P_{2}, P_{3}$ are the probabilities that the busiest door is the front door, the first rear door and the second rear door defined in Equations 5.51 to 5.53 and type B-ML.3, respectively.

\subsubsection{Method A vs. Method B and the Selection}

Two methods (A\& B) and their components have been developed to predict dwell time in the previous sections. Method $\mathrm{A}$ is based on the best dwell time regression function (i.e., type A-2.3) to examine the relationship between dwell time and the number of boarders and alighters as well as other variables. Method B is based on the revisions on busiest door selection method. Once the busiest door is selected on the basis of probability, dwell time is predicted by using the predicted number of boarders and alighters at that door as shown in Equations 5.45 and 5.46 for rigid-body buses or Equations 5.60 and 5.61 for articulated buses. In order to find enhanced methodology for use in this research, two methods are compared, based on their prediction performance in terms of the overall accuracy.

Overall accuracy can be determined by calculating one of the measures presented in Table 5.13. In this study, the mean absolute percentage error (MAPE) is used as a yardstick to select the better of the two methods. 
Table 5.13: Measures of Accuracy

\begin{tabular}{|c|l|}
\hline Measure & \multicolumn{1}{|c|}{ Equation } \\
\hline $\begin{array}{c}\text { MAE } \\
\text { Mean Absolute }\end{array}$ & $M A E=\frac{1}{N} \sum\left|x_{i}-x_{i}\right|$ \\
\hline $\begin{array}{c}\text { MAPE } \\
\text { Mean Absolute Percentage } \\
\text { Error }\end{array}$ & $M A P E=\left(\frac{1}{N} \sum_{i=1}^{N} \frac{\left|x_{i}-\dot{\Delta}\right|}{x_{i}}\right) \cdot 100$ \\
\hline $\begin{array}{c}\text { MRE } \\
\text { Maximum Relative Error }\end{array}$ & $M R E=\max _{i} \frac{\left|x_{i}-x_{i}\right|}{x_{i}}$ \\
\hline $\begin{array}{c}\text { RMSE } \\
\text { Root Mean Square Error }\end{array}$ & $R M S E=\sqrt{\frac{1}{N} \sum_{i=1}^{N}\left(x_{i}-\dot{x}_{i}\right)^{2}}$ \\
\hline
\end{tabular}

\subsubsection{Method A}

In method A, as shown in type A-2.3, dwell times are predicted as below:

$$
\begin{aligned}
& \left.d w e l l=\underset{t=13.921}{14.434}+\underset{t=54.071}{2.014}(\text { TOTAL_ON })-\underset{t=-17.944}{0.026}(\text { TOTAL_ON })^{2}+\underset{t=15.364}{0.516\left(T O T A L_{-}\right.} \text {OFFS }\right)+
\end{aligned}
$$

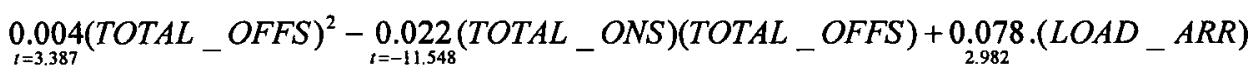

$$
\begin{aligned}
& \left.-5.047(L F)+\underset{t=5.492}{0.003(P U N T)}-\underset{t=-12.295}{4.748}(\text { DOORS })+\underset{t=10384}{2.087(S T O P} \_L O C A\right)+\underset{t=2.419}{0.196(S E A S O N)}
\end{aligned}
$$

\subsubsection{Method B}

While method A predicts dwell time without clustering data, method B clusters data into two samples, one for rigid-body bus and the other for articulated bus.

The values $t_{a} t_{b}$, are in accordance with the HCM (2000) suggestions (Exhibit 279, p.27-10) as shown in Table 5.14. 
Table 5.14: Average Service Time Selection(s/p)

\begin{tabular}{|c|c|c|c|c|}
\hline \multirow{2}{*}{ Bus Type } & \multicolumn{2}{|c|}{$\begin{array}{c}\text { Average dwell time per boarding } \\
\text { passenger through }\end{array}$} & \multicolumn{2}{c|}{$\begin{array}{c}\text { Average dwell time per alighting } \\
\text { passenger through }\end{array}$} \\
\cline { 2 - 5 } & Front door & Rear door & Front Door & Rear Door \\
\hline Rigid-Body Bus & 1.2 & NA & 0.9 & 0.9 \\
\hline Articulated Bus & 0.9 & 0.6 & 0.6 & 0.6 \\
\hline
\end{tabular}

\subsubsection{Rigid-Body Bus}

In the case of rigid-body bus, dwell times are predicted by using Equations 5.45 to 5.47 and the logistic regression type namely B-L.1. Table 5.15 shows a fraction extracted from the outputs of two dwell time prediction methods applied for rigid-body buses running on bus route 1 , the OC Transpo, Canada.

\subsubsection{Articulated Bus}

In case of articulated bus, dwell times are predicted by using Equations 5.60 and 5.61 and the logistic regression type namely B-ML3 whose multinomial logistic regression functions are:

$$
\begin{aligned}
& U_{1}=2.230+0.166^{*} \text { TOTAL_ONS }-0.513 * \text { TOTAL_OFFS }-0.006 * \text { TOTAL_ON }{ }^{2}+ \\
& 0.006^{*} \text { TOTAL_OFF }{ }^{2}+0.01 * \text { TOTAL_ONS *TOTAL_OFFS }-0.016^{*} \text { LOAD_ARR - } \\
& 0.172 * \text { TIME }+0.133 * \text { SEASON +0.601*STOP_LOCA } \\
& U_{2}=-0.178+0.19 * T O T A L \_O N S-0.032 *{ }^{*} \text { TOTAL_OFFS }-0.001^{*} \text { TOTAL_ON }{ }^{2} \\
& +0003 * L O A D \_A R R-1.05 * \text { TIME }-0.01 * S E A S O N+0.033 * S T O P{ }_{2} \text { LOCA }
\end{aligned}
$$


Table 5.15: Example Output of Dwell time Prediction of Method A and Method B Applied for Rigid-body-Bus

\begin{tabular}{|c|c|c|c|c|c|c|}
\hline $\begin{array}{c}\text { Bus } \\
\text { Stop }\end{array}$ & $\begin{array}{c}\text { Actual_Arr_ } \\
\text { Time }\end{array}$ & Expec_Time & Date & $\begin{array}{c}\text { Actual_ } \\
\text { Dwell } \\
\text { Time (s) }\end{array}$ & $\begin{array}{c}\text { Predicted_- } \\
\text { dwell_- } \\
\text { Method A } \\
\text { (s) }\end{array}$ & $\begin{array}{c}\text { Predicted_ } \\
\text { dwell_ } \\
\text { Method B(s) }\end{array}$ \\
\hline CH080 & 33105 & 32880 & $9 / 14 / 2004$ & 7 & 11.88 & 6.07 \\
\hline CF630 & 32700 & 32580 & $9 / 14 / 2004$ & 6 & &. \\
\hline CD920 & 33840 & 33600 & $9 / 16 / 2004$ & 19 & 27.84 & 24.03 \\
\hline RA190 & 30375 & 30540 & $9 / 24 / 2004$ & 2 & 4.39 & 2.65 \\
\hline CC190 & 29220 & 29280 & $10 / 5 / 2004$ & 42 & 33.67 & 41.6 \\
\hline RF075 & 31500 & 31500 & $10 / 6 / 2004$ & 5 & 7.29 & 2.46 \\
\hline CC190 & 33570 & 32880 & $10 / 8 / 2004$ & 7 & 10.04 & 6.35 \\
\hline CC190 & 30510 & 30480 & $10 / 8 / 2004$ & 28 & 18.49 & 22.69 \\
\hline CD920 & 33240 & 32400 & $10 / 8 / 2004$ & 15 & 26.3 & 18.29 \\
\hline CF630 & 29085 & 28980 & $10 / 8 / 2004$ & 4 & 10.07 & 4.21 \\
\hline CH080 & 29520 & 29280 & $10 / 8 / 2004$ & 9 & 8.34 & 4.8 \\
\hline CH080 & 32490 & 31680 & $10 / 8 / 2004$ & 12 & 15.26 & 7.59 \\
\hline CD920 & 30330 & 30000 & $10 / 14 / 200$ & 4 & 9.37 & 4.2 \\
\hline CH080 & 29595 & 29280 & $10 / 14 / 2004$ & 9 & 8.95 & 3.99 \\
\hline CH080 & 29460 & 29280 & $10 / 18 / 2004$ & 10 & 13.61 & 8.63 \\
\hline CD920 & 32850 & 32400 & $10 / 20 / 2004$ & 30 & 32.03 & 23 \\
\hline RA190 & 31680 & 31740 & $10 / 20 / 2004$ & 4 & 5.48 & 3.46 \\
\hline RA945 & 31140 & 31080 & $10 / 20 / 2004$ & 12 & 8.71 & 3.95 \\
\hline RA945 & 31110 & 31080 & $10 / 20 / 2004$ & 10 & 16.14 & 9.19 \\
\hline CH080 & 31980 & 31680 & $10 / 20 / 2004$ & 11 & 15.43 & 9.42 \\
\hline RA190 & 30480 & 30540 & $10 / 25 / 2004$ & 10 & 11.73 & 7.59 \\
\hline RA945 & 31125 & 31080 & $10 / 25 / 2004$ & 31 & 32.88 & 25.4 \\
\hline CF630 & 31605 & 31380 & $10 / 25 / 2004$ & 7 & 10.62 & 4.94 \\
\hline RA945 & 34635 & 34680 & $10 / 27 / 2004$ & 5 & 6.42 & 2.71 \\
\hline RA945 & 34560 & 34680 & $10 / 27 / 2004$ & 25 & 23.97 & 16.1 \\
\hline CF630 & 35025 & 35100 & $10 / 27 / 2004$ & 2 & 8.08 & 2.93 \\
\hline CD920 & 33690 & 33600 & $11 / 1 / 2004$ & 13 & 17.52 & 10.74 \\
\hline CH080 & 33105 & 32880 & $11 / 1 / 2004$ & 7 & 10.87 & 4.69 \\
\hline
\end{tabular}

Table 5.16 shows another fraction of the outputs of the two dwell time prediction methods applied for articulated buses running on bus route 95 , the OC Transpo, Canada. 
Table 5.16: Example Output of Dwell time Prediction of Method A and Method B Applied for Articulated Bus

\begin{tabular}{|c|c|c|c|c|c|c|}
\hline $\begin{array}{c}\text { Bus } \\
\text { Stop }\end{array}$ & Actual_Arr_Time & Expec_Time & Date & $\begin{array}{c}\text { Actual_- } \\
\text { Dwell_ } \\
\text { Time (s) }\end{array}$ & $\begin{array}{c}\text { Predicted } \\
\text { dwell_thod A } \\
\text { (s) }\end{array}$ & $\begin{array}{c}\text { Predicted } \\
\text { dwell } \\
\text { Method B } \\
\text { s) }\end{array}$ \\
\hline EE915 & 29475 & 29400 & $9 / 7 / 2004$ & 19 & 23.41 & 7.73 \\
\hline CJ900 & 30975 & 30720 & $9 / 7 / 2004$ & 9 & 9.91 & 4.9 \\
\hline CA920 & 30780 & 30420 & $9 / 7 / 2004$ & 7 & 14.67 & 6.64 \\
\hline CA920 & 31620 & 31440 & $9 / 8 / 2004$ & 9 & 10.61 & 5.38 \\
\hline CJ900 & 31830 & 31740 & $9 / 8 / 2004$ & 16 & 7.07 & 3.65 \\
\hline NI910 & 32415 & 32340 & $9 / 8 / 2004$ & 9 & 5.04 & 3.56 \\
\hline EB905 & 30615 & 30600 & $9 / 8 / 2004$ & 16 & 20.05 & 6.95 \\
\hline CD910 & 31245 & 31140 & $9 / 8 / 2004$ & 3 & 6.7 & 3.86 \\
\hline CJ900 & 33345 & 33300 & $9 / 9 / 2004$ & 4 & 4.6 & 3.23 \\
\hline EB905 & 32250 & 32160 & $9 / 9 / 2004$ & 8 & 15.51 & 8.23 \\
\hline CD910 & 32775 & 32700 & $9 / 9 / 2004$ & 24 & 18.24 & 7.31 \\
\hline EE915 & 35730 & 35820 & $9 / 10 / 2004$ & 26 & 16.07 & 5.86 \\
\hline EE915 & 31575 & 31500 & $9 / 10 / 2004$ & 21 & 11.69 & 5.89 \\
\hline EB905 & 31785 & 31680 & $9 / 10 / 2004$ & 7 & 7.83 & 4.62 \\
\hline CA920 & 32730 & 32520 & $9 / 10 / 2004$ & 8 & 11.41 & 7.12 \\
\hline CA920 & 30480 & 30240 & $9 / 10 / 2004$ & 11 & 11.02 & 4.41 \\
\hline N1910 & 32505 & 32340 & $9 / 10 / 2004$ & 2 & 3.27 & 2.8 \\
\hline N1910 & 32490 & 32340 & $9 / 10 / 2004$ & 3 & 2.28 & 2.86 \\
\hline N1910 & 31410 & 31080 & $9 / 10 / 2004$ & 3 & 4.26 & 3.25 \\
\hline CE940 & 32100 & 31980 & $9 / 10 / 2004$ & 3 & 3.14 & 2.74 \\
\hline EB905 & 30585 & 30600 & $9 / 10 / 2004$ & 5 & 8.71 & 5.81 \\
\hline N1910 & 35940 & 35940 & $9 / 14 / 2004$ & 2 & 2.92 & 3.1 \\
\hline CJ900 & 35400 & 35340 & $9 / 14 / 2004$ & 1 & 3.57 & 2.52 \\
\hline EE915 & 31575 & 31500 & $9 / 15 / 2004$ & 17 & 28.93 & 11.17 \\
\hline EB905 & 31770 & 31680 & $9 / 15 / 2004$ & 8 & 13.07 & 4.73 \\
\hline CE940 & 32115 & 31980 & $9 / 15 / 2004$ & 12 & 15.65 & 8.11 \\
\hline CJ900 & 32100 & 32100 & $9 / 17 / 2004$ & 25 & 9.14 & 4.93 \\
\hline N1910 & 32655 & 32700 & $9 / 17 / 2004$ & 6 & 5.53 & 4.27 \\
\hline CD910 & 31575 & 31500 & $9 / 17 / 2004$ & 21 & 20.94 & 5.94 \\
\hline CD910 & 33255 & 32880 & $9 / 17 / 2004$ & 11 & 13.09 & 8.34 \\
\hline C5900 & 33585 & 33420 & $9 / 20 / 2004$ & 2 & 3.88 & 2.53 \\
\hline
\end{tabular}

\subsubsection{Accuracy Performance of the Two Methods}

Obviously, the higher the value of MAPE, the poorer the prediction is. We can see from Table 5.17 that while method $\mathrm{A}$ is quite poor in predictions for rigid bus (i.e. 
MAPE up to $59.80 \%$ ) it works well for predicting dwell time for articulated buses with MAPE as low as $35.79 \%$. In contrast, method B outperforms method A in case of rigidbody bus (MAPE $=32.42 \%$ ) as compared to that of articulated bus (MAPE=45.72\%).

Table 5.17: Mean Absolute Prediction Error of Method A vs. Method B

\begin{tabular}{|l|c|c|c|}
\hline \multirow{2}{*}{ Bus Type } & \multicolumn{2}{|c|}{ MAPE (\%) } & \multirow{2}{*}{ 20\% sample size } \\
\cline { 2 - 3 } & Method A & Method B & \\
\hline Rigid-body-Bus & 59.80 & 32.42 & 473 cases \\
\hline Articulated Bus & 35.79 & 45.72 & 1846 cases \\
\hline
\end{tabular}

As a result, method $\mathrm{A}$ is suggested for predicting dwell time for an articulated bus while method B should be used for a rigid-body bus.

\subsection{Summary}

A comprehensive research methodology and proof of concept analyses on dwell time predictions are reported in this chapter.

Dwell time in previous works found in the literature was usually considered as delay and its values were defined by multiple regressions where the most influencing variables are total alighters and boarders. When using the models developed in the past to predict dwell time at a stop, the analyst is asked to enter the number of passengers boarding and alighting at that stop. Unfortunately, these numbers are not available because the bus has yet come to the stop. The dwell time prediction model developed in this chapter is a dynamic model because it can predict the number of boarding and alighting passengers. Based on the statistical pattern recognition technique, the historical 
data and the up-to-minute data transmission from the APC and AVL systems, the predictions of passengers' activities are predicted and updated dynamically by the two sub-modules, which were called the RBSM and the RASM.

Method A and method B were developed to examine the fact that dwell time has a complicated relationship with many characteristics of passenger activities and service activities. A series of regression types varying from multiple linear regression, namely non-linear regression, binary Logit regression, and multinomial Logistic regressions were applied to explore this relationship. Data collected by the APC system mounted on the OC Transpo buses were used as the inputs to these methods. The comparisons among them show that method A (i.e. non-linear regression model) outperformed method B (i.e., Multinomial Logit regression) in predicting dwell time for the articulated bus. On the other hand, method B (i.e. binary Logit) is suitable for the rigid-body bus.

The performance of boarding passenger prediction and alighting passenger prediction sub-modules is presented in the next chapter. 


\section{Chapter 6}

\section{MODEL PERFORMANCE}

\subsection{Introduction}

In the previous chapters, the study framework, the methodologies, the mathematical algorithms, and the detailed procedures for each constituent element of the developed model have been presented. Prior to application, it should be evaluated in terms of accuracy and reliability. From the accuracy perspective, the model's predictions on bus arrival time should be evaluated on the basis of several measurements of goodness-of-fit. Furthermore, these predictions would also be compared with predictions resulting from the use of other predictors, given the same input data. In terms of reliability, the developed model should be tested with different bus running scenarios in order to assess if it is a versatile model and if its predictions are reliable under any scenario.

This chapter presents information on the conversion of the developed mathematical prediction algorithms into computer programs, simulation of several bus operational scenarios, and evaluation of the developed model's performance based on simulated as well as real-world data. 


\subsection{Computer Program Development}

The developed model was coded in Matlab computer language, version 7.0. The computer program consists of two main parts, called "functions" by Matlab. Each function contains several sub-functions in order to make the whole program work efficiently. The structure of the computer program, its functions, and sub-functions are shown in Figure 6.1. The source codes for each can be seen in Appendix $\mathbf{C}$ of this document.

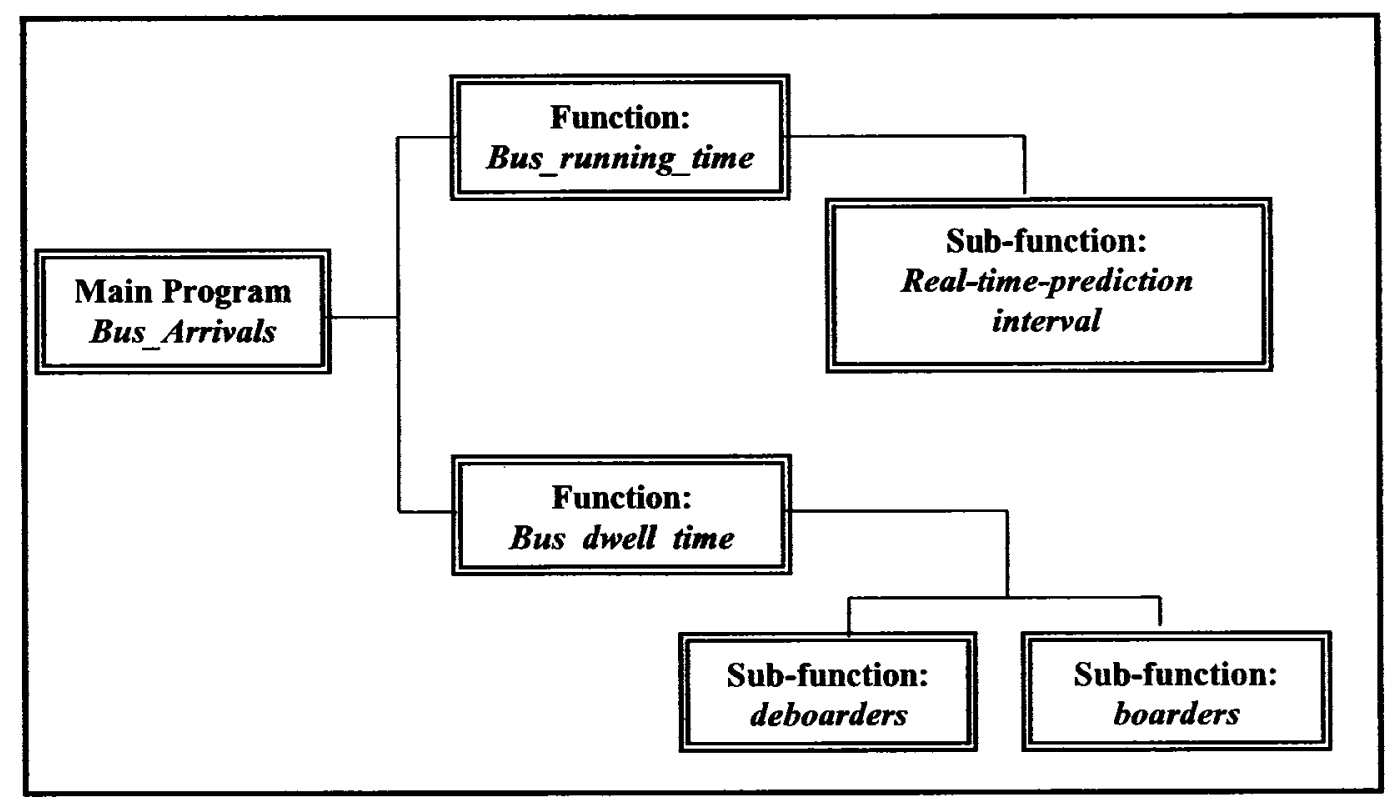

Figure 6.1: Structure and Constituent Functions of the Computer Program

\subsection{Simulations of Bus Operation Scenarios}

A number of bus operation scenarios that are difficult to carry out as field experiments were simulated. The simulated bus arrival times, dwell times, and passenger activities were used to test the developed model in term of its accuracy and reliability. 
Three bus operation scenarios were set up. These are: bus running under normal traffic condition, bus running under incident condition, and bus running under high passenger demand at some busy stops. It should be noted that the purpose of these simulations is to experience randomness of real-life bus operations generated by simulation tools. This approach goes beyond the analysis of a single operation case.

In order to obtain simulated data, transit routes were coded in suitable traffic software which was calibrated before being used for simulation purposes.

\subsubsection{VISSIM Simulator}

VISSIM is a popular microscopic traffic simulation software, Verkerhr In Stadten-SIMulation (PTV 2004). Like other traffic microsimulators such as CORSIM, SIMtraffic, etc., a key feature of VISSIM is the capability of simulating the randomness of traffic flow. The vehicle-following, lane-changing, and route selection components of VISSIM are recognized to mimic the real-world traffic operations. Furthermore, the flexibility and convenience of modelling interactions such as stopping, yielding, queuing, and passing, as well as the plentiful user-defined parameters, all make VISSIM to be a very useful traffic simulation tool.

VISSIM was chosen for this study due to not only attributes noted earlier but also owing to capability to simulate transit. Other simulators have limitations in this aspect. With VISSIM, a public transit planner can define different bus routes for the same road; each with corresponding time-based schedule or headway-based schedule. At each bus stop, passenger activities such as dwell time, number of boarders and de-boarders can be defined separately following either normal distribution or empirical distributions for 
every single bus route sharing the same bus stop. Furthermore, VISSIM allows the users to define various types of acceleration and deceleration characteristics of the buses under study as well as bus driver behaviour. These enable transit planners and operators to predict effects of locations of transit facilities and passenger activities, generation of the real-time traveller information, and the study of operational alternatives before actual implementation. Table 6.1 compares some transit-related features of VISSIM versus CORSIM, the other commonly used micro-simulation software.

Table 6.1: Comparison of VISSIM (version 4.10) and CORSIM (TSIS 5.1) for public transit simulation

\begin{tabular}{|l|l|l|}
\hline \multicolumn{1}{|c|}{ Module } & \multicolumn{1}{|c|}{ VISSIM } & \multicolumn{1}{c|}{ CORSIM } \\
\hline Dispatching buses & $\begin{array}{l}\text { - Headway-based schedule } \\
\text { - Time-based schedule }\end{array}$ & $\begin{array}{l}\text { - Headway-based schedule } \\
- \text { N/A }^{6}\end{array}$ \\
\hline Distribution of dwell time & $\begin{array}{l}\text { - Normal Distribution } \\
\text { - Empirical distribution }\end{array}$ & $\begin{array}{l}\text {-Mean dwell time and } \\
\text { distribution tables. }\end{array}$ \\
\hline Estimate dwell time & $\begin{array}{l}\text { - Additive method } \\
\text { - Maximum method }\end{array}$ & $\begin{array}{l}\text { - Additive method } \\
\text { - N/A }\end{array}$ \\
\hline $\begin{array}{l}\text { Estimate number of } \\
\text { boarders and de-boarders }\end{array}$ & $\begin{array}{l}\text { - Number of boarders and } \\
\text { de-boarders }\end{array}$ & - N/A \\
\hline $\begin{array}{l}\text { Estimate number of waiting } \\
\text { passenger }\end{array}$ & - Capability exists & - N/A \\
\hline $\begin{array}{l}\text { Calculate passenger waiting } \\
\text { time }\end{array}$ & - Capability exists & - N/A \\
\hline $\begin{array}{l}\text { Number of dedicated bus } \\
\text { lanes }\end{array}$ & - 2 or more & - only one \\
\hline
\end{tabular}

Notes

Headway-based schedule: Users can define headway (or service frequency) and start trip number. Time-based schedule: Users can define the time the bus is dispatched at all bus terminals.

3. Empirical distribution: Users can define empirical distribution of dwell time at any bus stop for any bus route based on actual dwell time.

4. Additive method: Dwell time is calculated by additional boarding and alighting times.

5. Maximum method: Dwell time is calculated based on the maximum service time for boarding or alighting passengers, otherwise sum of both. This is suitable for one directional door.

6. N/A : Not applicable 


\subsubsection{Transit Route Coding in VISSIM}

The two bus route segments of interest in this research, namely route 95 and route 1, were coded in VISSIM. Traffic and geometric data for each were provided by the City of Ottawa while the data on bus operations were obtained from APC systems of the OC Transpo. Several field surveys were also made at sites for which data were not available. A bitmap file of the routes' layout was imported into the simulator and the network geometry was built based on the background map using links and connectors. The traffic network coding required much effort because VISSIM specifies elaborate coding procedures for every single facility of the network. Other supplemental features such as number of lanes, speed limits, stop signs, right-turn-on-red, and actuated traffic signals, were also carefully coded. Furthermore, traffic and signal timings for intersections for the 2-hour peak period in the morning (8:00 a.m.-10:00 a.m.) were coded.

Since operation of the buses on selected routes was of much interest in this research, the kinetic characteristics of the actual buses (i.e., acceleration and deceleration rates) running on the OC Transpo network reported by Zargari (1997) and Zargari and Khan (2003) were used (see Table 6.2) instead of using VISSIM default parameters.

Table 6.2: Kinetic Characteristics of the $\mathrm{OC}$ bus

\begin{tabular}{|l|c|c|}
\hline \multicolumn{1}{|c|}{ Parameter } & $\begin{array}{c}\text { Maximum Acceleration } \\
\left(\mathbf{m} / \mathbf{s}^{\mathbf{2}}\right)\end{array}$ & $\begin{array}{c}\text { Maximum Deceleration } \\
\left(\mathbf{m} / \mathbf{s}^{\mathbf{2}}\right)\end{array}$ \\
\hline VISSIM defaults & 1.52 & 4.58 \\
\hline Zargari and Khan (2003) & 1.72 & 4.02 \\
\hline
\end{tabular}

In order to make the simulated bus operation to be more realistic, besides the selected bus stops for which the actual APC data on passenger activities (e.g., the number 
of boarders and alighters) were used as the VISSIM inputs, other supplemental bus stops were also included in the routes. For these stops, empirical distributions of dwell time at bus stops were used (e.g., mean, standard deviation). Also, real-world bus dispatching schedules were imported for each route. The two bus routes in the coded form are shown in Figure 6.2.

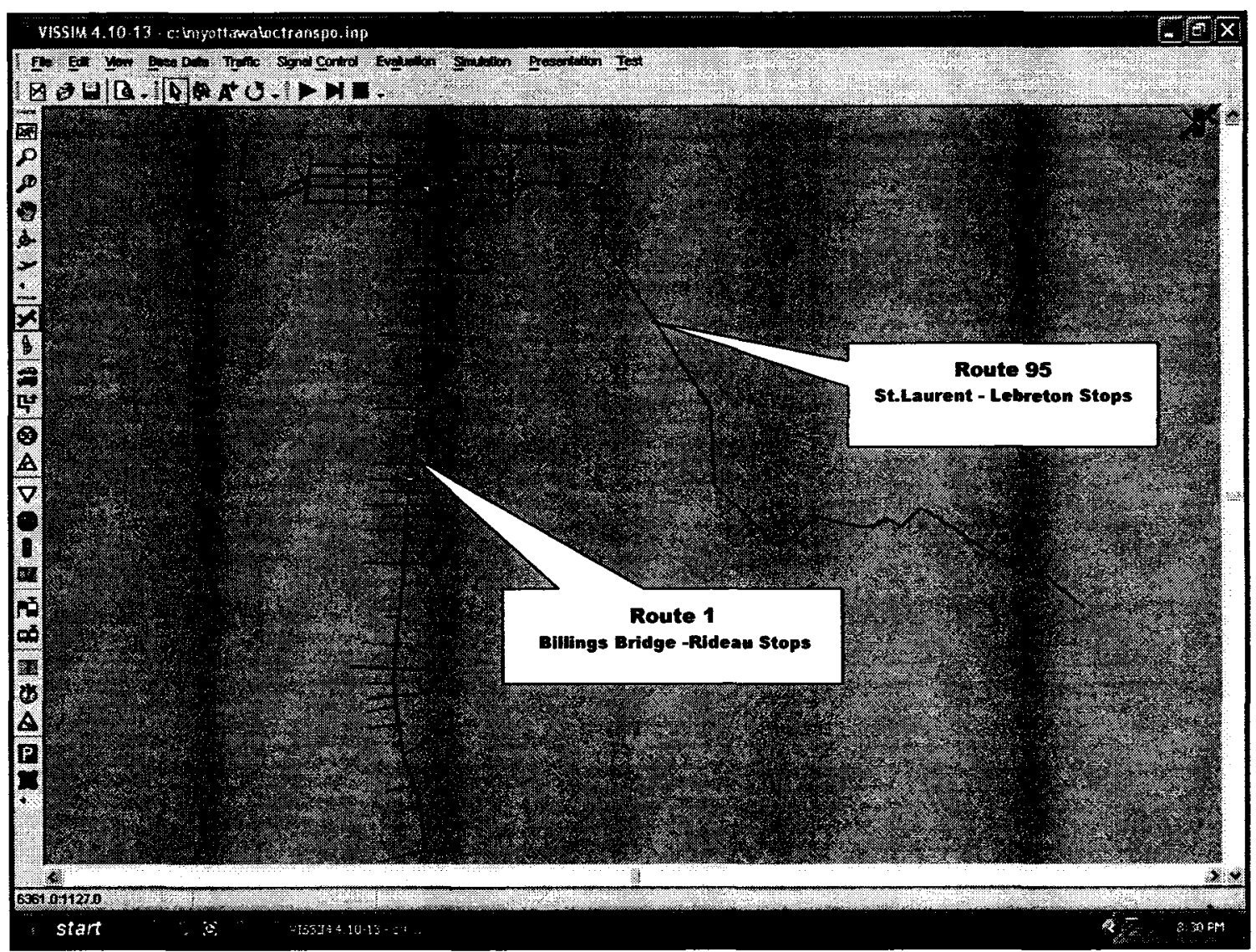

Figure 6.2: Bus Routes 95 and 1 coded in VISSIM 4.1

VISSIM can automatically record the history of every single bus running on the bus route at time steps as small as 0.1 second interval and tabulates the data into the builtin table, namely the Vehicle Record. The software can provide over 75 fields in a such a table varying from kinetic information of the vehicles to emissions, fuel consumption, 
passenger activities of public transit (i.e., dwell time, the number of boarders and deboarders, passenger waiting time, bus arrival time and departure time, etc.,), vehicle delay (e.g., queuing, number of stops, etc.,), and GPS data (e.g., X, Y, Z coordinates). Figure 6.3 shows the user-defined process in VISSIM for selecting transit bus parameters of the simulated network.

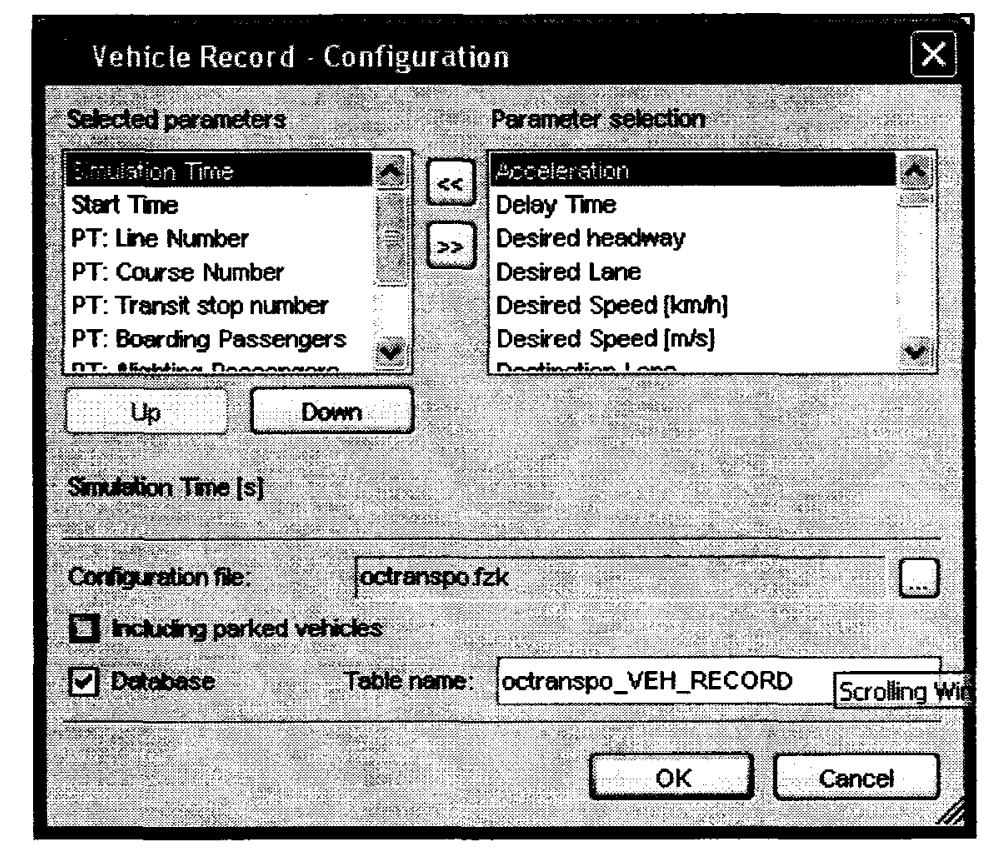

Figure 6.3 Vehicle Record-Configuration Tool in VISSIM

\subsubsection{Calibration and Validation}

Micro-simulation is useful but dangerous if it is not carried out properly (Fox, 2000). Although the purpose of simulations in this study is to take advantage of randomness of transportation data generated by VISSIM for the developed model testing, in order to enhance its value, the calibration is needed. The calibration process applied in this study follows the instructions provided by Dowling et al., (2004). 
The process started with on-screen animation so that coding errors could be easily detected. Each time an observed vehicle's behaviour appeared to be unrealistic such as unusual stop on the route, left turning without priority rule, unrealistic collision, or unrealistic congestion, the potential causes were analyzed and the relevant parameters were adjusted. This process was repeated several times in order to ensure that the simulated network is consistent, the coding is accurate, and the simulation is a reality.

Once the on-screen error checking was completed, the calibration process progressed to the next step. In doing so, the two most influencing parameters which have a major influence on safety distance between vehicles and thus affect the saturation flow rate were chosen for calibration. These parameters are $B X_{-} A D D$ and $B X_{-} M U L T$, where $B X_{-} A D D$ is the additive part and $B X_{-} M U L T$ is the multiplicity part of desired safety distance (VISSIM 4.1 User Manual, p.5-26). Please refer to Wiedemann et al., (1994) for further details.

Based on these parameters, the calibration targets were set up. Bus running times between a pair of stops (i.e., the links) generated by VISSIM were compared with actual running times of the $\mathrm{OC}$ Transpo buses. According to the calibration targets, the differences should be less than 15 percent for each link, and the number of such links should account for more than 85 percent of the total. These are suggested by Dowling et $a l .,(2002$, p.64). Details of the calibration process and results are presented in Appendix D1. Table 6.3 shows the calibrated values that were used for the follow-up steps. 
Table 6.3: VISSIM Calibrated Parameters

\begin{tabular}{|c|c|c|}
\hline Parameters & $\begin{array}{c}\text { Default } \\
\text { values }\end{array}$ & $\begin{array}{c}\text { Calibrated } \\
\text { values }\end{array}$ \\
\hline $\begin{array}{c}\text { Average stand still } \\
\text { distance (m) }\end{array}$ & 2.0 & 1.6 \\
\hline BX ADD (m) & 3.0 & 2.0 \\
BX_MULT (m) & 3.5 & 3.0 \\
\hline Gap acceptance (s) & 5 & $7-10$ \\
\hline
\end{tabular}
Note:
The calibrated value was based on the on-screen error checking

\subsection{Bus Operation Scenarios, Micro-simulation Runs, and the VISSIM Outputs}

The simulation model for the two bus routes and part of traffic network was coded and calibrated in VISSIM. This enabled the use of VISSIM to generate data on bus operations and passenger activities that correspond with transit and traffic conditions. Three scenarios were simulated. The first scenario related to routine traffic, and the hourly and daily traffic variations were simulated. The second scenario involved an incident on the bus route. The incident could be a one-lane closure or a slowdown zone for road maintenance, or it may be due to traffic accident. The last scenario was devoted to the simulations of passenger activities at some busy stops that were caused by special events such as Boxing Days, or a football match.

The number of simulation runs was also carefully considered. In VISSIM, each simulation run requires a random seed number, which is used by VISSIM to generate traffic volumes and vehicle arrival rates. Consequently, multiple runs in VISSIM with different random seeds provide variations in results. The necessary number of simulation runs (or sample size) can be established on the basis of confidence interval, sample 
standard deviation and the allowed error range of a parameter being statistically analyzed. For example, Zong et al., (2002) suggested the following equation:

$$
n=\left(\frac{z_{\alpha / 2} \sigma}{E}\right)^{2}
$$

Where: $\mathrm{n}=$ required number of simulation runs

$$
\begin{aligned}
& \sigma=\text { sample standard deviation } \\
& \alpha / 2=\text { threshold value for significance level } \\
& \mathrm{E}=\text { Allowed error range. }
\end{aligned}
$$

However, the main purpose of simulation runs in this research is to generate random data for model testing rather than using these for statistical and analytical purposes. Therefore, the above suggested approach was not applied.

If we refer to the mathematical models developed in previous chapters, we can see that they are all based on a statistical pattern recognition technique where the recognition of a neighbour is based on the similarities of its several features with that of a given focal point. In mathematical terms, each neighbour is a multi-dimensional point near the focal point. The dimensionality, the required sample size, and the recognition of neighbourhood are the difficulties to cope with for those who are applying statistical pattern recognition in practice. The difficulties are that at high dimensions, a point may be no longer the neighbour of a given focal point although it is so at low dimensions. This was referred to as "dimensionality curse" in the literature (Ernest, 1961, pp.94; Cleveland et al., 1988). For a better illustration, it should be interesting to read the following:

"Regions of high density may contain few samples, even for moderate sample sizes. For example, in the 10-dimensional unit multivariate normal distribution (Silverman, 1986), 99\% of the mass of the distribution 
is at points at a distance greater than 1.6, whereas in one dimension, $90 \%$ of the distribution lies between \pm 1.6 " (Webb Andrew, 2002, pp.116).

This means that if one wants to avoid non-recognition in a high dimensional space, the required sample size has to be increased. Table 6.4 presents suggestions by Silverman (1986) on required sample size based on dimensionality ${ }^{1}$.

Table 6.4: Dimensionality and Required Sample size

\begin{tabular}{|c|c|}
\hline Dimensionality & Required Sample size \\
\hline 1 & 4 \\
\hline 2 & 19 \\
\hline 3 & 67 \\
\hline 4 & 223 \\
\hline 5 & 768 \\
\hline 6 & 2790 \\
\hline 7 & 10700 \\
\hline 8 & 43700 \\
\hline 9 & 187000 \\
\hline 10 & 842000 \\
\hline
\end{tabular}

(Source: Silverman, 1986, pp. 94)

For the developed model, the maximum dimensionality is three. Therefore, according to Silverman (1986), the required sample size should be more than or equal to 67 simulation runs. In this study, 100 and 70 simulation runs were considered for route 1 and route 95, respectively. The number of runs for each scenario is shown in Table 6.5.

\footnotetext{
${ }^{1}$ Silverman (1986) suggested it for non-parametric density estimation in high dimensional space. Although our purpose is not the same, this reference would be valuable under the circumstance that information about required sample size for non-parametric regression in high dimensionality is difficult to find.
} 
Table 6.5: Scenario Design for Simulation

\begin{tabular}{|c|c|c|c|c|}
\hline \multirow[t]{2}{*}{ Scenario } & \multirow[t]{2}{*}{ Situation } & \multirow[t]{2}{*}{ Description } & \multicolumn{2}{|c|}{$\begin{array}{l}\text { Number of } \\
\text { simulation runs }\end{array}$} \\
\hline & & & Route 95 & Route 1 \\
\hline \multirow{3}{*}{1000} & 1100 & $\begin{array}{l}\text { Simulate traffic networks with } \\
\text { volumes } 15 \% \text { less than normal } \\
\text { traffic }\end{array}$ & N/A & 15 \\
\hline & 1200 & $\begin{array}{l}\text { Simulate network with normal } \\
\text { traffic volumes }\end{array}$ & 40 & 20 \\
\hline & 1300 & $\begin{array}{l}\text { Simulate network with volumes } \\
15 \% \text { more than normal traffic }\end{array}$ & N/A & 15 \\
\hline \multirow[t]{2}{*}{2000} & 2100 & $\begin{array}{l}\text { Simulate traffic network with } \\
\text { one-lane closed and normal } \\
\text { traffic volumes }\end{array}$ & N/A & 15 \\
\hline & 2200 & $\begin{array}{l}\text { Simulate traffic network with } \\
\text { slow-down zone with normal } \\
\text { traffic volumes }\end{array}$ & 15 & 20 \\
\hline 3000 & 3000 & $\begin{array}{l}\text { Simulate traffic network with } \\
\text { busy stops and normal traffic } \\
\text { volumes. }\end{array}$ & 15 & 15 \\
\hline \multicolumn{3}{|r|}{ Total } & 70 & 100 \\
\hline
\end{tabular}

Notes:

N/A: Not applicable because the buses run on the dedicated bus lanes. As a consequence, ordinary traffic variations would have not influence on bus transit.

Scenarios 1000 and 2000 were further divided into situations coded as 1100,1200 , 1300,2100 , and 2200 . In the situations 1100 and 1300 , daily traffic variations were simulated by increasing and decreasing normal traffic by 15 percent, respectively. Hourly traffic variations are based on the actual traffic counts and assumed to be the same for everyday.

Situation 2100 is for one-lane closure due to road construction zone on the segment of Bank St. from Holmwood to Gladstone stops. This situation does not apply to bus route 95 . 
Situation 2200 simulates slowdown zone. For bus route 1 , the speed limit is as low as $10-12 \mathrm{~km} / \mathrm{h}$ between Holmwood and Gladstone stops. For bus route 95 , this zone is in between Hurdman and Campus stations where speed limit is $20-25 \mathrm{~km} / \mathrm{h}$. These slowdown zones were effective for 2 hours and removed after that time.

Scenario 3000 simulates busy bus stops by increasing the number of boarding passengers by threefold. For bus route 1, these stops are Billings Bridge and Homlwood. For route 95, such stops are St. Laurent and Hudman.

For each simulation run, 15 minutes (i.e., 900 seconds) were added to the simulation time as a warm-up time, the time to fill-up the empty traffic network with vehicles so that meaningless outputs are excluded. The state of the bus is set up to be updated at every 0.5 simulation second. This can keep the necessary accuracy while reducing simulation time and the magnitude of each output file ${ }^{2}$. The output file for each simulation was recorded by VISSIM in a table, namely OC Transpo_VEH_RECORD in the form of Microsoft Access database.

Because the database is huge but only bus running times and passenger activities at stops are needed for the study, such information were filtered and re-tabulated. These tables are listed in Appendix D2.

If we consider each VISSIM output after each simulation run as a record of a service day of the buses, then we have 100-day database for route 1 and 70 days of records for route 95 . For these days, the situations mentioned in Table 6.5 may happen

\footnotetext{
${ }^{2}$ Each simulation nun by Intel Celeron Microprocessor $2.8 \mathrm{MHz}, 1 \mathrm{~GB}$ RAM, and Window Home XP environment, is about 3600 s for 8100 s actual time with $0.1 \mathrm{sec}$-update set, and about 1400 seconds with 0.5 sec-update set. As a consequence, the magnitude of the file reduces from $32 \mathrm{MB}$ to approximate 14-17 MB for each record, correspondingly.
} 
randomly, more or less frequently, due to the stochastic nature of traffic. In order to reflect these phenomena, the records were mixed up randomly. Also, by mixing the data, we could test the developed prediction model in term of its capability to recognize the similar bus running situations given a specified scenario.

\subsection{Model Testing and Comparison}

Like several models discussed in literature review, the developed model in this study is devoted to real-time bus arrival and related predictions. In order to study the relative merits of the developed model, it is necessary to compare it with other models in term of prediction accuracy and reliability. However, this comparison does not mean that the developed model is more or less advanced than others. Actually, it is aimed at informing the readers about the strengths and weakness of the compared models in case these are to be implemented in the field.

\subsubsection{Evaluation Criteria for Prediction Performance}

Three accuracy criteria shown in Table 6.6 are used to test the developed model as well as selected other predictors. 
Table 6.6: Accuracy Criteria for Model Testing

\begin{tabular}{|c|c|c|}
\hline Accuracy Criteria & Equation & Measurement \\
\hline $\begin{array}{c}\text { MAPE } \\
\text { Mean Absolute } \\
\text { Percentage Error }\end{array}$ & $M A P E=\left(\frac{1}{N} \sum_{i=1}^{N} \frac{\left|x_{i}-\hat{x_{i}}\right|}{x_{i}}\right) \cdot 100$ & $\begin{array}{l}\text { MAPE provides unit-free } \\
\text { measurement of the performance. } \\
\text { It can be used to compare } \\
\text { predictors across different test sets. }\end{array}$ \\
\hline $\begin{array}{c}\text { MRE } \\
\text { Maximum Relative Error }\end{array}$ & $M R E=\max _{i} \frac{\left|x_{i}-x_{i}\right|}{x_{i}}$ & $\begin{array}{l}\text { MRE distinguishes the magnitude } \\
\text { of the error from a predictor }\end{array}$ \\
\hline $\begin{array}{c}\text { RMSE } \\
\text { Root Mean Square Error }\end{array}$ & $R M S E=\sqrt{\frac{1}{N} \sum_{i=1}^{N}\left(x_{i}-x_{i}\right)^{2}}$ & $\begin{array}{l}\text { RMSE measures the goodness-of- } \\
\text { fit of the predictor. }\end{array}$ \\
\hline
\end{tabular}

Notes: $x$ - actual data; $x$-predicted data; $N$ - sample size

\subsubsection{Reference Predictors}

As discussed earlier, for reference purposes, the developed model should be compared with some other predictors, given the same input data. Two predictors were selected. The first one can be considered as the simplest model, namely the naïve model. The second reference model, which can be seen as one of the most advanced models in term of bus real-time arrival, was first developed by Reinhoudt et al. (1997), and further applied by Farhan (2002), and Shalaby et al. (2004). It is a Kalman filter-based model.

The reason to choose the first reference model is that it is a simple model without any computational difficulties. The estimation of a bus operation (i.e., running time or passenger activity) between a pair of stops at current time is simply calculated by taking historical data of the bus itself and that of the same-day data just before the prediction time.

The Kalman filter-based model aforementioned was chosen because it was successfully applied with both actual and simulated data for bus route 5 in downtown Toronto, Canada. Moreover, this model did outperform other models in a comparative 
assessment (Shalaby et al., 2004), especially, the ANN-based model - which is a very powerful prediction tool. For the sake of illustration, brief presentations about these predictors are made below. The details can be seen in Appendix D3.

Obviously, any prediction model that does not show consistent and more accurate forecast than the naïve model would have little value.

\subsubsection{The Naïve Model}

This predictor is rather simple in concept. Naive predictions were calculated in a manner that take into account both current condition and historical bus running times or passenger activities as follows

$$
r(k)=\left(\frac{r(k-1)}{r_{h i s}(k-1)}\right) \cdot r_{h i s}(k)
$$

Where:

$r(k)$ and $r_{h i s}(k)$ : running time or passenger activity of the bus for bus $k$ at current time and one-day before.

$r(k-1)$ and $r_{h i s}(k-1)$ : running time or passenger activity of the bus $(k-1)$ at current time and one-day before.

\subsubsection{The Kalman Filter-based Model}

The Kalman filter technique involves the state-space estimation. That is, the state of a subject in question at current time step is optimized (filtered) on the basis of the information about its historical states at previous discrete time steps and the latest 
information. Reinhoudt et al. (1997), Farhan (2002), and Shalaby et al. (2004), used this technique in their research where the authors considered the new state being filtered is the bus running time between a pair of stops; the newest received information at the current time step is the running time of the same-day previous bus, which has just finished the run right before the bus in question. The historical data are the means and variances of running times recorded for the buses on several days before.

According to Farhan (2002), the Kalman algorithm for bus running time prediction and passenger arrival rate is based on the system of four main equations as shown below.

$$
\begin{aligned}
& g(k+1)=\frac{e(k)+V A R\left[\text { local }_{\text {data }}\right]_{k+1}}{e(k)+2 V A R\left[\text { local }_{\text {data }}\right]_{k+1}} \\
& e(k+1)=V A R\left[\text { local }_{\text {data }}\right]_{k+1} \cdot g(k+1) \\
& a(k+1)=1-g(k+1) \\
& P(k+1)=a(k+1) \cdot \operatorname{art}(k)+g(k+1) \cdot \operatorname{art}_{1}(k+1)
\end{aligned}
$$

Where

g: filter-gain

a: loop-gain

e: filter error

P: prediction

art $(k)$ : actual running time or passenger arrival rate of previous bus at instant $(k)$

$\operatorname{art}_{1}(k+1)$ : actual running time or actual passenger arrival rate of the previous day at instant $(k+1)$ 
$V A R\left[\text { data }_{\text {local }}\right]_{k+1}$ : average variance of the last three days' data at time step $k+1$.

If we take a closer look at this system, we can see that the determination of filtergain (e.g., $g(k+1)$ ) plays the key role to update the prediction in Equations 6.4 and 6.5 . To determine $g(k+1)$, we need to know $e(k)$ (Eq. 6.2), which in turn, requires $g(k)$ to be defined (Eq.6.3). However, $g(k)$ then needs $e(k-1)$ to be estimated and so on. In other words, this is a recursive procedure where the bus running time being predicted at time step $k$ requires an update, prediction, and correction (filter) procedure beginning from the very first trip running on that link (e.g. time step 1, or initial estimation). In his study, Farhan used 3 day-historical data for updating the variable $V A R\left[\text { data }_{\text {local }}\right]_{k+1}$. In this research, 3-day historical data were also used. An Excel code was developed to apply this algorithm for bus running time prediction.

\subsubsection{Testing the Developed Model and Reference Predictors}

\subsubsection{Data issues}

The cross-validation technique is used in order to assess the accuracy of estimates. In this technique, the original sample data are mutually split into testing sample and learning sample. This technique is intended to avoid overly optimistic estimates when one uses the same data to construct the parameters for a prediction model and assess its performance. Although no parameters in our models are to be estimated (i.e., non- 
parametric $\operatorname{model}^{3}$ ), this technique is still applied in order to enhance the evaluations of model's performance.

There are various ways of performing data separation depending upon the magnitude of the original data. However, in a nutshell for the relatively small sample size that we have in hand, it is not practical to divide it in the way discussed above since it reduces the effective sample size. Instead, we consider the way based on a modified version of cross-validation technique, namely the leave-one-out cross validation (Hardle, 1990; Kwon et al., 2000). We treat one day as a test set and the remaining as historical data. By doing so, we can calculate the cross-validation evaluation criteria (e.g., MAPE, MRE, and RMSE) for the developed model and the reference predictors. In this study, 20 test sets, each for one time, were drawn randomly for the purpose of model testing.

As the developed model combines the running time module and dwell time module, the performance of each module, as well as the whole model for bus arrival, was estimated separately and reported in the following sections.

\subsubsection{Running Time Prediction Performance}

During the simulation time (i.e., 8100 seconds), each VISSIM record contained 9 trips $^{4,5}$ for bus route 1 and 35 trips for bus route 95 . Consequently, we have 180 simulated trips for route 1 and 700 simulated trips for route 95 , given 20 test sets for each route. Moreover, each trip belonging to route 1 has 3 running times (i.e., the time spent

\footnotetext{
${ }^{3}$ Please refer to chapter 4 , section 4.3 for discussions on parametric and non-parametric models.

${ }^{4}$ Under this circumstance, each trip is defined when a bus departs at the first stop and arrives at the last stop of the studied segment. For bus route 1, a trip starts when a simulated bus arrives at Billings Bridges stop and ends when the bus arrives at Rideau stop. For route 95, the two end stops are St.Laurent and Lebreton stops.

5 VISSIM uses the term "course" instead of "trip".
} 
by each trip between a pair of understudied stops). Also, bus route 95 has 5 running times. In theory, if we apply cross-validation technique regarding every running time between all consecutive stops as well as every trip, we will have to cope with 540 and 3500 running time predictions for route 1 and route 95, respectively. These are actually large numbers and beyond the scope of this thesis research.

In order to reduce the work load while we can maintain the generality in estimating the predictors (i.e., the developed model, the KF and the naïve model), only specified trips and running times of each route are selected for model testing. For route 1 , only the trips that start at 8:59 a.m. everyday at Billings Bridge station (i.e., trip 6) and only running times between Billings Bridge and Gladstone stops were considered. For bus route 95 , only the trips that start routinely at 8:59 a.m. at St. Laurent station (i.e., trip 24) and only the running times between St.Laurent and Mackenzie stations were studied. By doing so, the number of predictions reduces to 20 for each route.

To apply the running time module described in chapter 4, each 8:59 a.m. bus trip of route 1 was drawn from the 100-record database and considered as a test set. Running time of the 8:59 a.m. bus (i.e., trip 6) that is being predicted was defined by its pattern matrix containing three previous running times of 8:47 a.m. bus (trip 5), 8:37 a.m. bus (trip 4), and 8:27 a.m. bus (trip 3). The remaining data (i.e., 99 records) were treated as the historical pattern matrices. The differences between the actual running times and predicted running times in the test sets were calculated. The same procedure was also applied for the 8:59 a.m. bus trips at St.Laurent station of route 95 .

For the Kalman-filter based algorithm (KF), the record of 8:59 a.m. bus (trip 6) was drawn from the 100-record database and also considered as the test set. Based on the 
running time between Billings Bridges and Gladstone stops for trips 5, 4, 3, 2 ,1 and the historical running times experienced by 8:59 a.m. buses (trip 6) of 3 days ago, the running time was predicted and compared with the actual running time. For route 95 , the same steps were applied for 8:59 a.m. bus trips running between St.Laurent and Mackenzie stations. In the case the Naïve model, predictions were obtained from the use of Equation 6.1. The details for the use of data for testing the predictors are presented in Appendix D4.

Figures $6.4,6.5$ and Table 6.7 present the results of cross-validation MAPE, MRE, and RMSE of the three predictors applied to route 95.

Table 6.7: Cross-validation MAPE, MRE, and RMSE

\begin{tabular}{|l|c|c|c|}
\hline $\begin{array}{c}\text { Cross-validation } \\
\text { criteria }\end{array}$ & MAPE (\%) & MRE (\%) & RMSE (sec) \\
\hline Developed Model & 3.94 & 10.46 & 39.31 \\
\hline KF model & 9.64 & 24.76 & 82.50 \\
\hline Naïve model & 15.49 & 90.53 & 181.73 \\
\hline
\end{tabular}

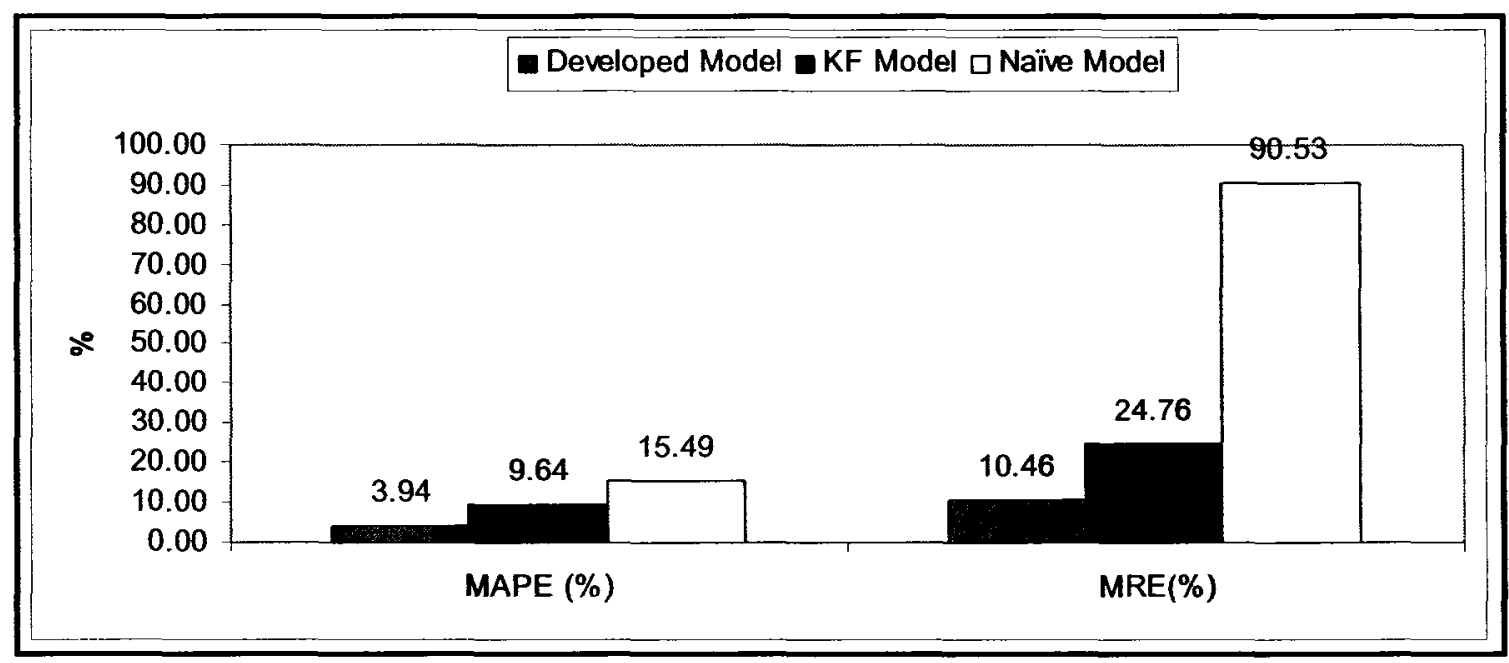

Figure 6.4: Route 95- Running Time Prediction: MAPE and MRE 


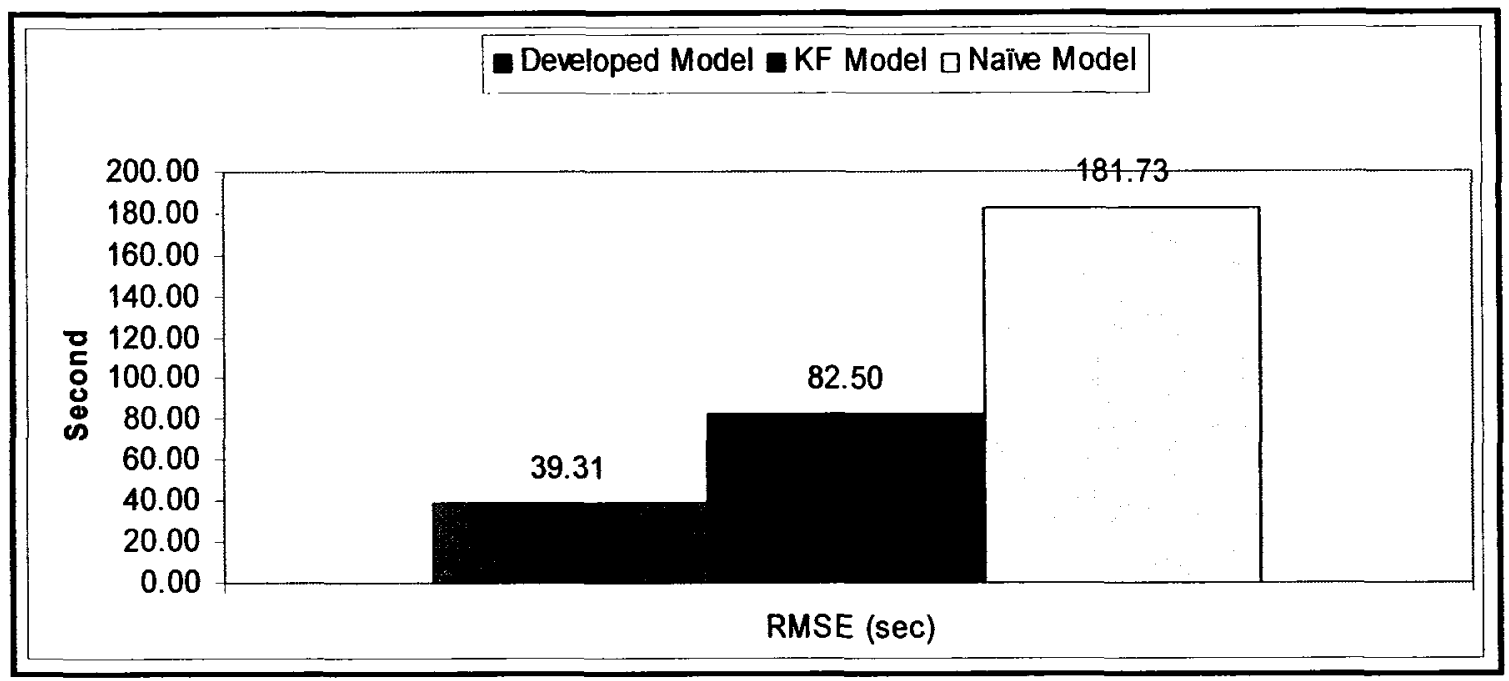

Figure 6.5: Route 95- Running Time Prediction: RMSE

From Figures 6.4 and 6.5, we can see that the developed model is superior to the two reference predictors when they were all applied to the Transitway route (i.e., Route 95). The error measurements for the developed model are only a half and a quarter compared to those of the Kalman Filter and the naïve predictor, respectively. This could be due to the fact that many types of impedance on bus running time were eliminated on the Transitway route so that the differences between situations are quite clear. Hence, the developed model recognized and classified the situations quite well.

For route 1, a mixed-traffic bus route, due to many frictions that influence the bus running time, different bus running situations may be alike (i.e., they may have quite similar pattern matrices). Therefore, it makes it difficult for the developed model to cope with these cases when recognizing and classifying similar situations. This observation is supported by Figures $6.6,6.7$ and Table 6.8 when the developed model and the reference predictors were applied for route 1. 
Table 6.8: Route 1- Running Time Prediction with Overall Situations: MAPE, MRE, and RMSE

\begin{tabular}{|l|c|c|c|}
\hline $\begin{array}{c}\text { Cross-validation } \\
\text { criteria }\end{array}$ & MAPE (\%) & MRE (\%) & RMSE (sec) \\
\hline Developed Model & 9.66 & 20.39 & 118.07 \\
\hline KF model & 10.32 & 43.10 & 154.29 \\
\hline Naïve Model & 13.59 & 35.37 & 168.90 \\
\hline
\end{tabular}

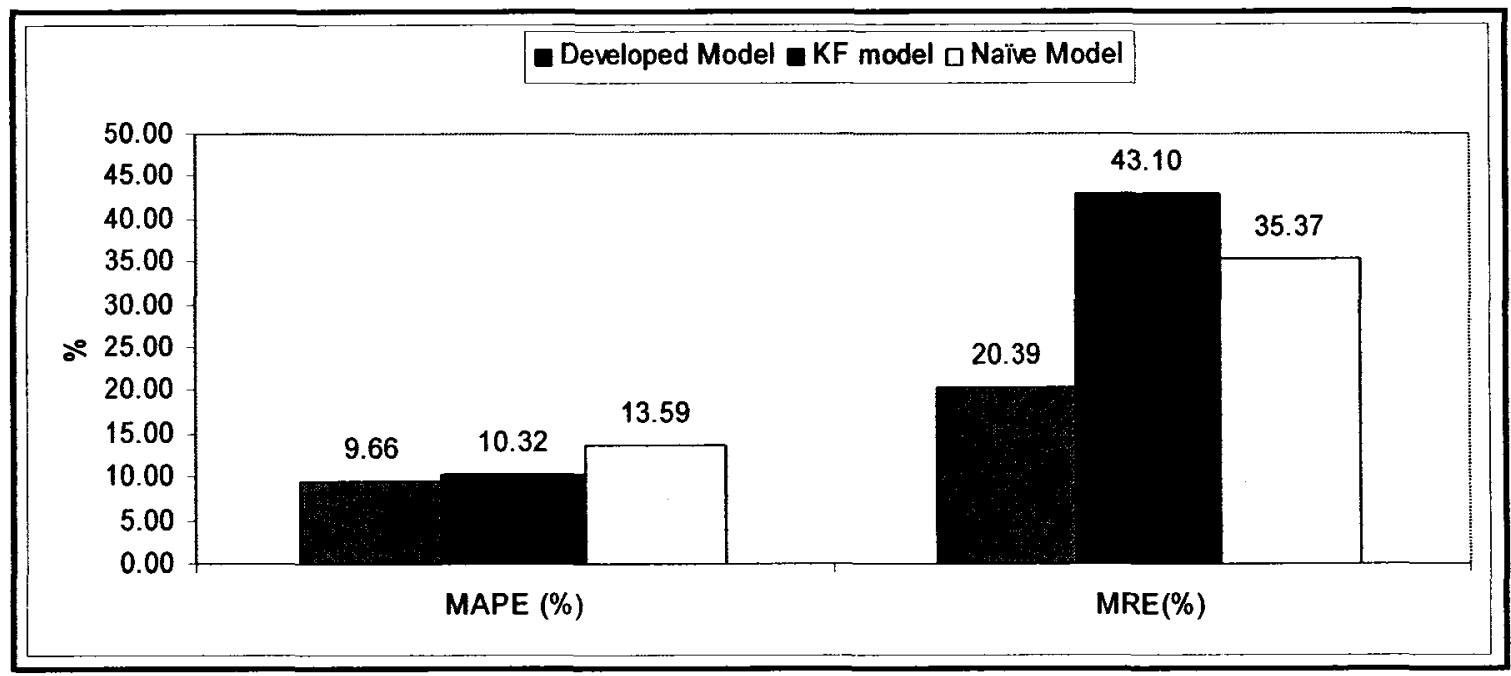

Figure 6.6: Route 1- Running Time Prediction with Overall Situations: MAPE and MRE

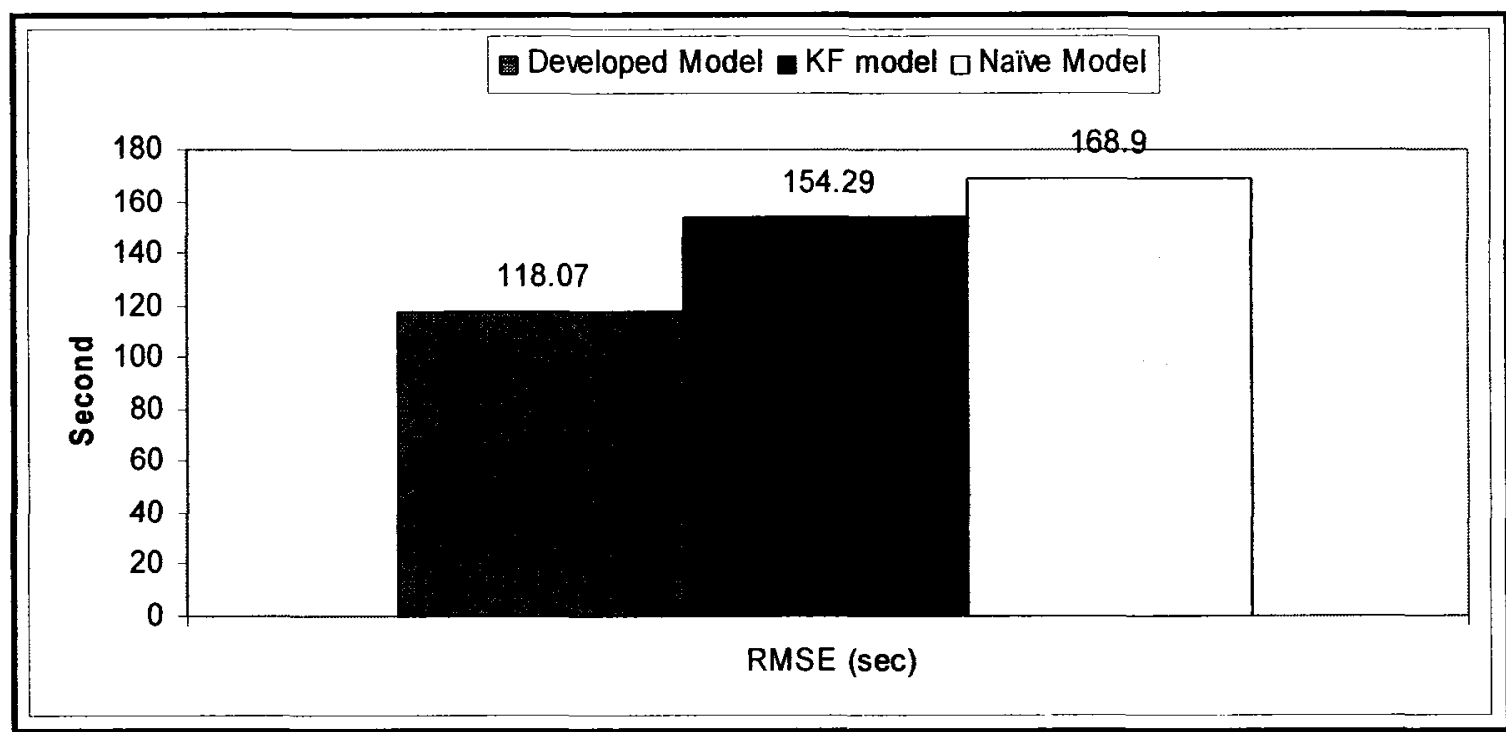

Figure 6.7 Route1- Running Time Prediction with Overall Situations: RMSE 
Results shown in figures 6.6 and 6.7 suggest that the developed model for bus running time still outperforms the reference predictors since all indicators are favorable. However, the differences were not as remarkable as in the case of the Transitway route.

In order to further explore the developed model, more comparisons were carried out. Fifteen running situations coded as 2200 (i.e., slowdown zone situation), each for one time, were extracted from database of route 1 . It should be noted that these situations were mixed up randomly with other situations in order to reflect the variable nature of traffic as well as to test the developed model's capability to recognize the similar situations in a mixed data.

For each predictor, by comparing the prediction results of MAPE, MRE and RMSE regarding these slowdown zone situations with those of 20 overall situations mentioned previously, we can see that the accuracy of the KF predictor changed considerably. Its MAPE increases from $10.32 \%$ to $19.03 \%$, the RMSE goes up from $154 \mathrm{~s}$ to 291 s (i.e., up to $88 \%$ ). In contrast, the prediction results of the developed model are still stable. The MAPE decreases from $9.66 \%$ to $8.76 \%$. Furthermore, the MRE and RMSE are almost the same as they were. Figures $6.8,6.9$, and 6.10 present these discrepancies. The comparisons point out that the developed model is more reliable than other predictors when dealing with abnormal traffic situations. 


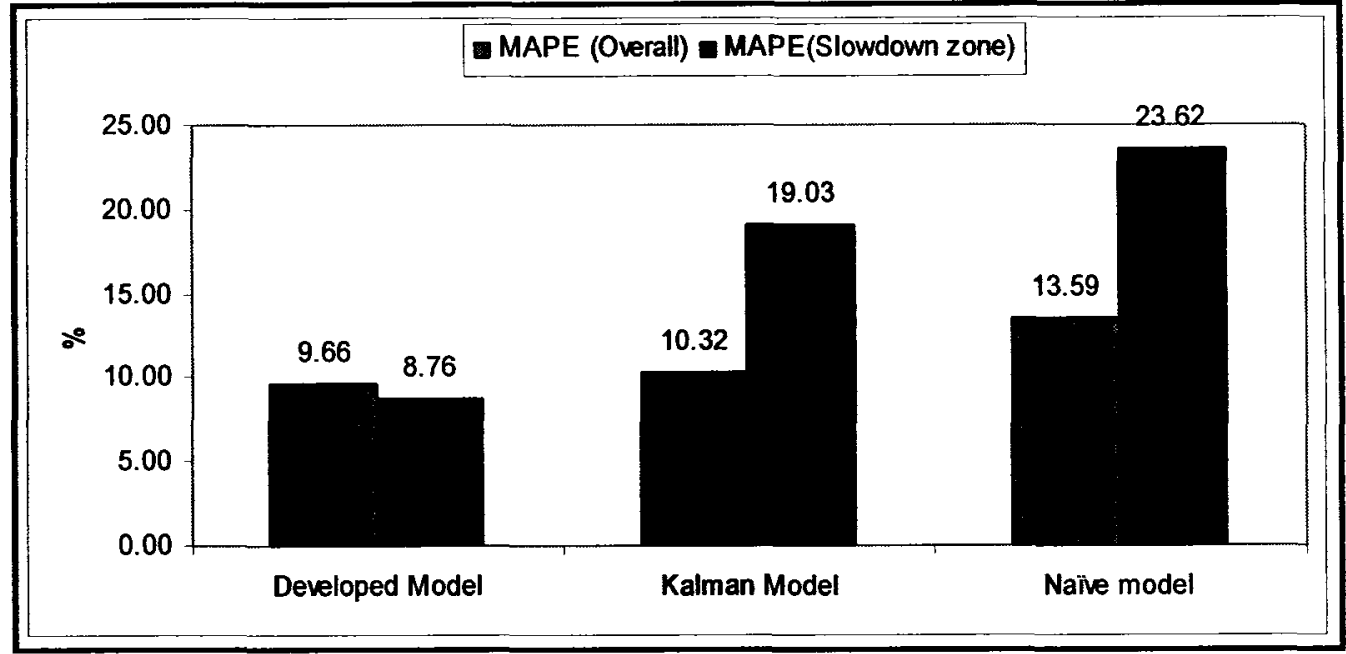

Figure 6.8: Route 1-The Differences of MAPE between the Overall and Slowdown zone Situations

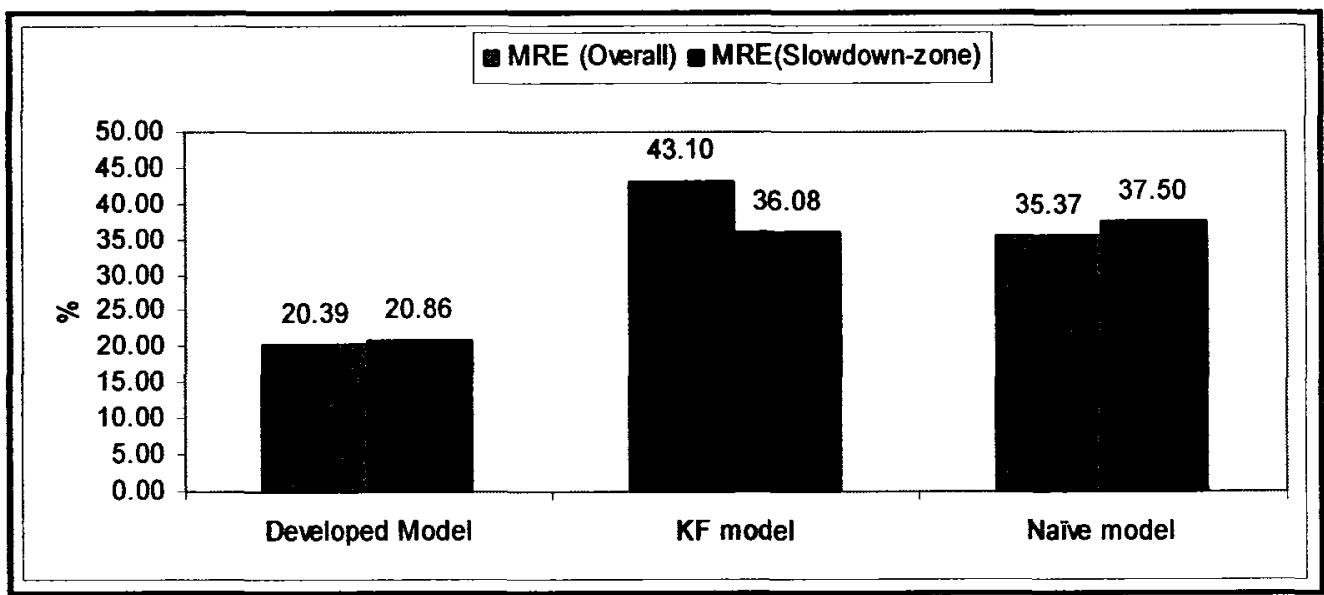

Figure 6.9: Route 1- The Differences of MRE between the Overall and Slowdown zone Situations

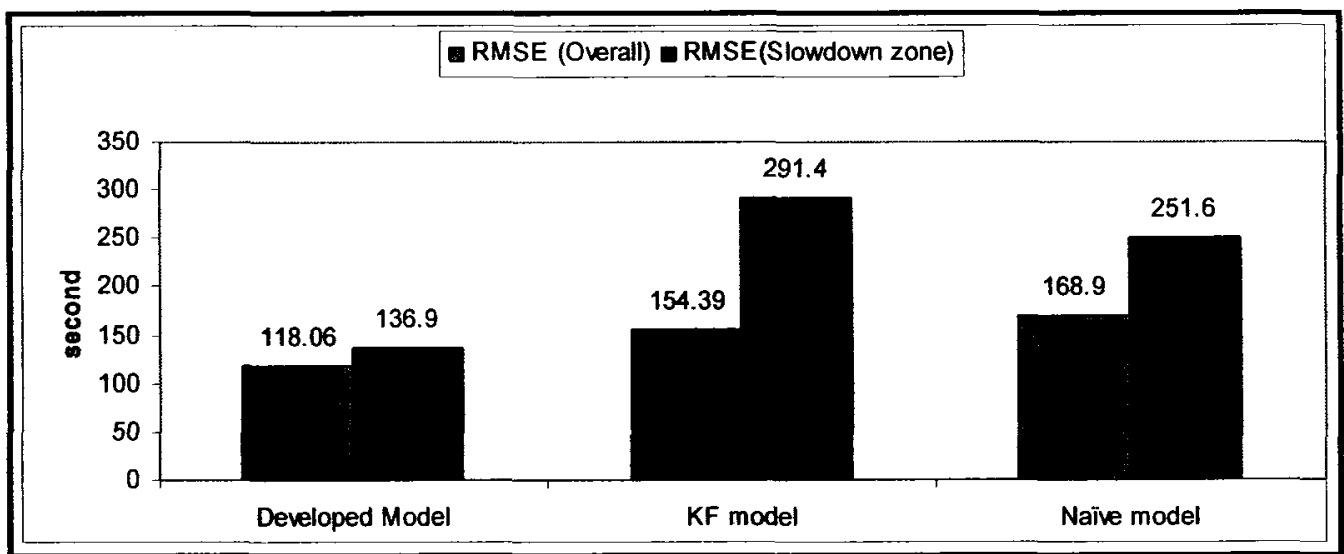

Figure 6.10: Route 1- The Differences of RMSE between the Overall and Slowdown zone Situations 
The poorer prediction performances of the reference predictors are explainable. In order to predict a current bus running time, the $\mathrm{KF}$ and the naĩve predictors were based on the historical data of several days before that would have no running situations similar to the present one. For instance, to predict running time of a situation that has Dcode $=$ 43 and Scode $=2200$ in table D4.1 (see Appendix D4), the KF model was based on the running times of 3 previous days which have different situations with Dcode-Scode as $78-3000,29-1300$, and 87-1100, respectively. As a consequence, the increase of prediction errors of these models is understandable.

Unlike the KF and the naïve models, the developed model searched through the whole database, recognized the most similar situations, and gathered them for estimations. For example, with the same case above (i.e., the situation coded as 43-2200), the automatic neighbour searching procedure ${ }^{6}$ searched through from 10 to 70 nearest neighbours around this case in order to find the optimal number of similar neighbours which provides the smallest cross-validation least-square prediction error (i.e. CR for short). It was found that 20 nearest neighbours offered smallest prediction error in this case, as shown in Figure 6.11. The figure was returned from the Matlab program coded for the developed model. Figure 6.12 depicts another example of such a neighbour searching for route 95 , case $54-2200$.

\footnotetext{
${ }^{6}$ Please refer to chapter 4 , section 4.4 for the details of this procedure.
} 


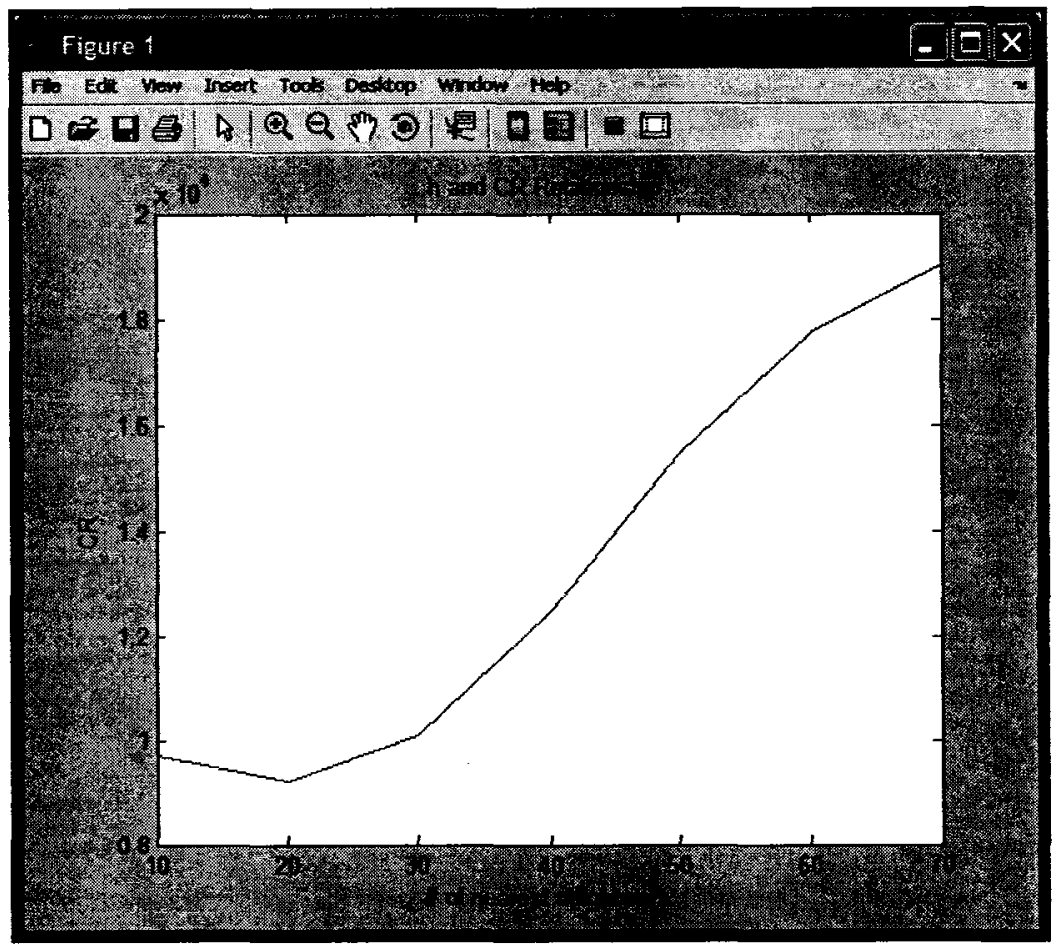

Figure 6.11: Nearest Neighbour and Cross-Validation Least-square Prediction Error (CR) for Bus Running Time Prediction. Case 43-2200, Route 1.

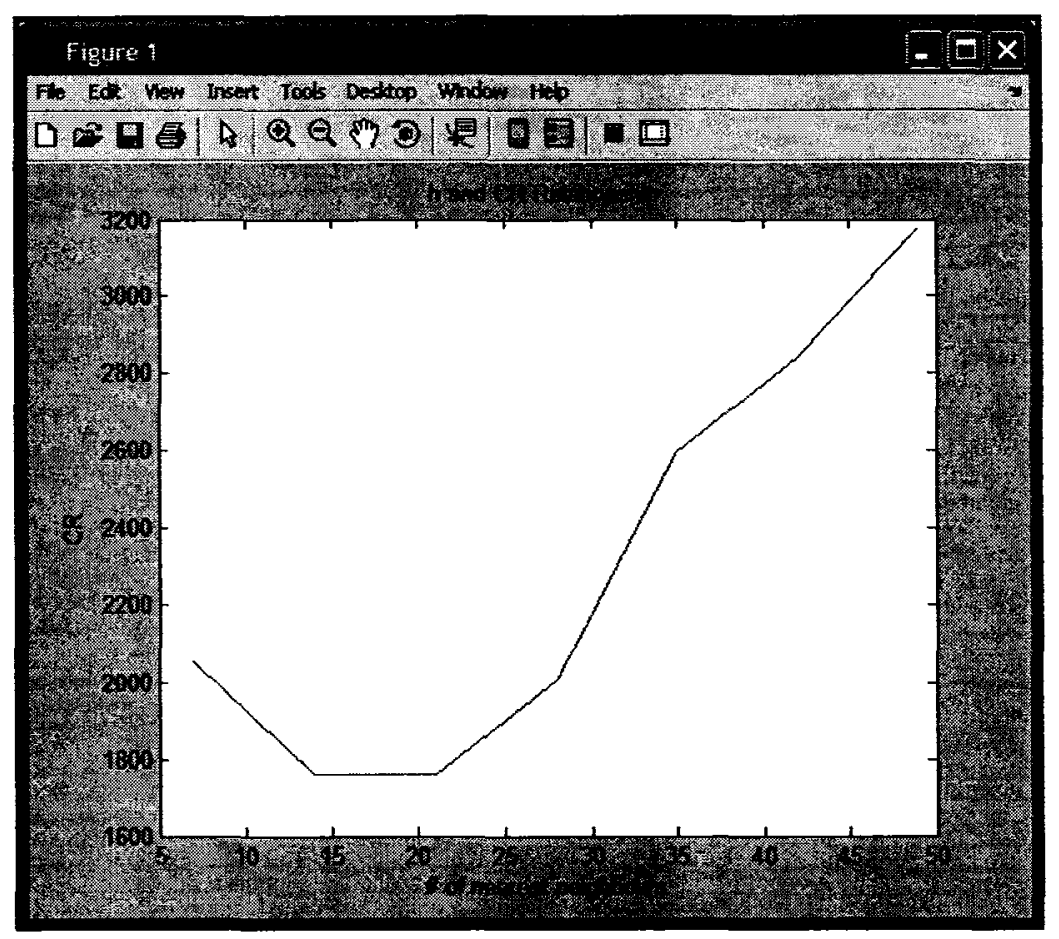

Figure 6.12: Nearest Neighbour and Cross-Validation Least-square Error (CR) for Bus Running Time Prediction. Case 54-2200, Route 95. 


\subsubsection{Boarding Passenger Prediction Performance}

As the number of boarders and de-boarders at the stops of route 1 are relatively small compared to that of route 95 , only boarding passenger data of route 95 were used to test the predictors ${ }^{7}$. Also, for the sake of reducing computational work, only the number of boarding passengers at Mackenzie station of the buses that begin their trips at 8:59 a.m. (trip 24) at St. Laurent station was predicted. The boarding passenger prediction submodule presented in chapter 5 (section 5.2.3) was applied for this purpose where the pattern matrix was defined by that of the previous bus (trip 20) served at Mackenzie station, the running time experienced by this bus, and the predicted running time of the bus itself (i.e., trip 24). The historical data of boarding passengers and running times of such buses were used as the historical pattern matrix.

The KF model developed by Farhan (2000) was applied to estimate the passenger arrival rate at a stop. The number of boarders for trip 24 then was predicted by multiplying this rate and the predicted running time. To apply the naive predictor, Equation 6.1 was used.

The details of each predictor are presented in Appendix 05.

Figures 6.13 and 6.14 depict the cross-validation MAPE, MRE, and RMSE of the three predictors applied to forecast the number of boarders for 20 test sets. As shown in the figures, the developed model is the best, followed by the Kalman Filter and the naïve predictor.

\footnotetext{
7 The average boarding passenger at stops of route 1 is only about $10-15 \mathrm{pas} / \mathrm{h} / \mathrm{stop}$, or it equals to $3-4$ pas/ bus/ stop. Therefore, even the difference between predicted and actual data of only 1 passenger/stop can cause a prediction error of up to $30 \%$ or more. This may lead to misjudgments on the predictors.
} 


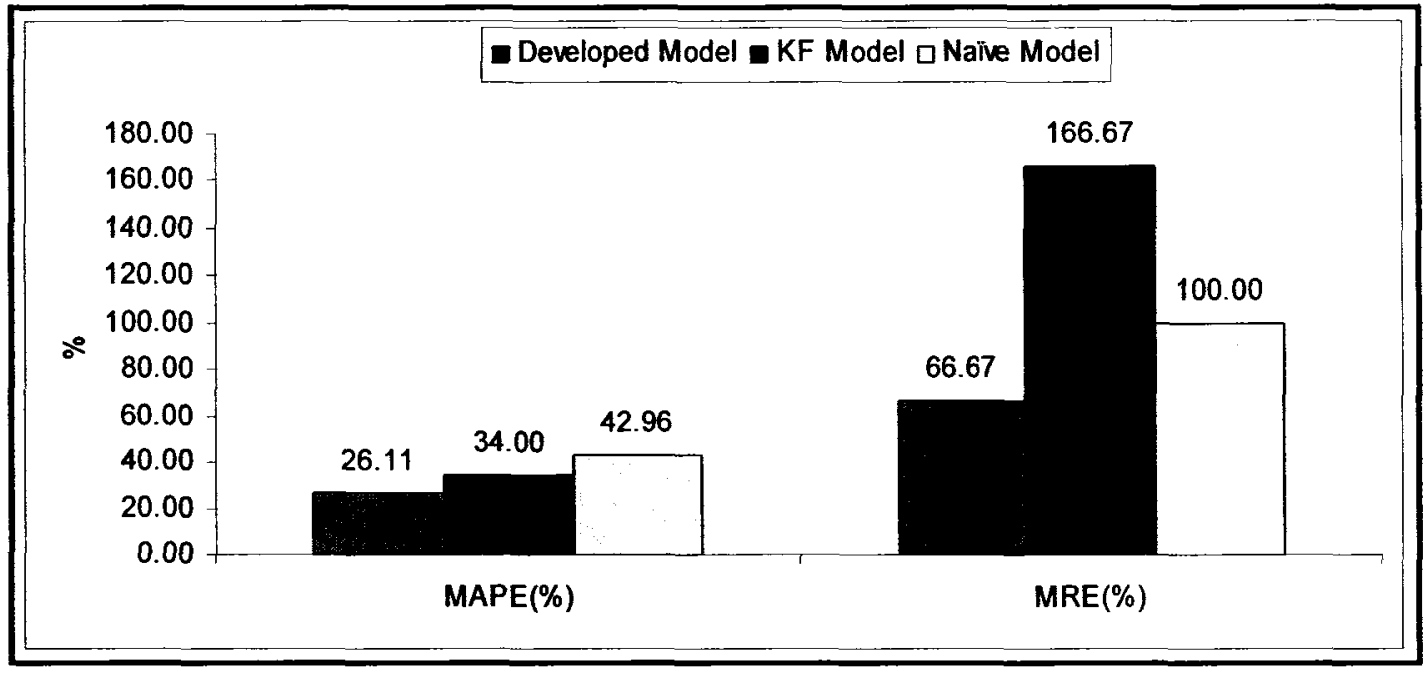

Figure 6.13: Route 95 - Boarding Passenger Prediction: MAPE and MRE

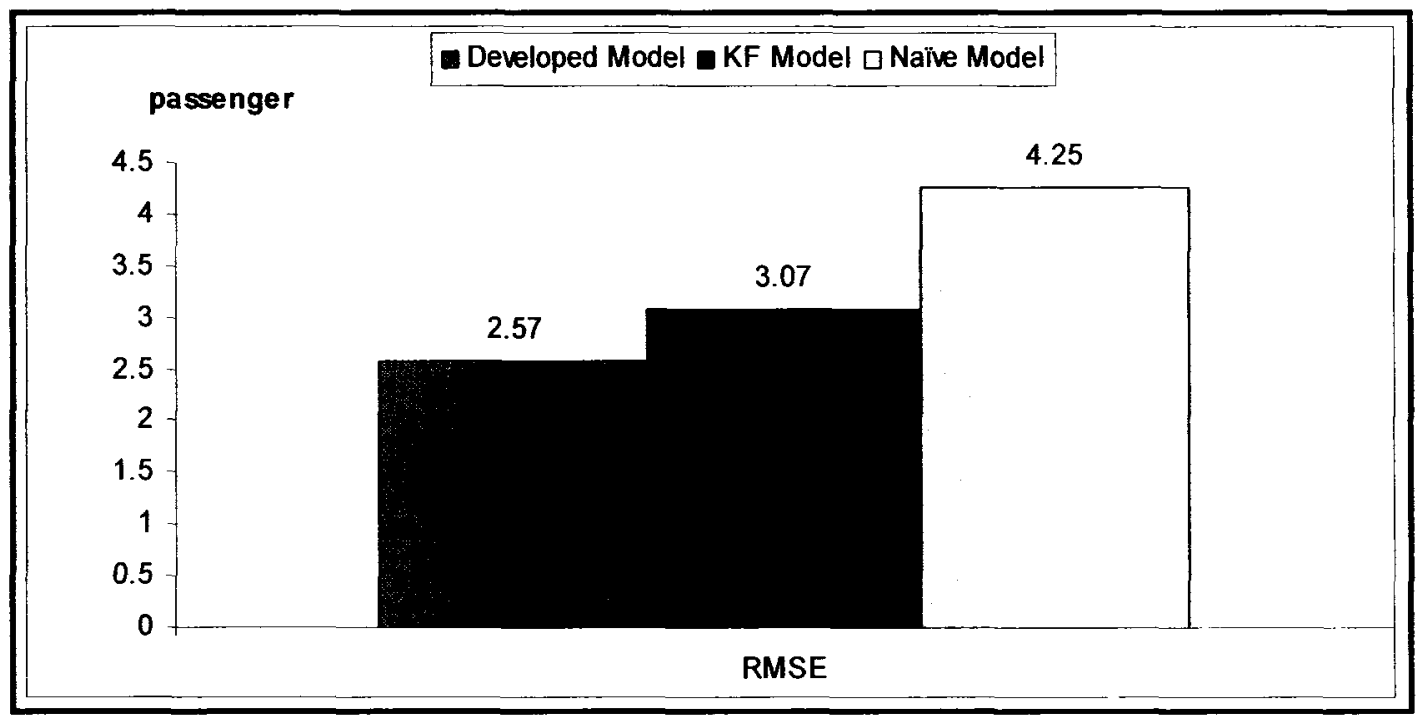

Figure 6.14: Route 95 - Boarding Passenger Prediction: RMSE

Figure 6.15 shows an example of a neighbour searching result for a boarding passenger prediction. It should be noted that while the KF predictor needs the controversial assumption of a uniform passenger arrival rate, there is no such assumption for the developed model. 


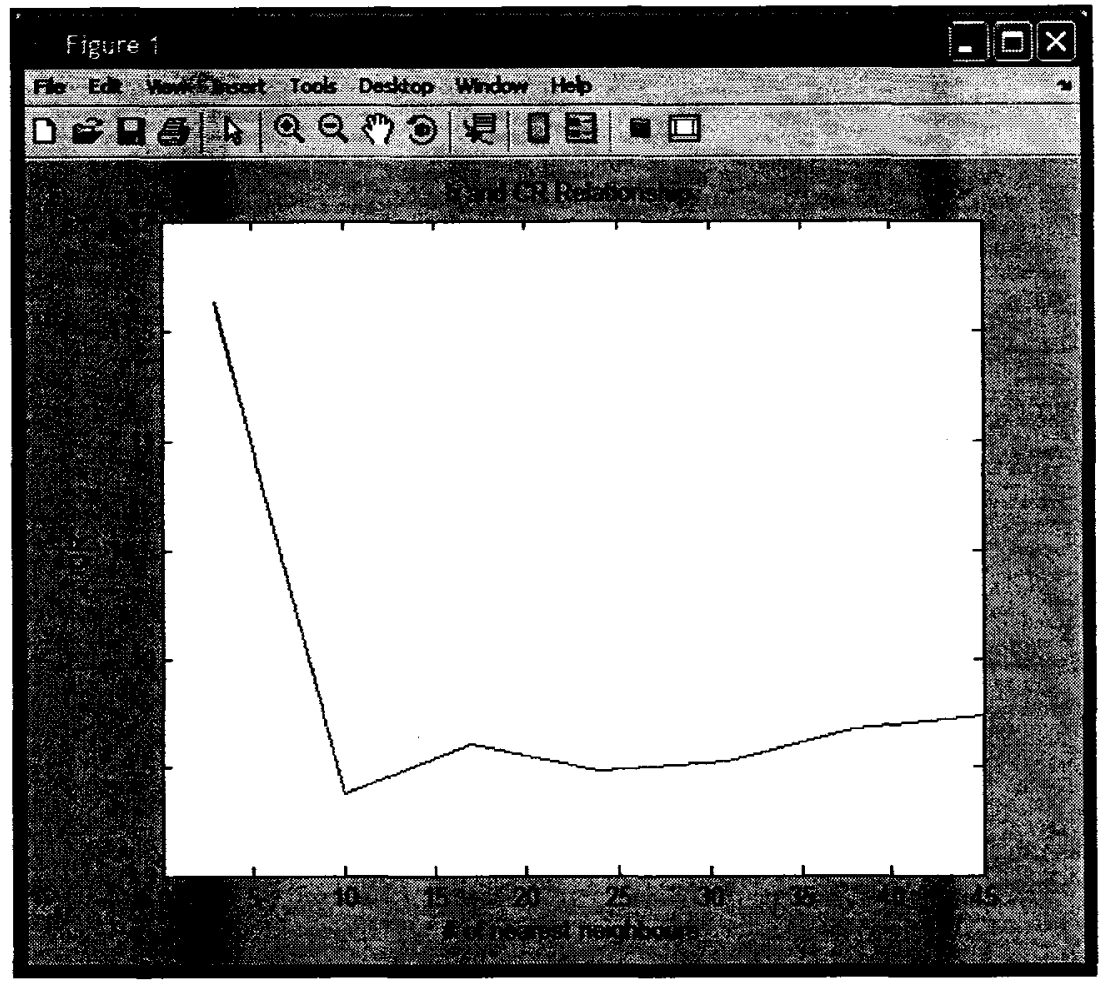

Figure 6.15: Nearest Neighbour and Cross-Validation Least-square Error (CR) for Boarding Passenger Prediction. Case 24-1200, Route 95.

\subsubsection{Alighting Passenger Prediction Performance}

The number of alighting passengers of the buses at Mackenzie station that start at 8:59 a.m. (trip 24) from St.Laurent station was used for testing the predictors. In doing so, the alighting passenger prediction sub-module in chapter 5 was used. Following the submodule, alighting passengers of the two previous buses (i.e., trips 20 and 19) that served at Mackenzie stop were used as the elements of the current pattern matrix defining the de-boarders of trip 24 that are being predicted. The historical data of alighting passengers of these buses was used as the historical pattern matrix for neighbour searching and prediction purposes. 
The Kalman Filter algorithm developed by Farhan et al., (2002) was not originally developed by the above authors for alighting passenger prediction. In this study, this method was modified with minor changes so that it can be used for alighting passenger prediction. For the naïve model, the predictions were based on Equation 6.1.

The details of each predictor are presented in Appendix D6.

Figures 6.16 and 6.17 depict the MAPE, MRE, and RMSE of the 3 predictors applied to forecast the number of de-boarders.

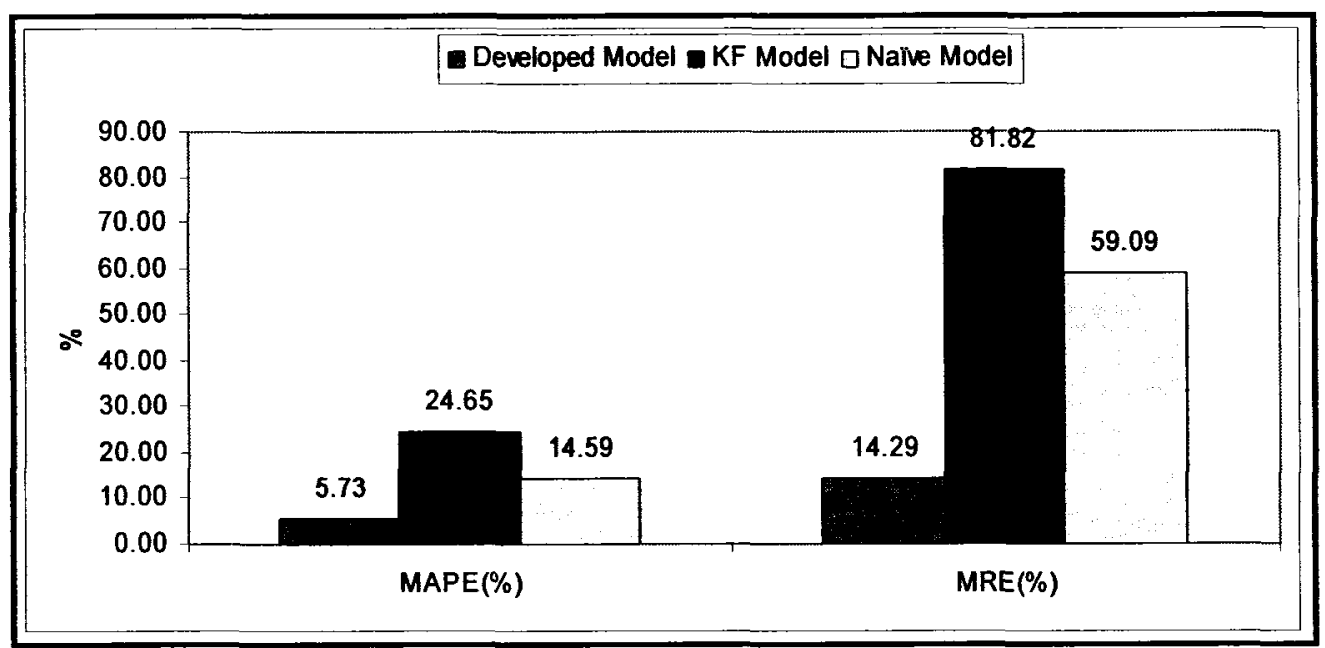

Figure 6.16: Route 95- Alighting Passenger Prediction: MAPE and MRE

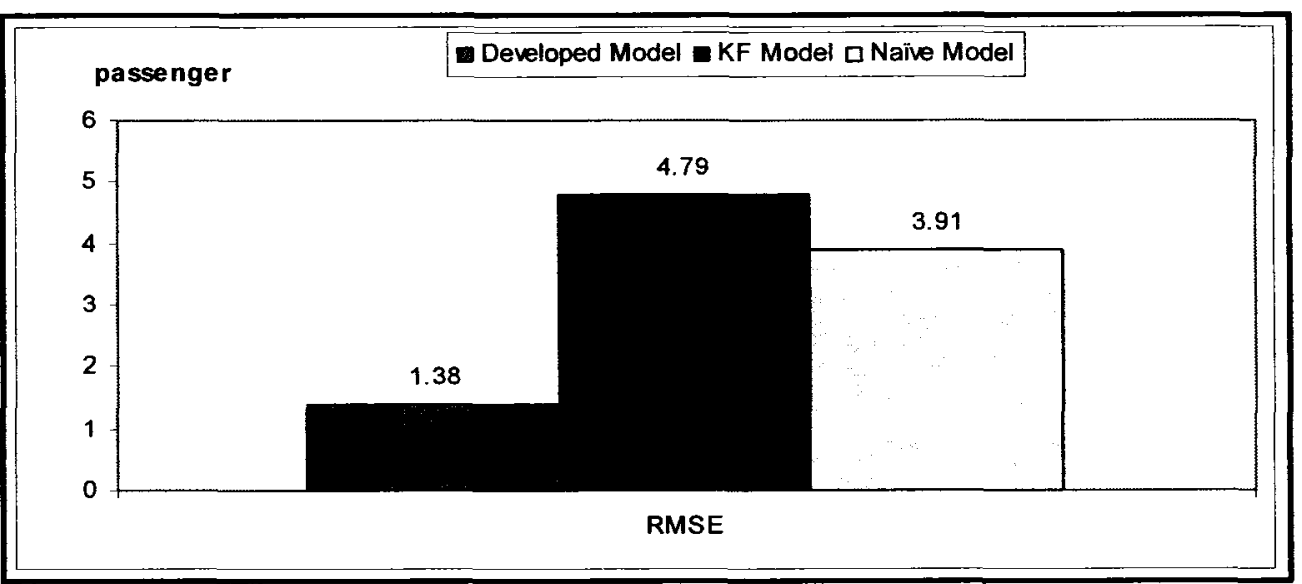

Figure 6.17: Route 95- Alighting Passenger Prediction: RMSE 


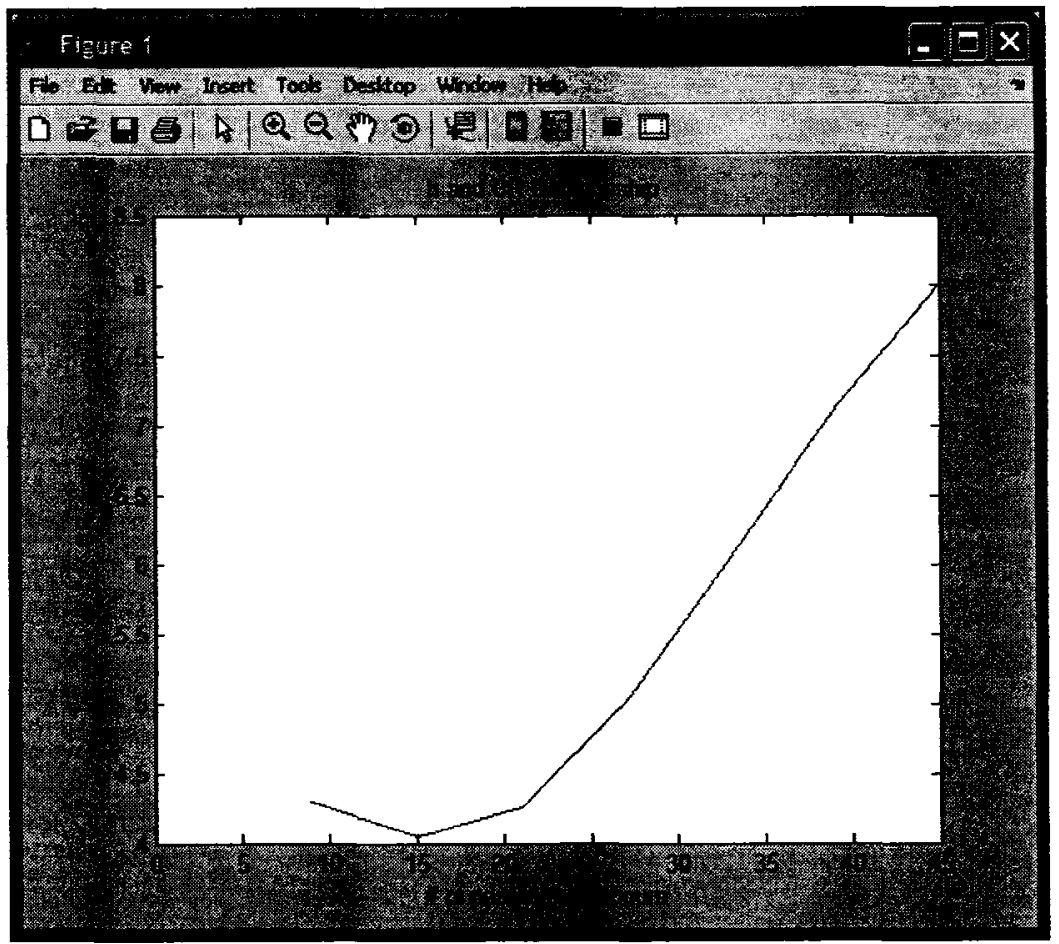

Figure 6.18: Nearest Neighbour and Cross-Validation Least-square Error (CR) for Alighting Passenger Prediction. Case 20-1200, Route 95.

The developed model outperformed the comparative predictors in term of alighting passenger prediction as shown in Figures 6.16 and 6.17. Figure 6.18 shows the result of an example of a neighbour searching.

\subsubsection{Prediction Performance with Actual Data}

Running time data and the information of the number of boarding and alighting passengers retrieved from the APC and AVL systems embedded on the buses of route 1 and route 95 were used to test the predictors. Figures from 6.19 to 6.26 present the MAPE, MPE, and RMSE of the 3 predictors applied to the selected segments and stops of route 95 and route 1 with different test sets. Please see Appendix D7 for details. 


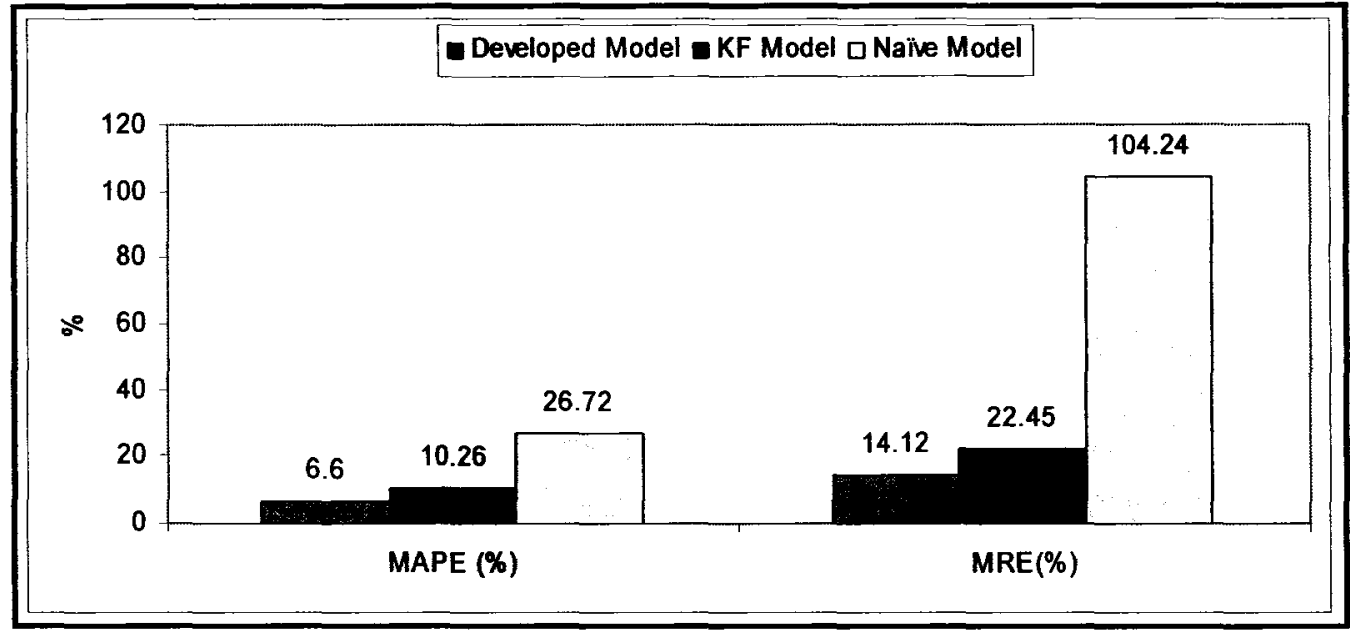

Figure 6.19: Route 95 - Running Time Prediction with Actual Data: MAPE and MRE

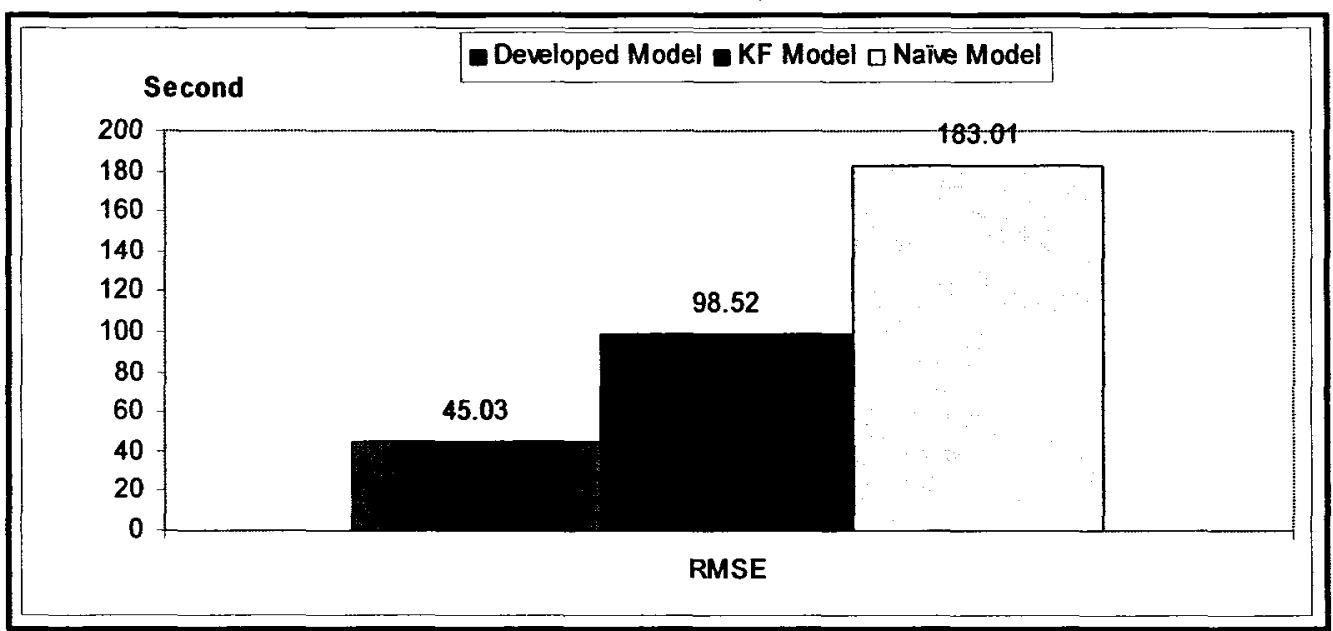

Figure 6.20: Route 95-Running Time Prediction with Actual Data: RMSE

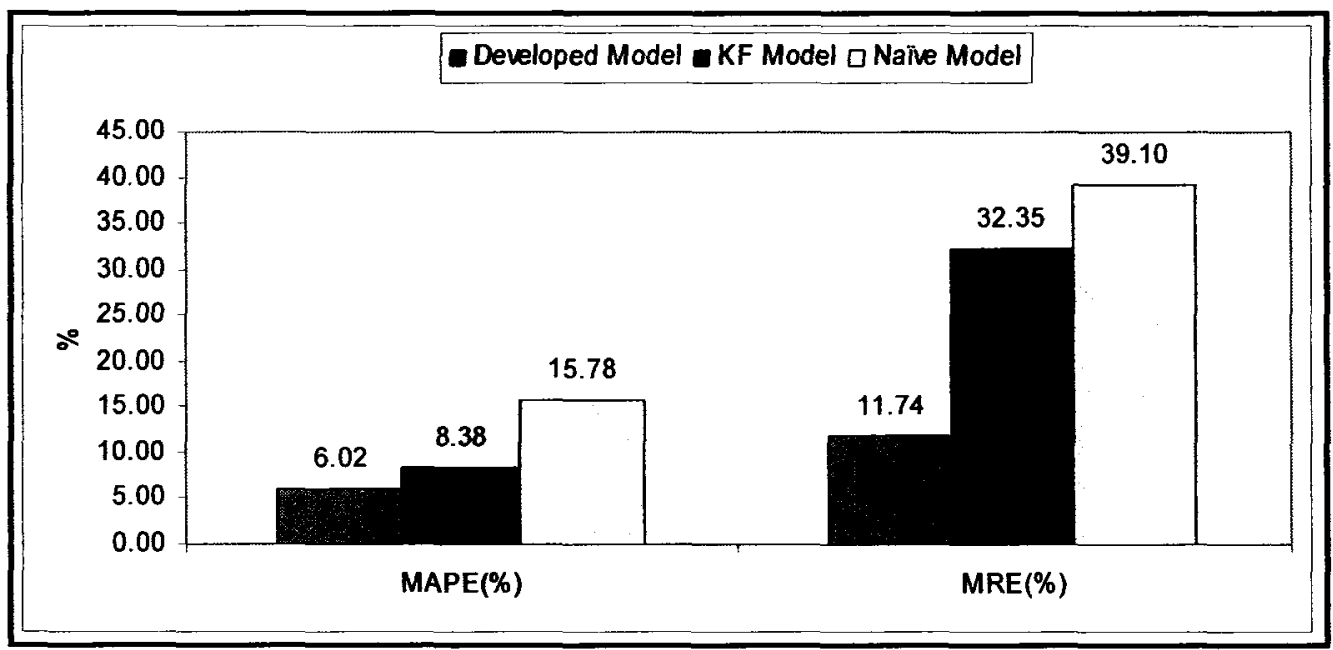

Figure 6.21: Route 1-Running time Prediction with Actual Data: MAPE and MRE 


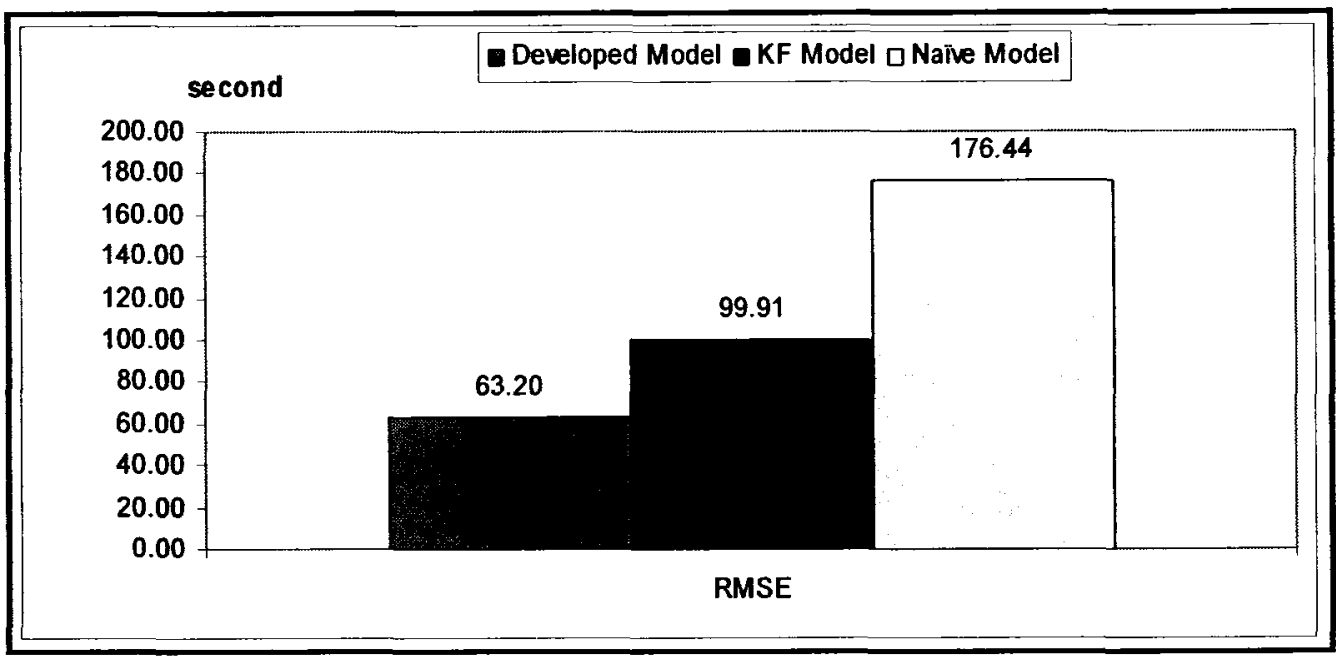

Figure 6.22: Route 1-Running Time Prediction with Actual Data: RMSE

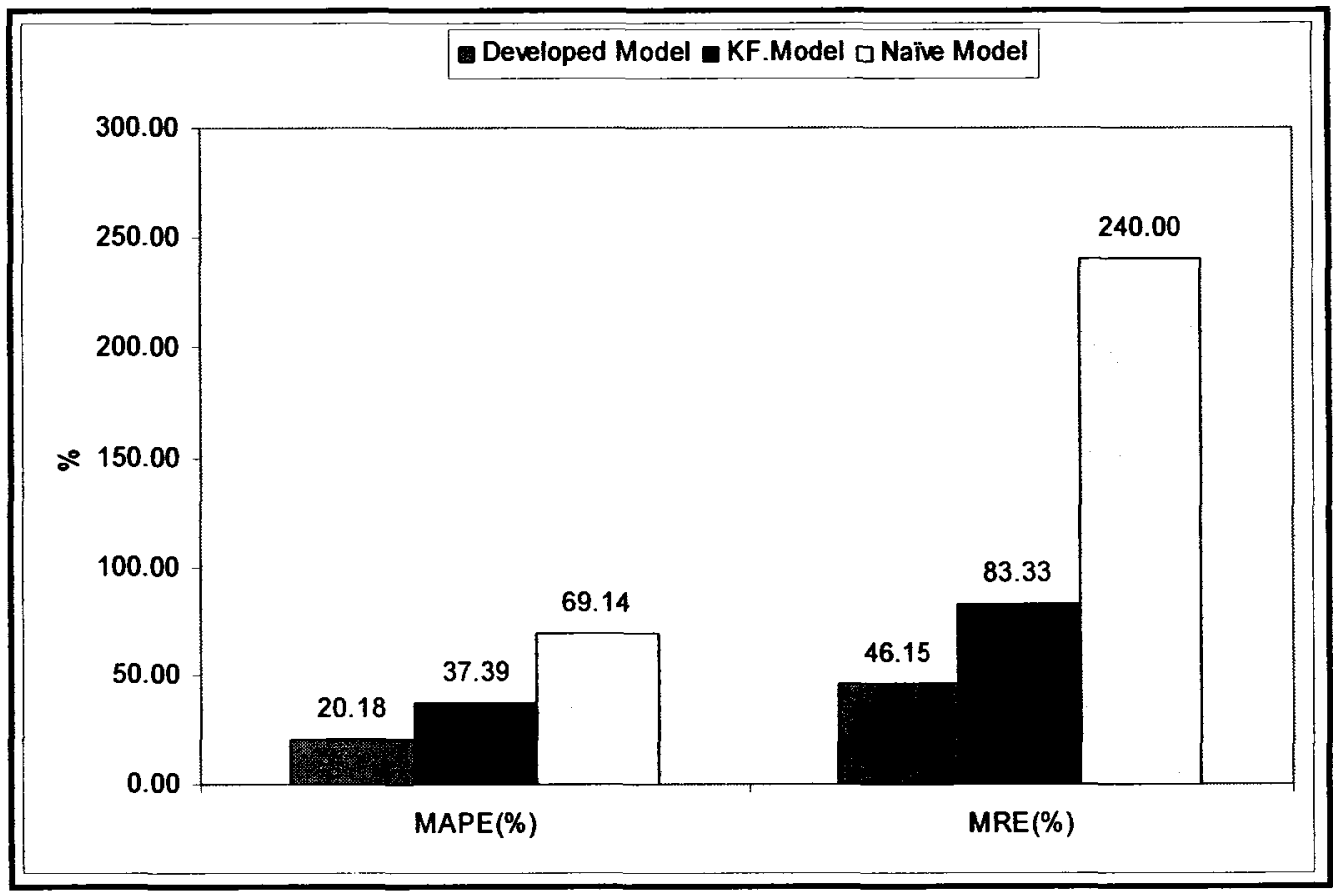

Figure 6.23: Route 95 - Boarding Passenger Prediction with Actual Data: MAPE and MRE 


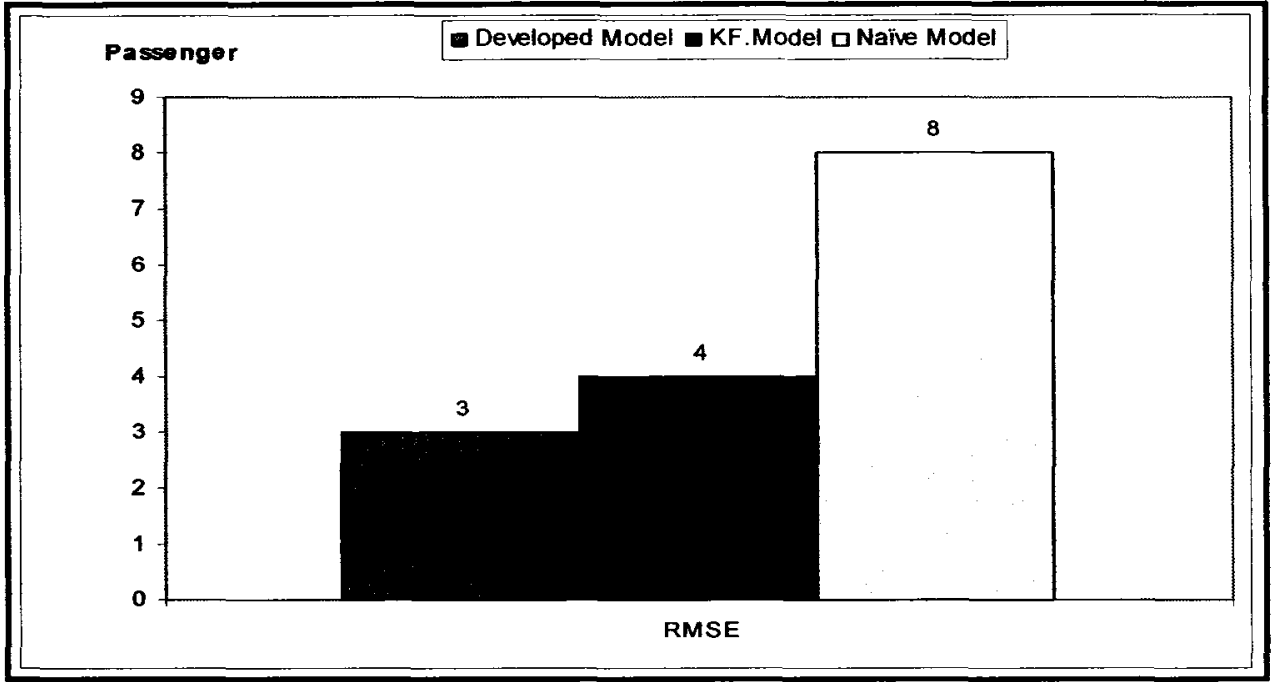

Figure 6.24: Route 95 - Boarding Passenger Prediction with Actual Data: RMSE

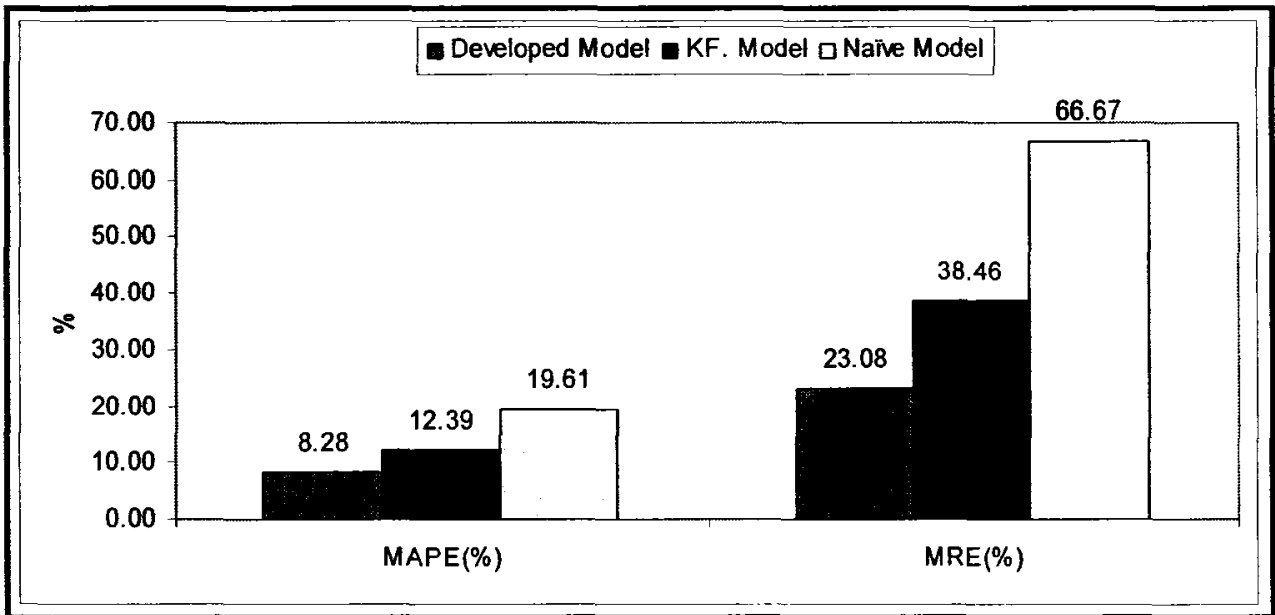

Figure 6.25: Route 95 - Alighting Passenger Prediction with Actual Data: MAPE and MRE

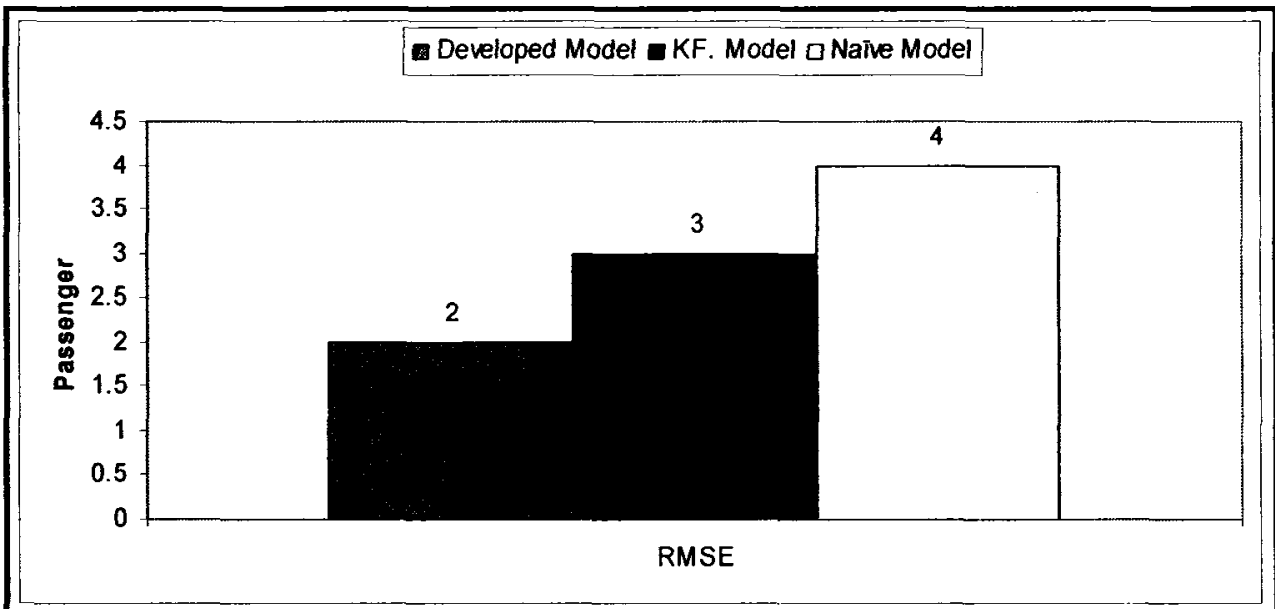

Figure 6.26: Route 95 - Alighting Passenger Prediction with Actual Data: RMSE 
The figures reveal that the developed model has the best prediction performance compared to the reference predictors. For running time prediction, the average MAPE is only about $6 \%$ for both routes. The MRE varies between $10 \%$ and $11 \%$, and the RMSE is in the range from 45 to 65 seconds. For boarding passenger predictions, the developed model's MAPE and MRE are as low as $20 \%$ and $46 \%$, respectively. For alighting passenger predictions, its MAPE and MRE are $8 \%$ and 23\%. All indicators reflect a favorable prediction model.

\subsubsection{Tukey Test for Performance Comparison}

The prediction performances of the three predictors were evaluated with different test sets. Although the charts presented earlier visually depict that the developed model outperformed the reference models, the comparisons based on the sound statistical analysis are the basis for reliable conclusions. In this part, Tukey's test was used to compare the MAPE values of the predictors. The details of Tukey procedure are presented in Appendix D8. Tables 6.9 and 6.10 present the results.

The results of the Tukey's procedure prove that the developed model has smallest MAPE compared to that of Kalman Filter and the naive models in most of the test sets at $95 \%$ confidence level. Therefore, the developed model can be considered as the preferred prediction model. 
Table 6.9: Studentized Range of the Test Sets

\begin{tabular}{|l|c|c|c|}
\hline \multicolumn{1}{|c|}{ Test sets } & $\boldsymbol{q}_{\text {a.05,357 }}$ & $\boldsymbol{M S E}$ & $\boldsymbol{w}$ \\
\hline Route 95: Simulated data of running times & 3.42 & 15.1 & 2.97 \\
\hline Route 1: Simulated data of running times & 3.42 & 8.72 & 2.25 \\
\hline $\begin{array}{l}\text { Route 1: Simulated data of running times for } \\
\text { slowdown-zone scenario }\end{array}$ & $3.43^{*}$ & 7.78 & 2.47 \\
\hline Route 95: Simulated data of boarding passengers & 3.42 & 79.74 & 6.82 \\
\hline Route 95: Simulated data of alighting passengers & 3.42 & 23.67 & 3.72 \\
\hline Route 1: Actual data of running times & $3.43^{*}$ & 6.55 & 2.26 \\
\hline Route 95: Actual data of running times & $3.43^{*}$ & 13.79 & 3.29 \\
\hline Route 95: Actual data of boarding passengers & $3.43^{*}$ & 114.46 & 9.47 \\
\hline Route 95: Actual data of alighting passengers & $3.43^{*}$ & 16.91 & 3.64 \\
\hline
\end{tabular}

Notes: MSE: Mean Square Error within groups; q: Studentized range distribution value (Netter, 1985); w: range

* Resulted from $q_{0.05,3,42}$

Table 6.10: Results of Tukey's Procedure at Significance Level $=0.05$

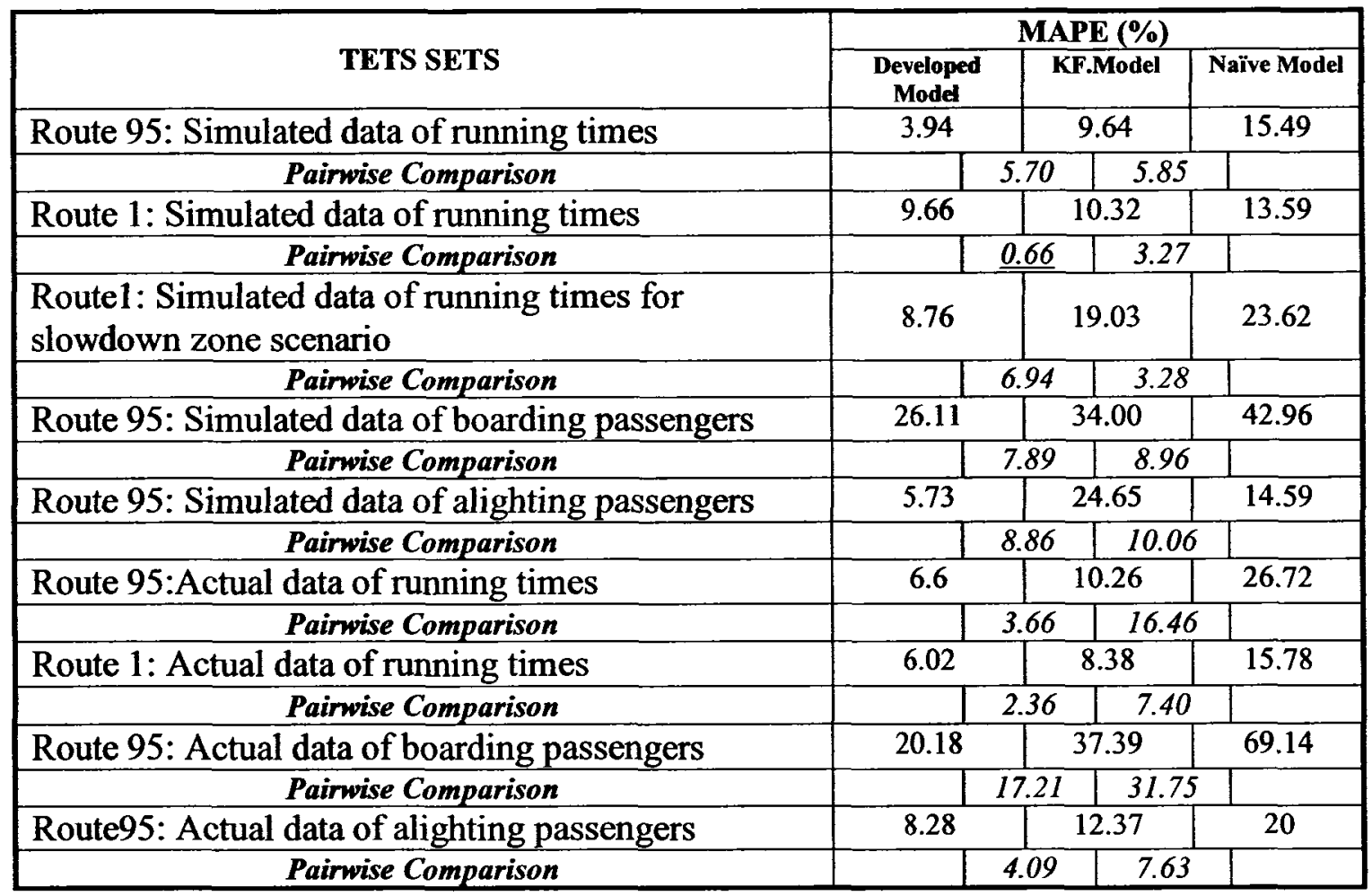




\subsubsection{Bus Arrival Time Prediction Performance}

Each component of the developed model was statistically tested and the performance results are encouraging. Therefore, the author is confident that the integrated model will outperform the reference predictors when applied to bus arrival time predictions.

In order to estimate performance of the integrated model for bus arrival time predictions, 15 full trips of route 1 that start at Billings Bridge station at 8:59 a.m. were selected randomly. For each trip, running times, dwell times, the number of boarding and alighting passengers, departure times, and arrival times of the buses at four selected stops (i.e., Billings Bridge, Holmwood, Gladstone, and Rideau stops) were predicted.

To predict bus arrival times at the stops, besides the use of the developed modules and sub-modules for predicting and updating bus running times on the links and dwell times at the stops, the updating predictions procedure presented in chapter 3 was applied in order to update arrival and departure time information of the buses at stops. The details of these predictions can be seen in Appendix D9.

Tables $6.11,6.12$ and 6.13 display the performance of the three predictors in term of bus arrival time at Holmwood, Gladstone and Rideau stops.

Table 6.11. Holmwood Stop: Bus Arrival Time Prediction Performance of the 3 Predictors

\begin{tabular}{|l|c|c|c|}
\hline $\begin{array}{c}\text { Cross-validation } \\
\text { criteria }\end{array}$ & MAPE (\%) & MRE (\%) & RMSE (sec) \\
\hline Developed Model & 8.60 & 20.14 & 62.31 \\
\hline KF model & 12.43 & 43.10 & 81.77 \\
\hline Naïve Model & 12.63 & 57.42 & 118.13 \\
\hline
\end{tabular}


Table 6.12. Gladstone Stop: Bus Arrival Time Prediction Performance of the 3 Predictors

\begin{tabular}{|l|c|c|c|c|c|c|}
\hline $\begin{array}{l}\text { Cross- } \\
\text { validation } \\
\text { Criteria }\end{array}$ & \multicolumn{2}{|c|}{ MAPE (\%) } & \multicolumn{2}{c|}{ MRE (\%) } & \multicolumn{2}{c|}{ RMSE(sec) } \\
\cline { 2 - 7 } & $1^{\text {st }}$ update & $2^{\text {nd }}$ update & $1^{\text {st }}$ update & $2^{\text {nd }}$ update & $I^{\text {st }}$ update & $2^{\text {nd }}$ update \\
\hline $\begin{array}{l}\text { Developed } \\
\text { Model }\end{array}$ & 5.58 & 2.93 & 16.82 & 7.95 & 85.18 & 40.69 \\
\hline KF. model & 8.93 & 3.85 & 17.24 & 8.79 & 97.86 & 50.95 \\
\hline $\begin{array}{l}\text { Naïve } \\
\text { Model }\end{array}$ & 10.53 & 7.46 & 42.64 & 23.41 & 160.53 & 100.90 \\
\hline
\end{tabular}

Table 6.13. Rideau Stop: Bus Arrival Time Prediction Performance of the 3 Predictors

\begin{tabular}{|l|c|c|c|c|c|c|c|c|c|}
\hline $\begin{array}{l}\text { Cross- } \\
\text { validation } \\
\text { Criteria }\end{array}$ & \multicolumn{3}{|c|}{ MAPE (\%) } & \multicolumn{4}{c|}{ MRE (\%) } & \multicolumn{3}{c|}{ RMSE (sec) } \\
\cline { 2 - 10 } & $\begin{array}{c}1^{\text {st }} \\
\text { update }\end{array}$ & $\begin{array}{c}2^{\text {nd }} \\
\text { update }\end{array}$ & $\begin{array}{c}\text { Last } \\
\text { update }\end{array}$ & $\begin{array}{c}1^{\text {st }} \\
\text { update }\end{array}$ & $\begin{array}{c}2^{\text {nd }} \\
\text { update }\end{array}$ & $\begin{array}{c}\text { Last } \\
\text { update }\end{array}$ & $\begin{array}{c}1^{\text {st }} \\
\text { update }\end{array}$ & $\begin{array}{c}2^{\text {nd }} \\
\text { update }\end{array}$ & $\begin{array}{c}\text { Last } \\
\text { update }\end{array}$ \\
\hline $\begin{array}{l}\text { Developed } \\
\text { Model }\end{array}$ & 5.25 & 3.32 & 2.58 & 12.75 & 11.87 & 4.17 & 97.16 & 70.43 & 47.19 \\
\hline $\begin{array}{l}\text { KF. } \\
\text { model }\end{array}$ & 6.27 & 4.52 & 3.99 & 15.04 & 9.41 & 8.68 & 120.80 & 68.61 & 60.25 \\
\hline $\begin{array}{l}\text { Naïve } \\
\text { Model }\end{array}$ & 10.22 & 6.17 & 6.87 & 27.11 & 12.33 & 12.55 & 196.63 & 123.64 & 127.33 \\
\hline
\end{tabular}

The results presented in the tables suggest that the developed model is the most preferable model, followed by the KF and the naive model. Also, the predicted arrival times at the stops become more accurate when the most updated bus information became available. For example, at Rideau stop, the average of MAPE of the 15 full-trips decreased from $5.25 \%$ to $3.32 \%$ and $2.58 \%$ corresponding to the first, the second and the last update. Table 6.14 shows a part of real-time prediction results for bus arrival times at the stops. 
Table 6.14: Numerical Examples of Real-time Bus Arrival Prediction Using Developed Model for 3 full trips (seconds)

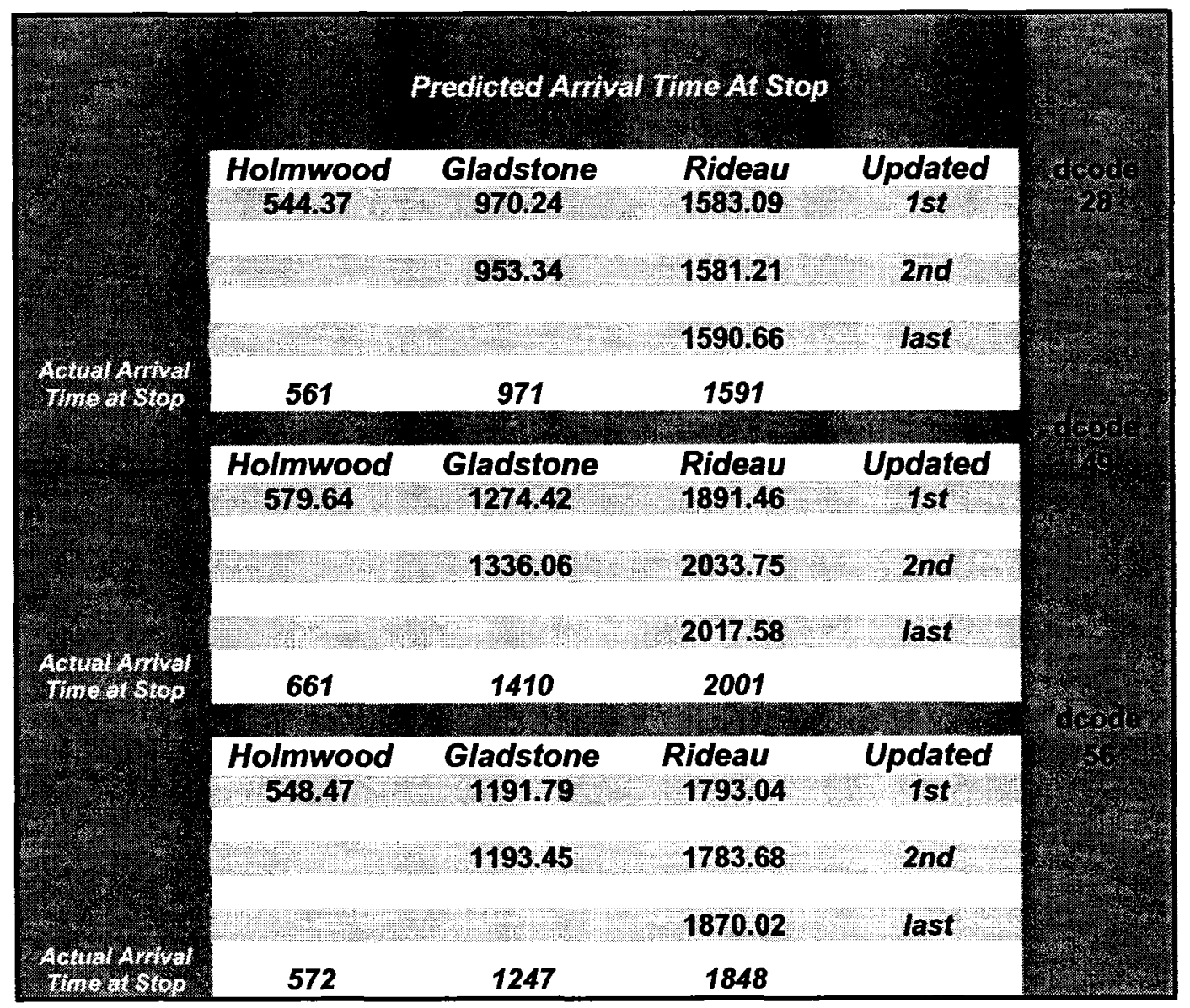

\subsection{Summary}

Different test sets were used to test different constituent modules as well as the integrated developed model in term of prediction capability. In order to compare the developed model with other predictors, the Kalman filter-based model selected from the literature and the naïve model were also used to predict bus running times and passenger activities. It was found that all modules of the developed model were statistically superior to the reference predictors, given the same input data. 
When applied for obtaining running time predictions, the running time prediction module worked satisfactorily, given its MAPE of only nearly $5 \%$ and $7 \%$ on average when using both actual and simulated data for route 95 and route 1 , respectively.

The real-time boarding passenger and alighting passenger sub-modules also worked quite well and all outperformed the reference predictors. The MAPE for boarding passenger prediction was from $20 \%$ to $26 \%$ and from $6 \%$ to $8 \%$ for alighting passenger estimation. It should be recalled that while other prediction models mentioned in the literature review need a controversial assumption on passenger arrival rate, the developed model does not require this assumption.

While the reference models showed instability when dealing with unusual event occurring in bus service activity, the developed model showed only small changes in its prediction performance. That is, it worked consistently.

For real-time bus arrival time prediction, the integrated model produced good results as can be appreciated with average MAPE as low as 3\%. The updating method helps to improve the accuracy of the model when new information becomes available and fed into the model. 


\section{Chapter 7}

\section{REAL-TIME PREDICTION INTERVAL, ON-LINE ADHERANCE EVALUATION AND BUS BUNCHING DETECTION}

\subsection{Introduction}

In this chapter, methods to define real-time prediction interval, bus on-time performance, and bus bunching detection are reported. In addition, further applications of the developed model are discussed. Finally, the spatial aspect and the level of detail for database are mentioned in order to facilitate model efficiency.

\subsection{Real-time Prediction Interval}

Prediction is a challenge and will never be a fixed value. Therefore, a prediction interval is important under any circumstances. In this study, prediction interval for bus running time carries the evidences of non-parametric regression and thus, it is complicated and time consuming.

Several methods have been developed to determine the prediction interval for non-parametric regression family. These methods can be seen in the books of Hardle (1990), Fan et al., (1996) or Loader et al., (1999). However, only the methods that are theoretically sound and can be applied easily are of interest in this research. In this 
section, an automatic computer-intensive procedure was applied to define prediction interval dynamically with each prediction.

According to Fan et al., (1996) the prediction intervals for pointwise locally weighted regression can be expressed as follows.

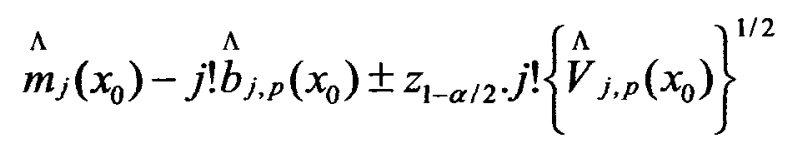

Where:

$\hat{m}_{j}\left(x_{0}\right)$ and $j$ have been explained in chapter 4

$z_{l-\alpha / 2}$ denotes the $(1-\alpha / 2)^{\text {th }}$ quantile of the standard Gaussian distribution

$\stackrel{\Lambda}{b}_{j, p}\left(x_{0}\right), \stackrel{\Lambda}{V}_{j, p}\left(x_{0}\right)$ the estimated conditional bias and variance

The estimates of bias and variance are obtained by calculating expectation and variance from equation defining $\beta$ as follows (Please see Equation 4.7, chapter 4)

$$
\begin{aligned}
& E\left(\stackrel{\Lambda}{\beta}\left(x_{0}\right) \mid X_{1}, X_{2}, \ldots, X_{n}\right)=\left(\boldsymbol{X}^{t} \boldsymbol{W} \boldsymbol{X}\right)^{-1} \boldsymbol{X}^{t} \boldsymbol{W} \boldsymbol{m}=\boldsymbol{\beta}+\left(\boldsymbol{X}^{t} \boldsymbol{W} \boldsymbol{X}\right)^{-1} \boldsymbol{X}^{t} \boldsymbol{W} \boldsymbol{r} \\
& \operatorname{Var}\left(\hat{\beta}\left(x_{0}\right) \mid X_{1}, X_{2}, \ldots, X_{n}\right)=\left(\boldsymbol{X}^{t} \boldsymbol{W} \boldsymbol{X}\right)^{-1}\left(\boldsymbol{X}^{t} \boldsymbol{I} \boldsymbol{X}\right)\left(\boldsymbol{X}^{t} \boldsymbol{W} \boldsymbol{X}\right)^{-1}
\end{aligned}
$$

Where: $\boldsymbol{m}=\left(m\left(X_{1}\right), m\left(X_{2}\right), \ldots, m\left(X_{n}\right)\right)^{t}, \boldsymbol{\beta}=\left(m\left(x_{0}\right), \ldots, m^{(p)}\left(x_{0}\right) / p !\right)^{t}$ and $\boldsymbol{r}=\boldsymbol{m}-X \boldsymbol{\beta}$, the residual of the local polynomial, and $\boldsymbol{\Pi}$ is square covariance diagonal matrix with $\Pi_{i, i}=K^{2}\left(\frac{X_{i}-x_{0}}{h}\right) \sigma^{2}\left(X_{i}\right) ;$ Please refer to chapter 4 for the definitions of $X$ and $\boldsymbol{W}$.

The bias in equation 7.2 and the variance in equation 7.3 cannot be directly usable because of unknown quantities $\boldsymbol{r}$ and $\boldsymbol{\Pi}$. Therefore, they need to be approximated through $\hat{b}_{j, p}\left(x_{0}\right), \stackrel{\Lambda}{V}_{j, p}\left(x_{0}\right)$ which are respectively the estimated conditional bias and 
variance. These are the decompositions of the estimator of mean squared error $(\stackrel{\Lambda}{M S} E)$ of $\stackrel{\Lambda}{\beta}_{j}=\hat{m}_{j}\left(x_{0}\right) / j !$ as shown below

$$
\stackrel{\Lambda}{M S E_{j, p}}\left(x_{0}, h\right)={\stackrel{\Lambda}{b^{2}}}_{j, p}\left(x_{0}\right)+\stackrel{\Lambda}{V}_{j, p}\left(x_{0}\right)
$$

According to Loader et al. (1999), the estimated bias and variance can be defined by the following equations.

$$
\stackrel{\Lambda}{V}_{j, p}\left(x_{0}\right) \approx \stackrel{\Lambda}{\sigma}^{2}\left(x_{0}\right)
$$

Where:

$$
\begin{aligned}
& \stackrel{\Lambda}{\sigma}^{2}\left(x_{0}\right)=\frac{1}{\operatorname{tr}(W)-\operatorname{tr}\left(\left(X^{t} W X\right)^{-1} X^{t} W^{2} X\right)} \sum_{i=1}^{n}\left(Y_{i}-\stackrel{\Lambda}{Y_{i}}\right)^{2} \cdot K\left(\frac{X_{i}-x_{0}}{h}\right) \\
& \stackrel{\Lambda}{b}, p_{j}\left(x_{0}\right)=\left(X^{t} W X\right)^{-1} X^{t} W \tau
\end{aligned}
$$

Where: $\tau$ is a row vector which replaces the residual vector in equation 7.2 in a higher order of polynomial.

While the estimator of variance can be approximated easily, it was found that the estimator of bias shown in equation 7.7 is unrealizable as it is based on a larger order of regression function with different pilot bandwidths (Fan et al., 1996). Therefore, it is an expensive and complicated computation.

In chapter 4, we applied leave-one-out method to define an optimal bandwidth, given a focal point. Based on that bandwidth, the estimator of mean square error $\stackrel{\wedge}{M S E}$ was defined as a by-product of the bandwidth computation. As thus, we can estimate the estimator of bias by using the system of equations 7.4, 7.5 and 7.6. Once the estimators 
of bias and variance have been determined, the prediction interval can be defined. The steps to determine a prediction interval are shown below:

Step 1: Determine the optimal bandwidth by leave-one-out method presented in chapter 4 .

Step 2: Based on the optimal bandwidth and the recognition of the neighbours around the given focal, determine the local regression function.

Step 3: Based on the local regression function, calculate mean squared error $\stackrel{\Lambda}{M S E}$ ) of the local neighbours in the domain of the optimal bandwidth.

Step 4: Determine the estimator of variance based on equations 7.6.

Step 5: Determine the estimator of bias based on $\stackrel{\Lambda}{M S E}$ and the estimator of variance.

Step 6: Estimate prediction interval based on equation 7.1 with $z$ statistic replaced by $t$ statistic due to the approximation of variance with the degree of freedom shown below (Fan et al., 1996).

$$
d_{n}\left(x_{0}\right)=\left\{\sum_{i=1}^{n} K_{h}\left(X_{i}-x_{0}\right)\right\}^{2} / \sum_{i=1}^{n} K_{h}^{2}\left(X_{i}-x_{0}\right)
$$

The overall procedure was coded in Matlab. Please see Appendix C.

Table 7.1 presents pointwise prediction intervals for running time test sets of route 1 with two levels of significance at 0.05 and 0.01 . As seen in the table, the predicted values were not the center of corresponding prediction intervals due to bias. The predicted values tend to be closer to the upper bounds of their prediction intervals. 
Table 7.1: Real-time Prediction Interval for Bus Running Time of Route 1 (Time in second)

\begin{tabular}{|c|c|c|c|c|c|}
\hline \multirow{2}{*}{ RCODE $^{*}$} & $\begin{array}{c}\text { Predicted } \\
\text { Running } \\
\text { time }\end{array}$ & \multicolumn{2}{|c|}{$\alpha=\mathbf{0}$} & \multicolumn{2}{c|}{$\alpha=0.01$} \\
\cline { 3 - 6 } & lower bound & upper bound & lower bound & upper bound \\
\hline 1 & 946 & 764 & 1003 & 707 & 1060 \\
\hline 7 & 962 & 735 & 1025 & 656 & 1095 \\
\hline 12 & 923 & 740 & 984 & 682 & 1041 \\
\hline 17 & 925 & 672 & 1014 & 591 & 1094 \\
\hline 18 & 949 & 755 & 1015 & 695 & 1075 \\
\hline 23 & 935 & 699 & 1007 & 629 & 1073 \\
\hline 28 & 859 & 606 & 950 & 525 & 1025 \\
\hline 33 & 912 & 718 & 975 & 659 & 1033 \\
\hline 37 & 951 & 751 & 1015 & 690 & 1076 \\
\hline 40 & 828 & 638 & 895 & 578 & 955 \\
\hline 46 & 896 & 655 & 975 & 579 & 1050 \\
\hline 50 & 909 & 742 & 967 & 688 & 1020 \\
\hline 57 & 899 & 596 & 1006 & 499 & 1103 \\
\hline 61 & 904 & 733 & 959 & 680 & 1012 \\
\hline 68 & 954 & 724 & 1033 & 651 & 1070 \\
\hline
\end{tabular}

* RCODE: a unique code set by the author for the purpose of computer programming

With a real-time prediction interval, a bus transit planner can capture the variation domain of the predicted bus running time, given a certain confidence level. Therefore, the provision of real-time prediction interval enables other applications of the developed model in the bus transit field. The following sections present the applications of this method for defining probabilities of the bus being on-time or in pair form.

\subsection{On-line Schedule Adherence Evaluation}

\subsubsection{Methodology}

As discussed in previous chapters, bus service providers and passengers need to know ahead of time whether the bus will be on-schedule or not. The model developed in 
this thesis research allows transit providers to know about bus arrival time as well as the prediction interval in real-time at any stop. However, the methodology to determine the probability of bus being on-time has yet to be developed.

Data for every 3 monthly periods that were retrieved from the AVL-APC systems in the OC Transpo were used to estimate bus on-time performance. Comparisons were made of the retrieved bus arrival times at bus stops with the scheduled times and then the differences were statistically analyzed. Such a difference is called the off-line adherence performance $(O A P)$ of the bus. The OC Transpo as well as other bus transit agencies throughout North America consider that the bus is on-time if its $O A P$ falls between 1 minute of earliness and 3 minutes of lateness (Stramathan et al., 1999; Shara, 2002). We call these values as the on-time limits and the time between the limits is on-time standard range.

To forecast the probability of the bus being on time in real-time, it is easy to apply the above on-time standard range, given a real-time bus arrival prediction. Now we call the difference between a scheduled arrival time and a real-time arrival time as Real-time Adherence Performance (RAP). Is the bus on-time given a predicted $R A P$ ? A simple answer is that if this RAP is within the on-time standard range, then the bus can be considered as on-time. However, in order to provide bus dispatchers more information about on-line arrival performance of the bus, further steps should be taken as follows

1. At each stop, the history of bus arrival times is collected and compared with scheduled arrival time.

2. Examine the density distribution of $O A P$ by assuming that it follows one of the following distributions: the Gaussian or Lognormal distributions. 
3. Find the best fit distribution using $\chi^{2}$ (Chi-square) test or Kolmogorov-Smirnov test (K-S test).

4. Determine the $R A P$ given a predicted bus arrival time and its prediction interval

5. Calculate the probability of the bus being on-time and other possibilities, given the best-fit distribution, the $R A P$, and the prediction interval.

\subsubsection{A Numerical Example}

For the sake of illustration, we select arrival times recorded at Gladstone stop of the buses that are scheduled to depart routinely at 8:59 a.m. (i.e., at the $1^{\text {st }}$ second) at Billings Bridge station and to arrive at Gladstone stop at 9:10 a.m. (i.e., at the $660^{\text {th }}$ second). The data were retrieved from the APC systems for one year period from 2004 to 2005.

1. The differences between historical arrival times and scheduled arrival times $(O A P)$ were calculated and arranged in the increasing order.

2. To fit the $O A P$ distribution, the Gaussian and Lognormal probability distributions were applied. Equations 7.8 and 7.9 present these distributions, respectively.

Lognormal Distribution

$\begin{cases}f(x)=\frac{\Delta h}{(x-\min ) \sqrt{2 \pi \theta^{2}}} & \exp \left(-\frac{[\ln (x-\min )-\varsigma]^{2}}{2 \theta^{2}}\right) \quad \mathrm{x}>\min \\ f(x)=0 & \text { if } \mathrm{x} \leq \min \end{cases}$

Gaussian distribution

$f(x)=\frac{\Delta h}{\sigma \sqrt{2 \pi}} \exp \left(-\frac{(x-\mu)^{2}}{2 \sigma^{2}}\right)$ 
Where:

$$
\begin{aligned}
& \min =\text { minimum } \mathrm{x} \\
& \Delta h=\text { interval length; } \Delta h=(\text { maximum } \mathrm{x}-\text { minimum } \mathrm{x}) / \text { total classes } \\
& \varsigma=\text { Location parameter of a lognormal distribution } \\
& \theta=\text { shape parameter } \\
& \sigma=\text { standard deviation } \\
& \mu=\text { mean }
\end{aligned}
$$

3. A null hypothesis $\left(\boldsymbol{H}_{0}\right)$ that the Gaussian or the Lognormal probability functions provide good fits for the distribution of $O A P$ was made and tested. For the chisquare test, let's call $\chi^{2}$ eti as the chi-square value estimated from the goodness-of-fit procedure. If $\chi^{2}$ eti is greater than $\chi^{2}$ critical at $\alpha=0.05$, then $\boldsymbol{H}_{\boldsymbol{o}}$ is rejected, meaning a poor fit. Otherwise, if $\chi^{2}{ }_{\text {eti }}$ is smaller than $\chi^{2}$ critical, $\boldsymbol{H}_{0}$ cannot be rejected (DO NOT REJECT), or a good fit is recognized. For the K-S test, the K-S estimated will be compared with $K-S$ critical at $\alpha=0.05$. If the $K-S$ estimated is smaller than $K-S$ critical, then $H o$ cannot be rejected (DO NOT REJECT).

4. For each type of distribution, the theoretical frequencies were calculated and compared to the observed frequencies of $O A P$. These discrepancies were then used to estimate $\chi^{2}$ and Kolmogorov-Simirnov test. Table 7.2 presents the results of the fitting procedures. These are the products of the StaFit 2.0 software. 
Table 7.2: Goodness-of-fit Test Summary $(\alpha=0.05)$

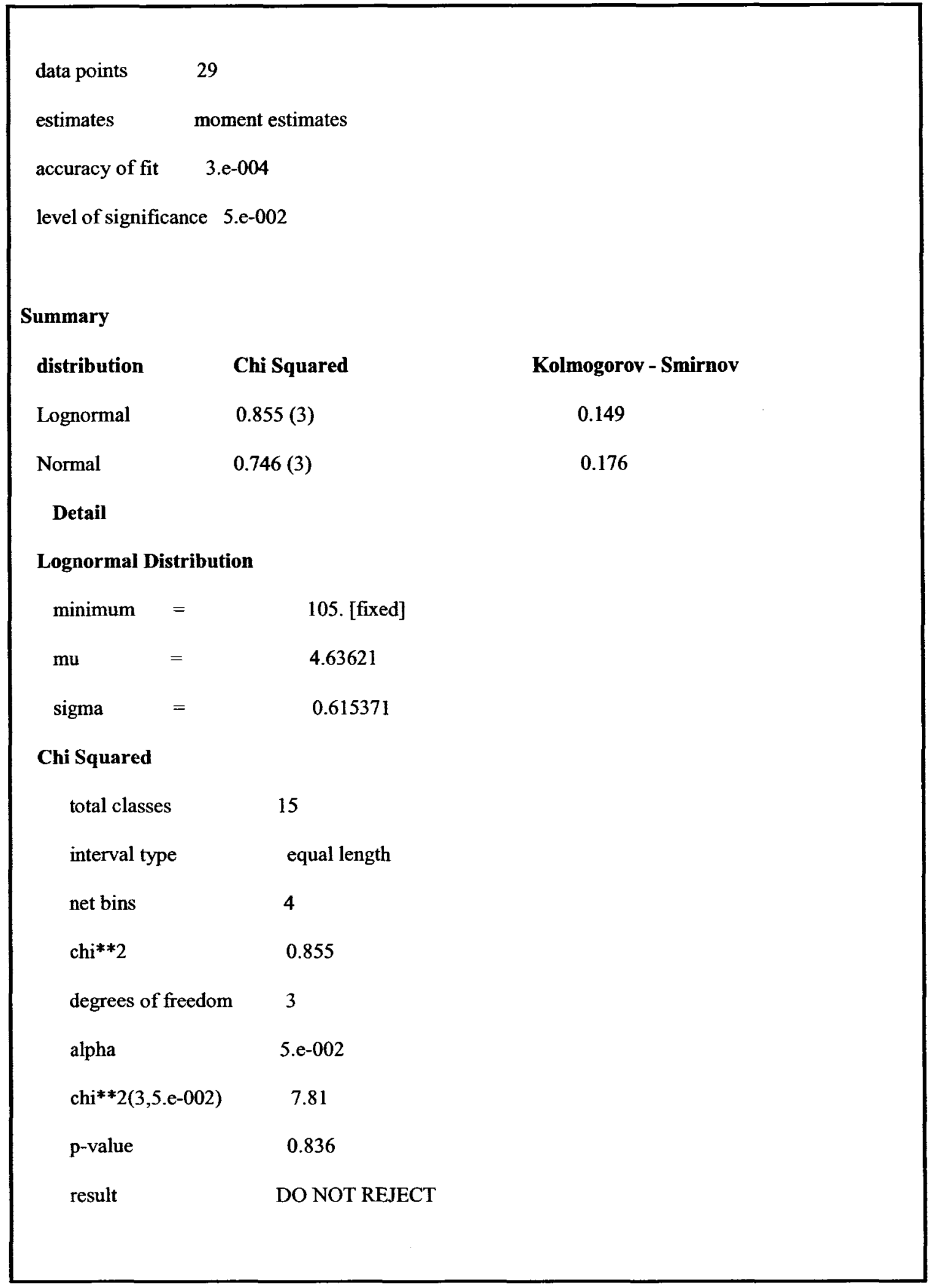




\begin{tabular}{|c|c|}
\hline \multicolumn{2}{|l|}{ Kolmogorov-Smirnov } \\
\hline data points & 29 \\
\hline K-S stat & 0.149 \\
\hline alpha & 5.e-002 \\
\hline K-S stat $(29,5 . e-002)$ & 0.246 \\
\hline p-value & 0.495 \\
\hline result & DO NOT REJECT \\
\hline \multicolumn{2}{|l|}{ Gaussian distribution } \\
\hline mean & 229.655 \\
\hline sigma & 83.107 \\
\hline \multicolumn{2}{|l|}{ Chi Squared } \\
\hline total classes & 15 \\
\hline interval type & equal length \\
\hline net bins & 4 \\
\hline $\mathrm{chi}^{* * 2}$ & 0.746 \\
\hline degrees of freedom & 3 \\
\hline alpha & 5.e-002 \\
\hline $\operatorname{chi}^{* * 2(3,5 . e-002)}$ & 7.81 \\
\hline $\mathrm{p}$-value & 0.862 \\
\hline result & DO NOT REJECT \\
\hline \multicolumn{2}{|l|}{ Kolmogorov-Smirnov } \\
\hline data points & 29 \\
\hline K-S stat & 0.176 \\
\hline alpha & 5.e-002 \\
\hline K-S stat $(29,5 . e-002)$ & 0.246 \\
\hline p-value & 0.296 \\
\hline result & DO NOT REJECT \\
\hline
\end{tabular}


5. The results show that both distributions were successfully in fitting the actual data at $\alpha=0.05$ (see Table 7.2 and Fig.7.1) but the Gaussian distribution gave a better fit. Therefore, the best-fit probability function is:

$$
f(x)=\frac{29}{83.107 \sqrt{2 \pi}} \exp \left(-\frac{(x-229.655)^{2}}{2(83.107)^{2}}\right)
$$

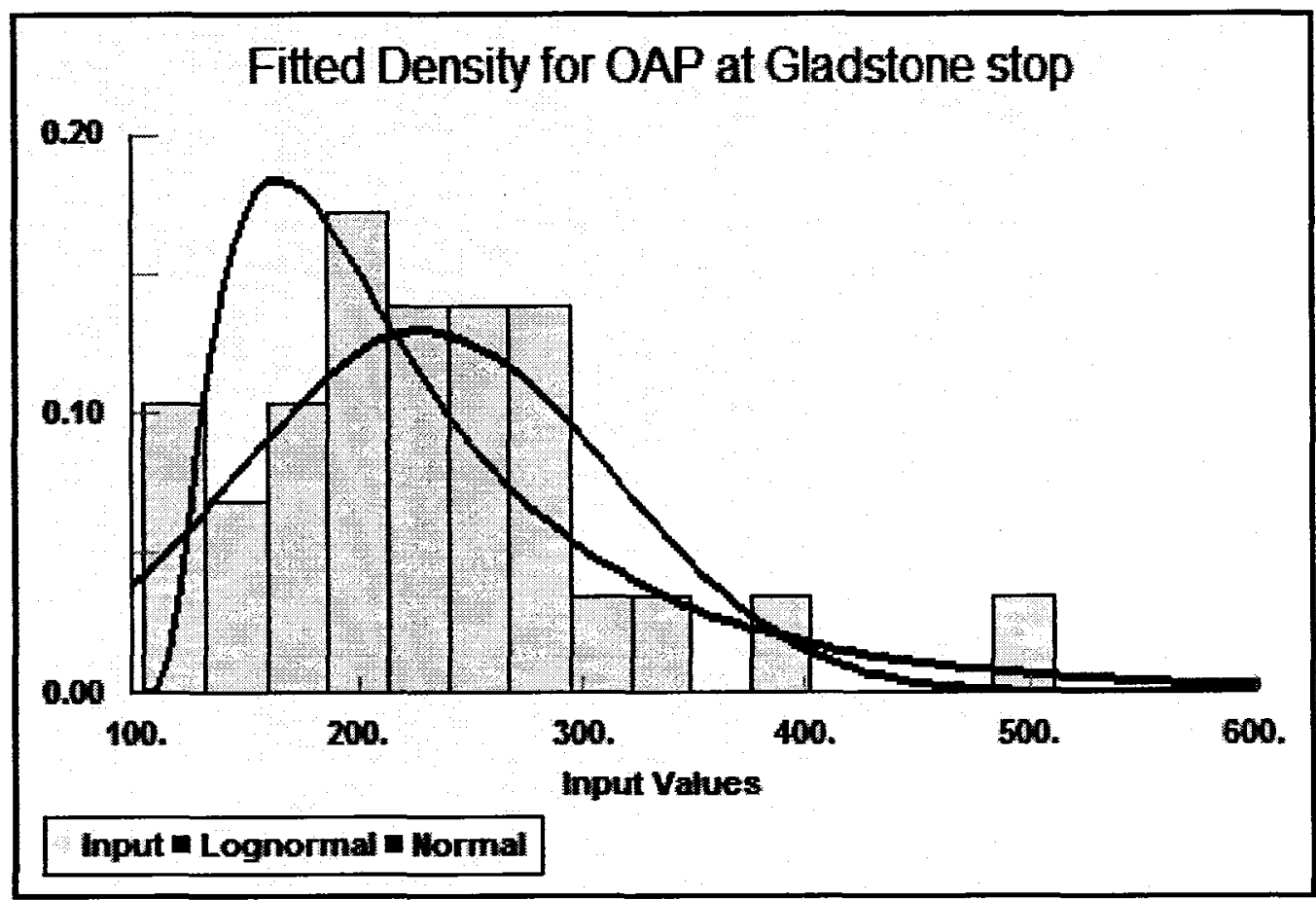

Figure 7.1: Fitted Density for OAP at Gladstone Stop- Route 1

Assume that a real-time prediction of bus arrival time, together with its prediction interval, has just been made as $946^{\text {th }}$ second at Gladstone stop (e.g., the case with $\mathrm{RCODE}=1, \alpha=0.05$, Table 7.1). The scheduled arrival time for this bus is at $660^{\text {th }}$ second. We calculate the differences between the lower prediction bound ( $764^{\text {th }}$ second), the predicted value $\left(946^{\text {th }}\right.$ second), and the upper prediction bound $\left(1003^{\text {rd }}\right.$ second), with the scheduled arrival time. These are $X_{1}=104 \mathrm{~s}, X_{2}=286 \mathrm{~s}$, and $X_{3}=343 \mathrm{~s}$, respectively. As 
discussed earlier, the bus is considered to be on time if the predicted arrival time compared to schedule times is 60 seconds ( 1 minute) of earliness or 180 seconds ( 3 minutes) of lateness. We denote the on-time limits as $O_{1}$ (lower limit) and $\mathrm{O}_{2}$ (upper limit). By applying Equation 7.10 with the values of $X$ and $O$, we can have the probabilities that the bus will be on-time or not. Figure 7.2 depicts the locations of those points.

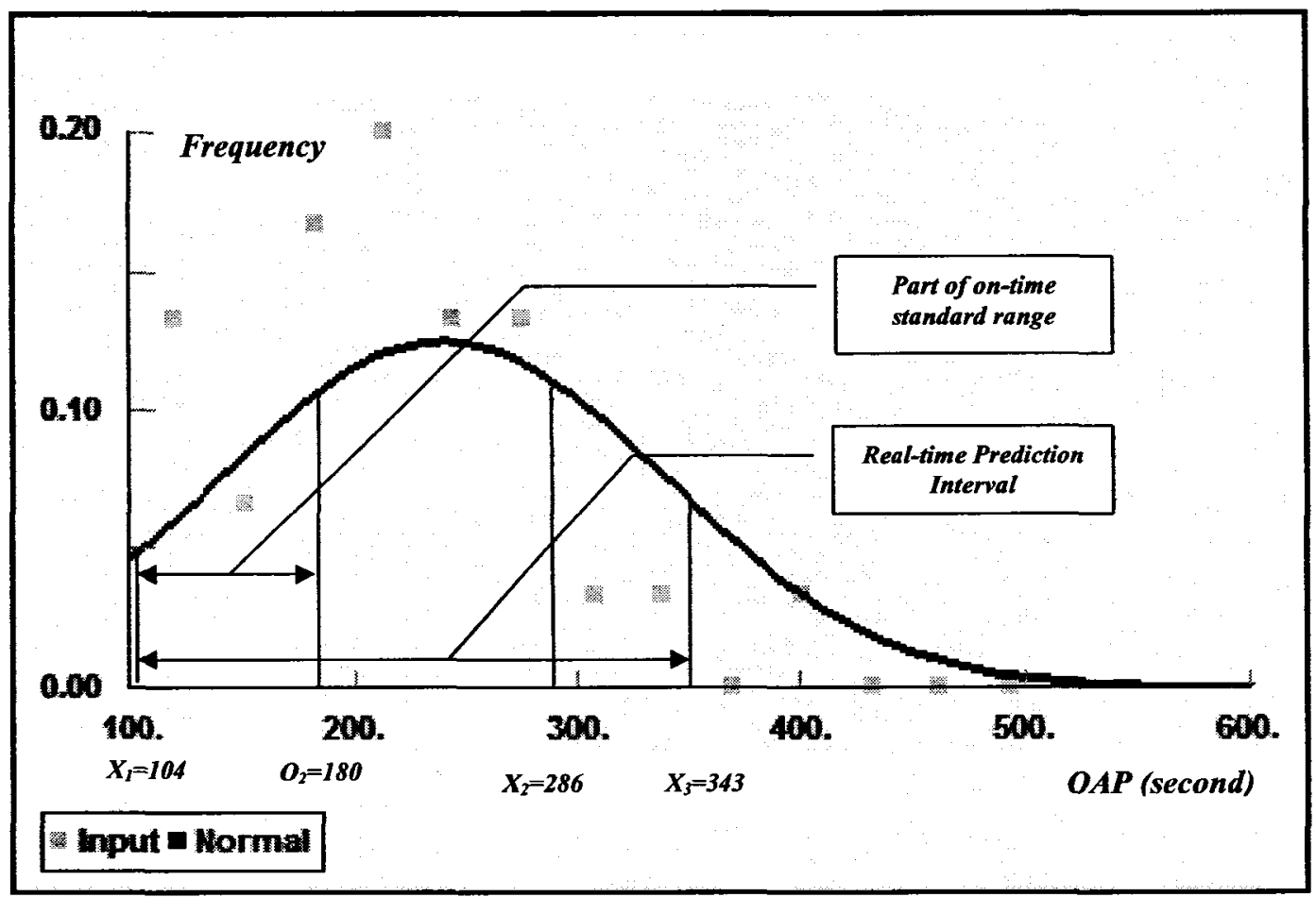

Figure 7.2: On-time Limits and Prediction Interval

In Figure 7.2, the lower limit $O_{J}$ is not presented since the lowest value of $O A P$ is 100 seconds of lateness. Therefore, there is no chance that the bus is on-time at the stop during the time range of $600^{\text {th }} \sim 760^{\text {th }}$ second. 
Because the predicted $R A P$ (i.e., $X_{2}=286$ seconds) is larger than the on-time upper limit ( $\mathrm{O}_{2}=180$ seconds) the bus is very likely not on-time. However, as the prediction lower bound $\left(X_{I}=104\right.$ seconds $)$ is smaller than the upper limit, there is still a chance the bus is on-time. The probability that the bus is on-time and its $R A P$ falls between the range $\mathrm{X}_{1} \mathrm{O}_{2}$ (i.e., $104 \sim 180$ seconds) is exactly the area bounded by the distribution curve (Equation 7.10) and part of the horizontal axis $X_{1} O_{2}$ as found below:

$$
P(104 \leq R A P \leq 180)=\int_{104}^{180} \frac{29}{83.107 \sqrt{2 \pi}} \exp \left(-\frac{(x-229.655)^{2}}{2(83.107)^{2}}\right) d x \approx 21 \%
$$

In other words, the probability that the bus is on-time is $21 \%$, with the bus arrival time is in between $764^{\text {th }} \sim 840^{\text {th }}$ second.

Similarly, the probability that the bus is behind the schedule and the lateness is within 180 to 343 seconds $\left(O_{2} X_{3}\right)$ is as follows

$$
P(180 \leq R A P \leq 343)=\int_{180}^{343} \frac{29}{83.107 \sqrt{2 \pi}} \exp \left(-\frac{(x-229.655)^{2}}{2(83.107)^{2}}\right) d x \approx 62 \%
$$

If we convert the $R A P$ into the arrival time, the probability that the bus is late is $62 \%$ with the bus arrival time in between $841^{s t} \sim 1003^{r d}$.

In general term, the probability that the bus is not on-time is $79 \%$. That is high enough to conclude that the bus is not on-time.

\subsubsection{Discussion}

1. Unlike the simple answer that the bus is certainly not on-time (e.g., probability that the bus is not on-time is equal to 1), if a predicted bus arrival time is out of the ontime standard range, the above example showed that there is still a chance the bus is on- 
schedule. This happens when an overlap between prediction interval and standard range exists. If the probability that the bus is not on-time is higher than that of the bus is ontime, there is potential that the bus is not-on time and vice versa.

2. If there is no overlap and the prediction upper bound $X_{3}$ is smaller than the ontime lower limit $\left(O_{1}\right)$, then the probability that the bus is not on-time and early is 1 . Similarly, if prediction lower bound $X_{I}$ is larger than the on-time upper limit $\left(\mathrm{O}_{2}\right)$, then the probability that the bus is not on-time and is late is 1 .

3. By combining the method to predict real-time prediction interval in previous section and the methodology developed in this section, bus dispatchers can quantitatively analyze all possibilities of bus adherence to the schedule. This would help them to make better decisions in managing their bus fleet.

\subsection{Real-time Bus Bunching Detection Method}

\subsubsection{Methodology}

Once bus arrival times at every stop can be predicted in real time, the tendency of buses to form in pairs (or bus bunching) can be detected. Knowledge in advance of a bus bunching will help bus dispatchers to make a proactive solution to prevent it. The decision should be based on the probability that bunching may take place and the experience of the bus dispatchers. In this section, a method to provide that probability is developed.

Given the $O A P$ distributions of bus $k$ and $k+1$ at a specified stop, the predicted bus arrival times of bus $k$ and bus $k+1$, and their prediction intervals, the probability of a 
bus bunching at the stop is positive if the upper prediction bound $\left(A R_{3, k}\right)$ of bus $k$ is larger than the lower prediction bound $\left(A R_{l, k+1}\right)$ of bus $k+1$ as shown in Figure 7.3. Otherwise, this probability is equal to zero as illustrated in Figure 7.4.

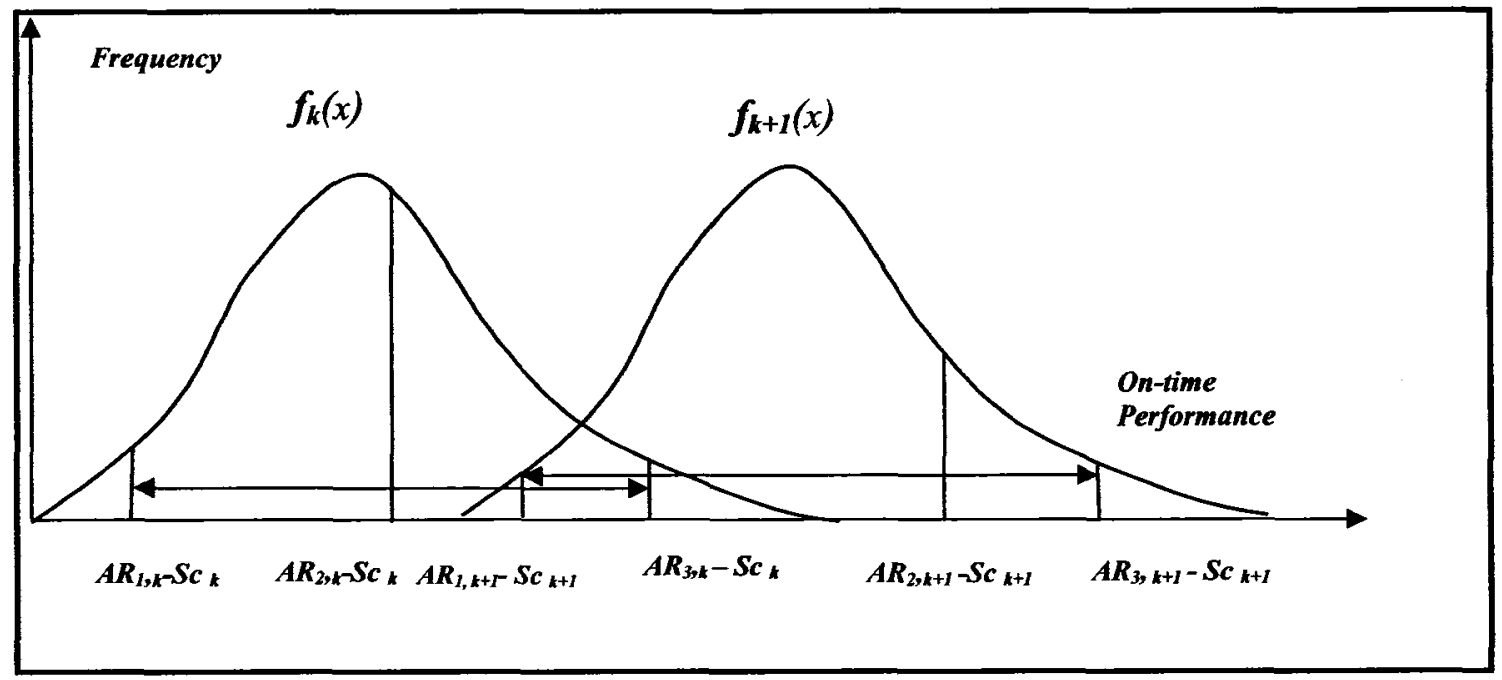

Figure 7.3: Tendency of Bus Bunching, $P>0$

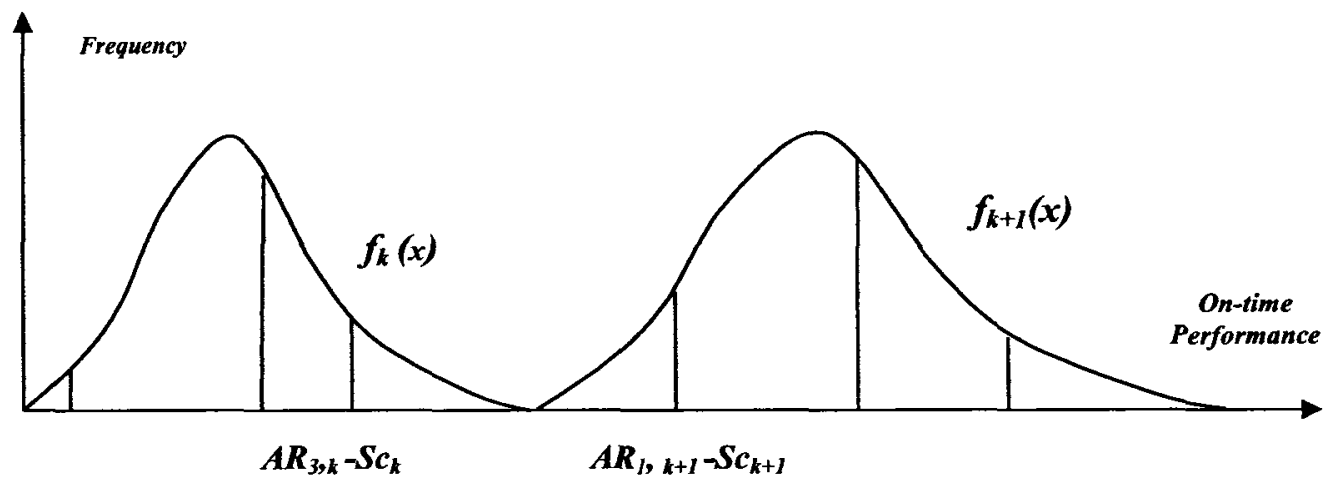

Figure 7.4: Tendency of bus Bunching, $P=0$

In Figure 7.3, the probability of bus bunching can be derived as follows: 


$$
P_{b}=\int_{A R_{1, k+1}-S c_{k}}^{A R_{3, k}-S c_{k}} f_{k}(x) d x . \int_{A R_{1, k+1}-S c_{k+1}}^{A R_{3, k}-S c_{k+1}} f_{k+1}(x) d x
$$

Where:

$A R_{1, j}$ and $A R_{3, j}(j=k, k+I)=$ the lower and the upper prediction bounds of a prediction interval of bus $j$

$S c_{k}$ and $S c_{k+1}=$ scheduled arrival times for bus $k$ and $k+1$

$f_{k}(x)$ and $f_{k+1}(x)=$ probability functions of $O A P$ of bus $k$ and $k+1$ at the stop

Obviously, the larger the $P_{b}$, the closer the bus dispatchers should pay attention to this phenomenon. The developed method for bus bunching detection is summarized as follows

1. Determine a pair of buses needed to be examined for bunching at a certain stop (e.g., bus $k$ and bus $k+1$ ).

2. Use the developed model to predict bus arrival times and the corresponding prediction intervals for each bus.

3. Determine the goodness-of-fit functions of $O A P$ for bus $k$ and $k+1$ at the stop (e.g., $f_{k}(O A P)$ and $\left.f_{k+1}(O A P)\right)$.

4. Compare the upper bound prediction of the preceding bus $\left(A R_{3}, k\right)$ with the lower prediction bound of the following bus $\left(A R_{l, k+l}\right)$.

If $A R_{1, k+1} \geq A R_{3, k}$ then $P_{b}=0$ or there is no chance the two buses can form a pair.

If $A R_{l, k+1}<A R_{3, k}$ then $P_{b}$ is found from Equation 7.11 


\subsubsection{A Numerical Example}

To illustrate the methodology described in the previous section, a pair of 8:56 a.m. and 8:59 a.m. buses departing from St.Laurent station of route 95 was selected. The scheduled arrival times of these buses at Mackenzie stop are 9:05 a.m. and 9:08 a.m., respectively. Historical data of bus arrival times recorded by the APC systems were used to examine the $O A P$ distributions of the two buses at Mackenzie stop. The real-time arrival times and the prediction intervals of these buses were predicted by using the developed model. We have to predict if the buses are forming into a pair or not. The parameters of the two buses are presented in Table 7.3.

Table 7.3: Parameters of the Two Consecutive Buses

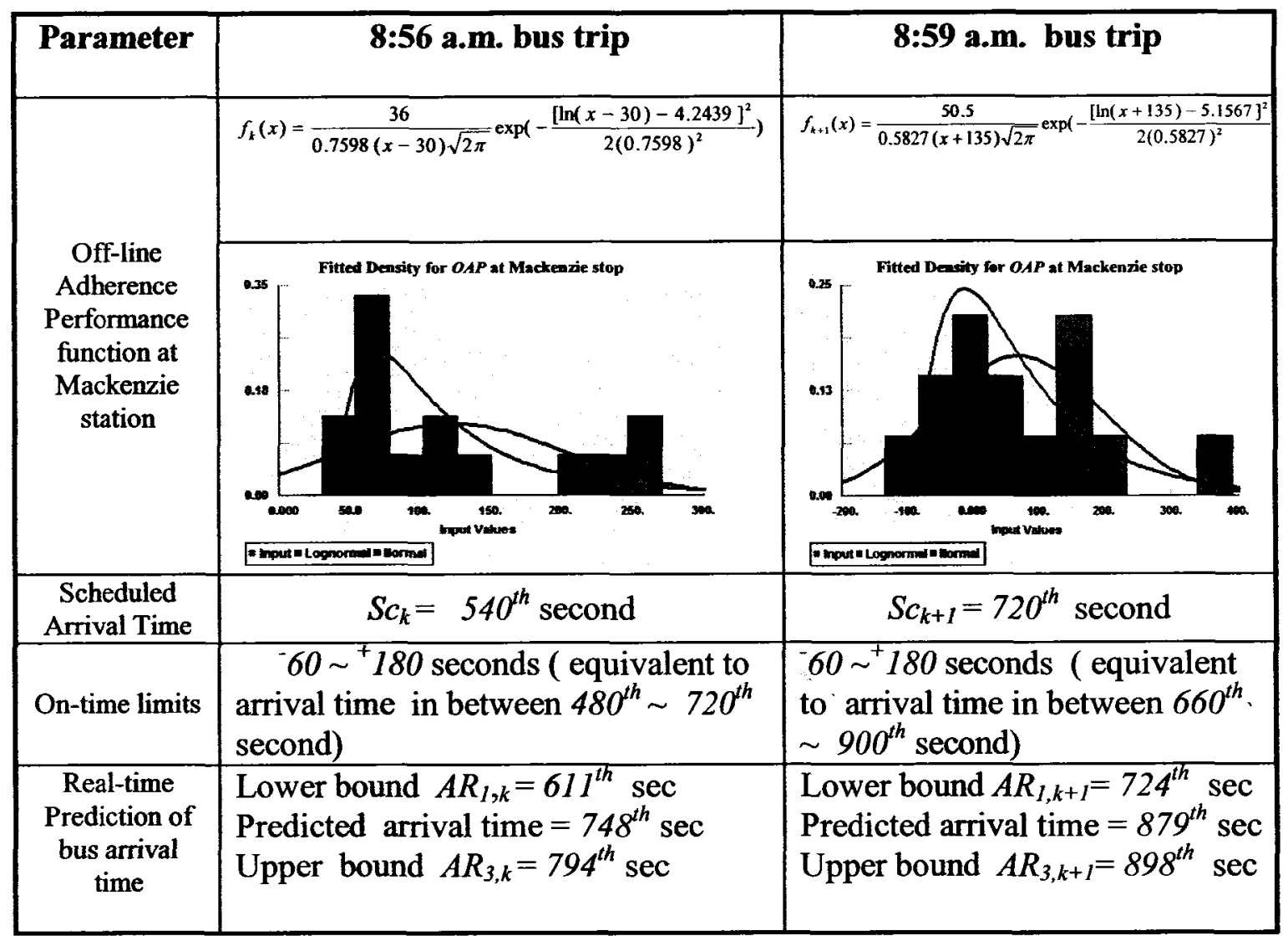


As shown in Table 7.3, $A R_{3, k}$ is larger than $A R_{l, k+1}$ so there is a chance that the two buses will form a pair at Mackenzie stop. The probability of this incidence is calculated as follows

$$
\begin{aligned}
P_{b} & =\int_{A R_{1, k+1}-S c_{c_{k}}}^{A R_{3,-}-S c_{k}} f_{k}(x) d x . \int_{A R_{1, k+1}-S c_{k+1}}^{A R_{3, k}-S c_{k+1}} f_{k+1}(x) d x=\int_{724-540}^{794-540} f_{k}(x) d x . \int_{724-720}^{794-720} f_{k+1}(x) d x= \\
P_{b} & =0.067^{*} 0.235=0.0154 \text { or } 1.54 \% .
\end{aligned}
$$

The probability is too small to be concerned about bus bunching at Mackenzie stop.

\subsection{Further Possible Applications by Using the Developed Model}

This section focuses on further possible applications of the developed model in the field of bus transit control. Instead of detailing the control methods currently used in transit agencies since it may lead to another field of bus transit and therefore divert the focus of the thesis out of its targets, this section aims at listing some real-time control models whose critical bases are the availability of real-time bus arrival and dwell time information at bus stops.

The bus control strategies are moving towards real-time responses to the sporadic service problems (Strathman et al., 2000). In the real-time bus control strategies, vehicle holding, short-turning, stop skipping, and speed modification are the tools to maintain the headway and service reliability given the knowledge of bus service problem in advance such as lateness, earliness or bunching. Having assumed that real-time bus running times and dwell times are available, many researchers did develop models for real-time bus 
control strategies for different purposes. The readers should refer to the work of Strathma et al., (2000) for automatic bus dispatching model, or the work of Ding et al., (2002); and Fu et al.,(2003, 2004) for optimizing headway variance. Those who are interested in speed modification can read the work of Shalaby et al., (2004). For service reliability or passenger utility, the works of Turnquist (1982) and Rabi et al., (2000) are suggested.

\subsection{Real-time Bus Arrival Information Broadcasting}

When arriving at a bus stop, passengers need arrival information of the next bus (buses) that is supposed to be broadcasted. Because broadcasted time changes as realtime arrivals are updated, passengers may get one or more than one bus arrival announcements.

In the developed model, the predicted bus arrival time of the current bus is based on the running times of previous buses. Therefore, if the headway is long, the prediction may be inaccurate. The author suggests that if the headway between buses is longer than 30 minutes, the real-time prediction should not be made and the announced arrival should be "no real-time information".

For the incoming stop (i.e., the stop closest to the bus), besides the real-time bus arrival time being announced, bus transit agencies should consider broadcasting the information about bus on-time possibility. The bus should be announced as "on-time" when the probability that the bus is on-time is larger than it is not so ${ }^{1}$. Otherwise, it should be broadcasted as "not on-time". For instance, in the numerical example in section 7.3.2, the probability that the bus is on-time is $21 \%$, while the probability that it is not so

\footnotetext{
1 The method to estimate this probability was developed in section 7.3
} 
is $79 \%$. Hence, the announcement at Gladstone stop should be as "Next bus is not ontime and will arrive in 4 minutes ${ }^{2 \prime}$. This would help passengers to know clearly that the expected bus is coming or it has already passed the stop.

\subsection{Level of Spatial and Temporal Detail of Data for the Developed Model}

A sound and well organized database is needed in order to facilitate the applications of the developed model. This section discusses a key aspect of a database for the model, the requirement of data capture and database design.

Table 7.4: Level of Spatial and Temporal Details for Data Capture

\begin{tabular}{|c|l|l|l|l|}
\hline Level & Description & $\begin{array}{c}\text { Event- } \\
\text { Independent } \\
\text { records }\end{array}$ & Event Records & $\begin{array}{c}\text { Between-stop } \\
\text { Performance } \\
\text { Data }\end{array}$ \\
\hline A & $\begin{array}{l}\text { AVL data } \\
\text { without real- } \\
\text { time Tracking }\end{array}$ & $\begin{array}{l}\text { Infrequency } \\
(60-120 \text { sec) }\end{array}$ & & \\
\hline B & $\begin{array}{l}\text { AVL data with } \\
\text { real-time } \\
\text { Tracking }\end{array}$ & $\begin{array}{l}\text { Infrequency } \\
(60-120 \text { sec) }\end{array}$ & each timepoint & \\
\hline C & $\begin{array}{l}\text { APC and event } \\
\text { recorder }\end{array}$ & & each stop & Each stop and \\
D & $\begin{array}{l}\text { Event recorder } \\
\text { with between } \\
\text { stop summaries }\end{array}$ & $\begin{array}{l}\text { Recorded } \\
\text { events and } \\
\text { summaries }\end{array}$ \\
\hline E & $\begin{array}{c}\text { Event } \\
\text { recorder/trip } \\
\text { recorder }\end{array}$ & Very frequent & all types & $\begin{array}{l}\text { All events, full } \\
\text { speed profiles }\end{array}$ \\
\hline
\end{tabular}

Furth et al., (2003) concluded that there are five levels of spatial and temporal detail, as illustrated in Table 7.4. Referring to the table and the data contents that the

\footnotetext{
${ }^{2}$ Number 4 is only an example. In fact, the announced time is the difference between the predicted arrival time and the current clock- time when the arrival time is announced.
} 
developed model requires, it is suggested that the level of spatial and temporal detail of data for the model should be level C and up. However, an integration of the APC system with the AVL technologies and a radio system is a requirement for the applications of the developed model for real-time bus arrival prediction. The reason is that on one hand, the on-board micro computer can match the vehicle to the route, and on the other hand the stop record can be sent over the air to the control center.

\subsection{Summary}

Chapter 7 is devoted to the methodologies that can predict the likelihood that the bus is on-time, late, early or bunching. These resulted from the conjunction of the realtime prediction interval presented in section 7.2 and the historical distribution of bus ontime performance at each bus stop. By knowing in advance the probabilities that the bus is not on-time, bus dispatcher can make a good decision to apply suitable control methods in order to keep service adherence.

The broadcasting of real-time bus arrival was also mentioned. As illustrated previously, even if a real-time bus arrival prediction falls within an on-time standard range, there is still a chance that the bus is not on-time and vice-versa since a prediction, in essence, is a probabilistic estimation. The announcement of if the bus is on-time or not should be broadcasted with the consideration of such probabilities.

For bus bunching detection, a method which is simple in application was developed. By applying this method, bus dispatchers can easily quantify a likelihood of bus bunching. As a consequence, they will have suitable proactive control methods to prevent it. 


\section{Chapter 8}

\section{CONCLUSIONS AND RECOMMENDATIONS}

\subsection{Conclusions}

The growing urban traffic congestion in medium and large size cities is forcing the city authorities to search for efficient solutions in order to reduce the adverse effects. It is widely recognized that among possible solutions, an advanced urban public transit system in the urban areas is potentially the most effective solution.

In a modern society where travellers value their time highly, the provision of bus real-time information is instrumental in improving bus transit's ridership. Therefore, it is important to both public transit providers and the passengers. For transit providers, the development of a real-time bus arrival information system (RETBAIS) is an important objective that they have to meet. For passengers, real-time bus arrival information is an important sign in their perception of a reliable, attractive, and convenient public transit system. Over the last twenty years, many transit providers have been equipping their bus fleets with the AVL and APC systems which are considered to be the technology backbone for the development of a RETBAIS. However, these expensive systems are underused. Moreover, the vital role of real-time bus arrival information has been adequately recognized by the transit providers only in the last few years.

This thesis research can be considered as a contribution to this new field of bus transit. All thesis research goals and objectives were addressed comprehensively in terms 
of methodologies that provide up-to-minute information of bus arrivals as well as to help bus dispatcher the on-line decision support tools for managing the bus fleet.

Urban bus transit has a major challenge that it has to strictly adhere to schedule but at the same time it is vulnerable to delays which are very complicated to manage in the urban context. In order to overcome the difficulties of the study of influencing factors on bus arrival time and also to use intensively the available data recoded in the AVL and APC systems, the research separated bus arrival time into two important parts, namely bus running time in a link and bus dwell time at a stop. These philosophies lead to the development of two modules.

Following the development of the running time prediction module, based on the basis of a statistical pattern recognition technique, namely the LOWESS method, and the similarities of bus running times experienced in the same period of time in the past, and in the same bus link, a prediction algorithm was formulated. In order to enhance the algorithm's prediction capability as well as to shorten the prediction time, the author applied leave-one-out cross validation technique and Nadaraya-Watson kernel regression in searching the similarities among variables. The entire algorithm is an automatic computer-intensive procedure for searching, matching and predicting bus running time whenever the AVL-APC data are available and updated. Tests made with simulated and actual data for various bus operation scenarios show that this module performed well, with the average mean relative error were just about $5 \%$ for Transitway route and $7 \%$ for mixed-traffic bus route. In this type of research, such relative error levels or even higher than these, would be acceptable to the consumer. Moreover, the module worked reliably when dealing with unusual events of bus operation. This characteristic was not found in 
the Kalman filter model, a reference model used in this study. It is useful to note that the Kalman filter model outperformed several other comparative models including the Artificial Neural Network-based model in term of bus running time prediction.

Following the development of bus dwell time prediction module, based on the logic that the number of boarding passengers and alighting passengers are the determinants for dwell time prediction, the research moved on to the development of a real-time passenger activity prediction algorithm. Four sub-modules were developed separately for predicting boarders, de-boarders and bus dwell time. The fist two submodules were also based on the same basis of statistical pattern recognition which was applied in the running time prediction module. In order to avoid using a controversial assumption on the distribution of passenger arrival rate, the algorithm developed for boarding passenger prediction used bus headway and boarding historical data as the elements for a pattern being recognized. It was found that both sub-modules outperformed the reference models and the results were acceptable with the average prediction error of about $22 \%$ for boarding prediction, and $6 \%$ for alighting prediction.

Unlike the first two sub-modules where the predictions were driven by the realtime data, the last two sub-modules were developed in the form of pre-defined functions by relating dwell time with the number of boarders, de-boarders, and other selected variables. The first sub-module of these two, namely regression sub-module, was based on the classical functions regressing dwell time to several variables whose data were retrieved form the APC systems. The author found that the regression type A-2.3 is the most suitable with its $R$-square of up to $63 \%$. In case of data shortage, the type A-2.6 is suggested. The last sub-module, namely the busiest door prediction sub-module, was 
based on the fact that alighting and boarding passengers' door choice influence dwell time and therefore the determination of the busiest door is important. Several types of binary and multinomial logistic regressions functions, which stem from the discrete choice theory, were examined with the retrieved APC data. The author suggested that binary logistic regression type B-L.1, together with Equations from 5.45 to 5.47 , is suitable for rigid-body bus while multiple regression (i.e., type A-2.3) should be used for articulated bus. This developed algorithm also solved the problem of bus stop skipping prediction.

When the two modules were integrated for predicting bus arrival times at every bus stop of the full-trips, the entire model worked satisfactorily. The average relative prediction error increased with the new data availability and varied from $3 \%$ to $8 \%$.

As extensions of the developed model, two algorithms were formulated, one for bus on-time performance evaluation and the other for real-time bus bunching detection. In order to use these algorithms, the author developed a method to delineate real-time bus arrival prediction interval. It is worth noting that most existing bus arrival time prediction models found in the literature could forecast only the mean value of arrival time. Therefore, how certain the prediction is and what the prediction interval is, were not provided by these models. The author of this thesis can quantitatively analyze all measures of bus on-time performance, which again were not found in previous models devoted to bus arrival time prediction.

On the basis of the nature of real-time bus arrival predictions, a method to broadcast bus arrivals to passengers was discussed. Bus arrival time broadcasting should be based on the sound probabilities that the bus will be on-time or not. Also, suggestions 
were made on the spatial characteristics and details of the database for the developed model where the integration of the APC system with AVL and a radio system is a prerequisite.

This research can be considered as a contribution to the development of real-time bus arrival information system. In this research, the term "real-time" was illustrated clearly. Here, a real-time prediction model meant to not only to be capable of producing prediction with real-time data but the predictive characteristics of the method itself. Specifically, one important feature for a real-time prediction model is accurate and rapid responsiveness. Therefore, any real-time model should be a computer-intensive one with fast calculation rate. The developed model met this requirement. It was found that a prediction was made by the developed model in only about 15 seconds. All prediction procedures of optimal searching, recognizing, and predicting were done automatically. Hence, this research can provide bus transit providers an accurate, fast, and reliable prediction model.

In the case of dwell time prediction, the term "real-time passenger activity prediction" was first introduced by the author and a methodology to predict it was comprehensively developed. The model can help bus transit planner to deal with highly busy bus stops as well as to predict bus dwell time in real-time that should be spent by the buses to serve such stops or to estimate the load factor for each bus, the waiting time of passengers at stop, and so on. Moreover, the algorithm developed in this study can solve a number of existing issues in term of dwell time estimation.

By using the methods developed by the author for analyzing bus on-time performance, the bus dispatcher can easily detect the possibilities of "not on-time bus 
arrivals" and "bus bunching". These capabilities would help transit agencies to provide passengers more accurate and reliable information as well as to manage their bus fleet economically and efficiently.

The entire model was developed in a manner that it solely used the AVL-APC data. No additional traffic data such as traffic volume, speed, density or so on, are needed. Moreover, because the data are updated at stops or timepoints (i.e., time-at-location ${ }^{1}$ ), tracking is not required (Furth et al, 2003). Therefore, the developed model is suitable even if a transit agency is still using the APC systems with the old AVL technologies (i.e., non GPS-based AVL technology).

In conclusion, the author believes that the real-time bus arrival time prediction model developed in this thesis will enhance the bus arrival information system and will be a contribution to public transit operation.

\subsection{Recommendations and Future Research}

The model developed in this thesis research based on the statistical pattern recognition technique is innovative and produces results that are clearly more accurate than obtainable from other models. As is the case with other methods, there are limitations of the developed model that should be understood prior to application. However, on the balance, the bus arrival prediction process would be considerably enhanced by adopting the model developed in this thesis. Based on the findings and the

\footnotetext{
'Time-at-location: a record of when a bus passed a predetermined point such as a stop or a timepoint. This data collection method is opposite to the location-at-time method - i.e., where a bus was at a given time. Time-at-location is strongly preferred to location-at-time data for management used of achieved AVL-APC data (Furth et al., 2003, p.42).
} 
difficulties experienced during the course of carrying out this research, the following recommendations are made.

The results found in chapter 3 raise the question "why the buses running on route 1 are mostly behind the schedule?" and "what should be done to solve that problem?" Based on the distribution of bus on time performance, it was found that the lateness of the buses have been caused not only by the traffic congestion but also by the schedule design. For example, the statistical mean of actual bus running times between Billings Bridge and Gladstone stops is about 15 minutes whereas the running time scheduled for the buses of this segment is only 11 minutes. With that set up, the probability for the bus "on-time" was only about $43 \%$. Therefore, the OC transpo should adjust the scheduled running times between stops closer to the actual means. Such an adjustment would be appreciated by their customers.

In chapter 4, the author used Euclidian distance to calculate the similarity among patterns. Mathematically speaking, the author actually treated the elements in a pattern matrix equally with the same weights. However, running times of the buses closer to the understudied bus may get more weights because they run under the most similar road and traffic condition with the bus of interest. Further study should be carried on with this standpoint in order to improve the developed model.

Due to constraints in data collection and simulation, a number of elements taken into a pattern in this study were eliminated in order to ensure a sound statistical inference. However, whenever the database is large, more elements should be added into the patterns so that it can improve the prediction results of the developed model. 
The elements in the form of categorical variables should be considered. For example, for a pattern of boarding passenger prediction, besides the elements which were presented, we can add categorical elements such as bus type, seating plan, and so on. The method to recognize such patterns should be further explored in order to make the developed model more versatile.

It is possible that matrix $\boldsymbol{B}=\left(\boldsymbol{X}^{T} \boldsymbol{W X}\right)$ in equation 4.7 will become a singular matrix so that no prediction can be made. In order to solve the problem, further study to convert the linear regression shown in chapter 4 into the form of ridge regression is needed.

Finally, data organization for the retrieved APC-AVL data as well as the data provisions for each module of the developed model requires further studies. 


\title{
REFERENCES
}

\author{
$* * *$
}

Abdelfattah, AliM., and Khan Ata M., (1998). Models for Predicting Bus Delays. Transportation Research Record 1623, pp. 8-15.

Abkowitz, Mark, Eiger Amir, and Engestein Israel, (1986). Optimal Control of Headway Variation on Transit Routes. Journal of Advanced Transportation, Vol.20 (1), pp.73-88.

Abkowitz, Mark, and Lepofsky, (1988). Implementing Headway Based Reliability Control on Transit Routes. Journal of Transportation Engineering, Vol.116, No.1, pp. 49- 63.

AC Transit. Rider Information. http://www.actransit.org

Akcelik \& Associates Pty Ltd. Micro-Simulation and Analytical Methods for Modeling Urban Traffic. October 2001.

Akcelic, R., and Besly M., (2001). Acceleration and Deceleration Models. The $23^{\text {rd }}$ Conference of Australian Institutes of Transport Research (CAITR 2001). Monash University, Melbourne, Australia, December 2001.

Alfa, Attahiru Sule, William B.Menzies, James Purcha, and R.McPherson, (1988). A regression Models for Bus Running Times in Suburban Areas of Winnipeg. Journal of Advanced Transportation, Vol. 21, pp. 227-237.

Andersen, Bob, (2005). Introducing Nonparametric Regression. Lecture notes. McGill University. 
Ben, Akiva, and Steven Lermark, (1985). Discrete Choice Analysis. The MIT Press

Bertini, R.L., and Ahmed M., Geneidy El., (2004). Modeling Transit Time Using Archived Bus Dispatch System Data. Journal of Transportation Engineering, ASCE Vol. 130, No. 1, January 1.

Berry, W., Feldman S., (1985). Multiple Regressions in Practice. Sage Publications.

Bodin, L.D., Ball M.O., Duguid R., and Mitchell M., (1985). The Vehicle Scheduling Problem with Interlining. Computer scheduling of Public Transport 2, Edited by Jean-Marc Rousseau, Elsevier Science Publishers B.V, pp.427-450.

Chang, Hyeon, Theo Arentze, and Harry Timmermans, (2001). Pattern Recognition in Complex Activity Travel Patterns; Comparison of Euclidian Distance, SignalProcessing, Theoretical and Multidimensional Sequence Alignment Methods. Transportation Research Record 1752, pp. 16-22.

Chapman, R.A., and Michel J.F, (1978). Modeling the Tendency of Buses to Form Pair. Transportation Science, Vol. 12, No. 2, pp.165-175. Transportation Research record 1752 , pp. 16-22.

Cathey, F.W., and D.J. Dailey, (2003). A Prescription for Transit Arrival/ Departure Prediction Using Automatic Vehicle Location Data. Transportation Research Part $C$, Vol.11, pp.241-264.

Chen, Mei, Liu Xiaobo, Xia Jingxin, and Chien Steven, (2004). A Dynamic BusArrival Time Prediction Model Based on APC Data. Computer-Aided Civil and Infrastructure Engineering, Vol. 19, pp. 364-376. 


\section{Chien, Steven I., Shoai M. Chowdhury, Kyriacos C., Mouskos, and Yuqing Ding,} (2000). Enhancements of CORSIM Model in Simulating Transit Operations. Journal of Transportation Engineering, Vol. 126, No. 5, September 1, pp. 396-404.

Chien, Steven I., Yuqing Ding, and Chienhung Wei., (2002). Dynamic Bus Arrival Time Prediction with Artificial Neural Networks. Journal of Transportation Engineering, Vol. 128, No.5, September 1, pp. 429-438.

Cleveland, William S., (1979). Robust Locally Weighted Regression and Smoothing Scatter Plots. Journal of the American Statistical Association, Vol.74, No.368, pp.829-836.

Cleveland, William S., (1988). Locally Weighted Regression: An Approach by Local Fitting. Journal of the American Statistical Association, Vol.88, pp.596-610.

Conover, W.J., (1971). Practical Nonparametric Statistics. John Wiley \&Sons Inc.

Dailey, D.J, S.D Maclean, F.W. Cathey, and Z.R. Wall, (2001). Transit Vehicle Arrival Prediction, Algorithm and Large-Scale Implementation. Transportation Research Record, 1771, pp. 46-51.

Daniel, L. Gerlough and Frank C. Barnes., (1971). Poisson and Other Distributions in Traffic. Eno Foundation for Transportation. Saugaruck, Conecticut.

Daniel, Wayne W., (1978). Applied Nonparametric Statistics. Houghton Mifflin Company.

Daniel, K. Boley, (1998). Passenger Counting Technologies and Procedures, TCRP synthesis 29 , TRB, Washington, DC. 
Davis, Gary A., Nacy L.Nihan, Mohammad M.Hamed, and Lesli N. Jacobson, (1990). Adaptive Forecasting of Freeway Traffic Congestion. Transportation Research Record, 1287, pp. 29-33.

Devroye, Luc, Laszlo Gyorfi, and Lugosi, (1996). A probabilistic Theory of Pattern Recognition. Springer Publication.

Dowling, R., Skabardonis A., and Alexiadis V., (2004). Traffic Analysis ToolboxVolume 3: Guidelines for Applying Traffic Simulation Software. Report FHWAHRT-04-040.

Ding, Yuquing, and Chien Steven, (2002). Improving Transit Quality and Headway Regularity with Real-Time Control. Transportation Research Record, 1760, pp. 161-170.

Dueker, Kenneth J., Thomas J. Kompel, and James G. Strathman, (2004). Determinants of Bus Dwell Time. Journal of Public Transportation, Vol.7, No.1, pp. 21-38. Website http://www.nctr.usf.edu/jpt/journalarticles.html

Ernest, Bellman R., (1961). Adaptive Control Process. Princeton, N.J, Princeton University Press.

Fan and Gijbels I., (1996). Local Polynomial Modeling and Its Applications. Chapman \& Hall Publisher.

Fang, Zhao and Min-Tang-Li., (2005). Calibration of Highway / Transit Speed relation For Improved Transit network Modeling in FSUTMS. BD-015-07, Final Report. Lehman Center for Transportation Research - Florida International University. 
Fang, Zhao and Nokil Park, (2004). Using Geographically Weighted Regression Models to Estimate Annual Average Daily Traffic. Transportation Research Record, 1879, pp. 99-107.

Farhan, Ali, (2002). Bus Arrival Time Prediction for Dynamic Operations Control and Passengers Information Systems. Thesis Dissertation of Masters of Applied Science, University of Toronto, Canada.

Fox, John, (1991). Regression Diagnostics. Series in Quantitative Applications in Social Sciences, Sage University Paper, Vol.79.

Fox, K., (2000). SMARTEST-new tools for evaluating ITS. Traffic Engineering and Control, Vol.41 (1), pp.20-22.

Fukotomi, Akihira, (2004). Simulation Approach to Study of Drving Behaviour, Fuel Consumption and Emissions. Master Thesis Dissertation, Carleton University, Ottawa-Canada.

Fu, Liping and Liu Qing, (2004). Real-time Optimization Model for Dynamic Scheduling of Transit Operations. Transportation Research Record, 1857, pp. 4855.

Fu, Liping and Yang X., (2003). Design and Implementation of Bus-Holding Control Strategies with Real-time Information. Transportation Research Record, 1791, pp. 6-12.

Furth, G. Peter, (2003). Use of Archived AVL-APC Data to Improve Transit Performance and Management: Review and Potential. Transit Cooperative Research Program Web Document 23 (Project H-28), TRB, Washington, DC. 
Gibbons, J. Dickinson., (1984). Nonparametric Methods for Quantitative Analysis. Second Edition. American Sciences Press, Inc.

Guether Richard P., and Kumares C. Sinha, (1983). Modeling Bus Delays due to Passenger Boarding and Alighting. Transportation Research Record, 915, pp. 7-13.

Gyorfi Lazlo, Hardle Wolfgang, Sarda Pascal, and Vieu Philippe, (1989). Nonparametric Curve Estimation from Time Series. Springer-Verlag.

Harris, Richard, (2000). A Primer of Multivariate Statistics. Third Edition, IEA Publisher

Hardle, Wolfgang, (1990). Applied Nonparametric Regression. Cambridge University Press.

Hay, William and Winkler Robert, (1971). Statistics, Probability, Inference, and Decision. Holt, Rinehart and Winston, Inc.

Helali, K.N and Khan A.M.., (1993). Regression Based Urban Arterial Road Delay Models. Canadian Journal of Civil Engineering, Vol. 20, No.1, Feb.1993, pp.3749.

Higham, Desmond and Higham Nicholas, (2000), MATLAB Guide. SIAM Philadelphia.

Horbury, Antoneta X., (1999). Using non-real-time Automatic Vehicle Location Data to Improve bus Services. Transportation research Part B. V33. pp. 559-579.

Ishak, S, C., and Alecsandru, (2004). Optimizing Traffic Prediction Performance of Neural Networks under Various Topological, Input, and Traffic Condition Settings. Journal of Transportation Engineering, Vol. 130, No.4, July 1, pp. 452-465. 
Iversen, G. R, and Norpoth Helmut, (1976). Analysis of Variance. Sage Publications.

Kalaputapu, R., and Demetsky, (1985). Application of Artificial Neural Networks and Automatic Vehicle Location Data for Bus Transit schedule behavior Modeling. Transportation Research Record 1497, pp.44-52.

Kimpe, J. Thomas., James G. Strathman, David Griffin, Steven Callas, and Richard L. Gehart, (2003). Automatic Passenger Counter Evaluation Implication for National Transit Database Reporting. Transportation Research Record 1835, pp. 93-99.

Kittelson, and Associates, Inc., (2003). Transit Capacity and Quality of Transit Manual. The Second Edition. TRB, Washington D.C

Klopfenstein, R., (1986). "Bus Communication System”. Transportation Research Board, National Research Council. Washington D.C.

Koutsopoulos, H.N, A.R. Odoni, and N.H.M Wilson, (1985). Determination of Headway as Function Time varying Characteristics on a Transit Network. Computer scheduling of Public Transport 2, Edited by Jean-Marc Rousseau, Elsevier Science Publishers B.V., 1985. pp. 391-413.

Kraft, W., and TH. Deutschman, (1974). Bus Passenger Service-time Distribution. Transportation Research Record, 625, pp.37-43.

Kwon, Coifman B., and Bickel P., (2000). Day to Day Travel Time Trends and Travel Time Prediction from Loop Detector Data. Transportation Research Record, 1717, pp. 120-128.

Leuzbatch, Wilhelm, (1988). Introduction to the Theory of Traffic Flow. Springer 
Levine, Jonathan C., and Gwo-Wei Torng, (1994). Dwell-time Effects of Low-Floor Bus Design. Journal of Transportation Engineering, Vol. 120, No.6, November, pp. 914-929.

Levinson, Herbert, (1983). Analyzing Transit Travel Time Performance. Transportation Research Record, 915, pp. 1-7.

Li, Weigang, Marlon W. Koendjbiharie, Ricardo C.deM.JuCa', Yaeko Yamashita, and Andrew Maciver, (?). Algorithms for Estimating Bus Arrival Times Using GPS Data. Retrieved from the Website

Linda, B., and Virginia C., (1992). Processing data-The survey Example. Series Quantitative Applications in Social Sciences, Sage University Paper 85.

Lewis P.A, and Orav. E.J.,(1980). Simulation Methodology for Statistic, Operation and Analysts, Engineers -Volume 1. Wadsworth \& Brooks/code Statistics/ Probability Series.

Lin, T., and N.H. Wilson, (1992). Dwell time Relationships for Light Rail Systems. Transportation Research Record, 1361, pp. 287-295.

Lin ,Wei Hua and Bertini Rober L., (2004). Modeling Schedule Recovery Process in Transit Operations for Bus Arrival Time Prediction. Journal of Advanced Transportation, Vol. 38, No.3, pp. 347-365.

Lin, Wei-Hua and Zeng Jian, (1999). Experimental Study of Real- Time Bus Arrival Time Prediction with GPS Data. Transportation Research Record, 1666, pp. 101109.

Loader, Clive, (1999). Local Regression and Likelihood. Statistics and Computing, Springer. 
Lyshevski, Sergey, (2003). Engineering and Scientific Computations Using MATLAB, John Wiley and Sons.

Maloney, Mark and Boyle Daniel, (1999). Components of Travel Time on the Glendale Beeline Bus Network. Transportation Research Record 1666, pp. 23-27.

Marshall, Leo F., Herbert S. Levison, Lawrence C. Lennon, and Jerry Cheng, (1990). Bus Service Times and Capacities in Manhattan. Transportation Research Record, 1266, pp. 189-196.

Maybeck, P.S.,(1979). Stochastic Models, Estimation, and Control. Volume 1. Academic Press.

McKnight, Claire, Herbert Levison, Kaan Ozbay, Cammile Kamga, and Rober E. Passwell, (2003). Impact of Congestion on Bus Operations and Costs. FHWA-NJ2003-008, Final Report.

McKnight C., and Passwell R.E, (1997). Impact of Congestion on New York Bus Service. UTRC for MTA NewYork City Transit.

Mei, Chen and Chien Steven I. J., (2001), Dynamic Freeway Travel-Time Prediction with Probe Vehicle Data. Transportation Research Record, 1768, pp. 157-161.

Menard, Scott, (2001). Applied Logistic Regression Analysis. $2^{\text {sd }}$ Edition, Sage University Paper. Series: Quantitative Applications in the Social Science.

Mishalani, Rabi G., Sungjoon, Lee and Mark, R. McCord, (2000). Evaluating RealTime Bus Arrival Information Systems. Transportation Research Record, 1731, pp. 81-87. 
Morgan, D., (2002). A Microscopic Simulation Laboratory for Advanced Public Transportation System Evaluation. Master Thesis Dissertation, MIT.

Neter J., Wasserman W., and Kutner M., (1985). Applied Linear Statistical Models. Second Edition, Richard D.IrWIN, Inc.

Normand R., Draper and Harry, Smith, (1998). Applied Regression Analysis. Wiley series in Probability and Statistics. Wiley Inter-science Publishers. Third Edition.

Okunieff, Paula E., (1997). AVL Systems for bus Transit. Synthesis of Transit Practice 24, TRR, National Academy Press, Washington D.C

Patnaik, J., Steven Chien, and Athanassios, Bladikas, (2004). Estimation of Bus Arrival Times Using APC Data. Journal of Public Transportation, Vol.7, No.1, pp. 1-19. Website: http://www.nctr.usf.edu/jpt/journalarticles.html

Pile, Randall, Jame Niemayer, and Rusell Chrishom, (1998). Transit Scheduling: Basic and Advanced Manuals. TCRP Report 30, TRB, Washington, DC.

Pedhazur, Elazar, (1997). Multiple Regression in Behavioral Research, Explanation and Prediction. Harcourt College Publishers, Third Edition.

PTV America, (2004). VISSIM User Manual. PTV Planung Transport Verkehr AG

PTV America, (2005). NEMA Editor Manual (Version3.3). PTV Planung Transport Verkehr AG

Rabi, Mishalani, Sungjoon Lee, and Mark R.McCord., (2000). Evaluating Real-time Bus Arrival Information Systems. Transportation Research Record 1731, pp. 8187. 
Rajbhandari Rajat, Chien Steven I., and Daniel Janice R., (2003). Estimation of Bus Dwell Times with Automatic Passenger Counter Information. Transportation Research Record, 1841, pp. 120-127.

Reinhoundt, Edwin M., and Veslatin S.A., (1997). A Dynamic Predicting Algorithm for Estimating Bus Arrival Time. Presents of the $8^{\text {th }}$ IFAC/IFIP/IFORS Symposium, Crete, Greece, June 1997. Vol3. pp. 1225-8.

Richard.P., and Dennis C.,(1984). Cross-validation of Regression Models. Journal of the American Statistical Association, Vol.79, No.387, pp.575-583.

Roess, Roger, Prassas Elena, and McShane William., (2004). Traffic Engineering, 3rd Edition, Prentice Hall.

Ruihong, Huang and Zhong-Ren Peng., (2002). Schedule-Based Path-Finding Algorithms for Transit Trip-Planning Systems. Transportation Research Record, 1783 , pp. $142-148$.

Schalkoff, Robert, (1992). Pattern Recognition, statistical, structural and neural Approaches. John Wiley \& Sons, Inc.

Schiavon, John, (1999). Understanding and Applying Advanced on Board Bus Electronics. TCRP Report 43. Transportation Research Board.

Schuldze, Thomas, and Fliees Thomas., (1997). Urban Traffic Simulation with Psychophysical Vehicle-Following Models. Proceedings of the 1997 Winter Simulation Conference.

Schweiger, L., (2003). Real-time Bus Arrival Information Systems, a Synthesis of Transit Practice. Transportation Research Board. Washington D.C 
Senevirante, P.N., (1988). Simulation of Fixed Bus Travel Time. Journal of Advanced Transportation, Vol. 22, pp. 39-53.

Senevirate, Prianka, (1990). Analysis of On-time Performance of Bus Services Using Simulation. Journal of Transportation Engineering, Vol.116, No.4, pp. 517-531.

Shalaby, A., Farhan Ali., (2004). Prediction Models of Bus Arrival and Departure Times Using AVL and APC Data. Journal of Public Transportation, Vol.7, No.1, pp. 41-60. Website: http://www.nctr.usf.edu/jpt/journalarticles.html

Shara R.J., (2002). Development of a Public Transit Information System Using GIS and ITS Technologies. Master Thesis Dissertation, Carleton University, OttawaCanada.

Silverman, B.W., (1986). Density Estimation for Statistic and Data Analysis. Monographs on Statistics and Applied Probability.

Skomal, Edward, (1981). Automatic Vehicle Locating System. Van Nostrand Reihold Company.

Smith, Brian L., and Michael J., Demetsky, (1994). Short-term Traffic Flow Prediction: Neural Network Approach. Transportation Research Record, 1453, pp. 98-104.

Son, Bongsoon, Kim Hyung Jin, Shin Chi huyn, and Lee sang keon, (2004). Intelligent Multimedia Solusion and security for the next generation Mobile Network-Bus Arrival Time Prediction Method for ITS Application. Lecture Notes in Computer Science. V.3215, pp.88-94. 
Stephan, R.S., Kenth A.B, and David W.S., (1994). Cross-validation of Multivariate Densities. Journal of the American Statistical Association, Vol.89, No.427, pp.807817.

Strathman, James G., Thomas J.Kimple, and Kentheth J. Dueker, (2000). Bus Transit Operations Control: Review and Experiment Involving Tri-Met's Automated Bus Dispatching System.

Sun, HongYu, Liu Henrry, Xiao Heng, He Rachel, and Ran Bin, (2003). Use of Local Linear Regression Model for Short-term Traffic Forecasting. Transportation Research Record, 1836, pp. 143-150.

Transportation research Board. Highway Capacity Manual. Special Report 209. TRB, National Research Council, Washington, D.C, 2000.

Transit Cooperative Research Program, (2001). Transit Capacity and Quality of Service Manual, Second Edition, TRB, Washington, DC.

Transit Cooperative Research Program, (2000). Strategies for Improved Traveler Information, TCRP Report 92, TRB, Washington, DC.

Transit Cooperative Research Program, (1998). Understanding and Applying Advanced On-Board Bus Electronics, Report 43, TRB, Washington, DC.

Turnquist, MA., (1982). Strategies for Improving Bud Transit Service Reliability. Evanston, IL: Northwestern University. (NTIS Report No. DOT/RSPA/DPB-50-8127).USDOT, Research and Special Programs Administration.

Ubitran and Levison, (1986). Bus Priority Improvement Study: Analysis Of bus Priority Proposal. November 1986 
Versteegrt, H.H, and Temprere C., MJ., (2003). PredicTime, a Stage of the Art and Functional Architecture. TNO- Inro Report 2003-07. Institute for Traffic and Transport, Logistic and Spatial Development, Nederland.

Vuchic, VuKan R., (2004). Urban Transit Operation, Planning, and Economics. John Willey and Sons, Inc.

Webb, Andrew, (2002). Statistical Pattern Recognition. John Wiley and Sons Ltd. Second Edition.

Wei, Hua Lin, Qingying Lu, and Joy Dahgren, (2002). Dynamic Procedure for ShortTerm Prediction of Traffic Conditions. Transportation Research Record, 1783, pp. 149-157.

Wei, Hua Lin, Amit Kulkarni, and Pitu Mirchandani, (2004). Short-term Arterial Travel Time Prediction for Advanced Traveler Information Systems. Intelligent Transportation System, Vol.8, pp. 143-154.

Weidemann, Reiter U., (1994). Micro-traffic simulation, the simulation system mission: Background and Actual State. Publication (no information).

Weigang, Li, Koendjbiharie Marlon, Ricardo C.de M.JuCa, Yaeko Yamashita, and Andrew Macive, (2004). Algorithm for Estimating Bus Arrival Times using GPS data. Retrieved from Website.

William, R. Schucany, (1995). Adaptive Bandwidth Choice for Kernel Regression. Journal of American Statistical Association, Vol.90, No.430, pp.535-540.

Zargari, S., (1997). Optimization of Integrated Multimodal Urban Transportation Corridors. Ph.D Thesis Dissertation, Carleton University, Ottawa, Canada. 
Zargari S., and Khan A.M., (2003). Optimization of Travel in Bus Rapid Transit-Based Multimodal Corridors. Journal of Public Transportation, Vol.6, No.2, pp.87-104.

Zhang, Wenyang and Lee Sik-Yum, (2000). Variable Bandwidth Selection in VaryingCoefficient Models. Journal of Multivariate Analysis, Vol. 74, pp.116-134.

Zhong, Ren Peng, Danlin Yu, and Edward Beimborn., (2002). Transit User Perceptions of the Benefits of Automatic Vehicle Location. Transportation Research Record, 1791, pp. 127-133.

Zong, Z., Tian, Thomas U., Roelof E., and Kevin B., (2002). Variations in Capacity and Delay Estimates from Microscopic Traffic Simulation Models. Transportation Research Record, 1802, pp. 23-31.

Yoon, Paul and Hwang, Ching-lai, (1995). Multiple Attribute Decision Making. An Introduction series Quantitative Applications in Social Sciences, Sage University Paper 104. 


\section{APPENDICIES}




\section{General}

\section{Appendix A}

This appendix shows the maps of bus route 95 and route 1 . The maps were retrieved from the Website www.octranspo.com

\section{Bus Route Maps}

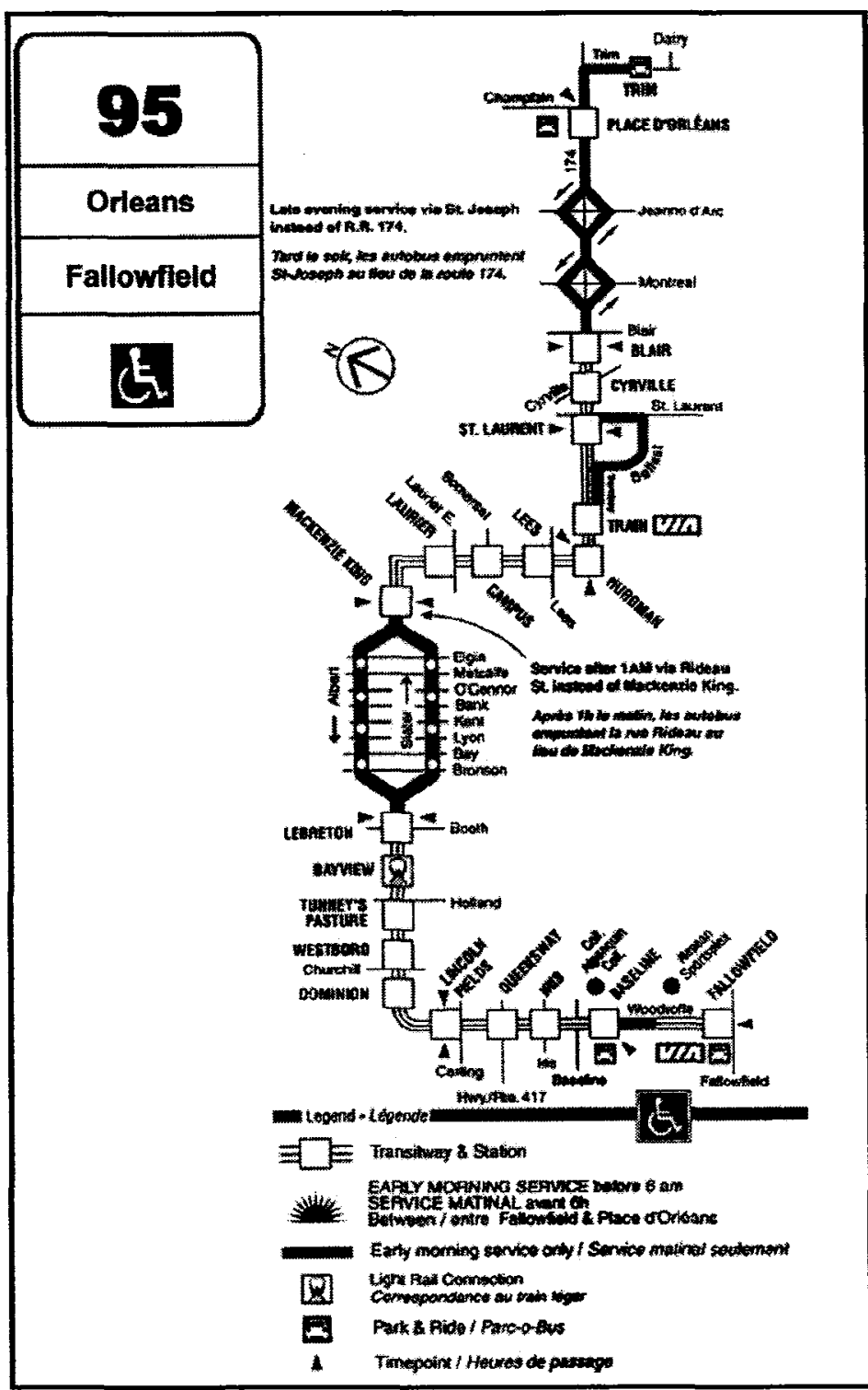

Figure A.1: Bus route 95 


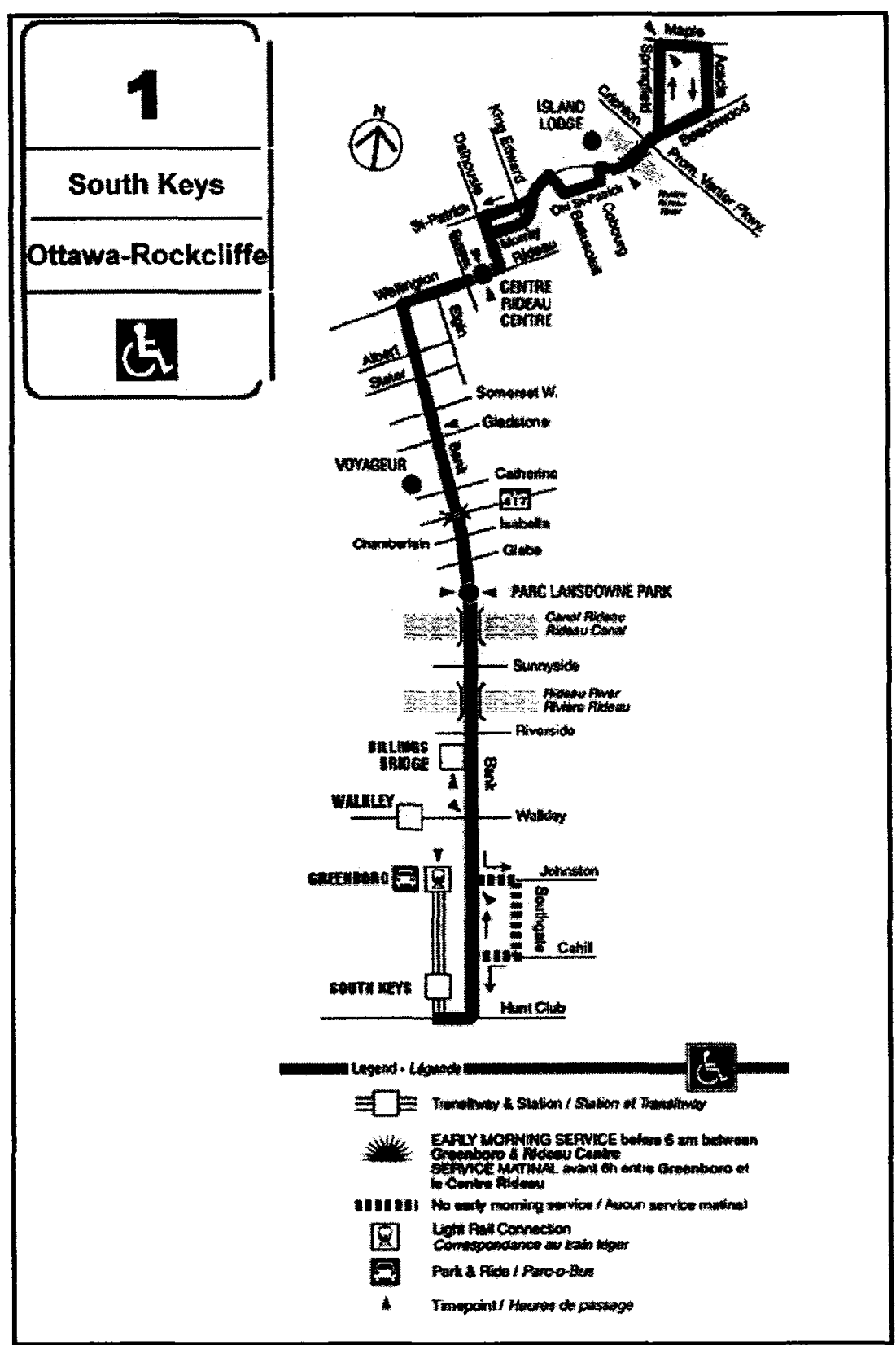

Figure A.2: Bus route 1 


\section{Appendix B1}

\section{General}

This Appendix covers the results of six regression types described in chapter 5 .

\section{SPSS results for Classical Regression Types}

Table B1:1 Model Summary for Type A-2.1

\begin{tabular}{|c|c|c|c|c|c|}
\hline \multicolumn{6}{|c|}{ Model Summary" } \\
\hline Model & $\mathbf{R}$ & R Square & $\begin{array}{l}\text { Adjusted } \\
\text { R Square }\end{array}$ & $\begin{array}{l}\text { Std. Error of } \\
\text { the Estimate }\end{array}$ & $\begin{array}{l}\text { Durbin- } \\
\text { Watson }\end{array}$ \\
\hline 1 & $.781^{a}$ & .610 & .609 & 7.35328 & \\
\hline 2 & $.781^{\mathrm{b}}$ & .610 & .609 & 7.35280 & \\
\hline 3 & $.781^{c}$ & .610 & .609 & 7.35339 & 1.800 \\
\hline \multicolumn{6}{|c|}{$\begin{array}{l}\text { a. Predictors: (Constant), DOORS, SEASON, PUNT, TotalOnPw, TOTAL_ } \\
\text { OFFS, STOP_LOCATE, LF, TOTAL_ONS, LOAD_ARR }\end{array}$} \\
\hline \multicolumn{6}{|c|}{$\begin{array}{l}\text { b. Predictors: (Constant), DOORS, SEASON, PUNT, TotalOnPw, TOTAL_ } \\
\text { OFFS, STOP_LOCATE, LF, TOTAL_ONS }\end{array}$} \\
\hline \multicolumn{6}{|c|}{$\begin{array}{l}\text { c. Predictors: (Constant), DOORS, PUNT, TotalOnPw, TOTAL_OFFS, } \\
\text { STOP_LOCATE, LF, TOTAL_ONS }\end{array}$} \\
\hline \multicolumn{6}{|c|}{ d. Dependent Variable: DWELL_3 } \\
\hline
\end{tabular}


Table B1.2: Parameters of Type A-2.1

\begin{tabular}{|c|c|c|c|c|c|c|c|c|}
\hline \multicolumn{9}{|c|}{ Coefficients } \\
\hline \multirow[b]{2}{*}{ Mod } & & \multicolumn{2}{|c|}{$\begin{array}{l}\text { Unstandardized } \\
\text { Coefficients }\end{array}$} & \multirow{2}{*}{$\begin{array}{c}\begin{array}{c}\text { Standardized } \\
\text { Coefficients }\end{array} \\
\text { Beta }\end{array}$} & \multirow[b]{2}{*}{$t$} & \multirow[b]{2}{*}{ Sig. } & \multicolumn{2}{|c|}{ Collinearity Statistics } \\
\hline & & B & Std. Error & & & & Tolerance & VIF \\
\hline \multirow[t]{10}{*}{1} & (Constant) & 13.346 & 1.070 & & 12.469 & .000 & & \\
\hline & TOTAL_OFFS & .438 & .015 & .250 & 28.700 & .000 & .843 & 1.186 \\
\hline & TotalOnPw & -.030 & .001 & -.413 & -20.587 & .000 & .159 & 6.287 \\
\hline & TOTAL_ONS & 1.928 & .037 & 1.054 & 51.417 & .000 & 152 & 6.558 \\
\hline & LOAD_ARR & .012 & .027 & .019 & .450 & .653 & .034 & 29.251 \\
\hline & LF & -1.532 & 1.575 & -.039 & -.973 & .331 & .040 & 25.161 \\
\hline & PUNT & .003 & .001 & .048 & 5.441 & .000 & .840 & 1.191 \\
\hline & SEASON & .118 & .084 & .011 & 1.414 & .158 & .990 & 1.010 \\
\hline & STOP_LOCATE & 1.975 & .206 & .083 & 9.573 & .000 & .846 & 1.182 \\
\hline & DOORS & -4.050 & .399 & -.150 & -10.152 & .000 & .295 & 3.386 \\
\hline \multirow[t]{9}{*}{2} & (Constant) & 12.961 & .645 & & 20.095 & .000 & & \\
\hline & TOTAL_OFFS & .439 & .015 & .251 & 28.871 & .000 & .851 & 1.176 \\
\hline & TotalonPw & -.030 & .001 & -.414 & .20 .673 & .000 & .160 & 6.255 \\
\hline & TOTAL_ONS & 1.929 & .037 & 1.055 & 51.657 & .000 & .154 & 6.508 \\
\hline & $\mathbf{L F}$ & -.840 & .344 & -.021 & -2.443 & .015 & .833 & 1.200 \\
\hline & PUNT & .003 & .001 & .047 & 5.437 & .000 & .840 & 4.191 \\
\hline & SEASON & .118 & .084 & .011 & 1.410 & .158 & .990 & 1.010 \\
\hline & STOP_LOCATE & 1.956 & .202 & .083 & 9.682 & .000 & .882 & 1.134 \\
\hline & DOORS & -3.902 & .227 & -.144 & -17.219 & .000 & .915 & 1.093 \\
\hline \multirow[t]{8}{*}{3} & (Constant) & 13.177 & .627 & & 21.024 & .000 & & \\
\hline & TOTAL_OFFS & .438 & .015 & .250 & 28.834 & .000 & .853 & 1.173 \\
\hline & TotalonPw & -.030 & .001 & -.413 & -20.636 & .000 & .160 & 6.248 \\
\hline & TOTAL_ONS & 1.928 & .037 & 1.054 & 51.635 & .000 & .154 & 6.500 \\
\hline & $\mathbf{L F}$ & -.830 & .344 & -.021 & -2.414 & .016 & .834 & 1.199 \\
\hline & PUNT & .003 & .001 & .048 & 5.456 & .000 & .840 & 1.191 \\
\hline & STOP_LOCATE & 1.957 & .202 & .083 & 9.684 & .000 & .882 & 4.134 \\
\hline & DOORS & -3.922 & .226 & -.145 & -17.342 & .000 & .919 & 1.089 \\
\hline
\end{tabular}

Table B1.3: Model Summary for Type A-2.2

\begin{tabular}{|l|r|r|r|r|r|}
\hline \multicolumn{7}{|c|}{ Model Summary } \\
\hline Model & \multicolumn{1}{|c|}{ R } & R Square & $\begin{array}{c}\text { Adjusted } \\
\text { R Square }\end{array}$ & $\begin{array}{c}\text { Std. Error of } \\
\text { the Estimate }\end{array}$ & $\begin{array}{c}\text { Durbin- } \\
\text { Watson }\end{array}$ \\
\hline 1 & $.786^{\mathrm{a}}$ & .618 & .618 & 7.26409 & \\
2 & $.786^{\mathrm{b}}$ & .618 & .618 & 7.26403 & 1.843 \\
\hline
\end{tabular}

a.

Predictors: (Constant), TotalOnCubic, PUNT, SEASON, DOORS, TOTAL_ OFFS, STOP_LOCATE, LF, TOTAL_ONS, LOAD_ARR, TotaIONPW

b. Predictors: (Constant), TotalOnCubic, PUNT, SEASON, DOORS, TOTAL_ OFFS, STOP_LOCATE, LF, TOTAL_ONS, TOtaIOnPw

c. Dependent Variable: DWELL_3 
Table B1.4: Parameters of Type A-2.2

\begin{tabular}{|c|c|c|c|c|c|c|c|c|}
\hline \multicolumn{9}{|c|}{ Coefficients ${ }^{\mathrm{a}}$} \\
\hline \multirow[b]{2}{*}{ Modr } & & \multicolumn{2}{|c|}{$\begin{array}{l}\text { Unstandardized } \\
\text { Coefficients }\end{array}$} & \multirow{2}{*}{$\begin{array}{c}\text { Standardized } \\
\text { Coefficients }\end{array}$} & \multirow[b]{2}{*}{$t$} & \multirow[b]{2}{*}{ Sig. } & \multicolumn{2}{|c|}{ Collinearity Statistics } \\
\hline & & B & Std. Error & & & & Tolerance & VIF \\
\hline 1 & (Constant) & 13.664 & 1.075 & & 12.706 & .000 & & \\
\hline & TOTAL_OFFS & .427 & .015 & .243 & 27.949 & .000 & .834 & 1.200 \\
\hline & TotalOnPw & -.071 & .006 & -.964 & -11.837 & .000 & .010 & 104.957 \\
\hline & TOTAL_ONS & 2.347 & .070 & 1.288 & 33.545 & .000 & .043 & 23.319 \\
\hline & LOAD_ARR & .026 & .027 & .041 & .956 & .339 & .034 & 29.035 \\
\hline & LF & -2.415 & 1.566 & -.061 & -1.542 & .123 & .040 & 25.093 \\
\hline & PUNT & .003 & .001 & .048 & 5.485 & .000 & .831 & 1.203 \\
\hline & SEASON & .153 & .084 & .015 & 1.824 & .068 & .985 & 1.015 \\
\hline & STOP_LOCATE & 1.848 & .205 & .078 & 8.995 & .000 & .839 & 1.192 \\
\hline & DOORS & -4.387 & .399 & -.161 & -10.989 & .000 & .295 & 3.392 \\
\hline & TotalOnCubic & .001 & .000 & .361 & 7.034 & .000 & .024 & 41.712 \\
\hline 2 & (Constant) & 12.843 & .649 & & 19.804 & .000 & & \\
\hline & TOTAL_OFFS & .429 & .015 & .244 & 28.173 & .000 & .841 & 1.189 \\
\hline & TotalOnPw & -.071 & .006 & -.966 & -11.873 & .000 & .010 & 104.851 \\
\hline & TOTAL_ONS & 2.351 & .070 & 1.290 & 33.657 & .000 & .043 & 23.241 \\
\hline & LF & -.954 & .344 & -.024 & -2.771 & .006 & .823 & 1.214 \\
\hline & PUNT & .003 & .001 & .048 & 5.490 & .000 & .831 & 1.203 \\
\hline & SEASON & .153 & .084 & .015 & 1.821 & .069 & .985 & 1.015 \\
\hline & STOP_LOCATE & 1.808 & .201 & .076 & 8.987 & .000 & .874 & 1.144 \\
\hline & DOORS & -4.073 & .227 & -149 & -17.953 & .000 & .913 & 1.095 \\
\hline & TotalOnCubic & .001 & .000 & .362 & 7.050 & .000 & .024 & 41.700 \\
\hline
\end{tabular}

Table B1.5: Parameters of Type A-2.4

\begin{tabular}{|l|r|r|r|r|}
\hline \multicolumn{9}{|c|}{ Parameter Estimates } \\
\hline & & & \multicolumn{2}{|c|}{$95 \%$ Confidence Interval } \\
\cline { 4 - 6 } Parameter & Estimate & Std. Error & Lower Bound & Upper Bound \\
\hline b1 & 31.287 & .384 & 30.534 & 32.039 \\
b2 & .346 & .014 & .319 & .374 \\
b3 & .795 & .005 & .784 & .805 \\
\hline
\end{tabular}


Table B1.6: Model Summary for Type A-2.4

\begin{tabular}{|c|c|c|c|}
\hline \multicolumn{4}{|c|}{ ANOVA $^{a}$} \\
\hline Source & $\begin{array}{l}\text { Sum of } \\
\text { Squares }\end{array}$ & df & $\begin{array}{c}\text { Mean } \\
\text { Squares }\end{array}$ \\
\hline Regression & 1627451 & 3 & 542483.7 \\
\hline Residual & 405012.0 & 6032 & 67.144 \\
\hline Uncorrected Total & 2032463 & 6035 & \\
\hline Corrected Total & 813235.7 & 6034 & \\
\hline \multicolumn{4}{|c|}{ Dependent variable: DWELL_3 } \\
\hline \multicolumn{4}{|c|}{$\begin{array}{l}\text { a. R squared }=1 \text { - (Residual Sum of Squares) } / \\
\text { (Corrected Sum of Squares) }=.502 .\end{array}$} \\
\hline
\end{tabular}

Table B1.7: Parameters of Type A-2.5

\begin{tabular}{|l|r|r|r|r|}
\hline \multicolumn{5}{|c|}{ Parameter Estimates } \\
\hline & & & \multicolumn{2}{|c|}{$95 \%$ Confidence Interval } \\
\cline { 3 - 5 } Parameter & Estimate & Std. Error & Lower Bound & Upper Bound \\
\hline b1 & 1184.802 & 1268661 & -2485838.131 & 2488207.734 \\
b2 & 91.856 & 998524.5 & -1957368.933 & 1957552.645 \\
b3 & -2.557 & 9811.205 & -19235.985 & 19230.870 \\
b4 & -.003 & 7.131 & -13.981 & 13.976 \\
\hline
\end{tabular}

Table B1.8: Model Summary for Type A-2.5

\begin{tabular}{|l|c|r|r|}
\hline \multicolumn{4}{|c|}{ ANOVA $^{\mathrm{a}}$} \\
\hline Source & Sum of & Mean \\
\hline Squares & df & Squares \\
Regression & 1643479 & 4 & 410869.8 \\
Uncorrected Total & 441527.8 & 6095 & 72.441 \\
Corrected Total & 2085007 & 6099 & \\
Dependent variable: DWELL_3 & 843898.3 & 6098 & \\
a. R squared = 1 - (Residual Sum of Squares) / \\
$\quad$ (Corrected Sum of Squares) = .477. \\
\hline
\end{tabular}


Table B1.9: Parameters of Type A-2.6

\begin{tabular}{|l|r|r|r|r|}
\hline \multicolumn{6}{|c|}{ Parameter Estimates } \\
\hline & & & \multicolumn{2}{|c|}{$95 \%$ Confidence Interval } \\
\cline { 4 - 6 } Parameter & Estimate & Std. Error & Lower Bound & Upper Bound \\
\hline b1 & -1.496 & 1.613 & -4.658 & 1.666 \\
b2 & 97.767 & 234.859 & -362.640 & 558.175 \\
b3 & 470.907 & 1253.074 & -1985.570 & 2927.383 \\
b4 & .561 & .117 & .332 & .790 \\
\hline
\end{tabular}

Table B1.10: Model Summary for Type A-2.6

\begin{tabular}{|c|c|c|c|}
\hline \multicolumn{4}{|c|}{ ANOVAa } \\
\hline Source & $\begin{array}{l}\text { Sum of } \\
\text { Squares }\end{array}$ & df & $\begin{array}{l}\text { Mean } \\
\text { Squares }\end{array}$ \\
\hline Regression & 1643120 & 4 & 410779.9 \\
\hline Residual & 387654.5 & 5996 & 64.652 \\
\hline Uncorrected Total & 2030774 & 6000 & \\
\hline Corrected Total & 819684.1 & 5999 & \\
\hline \multicolumn{4}{|c|}{ Dependent variable: DWELL_3 } \\
\hline \multicolumn{4}{|c|}{$\begin{array}{l}\text { a. } R \text { squared }=1-\text { (Residual Sum of Squares) } / \\
\text { (Corrected Sum of Squares) }=.527 \text {. }\end{array}$} \\
\hline
\end{tabular}

Table B1.11: Model Summary for Pilot Regression without Deletion of Outlier and Extreme values

\begin{tabular}{|c|c|c|c|c|c|c|c|c|c|c|}
\hline \multirow[b]{3}{*}{ Model } & \multirow[b]{3}{*}{$\mathbf{R}$} & \multirow[b]{3}{*}{ R Square } & \multirow[b]{3}{*}{$\begin{array}{l}\text { Adjusted } \\
\text { R Square }\end{array}$} & \multirow[b]{3}{*}{$\begin{array}{l}\text { Std. Error of } \\
\text { the Estimate }\end{array}$} & \multicolumn{5}{|c|}{ Model Summarf } & \multirow[b]{3}{*}{$\begin{array}{l}\text { Durbin- } \\
\text { Watson }\end{array}$} \\
\hline & & & & & \multicolumn{5}{|c|}{ Change Statistics } & \\
\hline & & & & & $\begin{array}{c}\text { R Square } \\
\text { Change }\end{array}$ & F Change & df1 & $d f 2$ & Sig. F Change & \\
\hline 1 & $.608^{\mathrm{a}}$ & .370 & .364 & 15.99027 & .370 & 65.929 & 8 & 898 & .000 & \multirow[b]{3}{*}{2.041} \\
\hline 2 & $.608^{b}$ & .370 & .365 & 15.98183 & .000 & .052 & 1 & 898 & .820 & \\
\hline 3 & $.607^{c}$ & .369 & .365 & 15.98773 & -.001 & 1.664 & 1 & 899 & .197 & \\
\hline \multicolumn{11}{|c|}{ a. Predictors: (Constant), BUS_TYPE, STOP_LOCATE, SEASON, TOTAL_ONS, PUNT, LF, TOTAL_OFFS, LOAD_DEP } \\
\hline \multicolumn{11}{|c|}{ b. Predictors: (Constant), BUS_TYPE, STOP_LOCATE, SEASON, TOTAL_ONS, LF, TOTAL_OFFS, LOAD_DEP } \\
\hline \multicolumn{11}{|c|}{ c. Predictors: (Constant), BUS_TYPE, STOP_LOCATE, TOTAL_ONS, LF, TOTAL_OFFS, LOAD_DEP } \\
\hline \multicolumn{11}{|c|}{ d. Dependent Variable: DWELL_1 } \\
\hline
\end{tabular}


Table B1.12: Coefficients for the Pilot Regression

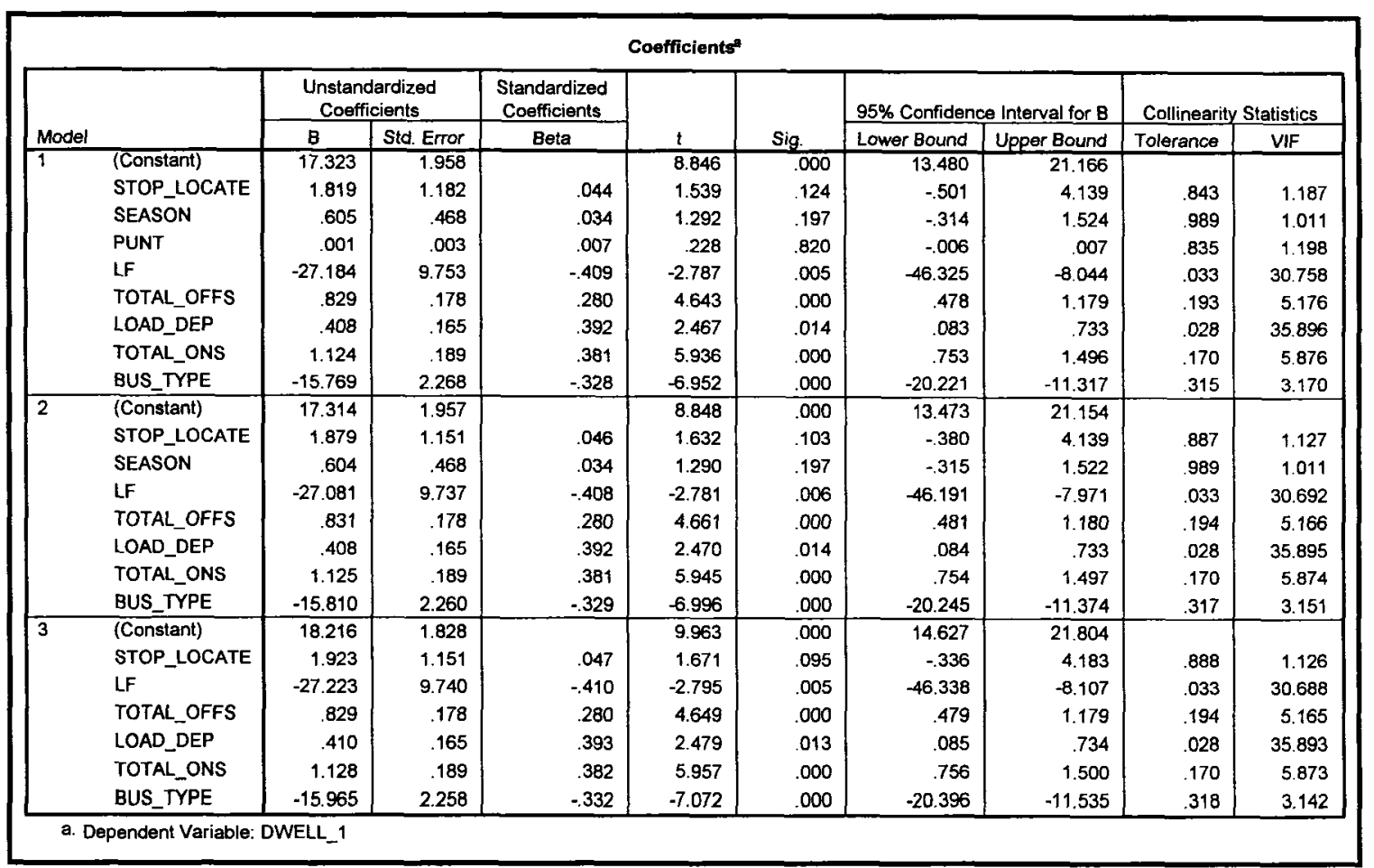




\section{Appendix B2}

\section{General}

This appendix presents the outputs of SPSS 13 applied for three types of binary logistic regressions. Each type has three tables recording the model summary, the omnibus tests, and model parameters.

\section{SPSS Results for Binary Logistic Regression}

Table B2.1: Model Summary for Type B-L.1

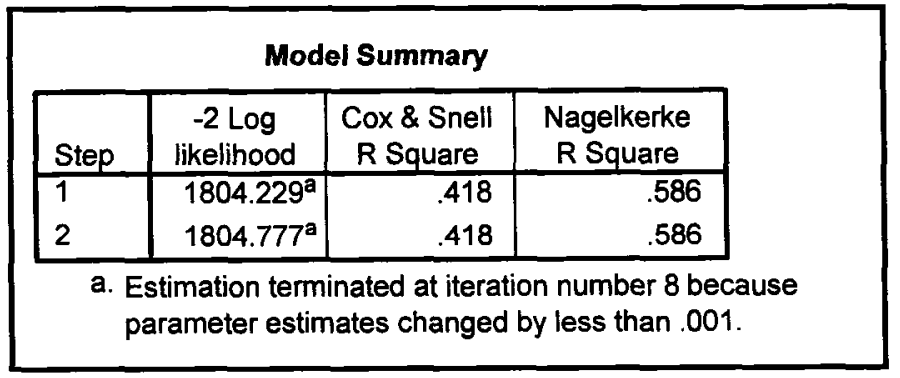

Table B2.2: Omnibus Tests of Model Coefficients of Type B-L.1

\begin{tabular}{|c|c|c|c|c|}
\hline \multicolumn{5}{|c|}{ Omnibus Tests of Model Coefficients } \\
\hline & & Chi-square & df & Sig. \\
\hline \multirow[t]{3}{*}{ Step 1} & Step & 1379.104 & 10 & .000 \\
\hline & Block & 1379.104 & 10 & .000 \\
\hline & Model & 1379.104 & 10 & .000 \\
\hline \multirow[t]{3}{*}{ Step $2^{a}$} & Step & -.548 & 2 & .760 \\
\hline & Block & 1378.557 & 8 & .000 \\
\hline & Model & 1378.557 & 8 & .000 \\
\hline \multicolumn{5}{|c|}{$\begin{array}{l}\text { A. A negative Chi-squares value indicates that the } \\
\text { Chi-squares value has decreased from the } \\
\text { previous step. }\end{array}$} \\
\hline
\end{tabular}


Table B2.3: Model Parameters of Type B-L.1

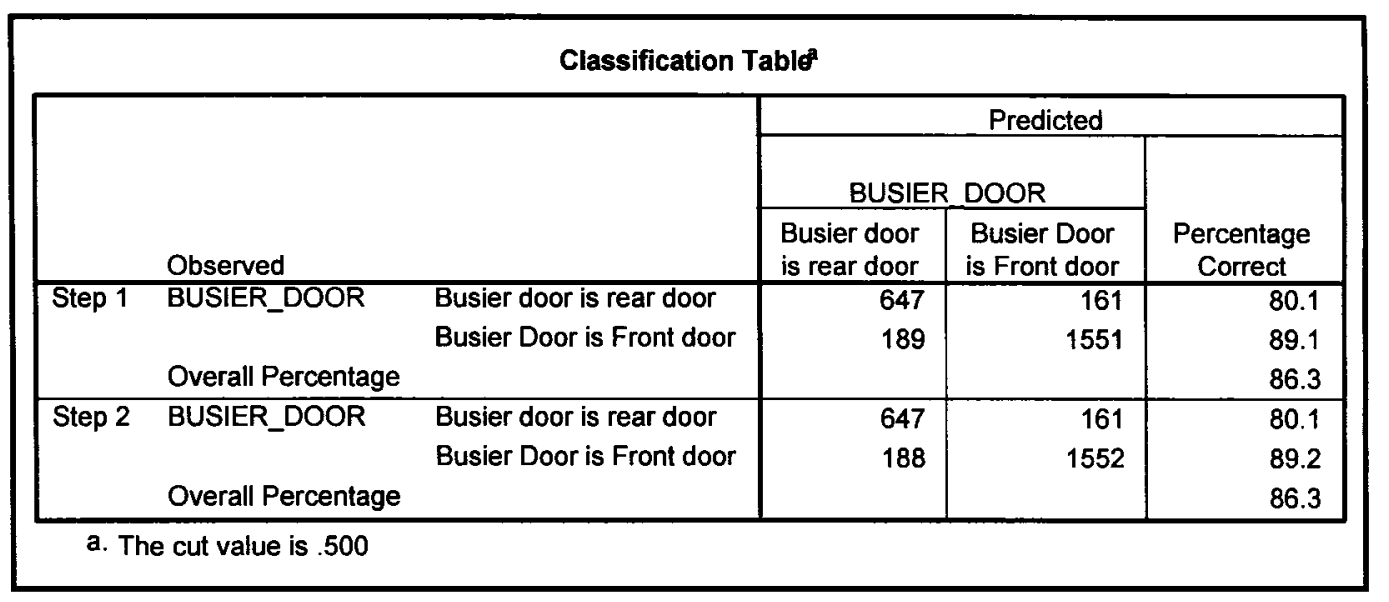

Table B2.4: Model Summary for Type B-L.2

\begin{tabular}{|c|c|c|c|}
\hline \multicolumn{4}{|c|}{ Model Summary } \\
\hline Step & $\begin{array}{l}-2 \text { Log } \\
\text { likelihood }\end{array}$ & $\begin{array}{l}\text { Cox \& Snell } \\
\text { R Square }\end{array}$ & $\begin{array}{l}\text { Nagelkerke } \\
\text { R Square }\end{array}$ \\
\hline 1 & $942.654^{\mathrm{a}}$ & .262 & .473 \\
\hline 2 & $942.743^{a}$ & .262 & .473 \\
\hline 3 & $944.813^{b}$ & .261 & .471 \\
\hline 4 & $948.127^{c}$ & .260 & .469 \\
\hline 5 & $948.378^{d}$ & .260 & .469 \\
\hline \multicolumn{4}{|c|}{$\begin{array}{l}\text { a. Estimation terminated at iteration number } 15 \text { because } \\
\text { parameter estimates changed by less than } .001 \text {. }\end{array}$} \\
\hline \multicolumn{4}{|c|}{$\begin{array}{l}\text { b. Estimation terminated at iteration number } 16 \text { because } \\
\text { parameter estimates changed by less than } .001 \text {. }\end{array}$} \\
\hline \multicolumn{4}{|c|}{$\begin{array}{l}\text { c. Estimation terminated at iteration number } 10 \text { because } \\
\text { parameter estimates changed by less than } .001 \text {. }\end{array}$} \\
\hline \multicolumn{4}{|c|}{$\begin{array}{l}\text { d. Estimation terminated at iteration number } 8 \text { because } \\
\text { parameter estimates changed by less than } .001 \text {. }\end{array}$} \\
\hline
\end{tabular}


Table B2.5: Model Parameters of Type B-L.2

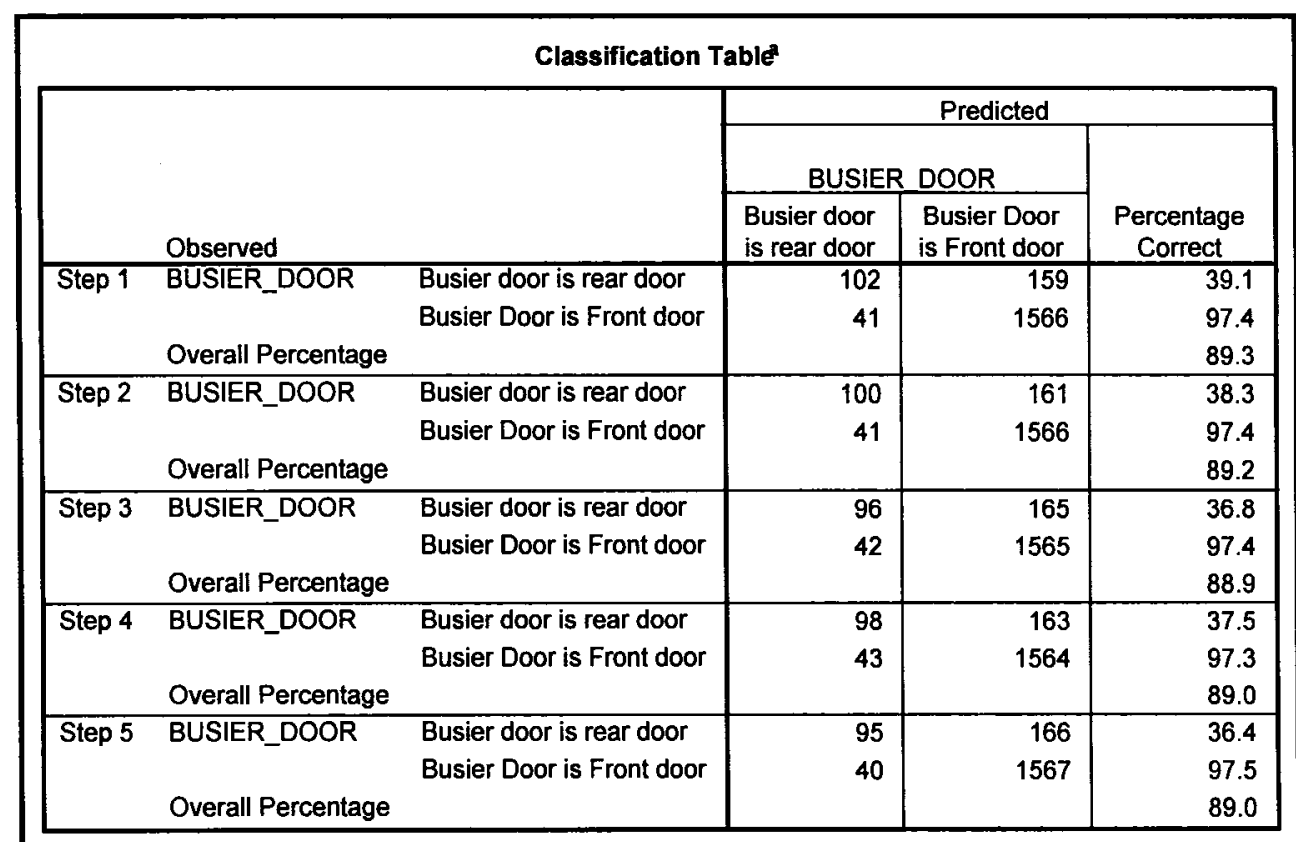

a. The cut value is .500

Table B2.6: Omnibus Tests of Model Coefficients of Type B-L.2

\begin{tabular}{|c|c|c|c|c|}
\hline \multicolumn{5}{|c|}{ Omnibus Tests of Model Coefficients } \\
\hline & & Chi-square & df & Sig. \\
\hline \multirow[t]{3}{*}{ Step 1} & Step & 568.401 & 12 & .000 \\
\hline & Block & 568.401 & 12 & .000 \\
\hline & Model & 568.401 & 12 & .000 \\
\hline \multirow[t]{3}{*}{ Step $2^{a}$} & Step & -.090 & 1 & .765 \\
\hline & Block & 568.311 & 11 & .000 \\
\hline & Model & 568.311 & 11 & .000 \\
\hline \multirow[t]{3}{*}{ Step $¥$} & Step & -2.070 & 2 & .355 \\
\hline & Block & 566.241 & 9 & .000 \\
\hline & Model & 566.241 & 9 & .000 \\
\hline \multirow[t]{3}{*}{ Step $4^{9}$} & Step & -3.314 & 3 & .346 \\
\hline & Block & 562.928 & 6 & .000 \\
\hline & Model & 562.928 & 6 & .000 \\
\hline \multirow[t]{3}{*}{ Step $5^{5}$} & Step & -.251 & 1 & .617 \\
\hline & Block & 562.677 & 5 & .000 \\
\hline & Model & 562.677 & 5 & .000 \\
\hline \multicolumn{5}{|c|}{$\begin{array}{l}\text { a. A negative Chi-squares value indicates that the } \\
\text { Chi-squares value has decreased from the } \\
\text { previous step. }\end{array}$} \\
\hline
\end{tabular}




\section{Appendix B3}

\section{General}

This appendix illustrate the outputs of the SPSS applied for Multinomial Logistic

Regression models proposed in chapter 5, section 5.3.6.2

\section{SPPSS Results for Multinomial Logistic Regression}

Table B3.1: Goodness-of-Fit for Type B-ML.1

\begin{tabular}{|l|c|c|c|}
\hline \multicolumn{4}{|c|}{ Goodness-of-Fit } \\
\hline & Chi-Square & df & \multicolumn{1}{|c|}{ Sig. } \\
\hline Pearson & 21001.411 & 12222 & .000 \\
Deviance & 10360.485 & 12222 & 1.000 \\
\hline
\end{tabular}

Table B3.2: Pseudo R-square for Type B-ML.1

\begin{tabular}{|l|r|}
\hline \multicolumn{2}{|c|}{ Pseudo R-Square } \\
\hline Cox and Snell & .333 \\
Nagelkerke & .379 \\
McFadden & .193 \\
\hline
\end{tabular}


Table B3.3: Model Parameters for Type B-ML.1

\begin{tabular}{|c|c|c|c|c|c|c|c|c|c|}
\hline \multicolumn{10}{|c|}{ Parameter Estimates } \\
\hline & & \multirow[b]{2}{*}{ B } & \multirow[b]{2}{*}{ Std. Error } & \multirow[b]{2}{*}{ Wald } & \multirow[b]{2}{*}{$d f$} & \multirow[b]{2}{*}{ Sig. } & \multirow[b]{2}{*}{$\operatorname{Exp}(B)$} & \multicolumn{2}{|c|}{$\begin{array}{c}95 \% \text { Confidence Interval for } \\
\text { Exp(B) }\end{array}$} \\
\hline \multicolumn{2}{|l|}{ Busiest_door ${ }^{\mathrm{B}}$} & & & & & & & Lower Bound & Upper Bound \\
\hline \multirow[t]{8}{*}{ door 2 busiest } & Intercept & -.133 & .123 & 1.172 & 7 & .279 & & & \\
\hline & $\begin{array}{l}\text { LOAD_ARR } \\
\text { LF }\end{array}$ & $\begin{array}{r}.004 \\
0^{\mathrm{b}}\end{array}$ & .002 & 2.911 & 1 & .088 & 1.004 & .999 & 1.008 \\
\hline & PUNT & .000 & .000 & .005 & 1 & .945 & 1.000 & 1.000 & 1.000 \\
\hline & SEASON & -.022 & .033 & .416 & 1 & .519 & .979 & .917 & 1.045 \\
\hline & STOP_LOCATE & .057 & .077 & .549 & 1 & .459 & 1.058 & .911 & 1.230 \\
\hline & TIME & -.114 & .044 & 6.803 & 1 & .009 & .892 & .819 & .972 \\
\hline & TOTAL_ONS &. .001 & .005 & .025 & 1 & .874 & .999 & .988 & 1.010 \\
\hline & TOTAL_OFFS & -.023 & .004 & 36.024 & 1 & .000 & .978 & .970 & .985 \\
\hline \multirow[t]{8}{*}{ Door 1 busiest } & Intercept & 1.302 & .121 & 115.269 & 1 & .000 & & & \\
\hline & LOAD_ARR & -.006 & .002 & 7.351 & $\begin{array}{l}1 \\
0\end{array}$ & .007 & .994 & .990 & .998 \\
\hline & LF & $0_{000}^{0}$ & 000 & .502 & $\begin{array}{l}0 \\
1\end{array}$ & 479 & 1.000 & 1000 & \\
\hline & $\begin{array}{l}\text { PUNT } \\
\text { SEASON }\end{array}$ & $\begin{array}{l}.000 \\
.067\end{array}$ & .032 & $\begin{array}{r}.002 \\
4.414\end{array}$ & 1 & .036 & $\begin{array}{l}1.000 \\
1.069\end{array}$ & $\begin{array}{l}1.000 \\
1.005\end{array}$ & $\begin{array}{l}1.001 \\
1.139\end{array}$ \\
\hline & $\begin{array}{l}\text { SEASUN } \\
\text { STOP LOCATE }\end{array}$ & .501 & .075 & 53.817 & 1 & .000 & 1.732 & 1.496 & 2.006 \\
\hline & TIME $^{-}$ & -.105 & .042 & 6.284 & 1 & .012 & .900 & .829 & .977 \\
\hline & TOTAL_ONS & .098 & .006 & 269.578 & 1 & .000 & 1.103 & 1.090 & 1.116 \\
\hline & TOTAL_OFFS & -.308 & .010 & 1013.492 & 1 & .000 & .735 & .721 & .749 \\
\hline
\end{tabular}

Table B3.1: Goodness-of-Fit for Type B-ML.2

\begin{tabular}{|l|r|r|r|}
\hline \multicolumn{4}{|c|}{ Goodness-of-Fit } \\
\hline & Chi-Square & df & \multicolumn{1}{|c|}{ Sig. } \\
\hline Pearson & 23844.277 & 10336 & .000 \\
Deviance & 8352.103 & 10336 & 1.000 \\
\hline
\end{tabular}

Table B3.2: Pseudo R-square for Type B-ML.2

\begin{tabular}{|l|r|}
\hline \multicolumn{2}{|c|}{ Pseudo R-Square } \\
\hline Cox and Snell & .321 \\
Nagelkerke & .372 \\
McFadden & .194 \\
\hline
\end{tabular}


Table B3.3: Model Parameters of Type B-ML.2

\begin{tabular}{|c|c|c|c|c|c|c|c|c|c|}
\hline \multicolumn{10}{|c|}{ Parameter Estimates } \\
\hline \multirow[b]{2}{*}{ Busiest_door ${ }^{\mathrm{a}}$} & & \multirow[b]{2}{*}{ B } & \multirow[b]{2}{*}{ Std. Enror } & \multirow[b]{2}{*}{ Wald } & \multirow[b]{2}{*}{$d f$} & \multirow[b]{2}{*}{ Sig. } & \multirow[b]{2}{*}{$\operatorname{Exp}(B)$} & \multicolumn{2}{|c|}{$\begin{array}{c}95 \% \text { Confidence Interval for } \\
\text { Exp(B) }\end{array}$} \\
\hline & & & & & & & & Lower Bound & Upper Bound \\
\hline \multirow[t]{9}{*}{ door 2 busiest } & Intercept & -.228 & .161 & 2.020 & 1 & .155 & & & \\
\hline & $\begin{array}{l}\text { LOAD_ARR } \\
\text { LF }\end{array}$ & $\begin{array}{c}.003 \\
0^{\mathrm{b}}\end{array}$ & .003 & 1.510 & $\begin{array}{l}1 \\
0\end{array}$ & .219 & 1.003 & .998 & 1.008 \\
\hline & PUNT & .000 & .000 & .003 & 1 & .957 & 1.000 & .999 & 1.001 \\
\hline & SEASON & -.011 & .039 & .082 & 1 & .775 & .989 & .916 & 1.067 \\
\hline & STOP_LOCATE & .034 & .090 & .139 & 1 & .710 & 1.034 & .867 & 1.234 \\
\hline & TIME & -.104 & .051 & 4.142 & 1 & .042 & .902 & .816 & .996 \\
\hline & TOTAL_ONS & .016 & .016 & 1.004 & 1 & .316 & 1.016 & .985 & 1.048 \\
\hline & TOTAL_OFFS & -.023 & .006 & 14.870 & 1 & .000 & .978 & .967 & .989 \\
\hline & Total_On_squared & .000 & .001 & .863 & 1 & .353 & 1.000 & .999 & 1.001 \\
\hline \multirow[t]{9}{*}{ Door 1 busiest } & Intercept & 1.602 & .146 & 121.095 & 1 & .000 & & & \\
\hline & LOAD_ARR & $\begin{array}{c}-.013 \\
0^{\mathrm{b}}\end{array}$ & .002 & 30.584 & 1 & .000 & .987 & .983 & .992 \\
\hline & $\begin{array}{l}\text { LF } \\
\text { PUNT }\end{array}$ & $\begin{array}{r}0^{b} \\
.000\end{array}$ & .000 & .004 & $\begin{array}{l}0 \\
1\end{array}$ & .947 & 1.000 & .999 & 1.001 \\
\hline & SEASON & .122 & .035 & 12.121 & 1 & .000 & 1.130 & 1.055 & 1.210 \\
\hline & STOP_LOCATE & .659 & .084 & 61.675 & 1 & .000 & 1.933 & 1.640 & 2.279 \\
\hline & TIME & -.116 & .046 & 6.455 & 1 & .011 & .890 & .814 & .974 \\
\hline & TOTAL_ONS & .150 & .015 & 95.282 & 1 & .000 & 1.161 & 1.127 & 1.197 \\
\hline & TOTAL_OFFS & -.300 & .010 & 869.260 & 1 & .000 & .741 & .726 & .756 \\
\hline & Total_On_squared & -.003 & .001 & 38.672 & 1 & .000 & .997 & .996 & .998 \\
\hline
\end{tabular}




\section{Appendix C}

\section{General}

This Appendix presents the MatLab source codes for the developed model. It includes the source code for bus running time, and boarding and de-boarding passenger predictions.

\section{Bus running time function}

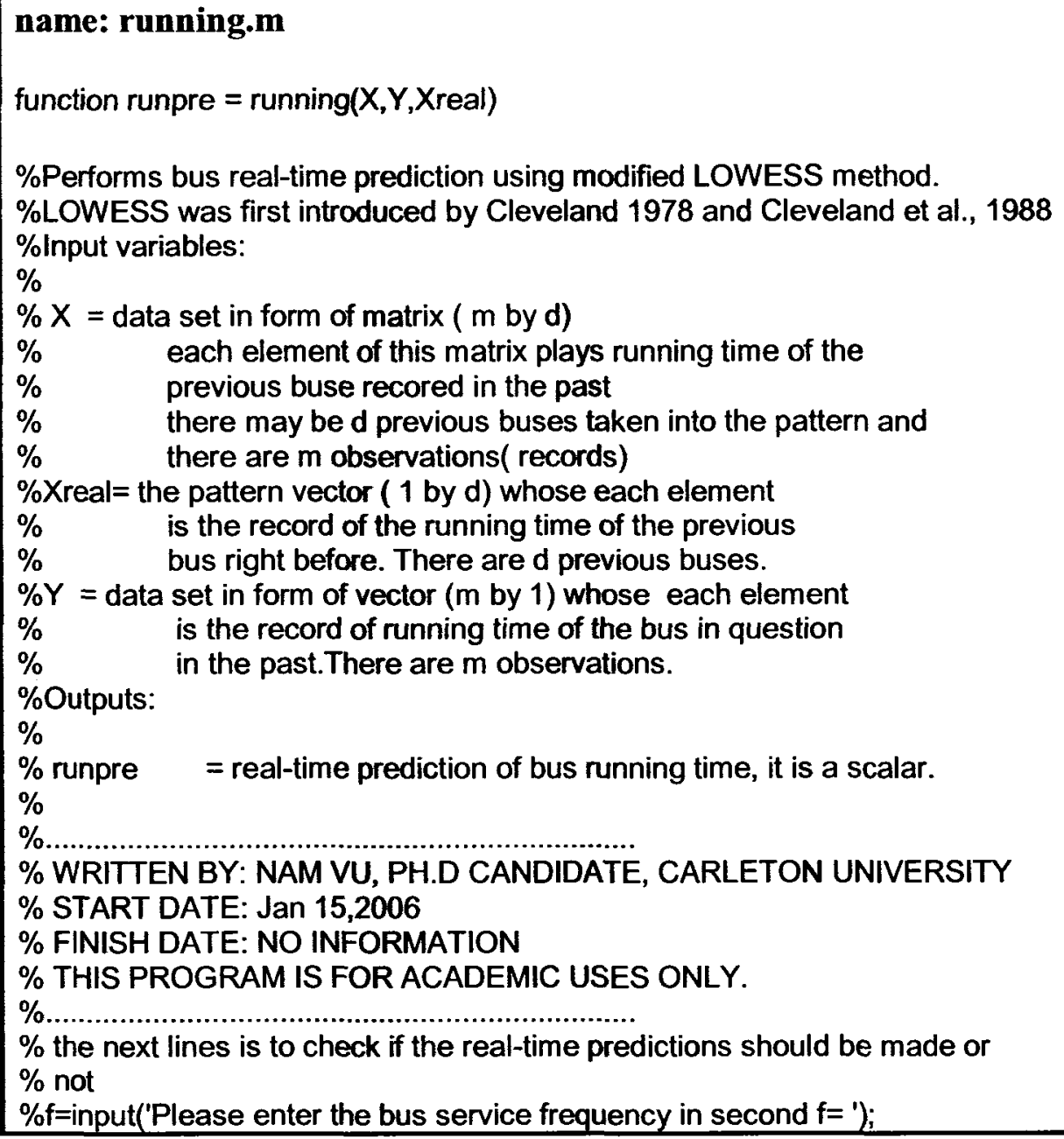


$\%$ first check for the prediction horizon (if $d$ smaller than 2 , there is no

$\%$ real-time prediction)

$\%$ if $f>1800$

$\%$ warning('the bus service frequency is too long');

$\%$ fprintf('Inl No real-time estimation being made');

$\%$ dum= input('InType any key to proceed');

$\%$ end

$\%$ clear f;

$\%$ NOW HERE IS THE MAIN PROGRAM

\%load Rpastpattern.dat;

$\%$ enter history data based on bus ID and service time

\%load Rpastdata.dat;

$\%$ enter history running times of the bus

\%load The Rcurrenpattern.dat

$\%$ the following lines is to find the optimal bandwidth $\mathrm{H}$ by applying

$\%$ leave-one-out method (see Wofgang 1996) and Nadaraya-Watson estimation

$\%$

$\%$ clear

$[\mathrm{m}, \mathrm{k}]=\operatorname{size}(\mathrm{X})$;

$\mathrm{hmin}=\operatorname{round}\left(0.1^{\star} \mathrm{m}\right)$;

$\mathrm{hmax}=\operatorname{round}\left(0.8^{\star} \mathrm{m}\right)$;

$\mathrm{s}=\operatorname{round}\left(0.1^{*} \mathrm{~m}\right)$;

for $h=h \min : \mathrm{s}: \mathrm{hmax}$

for $\mathrm{i}=1: 1: \mathrm{m}$

$Y$ test $=Y(i)$;

$Y_{-}$train $=Y$;

$Y$ train $(\mathrm{i})=\prod$;

test $1=X(i,:)$;

test_2=X;

test $2(i,:)=0 ; \%$ the training set after removing the ith row

for $n=1$ :size(test_2,1)

$d(n, 1)=$ sqrt(sum((test_2(n,:)-test_1(1,:)).^2));

end

disp(' The test set is')

disp(Y train);

disp( 'the Edist to the first second... neighbor')

$\operatorname{disp}\left(d^{\prime}\right)$;

$q=\operatorname{sort}(d)$;

bandwidth $=q(h) \%$ Select the $h$-nearest neighbours

$\mathrm{K} \_$weigh=d./bandwidth;

for $p=1$ :length(d)

if $K$ weigh $(p, 1)<1$

$w(\bar{p}, 1)=(70 / 81)^{ \pm}\left(1-\left(\operatorname{abs}\left(K \_w e i g h(p, 1)\right)^{\wedge} 3\right)^{\wedge} 3\right)$;

else

$w(p, 1)=0$;

end

end

w;

if $w==0$

warning('No neighbour has been found');

end

$y$ hat $=(Y$ train'* $w) /$ sum $(w)$; 


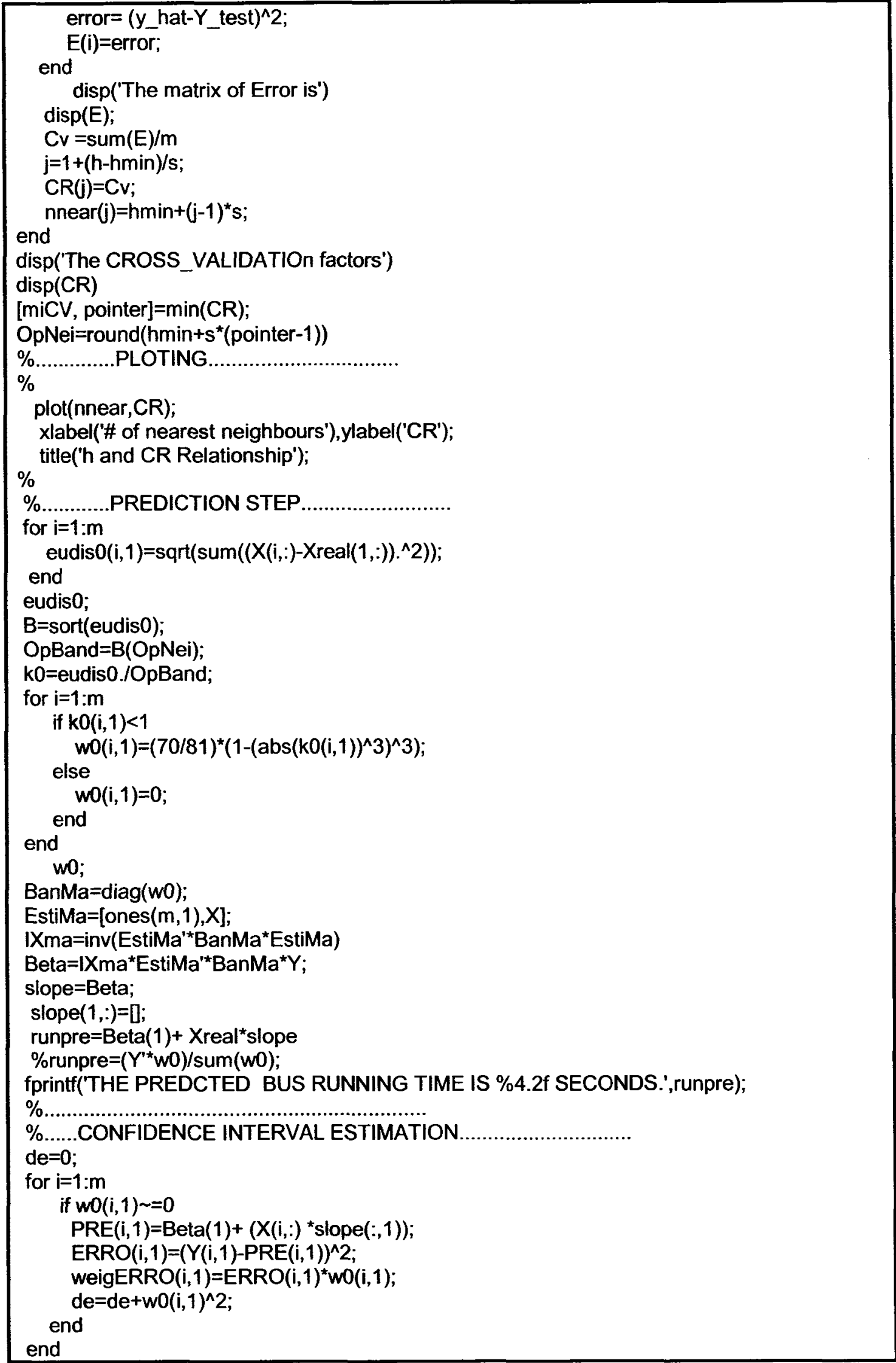


de $\%$ sum of the squared weights

PRE;\% Matrix of predicted values of neighbors around $x 0$

weigERRO; \% Matrix of weighted errors

MSE=sum(ERRO) \% Matrix of prediction error

ns=IXma*EstiMa"*BanMa*BanMa*EstiMa

us=EstiMa*BanMa*BanMa*EstiMa

$m s=1 /(\operatorname{trace}(\operatorname{BanMa})$-trace(ns))

sigma $=$ sum(weigERRO) ${ }^{\star} \mathrm{ms}$

bias $=(\mathrm{MSE}-$ sigma $) / \mathrm{OpNei}$

freDegree $=(\operatorname{sum}(w 0))^{\wedge} 2 / \mathrm{de}$

fprintf('the degree of freedom is \% $4.0 f$,freDegree)

critical=input('Please enter the confidence limit critical= ');

lower=runpre-abs(sqrt(bias))-critical'abs(sqrt(sigma))

upper=runpre-sqrt(bias)+critical ${ }^{*}$ sqrt(sigma)

fprintf('THE LOWER LIMIT IS \%4.2f seconds at 95 percent confidence level',lower);

fprintf('THE UPPER LIMIT IS \%4.2f seconds at 95 percent confidence level',upper);

fprintf('HAVE A NICE DAY')

\section{Bus boarder function}

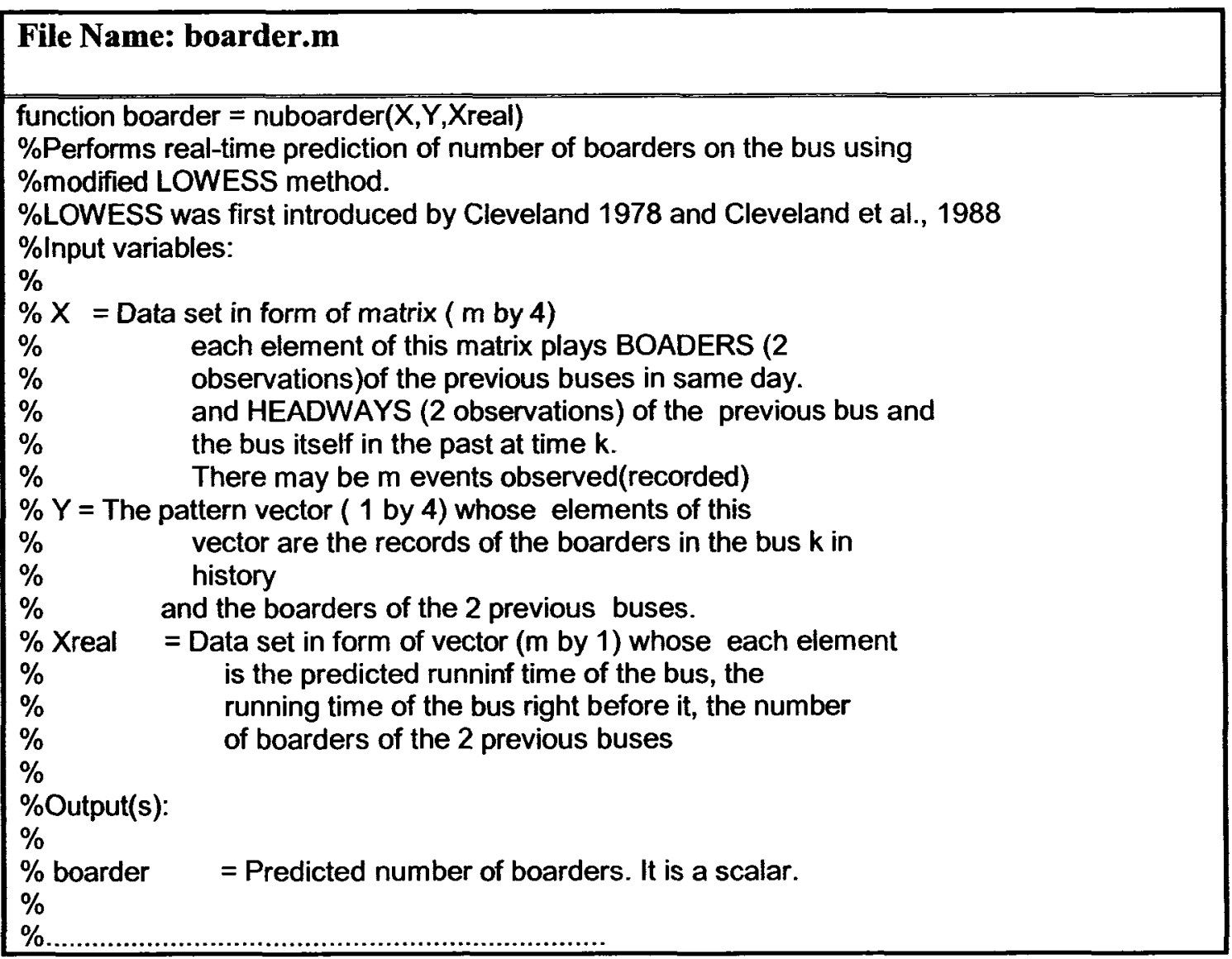




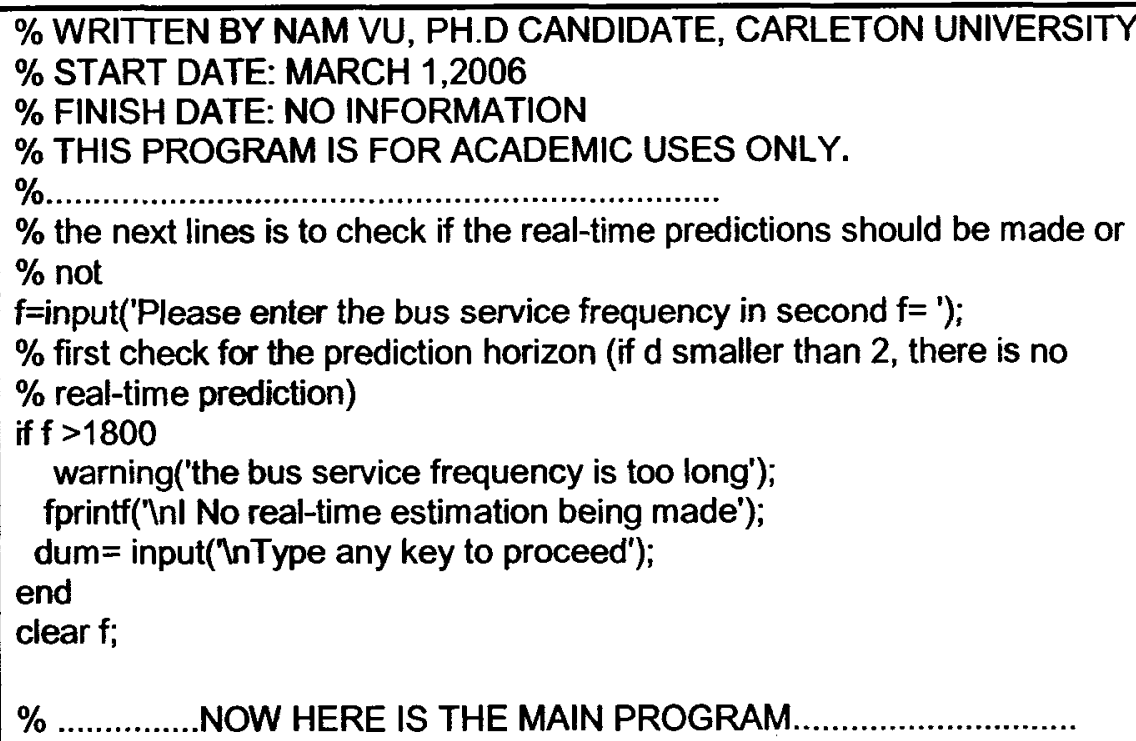

$\%$ We should first STANDARDIZE all variables

$[\mathrm{m}, \mathrm{k}]=\operatorname{size}(\mathrm{X})$;

for $j=1: k \%$ normarlize data of past pattern Matrix

$m X(1, j)=\operatorname{mean}(X(:, j))$;

$s X(1, j)=\operatorname{std}(X(:, j))$;

$X(:, j)=(X(:, j)-m X(1, j)) / s X(1, j)$

Xreal $(1, j)=($ Xreal $(1, j)-m X(1, j)) / s X(1, j) ; \%$ Current Pattern is also normalized end

$\% \mathrm{~m} Y=\operatorname{mean}(\mathrm{Y})$

$\% s Y=s t d(Y)$;

$\% \mathrm{Y}=(\mathrm{Y}-\mathrm{m} \mathrm{Y}) / \mathrm{s} \mathrm{Y}$

$\%$ Exhibit the inputs

$\mathrm{mX}$

$Y$

$\operatorname{disp}($ Xreal)

$\mathrm{X}$

$\mathrm{hmin}=\operatorname{round}\left(0.1^{*} \mathrm{~m}\right)$

hmax $=\operatorname{round}\left(0.7^{*} \mathrm{~m}\right)$;

$s=\operatorname{round}\left(0.1^{\star} \mathrm{m}\right)$;

for $h=h \min : \mathrm{s}: \mathrm{hmax}$

for $i=1: 1: \mathrm{m}$

$Y$ test $=Y(i)$;

$Y$ train $=Y$;

$Y_{-}$train(i)=[;

test_1 $1=X(i,:)$;

test_2=X;

test_2(i,:)= $\square ; \%$ the training set after removing the ith row for $n=1$ :size(test 2,1 ) 


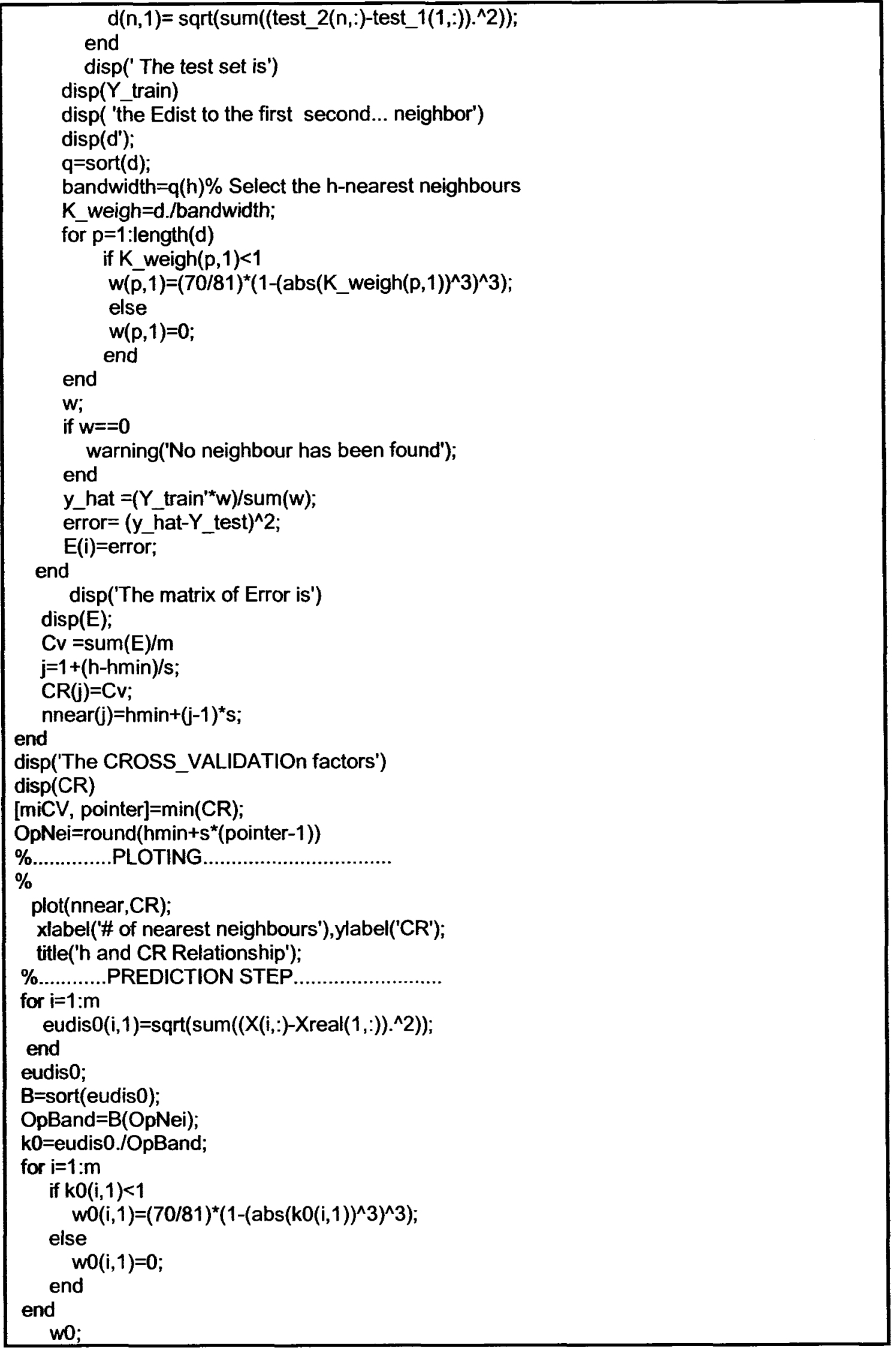


BanMa=diag (w0);

EstiMa=[ones $(\mathrm{m}, 1), \mathrm{X}]$;

IXma=inv(EstiMa*'BanMa`EstiMa);

disp(EstiMa');

Beta=IXma*EstiMa'*BanMa`Y;

disp(Beta);

boarder=round $\left(Y^{\prime *} w 0 /\right.$ sum(w0))

fprintf(2,'THE REAL-TIME PREDCTED \# of PASS OF THE BUS IS \%4.2f PAS.',boarder);

disp('Program complete.HAVE A NICE DAY' ');

\section{Bus de-boarder function}

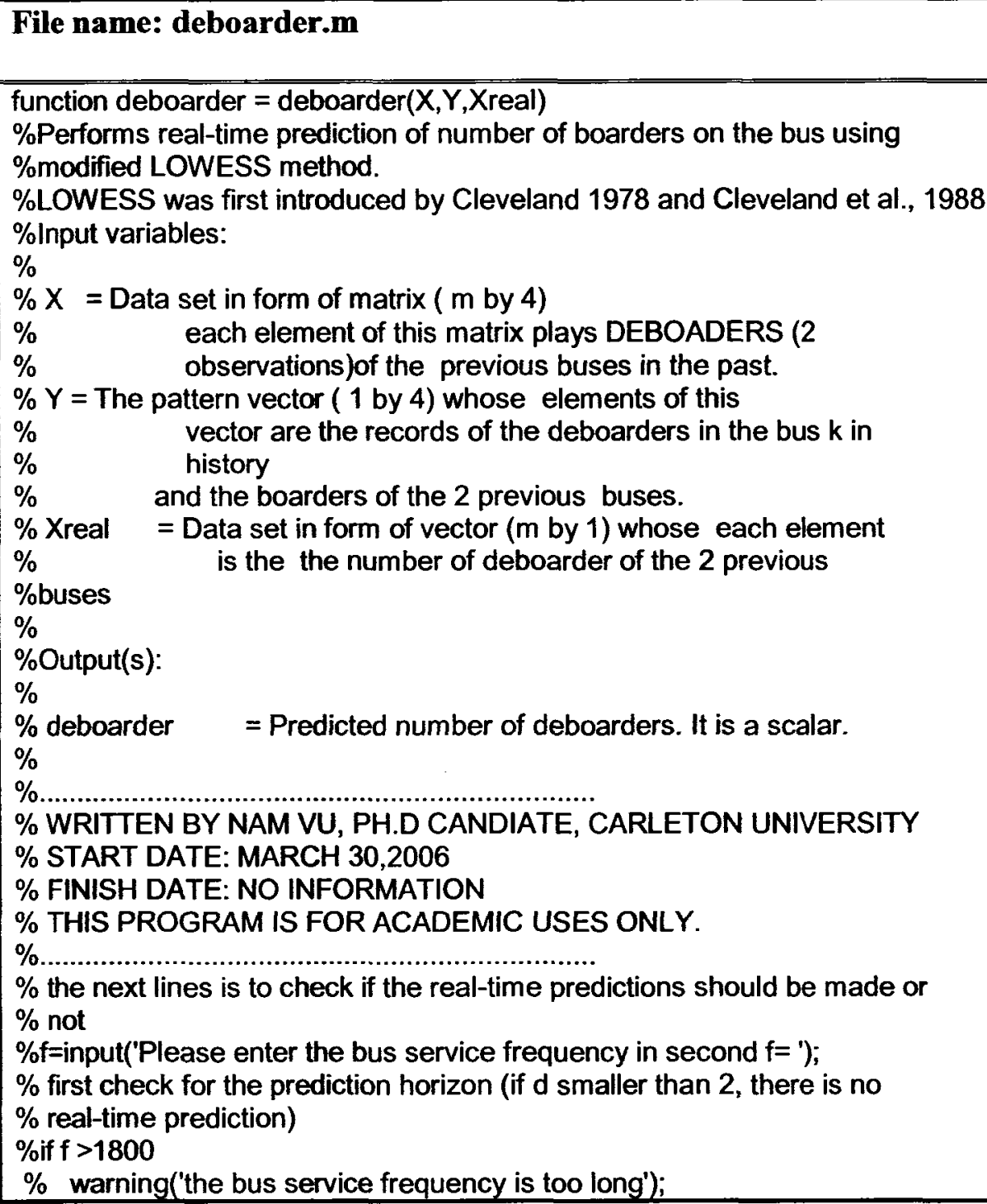




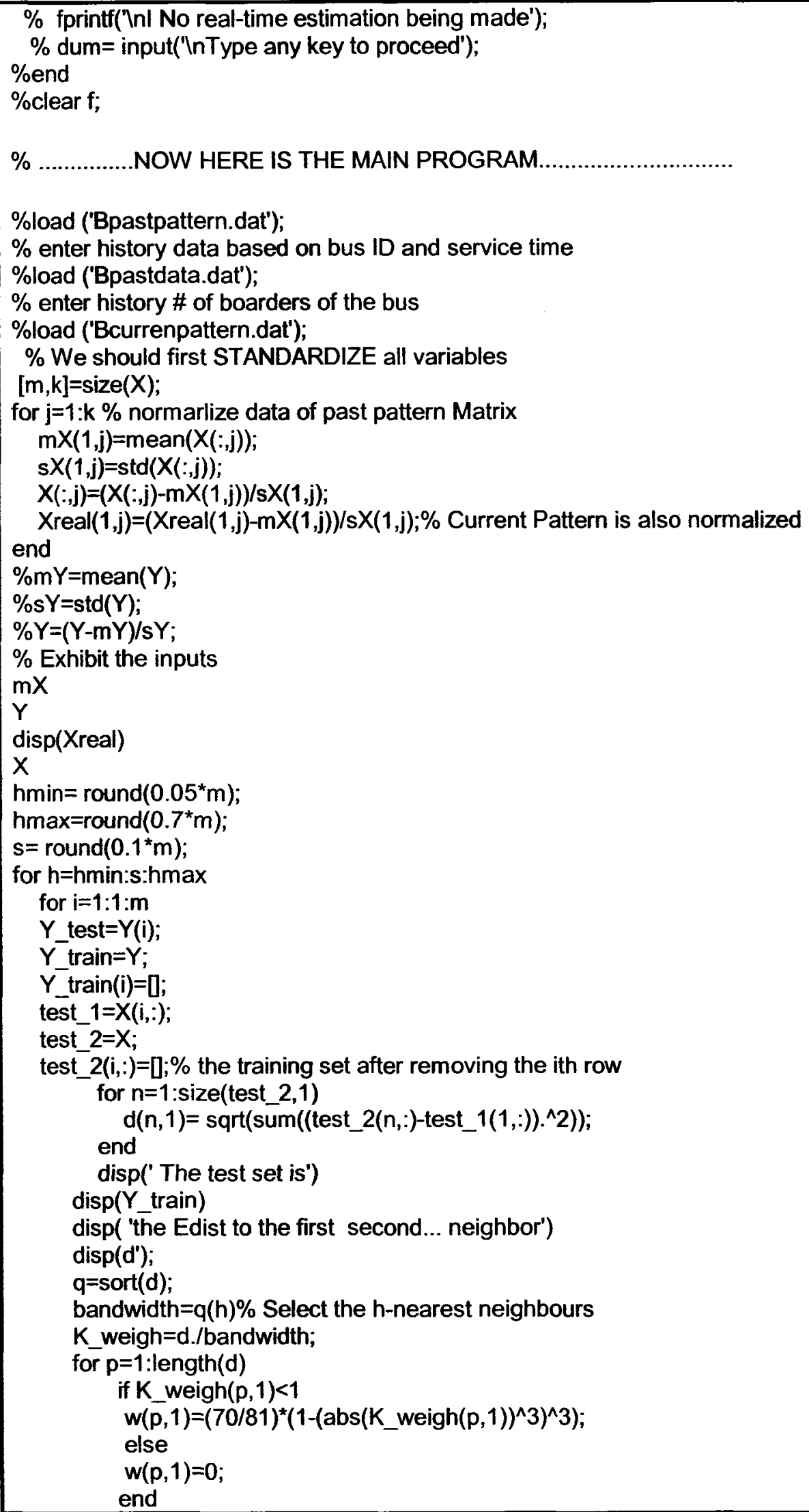




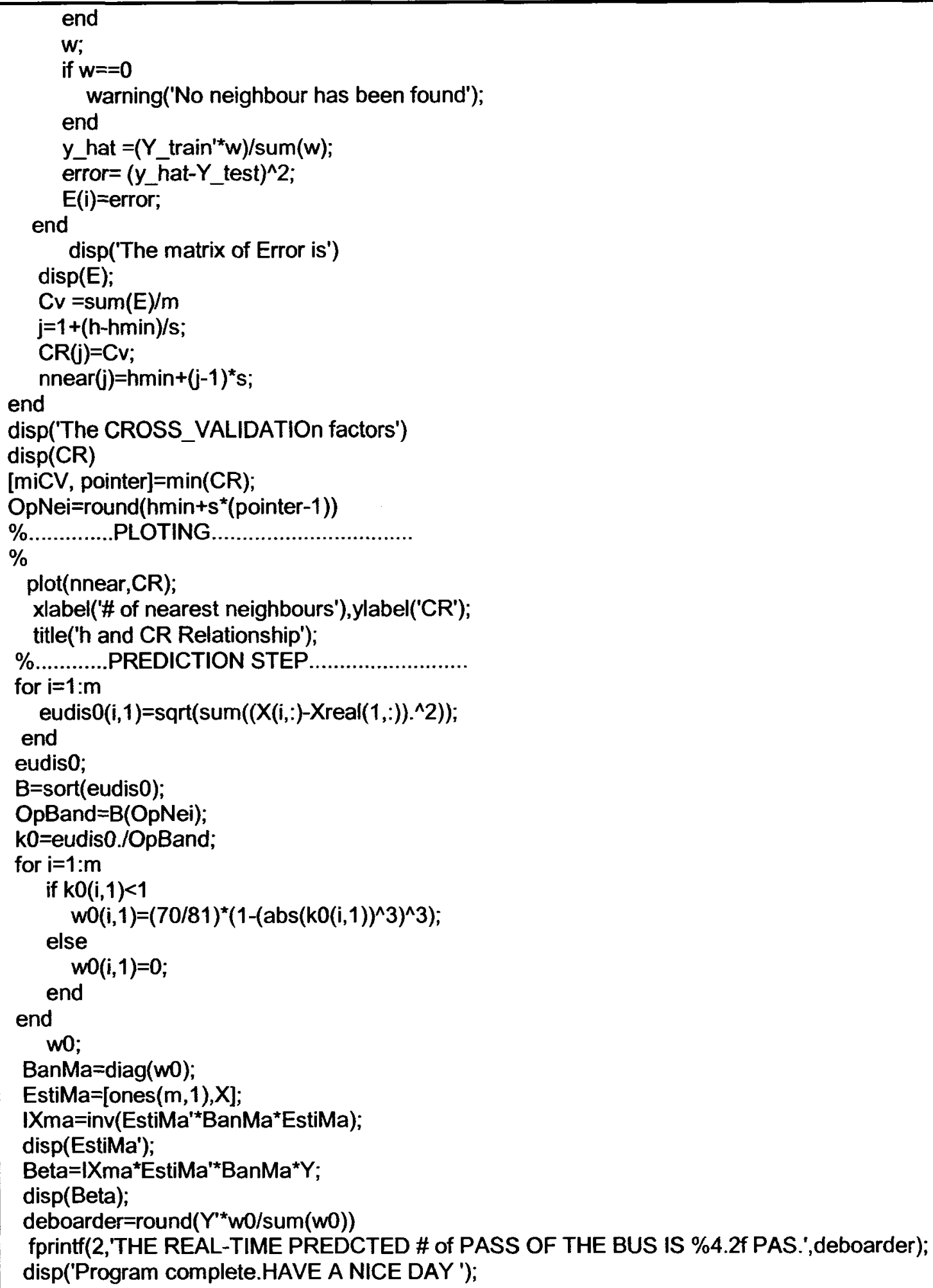




\section{Appendix D1}

\section{General}

This appendix presents the calibration procedure described in chapter 6 . First, two runs were made with VISSIM default parameters (see Table 6.3). By holding the same random seed numbers for each run and the same traffic volume, two other runs were also made with VISSIM but with calibrated values (see Table 6.3). The simulated results on bus travel times then were compared with corresponding actual bus travel times of each bus route provided by the OC Transpo at $95 \%$ confidence level.

\section{Travel time Measurement Configuration in VISSIM 4.1}

Simulated bus travel times were measured by a VISSIM's tool namely Travel time Measurement Configuration which records travel time of all buses running between stops. A travel time includes actual travel time, stop and go time, dwell time and all delays at intersections. Figure D1.1 shows this tool of VISSIM. 


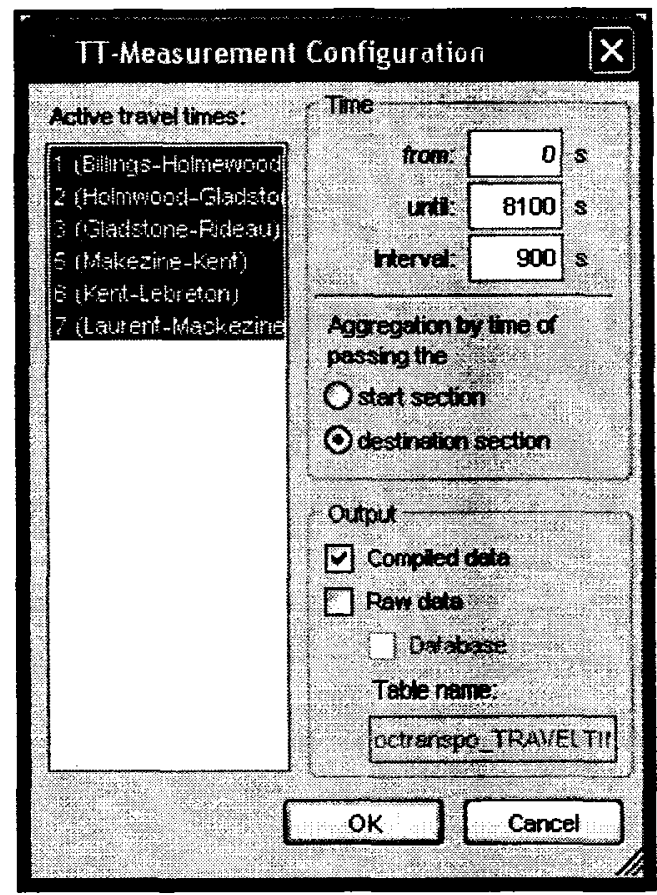

Figure D1.1: Travel time measurement tool in VISSIM

\section{Calibration Procedure}

Tables D1.1 to D1.6 present the comparisons between simulated bus travel times and the actual ones provided by the OC Transpo. As shown in the tables, the calibrated parameters provided closer bus running times to the actual data than the use of VISSIM default parameters did. However, if we use the default values suggested by VISSIM, the model still meets calibration targets. Therefore, no further calibration efforts were made after the first adjustment. The parameters used in this study are reasonable.

Tables D1.7- D1.10 tabulate VISSIM outputs during calibration process 


\section{APC-AVL data from OC Transpo}

Tables D1.11-D1.16 present actual bus travel time data provided by the OC Transpo

Table D1.1: VISSIM Calibration: Billings-Holmwood Link

\begin{tabular}{|c|c|c|c|c|c|c|c|c|}
\hline \multirow[b]{2}{*}{ Parameters (1) } & \multirow[b]{2}{*}{$\begin{array}{c}\text { Difference. } \\
\% \\
\text { (2) }\end{array}$} & \multirow[b]{2}{*}{$\begin{array}{l}\text { Significance } \\
\text { Level } \\
\text { (3) }\end{array}$} & \multicolumn{3}{|c|}{ Simulated data } & \multicolumn{3}{|c|}{ OC transpo data } \\
\hline & & & $\begin{array}{l}\text { \# of } \\
\text { Simulation } \\
\text { runs (4) }\end{array}$ & $\begin{array}{l}\text { Mean } \\
\text { (5) }\end{array}$ & $\begin{array}{l}\text { StD } \\
\text { (6) }\end{array}$ & $\begin{array}{l}\text { \# of APC } \\
\text { records } \\
\text { (7) }\end{array}$ & $\begin{array}{l}\text { Mean } \\
\text { (8) }\end{array}$ & $\begin{array}{l}\text { Standard } \\
\text { Dev. (9) }\end{array}$ \\
\hline $\begin{array}{l}\text { BX_ADD }=2.0 \\
\text { BX_MULT }=3.0\end{array}$ & 6.1 & 0.05 & 16 & $464.16 \mathrm{~s}$ & 31.99 & \multirow{2}{*}{37} & \multirow{2}{*}{435.6} & \multirow{2}{*}{48} \\
\hline $\begin{array}{l}\text { BX_ADD }=3.0 \\
\text { BX_MULT }=3.5\end{array}$ & 7.8 & 0.05 & 16 & 469.98 & 28.38 & & & \\
\hline
\end{tabular}

Table D1.2: VISSIM Calibration: Holmwood- Gladstone Link

\begin{tabular}{|c|c|c|c|c|c|c|c|c|}
\hline \multirow[b]{2}{*}{ Parameters (1) } & \multirow[b]{2}{*}{$\begin{array}{l}\text { Diff. \% } \\
\text { (2) }\end{array}$} & \multirow[b]{2}{*}{$\begin{array}{c}\text { Significance } \\
\text { Level } \\
\text { (3) }\end{array}$} & \multicolumn{3}{|c|}{ Simulated data } & \multicolumn{3}{|c|}{$O C$ transpo data } \\
\hline & & & $\begin{array}{l}\text { \# of } \\
\text { simulation } \\
\text { runs (4) } \\
\end{array}$ & $\begin{array}{l}\text { Mean } \\
\text { (5) }\end{array}$ & $\begin{array}{l}\text { Standard } \\
\text { Dev. (6) }\end{array}$ & $\begin{array}{l}\text { \# of APC } \\
\text { records } \\
\text { (7) } \\
\end{array}$ & $\begin{array}{l}\text { Mean } \\
\text { (8) }\end{array}$ & $\begin{array}{l}\text { Standard } \\
\text { Dev. (9) }\end{array}$ \\
\hline $\begin{array}{l}\text { BX_ADD }=2.0 \\
\text { BX } M U L T=3.0\end{array}$ & 9.49 & 0.05 & 16 & 395.85 & 36.21 & \multirow{2}{*}{38} & \multirow{2}{*}{437.4} & \multirow{2}{*}{$47.4 \mathrm{~s}$} \\
\hline $\begin{array}{l}\text { BX_ADD }=3.0 \\
\text { BX_MULT }=3.5\end{array}$ & 12.50 & 0.05 & 16 & 382.73 & 32.68 & & & \\
\hline
\end{tabular}

Table D1.3: VISSIM Calibration: Gladstone- Rideau Link

\begin{tabular}{|c|c|c|c|c|c|c|c|c|}
\hline \multirow[b]{2}{*}{ Parameters (1) } & \multirow[b]{2}{*}{$\begin{array}{l}\text { Difference } \\
\text { (2) }\end{array}$} & \multirow[b]{2}{*}{$\begin{array}{c}\text { Significance } \\
\text { Level } \\
\text { (3) }\end{array}$} & \multicolumn{3}{|c|}{ Simulated data } & \multicolumn{3}{|c|}{ OC transpo data } \\
\hline & & & $\begin{array}{l}\text { \# of } \\
\text { simulation } \\
\text { runs (4) }\end{array}$ & $\begin{array}{l}\text { Mean } \\
\text { (5) }\end{array}$ & $\begin{array}{l}\text { Standard } \\
\text { Dev. (6) }\end{array}$ & $\begin{array}{l}\text { \# of APC } \\
\text { records } \\
\text { (7) }\end{array}$ & $\begin{array}{l}\text { Mean } \\
\text { (8) }\end{array}$ & $\begin{array}{l}\text { Standard } \\
\text { Dev. (9) }\end{array}$ \\
\hline $\begin{array}{l}\text { BX_ADD }=2.0 \\
\text { BX_MULT }=3.0\end{array}$ & 5 & 0.05 & 15 & 595.88 & 67.52 & \multirow{2}{*}{35} & \multirow{2}{*}{$567.6 \mathrm{~s}$} & \multirow{2}{*}{$55.8 \mathrm{~s}$} \\
\hline $\begin{array}{l}\text { BX_ADD }=3.0 \\
\text { BX_MULT }=3.5\end{array}$ & 4.24 & 0.05 & 15 & 591.65 & 63.37 & & & \\
\hline
\end{tabular}

Table D1.4: VISSIM Calibration: Laurent- Mackenzie Link

\begin{tabular}{|c|c|c|c|c|c|c|c|c|}
\hline \multirow[b]{2}{*}{ Parameters (1) } & \multirow[b]{2}{*}{$\begin{array}{l}\text { Difference } \\
\text { (2) }\end{array}$} & \multirow[b]{2}{*}{$\begin{array}{c}\text { Signifieance } \\
\text { Level } \\
\text { (3) }\end{array}$} & \multicolumn{3}{|c|}{$\begin{array}{l}\text { Simulated data } \\
\end{array}$} & \multicolumn{3}{|c|}{ OC transpo data } \\
\hline & & & $\begin{array}{l}\text { \# of } \\
\text { simulation } \\
\text { runs (4) }\end{array}$ & $\begin{array}{l}\text { Mean } \\
\text { (5) }\end{array}$ & $\begin{array}{l}\text { Standard } \\
\text { Dev. } \\
\text { (6) }\end{array}$ & $\begin{array}{l}\text { \# of APC } \\
\text { records } \\
\text { (7) }\end{array}$ & $\begin{array}{l}\text { Mean } \\
\text { (8) }\end{array}$ & $\begin{array}{l}\text { Standard } \\
\text { Dev. (9) }\end{array}$ \\
\hline $\begin{array}{l}\text { BX_ADD }=2.0 \\
B X \text { MULT }=3.0\end{array}$ & 7.6 & 0.05 & 72 & 611.5 & 28.7 & \multirow{2}{*}{104} & \multirow{2}{*}{$568.2 \mathrm{~s}$} & \multirow{2}{*}{50.4} \\
\hline $\begin{array}{l}\text { BX_ADD }=3.0 \\
\text { BX_MULT }=3.5\end{array}$ & 8.9 & 0.05 & 72 & 618.8 & 29.1 & & & \\
\hline
\end{tabular}


Table D1.5: VISSIM Calibration: Mackenzie- Kent Link

\begin{tabular}{|c|c|c|c|c|c|c|c|c|}
\hline \multirow[b]{2}{*}{ Parameters (1) } & \multirow[b]{2}{*}{$\begin{array}{l}\text { Difference } \\
\text { (2) }\end{array}$} & \multirow[b]{2}{*}{$\begin{array}{c}\text { Significance } \\
\text { Level } \\
(3))\end{array}$} & \multicolumn{3}{|c|}{ Simulated data } & \multicolumn{3}{|c|}{ OC transpo data } \\
\hline & & & $\begin{array}{l}\text { \# of } \\
\text { simulation } \\
\text { runs (4) }\end{array}$ & $\begin{array}{l}\text { Mean } \\
\text { (5) }\end{array}$ & $\begin{array}{l}\text { Standa } \\
\text { Dev. (9) } \\
(6) \\
\end{array}$ & $\begin{array}{l}\text { \# of APC } \\
\text { records } \\
\text { (7) }\end{array}$ & $\begin{array}{l}\text { Mean } \\
\text { (8) }\end{array}$ & $\begin{array}{l}\text { Standard } \\
\text { Dev. (9)) }\end{array}$ \\
\hline $\begin{array}{l}\text { BX_ADD }=2.0 \\
\text { BX_MULT }=3.0\end{array}$ & 10.12 & 0.05 & 70 & 269.1 & 10.9 & \multirow{2}{*}{74} & \multirow{2}{*}{$299.4 \mathrm{~s}$} & \multirow{2}{*}{$30.6 \mathrm{~s}$} \\
\hline $\begin{array}{l}\text { BX_ADD }=3.0 \\
\text { BX_MULT }=3.5\end{array}$ & 15.34 & 0.05 & 70 & 253.5 & 12.2 & & & \\
\hline
\end{tabular}

Table D1.6: VISSIM Calibration: Kent- Lebreton Link

\begin{tabular}{|c|c|c|c|c|c|c|c|c|}
\hline \multirow[b]{2}{*}{ Parameters (1) } & \multirow[b]{2}{*}{$\begin{array}{l}\text { Difference } \\
\text { (2) }\end{array}$} & \multirow[b]{2}{*}{$\begin{array}{c}\text { Significance } \\
\text { Level } \\
\text { (3) }\end{array}$} & \multicolumn{3}{|c|}{ Simulated data } & \multicolumn{3}{|c|}{ OC transpo data } \\
\hline & & & $\begin{array}{l}\text { \# of } \\
\text { Simulation } \\
\text { runs (4) }\end{array}$ & $\begin{array}{l}\text { Mean } \\
\text { (5) }\end{array}$ & $\begin{array}{l}\text { Standard } \\
\text { Dev. } \\
\text { (6) }\end{array}$ & $\begin{array}{l}\text { \# of APC } \\
\text { records } \\
\text { (7) }\end{array}$ & $\begin{array}{l}\text { Mean } \\
\text { (8) }\end{array}$ & $\begin{array}{l}\text { Standard } \\
\text { Dev. (9) }\end{array}$ \\
\hline $\begin{array}{l}\text { BX_ADD }=2.0 \\
B X \text { MULT }=3.0\end{array}$ & 4.1 & 0.05 & 69 & 153.6 & 16.1 & \multirow{2}{*}{74} & \multirow{2}{*}{160.2} & \multirow{2}{*}{19.2} \\
\hline $\begin{array}{l}\text { BX_ADD }=3.0 \\
\text { BX_MULT }=3.5\end{array}$ & 2.4 & 0.05 & 69 & 156.2 & 16.7 & & & \\
\hline
\end{tabular}

Table D1.7: Simulated bus travel time data with VISSIM default values, Random seed $=1111$

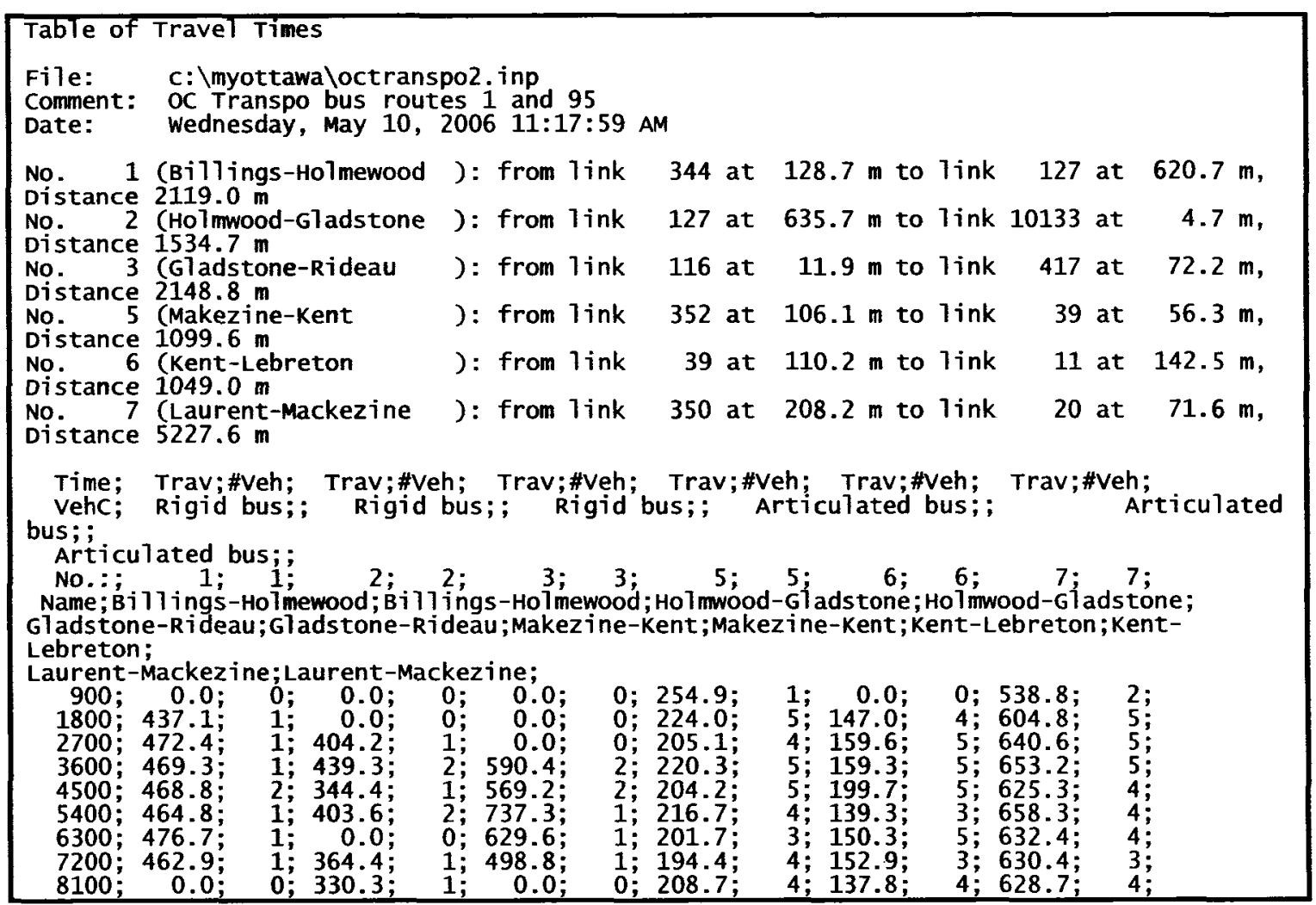


Table D1.8: Simulated bus travel time data with VISSIM defaults values, Random seed $=9999$

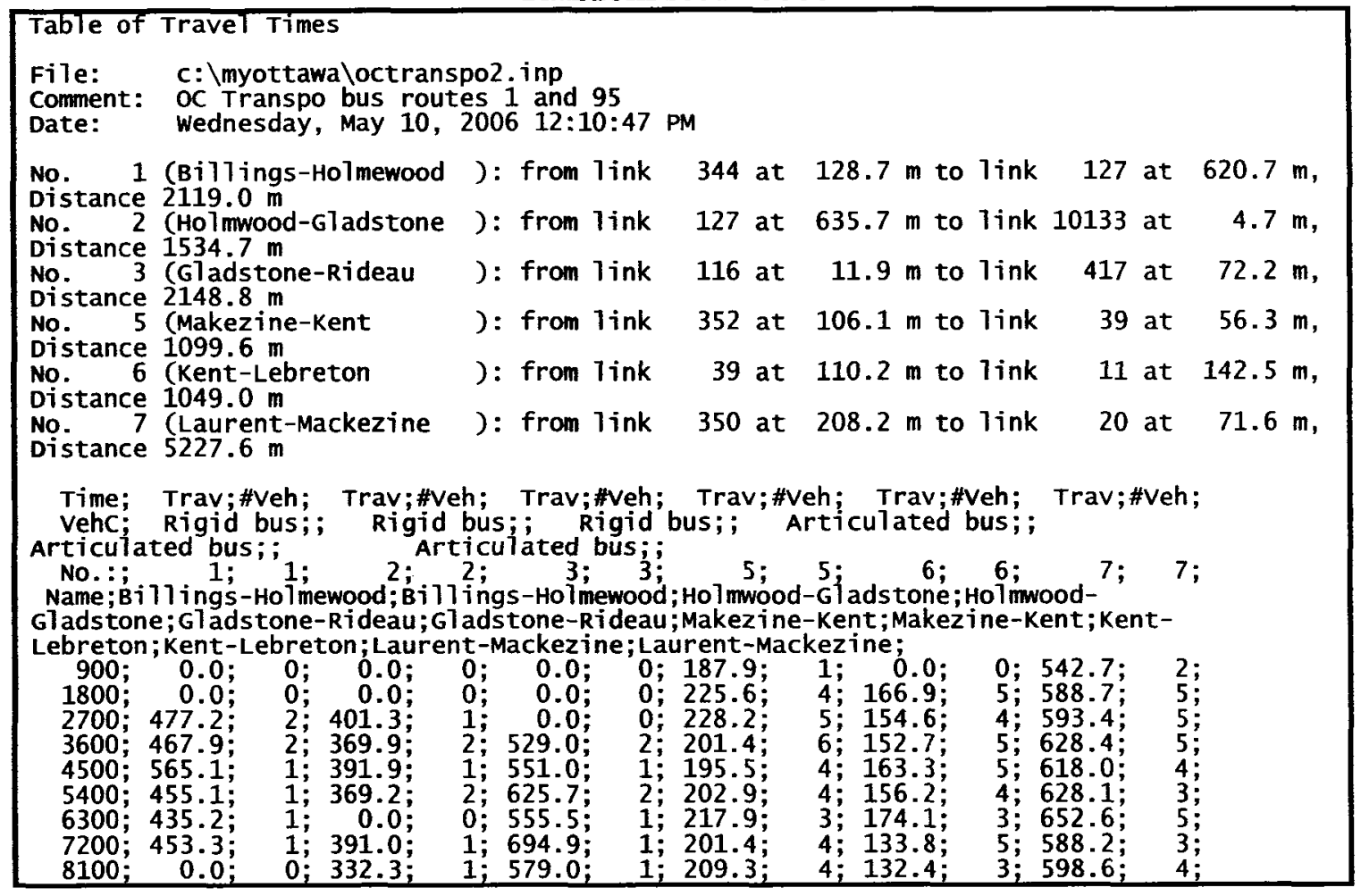

Table D1.9: Simulated bus travel time data with VISSIM Calibrated values, Random seed $=1111$

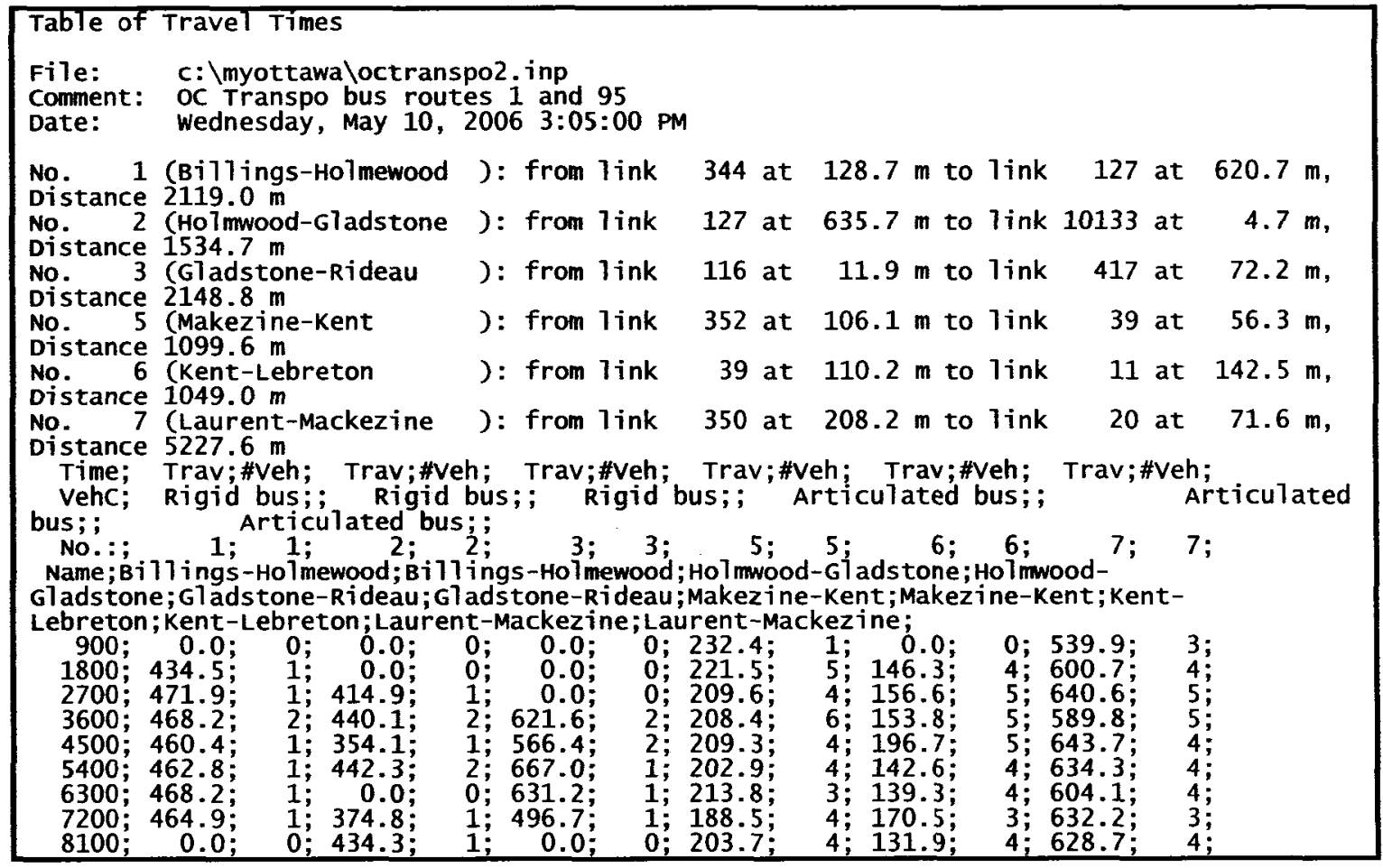


Table D1.10: Simulated Bus Travel Time data with VISSIM Calibrated Values, Random seed $=9999$

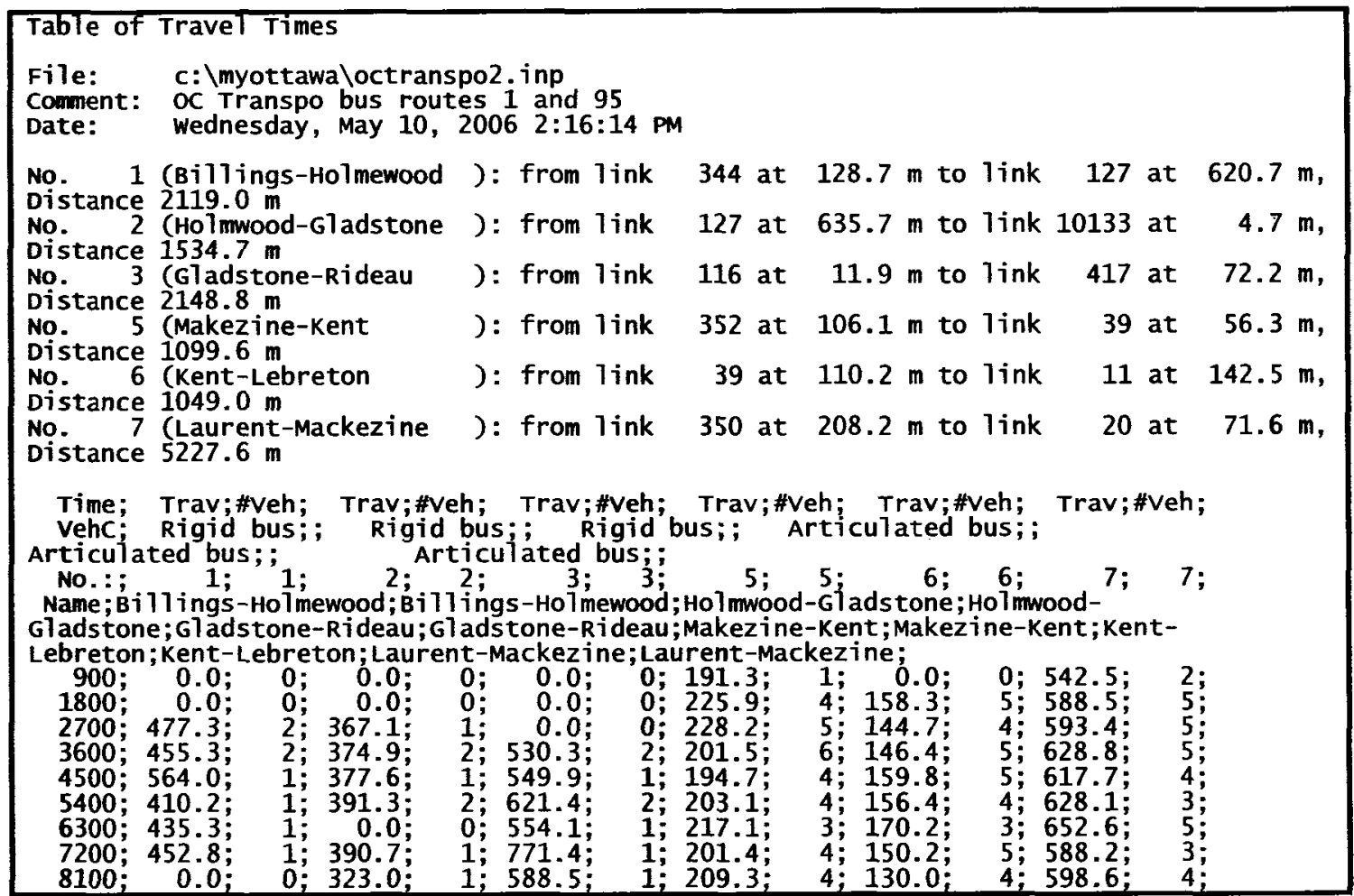

Table D1. 11: Actual Bus travel time: Billings- Holmwood stops

PROCESSED: 2006-05-09 13:11:35

BOOKING: APR05

FROM STOP: RA945 BILLINGS BRIDGE $4 \mathrm{C}$

25

TO STOP: CF630 BANK HOLMWOOD FS

ROUTE: 1 Bank - - Rockcliffe

Moving between stops

stop and go time

Idle time

Dwell time

Excess time

TOTAL

Layover time

Average sched time per trip

Total distance (KM)

Average moving speed (KM/HR)

Average total speed (KM/HR)

Total trips captured:
AUTOMATIC PASSENGER COUNTING SYSTEM REVENUE TIME UTILIZATION WEEKDAY SERVICE

PERIOD: 2005-04-24 TO 2005-06-

DAY TYPE: WEEKDAY

TIME: 08:00:00 TO 10:00:00

$\begin{array}{ccc}\begin{array}{c}\text { Average } \\ \text { Time } \\ \text { per }\end{array} & & \\ \text { Trip } & \text { Std } & \text { o of } \\ \text { Minutes } & \text { Dev } & \text { Time } \\ ---- & --- \\ 4.85 & 0.86 & 66.73 \\ 0.05 & 0.26 & 0.74 \\ 0.48 & 0.67 & 6.56 \\ 0.83 & 0.35 & 11.40 \\ 1.06 & 0.49 & 14.56 \\ -7.26 & 0 .-- & ---- \\ 7.26 & 100 \%\end{array}$

Reproduced with permission of the copyright owner. Further reproduction prohibited without permission. 
Table D1. 12: Actual Bus travel time: Holmwood- Gladstone stops

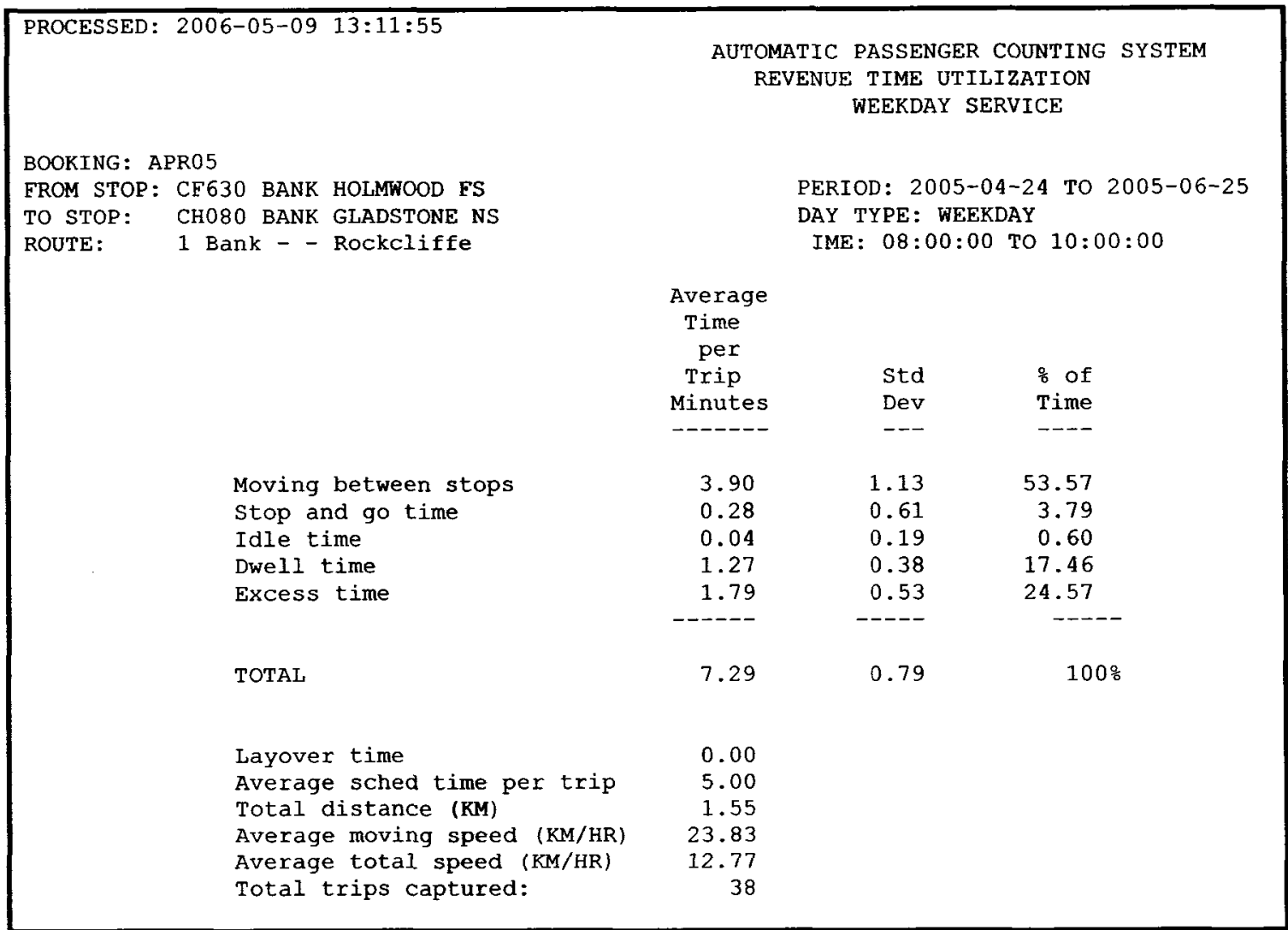

Table D1. 13: Actual Bus travel time: Gladstone-Rideau

PROCESSED: 2006-05-09 13:12:16

AUTOMATIC PASSENGER COUNTING SYSTEM REVENUE TIME UTILIZATION WEEKDAY SERVICE

BOOKING : APR05

FROM STOP: CHO80 BANK GLADSTONE NS

TO STOP: CD920 RIDEAU 3A

ROUTE: 1 Bank - - Rockcliffe
PERIOD: 2005-04-24 TO 2005-06-25 DAY TYPE: WEEKDAY

TIME: 08:00:00 TO 10:00:00

\begin{tabular}{|c|c|c|c|}
\hline & & \\
\hline Averag & $\begin{array}{l}\text { Time per } \\
\text { Trip }\end{array}$ & std & 응 of \\
\hline & Minutes & Dev & Time \\
\hline & $---\cdots$ & --- & \\
\hline Moving between stops & 6.43 & 2.05 & 67.93 \\
\hline Stop and go time & 0.58 & 1.46 & 6.12 \\
\hline Idle time & 0.30 & 0.54 & 3.12 \\
\hline Dwell time & 1.05 & 0.32 & 11.05 \\
\hline Excess time & 1.11 & 0.60 & 11.78 \\
\hline & ----- & ---- & $-\ldots$ \\
\hline TOTAL & 9.46 & 0.93 & $100 \%$ \\
\hline Layover time & 0.00 & & \\
\hline Average sched time per trip & 13.00 & & \\
\hline Total distance $(\mathrm{KM})$ & 2.10 & & \\
\hline Average moving speed (KM/HR) & 19.60 & & \\
\hline Average total speed (KM/HR) & 13.32 & & \\
\hline Total trips captured: & 35 & & \\
\hline
\end{tabular}


Table D1. 14: Actual Bus travel time: St.Laurent-Mackenzie stops

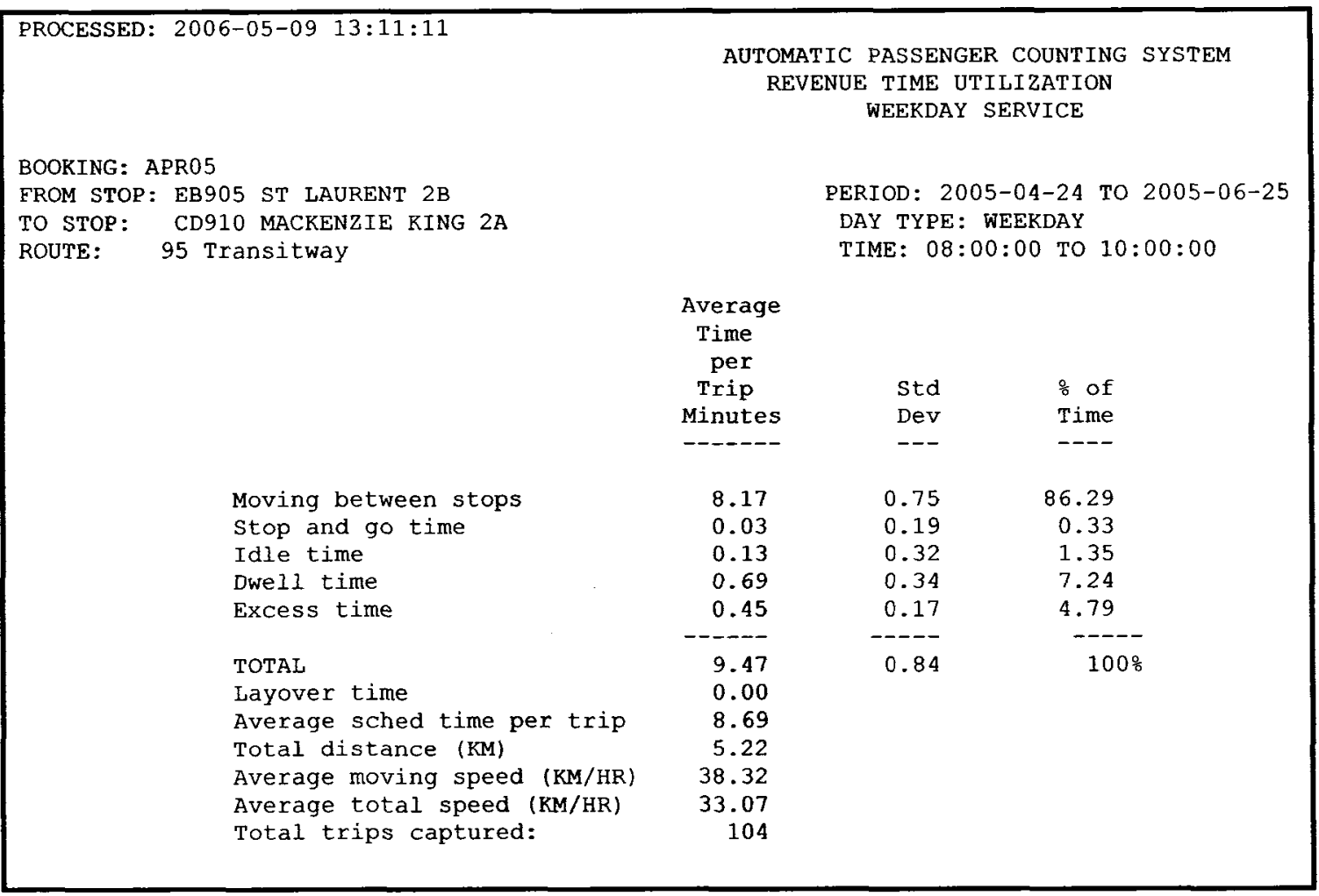

Table D1. 15: Actual Bus travel time: Mackenzie- Kent stops

PROCESSED: 2006-05-09 13:11:10

AUTOMATIC PASSENGER COUNTING SYSTEM REVENUE TIME UTILIZATION WEEKDAY SERVICE

BOORING: APR05

FROM STOP: CD910 MACKENZIE KING 2A

TO STOP: CA920 ALBERT KENT

ROUTE: 95 Transitway

PERIOD: 2005-04-24 TO 2005-06-25

DAY TYPE: WEEKDAY

TIME: 08:00:00 TO 10:00:00

Time

per

Trip

Minutes

Std $\quad \div$ of

-..--

Dev Time

Moving between stops

3.55

0.53

----

stop and go time

0.01

0.06

71.09

Idle time

Dwell time

0.19

0.14

Excess time

0.39

3.86

0.61

$0.31 \quad 12.75$

TOTAL

$0.29 \quad 12.16$

Layover time

4.99

$----$

Average sched time per trip

0.51

$100 \%$

Total distance (KM)

0.00

4.72

1.19

Average moving speed (KM/HR) 20.12

Average total speed (KM/HR) 14.30

Total trips captured:

30
74 
Table D1. 16: Actual Bus travel time: Kent - Lebreton stops

PROCESSED: 2006-05-11 11:40:57

BOOKING: APR05

FROM STOP: CA920 ALBERT KENT

TO STOP: CJ900 LEBRETON 1A

ROUTE: 95 Transitway
AUTOMATIC PASSENGER COUNTING SYSTEM REVENUE TIME UTILIZATION

WEEKDAY SERVICE

PERIOD: 2005-04-24 TO 2005-06-25

DAY TYPE: WEEKDAY

TIME : 08:00:00 TO 10:00:00

Average

Time

per

Trip

Minutes

Std

응

Minutes

Dev

Time

2.45

0.03

$---$

----

Moving between stops

stop and go time

Idle time

Dwell time

Excess time

TOTAL

Layover time

Average sched time per trip

Total distance ( $\mathrm{KM})$

Average moving speed (KM/HR)

Average total speed (KM/HR)

Total trips captured:
$0.36 \quad 91.56$

$0.19 \quad 1.14$

$0.00 \quad 0.00$

$0.08 \quad 3.37$

$0.08 \quad 3.93$

$0.32 \quad 100 \%$ 


\section{Appendix D2}

\section{General}

This Appendix presents the VISSIM's outputs following simulation and processing. There were 170 tables for the two bus routes. These record the operation information of the buses. Because the data are large ${ }^{1}$, only samples are printed out for the sake of illustration.

\section{Legend}

$\mathbf{t}=$ Arrival Time at stop (second)

ToD= Time of Day;

STime $=$ Start Time of the course (trip) at the first stop (second)

Line $=$ Bus line

Stp $=$ Stop Number

StpBd=Number of boarding Passengers

StpAlt $=$ Number of Alighting Passengers

StpDwl= Stop Dwell Time (second)

StpSveT $=$ Stop Service Time (second)

StpWP = Waiting Passenger

\begin{tabular}{|c|c|c|}
\hline Bus Station Name & Code (Stp) & Line \\
\hline St Laurent 2A & 51 & 95 \\
\hline Train 2A & 16 & 95 \\
\hline Hurdman & 20 & 95 \\
\hline Lees & 21 & 95 \\
\hline Campus & 50 & 95 \\
\hline Laurier & 25 & 95 \\
\hline Mackenrie King & 23 & 95 \\
\hline Billings Bridge & 1 & 1 \\
\hline Bank-Holmwood & 54 & 1 \\
\hline Bank-Gladstone & 3 & 1 \\
\hline Rideau Center & 53 & 1 \\
\hline
\end{tabular}

\footnotetext{
${ }^{1}$ It could be about 1200 pages if all of the above tables are printed out.
} 
Table D2.1: Route 95- Situation 1200

\begin{tabular}{|c|c|c|c|c|c|c|c|c|c|}
\hline 26 & $07: 45: 26.0$ & 0.5 & 95 & 1 & 51 & 0 & 8 & 27 & 27 \\
\hline 126.5 & $07: 47: 06.5$ & 0.5 & 95 & 1 & 16 & $\mathbf{0}$ & 8 & 15.4080185674615 & 15.4080185674615 \\
\hline 206 & $07: 48: 26.0$ & 180.5 & 95 & 2 & 51 & 10 & 8 & 52 & 52 \\
\hline 229 & $07: 48: 49.0$ & 0.5 & 95 & 1 & 20 & 14 & 9 & 64.5 & 64.5 \\
\hline 331.5 & $07: 50: 31.5$ & 180.5 & 95 & 2 & 16 & 10 & 8 & 16.6408717933666 & 16.6408717933666 \\
\hline 355.5 & $07: 50: 55.5$ & 0.5 & 95 & 1. & 21 & 14 & 9 & 20.7682394096475 & 20.7682394096475 \\
\hline 386.5 & $07: 51: 26.5$ & 360.5 & 95 & 3 & 51 & 11 & 8 & 54.5 & 54.5 \\
\hline 450.5 & $07: 52: 30.5$ & 0.5 & 95 & 1 & 50 & 14 & 9 & 41.1177085856324 & 41.1177085856324 \\
\hline 437.5 & $07: 52: 17.5$ & 180.5 & 95 & 2 & 20 & 12 & 12 & 67 & 67 \\
\hline 521.5 & $07: 53: 41.5$ & 0.5 & 95 & 1 & 25 & 14 & 9 & 11.8510497799656 & 11.8510497799656 \\
\hline 568.5 & $07: 54: 28.5$ & 180.5 & 95 & 2 & 21 & 12 & 12 & 17.6706932648818 & 17.6706932648818 \\
\hline 516 & $07: 53: 36.0$ & 360.5 & 95 & 3 & 16 & 11 & 8 & 13.0275490869938 & 13.0275490869938 \\
\hline 566 & $07: 54: 26.0$ & 540.5 & 95 & 4 & 51 & 7 & 8 & 44.5 & 44.5 \\
\hline 619.5 & 07:55:19.5 & 360.5 & 95 & 3 & 20 & 10 & 12 & 62 & 62 \\
\hline 652 & 07:55:52.0 & 0.5 & 95 & 1 & 23 & 18 & 18 & 97 & 97 \\
\hline 661 & 07:56:01.0 & 180.5 & 95 & 2 & 50 & 12 & 12 & 20.1698061319809 & 20.1698061319809 \\
\hline 711.5 & $07: 56: 51.5$ & 180.5 & 95 & 2 & 25 & 12 & 12 & 9.41338555159671 & 9.41338555159671 \\
\hline 683 & $07: 56: 23.0$ & 540.5 & 95 & 4 & 16 & 7 & 8 & 20.6037198994942 & 20.6037198994942 \\
\hline 734.5 & $07: 57: 14.5$ & 360.5 & 95 & 3 & 21 & 0 & 0 & 0 & 0 \\
\hline 747 & $07: 57: 27.0$ & 720.5 & 95 & 5 & 51 & 6 & 8 & 42 & 42 \\
\hline 792.5 & 07:58:12.5 & 540.5 & 95 & 4 & 20 & 11 & 11 & 62 & 62 \\
\hline 809.5 & 07:58:29.5 & 360.5 & 95 & 3 & 50 & 0 & 0 & 19.1968338518466 & 19.1968338518466 \\
\hline 820 & $07: 58: 40.0$ & 0.5 & 95 & 1 & 42 & 18 & 18 & 29.2409836316985 & 29.2409836316985 \\
\hline 773 & $07: 57: 53.0$ & 180.5 & 95 & 2 & 23 & 4 & 21 & 69.5 & 69.5 \\
\hline 865.5 & $07: 59: 25.5$ & 720.5 & 95 & 5 & 16 & 6 & 8 & 30.6395278375851 & 30.6395278375851 \\
\hline 883.5 & $07: 59: 43.5$ & 0.5 & 95 & 1 & 24 & 18 & 18 & 32.0886676006806 & 32.0886676006806 \\
\hline 859.5 & $07: 59: 19.5$ & 360.5 & 95 & 3 & 25 & 0 & 0 & 25.8094241290089 & 25.8094241290089 \\
\hline 918 & $08: 00: 18.0$ & 540.5 & 95 & 4 & 21 & 11 & 11 & 23.7811306750596 & 23.7811306750596 \\
\hline 926 & $08: 00: 26.0$ & 900.5 & 95 & 6 & 51 & 4 & 8 & 37 & 37 \\
\hline 941 & $08: 00: 41.0$ & 180.5 & 95 & 2 & 42 & 4 & 21 & 9.55396359766676 & 9.55396359766676 \\
\hline 953 & 08:00:53.0 & 0.5 & 95 & 1 & 7 & 13 & 25 & 102 & 102 \\
\hline 989 & $08: 01: 29.0$ & 720.5 & 95 & 5 & 20 & 18 & 11 & 79.5 & 79.5 \\
\hline 993.5 & $08: 01: 33.5$ & 180.5 & 95 & 2 & 24 & 4 & 21 & 26.2339346047374 & 26.2339346047374 \\
\hline 1013.5 & $08: 01: 53.5$ & 360.5 & 95 & 3 & 23 & 6 & 20 & 72 & 72 \\
\hline 1016.5 & $08: 01: 56.5$ & 540.5 & 95 & 4 & 50 & 11 & 11 & 25.8639488739813 & 25.8639488739813 \\
\hline 1038 & $08: 02: 18.0$ & 900.5 & 95 & 6 & 16 & 4 & 8 & 19.4601644732678 & 19.4601644732678 \\
\hline 1072 & $08: 02: 52.0$ & 540.5 & 95 & 4 & 25 & 11 & 11 & 29.635358426345 & 29.635358426345 \\
\hline 1074 & 08:02:54.0 & 180.5 & 95 & 2 & 7 & 2 & 17 & 54.5 & 54.5 \\
\hline 1085.5 & 08:03:05.5 & 0.5 & 95 & 1 & 15 & 13 & 25 & 34.943301091224 & 34.943301091224 \\
\hline 1105 & $08: 03: 25.0$ & 1080.5 & 95 & 7 & 51 & 19 & 8 & 74.5 & 74.5 \\
\hline
\end{tabular}

Reproduced with permission of the copyright owner. Further reproduction prohibited without permission. 


\begin{tabular}{|c|c|c|c|c|c|c|c|c|c|}
\hline 1135 & $08: 03: 55.0$ & 720.5 & 95 & 5 & 21 & 18 & 11 & 14.6498056895333 & 14.6498056895333 \\
\hline 1141 & 08:04:01.0 & 540.5 & 95 & 4 & 23 & 6 & 19 & 69.5 & 69.5 \\
\hline 1148.5 & $08: 04: 08.5$ & 900.5 & 95 & 6 & 20 & 8 & 10 & 52 & 52 \\
\hline 1182.5 & 08:04:42.5 & 180.5 & 95 & 2 & 15 & 2 & 17 & 29.3198984205231 & 29.3198984205231 \\
\hline 1180.5 & 08:04:40.5 & 360.5 & 95 & 3 & 42 & 6 & 20 & 7.28735577834652 & 7.28735577834652 \\
\hline 1234 & $08: 05: 34.0$ & 360.5 & 95 & 3 & 24 & 6 & 20 & 11.268640117897 & 11.268640117897 \\
\hline 1251 & $08: 05: 51.0$ & 1080.5 & 95 & 7 & 16 & 19 & 8 & 9.91379574376476 & 9.91379574376476 \\
\hline 1258 & $08: 05: 58.0$ & 0.5 & 95 & 1 & 52 & 13 & 25 & 7.94756682398519 & 7.94756682398519 \\
\hline 1228.5 & $08: 05: 28.5$ & 720.5 & 95 & 5 & 50 & 18 & 11 & 19.6868938284895 & 19.6868938284895 \\
\hline 1266.5 & 08:06:06.5 & 900.5 & 95 & 6 & 21 & 8 & 10 & 11.1554981443269 & 11.1554981443269 \\
\hline 1279.5 & $08: 06: 19.5$ & 720.5 & 95 & 5 & 25 & 18 & 11 & 40.5561034458096 & 40.5561034458096 \\
\hline 1286.5 & 08:06:26.5 & 1260.5 & 95 & 8 & 51 & 12 & 8 & 57 & 57 \\
\hline 1300.5 & 08:06:40.5 & 540.5 & 95 & 4 & 42 & 6 & 19 & 35.219331599476 & 35.219331599476 \\
\hline 1314 & 08:06:54.0 & 360.5 & 95 & 3 & 7 & 2 & 18 & 57 & 57 \\
\hline 1319 & $08: 06: 59.0$ & 180.5 & 95 & 2 & 52 & 2 & 17 & 33.9811784389407 & 33.9811784389407 \\
\hline 1349.5 & $08: 07: 29.5$ & 1080.5 & 95 & 7 & 20 & 6 & 15 & 59.5 & 59.5 \\
\hline 1354 & $08: 07: 34.0$ & 900.5 & 95 & 6 & 50 & 8 & 10 & 25.3888255677701 & 25.3888255677701 \\
\hline 1369 & $08: 07: 49.0$ & 540.5 & 95 & 4 & 24 & 6 & 19 & 5.49294724121229 & 5.49294724121229 \\
\hline 1375.5 & $08: 07: 55.5$ & 720.5 & 95 & 5 & 23 & 5 & 22 & 74.5 & 74.5 \\
\hline 1410.5 & 08:08:30.5 & 900.5 & 95 & 6 & 25 & 8 & 10 & 21.915413922672 & 21.915413922672 \\
\hline 1417 & 08:08:37.0 & 1260.5 & 95 & 8 & 16 & 12 & 8 & 19.9467728345976 & 19.9467728345976 \\
\hline 1422.5 & 08:08:42.5 & 360.5 & 95 & 3 & 15 & 2 & 18 & 13.9579757504817 & 13.9579757504817 \\
\hline 1433.5 & 08:08:53.5 & 540.5 & 95 & 4 & 7 & 3 & 18 & 59.5 & 59.5 \\
\hline 1472 & 08:09:32.0 & 1080.5 & 95 & 7 & 21 & 6 & 15 & 22.2812288908245 & 22.2812288908245 \\
\hline 1493.5 & $08: 09: 53.5$ & 900.5 & 95 & 6 & 23 & 5 & 17 & 62 & 62 \\
\hline 1467 & $08: 09: 27.0$ & 1440.5 & 95 & 9 & 51 & 8 & 8 & 47 & 47 \\
\hline 1525.5 & $08: 10: 25.5$ & 1260.5 & 95 & 8 & 20 & 5 & 13 & 52 & 52 \\
\hline 1542 & $08: 10: 42.0$ & 720.5 & 95 & 5 & 42 & 5 & 22 & 27.7101882253563 & 27.7101882253563 \\
\hline 1542 & $08: 10: 42.0$ & 540.5 & 95 & 4 & 15 & 3 & 18 & 14.9754120586199 & 14.9754120586199 \\
\hline 1559 & $08: 10: 59.0$ & 360.5 & 95 & 3 & 52 & 2 & 18 & 18.2594138743781 & 18.2594138743781 \\
\hline 1591.5 & $08: 11: 31.5$ & 1440.5 & 95 & 9 & 16 & 8 & 8 & 21.7979516728509 & 21.7979516728509 \\
\hline 1566.5 & $08: 11: 06.5$ & 1080.5 & 95 & 7 & 50 & 6 & 15 & 28.223327691404 & 28.223327691404 \\
\hline 1605.5 & $08: 11: 45.5$ & 720.5 & 95 & 5 & 24 & 5 & 22 & 2.03845422638412 & 2.03845422638412 \\
\hline 1624 & $08: 12: 04.0$ & 1080.5 & 95 & 7 & 25 & 6 & 15 & 29.4021079828607 & 29.4021079828607 \\
\hline 1641 & 08:12:21.0 & 1260.5 & 95 & 8 & 21 & 5 & 13 & 11.4381248583569 & 11.4381248583569 \\
\hline 1646 & $08: 12: 26.0$ & 1620.5 & 95 & 10 & 51 & 16 & 8 & 67 & 67 \\
\hline 1661 & $08: 12: 41.0$ & 900.5 & 95 & 6 & 42 & 5 & 17 & 21.4709414145211 & 21.4709414145211 \\
\hline 1674 & $08: 12: 54.0$ & 720.5 & 95 & 5 & 7 & 2 & 19 & 59.5 & 59.5 \\
\hline 1678.5 & 08:12:58.5 & 540.5 & 95 & 4 & 52 & 3 & 18 & 24.2338642213828 & 24.2338642213828 \\
\hline 1716.5 & 08:13:36.5 & 900.5 & 95 & 6 & 24 & 5 & 17 & 28.1892212176801 & 28.1892212176801 \\
\hline 1707.5 & 08:13:27.5 & 1440.5 & 95 & 9 & 20 & 10 & 12 & 62 & 62 \\
\hline 1727 & $08: 13: 47.0$ & 1260.5 & 95 & 8 & 50 & 5 & 13 & 11.8356045866382 & 11.8356045866382 \\
\hline
\end{tabular}

Reproduced with permission of the copyright owner. Further reproduction prohibited without permission. 


\begin{tabular}{|c|c|c|c|c|c|c|c|c|c|}
\hline 1731.5 & $08: 13: 51.5$ & 1080.5 & 95 & 7 & 23 & 7 & 21 & 77 & 77 \\
\hline 1768.5 & $08: 14: 28.5$ & 1260.5 & 95 & 8 & 25 & 5 & 13 & 52.8966901428382 & 52.8966901428382 \\
\hline 1783.5 & $08: 14: 43.5$ & 720.5 & 95 & 5 & 15 & 2 & 19 & 25.2340207650103 & 25.2340207650103 \\
\hline 1785.5 & $08: 14: 45.5$ & 1620.5 & 95 & 10 & 16 & 16 & 8 & 22.960157967385 & 22.960157967385 \\
\hline 1826 & $08: 15: 26.0$ & 1800.5 & 95 & 11 & 51 & 15 & 8 & 64.5 & 64.5 \\
\hline 1794.5 & $08: 14: 54.5$ & 900.5 & 95 & 6 & 7 & 0 & 15 & 44.5 & 44.5 \\
\hline 1837 & 08:15:37.0 & 1440.5 & 95 & 9 & 21 & 10 & 12 & 52.824036320219 & 52.824036320219 \\
\hline 1861 & 08:16:01.0 & 1260.5 & 95 & 8 & 23 & 4 & 18 & 62 & 62 \\
\hline 1897 & $08: 16: 37.0$ & 1620.5 & 95 & 10 & 20 & 8 & 14 & 62 & 62 \\
\hline 1901.5 & $08: 16: 41.5$ & 1080.5 & 95 & 7 & 42 & 7 & 21 & 13.7412054252605 & 13.7412054252605 \\
\hline 1906 & $08: 16: 46.0$ & 900.5 & 95 & 6 & 15 & 0 & 15 & 24.8785079302955 & 24.8785079302955 \\
\hline 1920.5 & 08:17:00.5 & 720.5 & 95 & 5 & 52 & 2 & 19 & 0 & 0 \\
\hline 1952.5 & $08: 17: 32.5$ & 1080.5 & 95 & 7 & 24 & 7 & 21 & 29.4062008271227 & 29.4062008271227 \\
\hline 1965 & $08: 17: 45.0$ & 1800.5 & 95 & 11 & 16 & 15 & 8 & 3.4983609929123 & 3.4983609929123 \\
\hline 1969 & 08:17:49.0 & 1440.5 & 95 & 9 & 50 & 10 & 12 & 1.6792013983905 & 1.6792013983905 \\
\hline 2003 & 08:18:23.0 & 1440.5 & 95 & 9 & 25 & 10 & 12 & 20.219276874692 & 20.219276874692 \\
\hline 2019 & 08:18:39.0 & 1260.5 & 95 & 8 & 42 & 4 & 18 & 25.3860101450702 & 25.3860101450702 \\
\hline 2022 & $08: 18: 42.0$ & 1620.5 & 95 & 10 & 21 & 8 & 14 & 17.1548377015565 & 17.1548377015565 \\
\hline 2006.5 & $08: 18: 26.5$ & 1980.5 & 95 & 12 & 51 & 24 & 8 & 87 & 87 \\
\hline 2034.5 & $08: 18: 54.5$ & 1080.5 & 95 & 7 & 7 & 7 & 19 & 72 & 72 \\
\hline 2039.5 & $08: 18: 59.5$ & 900.5 & 95 & 6 & 52 & 0 & 15 & 19.2632181295951 & 19.2632181295951 \\
\hline 2077.5 & 08:19:37.5 & 1260.5 & 95 & 8 & 24 & 4 & 18 & 21.8194859816282 & 21.8194859816282 \\
\hline 2059 & $08: 19: 19.0$ & 1800.5 & 95 & 11 & 20 & 8 & 14 & 62 & 62 \\
\hline 2095.5 & 08:19:55.5 & 1440.5 & 95 & 9 & 23 & 14 & 19 & 89.5 & 89.5 \\
\hline 2113 & 08:20:13.0 & 1620.5 & 95 & 10 & 50 & 8 & 14 & 20.908244199681 & 20.908244199681 \\
\hline 2138.5 & $08: 20: 38.5$ & 1080.5 & 95 & 7 & 15 & 7 & 19 & 14.7975736539457 & 14.7975736539457 \\
\hline 2153.5 & 08:20:53.5 & 1260.5 & 95 & 8 & 7 & 3 & 15 & 52 & 52 \\
\hline 2163 & 08:21:03.0 & 1620.5 & 95 & 10 & 25 & 8 & 14 & 35.3233184129663 & 35.3233184129663 \\
\hline 2167.5 & $08: 21: 07.5$ & 1980.5 & 95 & 12 & 16 & 24 & 8 & 20.5656558967728 & 20.5656558967728 \\
\hline 2185 & $08: 21: 25.0$ & 2160.5 & 95 & 13 & 51 & 11 & 8 & 54.5 & 54.5 \\
\hline 2186 & $08: 21: 26.0$ & 1800.5 & 95 & 11 & 21 & 8 & 14 & 17.2122399097436 & 17.2122399097436 \\
\hline 2235.5 & 08:22:15.5 & 1080.5 & 95 & 7 & 52 & 7 & 19 & 34.7580535812084 & 34.7580535812084 \\
\hline 2263.5 & $08: 22: 43.5$ & 1440.5 & 95 & 9 & 42 & 14 & 19 & 19.1026094806307 & 19.1026094806307 \\
\hline 2265 & 08:22:45.0 & 1260.5 & 95 & 8 & 15 & 3 & 15 & 27.0040373374394 & 27.0040373374394 \\
\hline 2278 & 08:22:58.0 & 1980.5 & 95 & 12 & 20 & 9 & 16 & 69.5 & 69.5 \\
\hline 2279.5 & $08: 22: 59.5$ & 1800.5 & 95 & 11 & 50 & 8 & 14 & 26.5560844710106 & 26.5560844710106 \\
\hline 2319 & 08:23:39.0 & 1440.5 & 95 & 9 & 24 & 14 & 19 & 22.707752223182 & 22.707752223182 \\
\hline 2312 & 08:23:32.0 & 2160.5 & 95 & 13 & 16 & 11 & 8 & 25.1572047241585 & 25.1572047241585 \\
\hline 2332 & 08:23:52.0 & 1620.5 & 95 & 10 & 23 & 11 & 21 & 87 & 87 \\
\hline 2337 & 08:23:57.0 & 1800.5 & 95 & 11 & 25 & 8 & 14 & 18.3323028581116 & 18.3323028581116 \\
\hline 2366.5 & $08: 24: 26.5$ & 2340.5 & 95 & 14 & 51 & 22 & 8 & 82 & 82 \\
\hline 2395 & $08: 24: 55.0$ & 1440.5 & 95 & 9 & 7 & 4 & 23 & 74.5 & 74.5 \\
\hline 2398.5 & $08: 24: 58.5$ & 1260.5 & 95 & 8 & 52 & 3 & 15 & 11.7219697401678 & 11.7219697401678 \\
\hline
\end{tabular}

Reproduced with permission of the copyright owner. Further reproduction prohibited without permission. 


\begin{tabular}{|c|c|c|c|c|c|c|c|c|c|}
\hline 2425 & $08: 25: 25.0$ & 2160.5 & 95 & 13 & 20 & 7 & 12 & 54.5 & 54.5 \\
\hline 2412 & $08: 25: 12.0$ & 1980.5 & 95 & 12 & 21 & 9 & 16 & 24.2663476350826 & 24.2663476350826 \\
\hline 2453.5 & $08: 25: 53.5$ & 1800.5 & 95 & 11 & 23 & 8 & 20 & 77 & 77 \\
\hline 2502.5 & $08: 26: 42.5$ & 1440.5 & 95 & 9 & 15 & 4 & 23 & 35.7978008883888 & 35.7978008883888 \\
\hline 2512.5 & $08: 26: 52.5$ & 1980.5 & 95 & 12 & 50 & 9 & 16 & 34.9375145429651 & 34.9375145429651 \\
\hline 2499.5 & $08: 26: 39.5$ & 1620.5 & 95 & 10 & 42 & 11 & 21 & 6.28853073269999 & 6.28853073269999 \\
\hline 2521 & $08: 27: 01.0$ & 2340.5 & 95 & 14 & 16 & 22 & 8 & 20.2395481154767 & 20.2395481154767 \\
\hline 2545.5 & $08: 27: 25.5$ & 2520.5 & 95 & 15 & 51 & 21 & 8 & 79.5 & 79.5 \\
\hline 2542.5 & $08: 27: 22.5$ & 2160.5 & 95 & 13 & 21 & 7 & 12 & 27.474141306356 & 27.474141306356 \\
\hline 2552.5 & $08: 27: 32.5$ & 1620.5 & 95 & 10 & 24 & 11 & 21 & 20.4017029958996 & 20.4017029958996 \\
\hline 2577.5 & $08: 27: 57.5$ & 1980.5 & 95 & 12 & 25 & 9 & 16 & 23.2010066579892 & 23.2010066579892 \\
\hline 2621 & $08: 28: 41.0$ & 1800.5 & 95 & 11 & 42 & 8 & 20 & 16.2928305636922 & 16.2928305636922 \\
\hline 2628 & $08: 28: 48.0$ & 2340.5 & 95 & 14 & 20 & 12 & 16 & 77 & 77 \\
\hline 2634 & $08: 28: 54.0$ & 1620.5 & 95 & 10 & 7 & 5 & 22 & 74.5 & 74.5 \\
\hline 2642 & 08:29:02.0 & 2160.5 & 95 & 13 & 50 & 7 & 12 & 4.9348794933683 & 4.9348794933683 \\
\hline 2674 & $08: 29: 34.0$ & 1800.5 & 95 & 11 & 24 & 8 & 20 & 22.9099954820845 & 22.9099954820845 \\
\hline 2676 & 08:29:36.0 & 2160.5 & 95 & 13 & 25 & 7 & 12 & 23.468804485881 & 23.468804485881 \\
\hline 2693 & $08: 29: 53.0$ & 1980.5 & 95 & 12 & 23 & 16 & 24 & 107 & 107 \\
\hline 2698 & 08:29:58.0 & 2520.5 & 95 & 15 & 16 & 21 & 8 & 22.6049955504088 & 226049955504088 \\
\hline 2700.5 & $08: 30: 00.5$ & 1440.5 & 95 & 9 & 52 & 4 & 23 & 0 & 0 \\
\hline 2726.5 & $08: 30: 26.5$ & 2700.5 & 95 & 16 & 51 & 18 & 8 & 72 & 72 \\
\hline 2740 & $08: 30: 40.0$ & 1620.5 & 95 & 10 & 15 & 5 & 22 & 21.1668147437831 & 21.1668147437831 \\
\hline 2767 & $08: 31: 07.0$ & 2340.5 & 95 & 14 & 21 & 12 & 16 & 36.6406023740624 & 36.6406023740624 \\
\hline 2754 & 08:30:54.0 & 1800.5 & 95 & 11 & 7 & 2 & 20 & 62 & 62 \\
\hline 2812.5 & $08: 31: 52.5$ & 2160.5 & 95 & 13 & 23 & 1 & 19 & 57 & 57 \\
\hline 2809 & $08: 31: 49.0$ & 2520.5 & 95 & 15 & 20 & 13 & 15 & 77 & 77 \\
\hline 2873 & $08: 32: 53.0$ & 2700.5 & 95 & 16 & 16 & 18 & 8 & 25.8976918188092 & 25.8976918188092 \\
\hline 2878 & $08: 32: 58.0$ & 1620.5 & 95 & 10 & 52 & 5 & 22 & 32.1622888171239 & 32.1622888171239 \\
\hline 2878 & $08: 32: 58.0$ & 2340.5 & 95 & 14 & 50 & 12 & 16 & 5.67213631849137 & 5.67213631849137 \\
\hline 2862.5 & $08: 32: 42.5$ & 1980.5 & 95 & 12 & 42 & 16 & 24 & 9.55724702847379 & 9.55724702847379 \\
\hline 2867.5 & $08: 32: 47.5$ & 1800.5 & 95 & 11 & 15 & 2 & 20 & 6.78266611184534 & 6.78266611184534 \\
\hline 2906.5 & $08: 33: 26.5$ & 2880.5 & 95 & 17 & 51 & 21 & 8 & 79.5 & 79.5 \\
\hline 2913 & 08:33:33.0 & 2340.5 & 95 & 14 & 25 & 12 & 16 & 25.7887515919864 & 25.7887515919864 \\
\hline 2913.5 & $08: 33: 33.5$ & 1980.5 & 95 & 12 & 24 & 16 & 24 & 7.00857075452838 & 7.00857075452838 \\
\hline 2949 & $08: 34: 09.0$ & 2520.5 & 95 & 15 & 21 & 13 & 15 & 22.5148356183951 & 22.5148356183951 \\
\hline 2957.5 & $08: 34: 17.5$ & 1800.5 & 95 & 11 & 52 & 2 & 20 & 17.7503283888678 & 17.7503283888678 \\
\hline 2980.5 & $08: 34: 40.5$ & 2160.5 & 95 & 13 & 42 & 1 & 19 & 39.1189068557031 & 39.1189068557031 \\
\hline 2989.5 & $08: 34: 49.5$ & 2700.5 & 95 & 16 & 20 & 13 & 15 & 77 & 77 \\
\hline 2993.5 & $08: 34: 53.5$ & 1980.5 & 95 & 12 & 7 & 3 & 28 & 84.5 & 84.5 \\
\hline 3045.5 & $08: 35: 45.5$ & 2520.5 & 95 & 15 & 50 & 13 & 15 & 26.1703266971289 & 26.1703266971289 \\
\hline 3060.5 & $08: 36: 00.5$ & 2880.5 & 95 & 17 & 16 & 21 & 8 & 12.9772107090858 & 12.9772107090858 \\
\hline 3052.5 & $08: 35: 52.5$ & 2340.5 & 95 & 14 & 23 & 7 & 25 & 87 & \\
\hline
\end{tabular}

Reproduced with permission of the copyright owner. Further reproduction prohibited without permission. 


\begin{tabular}{|c|c|c|c|c|c|c|c|c|c|}
\hline 3086 & $08: 36: 26.0$ & 3060.5 & 95 & 18 & 51 & 28 & 8 & 97 & 97 \\
\hline 3101.5 & $08: 36: 41.5$ & 2520.5 & 95 & 15 & 25 & 13 & 15 & 24.9566980658624 & 24.9566980658624 \\
\hline 3092.5 & $08: 36: 32.5$ & 2160.5 & 95 & 13 & 24 & 1 & 19 & 19.5688205404824 & 19.5688205404824 \\
\hline 3119 & $08: 36: 59.0$ & 1980.5 & 95 & 12 & 15 & 3 & 28 & 19.2069067504596 & 19.2069067504596 \\
\hline 3131.5 & $08: 37: 11.5$ & 2700.5 & 95 & 16 & 21 & 13 & 15 & 28.5398107125153 & 28.5398107125153 \\
\hline 3163.5 & $08: 37: 43.5$ & 2880.5 & 95 & 17 & 20 & 9 & 15 & 67 & 67 \\
\hline 3172.5 & $08: 37: 52.5$ & 2520.5 & 95 & 15 & 23 & 3 & 25 & 77 & 77 \\
\hline 3173.5 & 08:37:53.5 & 2160.5 & 95 & 13 & 7 & 1 & 14 & 44.5 & 44.5 \\
\hline 3218.5 & $08: 38: 38.5$ & 2340.5 & 95 & 14 & 42 & 7 & 25 & 43.3106773439273 & 43.3106773439273 \\
\hline 3239 & $08: 38: 59.0$ & 1980.5 & 95 & 12 & 52 & 3 & 28 & 7.28084833846673 & 7.28084833846673 \\
\hline 3236.5 & $08: 38: 56.5$ & 2700.5 & 95 & 16 & 50 & 13 & 15 & 33.0503709327271 & 33.0503709327271 \\
\hline 3267 & $08: 39: 27.0$ & 3240.5 & 95 & 19 & 51 & 19 & 9 & 77 & 77 \\
\hline 3256.5 & $08: 39: 16.5$ & 3060.5 & 95 & 18 & 16 & 28 & 8 & 11.7016795737678 & 11.7016795737678 \\
\hline 3283.5 & $08: 39: 43.5$ & 2160.5 & 95 & 13 & 15 & 1 & 14 & 38.4944297014503 & 38.4944297014503 \\
\hline 3294.5 & $08: 39: 54.5$ & 2880.5 & 95 & 17 & 21 & 9 & 15 & 45.2037699409313 & 45.2037699409313 \\
\hline 3300.5 & $08: 40: 00.5$ & 2700.5 & 95 & 16 & 25 & 13 & 15 & 26.6685695871849 & 26.6685695871849 \\
\hline 3339 & $08: 40: 39.0$ & 2520.5 & 95 & 15 & 42 & 3 & 25 & 0.143383196888053 & 0.143383196888053 \\
\hline 3332.5 & $08: 40: 32.5$ & 2340.5 & 95 & 14 & 24 & 7 & 25 & 18.2476665302477 & 18.2476665302477 \\
\hline 3357 & 08:40:57.0 & 3060.5 & 95 & 18 & 20 & 5 & 18 & 64.5 & 64.5 \\
\hline 3393 & $08: 41: 33.0$ & 2520.5 & 95 & 15 & 24 & 3 & 25 & 17.5281466034446 & 17.5281466034446 \\
\hline 3412.5 & $08: 41: 52.5$ & 2340.5 & 95 & 14 & 7 & 4 & 22 & 72 & 72 \\
\hline 3413.5 & $08: 41: 53.5$ & 2700.5 & 95 & 16 & 23 & 7 & 24 & 84.5 & 84.5 \\
\hline 3416 & $08: 41: 56.0$ & 2880.5 & 95 & 17 & 50 & 9 & 15 & 0 & 0 \\
\hline 3420 & $08: 42: 00.0$ & 3240.5 & 95 & 19 & 16 & 19 & 9 & 35.5914647586737 & 35.5914647586737 \\
\hline 3447 & $08: 42: 27.0$ & 2880.5 & 95 & 17 & 25 & 9 & 15 & 35.7159251832657 & 35.7159251832657 \\
\hline 3436 & $08: 42: 16.0$ & 2160.5 & 95 & 13 & 52 & 1 & 14 & 42.2990516638717 & 42.2990516638717 \\
\hline 3472 & $08: 42: 52.0$ & 2520.5 & 95 & 15 & 7 & 0 & 20 & 57 & 57 \\
\hline 3485 & $08: 43: 05.0$ & 3060.5 & 95 & 18 & 21 & 5 & 18 & 55.1546256119374 & 55.1546256119374 \\
\hline 3505.5 & $08: 43: 25.5$ & 3480.5 & 95 & 20 & 51 & 29 & 8 & 99.5 & 99.5 \\
\hline 3525 & $08: 43: 45.0$ & 2340.5 & 95 & 14 & 15 & 4 & 22 & 49.2666795560478 & 49.2666795560478 \\
\hline 3533.5 & $08: 43: 53.5$ & 2880.5 & 95 & 17 & 23 & 0 & 23 & 64.5 & 64.5 \\
\hline 3547 & 08:44:07.0 & 3240.5 & 95 & 19 & 20 & 11 & 16 & 74.5 & 74.5 \\
\hline 3581 & $08: 44: 41.0$ & 2700.5 & 95 & 16 & 42 & 7 & 24 & 28.303029537133 & 28.303029537133 \\
\hline 3589 & $08: 44: 49.0$ & 2520.5 & 95 & 15 & 15 & 0 & 20 & 14.7797399089687 & 14.7797399089687 \\
\hline 3615 & $08: 45: 15.0$ & 3060.5 & 95 & 18 & 50 & 5 & 18 & 24.6661945810962 & 24.6661945810962 \\
\hline 3643 & $08: 45: 43.0$ & 2700.5 & 95 & 16 & 24 & 7 & 24 & 31.0556529278476 & 31.0556529278476 \\
\hline 3670 & $08: 46: 10.0$ & 3060.5 & 95 & 18 & 25 & 5 & 18 & 10.8625354505967 & 10.8625354505967 \\
\hline 3676.5 & $08: 46: 16.5$ & 3480.5 & 95 & 20 & 16 & 29 & 8 & 12.1156619534737 & 12.1156619534737 \\
\hline 3687 & $08: 46: 27.0$ & 3240.5 & 95 & 19 & 21 & 11 & 16 & 20.293115927708 & 20.293115927708 \\
\hline 3701.5 & $08: 46: 41.5$ & 2880.5 & 95 & 17 & 42 & 0 & 23 & 22.8139938790113 & 22.8139938790113 \\
\hline 3679.5 & $08: 46: 19.5$ & 2340.5 & 95 & 14 & 52 & 4 & 22 & 27.6033627495755 & 27.6033627495755 \\
\hline
\end{tabular}

Reproduced with permission of the copyright owner. Further reproduction prohibited without permission. 


\begin{tabular}{|c|c|c|c|c|c|c|c|c|c|}
\hline 3713 & 08:46:53.0 & 2700.5 & 95 & 16 & 7 & 1 & 21 & 62 & 62 \\
\hline 3719 & $08: 46: 59.0$ & 2520.5 & 95 & 15 & 52 & 0 & 20 & 15.1548953894126 & 15.1548953894126 \\
\hline 3746 & $08: 47: 26.0$ & 3720.5 & 95 & 21 & 51 & 21 & 8 & 79.5 & 79.5 \\
\hline 3772.5 & $08: 47: 52.5$ & 3060.5 & 95 & 18 & 23 & 8 & 23 & 84.5 & 84.5 \\
\hline 3775.5 & $08: 47: 55.5$ & 3480.5 & 95 & 20 & 20 & 14 & 19 & 89.5 & 89.5 \\
\hline 3758.5 & $08: 47: 38.5$ & 2880.5 & 95 & 17 & 24 & 0 & 23 & 15.6802729934569 & 15.6802729934569 \\
\hline 3784.5 & $08: 48: 04.5$ & 3240.5 & 95 & 19 & 50 & 11 & 16 & 29.2068057900778 & 29.2068057900778 \\
\hline 3822.5 & $08: 48: 42.5$ & 2700.5 & 95 & 16 & 15 & 1 & 21 & 21.189326216139 & 21.189326216139 \\
\hline 3845 & $08: 49: 05.0$ & 3240.5 & 95 & 19 & 25 & 11 & 16 & 0 & 0 \\
\hline 3834 & $08: 48: 54.0$ & 2880.5 & 95 & 17 & 7 & 2 & 16 & 52 & 52 \\
\hline 3894.5 & $08: 49: 54.5$ & 3240.5 & 95 & 19 & 23 & 3 & 25 & 77 & 77 \\
\hline 3899.5 & $08: 49: 59.5$ & 3720.5 & 95 & 21 & 16 & 21 & 8 & 37.0796820626057 & 37.0796820626057 \\
\hline 3926 & $08: 50: 26.0$ & 3480.5 & 95 & 20 & 21 & 14 & 19 & 12.2899039012735 & 12.2899039012735 \\
\hline 3939.5 & $08: 50: 39.5$ & 3060.5 & 95 & 18 & 42 & 8 & 23 & 34.7271146053383 & 34.7271146053383 \\
\hline 3945.5 & $08: 50: 45.5$ & 2880.5 & 95 & 17 & 15 & 2 & 16 & 26.6992100535395 & 26.6992100535395 \\
\hline 3959 & 08:50:59.0 & 2700.5 & 95 & 16 & 52 & 1 & 21 & 31.5222598313149 & 31.5222598313149 \\
\hline 4010.5 & $08: 51: 50.5$ & 3480.5 & 95 & 20 & 50 & 14 & 19 & 17.5260660874827 & 17.5260660874827 \\
\hline 3986 & $08: 51: 26.0$ & 3960.5 & 95 & 22 & 51 & 17 & 8 & 69.5 & 69.5 \\
\hline 4008 & $08: 51: 48.0$ & 3060.5 & 95 & 18 & 24 & 8 & 23 & 32.2580614143162 & 32.2580614143162 \\
\hline 4026.5 & 08:52:06.5 & 3720.5 & 95 & 21 & 20 & 15 & 16 & 84.5 & 84.5 \\
\hline 4057 & $08: 52: 37.0$ & 3480.5 & 95 & 20 & 25 & 14 & 19 & 20.1878627712418 & 20.1878627712418 \\
\hline 4079 & $08: 52: 59.0$ & 2880.5 & 95 & 17 & 52 & 2 & 16 & 13.5816157059634 & 13.5816157059634 \\
\hline 4062 & $08: 52: 42.0$ & 3240.5 & 95 & 19 & 42 & 3 & 25 & 26.625886904377 & 26.625886904377 \\
\hline 4072 & 08:52:52.0 & 3060.5 & 95 & 18 & 7 & 1 & 22 & 64.5 & 64.5 \\
\hline 4131 & 08:53:51.0 & 3480.5 & 95 & 20 & 23 & 5 & 29 & 92 & 92 \\
\hline 4123.5 & $08: 53: 43.5$ & 3240.5 & 95 & 19 & 24 & 3 & 25 & 25.9107241235012 & 25.9107241235012 \\
\hline 4128 & $08: 53: 48.0$ & 3960.5 & 95 & 22 & 16 & 17 & 8 & 23.8041358846617 & 23.8041358846617 \\
\hline 4175 & $08: 54: 35.0$ & 3720.5 & 95 & 21 & 21 & 15 & 16 & 21.6590086922245 & 21.6590086922245 \\
\hline 4185.5 & $08: 54: 45.5$ & 3060.5 & 95 & 18 & 15 & 1 & 22 & 53.3741020803076 & 53.3741020803076 \\
\hline 4194.5 & $08: 54: 54.5$ & 3240.5 & 95 & 19 & 7 & 1 & 19 & 57 & 57 \\
\hline 4226.5 & $08: 55: 26.5$ & 4200.5 & 95 & 23 & 51 & 12 & 8 & 57 & 57 \\
\hline 4272 & $08: 56: 12.0$ & 3720.5 & 95 & 21 & 50 & 15 & 16 & 9.47203578202862 & 9.47203578202862 \\
\hline 4241 & $08: 55: 41.0$ & 3960.5 & 95 & 22 & 20 & 16 & 15 & 84.5 & 84.5 \\
\hline 4298.5 & $08: 56: 38.5$ & 3480.5 & 95 & 20 & 42 & 5 & 29 & 16.5083276733241 & 16.5083276733241 \\
\hline 4306 & $08: 56: 46.0$ & 3240.5 & 95 & 19 & 15 & 1 & 19 & 19.2484204738052 & 19.2484204738052 \\
\hline 4311.5 & $08: 56: 51.5$ & 3720.5 & 95 & 21 & 25 & 15 & 16 & 25.0472432560491 & 25.0472432560491 \\
\hline 4340 & $08: 57: 20.0$ & 3060.5 & 95 & 18 & 52 & 1 & 22 & 36.2744676634914 & 36.2744676634914 \\
\hline 4352 & $08: 57: 32.0$ & 3480.5 & 95 & 20 & 24 & 5 & 29 & 3.74275448956048 & 3.74275448956048 \\
\hline 4361 & $08: 57: 41.0$ & 4200.5 & 95 & 23 & 16 & 12 & 8 & 23.6964912551724 & 23.6964912551724 \\
\hline 4375.5 & 08:57:55.5 & 3720.5 & 95 & 21 & 23 & 11 & 27 & 102 & 102 \\
\hline 4389.5 & 08:58:09.5 & 3960.5 & 95 & 22 & 21 & 16 & 15 & 12.9703745153732 & 12.9703745153732 \\
\hline 4433.5 & $08: 58: 53.5$ & 3480.5 & 95 & 20 & 7 & 4 & 24 & 77 & 77 \\
\hline
\end{tabular}

Reproduced with permission of the copyright owner. Further reproduction prohibited without permission. 


\begin{tabular}{|c|c|c|c|c|c|c|c|c|c|}
\hline 4439.5 & $08: 58: 59.5$ & 3240.5 & 95 & 19 & 52 & 1 & 19 & 26.1875991525283 & 26.1875991525283 \\
\hline 4477 & $08: 59: 37.0$ & 3960.5 & 95 & 22 & 50 & 16 & 15 & 36.3476847942022 & 36.3476847942022 \\
\hline 4465 & 08:59:25.0 & 4440.5 & 95 & 24 & 51 & 21 & 8 & 79.5 & 79.5 \\
\hline 4479 & $08: 59: 39.0$ & 4200.5 & 95 & 23 & 20 & 13 & 14 & 74.5 & 74.5 \\
\hline 4539 & 09:00:39.0 & 3720.5 & 95 & 21 & 42 & 11 & 27 & 30.372170229074 & 30.372170229074 \\
\hline 4543 & 09:00:43.0 & 3960.5 & 95 & 22 & 25 & 16 & 15 & 14.0515556003056 & 14.0515556003056 \\
\hline 4544.5 & $09: 00: 44.5$ & 3480.5 & 95 & 20 & 15 & 4 & 24 & 32.0448772867643 & 32.0448772867643 \\
\hline 4603 & 09:01:43.0 & 3720.5 & 95 & 21 & 24 & 11 & 27 & 31.1350461911719 & 31.1350461911719 \\
\hline 4613 & 09:01:53.0 & 3960.5 & 95 & 22 & 23 & 3 & 26 & 79.5 & 79.5 \\
\hline 4620.5 & 09:02:00.5 & 4440.5 & 95 & 24 & 16 & 21 & 8 & 15.7389234860793 & 15.7389234860793 \\
\hline 4621.5 & 09:02:01.5 & 4200.5 & 95 & 23 & 21 & 13 & 14 & 12.2836130248426 & 12.2836130248426 \\
\hline 4673.5 & $09: 02: 53.5$ & 3720.5 & 95 & 21 & 7 & 1 & 27 & 77 & 77 \\
\hline 4677.5 & $09: 02: 57.5$ & 3480.5 & 95 & 20 & 52 & 4 & 24 & 39.279605069313 & 39.279605069313 \\
\hline 4706 & 09:03:26.0 & 4680.5 & 95 & 25 & 51 & 20 & 8 & 77 & 77 \\
\hline 4712 & 09:03:32.0 & 4200.5 & 95 & 23 & 50 & 13 & 14 & 4.15574188593224 & 4.15574188593224 \\
\hline 4730.5 & 09:03:50.5 & 4440.5 & 95 & 24 & 20 & 12 & 16 & 77 & 77 \\
\hline 4748.5 & 09:04:08.5 & 4200.5 & 95 & 23 & 25 & 13 & 14 & 5.06061638903156 & 5.06061638903156 \\
\hline 4785 & $09: 04: 45.0$ & 3720.5 & 95 & 21 & 15 & 1 & 27 & 17.0526883141438 & 17.0526883141438 \\
\hline 4781 & $09: 04: 41.0$ & 3960.5 & 95 & 22 & 42 & 3 & 26 & 8.0156513865455 & 8.0156513865455 \\
\hline 4833.5 & 09:05:33.5 & 3960.5 & 95 & 22 & 24 & 3 & 26 & 17.1927505673281 & 17.1927505673281 \\
\hline 4855.5 & 09:05:55.5 & 4200.5 & 95 & 23 & 23 & 7 & 23 & 82 & 82 \\
\hline 4856.5 & $09: 05: 56.5$ & 4680.5 & 95 & 25 & 16 & 20 & 8 & 5.29336716021017 & 5.29336716021017 \\
\hline 4876 & $09: 06: 16.0$ & 4440.5 & 95 & 24 & 21 & 12 & 16 & 16.2282314832981 & 16.2282314832981 \\
\hline 4919 & 09:06:59.0 & 3720.5 & 95 & 21 & 52 & 1 & 27 & 22.3055422753444 & 22.3055422753444 \\
\hline 4914 & $09: 06: 54.0$ & 3960.5 & 95 & 22 & 7 & 2 & 21 & 64.5 & 64.5 \\
\hline 4951 & 09:07:31.0 & 4680.5 & 95 & 25 & 20 & 11 & 16 & 74.5 & 74.5 \\
\hline 4968.5 & $09: 07: 48.5$ & 4440.5 & 95 & 24 & 50 & 12 & 16 & 0 & 0 \\
\hline 4947.5 & $09: 07: 27.5$ & 4920.5 & 95 & 26 & 51 & 16 & 8 & 67 & 67 \\
\hline 5001.5 & 09:08:21.5 & 4440.5 & 95 & 24 & 25 & 12 & 16 & 20.9663978509401 & 20.9663978509401 \\
\hline 5021.5 & $09: 08: 41.5$ & 4200.5 & 95 & 23 & 42 & 7 & 23 & 0 & 0 \\
\hline 5030 & $09: 08-50.0$ & 3960.5 & 95 & 22 & 15 & 2 & 21 & 12.3463063881264 & 12.3463063881264 \\
\hline 5076 & 09:09:36.0 & 4200.5 & 95 & 23 & 24 & 7 & 23 & 12.6588642997845 & 12.6588642997845 \\
\hline 5089 & $09: 09: 49.0$ & 4680.5 & 95 & 25 & 21 & 11 & 16 & 29.6445976483914 & 29.6445976483914 \\
\hline 5090.5 & 09:09:50.5 & 4920.5 & 95 & 26 & 16 & 16 & 8 & 20.4897853294716 & 20.4897853294716 \\
\hline 5094.5 & $09: 09: 54.5$ & 4440.5 & 95 & 24 & 23 & 5 & 26 & 84.5 & 84.5 \\
\hline 5158.5 & $09: 10: 58.5$ & 3960.5 & 95 & 22 & 52 & 2 & 21 & 3.77946960685088 & 3.77946960685088 \\
\hline 5155 & $09: 10: 55.0$ & 4200.5 & 95 & 23 & 7 & 1 & 21 & 62 & 62 \\
\hline 5185 & $09: 11: 25.0$ & 5160.5 & 95 & 27 & 51 & 20 & 8 & 77 & 77 \\
\hline 5193.5 & $09: 11: 33.5$ & 4680.5 & 95 & 25 & 50 & 11 & 16 & 19.6499393782459 & 19.6499393782459 \\
\hline 5202.5 & $09: 11: 42.5$ & 4920.5 & 95 & 26 & 20 & 15 & 15 & 82 & 82 \\
\hline 5244 & $09: 12: 24.0$ & 4680.5 & 95 & 25 & 25 & 11 & 16 & 16.5429576075664 & 16.5429576075664 \\
\hline 5264.5 & $09: 12: 44.5$ & 4440.5 & 95 & 24 & 42 & 5 & 26 & 20.9501583037169 & 20.9501583037169 \\
\hline
\end{tabular}

Reproduced with permission of the copyright owner. Further reproduction prohibited without permission. 


\begin{tabular}{|c|c|c|c|c|c|c|c|c|c|}
\hline 5270 & $09: 12: 50.0$ & 4200.5 & 95 & 23 & 15 & 1 & 21 & 25.3010242162322 & 25.3010242162322 \\
\hline 5320.5 & $09: 13: 40.5$ & 4440.5 & 95 & 24 & 24 & 5 & 26 & 24.0670833095815 & 24.0670833095815 \\
\hline 5333 & $09: 13: 53.0$ & 4680.5 & 95 & 25 & 23 & 5 & 25 & 82 & 82 \\
\hline 5335 & $09: 13: 55.0$ & 5160.5 & 95 & 27 & 16 & 20 & 8 & 8.99609205785535 & 8.99609205785535 \\
\hline 5349.5 & $09: 14: 09.5$ & 4920.5 & 95 & 26 & 21 & 15 & 15 & 32.580970474208 & 32.580970474208 \\
\hline 5395.5 & $09: 14: 55.5$ & 4440.5 & 95 & 24 & 7 & 1 & 21 & 62 & 62 \\
\hline 5400.5 & 09:15:00.5 & 4200.5 & 95 & 23 & 52 & 1 & 21 & 13.61664373854 & 13.61664373854 \\
\hline 5433 & $09: 15: 33.0$ & 5160.5 & 95 & 27 & 20 & 14 & 16 & 82 & 82 \\
\hline 5426.5 & $09: 15: 26.5$ & 5400.5 & 95 & 28 & 51 & 23 & 8 & 84.5 & 84.5 \\
\hline 5460.5 & $09: 16: 00.5$ & 4920.5 & 95 & 26 & 50 & 15 & 15 & 8.74029392445983 & 8.74029392445983 \\
\hline 5500.5 & $09: 16: 40.5$ & 4680.5 & 95 & 25 & 42 & 5 & 25 & 9.18784937144202 & 9.18784937144202 \\
\hline 5500.5 & $09: 16: 40.5$ & 4920.5 & 95 & 26 & 25 & 15 & 15 & 25.3067173453278 & 25.3067173453278 \\
\hline 5510 & $09: 16: 50.0$ & 4440.5 & 95 & 24 & 15 & 1 & 21 & 19.488750675755 & 19.488750675755 \\
\hline 5588 & 09:18:08.0 & 5400.5 & 95 & 28 & 16 & 23 & 8 & 0.10553875567286 & 0.10553875567286 \\
\hline 5554 & $09: 17: 34.0$ & 4680.5 & 95 & 25 & 24 & 5 & 25 & 11.3271640958308 & 11.3271640958308 \\
\hline 5574.5 & $09: 17: 54.5$ & 4920.5 & 95 & 26 & 23 & 5 & 25 & 82. & 82 \\
\hline 5578.5 & $09: 17: 58.5$ & 5160.5 & 95 & 27 & 21 & 14 & 16 & 14.5694630291844 & 14.5694630291844 \\
\hline 5634 & 09:18:54.0 & 4680.5 & 95 & 25 & 7 & 1 & 21 & 62 & 62 \\
\hline 5641 & $09: 19=01.0$ & 4440.5 & 95 & 24 & 52 & 1 & 21 & 28.9065577999884 & 28.9065577999884 \\
\hline 5666 & $09: 19: 26.0$ & 5640.5 & 95 & 29 & 51 & 19 & 8 & 74.5 & 74.5 \\
\hline 5666.5 & $09: 19: 26.5$ & 5160.5 & 95 & 27 & 50 & 14 & 16 & 0 & 0 \\
\hline 5682.5 & $09: 19: 42.5$ & 5400.5 & 95 & 28 & 20 & 17 & 17 & 92 & 92 \\
\hline 5741 & $09: 20: 41.0$ & 4920.5 & 95 & 26 & 42 & 5 & 25 & 12.8237554008622 & 12.8237554008622 \\
\hline 5697 & $09: 19: 57.0$ & 5160.5 & 95 & 27 & 25 & 14 & 16 & 6.39417429101949 & 6.39417429101949 \\
\hline 5745.5 & $09: 20: 45.5$ & 4680.5 & 95 & 25 & 15 & 1 & 21 & 14.9165469343161 & 14.9165469343161 \\
\hline 5795.5 & $09: 21: 35.5$ & 4920.5 & 95 & 26 & 24 & 5 & 25 & 22.9048427862369 & 22.9048427862369 \\
\hline 5804.5 & $09: 21: 44.5$ & 5640.5 & 95 & 29 & 16 & 0 & 0 & 0 & 0 \\
\hline 5812 & $09: 21: 52.0$ & 5160.5 & 95 & 27 & 23 & 8 & 26 & 92 & 92 \\
\hline 5843 & $09: 22: 23.0$ & 5400.5 & 95 & 28 & 21 & 17 & 17 & 3.2860347695154 & 3.2860347695154 \\
\hline 5879 & $09: 22: 59.0$ & 4680.5 & 95 & 25 & 52 & 1 & 21 & 37.8215949947311 & 37.8215949947311 \\
\hline 5874 & $09: 22: 54.0$ & 4920.5 & 95 & 26 & 7 & 0 & 21 & 59.5 & 59.5 \\
\hline 5891.5 & $09: 23: 11.5$ & 5640.5 & 95 & 29 & 20 & 8 & 16 & 67 & 67 \\
\hline 5906 & $09: 23: 26.0$ & 5880.5 & 95 & 30 & 51 & 12 & 8 & 57 & 57 \\
\hline 5924.5 & $09: 23: 44.5$ & 5400.5 & 95 & 28 & 50 & 17 & 17 & 18.4728386632098 & 18.4728386632098 \\
\hline 5985 & $09: 24: 45.0$ & 4920.5 & 95 & 26 & 15 & 0 & 21 & 23.7798581361778 & 23.7798581361778 \\
\hline 5975 & $09: 24: 35.0$ & 5400.5 & 95 & 28 & 25 & 17 & 17 & 26.1032905974223 & 26.1032905974223 \\
\hline 5980.5 & $09: 24: 40.5$ & 5160.5 & 95 & 27 & 42 & 8 & 26 & 3.44662979712462 & 3.44662979712462 \\
\hline 6022.5 & $09: 25: 22.5$ & 5640.5 & 95 & 29 & 21 & 8 & 16 & 4.42756581463529 & 4.42756581463529 \\
\hline 6034 & $09: 25: 34.0$ & 5160.5 & 95 & 27 & 24 & 8 & 26 & 27.1119473786949 & 27.1119473786949 \\
\hline 6035.5 & $09: 25: 35.5$ & 5880.5 & 95 & 30 & 16 & 12 & 8 & 13.9237116884215 & 13.9237116884215 \\
\hline 6055.5 & $09: 25: 55.5$ & 5400.5 & 95 & 28 & 23 & 7 & 29 & 97 & 97 \\
\hline 6102 & $09: 26: 42.0$ & 5640.5 & 95 & 29 & 50 & 8 & 16 & 23.9712883692326 & 23.9712883692326 \\
\hline 6114 & $09: 26: 54.0$ & 5160.5 & 95 & 27 & 7 & 3 & 24 & 74.5 & 74.5 \\
\hline
\end{tabular}

Reproduced with permission of the copyright owner. Further reproduction prohibited without permission. 


\begin{tabular}{|c|c|c|c|c|c|c|c|c|c|}
\hline 6120 & $09: 27: 00.0$ & 4920.5 & 95 & 26 & 52 & 0 & 21 & 19.6718139861789 & 19.6718139861789 \\
\hline 6138 & $09: 27: 18.0$ & 5880.5 & 95 & 30 & 20 & 7 & 14 & 59.5 & 59.5 \\
\hline 6156 & $09: 27: 36.0$ & 5640.5 & 95 & 29 & 25 & 8 & 16 & 38.0878146071646 & 38.0878146071646 \\
\hline 6146.5 & $09: 27: 26.5$ & 6120.5 & 95 & 31 & 51 & 5 & 8 & 39.5 & 39.5 \\
\hline 6219.5 & $09: 28: 39.5$ & 5160.5 & 95 & 27 & 15 & 3 & 24 & 6.66599889247703 & 6.66599889247703 \\
\hline 6222 & $09: 28: 42.0$ & 5400.5 & 95 & 28 & 42 & 7 & 29 & 10.7026956547843 & 10.7026956547843 \\
\hline 6250 & 09:29:10.0 & 5880.5 & 95 & 30 & 21 & 0 & 0 & 0 & 0 \\
\hline 6276 & $09: 29: 36.0$ & 5400.5 & 95 & 28 & 24 & 7 & 29 & 13.4475356103968 & 13.4475356103968 \\
\hline 6264 & 09:29:24.0 & 6120.5 & 95 & 31 & 16 & 5 & 8 & 18.2622089357901 & 18.2622089357901 \\
\hline 6293.5 & $09: 29: 53.5$ & 5640.5 & 95 & 29 & 23 & 8 & 23 & 84.5 & 84.5 \\
\hline 6326 & $09: 30: 26.0$ & 5160.5 & 95 & 27 & 52 & 3 & 24 & 31.940801226161 & 31.940801226161 \\
\hline 6323.5 & $09: 30: 23.5$ & 5880.5 & 95 & 30 & 50 & 0 & 0 & 13.2403125421837 & 13.2403125421837 \\
\hline 6355 & $09: 30: 55.0$ & 5400.5 & 95 & 28 & 7 & 3 & 25 & 77 & 77 \\
\hline 6366.5 & $09: 31: 06.5$ & 5880.5 & 95 & 30 & 25 & 0 & 0 & 4.59490935936899 & 4.59490935936899 \\
\hline 6378.5 & $09: 31: 18.5$ & 6120.5 & 95 & 31 & 20 & 10 & 12 & 62 & 62 \\
\hline 6387.5 & $09: 31: 27.5$ & 6360.5 & 95 & 32 & 51 & 8 & 8 & 47 & 47 \\
\hline 6412.5 & $09: 31: 52.5$ & 5880.5 & 95 & 30 & 23 & 3 & 20 & 64.5 & 64.5 \\
\hline 6465 & $09: 32: 45.0$ & 5400.5 & 95 & 28 & 15 & 3 & 25 & 31.1792059609331 & 31.1792059609331 \\
\hline 6459.5 & $09: 32: 39.5$ & 5640.5 & 95 & 29 & 42 & 8 & 23 & 10.5236023096192 & 10.5236023096192 \\
\hline 6510.5 & $09: 33: 30.5$ & 6120.5 & 95 & 31 & 21 & 10 & 12 & 17.7938943048758 & 17.7938943048758 \\
\hline 6512.5 & $09: 33: 32.5$ & 6360.5 & 95 & 32 & 16 & 8 & 8 & 16.9861651926325 & 16.9861651926325 \\
\hline 6513.5 & $09: 33: 33.5$ & 5640.5 & 95 & 29 & 24 & 8 & 23 & 3.92747389807214 & 3.92747389807214 \\
\hline 6579 & $09: 34: 39.0$ & 5880.5 & 95 & 30 & 42 & 3 & 20 & 15.4358496228558 & 15.4358496228558 \\
\hline 6601 & $09: 35: 01.0$ & 5400.5 & 95 & 28 & 52 & 3 & 25 & 21.8043619229966 & 21.8043619229966 \\
\hline 6607.5 & $09: 35: 07.5$ & 6120.5 & 95 & 31 & 50 & 10 & 12 & 33.125921396223 & 33.125921396223 \\
\hline 6621.5 & $09: 35: 21.5$ & 6360.5 & 95 & 32 & 20 & 9 & 12 & 59.5 & 59.5 \\
\hline 6593.5 & $09: 34: 53.5$ & 5640.5 & 95 & 29 & 7 & 4 & 21 & 69.5 & 69.5 \\
\hline 6626 & $09: 35: 26.0$ & 6600.5 & 95 & 33 & 51 & 9 & 8 & 49.5 & 49.5 \\
\hline 6633 & $09: 35: 33.0$ & 5880.5 & 95 & 30 & 24 & 3 & 20 & 23.2027227829131 & 23.2027227829131 \\
\hline 6673.5 & $09: 36: 13.5$ & 6120.5 & 95 & 31 & 25 & 10 & 12 & 0.142594699579 & 0.142594699579 \\
\hline 6705 & $09: 36: 45.0$ & 5640.5 & 95 & 29 & 15 & 4 & 21 & 23.5039948765551 & 23.5039948765551 \\
\hline 6713.5 & $09: 36: 53.5$ & 5880.5 & 95 & 30 & 7 & 0 & 16 & 47 & 47 \\
\hline 6750.5 & $09: 37: 30.5$ & 6600.5 & 95 & 33 & 16 & 9 & 8 & 9.2634927830178 & 9.2634927830178 \\
\hline 6776.5 & $09: 37: 56.5$ & 6120.5 & 95 & 31 & 23 & 16 & 19 & 94.5 & 94.5 \\
\hline 6747.5 & $09: 37: 27.5$ & 6360.5 & 95 & 32 & 21 & 9 & 12 & 18.2914109014894 & 18.2914109014894 \\
\hline 6822 & 09:38:42.0 & 5880.5 & 95 & 30 & 15 & 0 & 16 & 28.8570505891686 & 28.8570505891686 \\
\hline 6839 & 09:38:59.0 & 5640.5 & 95 & 29 & 52 & 4 & 21 & 32.1118785517802 & 32.1118785517802 \\
\hline 6844.5 & $09: 39: 04.5$ & 6360.5 & 95 & 32 & 50 & 9 & 12 & 40.5978623003054 & 40.5978623003054 \\
\hline 6865.5 & $09: 39: 25.5$ & 6840.5 & 95 & 34 & 51 & 5 & 8 & 39.5 & 39.5 \\
\hline 6851 & $09: 39: 11.0$ & 6600.5 & 95 & 33 & 20 & 9 & 13 & 62 & 62 \\
\hline 6917.5 & $09: 40: 17.5$ & 6360.5 & 95 & 32 & 25 & 9 & 12 & 25.1264076828181 & 25.1264076828181 \\
\hline 6958.5 & $09: 40: 58.5$ & 5880.5 & 95 & 30 & 52 & 0 & 16 & 40.5808391462845 & 40.5808391462845 \\
\hline 6947 & $09: 40: 47.0$ & 6120.5 & 95 & 34 & 42 & 16 & 19 & 9.528538795536 & 9.528538795536 \\
\hline
\end{tabular}

Reproduced with permission of the copyright owner. Further reproduction prohibited without permission. 


\begin{tabular}{|c|c|c|c|c|c|c|c|c|c|}
\hline 6976.5 & $09: 41: 16.5$ & 6840.5 & 95 & 34 & 16 & 5 & 8 & 36.5970839685049 & 36.5970839685049 \\
\hline 6996 & $09: 41: 36.0$ & 6120.5 & 95 & 31 & 24 & 16 & 19 & 0 & 0 \\
\hline 7015.5 & $09: 41: 55.5$ & 6360.5 & 95 & 32 & 23 & 4 & 20 & 67 & 67 \\
\hline 6978.5 & $09: 41: 18.5$ & 6600.5 & 95 & 33 & 21 & 9 & 13 & 33.2707220892744 & 33.2707220892744 \\
\hline 7075.5 & $09: 42: 55.5$ & 6120.5 & 95 & 31 & 7 & 5 & 24 & 79.5 & 79.5 \\
\hline 7087 & $09: 43: 07.0$ & 6600.5 & 95 & 33 & 50 & 9 & 13 & 15.3258228142802 & 15.3258228142802 \\
\hline 7100 & $09: 43: 20.0$ & 6840.5 & 95 & 34 & 20 & 11 & 12 & 64.5 & 64.5 \\
\hline 7106 & $09: 43: 26.0$ & 7080.5 & 95 & 35 & 51 & 7 & 8 & 44.5 & 44.5 \\
\hline 7133 & 09:43:53.0 & 6600.5 & 95 & 33 & 25 & 9 & 13 & 35.6970750506626 & 35.6970750506626 \\
\hline 7182 & $09: 44: 42.0$ & 6360.5 & 95 & 32 & 42 & 4 & 20 & 28.2816946303335 & 28.2816946303335 \\
\hline 7195 & 09:44:55.0 & 6120.5 & 95 & 31 & 15 & 5 & 24 & 4.44997661544161 & 4.44997661544161 \\
\hline 7213 & $09: 45: 13.0$ & 7080.5 & 95 & 35 & 16 & 0 & 0 & 0 & 0 \\
\hline 7226 & $09: 45: 26.0$ & 6840.5 & 95 & 34 & 21 & 11 & 12 & 20.0434564677048 & 20.0434564677048 \\
\hline 7246 & $09: 45: 46.0$ & 6360.5 & 95 & 32 & 24 & 4 & 20 & 19.5910203146401 & 19.5910203146401 \\
\hline 7253.5 & $09: 45: 53.5$ & 6600.5 & 95 & 33 & 23 & 9 & 20 & 79.5 & 79.5 \\
\hline 7300 & $09: 46: 40.0$ & 7080.5 & 95 & 35 & 20 & 10 & 12 & 62 & 62 \\
\hline 7314 & $09: 46: 54.0$ & 6360.5 & 95 & 32 & 7 & 2 & 16 & 52 & 52 \\
\hline 7321.5 & $09: 47: 01.5$ & 6120.5 & 95 & 31 & 52 & 5 & 24 & 19.0624231850498 & 19.0624231850498 \\
\hline 7351 & $09: 47: 31.0$ & 6840.5 & 95 & 34 & 25 & 11 & 12 & 17.5248523435433 & 17.5248523435433 \\
\hline 7318 & 09:46:58.0 & 6840.5 & 95 & 34 & 50 & 11 & 12 & 3.58440917993988 & 3.58440917993988 \\
\hline 7421.5 & $09: 48: 41.5$ & 6600.5 & 95 & 33 & 42 & 9 & 20 & 2.43932507561824 & 2.43932507561824 \\
\hline 7425 & $09: 48: 45.0$ & 7080.5 & 95 & 35 & 21 & 10 & 12 & 3.00675717633991 & 3.00675717633991 \\
\hline 7425.5 & $09: 48: 45.5$ & 6360.5 & 95 & 32 & 15 & 2 & 16 & 35.1387273006741 & 35.1387273006741 \\
\hline 7474.5 & $09: 49: 34.5$ & 6600.5 & 95 & 33 & 24 & 9 & 20 & 32.9090432907553 & 32.9090432907553 \\
\hline 7492 & $09: 49: 52.0$ & 6840.5 & 95 & 34 & 23 & 6 & 19 & 69.5 & 69.5 \\
\hline 7502.5 & 09:50:02.5 & 7080.5 & 95 & 35 & 50 & 10 & 12 & 19.7813045612462 & 19.7813045612462 \\
\hline 7552 & $09: 50: 52.0$ & 7080.5 & 95 & 35 & 25 & 10 & 12 & 8.84483974387476 & 8.84483974387476 \\
\hline 7554.5 & $09: 50: 54.5$ & 6600.5 & 95 & 33 & 7 & 1 & 20 & 59.5 & 59.5 \\
\hline 7612.5 & $09: 51: 52.5$ & 7080.5 & 95 & 35 & 23 & 6 & 20 & 72 & 72 \\
\hline 7620.5 & $09: 52: 00.5$ & 6360.5 & 95 & 32 & 52 & 2 & 16 & 13.1094724464612 & 13.1094724464612 \\
\hline 7660.5 & $09: 52: 40.5$ & 6840.5 & 95 & 34 & 42 & 6 & 19 & 16.0723240200001 & 16.0723240200001 \\
\hline 7663.5 & $09: 52: 43.5$ & 6600.5 & 95 & 33 & 15 & 1 & 20 & 12.1277774792325 & 12.1277774792325 \\
\hline 7712.5 & $09: 53: 32.5$ & 6840.5 & 95 & 34 & 24 & 6 & 19 & 34.7761975694417 & 34.7761975694417 \\
\hline 7793.5 & 09:54:53.5 & 6840.5 & 95 & 34 & 7 & 5 & 18 & 64.5 & 64.5 \\
\hline 7779 & 09:54:39.0 & 7080.5 & 95 & 35 & 42 & 6 & 20 & 36.944007232616 & 36.944007232616 \\
\hline 7799.5 & $09: 54: 59.5$ & 6600.5 & 95 & 33 & 52 & 1. & 20 & 7.50651086312656 & 7.50651086312656 \\
\hline 7848.5 & $09: 55: 48.5$ & 7080.5 & 95 & 35 & 24 & 6 & 20 & 43.3738742069109 & 43.3738742069109 \\
\hline 7973.5 & $09: 57: 53.5$ & 7080.5 & 95 & 35 & 7 & 0 & 18 & 52 & 52 \\
\hline
\end{tabular}

Reproduced with permission of the copyright owner. Further reproduction prohibited without permission. 
Table D2.2: Route1 -Situation 1200

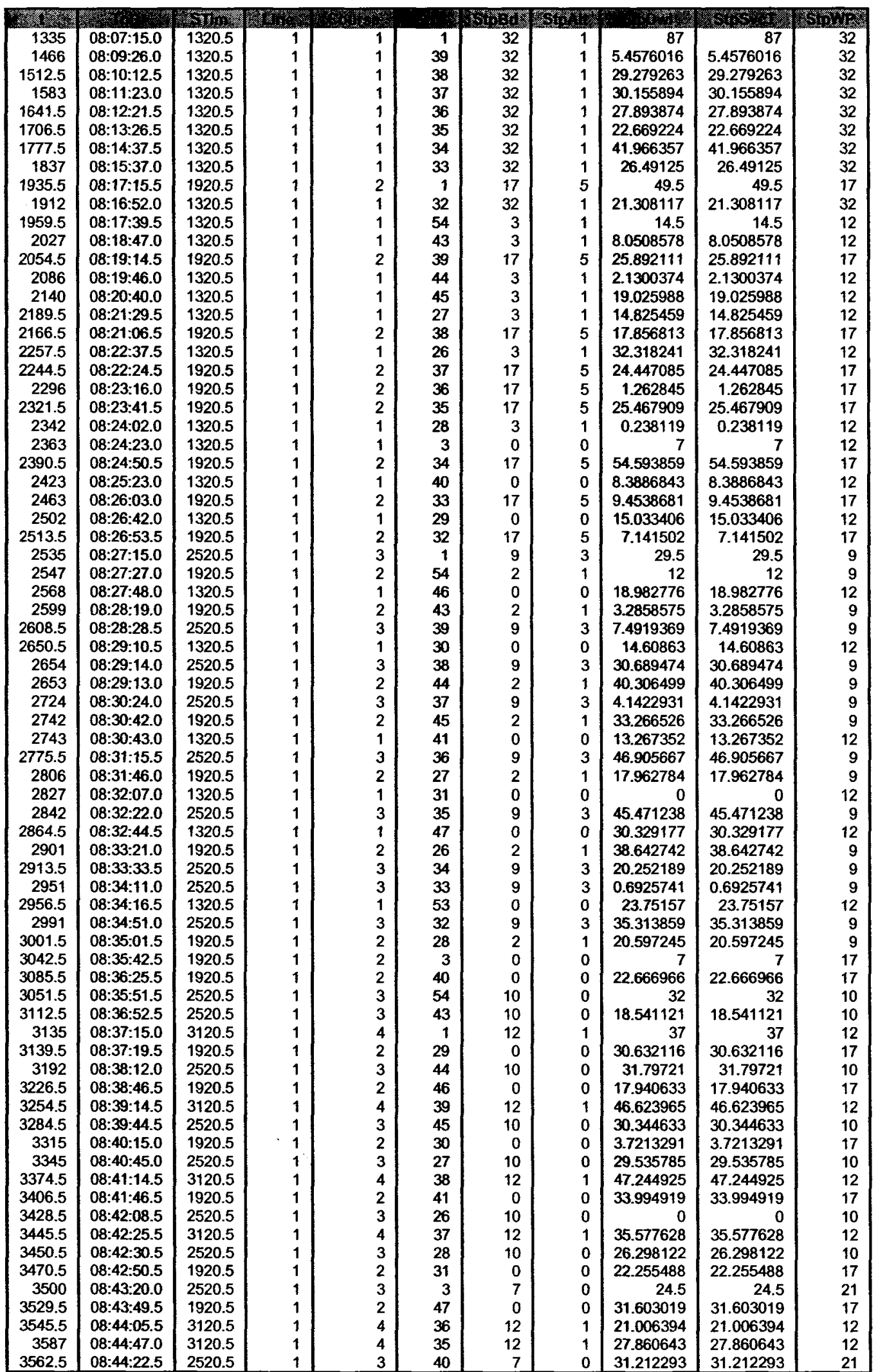




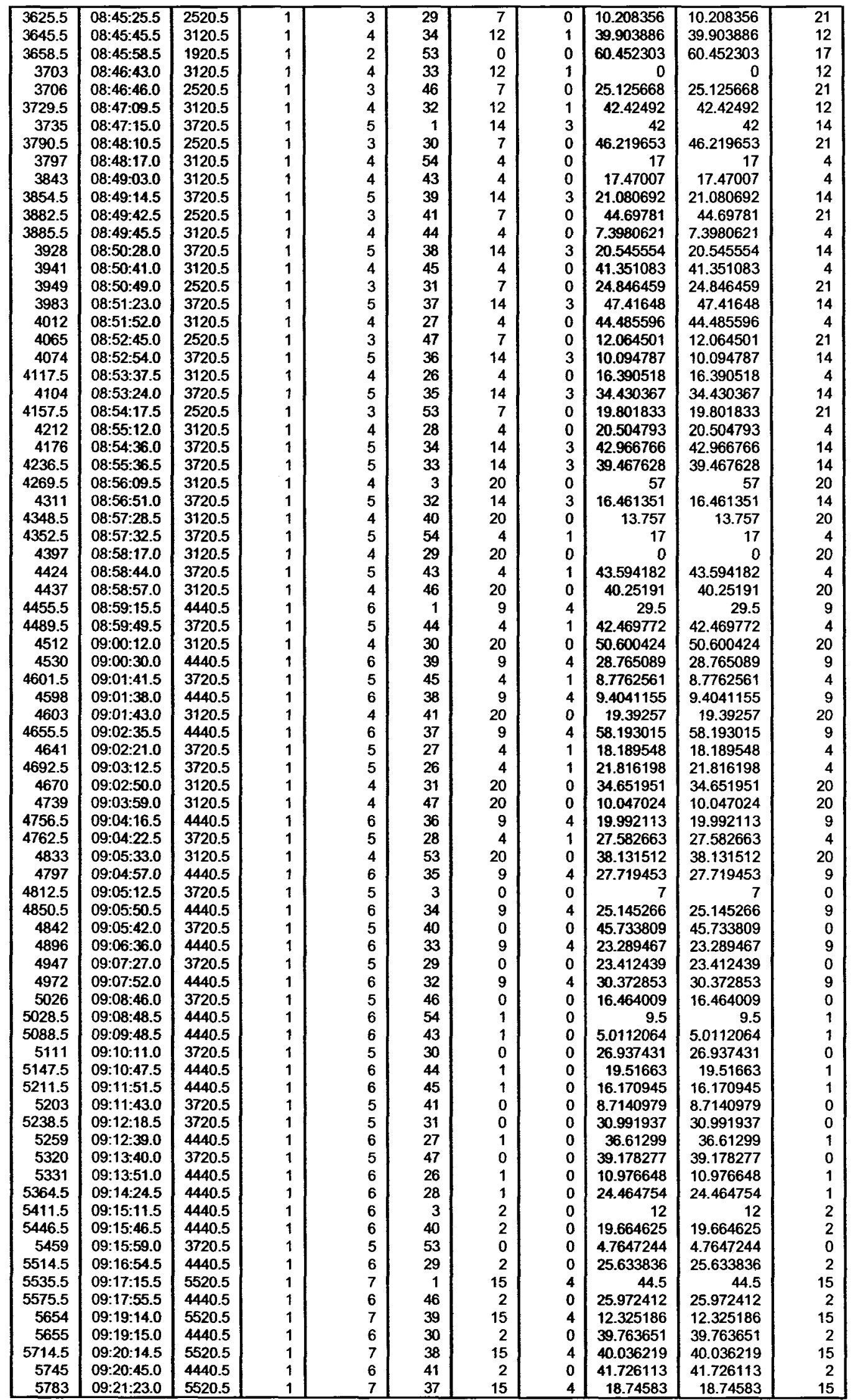




\begin{tabular}{|c|c|c|c|c|c|c|c|c|c|c|}
\hline 5809.5 & $09: 21: 49.5$ & 4440.5 & 1 & 6 & 31 & 2 & 0 & 20.321632 & 20.321632 & 2 \\
\hline 5829 & 09:22:09.0 & 5520.5 & 1 & 7 & 36 & 15 & 4 & 30.83984 & 30.83984 & 15 \\
\hline 5866 & $09: 22: 46.0$ & 4440.5 & 1 & 6 & 47 & 2 & 0 & 15.730392 & 15.730392 & 2 \\
\hline 5879.5 & $09: 22: 59.5$ & 5520.5 & 1 & 7 & 35 & 15 & 4 & 22.113042 & 22.113042 & 15 \\
\hline 5927.5 & $09: 23: 47.5$ & 5520.5 & 1 & 7 & 34 & 15 & 4 & 14.155327 & 14.155327 & 15 \\
\hline 5957.5 & $09: 24: 17.5$ & 4440.5 & 1 & 6 & 53 & 2 & 0 & 37.11808 & 37.11808 & 2 \\
\hline 5974 & $09: 24: 34.0$ & 5520.5 & 1 & 7 & 33 & 15 & 4 & 25.927102 & 25.927102 & 15 \\
\hline 6051 & $09: 25: 51.0$ & 5520.5 & 1 & 7 & 32 & 15 & 4 & 19.132478 & 19.132478 & 15 \\
\hline 6095.5 & $09: 26: 35.5$ & 5520.5 & 1 & 7 & 54 & 3 & 1 & 14.5 & 14.5 & 3 \\
\hline 6164.5 & $09: 27: 44.5$ & 5520.5 & 1 & 7 & 43 & 3 & 1 & 52.390536 & 52.390536 & 3 \\
\hline 6238.5 & $09: 28: 58.5$ & 5520.5 & 1 & 7 & 44 & 3 & 1 & 0 & 0 & 3 \\
\hline 6281.5 & $09: 29: 41.5$ & 5520.5 & 1 & 7 & 45 & 3 & 1 & 6.4722438 & 6.4722438 & 3 \\
\hline 6318 & $09: 30: 18.0$ & 5520.5 & 1 & 7 & 27 & 3 & 1 & 18.891125 & 18.891125 & 3 \\
\hline 6385 & $09: 31: 25.0$ & 5520.5 & 1 & 7 & 26 & 3 & 1 & 8.2249188 & 8.2249188 & 3 \\
\hline 6415 & $09: 31: 55.0$ & 5520.5 & 1 & 7 & 28 & 3 & 1 & 27.883884 & 27.883884 & 3 \\
\hline 6462.5 & $09: 32: 42.5$ & 5520.5 & 1 & 7 & 3 & 4 & 0 & 17 & 17 & 4 \\
\hline 6502.5 & $09: 33: 22.5$ & 5520.5 & 1 & 7 & 40 & 4 & 0 & 26.902577 & 26.902577 & 4 \\
\hline 6569 & $09: 34: 29.0$ & 5520.5 & 1 & 7 & 29 & 4 & 0 & 8.6644114 & 8.6644114 & 4 \\
\hline 6645.5 & $09: 35: 45.5$ & 5520.5 & 1 & 7 & 46 & 4 & 0 & 15.449596 & 15.449596 & 4 \\
\hline 6675 & $09: 36: 15.0$ & 6660.5 & 1 & 8 & 1 & 16 & 2 & 47 & 47 & 16 \\
\hline 6735.5 & $09: 37: 15.5$ & 5520.5 & 1 & 7 & 30 & 4 & 0 & 49.522318 & 49.522318 & 4 \\
\hline 6795 & $09: 38: 15.0$ & 6660.5 & 1 & 8 & 39 & 16 & 2 & 25.28876 & 25.28876 & 16 \\
\hline 6826.5 & $09: 38: 46.5$ & 5520.5 & 1 & 7 & 41 & 4 & 0 & 21.891848 & 21.891848 & 4 \\
\hline 6897 & $09: 39: 57.0$ & 6660.5 & 1 & 8 & 38 & 16 & 2 & 13.235967 & 13.235967 & 16 \\
\hline 6907 & $09: 40: 07.0$ & 5520.5 & 1 & 7 & 31 & 4 & 0 & 17.445411 & 17.445411 & 4 \\
\hline 6960 & $09: 41: 00.0$ & 5520.5 & 1 & 7 & 47 & 4 & 0 & 12.745853 & 12.745853 & 4 \\
\hline 6980.5 & $09: 41: 20.5$ & 6660.5 & 1 & 8 & 36 & 16 & 2 & 25.938058 & 25.938058 & 16 \\
\hline 6940.5 & $09: 40: 40.5$ & 6660.5 & 1 & 8 & 37 & 16 & 2 & 8.755851 & 8.755851 & 16 \\
\hline 7027 & $09: 42: 07.0$ & 6660.5 & 1 & 8 & 35 & 16 & 2 & 30.233526 & 30.233526 & 16 \\
\hline 7080.5 & 09:43:00.5 & 5520.5 & 1 & 7 & 53 & 4 & 0 & 37.665976 & 37.665976 & 4 \\
\hline 7089 & $09: 43: 09.0$ & 6660.5 & 1 & 8 & 34 & 16 & 2 & 24.391749 & 24.391749 & 16 \\
\hline 7131.5 & $09: 43: 51.5$ & 6660.5 & 1 & 8 & 33 & 16 & 2 & 1.7780939 & 1.7780939 & 16 \\
\hline 7160.5 & $09: 44: 20.5$ & 6660.5 & 1 & 8 & 32 & 16 & 2 & 29.271663 & 29.271663 & 16 \\
\hline 7216 & $09: 45: 16.0$ & 6660.5 & 1 & 8 & 54 & 8 & 0 & 27 & 27 & 8 \\
\hline 7273 & $09: 46: 13.0$ & 6660.5 & 1 & 8 & 43 & 8 & 0 & 5.1971143 & 5.1971143 & 8 \\
\hline 7306.5 & $09: 46: 46.5$ & 6660.5 & 1 & 8 & 44 & 8 & 0 & 25.060252 & 25.060252 & 8 \\
\hline 7381 & $09: 48: 01.0$ & 6660.5 & 1 & 8 & 45 & 8 & 0 & 11.438539 & 11.438539 & 8 \\
\hline 7423 & $09: 48: 43.0$ & 6660.5 & 1 & 8 & 27 & 8 & 0 & 11.162477 & 11.162477 & 8 \\
\hline 7462.5 & $09: 49: 22.5$ & 6660.5 & 1 & 8 & 26 & 8 & 0 & 25.012257 & 25.012257 & 8 \\
\hline 7514.5 & $09: 50: 14.5$ & 6660.5 & 1 & 8 & 28 & 8 & 0 & 11.078448 & 11.078448 & 8 \\
\hline 7549 & $09: 50: 49.0$ & 6660.5 & 1 & 8 & 3 & 3 & 0 & 14.5 & 14.5 & 3 \\
\hline 7586.5 & $09: 51: 26.5$ & 6660.5 & 1 & 8 & 40 & 3 & 0 & 34.01282 & 34.01282 & 3 \\
\hline 7680 & 09:53:00.0 & 6660.5 & 1 & 8 & 29 & 3 & 0 & 29.961759 & 29.961759 & 3 \\
\hline 7738 & 09:53:58.0 & 6660.5 & 1 & 8 & 46 & 3 & 0 & 18.006331 & 18.006331 & 3 \\
\hline 7811 & 09:55:11.0 & 6660.5 & 1 & 8 & 30 & 3 & 0 & 20.333498 & 20.333498 & 3 \\
\hline 7815 & $09: 55: 15.0$ & 7800.5 & 1 & 9 & 1 & 11 & 2 & 34.5 & 34.5 & 11 \\
\hline 7903 & $09: 56: 43.0$ & 6660.5 & 1 & 8 & 41 & 3 & 0 & 31.899362 & 31.899362 & 3 \\
\hline 7934.5 & $09: 57: 14.5$ & 7800.5 & 1 & 9 & 39 & 11 & 2 & 13.838277 & 13.838277 & 11 \\
\hline 7971 & $09: 57: 51.0$ & 6660.5 & 1 & 8 & 31 & 3 & 0 & 29.420716 & 29.420716 & 3 \\
\hline 7994 & $09: 58: 14.0$ & 7800.5 & 1 & 9 & 38 & 11 & 2 & 26.232242 & 26.232242 & 11 \\
\hline 8035.5 & 09:58:55.5 & 6660.5 & 1 & 8 & 47 & 3 & 0 & 35.974127 & 35.974127 & 3 \\
\hline 8062.5 & $09: 59: 22.5$ & 7800.5 & 1 & 9 & 37 & 11 & 2 & 40.146187 & 40.146187 & 11 \\
\hline
\end{tabular}




\section{Appendix D3}

\section{General}

This Appendix presents the basic concepts of the Kalman filter (KF) technique, and the applications of this technique to bus running time prediction developed by Reihoundt (1997), Farhan (2002) and Sahalaby et al. (2004).

\section{Fundamental Concepts of Kalman Filter Technique}

To illustrate how the Kalman filter works, Maybeck (1979, pp 8-15) presented a very clear example, as follows.

Suppose that a measurement of any one-dimensional location of a moving point is taken to be $z_{l}$ at time $t_{l}$ and the standard deviation $s d_{z l}$. Thus, the conditional probability of $x\left(t_{1}\right)$, the position at time $t_{1}$ conditioned on the observed value of the measurement being $z_{l}$ is normally distributed with mean $z_{1}$ and standard deviation of $s d_{z l}$.

Based on the conditional probability density, the best estimate of the point location

$$
\hat{x}\left(t_{1}\right)=z_{1}
$$

And the variance of the error in estimate is

$$
s d_{x}^{2}\left(t_{1}\right)=s d_{21}^{2}
$$

Suppose that there is another more accurate measurement of the point location $z_{2}$ of the true position but taken at time $t_{2} \equiv t_{1}$ with new standard variation $s d_{z 2}$. 
With two measurements availabble for the same point location, the problem is how to give the best prediction $x\left(t_{2}\right)$, given $t_{2}=t_{1}$ and already known $z_{1}$ and $z_{2}$. The Kalman filter assumes that the conditional probability density of the new position at time $t_{2}$ is a Gaussian density with mean $\mu$ and standard deviation $s d$ where

$$
\begin{aligned}
& \mu=\left[s d_{z 2}^{2} /\left(s d_{z 1}^{2}+s d_{z 2}^{2}\right)\right] z_{1}+\left[s d_{z 1}^{2} /\left(s d_{z 1}^{2}+s d_{z 2}^{2}\right)\right] z_{2} \\
& 1 / s d^{2}=\left(1 / s d_{z 1}^{2}\right)+\left(1 / s d_{z 2}^{2}\right)
\end{aligned}
$$

The best estimation is, of course, when $\stackrel{\Lambda}{x}\left(t_{2}\right)=\mu$ or

$$
\hat{x}\left(t_{2}\right)=\left[s d_{z 2}^{2} /\left(s d_{z 1}^{2}+s d_{z 2}^{2}\right)\right] z_{1}+\left[s d_{z 1}^{2} /\left(s d_{z 1}^{2}+s d_{z 2}^{2}\right)\right] z_{2}=\left[s d_{z 1}^{2} /\left(s d_{z 1}^{2}+s d_{z 2}^{2}\right)\right]\left(z_{2}-z_{1}\right)
$$

Therefore,

$$
\hat{x}\left(t_{2}\right)=\hat{x}\left(t_{1}\right)+K\left(t_{2}\right)\left[z_{2}-\hat{x}\left(t_{1}\right)\right]
$$

where: $\mathrm{K}$ is a Kalman Gain

$$
K\left(t_{2}\right)=\left[s d_{z 1}^{2} /\left(s d_{z 1}^{2}+s d_{z 2}^{2}\right)\right]
$$

Now the new variance is updated as follows

$$
s d_{x}^{2}\left(t_{2}\right)=s d_{x}^{2}\left(t_{1}\right)-K\left(t_{2}\right) s d_{x}^{2}\left(t_{1}\right)
$$

In conclusion, the system of equations says that the optimal estimate of new state at time $t_{2}, \hat{x}\left(t_{2}\right)$, is equal to the best prediction of its value before $z_{2}$ is actually taken, $\hat{x}\left(t_{1}\right)$, plus a correction term of an optimal weighing value multiplied by the difference between $z_{2}$ and $\stackrel{\Lambda}{x}\left(t_{1}\right)$. 


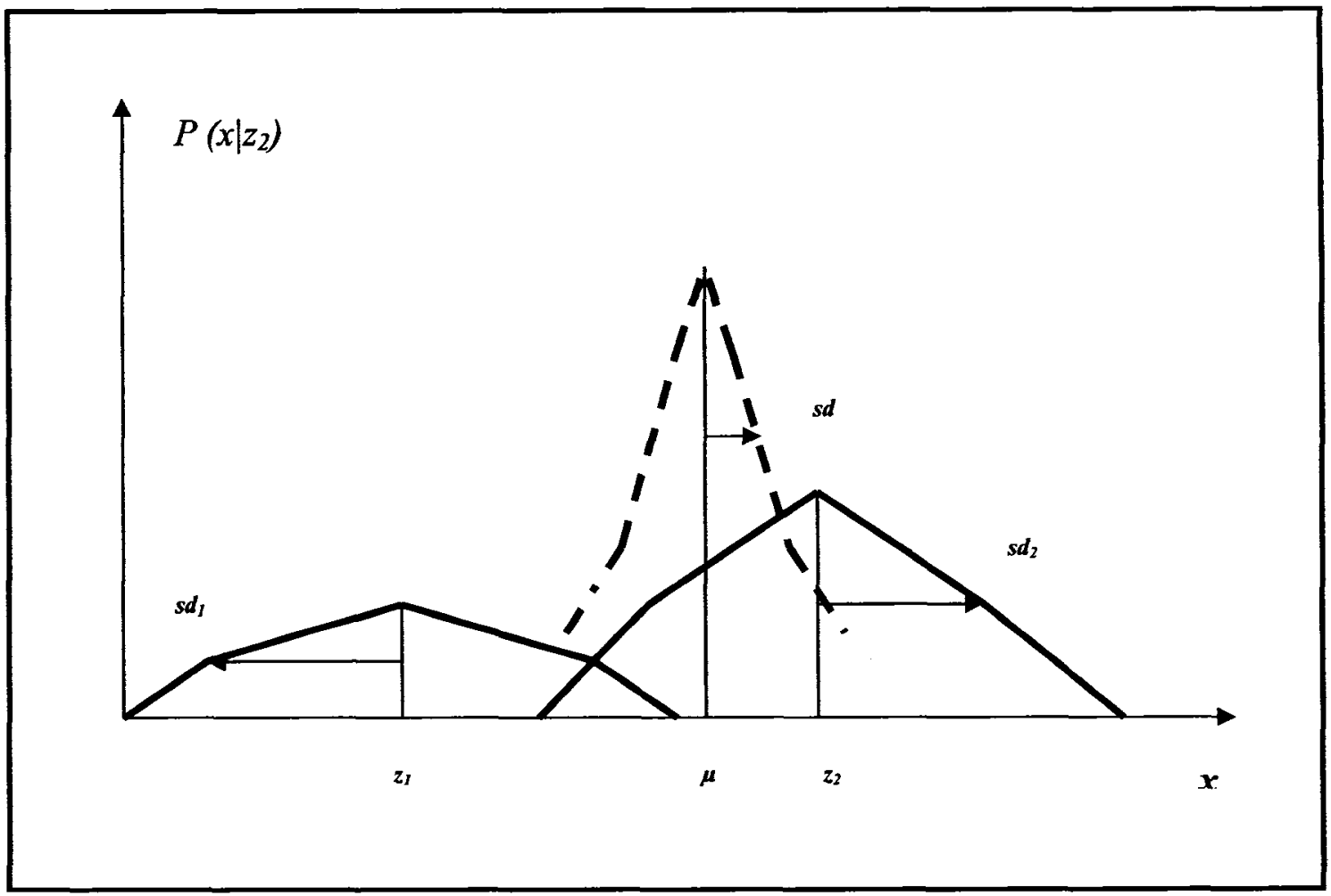

Figure D3.1: Kalman Filter Technique- Basic Concepts (Source: Maybeck 1979)

\section{The KF Model for Bus running time Prediction}

As mentioned in chapter 6 , the Kalman Filter algorithm for bus link running time is the system of 4 main equations

$$
\begin{aligned}
& g(k+1)=\frac{e(k)+V A R\left[\text { data }_{\text {out }}\right]_{k+1}}{e(k)+V A R\left[\text { data }_{\text {out }}\right]_{k+1}+V A R\left[\text { data }_{\text {in }}\right]_{k+1}} \\
& e(k+1)=V A R\left[\text { data }_{\text {in }}\right]_{k+1} \cdot g(k+1) \\
& a(k+1)=1-g(k+1) \\
& P(k+1)=a(k+1) \cdot \operatorname{art}(k)+g(k+1) \cdot \operatorname{art}_{1}(k+1)
\end{aligned}
$$

Where

g: filter-gain 
a: loop-gain

e: filter error

P: prediction

$\operatorname{art}(k)$ : actual running time of previous bus at instant(k)

$\operatorname{art}_{l}(k+1)$ : actual running time of the previous day at instant $(\mathrm{k}+1)$

$V A R\left[\text { data }_{i n}\right]_{k+1}=$ Variance of the last three days data at time step $\mathrm{k}+1$.

$V A R\left[\text { data }_{o u t}\right]_{k+1}=$ Variance of Prediction

One difficulty we have to cope with is that the estimation of prediction variance. This has not been available both in the predicted form or in the form of actual observation. Farhan (2002) assumed as follows

$$
V A R\left[\text { data }_{\text {oud }}\right]=V A R\left[\text { data }_{\text {in }}\right]=V A R\left[\text { local }_{\text {data }}\right]
$$

Substitute VAR[local data] into Equations D3.9 and D3.11, then we have Equations 6.1 and 6.2 in chapter 6.

To calculate $V A R\left[\right.$ data $\left._{i n}\right]$ the following equation is applied

$V A R\left[\right.$ data $\left._{\text {in }}\right]=\mathrm{E}\left\{\left[\operatorname{art}_{i}(k+1)-E\left[\operatorname{art}_{i}(k+1)\right]^{2}\right)\right.$

Where $i=$ number of observed day at time step $(k+1)$ 


\section{Appendix D4}

\section{General}

This Appendix presents database for bus routes 1 and 95 as well as the predictions which are obtained from the developed model, the $\mathrm{KF}$ and the naive predictors.

\section{Database for Route1}

Table D4.1: Database for Route 1, segment Billings-Gladstone

\begin{tabular}{|c|c|c|c|c|c|}
\hline paos & s3 y be & A. & r. & 28 & $8 \%=$ \\
\hline 71 & 3000 & 950 & 902.5 & 938 & 813 \\
\hline 72 & 3000 & 747.5 & 833.5 & 959 & 788.5 \\
\hline 90 & 1100 & 905 & 975 & 1104 & 925.5 \\
\hline 52 & 2200 & 1403 & 1318 & 1323 & 1451 \\
\hline 38 & 2200 & 1252 & 1407 & 1367 & 1401 \\
\hline 23 & 1300 & 905 & 977.5 & 904.5 & 1038 \\
\hline 22 & 1300 & 806.5 & 1056 & 1052 & 914.5 \\
\hline 25 & 1300 & 912.5 & 880.5 & 977.5 & 886.5 \\
\hline 39 & 2200 & 1273 & 1356 & 1239 & 1287 \\
\hline 5 & 1200 & 912.5 & 1049 & 971 & 865.5 \\
\hline 11 & 1200 & 986.5 & 1055 & 886 & 996.5 \\
\hline 28 & 1300 & 1002 & 961.5 & 889 & 889.5 \\
\hline 50 & 2200 & 1355 & 1424 & 1167 & 1385 \\
\hline 53 & 2200 & 1229 & 1332 & 1357 & 1214 \\
\hline 73 & 3000 & 1009 & 824.5 & 961.5 & 830.5 \\
\hline 54 & 2200 & 1362 & 1421 & 1490 & 1460 \\
\hline 74 & 3000 & 851.5 & 782 & 805 & 789 \\
\hline 61 & 2100 & 921 & 974 & 1078 & 924 \\
\hline 6 & 1200 & 915.5 & 841 & 865.5 & 917 \\
\hline 70 & 2100 & 946.5 & 1036 & 1083 & 934.5 \\
\hline 26 & 1300 & 863.5 & 812.5 & 995.5 & 956 \\
\hline 68 & 2100 & 990 & 1047 & 998 & 832.5 \\
\hline 21 & 1300 & 998 & 981.5 & 983 & 932.5 \\
\hline 59 & 2100 & 1110 & 834 & 905 & 830 \\
\hline 8 & 1200 & 1023 & 1041 & 997.5 & 910.5 \\
\hline
\end{tabular}




\begin{tabular}{|c|c|c|c|c|c|}
\hline 92 & 1100 & 880 & 949 & 879.5 & 918.5 \\
\hline 94 & 1100 & 862 & 941 & 972 & 914.5 \\
\hline 57 & 2100 & 1065 & 851.5 & 999 & 941.5 \\
\hline 4 & 1200 & 968.5 & 859.5 & 866 & 912.5 \\
\hline 3 & 1200 & 852.5 & 912.5 & 792 & 833 \\
\hline 16 & 1200 & 892.5 & 927 & 985.5 & 1018 \\
\hline 75 & 3000 & 716.5 & 774.5 & 827.5 & 821 \\
\hline 40 & 2200 & 1396 & 1219 & 1358 & 1281 \\
\hline 55 & 2200 & 1166 & 1178 & 1471 & 1438 \\
\hline 96 & 1100 & 1101 & 841 & 1025 & 934 \\
\hline 76 & 3000 & 730.5 & 773.5 & 827.5 & 821 \\
\hline 37 & 2200 & 1358 & 1176 & 1254 & 1276 \\
\hline 10 & 1200 & 864.5 & 953.5 & 896 & 818.5 \\
\hline 30 & 1300 & 975 & 856.5 & 1093 & 933.5 \\
\hline 45 & 2200 & 1228 & 1366 & 1488 & 1421 \\
\hline 35 & 1300 & 964 & 933 & 1099 & 1021 \\
\hline 12 & 1200 & 861.5 & 848 & 1073 & 959.5 \\
\hline 9 & 1200 & 967.5 & 860 & 925.5 & 1011 \\
\hline 65 & 2100 & 749 & 953 & 806 & 936 \\
\hline 58 & 2100 & 989 & 856.5 & 907.5 & 935.5 \\
\hline 34 & 1300 & 860.5 & 944.5 & 761.5 & 1003 \\
\hline 77 & 3000 & 1022 & 787.5 & 832 & 888.5 \\
\hline 95 & 1100 & 1104 & 1018 & 980.5 & 1042 \\
\hline 69 & 2100 & 771.5 & 848.5 & 869 & 1045 \\
\hline 17 & 1200 & 1019 & 968 & 1016 & 953.5 \\
\hline 97 & 1100 & 868 & 1039 & 995 & 1030 \\
\hline 93 & 1100 & 843 & 1041 & 1089 & 1027 \\
\hline 60 & 2100 & 880.5 & 956 & 962.5 & 1050 \\
\hline 87 & 1100 & 842 & 918 & 907 & 933 \\
\hline 29 & 1300 & 1095 & 946 & 875 & 952.5 \\
\hline 78 & 3000 & 953 & 837 & 798.5 & 884 \\
\hline 43 & 2200 & 1141 & 1231 & 1346 & 1193 \\
\hline 46 & 2200 & 1394 & 1400 & 1250 & 1344 \\
\hline 24 & 1300 & 1111 & 1044 & 1083 & 945.5 \\
\hline 19 & 1200 & 930.5 & 896 & 1005 & 930 \\
\hline 51 & 2200 & 1124 & 1358 & 1239 & 1307 \\
\hline 62 & 2100 & 911.5 & 860.5 & 1094 & 833 \\
\hline 44 & 2200 & 1362 & 1343 & 1473 & 1168 \\
\hline 36 & 2200 & 1275 & 1397 & 1417 & 1411 \\
\hline 32 & 1300 & 956 & 949 & 1005 & 1015 \\
\hline 47 & 2200 & 1404 & 1223 & 1132 & 1269 \\
\hline 66 & 2100 & 1041 & 1155 & 1103 & 927.5 \\
\hline 56 & 2100 & 877.5 & 816.5 & 892.5 & 848.5 \\
\hline 79 & 3000 & 660.5 & 775.5 & 857 & 874.5 \\
\hline 27 & 1300 & 1023 & 1001 & 1106 & 1026 \\
\hline 88 & 1100 & 995 & 851.5 & 913.5 & 912 \\
\hline 74 & 3000 & 1040 & 988.5 & 1136 & 973.5 \\
\hline
\end{tabular}


Note:

\begin{tabular}{|c|c|c|c|c|c|}
\hline 1 & 1200 & 926.5 & 1036 & 1098 & 935.5 \\
\hline 80 & 3000 & 962 & 1008 & 979 & 899 \\
\hline 81 & 3000 & 831.5 & 781.5 & 823.5 & 876 \\
\hline 89 & 1100 & 992.5 & 1055 & 1067 & 969 \\
\hline 42 & 2200 & 1359 & 1416 & 1263 & 1318 \\
\hline 82 & 3000 & 961.5 & 889 & 928 & 820.5 \\
\hline 49 & 2200 & 1236 & 1338 & 1352 & 1345 \\
\hline 2 & 1200 & 864.5 & 1032 & 1047 & 1053 \\
\hline 98 & 1100 & 933.5 & 918.5 & 964.5 & 1055 \\
\hline 67 & 2100 & 970.5 & 967 & 863 & 815.5 \\
\hline 99 & 1100 & 962.5 & 956.5 & 860 & 922.5 \\
\hline 64 & 2100 & 1104 & 1160 & 1122 & 931.5 \\
\hline 63 & 2100 & 982.5 & 861 & 985.5 & 906.5 \\
\hline 33 & 1300 & 988.5 & 1043 & 985 & 1005 \\
\hline 18 & 1200 & 897 & 940 & 1108 & 949 \\
\hline 83 & 3000 & 952 & 853.5 & 1035 & 819.5 \\
\hline 48 & 2200 & 1471 & 1400 & 1270 & 1290 \\
\hline 20 & 1200 & 860 & 845 & 1005 & 920 \\
\hline 31 & 1300 & 926 & 982.5 & 983.5 & 1040 \\
\hline 91 & 1100 & 965 & 942.5 & 860 & 929.5 \\
\hline 100 & 1100 & 906.5 & 1029 & 1043 & 1049 \\
\hline 84 & 3000 & 963 & 832 & 802.5 & 774 \\
\hline 13 & 1200 & 798 & 977.5 & 871.5 & 917 \\
\hline 14 & 1200 & 999.5 & 959.5 & 811 & 1056 \\
\hline 101 & 1100 & $\mathbf{9 0 6 . 5}$ & 1029 & 1043 & 1049 \\
\hline 15 & 1200 & 887 & 837.5 & 881.5 & 918 \\
\hline 85 & 3000 & 1005 & 945 & 889 & 836 \\
\hline 7 & 1200 & 916.5 & 883 & 788.5 & 938.5 \\
\hline
\end{tabular}

1. Dcode= Date code; Scode $=$ Situation code (Please refer to Table 6.5 for the codes); R1, R2, R3, and R4 = running time of trip $6,5,4$, and 3 .

\section{Predictions Resulted from the Proposed Model and Reference Predictors for Route1}

The results of the proposed model were returned from the Matlab program coded for the bus running time prediction module (Please see Appendix C). In this section, the example of Matlab outputs is partially presented for the sake of illustration. The predictions obtained from the developed model as well as those of reference predictors are tabulated in Tables D4.38 and D4.39. 


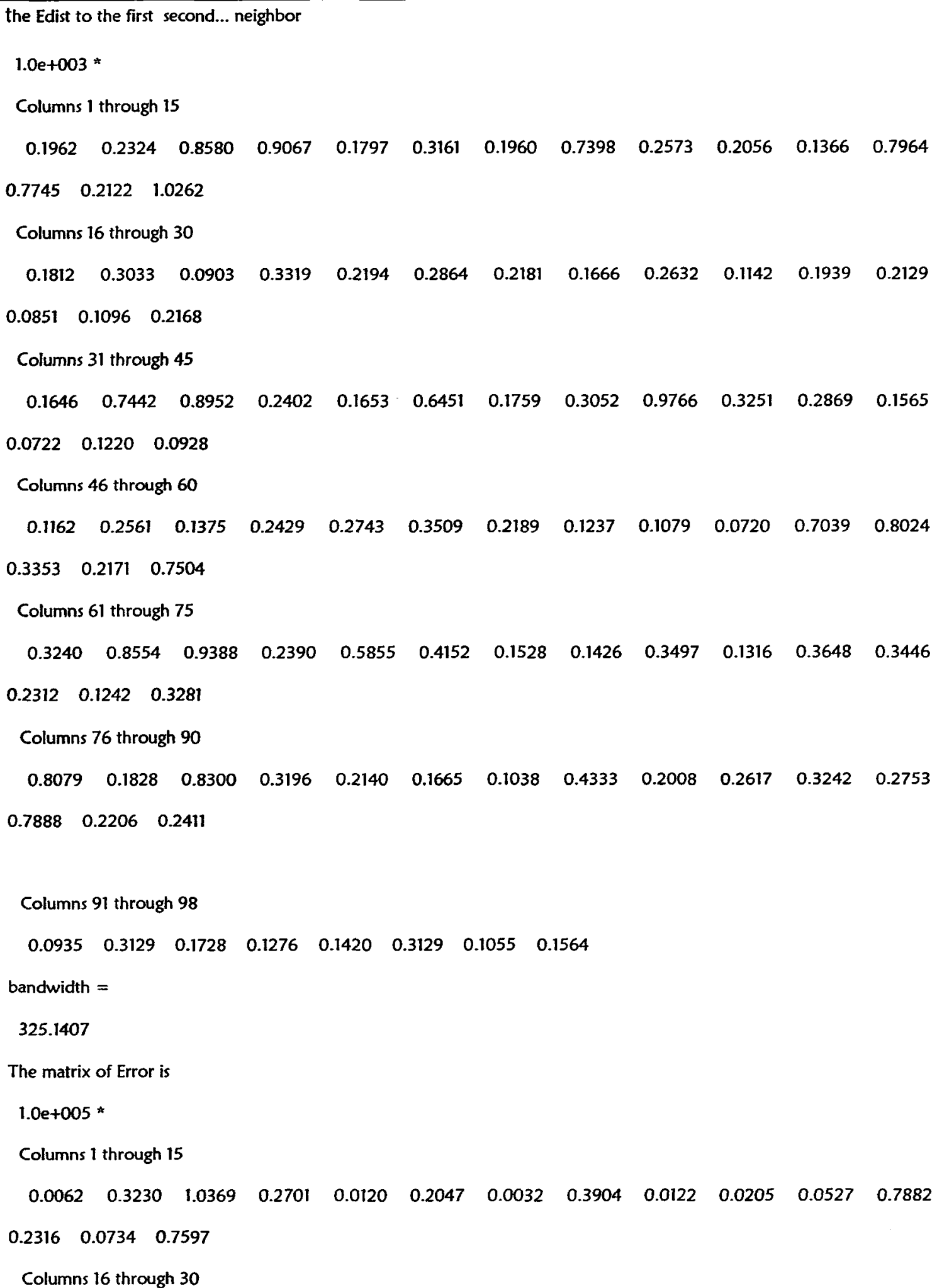




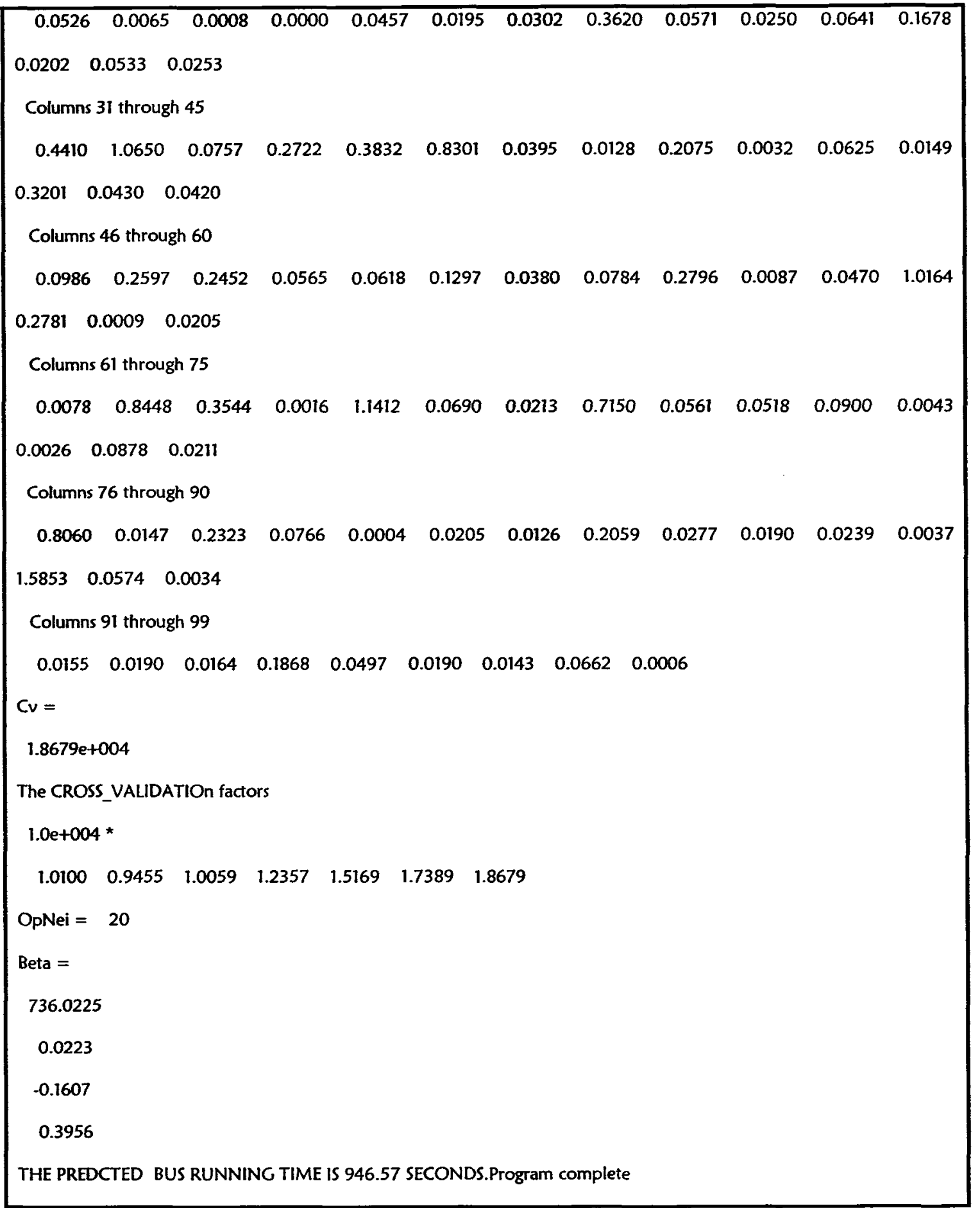




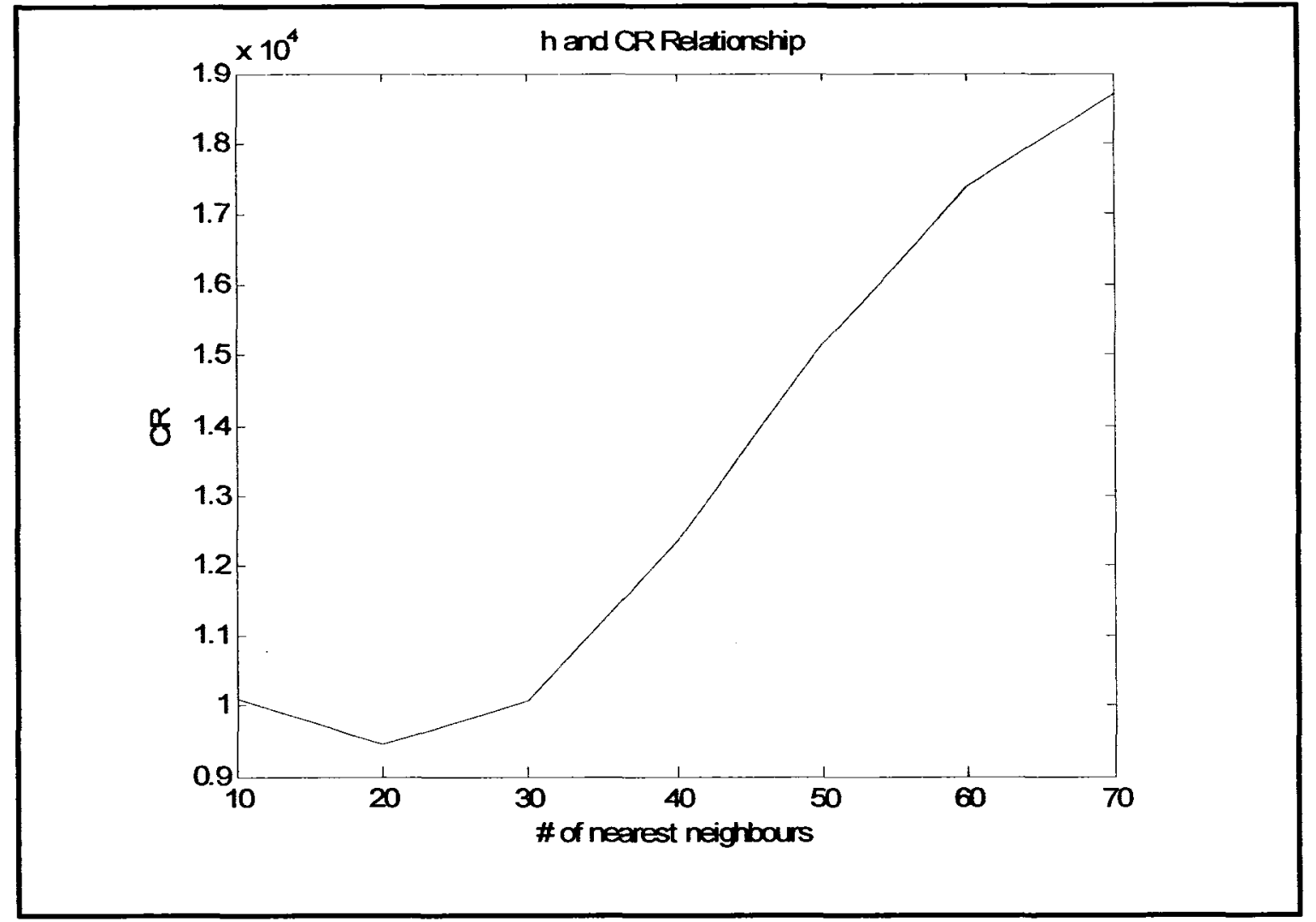

Figure D4.1: An Example of the Matlab Outputs (Case 90-1100, R1)

\section{Kalman Filter model's prediction results for Route1}


Table D4.3: Kalman Filter for test case $\mathbf{9 0 - 1 1 0 0}$

\begin{tabular}{|c|c|c|c|c|c|c|c|c|c|c|c|c|c|c|}
\hline time & trip & art3 & art2 & art1 & Average & $\Delta 3$ & $\Delta 2$ & $\Delta 1$ & Var[data]n & $g(k+1)$ & $e(k+1)$ & $a(k+1)$ & Predict & Observed \\
\hline $8: 17$ & 2 & 0 & 0 & 0 & 1005 & $1 E+06$ & $1 E+06$ & $1 E+06$ & $1 E+06$ & 0.5 & 0 & 0.5 & & 1005 \\
\hline $8: 27$ & 3 & 938.5 & 813 & 788.5 & 846.667 & 8433.36 & 1133.44 & 3383.36 & 4783.4 & 0.5 & 2391.7 & 0.5 & 896.75 & 925.5 \\
\hline $8: 37$ & 4 & 788.5 & 889 & 833.5 & 837 & 2352.25 & 2704 & 12.25 & 2528.13 & 0.66056 & 1669.98 & 0.33944 & 864.728 & 1104 \\
\hline $8: 47$ & 5 & 883 & 902.5 & 833.5 & 873 & 100 & 870.25 & 1560.25 & 485.125 & 0.81626 & 395.986 & 0.18374 & 883.203 & 975 \\
\hline $8: 59$ & 6 & 916.5 & 950 & 747.5 & 871.333 & 2040.03 & 6188.44 & 15334.7 & 4114.24 & 0.52296 & 2151.57 & 0.47704 & 856 & 905 \\
\hline
\end{tabular}

Table D4.4: Kalman Filter for test case 50-2200

\begin{tabular}{|c|c|c|c|c|c|c|c|c|c|c|c|c|c|c|}
\hline time & trip & art3 & $\operatorname{art2}$ & art1 & Average & $\Delta \mathbf{3}$ & $\Delta \mathbf{2}$ & $\Delta 1$ & Var[data]n & $g(k+1)$ & $\theta(k+1)$ & $a(k+1)$ & Predict & Observed \\
\hline $8: 17$ & 2 & 0 & 0 & 0 & 1005 & $1 E+06$ & $1 E+06$ & $1 E+06$ & $1 E+06$ & 0.5 & 0 & 0.5 & & 1005 \\
\hline $8: 27$ & 3 & 865.5 & 996.5 & 889.5 & 917.167 & 2669.44 & 6293.78 & 765.444 & 4481.61 & 0.5 & 2240.81 & 0.5 & 947.25 & 1423.5 \\
\hline $8: 37$ & 4 & 971 & 886 & 889 & 915.333 & 3098.78 & 860.444 & 693.444 & 1979.61 & 0.68071 & 1347.54 & 0.31929 & 1059.66 & 1167 \\
\hline $8: 47$ & 5 & 1049 & 1054.5 & 961.5 & 1021.67 & 747.111 & 1078.03 & 3620.03 & 912.569 & 0.71237 & 650.084 & 0.28763 & 1020.61 & 1384.5 \\
\hline $8: 59$ & 6 & 912.5 & 986.5 & 1002 & 967 & 2970.25 & 380.25 & 1225 & 1675.25 & 0.58125 & 973.737 & 0.41875 & 1162 & 1354.5 \\
\hline
\end{tabular}

Table D4.5: Kalman Filter for test case 6-1200

\begin{tabular}{|c|c|c|c|c|c|c|c|c|c|c|c|c|c|c|}
\hline time & trip & art3 & art2 & art1 & Average & $\Delta 3$ & $\Delta 2$ & $\Delta 1$ & Var[data]n & $g(k+1)$ & $e(k+1)$ & $a(k+1)$ & Predict & Observed \\
\hline $8: 17$ & 2 & 0 & 0 & 0 & 1005 & $1 E+06$ & $1 E+06$ & $1 E+06$ & $1 E+06$ & 0.5 & 0 & 0.5 & & 1005 \\
\hline $8: 27$ & 3 & 1459.5 & 789 & 924 & 1057.5 & 161604 & 72092.3 & 17822.3 & 116848 & 0.5 & 58424.1 & 0.5 & 964.5 & 917 \\
\hline $8: 37$ & 4 & 1490 & 805 & 1077.5 & 1124.17 & 133834 & 101867 & 2177.78 & 117851 & 0.59932 & 70630.1 & 0.40068 & 1013.19 & 865.5 \\
\hline $8: 47$ & 5 & 1421 & 782 & 974 & 1059 & 131044 & 76729 & 7225 & 103887 & 0.62685 & 65121.1 & 0.37315 & 933.513 & 841 \\
\hline $8: 59$ & 6 & 1361.5 & 851.5 & 921 & 1044.67 & 100383 & 37313.4 & 15293.4 & 68848.4 & 0.66054 & 45477.2 & 0.33946 & 893.8 & 915.5 \\
\hline
\end{tabular}


Table D4.6: Kalman Filter for test case 26-1300

\begin{tabular}{|c|c|c|c|c|c|c|c|c|c|c|c|c|c|c|}
\hline time & trip & art3 & art2 & art1 & Average & $\Delta 3$ & $\Delta \mathbf{2}$ & $\Delta 1$ & Var[data]n & $g(k+1)$ & $e(k+1)$ & $a(k+1)$ & Predict & Observed \\
\hline $8: 17$ & 2 & 0 & 0 & 0 & 1005 & $1 E+06$ & $1 E+06$ & $1 E+06$ & $1 E+06$ & 0.5 & 0 & 0.5 & & 1005 \\
\hline $8: 27$ & 3 & 924 & 917 & 934.5 & 925.167 & 1.36111 & 66.6944 & 87.1111 & 34.0278 & 0.5 & 17.0139 & 0.5 & 969.75 & 956 \\
\hline $8: 37$ & 4 & 1077.5 & 865.5 & 1083 & 1008.67 & 4738.03 & 20496.7 & 5525.44 & 12617.4 & 0.50034 & 6312.93 & 0.49966 & 1019.54 & 995.5 \\
\hline $8: 47$ & 5 & 974 & 841 & 1036 & 950.333 & 560.111 & 11953.8 & 7338.78 & .6256 .94 & 0.66766 & 4177.5 & 0.33234 & 1022.54 & 812.5 \\
\hline $8: 59$ & 6 & 921 & 915.5 & 946.5 & 927.667 & 44.4444 & 148.028 & 354.694 & 96.2361 & 0.97798 & 94.1168 & 0.02202 & 943.5 & 863.5 \\
\hline
\end{tabular}

Table D4.7: Kalman Filter for test case 21-1300

\begin{tabular}{|c|c|c|c|c|c|c|c|c|c|c|c|c|c|c|}
\hline time & trip & art3 & art2 & art1 & Average & $\Delta \mathbf{3}$ & $\Delta 2$ & $\Delta 1$ & Var[data]n & $g(k+1)$ & $e(k+1)$ & $a(k+1)$ & Predict & Observed \\
\hline $8: 17$ & 2 & 0 & 0 & 0 & 1005 & $1 E+06$ & $1 E+06$ & $1 E+06$ & $1 E+06$ & 0.5 & 0 & 0.5 & & 1005 \\
\hline $8: 27$ & 3 & 934.5 & 956 & 832.5 & 907.667 & 720.028 & 2336.11 & 5650.03 & 1528.07 & 0.5 & 764.035 & 0.5 & 918.75 & 932.5 \\
\hline $8: 37$ & 4 & 1083 & 995.5 & 998 & 1025.5 & 3306.25 & 900 & 756.25 & 2103.13 & 0.57686 & 1213.21 & 0.42314 & 970.284 & 983 \\
\hline $8: 47$ & 5 & 1036 & 812.5 & 1047 & 965.167 & 5017.36 & 23307.1 & 6696.69 & 14162.2 & 0.52054 & 7371.96 & 0.47946 & 1016.31 & 981 \\
\hline $8: 59$ & 6 & 946.5 & 863.5 & 990 & 933.333 & 173.361 & 4876.69 & 3211.11 & 2525.03 & 0.79673 & 2011.76 & 0.20327 & 988.2 & 998 \\
\hline
\end{tabular}

Table D4.8: Kalman Filter for test case 8-1200

\begin{tabular}{|c|c|c|c|c|c|c|c|c|c|c|c|c|c|c|}
\hline time & trip & art3 & art2 & art1 & Average & $\Delta \mathbf{3}$ & $\Delta 2$ & $\Delta 1$ & Var[data]n & $g(k+1)$ & $e(k+1)$ & $a(k+1)$ & Predlct & Observed \\
\hline $8: 17$ & 2 & 0 & 0 & 0 & 1005 & $1 E+06$ & $1 E+06$ & $1 E+06$ & $1 E+06$ & 0.5 & 0 & 0.5 & & 1005 \\
\hline $8: 27$ & 3 & 832.5 & 932 & 830 & 864.833 & 1045.44 & 4511.36 & 1213.36 & 2778.4 & 0.5 & 1389.2 & 0.5 & 917.5 & 910.5 \\
\hline $8: 37$ & 4 & 998 & 983 & 905 & 962 & 1296 & 441 & 3249 & 868.5 & 0.72219 & 627.219 & 0.27781 & 906.528 & 997.5 \\
\hline $8: 47$ & 5 & 1047 & 981 & 834 & 954 & 8649 & 729 & 14400 & 4689 & 0.53134 & 2491.47 & 0.46866 & 910.625 & 1040.5 \\
\hline $8: 59$ & 6 & 990 & 998 & 1109.5 & 1032.5 & 1806.25 & 1190.25 & 5929 & 1498.25 & 0.72699 & 1089.22 & 0.27301 & 1091 & 1023 \\
\hline
\end{tabular}


Table D4.9: Kalman Filter for test case 57-2100

\begin{tabular}{|c|c|c|c|c|c|c|c|c|c|c|c|c|c|c|}
\hline time & trip & art3 & art2 & art1 & Average & $\Delta 3$ & $\Delta 2$ & $\Delta 1$ & Var[data]n & $g(k+1)$ & $e(k+1)$ & $a(k+1)$ & Predict & Observed \\
\hline $8: 17$ & 2 & 0 & 0 & 0 & 1005 & $1 E+06$ & $1 E+06$ & $1 E+06$ & $1 E+06$ & 0.5 & 0 & 0.5 & & 1005 \\
\hline $8: 27$ & 3 & 910.5 & 918.5 & 914.5 & 914.5 & 16 & 16 & 0 & 16 & 0.5 & 8 & 0.5 & 959.75 & 941.5 \\
\hline $8: 37$ & 4 & 997.5 & 879.5 & 972 & 949.667 & 2288.03 & 4923.36 & 498.778 & 3605.69 & 0.50055 & 1804.85 & 0.49945 & 956.767 & 999 \\
\hline $8: 47$ & 5 & 1040 & 949 & 941 & 976.667 & 4011.11 & 765.444 & 1272.11 & 2388.28 & 0.63712 & 1521.61 & 0.36288 & 962.047 & 851.5 \\
\hline $8: 59$ & 6 & 1022.5 & 880 & 862 & 921.5 & 10201 & 1722.25 & 3540.25 & 5961.63 & 0.55659 & 3318.16 & 0.44341 & 857.3 & 1065 \\
\hline
\end{tabular}

Table D4.10: Kalman Filter for test case 3-1200

\begin{tabular}{|c|c|c|c|c|c|c|c|c|c|c|c|c|c|c|}
\hline time & trip & art3 & art2 & art1 & Average & $\Delta 3$ & $\Delta 2$ & $\Delta 1$ & Var[data]n & $g(k+1)$ & $e(k+1)$ & $a(k+1)$ & Predict & Observed \\
\hline $8: 17$ & 2 & 0 & 0 & 0 & 1005 & $1 E+06$ & $1 E+06$ & $1 E+06$ & $1 E+06$ & 0.5 & 0 & 0.5 & & 1005 \\
\hline 27 & 3 & 914.5 & 941.5 & 912.5 & 922.833 & 69.4444 & 348.444 & 106.778 & 208.944 & & 104.472 & 0.5 & 58.75 & 833 \\
\hline & 4 & 72 & & & 945. & 693.444 & 4.44 & .78 & & 0.51434 & & 0.48566 & .973 & 792 \\
\hline 47 & 5 & 941 & 851.5 & 859.5 & & & 1056.25 & 600.25 & & & & 0.41277 & 831.638 & 912.5 \\
\hline $8: 59$ & 6 & 862 & 1065 & 968.5 & 965.167 & 10643.4 & 9966.69 & 11.1111 & 10305 & 0.52889 & 5450.27 & 0.47111 & 942.1 & 852.5 \\
\hline
\end{tabular}

Table D4.11: Kalman Filter for test case 69-2100

\begin{tabular}{|c|c|c|c|c|c|c|c|c|c|c|c|c|c|c|}
\hline time & trip & art3 & art2 & art1 & Average & $\Delta \mathbf{3}$ & $\Delta 2$ & $\Delta 1$ & Var[data]n & $g(k+1)$ & $e(k+1)$ & $a(k+1)$ & Predict & Observed \\
\hline $8: 17$ & 2 & 0 & 0 & 0 & 1005 & $1 E+06$ & $1 E+06$ & $1 E+06$ & $1 E+06$ & 0.5 & 0 & 0.5 & & 1005 \\
\hline $8: 27$ & 3 & 1002.5 & 888.5 & 1041.5 & 977.5 & 625 & 7921 & 4096 & 4273 & 0.5 & 2136.5 & 0.5 & 1023.25 & 1044.5 \\
\hline $8: 37$ & 4 & 761.5 & 832 & 980.5 & 858 & 9312.25 & 676 & 15006.3 & 4994.13 & 0.5881 & 2937.07 & 0.4119 & 1006.86 & 869 \\
\hline $8: 47$ & 5 & 860.5 & 1021.5 & 1103.5 & 995.167 & 18135.1 & 693.444 & 11736.1 & 9414.28 & 0.56747 & 5342.32 & 0.43253 & 1002.07 & 848.5 \\
\hline $8: 59$ & 6 & 860.5 & 1021.5 & 1103.5 & 995.167 & 18135.1 & 693.444 & 11736.1 & 9414.28 & 0.61051 & 5747.53 & 0.38949 & 1004 & 771.5 \\
\hline
\end{tabular}


Table D4.12: Kalman Filter for test case 87-1100

\begin{tabular}{|c|c|c|c|c|c|c|c|c|c|c|c|c|c|c|}
\hline time & trip & art3 & art2 & art1 & Average & $\Delta 3$ & $\Delta 2$ & $\Delta 1$ & Var[data]n & $g(k+1)$ & $e(k+1)$ & $a(k+1)$ & Predict & Observed \\
\hline $8: 17$ & 2 & 0 & 0 & 0 & 1005 & $1 \mathrm{E}+06$ & $1 E+06$ & $1 \mathrm{E}+06$ & $1 \mathrm{E}+06$ & 0.5 & 0 & 0.5 & & 1005 \\
\hline $8: 27$ & 3 & 1029.5 & 1027 & 1049.5 & 1035.33 & 34.0278 & 69.4444 & 200.694 & 51.7361 & 0.5 & 25.8681 & 0.5 & 1027.25 & 933 \\
\hline $8: 37$ & 4 & 995 & 1089 & 962.5 & 1015.5 & 420.25 & 5402.25 & 2809 & 2911.25 & 0.50221 & 1462.06 & 0.49779 & 947.815 & 907 \\
\hline $8: 47$ & 5 & 968 & 1039 & 1041 & 1016 & 2304 & 529 & 625 & 1416.5 & 0.6702 & 949.342 & 0.3298 & 996.807 & 918 \\
\hline $8: 59$ & 6 & 868 & 843 & 880.5 & 863.833 & 17.3611 & 434.028 & 277.778 & 225.694 & 0.83887 & 189.329 & 0.16113 & 886.5 & 842 \\
\hline
\end{tabular}

Table D4.13: Kalman Filter for test case 78-3000

\begin{tabular}{|c|c|c|c|c|c|c|c|c|c|c|c|c|c|c|}
\hline time & trip & art3 & art2 & art1 & Average & $\Delta \mathbf{3}$ & $\Delta 2$ & $\Delta 1$ & Var[data]n & $g(k+1)$ & $e(k+1)$ & $a(k+1)$ & Predict & Observed \\
\hline $8: 17$ & 2 & 0 & 0 & 0 & 1005 & $1 E+06$ & $\overline{1 E+06}$ & $1 E+06$ & $1 E+06$ & 0.5 & 0 & 0.5 & & 1005 \\
\hline $8: 27$ & 3 & 1049.5 & 933 & 952.5 & 978.333 & 5064.69 & 2055.11 & 667.361 & 3559.9 & 0.5 & 1779.95 & 0.5 & 978.75 & 884 \\
\hline $8: 37$ & 4 & 962.5 & 907 & 875 & 914.833 & 2272.11 & 61.3611 & 1586.69 & 1166.74 & 0.71636 & 835.802 & 0.28364 & 877.553 & 798.5 \\
\hline $8: 47$ & 5 & $\frac{90.0}{956}$ & 918 & 946 & 940 & 256 & 484 & 36 & 370 & 0.7652 & 283.124 & 0.2348 & 911.367 & 837 \\
\hline $8: 59$ & 6 & 880.5 & 842 & 1095 & 939.167 & 3441.78 & 9441.36 & 24284 & 6441.57 & 0.51075 & 3290.04 & 0.48925 & 968.8 & 953 \\
\hline
\end{tabular}

Table D4.14: Kalman Filter for test case $\mathbf{4 3 - 2 2 0 0}$

\begin{tabular}{|c|c|c|c|c|c|c|c|c|c|c|c|c|c|c|}
\hline time & trip & art3 & art2 & art1 & Average & $\Delta \mathbf{3}$ & $\Delta \mathbf{2}$ & $\Delta 1$ & Var[data]n & $g(k+1)$ & $\theta(k+1)$ & $a(k+1)$ & Predict & Observed \\
\hline $8: 17$ & 2 & 0 & 0 & 0 & 1005 & $1 E+06$ & $1 E+06$ & $1 E+06$ & $1 E+06$ & 0.5 & 0 & 0.5 & & 1005 \\
\hline $8: 27$ & 3 & 933 & 952.5 & 884 & 923.167 & 96.6944 & 860.444 & 1534.03 & 478.569 & 0.5 & 239.285 & 0.5 & 944.5 & 1192.5 \\
\hline $8: 37$ & 4 & 907 & 875 & 798.5 & 860.167 & 2193.36 & 220.028 & 3802.78 & 1206.69 & 0.5451 & 657.772 & 0.4549 & 977.73 & 1345.5 \\
\hline $8: 47$ & 5 & 918 & 946 & 837 & 900.333 & 312.111 & 2085.44 & 4011.11 & 1198.78 & 0.60764 & 728.429 & 0.39236 & 1036.51 & 1230.5 \\
\hline $8: 59$ & 6 & 842 & 1095 & 953 & 963.333 & 14721.8 & 17336.1 & 106.778 & 16028.9 & 0.51111 & 8192.53 & 0.48889 & 1089 & 1141 \\
\hline
\end{tabular}


Table D4.15: Kalman Filter for test case 24-1300

\begin{tabular}{|c|c|c|c|c|c|c|c|c|c|c|c|c|c|c|}
\hline tlme & trlp & art3 & art2 & art1 & Average & $\Delta \mathbf{3}$ & $\Delta 2$ & $\Delta 1$ & Var[data]n & $g(k+1)$ & $\theta(k+1)$ & $a(k+1)$ & Predict & Observed \\
\hline $8: 17$ & 2 & 0 & 0 & 0 & 1005 & $1 E+06$ & $1 E+06$ & $1 E+06$ & $1 E+06$ & 0.5 & 0 & 0.5 & & 1005 \\
\hline $8: 27$ & 3 & 884 & 1192.5 & 1343.5 & 1140 & 65536 & 2756.25 & 41412.3 & 34146.1 & 0.5 & 17073.1 & 0.5 & 1174.25 & 945.5 \\
\hline $8: 37$ & 4 & 798.5 & 1345.5 & 1250 & 1131.33 & 110778 & 45867.4 & 14081.8 & 78322.7 & 0.54914 & 43010.1 & 0.45086 & 1112.71 & 1082.5 \\
\hline $8: 47$ & 5 & 837 & 1230.5 & 1399.5 & 1155.67 & 101548 & 5600.03 & 59454.7 & 53574.2 & 0.64322 & 34459.8 & 0.35678 & 1286.4 & 1044 \\
\hline $8: 59$ & 6 & 953 & 1141 & 1394 & 1162.67 & 43960.1 & 469.444 & 53515.1 & 22214.8 & 0.71841 & 15959.2 & 0.28159 & 1295 & 1111 \\
\hline
\end{tabular}

Table D4.16: Kalman Filter for test case 51-2200

\begin{tabular}{|c|c|c|c|c|c|c|c|c|c|c|c|c|c|c|}
\hline time & trip & art3 & art2 & art1 & Average & $\Delta \mathbf{3}$ & $\Delta 2$ & $\Delta 1$ & Var[data]n & $g(k+1)$ & $\theta(k+1)$ & $a(k+1)$ & Predict & Observed \\
\hline $8: 17$ & 2 & 0 & 0 & 0 & 1005 & $1 E+06$ & $1 E+06$ & $1 E+06$ & $1 E+06$ & 0.5 & 0 & 0.5 & & 1005 \\
\hline $8: 27$ & 3 & 343.5 & 945.5 & 930 & & & 16256.3 & & 13.3 & 0.5 & 22356.6 & 0.5 & 967.5 & 1306.5 \\
\hline $8: 37$ & 4 & & & 1005 & & & & & & & & 0.23488 & 1075.82 & 8.5 \\
\hline $8: 47$ & 5 & 1399.5 & & 896 & & & 4784.03 & & & & & & & 1357.5 \\
\hline $8: 59$ & 6 & 1141 & 1394 & 930.5 & 1155.17 & 200.694 & 57041.4 & 50475.1 & 28621 & 0.64524 & 18467.4 & 0.35476 & 1082 & 1124 \\
\hline
\end{tabular}

Table D4.17: Kalman Filter for test case 56-2100

\begin{tabular}{|c|c|c|c|c|c|c|c|c|c|c|c|c|c|c|}
\hline time & trip & art3 & art2 & art1 & Average & $\mathbf{\Delta 3}$ & $\Delta \mathbf{2}$ & $\Delta 1$ & Var[data]n & $g(k+1)$ & $\theta(k+1)$ & $a(k+1)$ & Predict & Observed \\
\hline $8: 17$ & 2 & 0 & 0 & 0 & 1005 & $1 E+06$ & $1 E+06$ & $1 E+06$ & $1 E+06$ & 0.5 & 0 & 0.5 & & 1005 \\
\hline $8: 27$ & 3 & 1015.2 & 1269 & 927.5 & 1070.57 & 3065.47 & 39375.8 & 20468.1 & 21220.6 & 0.5 & 10610.3 & 0.5 & 966.25 & 848.5 \\
\hline $8: 37$ & 4 & 1005 & 1132 & 1102.5 & 1079.83 & 5600.03 & 2721.36 & 513.778 & 4160.69 & 0.78023 & 3246.28 & 0.21977 & 1046.68 & 892.5 \\
\hline $8: 47$ & 5 & 949 & 1223 & 1154.5 & 1108.83 & 25546.7 & 13034 & 2085.44 & 19290.4 & 0.53881 & 10393.8 & 0.46119 & 1033.67 & 816.5 \\
\hline $8: 59$ & 6 & 956 & 1404 & 1041 & 1133.67 & 31565.4 & 73080.1 & 8587.11 & 52322.8 & 0.54517 & 28525.1 & 0.45483 & 938.9 & 877.5 \\
\hline
\end{tabular}


Table D4.18: Kalman Filter for test case 33-1300

\begin{tabular}{|c|c|c|c|c|c|c|c|c|c|c|c|c|c|c|}
\hline $\operatorname{t} \operatorname{mm} \theta$ & trip & art3 & $\operatorname{art2}$ & art1 & Average & $\Delta \mathbf{3}$ & $\Delta \mathbf{2}$ & $\Delta 1$ & Var[data]n & $g(k+1)$ & $e(k+1)$ & $a(k+1)$ & Predict & Observed \\
\hline $8: 17$ & 2 & 0 & 0 & 0 & 1005 & $1 E+06$ & $1 E+06$ & $1 E+06$ & $1 E+06$ & 0.5 & 0 & 0.5 & & 1005 \\
\hline $8: 27$ & 3 & 922.5 & 931.5 & 906.5 & 920.167 & 5.44444 & 128.444 & 186.778 & 66.9444 & 0.5 & 33.4722 & 0.5 & 955.75 & 1005 \\
\hline $8: 37$ & 4 & 860 & 1122 & 985.5 & 989.167 & 16684 & 17644.7 & 13.4444 & 17164.4 & 0.50049 & 8590.54 & 0.49951 & 995.241 & 985 \\
\hline $8: 47$ & 5 & 956.5 & 1159.5 & 861 & 992.333 & 1284.03 & 27944.7 & 17248.4 & 14614.4 & 0.61357 & 8966.99 & 0.38643 & 908.917 & 1042.5 \\
\hline $8: 59$ & 6 & 962.5 & 1104 & 982.5 & 1016.33 & 2898.03 & 7685.44 & 1144.69 & 5291.74 & 0.72933 & 3859.42 & 0.27067 & 998.7 & 988.5 \\
\hline
\end{tabular}

Table D4.19: Kalman Filter for test case 27-1300

\begin{tabular}{|c|c|c|c|c|c|c|c|c|c|c|c|c|c|c|}
\hline time & trip & art3 & art2 & art1 & Average & $\Delta \mathbf{3}$ & $\Delta 2$ & $\Delta 1$ & Var[data]n & $g(k+1)$ & $e(k+1)$ & $a(k+1)$ & Predict & Observed \\
\hline $8: 17$ & 2 & 0 & 0 & 0 & 1005 & $1 E+06$ & $1 E+06$ & $1 E+06$ & $1 E+06$ & 0.5 & 0 & 0.5 & & 1005 \\
\hline $8: 27$ & 3 & 927.5 & 848.5 & 874.5 & 883.5 & 1936 & 1225 & 81 & 1580.5 & 0.5 & 790.25 & 0.5 & 939.75 & 1025.5 \\
\hline $8: 37$ & 4 & 1102.5 & 892.5 & 857 & 950.667 & 23053.4 & 3383.36 & 8773.44 & 13218.4 & 0.51451 & 6801.01 & 0.48549 & 938.805 & 1106 \\
\hline $8: 47$ & 5 & 1154.5 & 816.5 & 775.5 & 915.5 & 57121 & 9801 & 19600 & 33461 & 0.54613 & 18273.9 & 0.45387 & 925.506 & 1001 \\
\hline $8: 59$ & 6 & 1041 & 877.5 & 660.5 & 859.667 & 32881.8 & 318.028 & 39667.4 & 16599.9 & 0.67751 & 11246.6 & 0.32249 & 770.3 & 1023 \\
\hline
\end{tabular}

Table D4.20: Kalman Filter for test case $\mathbf{7 4 - 3 0 0 0}$

\begin{tabular}{|c|c|c|c|c|c|c|c|c|c|c|c|c|c|c|}
\hline time & trip & art3 & art2 & art1 & Average & $\Delta 3$ & $\Delta 2$ & $\Delta 1$ & Var[data]n & $g(k+1)$ & $e(k+1)$ & $a(k+1)$ & Predict & Observed \\
\hline $8: 17$ & 2 & 0 & 0 & 0 & 890 & 792100 & 792100 & 792100 & 792100 & 0.5 & 0 & 0.5 & & 1005 \\
\hline $8: 27$ & 3 & 874.5 & 1025.5 & 912 & 937.333 & 3948.03 & 7773.36 & 641.778 & 5860.69 & 0.5 & 2930.35 & 0.5 & 958.5 & 973.5 \\
\hline $8: 37$ & 4 & 857 & 1106 & 913.5 & 958.833 & 10370 & 21658 & 2055.11 & 16014 & 0.54191 & 8678.19 & 0.45809 & 940.985 & 1136 \\
\hline $8: 47$ & 5 & 775.5 & 1001 & 851.5 & 876 & 10100.3 & 15625 & 600.25 & 12862.6 & 0.62612 & 8053.6 & 0.37388 & 957.868 & 988.5 \\
\hline $8: 59$ & 6 & 660.5 & 1023 & 995 & 892.833 & 53978.8 & 16943.4 & 10438 & 35461.1 & 0.55099 & 19538.6 & 0.44901 & 992.1 & 1040 \\
\hline
\end{tabular}


Table D4.21: Kalman Filter for test case $\mathbf{9 9 - 1 1 0 0}$

\begin{tabular}{|c|c|c|c|c|c|c|c|c|c|c|c|c|c|c|}
\hline time & trip & $\operatorname{art3}$ & art2 & art1 & Average & $\Delta \mathbf{3}$ & $\Delta 2$ & $\Delta 1$ & Var[data]n & $g(k+1)$ & $\theta(k+1)$ & $a(k+1)$ & Predict & Observed \\
\hline $8: 17$ & 2 & 0 & 0 & 0 & 890 & 792100 & 792100 & 792100 & 792100 & 0.5 & 0 & 0.5 & & 1005 \\
\hline $8: 27$ & 3 & 1053 & 1055 & 815.5 & 974.5 & 6162.25 & 6480.25 & 25281 & 6321.25 & 0.5 & 3160.63 & 0.5 & 910.25 & 922.5 \\
\hline $8: 37$ & 4 & 1047 & 964.5 & 863 & 958.167 & 7891.36 & 40.1111 & 9056.69 & 3965.74 & 0.64247 & 2547.87 & 0.35753 & 884.273 & 860 \\
\hline $8: 47$ & 5 & 1032 & 918.5 & 967 & 972.5 & 3540.25 & 2916 & 30.25 & 3228.13 & 0.64148 & 2070.79 & 0.35852 & 928.639 & 956.5 \\
\hline $8: 59$ & 6 & 864.5 & 933.5 & 970.5 & 922.833 & 3402.78 & 113.778 & 2272.11 & 1758.28 & 0.68531 & 1204.97 & 0.31469 & 966.1 & 962.5 \\
\hline
\end{tabular}

Table D4.22: Kalman Filter for test case 91-1100

\begin{tabular}{|c|c|c|c|c|c|c|c|c|c|c|c|c|c|c|}
\hline time & trip & art3 & art2 & art1 & Average & $\Delta \mathbf{3}$ & $\Delta \mathbf{2}$ & $\Delta 1$ & Var[data]n & $g(k+1)$ & $e(k+1)$ & $a(k+1)$ & Predict & Observed \\
\hline $8: 17$ & 2 & 0 & 0 & 0 & 890 & 792100 & 792100 & 792100 & 792100 & 0.5 & 0 & 0.5 & & 1005 \\
\hline $8: 27$ & 3 & 1290 & 920 & 1039.5 & 1083.17 & 42780 & 26623.4 & 1906.78 & 34701.7 & 0.5 & 17350.8 & 0.5 & 1022.25 & 929.5 \\
\hline $8: 37$ & 4 & 1269.5 & 1005 & 983.5 & 1086 & 33672.3 & 6561 & 10506.3 & 20116.6 & 0.65066 & 13089 & 0.34934 & 964.635 & 860 \\
\hline $8: 47$ & 5 & 1400 & 845 & 982.5 & 1075.83 & 105084 & 53284 & 8711.11 & 79184 & 0.53817 & 42614.5 & 0.46183 & 925.926 & 942.5 \\
\hline $8: 59$ & 6 & 1471 & 860 & 926 & 1085.67 & 148482 & 50925.4 & 25493.4 & 99703.6 & 0.58804 & 58629.6 & 0.41196 & 932.8 & 965 \\
\hline
\end{tabular}

Table D4.23: Kalman Filter for test case 52-2200

\begin{tabular}{|c|c|c|c|c|c|c|c|c|c|c|c|c|c|c|}
\hline time & trip & art3 & art2 & art1 & Average & $\Delta \mathbf{3}$ & $\Delta 2$ & $\Delta 1$ & Var[data]n & $g(k+1)$ & $e(k+1)$ & $a(k+1)$ & Predict & Observed \\
\hline $8: 17$ & 2 & 0 & 0 & 0 & 1005 & $1 E+06$ & $1 E+06$ & $1 E+06$ & $1 E+06$ & 0.5 & 0 & 0.5 & & 1005 \\
\hline $8: 27$ & 3 & 813 & 788.5 & 925.5 & 842.333 & 860.444 & 2898.03 & 6916.69 & 1879.24 & 0.5 & 939.618 & 0.5 & 965.25 & 1450 \\
\hline $8: 37$ & 4 & 938 & 959 & 1104 & 1000.33 & 3885.44 & 1708.44 & 10746.8 & 2796.94 & 0.57191 & 1599.59 & 0.42809 & 1252.12 & 1322.5 \\
\hline $8: 47$ & 5 & 902.5 & 833.5 & 975 & 903.667 & 1.36111 & 4923.36 & 5088.44 & 2462.36 & 0.62259 & 1533.03 & 0.37741 & 1106.15 & 1317.5 \\
\hline $8: 59$ & 6 & 950 & 747.5 & 905 & 867.5 & 6806.25 & 14400 & 1406.25 & 10603.1 & 0.53371 & 5658.98 & 0.46629 & 1097 & 1421 \\
\hline
\end{tabular}


Table D4.24: Kalman Filter for test case 38-2200

\begin{tabular}{|c|c|c|c|c|c|c|c|c|c|c|c|c|c|c|}
\hline time & trip & art3 & art2 & art1 & Average & $\Delta \mathbf{3}$ & $\Delta 2$ & $\Delta 1$ & Var[data]n & $g(k+1)$ & $e(k+1)$ & $a(k+1)$ & Predict & Observed \\
\hline $8: 17$ & 2 & 0 & 0 & 0 & 1005 & $1 E+06$ & $1 E+06$ & $1 E+06$ & $1 E+06$ & 0.5 & 0 & 0.5 & & 1005 \\
\hline $8: 27$ & 3 & 788.5 & 925.5 & 1450.5 & 1054.83 & 70933.4 & 16727.1 & 156552 & 43830.3 & 0.5 & 21915.1 & 0.5 & 1227.75 & 1400 \\
\hline $8: 37$ & 4 & 959 & 1104 & 1322.5 & 1128.5 & 28730.3 & 600.25 & 37636 & 14665.3 & 0.71382 & 10468.4 & 0.28618 & 1344.68 & 1367 \\
\hline $8: 47$ & 5 & 833.5 & 975 & 1317.5 & 1042 & 43472.3 & 4489 & 75900.3 & 23980.6 & 0.58958 & 14138.5 & 0.41042 & 1337.82 & 1406.5 \\
\hline $8: 59$ & 6 & 747.5 & 905 & 1402.5 & 1018.33 & 73350.7 & 12844.4 & 147584 & 43097.6 & 0.57046 & 24585.3 & 0.42954 & 1404 & 1252 \\
\hline
\end{tabular}

Table D4.25: Kalman Filter for test case 39-2200

\begin{tabular}{|c|c|c|c|c|c|c|c|c|c|c|c|c|c|c|}
\hline time & trip & art3 & art2 & art1 & Average & $\Delta \mathbf{3}$ & $\Delta 2$ & $\Delta 1$ & Var[data]n & $g(k+1)$ & $e(k+1)$ & $a(k+1)$ & Predict & Observed \\
\hline $8: 17$ & 2 & 0 & 0 & 0 & 1005 & $1 E+06$ & $1 E+06$ & $1 E+06$ & $1 E+06$ & 0.5 & 0 & 0.5 & & 1005 \\
\hline $8: 27$ & 3 & 1038 & 914.5 & 886.5 & 946.333 & 8402.78 & 1013.36 & 3580.03 & 4708.07 & 0.5 & 2354.03 & 0.5 & 945.75 & 1286.5 \\
\hline $8: 37$ & 4 & 904.5 & 1052 & 977.5 & 978 & 5402.25 & 5476 & 0.25 & 5439.13 & 0.58895 & 3203.37 & 0.41105 & 1104.51 & 1238.5 \\
\hline $8: 47$ & 5 & 977.5 & 1056 & 880.5 & 971.333 & 38.0278 & 7168.44 & 8250.69 & 3603.24 & 0.65386 & 2356.02 & 0.34614 & 1004.42 & 1356 \\
\hline $8: 59$ & 6 & 905 & 806.5 & 912.5 & 874.667 & 920.111 & 4646.69 & 1431.36 & 2783.4 & 0.64869 & 1805.55 & 0.35131 & 1068 & 1273 \\
\hline
\end{tabular}

Table D4.26: Kalman Filter for test case 54-2200

\begin{tabular}{|c|c|c|c|c|c|c|c|c|c|c|c|c|c|c|}
\hline time & trip & art3 & art2 & art1 & Average & $\Delta 3$ & $\Delta 2$ & $\Delta 1$ & Var[data]n & $g(k+1)$ & $e(k+1)$ & $a(k+1)$ & Predict & Observed \\
\hline $8: 17$ & 2 & 0 & 0 & 0 & 1005 & $1 E+06$ & $1 E+06$ & $1 E+06$ & $1 E+06$ & 0.5 & 0 & 0.5 & & 1005 \\
\hline $8: 27$ & 3 & 1384.5 & 1213.5 & 830.5 & 1142.83 & 58402.8 & 4993.78 & 97552.1 & 31698.3 & 0.5 & 15849.1 & 0.5 & 917.75 & 1459.5 \\
\hline $8: 37$ & 4 & 1167 & 1356.5 & 961.5 & 1161.67 & 28.4444 & 37960 & 40066.7 & 18994.2 & 0.64719 & 12293 & 0.35281 & 1137.2 & 1490 \\
\hline $8: 47$ & 5 & 1423.5 & 1332 & 824.5 & 1193.33 & 52976.7 & 19228.4 & 136038 & 36102.6 & 0.57274 & 20677.4 & 0.42726 & 1108.84 & 1421 \\
\hline $8: 59$ & 6 & 1354.5 & 1228.5 & 1008.5 & 1197.17 & 24753.8 & 981.778 & 35595.1 & 12867.8 & 0.72275 & 9300.25 & 0.27725 & 1123 & 1362 \\
\hline
\end{tabular}


Table D4.27: Kalman Filter for test case 40-2200

\begin{tabular}{|c|c|c|c|c|c|c|c|c|c|c|c|c|c|c|}
\hline time & trip & art3 & art2 & art1 & Average & $\Delta 3$ & $\Delta 2$ & $\Delta 1$ & Var[data]n & $g(k+1)$ & $e(k+1)$ & $a(k+1)$ & Predict & Observed \\
\hline $8: 17$ & 2 & 0 & 0 & 0 & 1005 & $1 E+06$ & $1 E+06$ & $1 E+06$ & $1 E+06$ & 0.5 & 0 & 0.5 & & 1005 \\
\hline $8: 27$ & 3 & 833 & 1017.5 & 821 & 890.5 & 3306.25 & 16129 & 4830.25 & 9717.63 & 0.5 & 4858.81 & 0.5 & 913 & 1280.5 \\
\hline $8: 37$ & 4 & 792 & 985.5 & 827.5 & 868.333 & 5826.78 & 13728 & 1667.36 & 9777.4 & 0.59951 & 5861.65 & 0.40049 & 1008.92 & 1358 \\
\hline $8: 47$ & 5 & 912.5 & 927 & 774.5 & 871.333 & 1694.69 & 3098.78 & 9376.69 & 2396.74 & 0.77506 & 1857.62 & 0.22494 & 905.751 & 1218.5 \\
\hline $8: 59$ & 6 & 852.5 & 892.5 & 716.5 & 820.5 & 1024 & 5184 & 10816 & 3104 & 0.61516 & 1909.45 & 0.38484 & 909.7 & 1396 \\
\hline
\end{tabular}

Table D4.28: Kalman Filter for test case 55-2200

\begin{tabular}{|c|c|c|c|c|c|c|c|c|c|c|c|c|c|c|}
\hline time & trip & art3 & art2 & art1 & Average & $\Delta \mathbf{3}$ & $\Delta \mathbf{2}$ & $\Delta 1$ & Var[data]n & $g(k+1)$ & $e(k+1)$ & $a(k+1)$ & Predict & Observed \\
\hline $8: 17$ & 2 & 0 & 0 & 0 & 1005 & $1 E+06$ & $1 E+06$ & $1 E+06$ & $1 E+06$ & 0.5 & 0 & 0.5 & & 1005 \\
\hline $8: 27$ & 3 & 1017.5 & 821 & 1280.5 & 1039.67 & 491.361 & 47815.1 & 58000.7 & 24153.2 & 0.5 & 12076.6 & 0.5 & 1142.75 & 1438 \\
\hline $8: 37$ & 4 & 985.5 & 827.5 & 1358 & 1057 & 5112.25 & 52670.3 & 90601 & 28891.3 & 0.58644 & 16942.9 & 0.41356 & 1391.09 & 1470.5 \\
\hline $8: 47$ & 5 & 927 & 774.5 & 1218.5 & 973.333 & 2146.78 & 39534.7 & 60106.7 & 20840.7 & 0.6445 & 13431.9 & 0.3555 & 1308.09 & 1177.5 \\
\hline $8: 59$ & 6 & 892.5 & 716.5 & 1395.5 & 1001.5 & 11881 & 81225 & 155236 & 46553 & 0.56304 & 26211.1 & 0.43696 & 1300 & 1166 \\
\hline
\end{tabular}

Table D4.29: Kalman Filter for test case 37-2200

\begin{tabular}{|c|c|c|c|c|c|c|c|c|c|c|c|c|c|c|}
\hline time & trip & art3 & art2 & art1 & Average & $\overline{\Delta \mathbf{3}}$ & $\Delta \mathbf{2}$ & $\Delta 1$ & Var[data]n & $g(k+1)$ & $e(k+1)$ & $a(k+1)$ & Predict & Observed \\
\hline $8: 17$ & 2 & 0 & 0 & 0 & 1005 & $1 E+06$ & $1 E+06$ & $1 E+06$ & $1 E+06$ & 0.5 & 0 & 0.5 & & 1005 \\
\hline $8: 27$ & 3 & 1438 & 934 & 821 & 1064.33 & 139627 & 16986.8 & 59211.1 & 78306.8 & 0.5 & 39153.4 & 0.5 & 913 & 1275.5 \\
\hline $8: 37$ & 4 & 1470.5 & 1025 & 827.5 & 1107.67 & 131648 & 6833.78 & 78493.4 & 69240.9 & 0.61021 & 42251.3 & 0.38979 & 1002.13 & 1254 \\
\hline $8: 47$ & 5 & 1177.5 & 841 & 773.5 & 930.667 & 60926.7 & 8040.11 & 24701.4 & 34483.4 & 0.68995 & 23791.8 & 0.31005 & 922.48 & 1176 \\
\hline $8: 59$ & 6 & 1166 & 1100.5 & 730.5 & 999 & 27889 & 10302.3 & 72092.3 & 19095.6 & 0.69192 & 13212.7 & 0.30808 & 867.7 & 1358 \\
\hline
\end{tabular}


Table D4.30: Kalman Filter for test case 45-2200

\begin{tabular}{|c|c|c|c|c|c|c|c|c|c|c|c|c|c|c|}
\hline time & trip & art3 & art2 & art1 & Average & $\Delta 3$ & $\Delta 2$ & $\Delta 1$ & Var[data]n & $g(k+1)$ & $\theta(k+1)$ & $a(k+1)$ & Predict & Observed \\
\hline $8: 17$ & 2 & 0 & 0 & 0 & 1005 & $1 E+06$ & $1 E+06$ & $1 E+06$ & $1 E+06$ & 0.5 & 0 & 0.5 & & 1005 \\
\hline $8: 27$ & 3 & 1275.5 & 818.5 & 933.5 & 1009.17 & 70933.4 & 36353.8 & 5725.44 & 53643.6 & 0.5 & 26821.8 & 0.5 & 969.25 & 1420.5 \\
\hline $8: 37$ & 4 & 1254 & 896 & 1092.5 & 1080.83 & 29986.7 & 34163.4 & 136.111 & 32075 & 0.64742 & 20766 & 0.35258 & 1208.15 & 1487.5 \\
\hline $8: 47$ & 5 & 1176 & 953.5 & 856.5 & 995.333 & 32640.4 & 1750.03 & 19274.7 & 17195.2 & 0.68825 & 11834.6 & 0.31175 & 1053.22 & 1365.5 \\
\hline $8: 59$ & 6 & 1357.5 & 86.5 & 975 & 806.333 & 303785 & 518160 & 28448.4 & 410972 & 0.5071 & 208403 & 0.4929 & 1167 & 1228 \\
\hline
\end{tabular}

Table D4.31: Kalman Filter for test case 43-2200

\begin{tabular}{|c|c|c|c|c|c|c|c|c|c|c|c|c|c|c|}
\hline time & trip & art3 & art2 & art1 & Average & $\mathbf{\Delta 3}$ & $\Delta \mathbf{2}$ & $\Delta 1$ & Var[data]n & $g(k+1)$ & $e(k+1)$ & $a(k+1)$ & Predict & Observed \\
\hline $8: 17$ & 2 & 0 & 0 & 0 & 1005 & $1 E+06$ & $1 E+06$ & $1 E+06$ & $1 E+06$ & 0.5 & 0 & 0.5 & & 1005 \\
\hline $8: 27$ & 3 & 933 & 952.5 & 884 & 923.167 & 96.6944 & 860.444 & 1534.03 & 478.569 & 0.5 & 239.285 & 0.5 & 944.5 & 1192.5 \\
\hline $8: 37$ & 4 & 907 & 875 & 798.5 & 860.167 & 2193.36 & 220.028 & 3802.78 & 1206.69 & 0.5451 & 657.772 & 0.4549 & 977.73 & 1345.5 \\
\hline $8: 47$ & 5 & 918 & 946 & 837 & 900.333 & 312.111 & 2085.44 & 4011.11 & 1198.78 & 0.60764 & 728.429 & 0.39236 & 1036.51 & 1230.5 \\
\hline $8: 59$ & 6 & 842 & 1095 & 953 & 963.333 & 14721.8 & 17336.1 & 106.778 & 16028.9 & 0.511111 & 8192.53 & 0.48889 & 1089 & 1141 \\
\hline
\end{tabular}

Table D4.32: Kalman Filter for test case 46-2200

\begin{tabular}{|c|c|c|c|c|c|c|c|c|c|c|c|c|c|c|}
\hline $\operatorname{time}$ & trip & art3 & art2 & art1 & Average & $\Delta \mathbf{3}$ & $\Delta 2$ & $\Delta 1$ & Var[data]n & $g(k+1)$ & $e(k+1)$ & $a(k+1)$ & Predict & Observed \\
\hline $8: 17$ & 2 & 0 & 0 & 0 & 1005 & $1 E+06$ & $1 E+06$ & $1 E+06$ & $1 E+06$ & 0.5 & 0 & 0.5 & & 1005 \\
\hline $8: 27$ & 3 & 952.5 & 884 & 1192.5 & 1009.67 & 3268.03 & 15792.1 & 33428 & 9530.07 & 0.5 & 4765.03 & 0.5 & 1098.75 & 1343.5 \\
\hline $8: 37$ & 4 & 875 & 798.5 & 1345.5 & 1006.33 & 17248.4 & 43194.7 & 115034 & 30221.6 & 0.53654 & 16215 & 0.46346 & 1344.57 & 1250 \\
\hline $8: 47$ & 5 & 946 & 837 & 1230.5 & 1004.5 & 3422.25 & 28056.3 & 51076 & 15739.3 & 0.66999 & 10545.2 & 0.33001 & 1236.94 & 1399.5 \\
\hline $8: 59$ & 6 & 1095 & 953 & 1141 & 1063 & 1024 & 12100 & 6084 & 6562 & 0.72276 & 4742.76 & 0.27724 & 1213 & 1394 \\
\hline
\end{tabular}


Table D4.33: Kalman Filter for test case 51-2200

\begin{tabular}{|c|c|c|c|c|c|c|c|c|c|c|c|c|c|c|}
\hline $\operatorname{tim} \theta$ & trip & $\operatorname{art3}$ & $\operatorname{art2}$ & art1 & Average & $\Delta 3$ & $\Delta 2$ & $\Delta 1$ & Var[data]n & $g(k+1)$ & $\theta(k+1)$ & $a(k+1)$ & Predlct & Observed \\
\hline $8: 17$ & 2 & 0 & 0 & 0 & 1005 & $1 E+06$ & $1 E+06$ & $1 E+06$ & $1 E+06$ & 0.5 & 0 & 0.5 & & 1005 \\
\hline $8: 27$ & 3 & 1343.5 & 945.5 & 930 & 1073 & 73170.3 & 16256.3 & 20449 & 44713.3 & 0.5 & 22356.6 & 0.5 & 967.5 & 1306.5 \\
\hline $8: 37$ & 4 & 1250 & 1082.5 & 1005 & 1112.5 & 18906.3 & 900 & 11556.3 & 9903.13 & 0.76512 & 7577.1 & 0.23488 & 1075.82 & 1238.5 \\
\hline $8: 47$ & 5 & 1399.5 & 1044 & 896 & 1113.17 & 81986.8 & 4784.03 & 47161.4 & 43385.4 & 0.54016 & 23434.8 & 0.45984 & 1053.5 & 1357.5 \\
\hline $8: 59$ & 6 & 1141 & 1394 & 930.5 & 1155.17 & 200.694 & 57041.4 & 50475.1 & 28621 & 0.64524 & 18467.4 & 0.35476 & 1082 & 1124 \\
\hline
\end{tabular}

Table D4.34: Kalman Filter for test case 44-2200

\begin{tabular}{|c|c|c|c|c|c|c|c|c|c|c|c|c|c|c|}
\hline time & trip & art3 & art2 & art1 & Average & $\mathbf{\Delta 3}$ & $\Delta 2$ & $\Delta 1$ & Var[data]n & $g(k+1)$ & $\theta(k+1)$ & $a(k+1)$ & Predict & Observed \\
\hline $8: 17$ & 2 & 0 & 0 & 0 & 1005 & $1 E+06$ & $1 E+06$ & $1 E+06$ & $1 E+06$ & 0.5 & 0 & 0.5 & & 1005 \\
\hline $8: 27$ & 3 & 930 & 1306.5 & 833 & 1023.17 & 8680.03 & 80277.8 & 36163.4 & 44478.9 & 0.5 & 22239.5 & 0.5 & 919 & 1168 \\
\hline $8: 37$ & 4 & 1005 & 1238.5 & 1094 & 1112.5 & 11556.3 & 15876 & 342.25 & 13716.1 & 0.72386 & 9928.61 & 0.27614 & 1114.43 & 1472.5 \\
\hline $8: 47$ & 5 & 896 & 1357.5 & 860.5 & 1038 & 20164 & 102080 & 31506.3 & 61122.1 & 0.53756 & 32856.8 & 0.46244 & 1143.51 & 1342.5 \\
\hline $8: 59$ & 6 & 930.5 & 1124 & 911.5 & 988.667 & 3383.36 & 18315.1 & 5954.69 & 10849.2 & 0.80113 & 8691.68 & 0.19887 & 997.2 & 1362 \\
\hline
\end{tabular}

Table D4.35: Kalman Filter for test case 47-2200

\begin{tabular}{|c|c|c|c|c|c|c|c|c|c|c|c|c|c|c|}
\hline time & trip & art3 & art2 & $\operatorname{art1}$ & Average & $\Delta 3$ & $\Delta 2$ & $\Delta 1$ & Var[data]n & $g(k+1)$ & $e(k+1)$ & $a(k+1)$ & Predlct & Observed \\
\hline $8: 17$ & 2 & 0 & 0 & 0 & 1005 & $1 E+06$ & $1 E+06$ & $1 E+06$ & $1 E+06$ & 0.5 & 0 & 0.5 & & 1005 \\
\hline $8: 27$ & 3 & 1168 & 1410.5 & 1015.2 & 1197.9 & 894.01 & 45198.8 & 33379.3 & 23046.4 & 0.5 & 11523.2 & 0.5 & 1010.1 & 1269 \\
\hline $8: 37$ & 4 & 1472.5 & 1416.5 & 1005 & 1298 & 30450.3 & 14042.3 & 85849 & 22246.3 & 0.60286 & 13411.3 & 0.39714 & 1109.85 & 1132 \\
\hline $8: 47$ & $\frac{4}{5}$ & 1342.5 & 1397 & 949 & 1229.5 & 12769 & 28056.3 & 78680.3 & 20412.6 & 0.62364 & 12730.1 & 0.37636 & 1017.87 & 1223 \\
\hline $8: 59$ & $\frac{4}{6}$ & 1362 & 1275 & 956 & 1197.67 & 27005.4 & 5980.44 & 58402.8 & 16492.9 & 0.63923 & 10542.8 & 0.36077 & 1052 & 1404 \\
\hline
\end{tabular}


Table D4.36: Kalman Filter for test case 42-2200

\begin{tabular}{|c|c|c|c|c|c|c|c|c|c|c|c|c|c|c|}
\hline time & trip & art3 & art2 & art1 & Average & $\Delta 3$ & $\Delta 2$ & $\Delta 1$ & Var[data]n & $g(k+1)$ & $e(k+1)$ & $a(k+1)$ & Predict & Observed \\
\hline $8: 17$ & 2 & 0 & 0 & 0 & 1005 & $1 E+06$ & $1 E+06$ & $1 E+06$ & $1 E+06$ & 0.5 & 0 & 0.5 & & 1005 \\
\hline $8: 27$ & 3 & 899 & 876 & 969 & 914.667 & 245.444 & 1495.11 & 2952.11 & 870.278 & 0.5 & 435.139 & 0.5 & 987 & 1318 \\
\hline $8: 37$ & 4 & 781.5 & 823.5 & 1066.5 & 890.5 & 11881 & 4489 & 30976 & 8185 & 0.51295 & 4198.47 & 0.48705 & 1188.99 & 1263 \\
\hline $8: 47$ & 5 & 1008 & 781.5 & 1054.5 & 948 & 3600 & 27722.3 & 11342.3 & 15661.1 & 0.5591 & 8756.12 & 0.4409 & 1146.43 & 1415.5 \\
\hline $8: 59$ & 6 & 962 & 831.5 & 992.5 & 928.667 & 1111.11 & 9441.36 & 4074.69 & 5276.24 & 0.72674 & 3834.46 & 0.27326 & 1108 & 1359 \\
\hline
\end{tabular}

Table D4.37: Kalman Filter for test case 48-2200

\begin{tabular}{|c|c|c|c|c|c|c|c|c|c|c|c|c|c|c|}
\hline time & trip & art3 & art2 & art1 & Average & $\Delta \mathbf{3}$ & $\Delta 2$ & $\Delta 1$ & Var[data]n & $\mathbf{g}(\mathbf{k}+1)$ & $\theta(k+1)$ & $a(k+1)$ & Predict & Observed \\
\hline $8: 17$ & 2 & 0 & 0 & 0 & 1005 & $1 E+06$ & $1 E+06$ & $1 E+06$ & $1 E+06$ & 0.5 & 0 & 0.5 & & 1005 \\
\hline $8: 27$ & 3 & 1005 & 949 & 819.5 & 924.5 & 6480.25 & 600.25 & 11025 & 3540.25 & 0.5 & 1770.13 & 0.5 & 912.25 & 1290 \\
\hline $8: 37$ & 4 & 985 & 1107.5 & 1035 & 1042.5 & 3306.25 & 4225 & 56.25 & 3765.63 & 0.59515 & 2241.13 & 0.40485 & 1138.24 & 1269.5 \\
\hline $8: 47$ & 5 & 1042.5 & 940 & 853.5 & 945.333 & 9441.36 & 28.4444 & 8433.36 & 4734.9 & 0.59569 & 2820.51 & 0.40431 & 1021.69 & 1400 \\
\hline $8: 59$ & 6 & 988.5 & 897 & 952 & 945.833 & 1820.44 & 2384.69 & 38.0278 & 2102.57 & 0.70073 & 1473.33 & 0.29927 & 1086 & 1471 \\
\hline
\end{tabular}


Table D4.38: Prediction Error of the Predictors (Overall situation)

\begin{tabular}{|c|c|c|c|c|c|c|c|c|}
\hline Dcode & Scode & Actual & Developed & Mar: & $\begin{array}{l}\text { Naive } \\
\text { Model }\end{array}$ & (2x+5. in & de & d3 \\
\hline 90 & 1100 & 905 & 946 & 856 & 874.4 & 0.045303867 & 0.05414 & 0.03381 \\
\hline 50 & 2200 & 1354.5 & 1377.6 & 1068.3 & 1484 & 0.017054264 & 0.2113 & 0.09561 \\
\hline 6 & 1200 & 915.5 & 917.61 & 893.84 & 795.25 & 0.002304752 & 0.02366 & 0.13135 \\
\hline 26 & 1300 & 863.5 & 1009.84 & 943.55 & 742.31 & 0.169473075 & 0.0927 & 0.14035 \\
\hline 21 & 1300 & 998 & 931.29 & 988.17 & 928.06 & 0.066843687 & 0.00985 & 0.07008 \\
\hline 8 & 1200 & 1022.5 & 952.37 & 1090 & 1384.2 & 0.068586797 & 0.06601 & 0.35374 \\
\hline 57 & 2100 & 1065 & 941.96 & 857 & 780.01 & 0.115530516 & 0.19531 & 0.2676 \\
\hline 3 & 1200 & 852.5 & 889.85 & 942 & 1028.22 & 0.043812317 & 0.10499 & 0.20612 \\
\hline 69 & 2100 & 771.5 & 928.82 & 1104 & 920.21 & 0.203914452 & 0.43098 & 0.19275 \\
\hline 87 & 1100 & 842 & 945.54 & 886 & 845.5 & 0.122969121 & 0.05226 & 0.00416 \\
\hline 78 & 3000 & 953 & 822.44 & 968 & 968.83 & 0.136998951 & 0.01574 & 0.01661 \\
\hline$\overline{43}$ & 2200 & 1141 & 1296 & 1088 & 1401.03 & 0.135845749 & 0.04645 & 0.2279 \\
\hline 24 & 1300 & 1111 & 943.17 & 1212.7 & 1039 & 0.151062106 & 0.09154 & 0.06481 \\
\hline 51 & 2200 & 1124 & 1358.5 & 1082 & 1409.7 & 0.203291815 & 0.03737 & 0.25418 \\
\hline 56 & 2100 & 877.5 & 874.29 & 938.89 & 736.22 & 0.00365812 & 0.06996 & 0.161 \\
\hline 33 & 1300 & 988.5 & 950.32 & 998.74 & 1189.6 & 0.038624178 & 0.01036 & 0.20344 \\
\hline 99 & 1100 & 962.5 & 896 & 966.09 & 959.56 & 0.069090909 & 0.00373 & 0.00305 \\
\hline 91 & 1100 & $\overline{96}$ & 902.38 & 932.8 & 888.3 & 0.064891192 & 0.03337 & 0.07948 \\
\hline 44 & 2200 & 1362 & 1132.69 & 997 & 1422.5 & 0.164236417 & 0.26799 & 0.04442 \\
\hline 27 & 1300 & 1023 & 911.27 & 770.31 & 852.56 & 0.109217986 & 0.24701 & 0.16661 \\
\hline
\end{tabular}

Note: d1,d2, d3: The absolute relative error (\%) of the Developed Model, the Kalman Filter and the Naivve predictor, respectively. 
Table D4.39: Prediction Error of the Predictors (situation coded as 2200)

\begin{tabular}{|c|c|c|c|c|c|c|c|c|}
\hline 52 & 2200 & 1402.5 & 1290.21 & 1097 & 1222.91 & 0.080064 & 0.217825 & 0.12805 \\
\hline 38 & 2200 & 1251.5 & 1306.05 & 1404.02 & 1497.24 & 0.038895 & 0.108749 & 0.175216 \\
\hline 39 & 2200 & 1272.5 & 1331.72 & 1068.3 & 795.25 & 0.042225 & 0.145597 & 0.340285 \\
\hline 50 & 2200 & 1354.5 & 1377.61 & 1162.2 & 1483.46 & 0.016478 & 0.137112 & 0.09195 \\
\hline 40 & 2200 & 1395.5 & 1227.24 & 909.69 & 1127.25 & 0.119971 & 0.346389 & 0.191266 \\
\hline 37 & 2200 & 1357.5 & 1211 & 867.75 & 1384.2 & 0.104456 & 0.349198 & 0.019037 \\
\hline 57 & 2200 & 1065 & 941.96 & 857 & 780.01 & 0.087729 & 0.148307 & 0.203201 \\
\hline 54 & 2200 & 1361.5 & 1259.5 & 1122.9 & 1028.22 & 0.072727 & 0.170125 & 0.237633 \\
\hline 46 & 2200 & 1394 & 1332 & 1212.7 & 920.21 & 0.044207 & 0.129269 & 0.337818 \\
\hline 44 & 2200 & 1362 & 1132.69 & 997.21 & 1422.5 & 0.163501 & 0.2601 & 0.043137 \\
\hline 47 & 2200 & 1404 & 1187 & 1052 & 1233.32 & 0.154724 & 0.25098 & 0.121697 \\
\hline 42 & 2200 & 1359 & 1332 & 1108.1 & 1332.27 & 0.019251 & 0.178895 & 0.019059 \\
\hline 48 & 2200 & 1471 & 1306.9 & 1086.1 & 1562 & 0.117005 & 0.274439 & 0.064884 \\
\hline 51 & 2200 & 1124 & 1358.5 & 1082 & 1409.7 & 0.167201 & 0.029947 & 0.203708 \\
\hline
\end{tabular}

Note: $\mathrm{d} 1, \mathrm{~d} 2, \mathrm{~d} 3$ : The absolute relative error (\%) of the Developed Model, the Kalman Filter and the Naïve Models, respectively. 


\section{Database for Route 95}

Table D4.40: Database for Route 95, segment Laurent-Mackenzie (70 simulation runs)

\begin{tabular}{|c|c|c|c|c|c|}
\hline Dcode & Scode & Re & R2 & R3 & R4 \\
\hline 54 & 2200 & 794.5 & 681.5 & 677 & 747 \\
\hline 58 & 2200 & 681.5 & 798 & 662 & 741.5 \\
\hline 33 & 1200 & 571 & 537.5 & 561.5 & 605.5 \\
\hline 42 & 1200 & 867 & 747 & 746 & 686.5 \\
\hline 47 & 1200 & 747 & 866.5 & 747 & 686.5 \\
\hline 62 & 2200 & 699 & 672.5 & 673 & 642.5 \\
\hline 48 & 1200 & 746 & 866.5 & 747 & 808.5 \\
\hline 36 & 1200 & 746.5 & 755 & 748 & 686 \\
\hline 16 & 3000 & 632 & 632.5 & 648.5 & 592 \\
\hline 52 & 2200 & 781.5 & 785 & 686.5 & 740 \\
\hline 3 & 3000 & 803.5 & 647.5 & 795 & 731.5 \\
\hline 2 & 3000 & 673.5 & 666 & 687 & 633.5 \\
\hline 7 & 3000 & 698.5 & 677 & 666.5 & 725 \\
\hline 21 & 1200 & 563 & 638 & 548.5 & 526 \\
\hline 28 & 1200 & 562 & 657 & 561.5 & 511.5 \\
\hline 19 & 1200 & 553.5 & 547.5 & 546 & 613 \\
\hline 4 & 3000 & 782 & 792 & 680.5 & 619 \\
\hline 11 & 3000 & 685 & 753.5 & 797 & 641.5 \\
\hline 45 & 1200 & 747 & 773 & 747.5 & 686 \\
\hline 39 & 1200 & 746 & 746.5 & 643.5 & 698.5 \\
\hline 5 & 3000 & 690 & 770.5 & 679.5 & 735.5 \\
\hline 55 & 2200 & 685 & 795 & 677.5 & 737.5 \\
\hline 38 & 1200 & 746.5 & 747 & 763 & 688 \\
\hline 13 & 3000 & 668 & 792.5 & 677 & 728.5 \\
\hline 64 & 2200 & 694 & 830.5 & 755 & 752 \\
\hline 14 & 3000 & 689 & 674 & 683 & 627 \\
\hline 10 & 3000 & 672.5 & 661.5 & 667 & 615.5 \\
\hline 29 & 1200 & 559 & 548 & 563 & 614.5 \\
\hline 35 & 1200 & 548 & 521.5 & 559.5 & 499.5 \\
\hline 53 & 2200 & 820 & 694.5 & 671.5 & 743.5 \\
\hline 17 & 1200 & 550 & 526 & 550.5 & 589.5 \\
\hline 6 & 3000 & 720 & 810 & 750 & 700 \\
\hline 12 & 3000 & 689.5 & 795.5 & 680 & 717 \\
\hline 18 & 1200 & 550 & 641.5 & 571.5 & 525 \\
\hline 31 & 1200 & 558 & 558.5 & 557 & 509 \\
\hline 56 & 2200 & 805.5 & 699 & 672.5 & 728 \\
\hline 8 & 3000 & 681 & 647.5 & 691.5 & 743 \\
\hline 49 & 1200 & 748.5 & 747.5 & 746 & 686.5 \\
\hline
\end{tabular}




\begin{tabular}{|c|c|c|c|c|c|}
\hline 9 & 3000 & 679.5 & 804.5 & 682 & 745 \\
\hline 59 & 2200 & 778.5 & 781 & 687 & 740 \\
\hline 41 & 1200 & 748 & 754.5 & 638.5 & 809.5 \\
\hline 46 & 1200 & 747 & 766.5 & 746.5 & 807 \\
\hline 57 & 2200 & 671 & 691.5 & 683 & 734 \\
\hline 51 & 2200 & 694.5 & 777.5 & 686 & 734.5 \\
\hline 1 & 3000 & 702 & 676.5 & 676.5 & 737 \\
\hline 22 & 1200 & 556 & 540.5 & 554 & 502 \\
\hline 40 & 1200 & 747.5 & 746.5 & 746 & 708 \\
\hline 63 & 2200 & 798 & 796.5 & 675 & 732 \\
\hline 50 & 1200 & 820 & 788 & 777 & 790 \\
\hline 61 & 2200 & 683 & 763.5 & 688 & 833.5 \\
\hline 43 & 1200 & 748 & 746.5 & 644.5 & 811.5 \\
\hline 24 & 1200 & 557 & 542 & 564 & 580 \\
\hline 25 & 1200 & 566 & 535.5 & 554.5 & 612.5 \\
\hline 37 & 1200 & 746 & 869 & 747.5 & 707.5 \\
\hline 65 & 2200 & 685 & 772 & 686 & 712 \\
\hline 44 & 1200 & 646 & 746.5 & 748.5 & 686.5 \\
\hline 60 & 2200 & 686 & 678 & 681 & 722.5 \\
\hline 20 & 1200 & 554 & 538.5 & 561.5 & 509 \\
\hline 34 & 1200 & 553.5 & 657.5 & 554.5 & 514.5 \\
\hline 26 & 1200 & 553 & 640 & 555.5 & 496.5 \\
\hline 23 & 1200 & 569.5 & 548.5 & 567 & 609 \\
\hline 30 & 1200 & 565.5 & 552.5 & 568.5 & 631 \\
\hline 32 & 1200 & 572.5 & 569.5 & 675.5 & 513 \\
\hline 27 & 1200 & 573.5 & 553.5 & 551.5 & 513 \\
\hline 15 & 3000 & 655 & 673 & 648 & 632 \\
\hline 66 & 1200 & 755.5 & 866.5 & 633.5 & 685.5 \\
\hline 67 & 1200 & 747 & 746.5 & 747.5 & 809.5 \\
\hline 68 & 1200 & 756.5 & 866.5 & 755.5 & 931.5 \\
\hline 69 & 1200 & 747 & 746 & 747.5 & 806.5 \\
\hline 70 & 1200 & 746.5 & 748.5 & 748 & 696 \\
\hline
\end{tabular}




\section{Route 95- Running time Predictions of Developed Model and the Reference Predictors}

The results of the developed model were returned from the Matlab program (Please see Appendix C). The predictions of the developed model as well as that of reference predictors are presented in the Table D4.62.

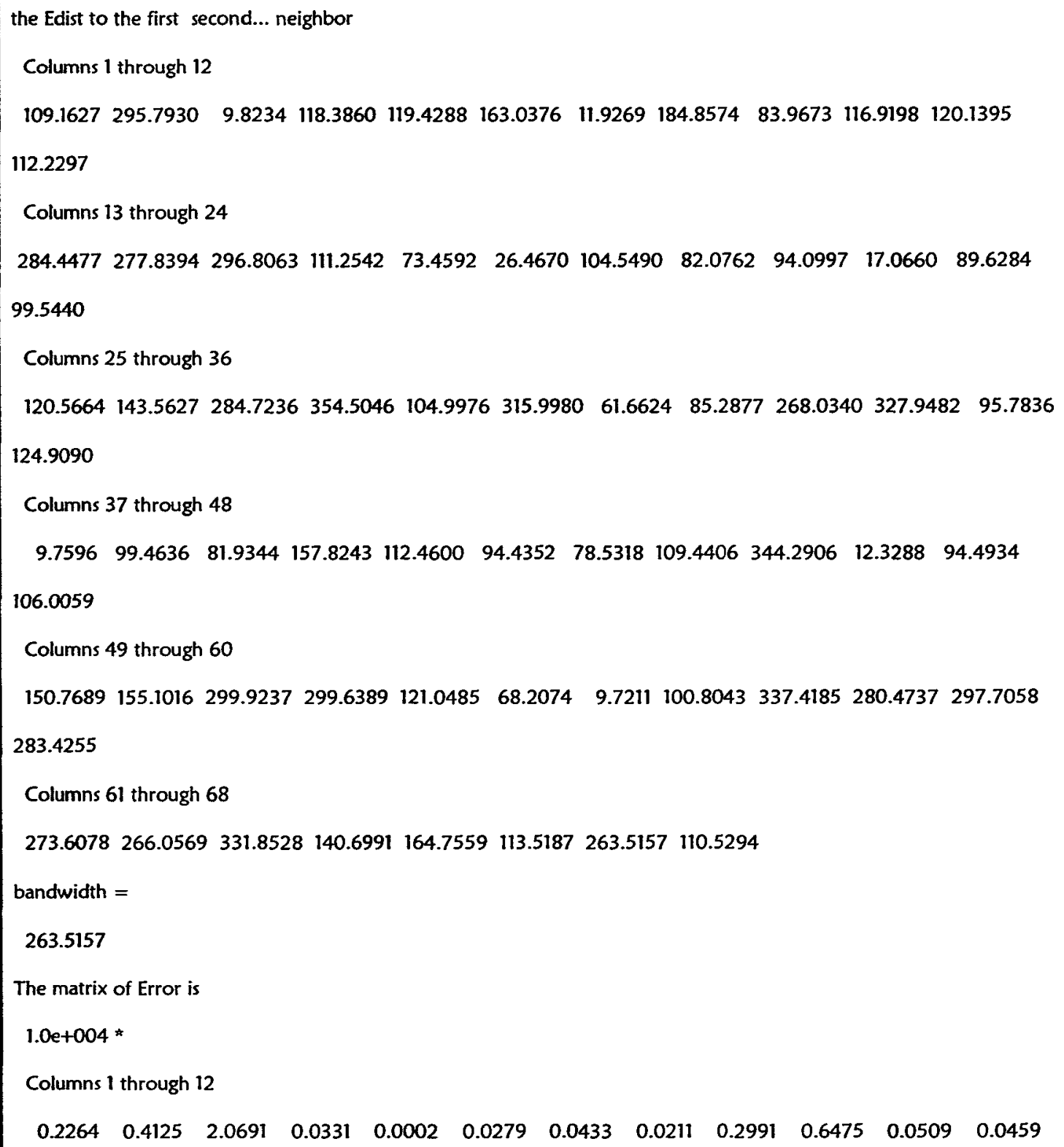




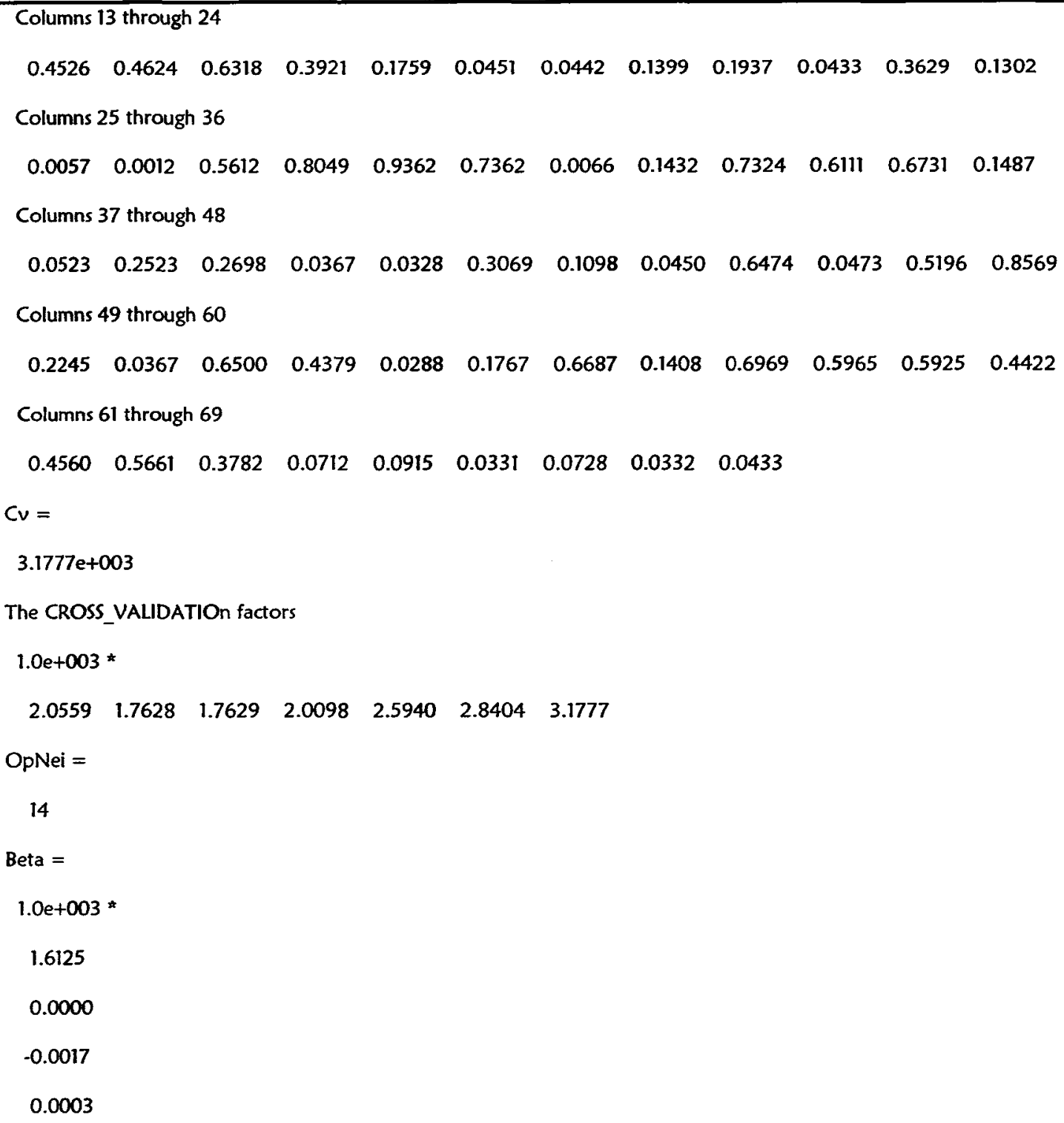

Figure D4.2: An Example of the Matlab Outputs (Case 54-2200, R95) 
Table D4.42: Kalman Filter for case 54-2200

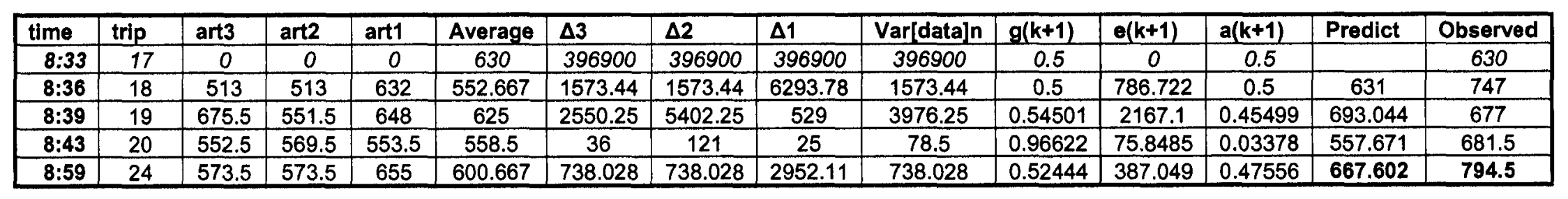

Table D4.43: Kalman Filter for case 47-1200

\begin{tabular}{|c|c|c|c|c|c|c|c|c|c|c|c|c|c|c|}
\hline time & trip & art3 & art2 & art1 & Average & $\Delta 3$ & $\Delta \mathbf{2}$ & $\Delta 1$ & Var[data]n & $g(k+1)$ & $\theta(k+1)$ & $a(k+1)$ & Predict & Observed \\
\hline $8: 33$ & 17 & 0 & 0 & 0 & 630 & 396900 & 396900 & 396900 & 396900 & 0.5 & 0 & 0.5 & & 630 \\
\hline $8: 36$ & 18 & 605.5 & 686.5 & 686.5 & 659.5 & 2916 & 729 & 729 & 1822.5 & 0.5 & 911.25 & 0.5 & 658.25 & 686.5 \\
\hline $8: 39$ & 19 & 662 & 561.5 & 746 & 656.5 & 30.25 & 9025 & 8010.25 & 4527.63 & 0.54572 & 2470.8 & 0.45428 & 718.97 & 746 \\
\hline $8: 43$ & 20 & 789 & 537.5 & 747 & 691.167 & 9571.36 & 23613.4 & 3117.36 & 16592.4 & 0.53465 & 8871.1 & 0.46535 & 746.535 & 866.5 \\
\hline $8: 59$ & 24 & 681.5 & 571 & 867 & 706.5 & 625 & 18360.3 & 25760.3 & 9492.63 & 0.65923 & 6257.82 & 0.34077 & 866.83 & 747 \\
\hline
\end{tabular}

Table D4.44: Kalman Filter for case 48-1200

\begin{tabular}{|c|c|c|c|c|c|c|c|c|c|c|c|c|c|c|}
\hline time & trip & art3 & art2 & art1 & Average & $\Delta 3$ & $\Delta 2$ & $\Delta 1$ & Var[data]n & $g(k+1)$ & $e(k+1)$ & $a(k+1)$ & Predict & Observed \\
\hline $8: 33$ & 17 & 0 & 0 & 0 & 630 & 396900 & 396900 & 396900 & 396900 & 0.5 & 0 & 0.5 & & 630 \\
\hline $8: 36$ & 1 & 686.5 & 686.5 & 642.5 & 671.833 & 215.111 & 215.111 & 860.444 & 215.111 & 0.5 & 107.556 & 0.5 & 636.25 & 808.5 \\
\hline $8: 39$ & 19 & 746 & 747 & 673 & 722 & 576 & 625 & 2401 & 600.5 & 0.5411 & 324.929 & 0.4589 & 735.181 & 747 \\
\hline $8: 43$ & 20 & 747 & 866.5 & 672.5 & 762 & 225 & 10920.3 & 8010.25 & 5572.63 & 0.51416 & 2865.24 & 0.48584 & 708.695 & 866.5 \\
\hline $8: 59$ & 24 & 867 & 747 & 699 & 771 & 9216 & 576 & 5184 & 4896 & 0.61319 & 3002.16 & 0.38681 & 763.791 & 746 \\
\hline
\end{tabular}


Table D4.45: Kalman Filter for case 36-1200

\begin{tabular}{|c|c|c|c|c|c|c|c|c|c|c|c|c|c|c|}
\hline time & trip & art3 & art2 & art1 & Average & $\Delta 3$ & $\Delta 2$ & $\Delta 1$ & Var[data]n & $g(k+1)$ & $\theta(k+1)$ & $a(k+1)$ & Predlct & Observed \\
\hline $8: 33$ & 17 & 0 & 0 & 0 & 630 & 396900 & 396900 & 396900 & 396900 & 0.5 & 0 & 0.5 & & 630 \\
\hline $8: 36$ & 18 & 686.5 & 642.5 & 808.5 & 712.5 & 676 & 4900 & 9216 & 2788 & 0.5 & 1394 & 0.5 & 719.25 & 686 \\
\hline $8: 39$ & 19 & 747 & 673 & 747 & 722.333 & 608.444 & 2433.78 & 608.444 & 1521.1111 & 0.65712 & 999.546 & 0.34288 & 726.084 & 748 \\
\hline $8: 43$ & 20 & 866.5 & 672.5 & 866.5 & 801.833 & 4181.78 & 16727.1 & 4181.78 & 10454.444 & 0.52281 & 5465.71 & 0.47719 & 809.953 & 755 \\
\hline $8: 59$ & 24 & 747 & 699 & 746 & 730.667 & 266.778 & 1002.78 & 235.111 & 634.77778 & 0.90575 & 574.952 & 0.09425 & 746.848 & 746.5 \\
\hline
\end{tabular}

Table D4.46: Kalman Filter for case $\mathbf{1 6 - 3 0 0 0}$

\begin{tabular}{|c|c|c|c|c|c|c|c|c|c|c|c|c|c|c|}
\hline time & trip & art3 & art2 & art1 & Average & $\Delta 3$ & $\Delta 2$ & $\Delta 1$ & Var[data]n & $g(k+1)$ & $\theta(k+1)$ & $a(k+1)$ & Predict & Observed \\
\hline $8: 33$ & 17 & 0 & 0 & 0 & 630 & 396900 & 396900 & 396900 & 396900 & 0.5 & 0 & 0.5 & & 630 \\
\hline $8: 36$ & 18 & 642.5 & 808.5 & 686 & 712.3333 & 4876.69 & 9248.03 & 693.444 & 7062.3611 & 0.5 & 3531.18 & 0.5 & 658 & 592 \\
\hline $8: 39$ & 19 & 673 & 747 & 748 & 722.6667 & 2466.78 & 592.111 & 641.778 & 1529.4444 & 0.76792 & 1174.49 & 0.23208 & 711.795 & 648.5 \\
\hline $8: 59$ & 24 & 699 & 746 & 746.5 & 730.5 & 992.25 & 240.25 & 256 & 616.25 & 0.90101 & 555.245 & 0.09899 & 735.215 & 632 \\
\hline
\end{tabular}

Table D4.47: Kalman Filter for case 21-1200

\begin{tabular}{|c|c|c|c|c|c|c|c|c|c|c|c|c|c|c|}
\hline time & trip & $\operatorname{art3}$ & art2 & art1 & Average & $\Delta 3$ & $\Delta 2$ & $\Delta 1$ & Var[data]n & $g(k+1)$ & $\theta(k+1)$ & $a(k+1)$ & Predict & Observed \\
\hline $8: 33$ & 17 & 0 & 0 & 0 & 630 & 396900 & 396900 & 396900 & 396900 & 0.5 & 0 & 0.5 & & 630 \\
\hline $8: 36$ & 18 & 731.5 & 633.5 & 725 & 696.667 & 1213.36 & 3990.03 & 802.778 & 2601.6944 & 0.5 & 1300.85 & 0.5 & 677.5 & 526 \\
\hline $8: 39$ & 19 & 795 & 687 & 666.5 & 716.167 & 6214.69 & 850.694 & 2466.78 & 3532.6944 & 0.57774 & 2040.99 & 0.42226 & 607.173 & 548.5 \\
\hline $8: 43$ & 20 & 647.5 & 666 & 677 & 663.5 & 256 & 6.25 & 182.25 & 131.125 & 0.94307 & 123.66 & 0.05693 & 669.684 & 638 \\
\hline $8: 59$ & 24 & 647.5 & 666 & 677 & 663.5 & 256 & 6.25 & 182.25 & 131.125 & 0.66022 & 86.5712 & 0.33978 & 663.749 & 563 \\
\hline
\end{tabular}


Table D4.48: Kalman Filter for case 19-1200

\begin{tabular}{|c|c|c|c|c|c|c|c|c|c|c|c|c|c|c|}
\hline time & trip & art3 & $\operatorname{art2}$ & art1 & Average & $\Delta \mathbf{3}$ & $\Delta 2$ & $\Delta 1$ & Var[data]n & $g(k+1)$ & $e(k+1)$ & $a(k+1)$ & Predict & Observed \\
\hline $8: 33$ & 17 & 0 & 0 & 0 & 630 & 396900 & 396900 & 396900 & 396900 & 0.5 & 0 & 0.5 & & 630 \\
\hline $8: 36$ & 18 & 725 & 526 & 511.5 & 587.5 & 18906.3 & 3782.25 & 5776 & 11344.25 & 0.5 & 5672.13 & 0.5 & 570.75 & 613 \\
\hline $8: 39$ & 19 & 666.5 & 548.5 & 561.5 & 592.1667 & 5525.44 & 1906.78 & 940.444 & 3716.1111 & 0.71642 & 2662.3 & 0.28358 & 576.104 & 546 \\
\hline $8: 43$ & 20 & 677 & 638 & 657 & 657.3333 & 386.778 & 373.778 & 0.11111 & 380.27778 & 0.8889 & 338.029 & 0.1111 & 644.668 & 547.5 \\
\hline $8: 59$ & 24 & 698.5 & 563 & 562 & 607.8333 & 8220.44 & 2010.03 & 2100.69 & 5115.2361 & 0.51599 & 2639.42 & 0.48401 & 554.982 & 553.5 \\
\hline
\end{tabular}

Table D4.49: Kalman Filter for case 45-1200

\begin{tabular}{|c|c|c|c|c|c|c|c|c|c|c|c|c|c|c|}
\hline time & trip & art3 & art2 & art1 & Average & $\Delta 3$ & $\Delta 2$ & $\Delta 1$ & Var[data]n & $g(k+1)$ & $\theta(k+1)$ & $a(k+1)$ & Predlct & Observed \\
\hline $8: 33$ & 17 & 0 & 0 & 0 & 630 & 396900 & 396900 & 396900 & 396900 & 0.5 & 0 & 0.5 & & 630 \\
\hline $8: 36$ & 18 & 613 & 619 & 641.5 & 624.5 & 132.25 & 30.25 & 289 & 81.25 & 0.5 & 40.625 & 0.5 & 635.75 & 686 \\
\hline $8: 39$ & 19 & 546 & 680.5 & 797 & 674.5 & 16512.3 & 36 & 15006.3 & 8274.125 & 0.50122 & 4147.19 & 0.49878 & 741.636 & 747.5 \\
\hline $8: 43$ & 20 & 547.5 & 792 & 753.5 & 697.667 & 22550 & 8898.78 & 3117.36 & 15724.403 & 0.55825 & 8778.21 & 0.44175 & 750.85 & 773 \\
\hline $8: 59$ & 24 & 553.5 & 782 & 685 & 673.5 & 14400 & 11772.3 & 132.25 & 13086.125 & 0.62558 & 8186.43 & 0.37442 & 717.949 & 747 \\
\hline
\end{tabular}

Table D4.50: Kalman Filter for case 39-1200

\begin{tabular}{|c|c|c|c|c|c|c|c|c|c|c|c|c|c|c|}
\hline time & trip & art3 & art2 & art1 & Average & $\Delta \mathbf{3}$ & $\Delta 2$ & $\Delta 1$ & Var[data]n & $g(k+1)$ & $e(k+1)$ & $a(k+1)$ & Predict & Observed \\
\hline $8: 33$ & 17 & 0 & 0 & 0 & 630 & 396900 & 396900 & 396900 & 396900 & 0.5 & 0 & 0.5 & & 630 \\
\hline $8: 36$ & 18 & 619 & 641.5 & 686 & 648.833 & 890.028 & 53.7778 & 1381.36 & 471.903 & 0.5 & 235.951 & 0.5 & 658 & 698.5 \\
\hline $8: 39$ & 19 & 680.5 & 797 & 747.5 & 741.667 & 3741.36 & 3061.78 & 34.0278 & 3401.57 & 0.51676 & 1757.8 & 0.48324 & 723.821 & 643.5 \\
\hline $8: 43$ & 20 & 792 & 753.5 & 773 & 772.833 & 367.361 & 373.778 & 0.02778 & 370.569 & 0.85171 & 315.617 & 0.14829 & 753.796 & 746.5 \\
\hline $8: 59$ & 24 & 782 & 685 & 747 & 738 & 1936 & 2809 & 81 & 2372.5 & 0.53118 & 1260.23 & 0.46882 & 746.766 & 746 \\
\hline
\end{tabular}


Table D4.51: Kalman Filter for case 38-1200

\begin{tabular}{|c|c|c|c|c|c|c|c|c|c|c|c|c|c|c|}
\hline $\operatorname{tim} \theta$ & trlp & art3 & art2 & art1 & Average & $\Delta 3$ & $\Delta \mathbf{2}$ & $\Delta 1$ & Var[data]n & $g(k+1)$ & $e(k+1)$ & $a(k+1)$ & Predlct & Observed \\
\hline $8: 33$ & 17 & 0 & 0 & 0 & 630 & 396900 & 396900 & 396900 & 396900 & 0.5 & 0 & 0.5 & & 630 \\
\hline $8: 36$ & 18 & 735.5 & 735.5 & 737.5 & 736.167 & 0.44444 & 0.44444 & 1.77778 & 0.44444 & 0.5 & 0.22222 & 0.5 & 683.75 & 688 \\
\hline $8: 39$ & 19 & 643.5 & 679.5 & 677.5 & 666.833 & 544.444 & 160.444 & 113.778 & 352.444 & 0.50016 & 176.278 & 0.49984 & 682.748 & 763 \\
\hline $8: 43$ & 20 & 746.5 & 770.5 & 795 & 770.667 & 584.028 & 0.02778 & 592.111 & 292.028 & 0.61592 & 179.866 & 0.38408 & 782.709 & 747 \\
\hline $8: 59$ & 24 & 746 & 690 & 685 & 707 & 1521 & 289 & 484 & 905 & 0.5452 & 493.402 & 0.4548 & 713.198 & 746.5 \\
\hline
\end{tabular}

Table D4.52: Kalman Filter for case 64-2200

\begin{tabular}{|c|c|c|c|c|c|c|c|c|c|c|c|c|c|c|}
\hline time & trip & $\operatorname{art3}$ & art2 & art1 & Average & $\Delta \mathbf{3}$ & $\Delta \mathbf{2}$ & $\Delta 1$ & Var[data]n & $g(k+1)$ & $e(k+1)$ & $a(k+1)$ & Predict & Observed \\
\hline $8: 33$ & 17 & 0 & 0 & 0 & 630 & 396900 & 396900 & 396900 & 396900 & 0.5 & 0 & 0.5 & & 630 \\
\hline $8: 36$ & 18 & 737.5 & 688 & 728.5 & 718 & 380.25 & 900 & 110.25 & 640.125 & 0.5 & 320.063 & 0.5 & 679.25 & 752 \\
\hline $8: 39$ & 19 & 677.5 & 763 & 677 & 705.833 & 802.778 & 3268.03 & 831.361 & 2035.4 & 0.53645 & 1091.88 & 0.46355 & 711.767 & 755 \\
\hline $8: 43$ & 20 & 795 & 747 & 792.5 & 778.167 & 283.361 & 971.361 & 205.444 & 627.361 & 0.73265 & 459.637 & 0.26735 & 782.474 & 830.5 \\
\hline $8: 59$ & 24 & 685 & 746.5 & 668 & 699.833 & 220.028 & 2177.78 & 1013.36 & 1198.9 & 0.58043 & 695.877 & 0.41957 & 736.18 & 694 \\
\hline
\end{tabular}

Table D4.53: Kalman Filter for case 29-1200

\begin{tabular}{|c|c|c|c|c|c|c|c|c|c|c|c|c|c|c|}
\hline time & trip & art3 & art2 & art1 & Average & $\Delta \mathbf{3}$ & $\Delta \mathbf{2}$ & $\Delta 1$ & Var[data]n & $g(k+1)$ & $e(k+1)$ & $a(k+1)$ & Predict & Observed \\
\hline $8: 33$ & 17 & 0 & 0 & 0 & 630 & 396900 & 396900 & 396900 & 396900 & 0.5 & 0 & 0.5 & & 630 \\
\hline $8: 36$ & 18 & 752 & 627 & 615.5 & 664.833 & 7598.03 & 1431.36 & 2433.78 & 4514.69 & 0.5 & 2257.35 & 0.5 & 622.75 & 614.5 \\
\hline $8: 39$ & 19 & 755 & 683 & 667 & 701.667 & 2844.44 & 348.444 & 1201.78 & 1596.44 & 0.70709 & 1128.83 & 0.29291 & 651.622 & 563 \\
\hline $8: 43$ & 20 & 830.5 & 674 & 61.5 & 522 & 95172.3 & 23104 & 212060 & 59138.1 & 0.50473 & 29848.6 & 0.49527 & 309.879 & 548 \\
\hline $8: 59$ & 24 & 694 & 689 & 672.5 & 685.167 & 78.0278 & 14.6944 & 160.444 & 46.3611 & 0.99845 & 46.2893 & 0.00155 & 672.307 & 559 \\
\hline
\end{tabular}


Table D4.54: Kalman Filter for case 6-3000

\begin{tabular}{|c|c|c|c|c|c|c|c|c|c|c|c|c|c|c|}
\hline time & trip & art3 & art2 & art1 & Average & $\Delta 3$ & $\Delta 2$ & $\Delta 1$ & Var[data]n & $g(k+1)$ & $\theta(k+1)$ & $a(k+1)$ & Predict & Observed \\
\hline $8: 33$ & 17 & 0 & 0 & 0 & 630 & 396900 & 396900 & 396900 & 396900 & 0.5 & 0 & 0.5 & & 630 \\
\hline $8 ; 36$ & 18 & 499.5 & 743.5 & 589.5 & 610.833 & 12395.1 & 17600.4 & 455.111 & 14997.8 & 0.5 & 7498.89 & 0.5 & 609.75 & 700 \\
\hline $8: 39$ & 19 & 559.5 & 671.5 & 550.5 & 593.833 & 1178.78 & 6032.11 & 1877.78 & 3605.44 & 0.75489 & 2721.73 & 0.24511 & 587.143 & 750 \\
\hline $8: 43$ & 20 & 521.5 & 694.5 & 526 & 580.667 & 3500.69 & 12958 & 2988.44 & 8229.36 & 0.57095 & 4698.56 & 0.42905 & 622.107 & 810 \\
\hline $8: 59$ & 24 & 548 & 820 & 550 & 639.333 & 8341.78 & 32640.4 & 7980.44 & 20491.1 & 0.55143 & 11299.4 & 0.44857 & 666.629 & 720 \\
\hline
\end{tabular}

Table D4.55: Kalman Filter for case 56-2200

\begin{tabular}{|c|c|c|c|c|c|c|c|c|c|c|c|c|c|c|}
\hline time & trip & art3 & art2 & art1 & Average & $\Delta 3$ & $\Delta 2$ & $\Delta 1$ & Var[data]n & $g(k+1)$ & $\theta(k+1)$ & $a(k+1)$ & Predict & Observed \\
\hline $8: 33$ & 17 & 0 & 0 & 0 & 630 & 396900 & 396900 & 396900 & 396900 & 0.5 & 0 & 0.5 & & 630 \\
\hline $8: 36$ & 18 & 717 & 525 & 509 & 583.667 & 17777.8 & 3441.78 & 5575.11 & 10609.8 & 0.5 & 5304.89 & 0.5 & 569.5 & 728 \\
\hline $8: 39$ & 19 & 680 & 571.5 & 557 & 602.833 & 5954.69 & 981.778 & 2100.69 & 3468.24 & 0.71668 & 2485.61 & 0.28332 & 605.448 & 672.5 \\
\hline $8: 43$ & 20 & 795.5 & 641.5 & 558.5 & 665.167 & 16986.8 & 560.111 & 11377.8 & 8773.44 & 0.56204 & 4931.02 & 0.43796 & 608.427 & 699 \\
\hline $8: 59$ & 24 & 689.5 & 550 & 558 & 599.167 & 8160.11 & 2417.36 & 1694.69 & 5288.74 & 0.65898 & 3485.16 & 0.34102 & 606.084 & 805.5 \\
\hline
\end{tabular}

Table D4.56: Kalman Filter for case 8-3000

\begin{tabular}{|c|c|c|c|c|c|c|c|c|c|c|c|c|c|c|}
\hline time & trip & art3 & art2 & art1 & Average & $\Delta \mathbf{3}$ & $\Delta 2$ & $\Delta 1$ & Var[data]n & $g(k+1)$ & $e(k+1)$ & $a(k+1)$ & Predict & Observed \\
\hline $8: 33$ & 17 & 0 & 0 & 0 & 630 & 396900 & 396900 & 396900 & 396900 & 0.5 & 0 & 0.5 & & 630 \\
\hline $8: 36$ & 18 & 525 & 509 & 728 & 587.333 & 3885.44 & 6136.11 & 19787.1 & 5010.78 & 0.5 & 2505.39 & 0.5 & 679 & 743 \\
\hline $8: 39$ & 19 & 571.5 & 557 & 672.5 & 600.333 & 831.361 & 1877.78 & 5208.03 & 1354.57 & 0.74023 & 1002.7 & 0.25977 & 690.814 & 691.5 \\
\hline $8: 43$ & 20 & 641.5 & 558.5 & 699 & 633 & 72.25 & 5550.25 & 4356 & 2811.25 & 0.57567 & 1618.36 & 0.42433 & 695.818 & 647.5 \\
\hline $8: 59$ & 24 & 550 & 558 & 805.5 & 637.833 & 7714.69 & 6373.36 & 28112.1 & 7044.03 & 0.55152 & 3884.92 & 0.44848 & 734.64 & 681 \\
\hline
\end{tabular}


Table D4.57: Kalman Filter for case 49-1200

\begin{tabular}{|c|c|c|c|c|c|c|c|c|c|c|c|c|c|c|}
\hline time & trip & art3 & art2 & art1 & Average & $\Delta 3$ & $\Delta 2$ & $\Delta 1$ & Var[data]n & $g(k+1)$ & $e(k+1)$ & $a(k+1)$ & Predict & Obbserved \\
\hline $8: 33$ & 17 & 0 & 0 & 0 & 630 & 396900 & 396900 & 396900 & 396900 & 0.5 & 0 & 0.5 & & 630 \\
\hline $8: 36$ & 18 & 509 & 728 & 743 & 660 & 22801 & 4624 & 6889 & 13712.5 & 0.5 & 6856.25 & 0.5 & 686.5 & 686.5 \\
\hline $8: 39$ & 19 & 557 & 672.5 & 691.5 & 640.333 & 6944.44 & 1034.69 & 2618.03 & 3989.57 & 0.73108 & 2916.68 & 0.26892 & 690.155 & 746 \\
\hline $8: 43$ & 20 & 558.5 & 699 & 647.5 & 635 & 5852.25 & 4096 & 156.25 & 4974.13 & 0.61336 & 3050.92 & 0.38664 & 685.584 & 747.5 \\
\hline $8: 59$ & 24 & 558 & 805.5 & 681 & 681.5 & 15252.3 & 15376 & 0.25 & 15314.1 & 0.54529 & 8350.7 & 0.45471 & 711.238 & 748.5 \\
\hline
\end{tabular}

Table D4.58: Kalman Filter for case 59-2200

\begin{tabular}{|c|c|c|c|c|c|c|c|c|c|c|c|c|c|c|}
\hline time & trip & art3 & art2 & art1 & Average & $\Delta \mathbf{3}$ & $\Delta 2$ & $\Delta 1$ & Var[data]n & $g(k+1)$ & $e(k+1)$ & $a(k+1)$ & Predict & Observed \\
\hline $8: 33$ & 17 & 0 & 0 & 0 & 630 & 396900 & 396900 & 396900 & 396900 & 0.5 & 0 & 0.5 & & 630 \\
\hline $8: 36$ & 18 & 743 & 686.5 & 745 & 724.833 & 330.028 & 1469.44 & 406.694 & 899.736 & 0.5 & 449.868 & 0.5 & 687.5 & 740 \\
\hline $8: 39$ & 19 & 691.5 & 746 & 682 & 706.5 & 225 & 1560.25 & 600.25 & 892.625 & 0.60064 & 536.143 & 0.39936 & 705.163 & 687 \\
\hline $8: 43$ & 20 & 647.5 & 747.5 & 804.5 & 733.167 & 7338.78 & 205.444 & 5088.44 & 3772.11 & 0.53318 & 2011.2 & 0.46682 & 749.648 & 781 \\
\hline $8: 59$ & 24 & 681 & 748.5 & 679.5 & 703 & 484 & 2070.25 & 552.25 & 1277.13 & 0.72026 & 919.866 & 0.27974 & 707.893 & 778.5 \\
\hline
\end{tabular}

Table D4.59: Kalman Filter for case 24-1200

\begin{tabular}{|c|c|c|c|c|c|c|c|c|c|c|c|c|c|c|}
\hline time & trip & art3 & $\operatorname{art2}$ & art1 & Average & $\Delta 3$ & $\Delta \mathbf{2}$ & $\Delta 1$ & Var[data]n & $g(k+1)$ & $e(k+1)$ & $a(k+1)$ & Predict & Observed \\
\hline $8: 33$ & 17 & 0 & 0 & 0 & 630 & 396900 & 396900 & 396900 & 396900 & 0.5 & 0 & 0.5 & & 630 \\
\hline $8: 36$ & 18 & 790 & 833.5 & 811.5 & 811.667 & 469.444 & 476.694 & 0.02778 & 473.069 & 0.5 & 236.535 & 0.5 & 720.75 & 580 \\
\hline $8: 39$ & 19 & 777 & 688 & 644.5 & 703.167 & 5451.36 & 230.028 & 3441.78 & 2840.69 & 0.51998 & 1477.12 & 0.48002 & 613.539 & 564 \\
\hline $8: 43$ & 20 & 788 & 763.5 & 746.5 & 766 & 484 & 6.25 & 380.25 & 245.125 & 0.8754 & 214.584 & 0.1246 & 723.761 & 542 \\
\hline $8: 59$ & 24 & 820 & 683 & 748 & 750.333 & 4853.44 & 4533.78 & 5.44444 & 4693.61 & 0.51117 & 2399.25 & 0.48883 & 647.302 & 557 \\
\hline
\end{tabular}


Table D4.50: Kalman Filter for case 25-1200

\begin{tabular}{|c|c|c|c|c|c|c|c|c|c|c|c|c|c|c|}
\hline time & trip & art3 & art2 & art1 & Average & $\Delta 3$ & $\Delta 2$ & $\Delta 1$ & Var[data]n & $g(k+1)$ & $e(k+1)$ & $a(k+1)$ & Predict & Observed \\
\hline $8: 33$ & 17 & 0 & 0 & 0 & 630 & 396900 & 396900 & 396900 & 396900 & 0.5 & 0 & 0.5 & & 630 \\
\hline $8: 36$ & 18 & 833.5 & 811.5 & 580 & 741.667 & 8433.36 & 4876.69 & 26136.1 & 6655.028 & 0.5 & 3327.51 & 0.5 & 605 & 612.5 \\
\hline $8: 39$ & 19 & 688 & 644.5 & 564 & 632.167 & 3117.36 & 152.111 & 4646.69 & 1634.736 & 0.7522 & 1229.65 & 0.2478 & 576.018 & 554.5 \\
\hline $8: 43$ & 20 & 763.5 & 746.5 & 542 & 684 & 6320.25 & 3906.25 & 20164 & 5113.25 & 0.55367 & 2831.04 & 0.44633 & 547.579 & 535.5 \\
\hline $8: 59$ & 24 & 683 & 748 & 557 & 662.667 & 413.444 & 7281.78 & 11165.4 & 3847.611 & 0.63448 & 2441.21 & 0.36552 & 549.141 & 566 \\
\hline
\end{tabular}

Table D4.61: Kalman Filter for case 20-1200

\begin{tabular}{|c|c|c|c|c|c|c|c|c|c|c|c|c|c|c|}
\hline time & trip & art3 & art2 & art1 & Average & $\Delta 3$ & $\Delta \mathbf{2}$ & $\Delta 1$ & Var[data]n & $g(k+1)$ & $e(k+1)$ & $a(k+1)$ & Predict & Observed \\
\hline $8: 33$ & 17 & 0 & 0 & 0 & 630 & 396900 & 396900 & 396900 & 396900 & 0.5 & 0 & 0.5 & & 630 \\
\hline $8: 36$ & 18 & 712 & 686.5 & 722.5 & 707 & 25 & 420.25 & 240.25 & 222.625 & 0.5 & 111.313 & 0.5 & 676.25 & 509 \\
\hline $8: 39$ & 19 & 686 & 748.5 & 681 & 705.167 & 367.361 & 1877.78 & 584.028 & 1122.57 & 0.52362 & 587.798 & 0.47638 & 599.062 & 561.5 \\
\hline $8: 43$ & 20 & 772 & 746.5 & 678 & 732.167 & 1586.69 & 205.444 & 2934.03 & 896.069 & 0.62349 & 558.691 & 0.37651 & 634.137 & 538.5 \\
\hline $8: 59$ & 24 & 685 & 646 & 686 & 672.333 & 160.444 & 693.444 & 186.778 & 426.944 & 0.69776 & 297.903 & 0.30224 & 641.419 & 554 \\
\hline
\end{tabular}


Table D4.62: Prediction Error of the Predictors

\begin{tabular}{|c|c|c|c|c|c|c|c|c|}
\hline 54 & 2200 & 794.5 & 721.66 & 667.6 & 663.32 & 0.0917 & 0.1597 & 0.165110132 \\
\hline 47 & 1200 & 747 & 745.72 & 866.83 & 1005.69 & 0.0017 & 0.1604 & 0.346305221 \\
\hline 48 & 1200 & 746 & 695.64 & 763.79 & 900.06 & 0.0675 & 0.0238 & 0.206514745 \\
\hline 36 & 1200 & 746.5 & 731.6 & 746.85 & 649.93 & 0.02 & 0.0005 & 0.129363697 \\
\hline 16 & 3000 & 632 & 630.23 & 735.21 & 529.45 & 0.0028 & 0.1633 & 0.162262658 \\
\hline 21 & 1200 & 563 & 548.75 & 663.75 & 658.26 & 0.0253 & 0.179 & 0.16920071 \\
\hline 19 & 1200 & 553.5 & 563.92 & 554.98 & 468.33 & 0.0188 & 0.0027 & 0.153875339 \\
\hline 45 & 1200 & 747 & 716.24 & 717.47 & 70.72 & 0.0412 & 0.0395 & 0.905327979 \\
\hline 39 & 1200 & 746 & 696.85 & 746.17 & 721.39 & 0.0659 & 0.0002 & 0.032989276 \\
\hline 38 & 1200 & 746.5 & 746.24 & 713.2 & 643.64 & 0.0003 & 0.0446 & 0.137789685 \\
\hline 64 & 2200 & 694 & 760.47 & 736.18 & 700 & 0.0958 & 0.0608 & 0.008645533 \\
\hline 29 & 1200 & 559 & 566.51 & 672.31 & 557.11 & 0.0134 & 0.2027 & 0.003381038 \\
\hline 6 & 3000 & 720 & 739.9 & 666.63 & 846.95 & 0.0276 & 0.0741 & 0.176319444 \\
\hline 56 & 2200 & 805.5 & 721.24 & 606.08 & 698.37 & 0.1046 & 0.2476 & 0.132998138 \\
\hline 8 & 3000 & 681 & 731.69 & 734.64 & 746.15 & 0.0744 & 0.0788 & 0.095668135 \\
\hline 49 & 1200 & 748.5 & 730.5 & 711.24 & 786.17 & 0.024 & 0.0498 & 0.050327321 \\
\hline 59 & 2200 & 778.5 & 713.85 & 707.89 & 659.65 & 0.083 & 0.0907 & 0.152665382 \\
\hline 24 & 1200 & 557 & 562.93 & 647.3 & 543.08 & 0.0106 & 0.1621 & 0.024991023 \\
\hline 25 & 1200 & 566 & 557.38 & 549.14 & 550.32 & 0.0152 & 0.0298 & 0.02770318 \\
\hline 20 & 1200 & $\overline{554}$ & 555.73 & 641.42 & 544.85 & 0.0031 & 0.1578 & 0.016516245 \\
\hline
\end{tabular}

Note: $\mathrm{d} 1, \mathrm{~d} 2$, d3: The absolute relative error (\%) of the Developed model, the Kalman Filter and the Naïve models, respectively 


\section{Appendix D5}

\section{General}

This Appendix presents the database, the prediction results of the developed

model, the Kalman Filter and the naïve models applied for boarding passenger prediction.

\section{Database for the Developed Model and Kalman Filter Model}

Table D5.1: Database of Boarding Passengers at Mackenzie Station (Route 95)

\begin{tabular}{|c|c|c|c|c|c|}
\hline bis & 101 & is & 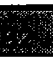 & 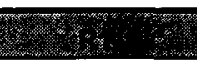 & 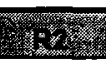 \\
\hline 54 & 2200 & 3 & 5 & $794.5(721.16)$ & 681.5 \\
\hline 58 & 2200 & 2 & 8 & 681.5 & 798 \\
\hline 33 & 1200 & 7 & 5 & 571 & 537.5 \\
\hline 42 & 1200 & 12 & 6 & 867 & 747 \\
\hline 47 & 1200 & 9 & 7 & $747(745.72)$ & 866.5 \\
\hline 62 & 2200 & 1 & 6 & 699 & 672.5 \\
\hline 48 & 1200 & 11 & 10 & $746(695.64)$ & 866.5 \\
\hline 36 & 1200 & 6 & 8 & $746.5(730.63)$ & 755 \\
\hline 16 & 3000 & 9 & 9 & $632(630.23)$ & 632.5 \\
\hline 52 & 2200 & 8 & 9 & 781.5 & 785 \\
\hline 3 & 3000 & 12 & 3 & 803.5 & 647.5 \\
\hline 2 & 3000 & 7 & 9 & 673.5 & 666 \\
\hline 7 & 3000 & 3 & 6 & 698.5 & 677 \\
\hline 21 & 1200 & 7 & 11 & $563(548.75)$ & 638 \\
\hline 28 & 1200 & 8 & 11 & 562 & 657 \\
\hline 19 & 1200 & 7 & 7 & $553.5(563.92)$ & 547.5 \\
\hline 4 & 3000 & 5 & 16 & 782 & 792 \\
\hline 11 & 3000 & 1 & 8 & 685 & 753.5 \\
\hline 45 & 1200 & 10 & 8 & $747(716.24)$ & 773 \\
\hline 39 & 1200 & 7 & 10 & $746(696.85)$ & 746.5 \\
\hline 5 & 3000 & 6 & 6 & 690 & 770.5 \\
\hline 55 & 2200 & 6 & 9 & 685 & 795 \\
\hline 38 & 1200 & 9 & 9 & $746.5(746.24)$ & 747 \\
\hline 13 & 3000 & 7 & 9 & 668 & 792.5 \\
\hline 64 & 2200 & 12 & 9 & $694(760.47)$ & 830.5 \\
\hline 14 & 3000 & 7 & 4 & 689 & 674 \\
\hline 10 & 3000 & 4 & 0 & 672.5 & 661.5 \\
\hline 29 & 1200 & 7 & 7 & $559(566.51)$ & 548 \\
\hline 35 & 1200 & 6 & 6 & 548 & 521.5 \\
\hline
\end{tabular}




\begin{tabular}{|c|c|c|c|c|c|}
\hline 53 & 2200 & 11 & 7 & 820 & 694.5 \\
\hline 17 & 1200 & 5 & 5 & 550 & 526 \\
\hline 6 & 3000 & 6 & 6 & $720(739.39)$ & 810 \\
\hline 12 & 3000 & 9 & 6 & 689.5 & 795.5 \\
\hline 18 & 1200 & 9 & 11 & 550 & 641.5 \\
\hline 31 & 1200 & 7 & 5 & 558 & 558.5 \\
\hline 56 & 2200 & 7 & 4 & $805.5(721.24)$ & 699 \\
\hline 8 & 3000 & 8 & 6 & $681(731.69)$ & 647.5 \\
\hline 49 & 1200 & 6 & 4 & $748.5(730.5)$ & 747.5 \\
\hline 9 & 3000 & 7 & 8 & 679.5 & 804.5 \\
\hline 59 & 2200 & 12 & 7 & $778.5(713.85)$ & 781 \\
\hline 41 & 1200 & 1 & 12 & 748 & 754.5 \\
\hline 46 & 1200 & 10 & 4 & 747 & 766.5 \\
\hline 57 & 2200 & 2 & 5 & 671 & 691.5 \\
\hline 51 & 2200 & 9 & 10 & 694.5 & 777.5 \\
\hline 1 & 3000 & 8 & 10 & 702 & 676.5 \\
\hline 22 & 1200 & 8 & 7 & 556 & 540.5 \\
\hline 40 & 1200 & 10 & 5 & 747.5 & 746.5 \\
\hline 63 & 2200 & 14 & 10 & 798 & 796.5 \\
\hline 50 & 1200 & 9 & 5 & 820 & 788 \\
\hline 61 & 2200 & 9 & 10 & 683 & 763.5 \\
\hline 43 & 1200 & 5 & 12 & 748 & 746.5 \\
\hline 24 & 1200 & 8 & 13 & $557(562.93)$ & 542 \\
\hline 25 & 1200 & 7 & 2 & $566(557.38)$ & 535.5 \\
\hline 37 & 1200 & 1 & 5 & 746 & 869 \\
\hline 65 & 2200 & 9 & 9 & 685 & 772 \\
\hline 44 & 1200 & 6 & 5 & 646 & 746.5 \\
\hline 60 & 2200 & 3 & 4 & 686 & 678 \\
\hline 20 & 1200 & 8 & 10 & $554(555.73)$ & 538.5 \\
\hline 34 & 1200 & 10 & 8 & 553.5 & 657.5 \\
\hline 26 & 1200 & 8 & 5 & 553 & 640 \\
\hline 23 & 1200 & 6 & 7 & 569.5 & 548.5 \\
\hline 30 & 1200 & 5 & 7 & 565.5 & 552.5 \\
\hline 32 & 1200 & 12 & 3 & 572.5 & 569.5 \\
\hline 27 & 1200 & 6 & 7 & 573.5 & 553.5 \\
\hline 15 & 3000 & 11 & 9 & 655 & 673 \\
\hline 66 & 1200 & 5 & 12 & 746.5 & 748.5 \\
\hline 67 & 1200 & 2 & 10 & 755.5 & 866.5 \\
\hline 68 & 1200 & 4 & 10 & 747 & 765.5 \\
\hline 69 & 1200 & 10 & 11 & 756.5 & 866.5 \\
\hline 70 & 1200 & 4 & 8 & 747 & 746 \\
\hline
\end{tabular}

Note:

1. $R 1, R 2, B 1$, and $B 2=$ Running time and Boarding passengers of trip 24 and trip 20 respectively.

2. Numbers in brackets are the predicted running times of the bus in question. These, along with B1, B2, and R2, are the elements of the pattern matrix defining the boarding passenger being predicted. 
Table D5.2: Database for Kalman Filter Predictor

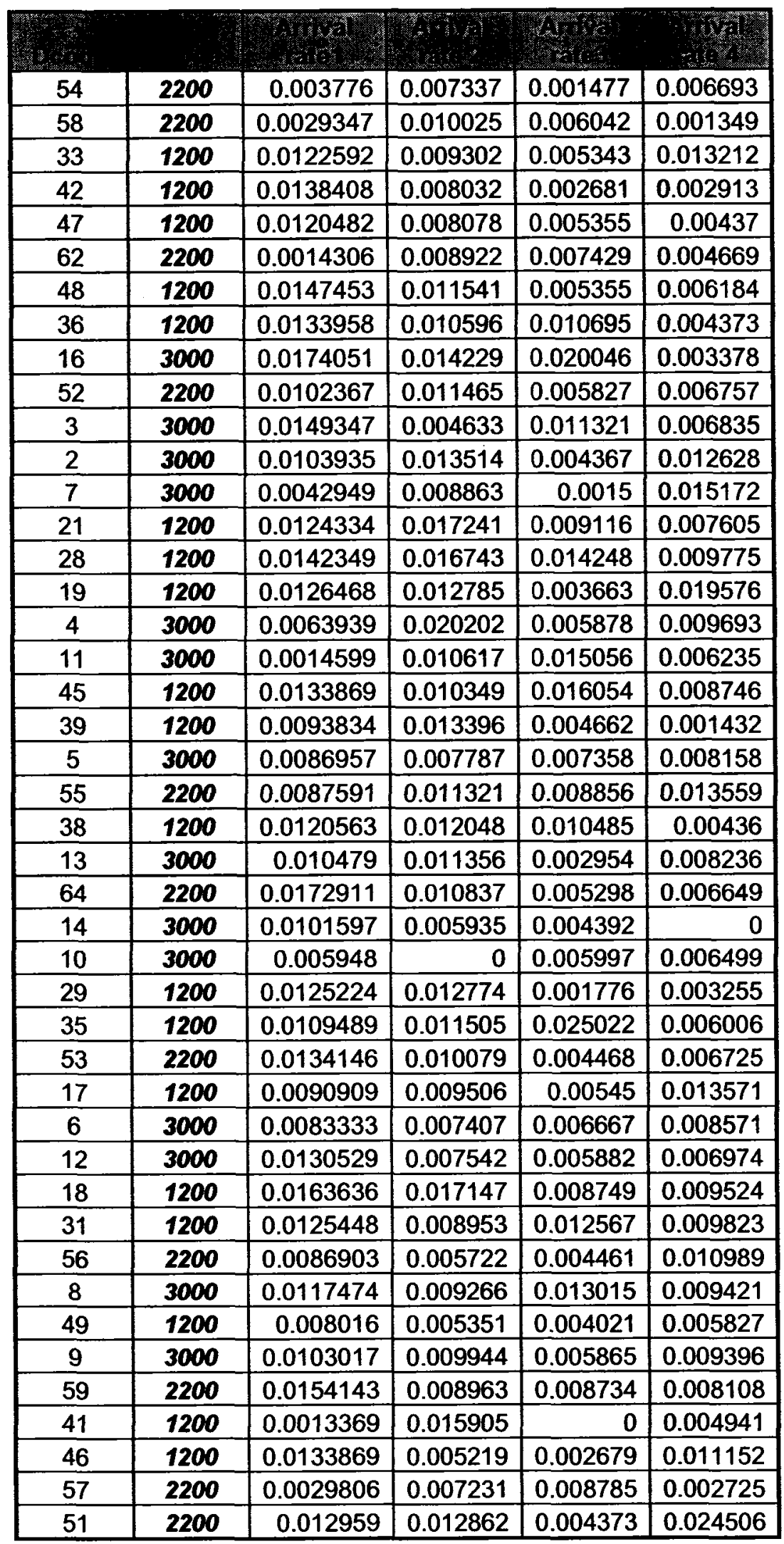




\begin{tabular}{|r|r|r|r|r|r|}
\hline 1 & 3000 & 0.011396 & 0.014782 & 0.007391 & 0.006784 \\
\hline 22 & 1200 & 0.0143885 & 0.012951 & 0.021661 & 0.003984 \\
\hline 40 & 1200 & 0.0133779 & 0.006698 & 0.010724 & 0.004237 \\
\hline 63 & 2200 & 0.0175439 & 0.012555 & 0.001481 & 0.009563 \\
\hline 50 & 1200 & 0.0109756 & 0.006345 & 0.005148 & 0.006329 \\
\hline 61 & 2200 & 0.0131772 & 0.013098 & 0 & 0.011998 \\
\hline 43 & 1200 & 0.0066845 & 0.016075 & 0.001552 & 0.011091 \\
\hline 24 & 1200 & 0.0071813 & 0.023985 & 0.001773 & 0.006897 \\
\hline 25 & 1200 & 0.0123675 & 0.003735 & 0.00541 & 0.006531 \\
\hline 37 & 1200 & 0.0013405 & 0.005754 & 0.009365 & 0.00424 \\
\hline 65 & 2200 & 0.0131387 & 0.011658 & 0.004373 & 0.005618 \\
\hline 44 & 1200 & 0.0092879 & 0.006698 & 0.005344 & 0.005827 \\
\hline 60 & 2200 & 0.0043732 & 0.0059 & 0.007342 & 0.005536 \\
\hline 20 & 1200 & 0.0108303 & 0.01857 & 0.003562 & 0.011788 \\
\hline 34 & 1200 & 0.0180668 & 0.012167 & 0.007214 & 0.007775 \\
\hline 26 & 1200 & 0.0144665 & 0.007813 & 0.010801 & 0.008056 \\
\hline 23 & 1200 & 0.0105356 & 0.012762 & 0 & 0.00821 \\
\hline 30 & 1200 & 0.0088417 & 0.01267 & 0.008795 & 0.012678 \\
\hline 32 & 1200 & 0.0209607 & 0.005268 & 0.013323 & 0.003899 \\
\hline 27 & 1200 & 0.0104621 & 0.012647 & 0.012693 & 0.001949 \\
\hline 15 & 3000 & 0.0167939 & 0.013373 & 0.018519 & 0.004747 \\
\hline
\end{tabular}

\section{Prediction Results of the Developed Model}

The following table shows part of an output returned from Matlab source code for the sake of illustration. All predictions are tabulated in Table D5.23

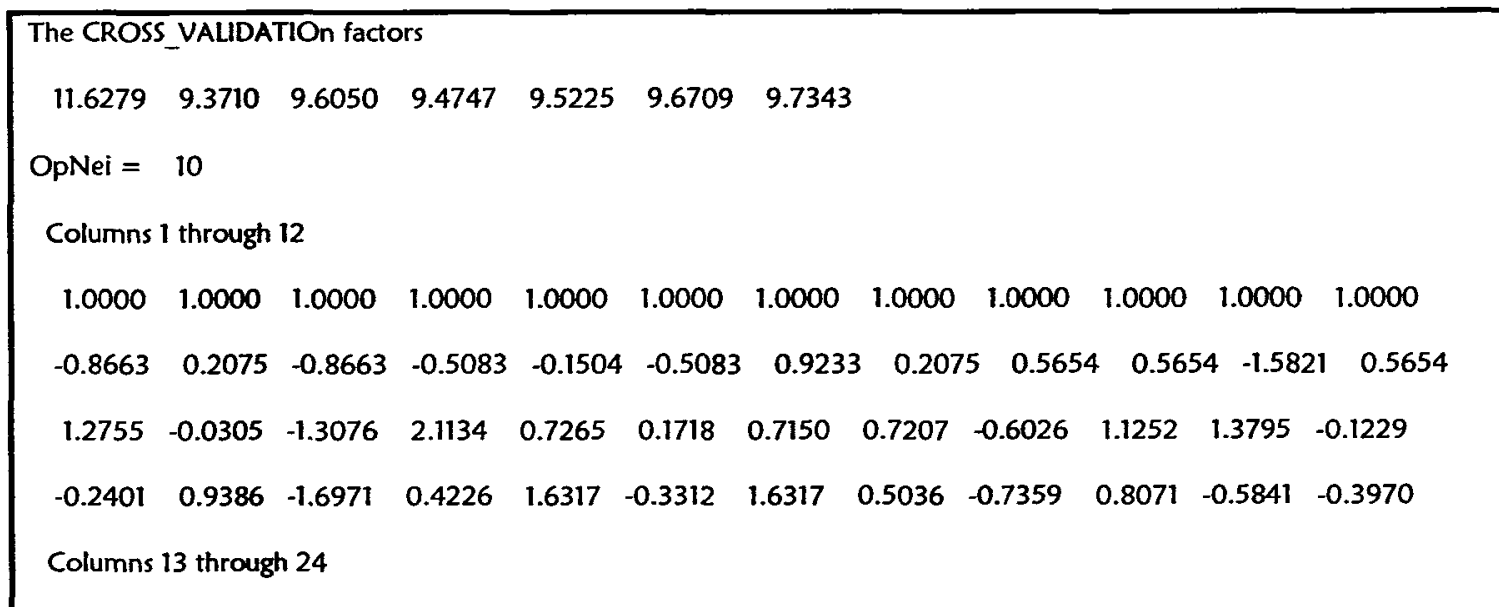




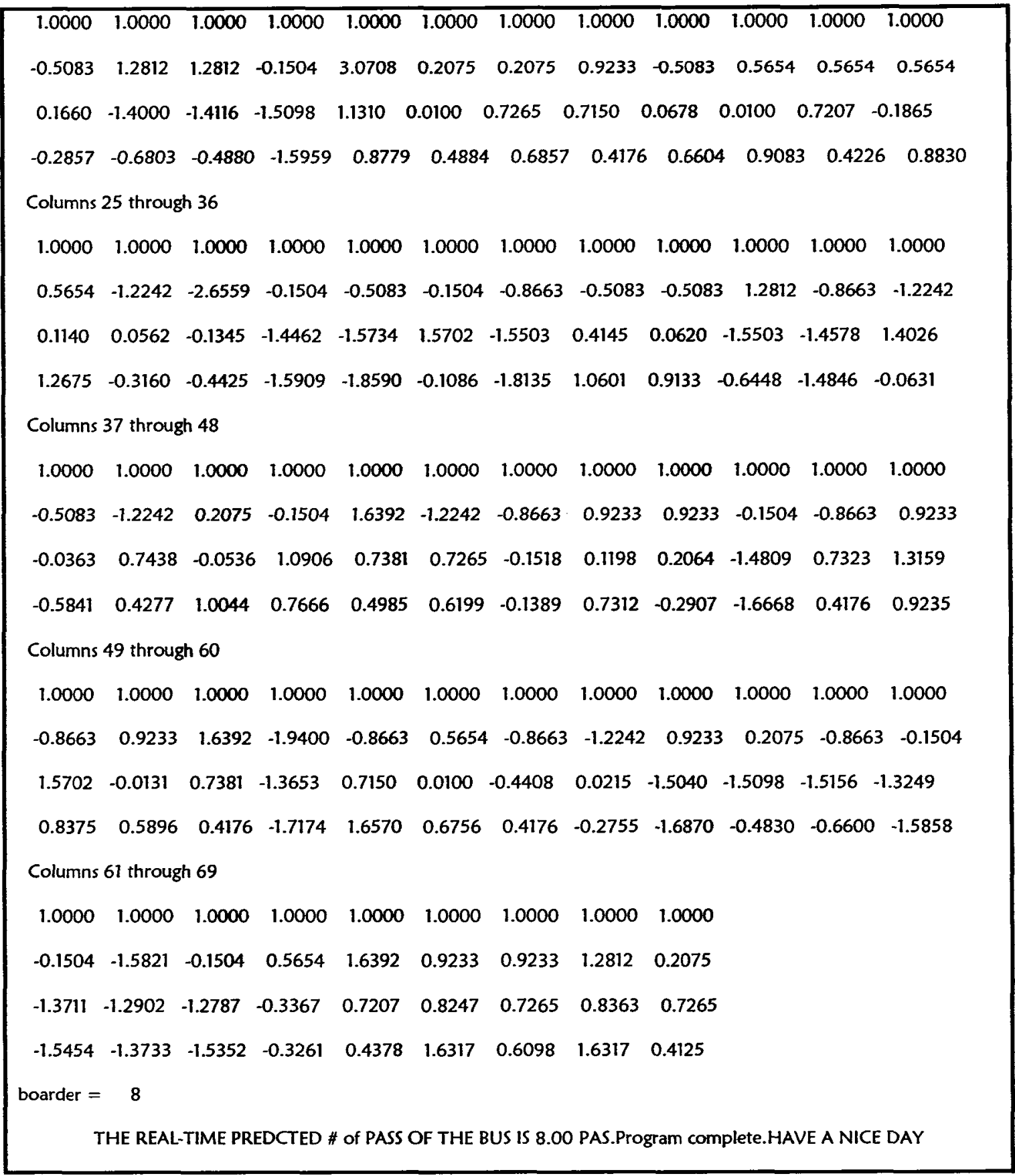

Figure D5.1: An Example of the Matlab outputs for Boarding Passenger Prediction (Case 24-1200) 
Table D5.3: Kalman Filter for test case 54-2200

\begin{tabular}{|c|c|c|c|c|c|c|c|c|c|c|c|c|c|c|c|}
\hline time & trip & art3 & art2 & art1 & Average & $\Delta \mathbf{3}$ & $\Delta 2$ & $\Delta 1$ & $\overline{\text { Var[data]n }}$ & $g(k+1)$ & $\theta(k+1)$ & $a(k+1)$ & Predict & Observed & Boarders \\
\hline $8: 33$ & 17 & 0 & 0 & 0 & 0.025 & 0.0006 & 0.0006 & 0.0006 & 0.0006 & 0.5 & 0 & 0.5 & & 0.025 & \\
\hline $8: 36$ & 18 & 0.0039 & 0.00195 & 0.00475 & 0.00353 & $\begin{array}{c}1.3 \mathrm{E}- \\
07\end{array}$ & $\begin{array}{c}2.5 \mathrm{E}- \\
06\end{array}$ & $\begin{array}{c}.5 \mathrm{E}- \\
06\end{array}$ & 1.3E-06 & 0.5 & $\begin{array}{c}6.6 \mathrm{E}- \\
07\end{array}$ & 0.5 & 0.01487 & 0.00669 & \\
\hline $8: 39$ & 19 & 0.01332 & 0.01269 & 0.01852 & 0.01485 & $\begin{array}{c}2.3 \mathrm{E}- \\
06\end{array}$ & $\begin{array}{c}4.6 \mathrm{E}- \\
06\end{array}$ & $\begin{array}{c}1.3 \mathrm{E}- \\
05\end{array}$ & $3.5 E-06$ & 0.54337 & $\begin{array}{c}1.9 \mathrm{E}- \\
06\end{array}$ & 0.45663 & 0.01312 & 0.00148 & \\
\hline $8: 43$ & 20 & 0.00527 & 0.01265 & 0.01337 & 0.01043 & $\begin{array}{c}2.7 \mathrm{E}- \\
05\end{array}$ & $\begin{array}{c}4.9 \mathrm{E}- \\
06\end{array}$ & $\begin{array}{c}\text { 8.7E- } \\
06\end{array}$ & 1.6E-05 & 0.52822 & $\begin{array}{c}8.3 \mathrm{E}- \\
06\end{array}$ & 0.47178 & 0.00776 & 0.00734 & \\
\hline $8: 59$ & 24 & 0.02096 & 0.01046 & 0.01679 & 0.01607 & $\begin{array}{c}2.4 \mathrm{E}- \\
05\end{array}$ & $\begin{array}{c}.1 \mathrm{E}- \\
05\end{array}$ & $\begin{array}{c}5.2 \mathrm{E}- \\
07\end{array}$ & $2.8 \mathrm{E}-05$ & 0.56542 & $\begin{array}{c}1.6 \mathrm{E}- \\
05\end{array}$ & 0.43458 & 0.013 & 0.004 & 8 \\
\hline
\end{tabular}

Table D5.4: Kalman Filter for test case 47-1200

\begin{tabular}{|c|c|c|c|c|c|c|c|c|c|c|c|c|c|c|c|}
\hline time & trip & art3 & art2 & art1 & Average & $\Delta 3$ & $\Delta 2$ & $\Delta 1$ & Var[data]n & $g(k+1)$ & $e(k+1)$ & $a(k+1)$ & Predict & Observed & Boarders \\
\hline $8: 33$ & 17 & 0 & 0 & 0 & 0.025 & 0.0006 & 0.0006 & 0.0006 & 0.0006 & 0.5 & 0 & 0.5 & & 0.025 & \\
\hline $8: 36$ & 18 & 0.00135 & 0.01321 & 0.00291 & 0.00582 & 2E-05 & $\begin{array}{c}5.5 \mathrm{E}- \\
05\end{array}$ & $\begin{array}{c}8.5 \mathrm{E}- \\
06\end{array}$ & 3.7E-05 & 0.5 & $\begin{array}{c}1.9 \mathrm{E}- \\
05\end{array}$ & 0.5 & 0.01396 & 0.00437 & \\
\hline $8: 39$ & 19 & 0.00604 & 0.00534 & 0.00268 & 0.00469 & $\begin{array}{c}1.8 \mathrm{E}- \\
06\end{array}$ & $\begin{array}{c}4.3 \mathrm{E}- \\
07\end{array}$ & $4 E-06$ & 1.1E-06 & 0.946 & $\begin{array}{c}1.1 \mathrm{E}- \\
06\end{array}$ & 0.054 & 0.00277 & 0.00536 & \\
\hline $8: 43$ & 20 & 0.01003 & 0.0093 & 0.00803 & 0.00912 & $\begin{array}{l}8.2 \mathrm{E}- \\
07\end{array}$ & $\begin{array}{c}3.3 \mathrm{E}- \\
08\end{array}$ & $\begin{array}{c}1.2 \mathrm{E}- \\
06\end{array}$ & 4.3E-07 & 0.77802 & $\begin{array}{c}3.3 \mathrm{E}- \\
07\end{array}$ & 0.22198 & 0.00744 & 0.00808 & \\
\hline $8: 59$ & 24 & 0.00293 & 0.01226 & 0.01384 & 0.00968 & $\begin{array}{c}4.5 \mathrm{E}- \\
05\end{array}$ & $\begin{array}{c}6.7 E- \\
06\end{array}$ & $\begin{array}{c}1.7 \mathrm{E}- \\
05\end{array}$ & 2.6E-05 & 0.50316 & $\begin{array}{c}1.3 \mathrm{E}- \\
05\end{array}$ & 0.49684 & 0.011 & 0.012 & 10 \\
\hline
\end{tabular}

Table D5.5: Kalman Filter for test case 48-1200

\begin{tabular}{|c|c|c|c|c|c|c|c|c|c|c|c|c|c|c|c|}
\hline time & trip & art3 & $\operatorname{art2}$ & art1 & Average & $\Delta \mathbf{3}$ & $\Delta 2$ & $\Delta 1$ & Var[data]n & $g(k+1)$ & $\theta(k+1)$ & $a(k+1)$ & Predict & Observed & Boarders \\
\hline $8: 33$ & 17 & 0 & 0 & 0 & 0.025 & 0.0006 & 0.0006 & 0.0006 & 0.0006 & 0.5 & 0 & 0.5 & & 0.025 & \\
\hline $8: 36$ & 18 & 0.00291 & 0.00437 & 0.00467 & 0.00398 & $\begin{array}{c}1.1 \mathrm{E}- \\
06\end{array}$ & $\begin{array}{c}1.5 \mathrm{E}- \\
07\end{array}$ & $\begin{array}{c}4.7 \mathrm{E}- \\
07\end{array}$ & $6.5 \mathrm{E}-07$ & 0.5 & $\begin{array}{c}3.2 \mathrm{E}- \\
07\end{array}$ & 0.5 & 0.01483 & 0.00618 & \\
\hline $8: 39$ & 19 & 0.00604 & 0.00743 & 0.00536 & 0.00628 & $\begin{array}{c}5.4 \mathrm{E}- \\
08\end{array}$ & $\begin{array}{c}1.3 \mathrm{E}- \\
06\end{array}$ & $\begin{array}{c}8.5 \mathrm{E}- \\
07\end{array}$ & $6.9 \mathrm{E}-07$ & 0.59477 & $\begin{array}{c}4.1 E- \\
07\end{array}$ & 0.40523 & 0.00569 & 0.00536 & \\
\hline $8: 43$ & 20 & 0.00803 & 0.00808 & 0.00892 & 0.00834 & $\begin{array}{c}9.7 \mathrm{E}- \\
08\end{array}$ & $\begin{array}{c}7.1 \mathrm{E}- \\
08\end{array}$ & $\begin{array}{c}3.3 \mathrm{E}- \\
07\end{array}$ & $8.4 E-08$ & 0.85511 & $\begin{array}{c}7.2 \mathrm{E}- \\
08\end{array}$ & 0.14489 & 0.00841 & 0.01154 & \\
\hline $8: 59$ & 24 & 0.01384 & 0.01205 & 0.00143 & 0.00911 & $\begin{array}{c}2.2 \mathrm{E}- \\
05\end{array}$ & $\begin{array}{c}8.7 E- \\
06\end{array}$ & $\begin{array}{c}5.9 \mathrm{E}- \\
05\end{array}$ & 1.6E-05 & 0.50115 & $\begin{array}{c}7.8 \mathrm{E}- \\
06\end{array}$ & 0.49885 & 0.006 & 0.015 & 5 \\
\hline
\end{tabular}


Table D5.6: Kalman Filter for test case $\mathbf{3 6 - 1 2 0 0}$

\begin{tabular}{|c|c|c|c|c|c|c|c|c|c|c|c|c|c|c|c|}
\hline time & trip & art3 & art2 & art1 & Average & $\Delta 3$ & $\Delta \mathbf{2}$ & $\Delta 1$ & Var[data]n & $g(k+1)$ & $\theta(k+1)$ & $a(k+1)$ & Predict & Observed & Boarders \\
\hline $8: 33$ & 17 & 0 & 0 & 0 & 0.025 & 0.0006 & 0.0006 & 0.0006 & 0.0006 & 0.5 & 0 & 0.5 & & 0.025 & \\
\hline $8: 36$ & 18 & 0.00437 & 0.00469 & 0.00618 & 0.00508 & $\begin{array}{c}5.1 \mathrm{E}- \\
07 \\
\end{array}$ & $\begin{array}{c}1.5 \mathrm{E}- \\
07\end{array}$ & $\begin{array}{c}1.2 \mathrm{E}- \\
06\end{array}$ & $3.3 E-07$ & 0.5 & $\begin{array}{c}1.6 \mathrm{E}- \\
07\end{array}$ & 0.5 & 0.01559 & 0.00437 & \\
\hline $8: 39$ & 19 & 0.00536 & 0.00743 & 0.00536 & 0.00605 & $\begin{array}{c}4.8 \mathrm{E}- \\
07\end{array}$ & $\begin{array}{c}1.9 \mathrm{E}- \\
06\end{array}$ & $\begin{array}{c}4.8 \mathrm{E}- \\
07\end{array}$ & $1.2 E-06$ & 0.53226 & $\begin{array}{c}6.4 \mathrm{E}- \\
07\end{array}$ & 0.46774 & 0.0049 & 0.0107 & \\
\hline $8: 43$ & 20 & 0.00808 & 0.00892 & 0.01154 & 0.00951 & $\begin{array}{c}2.1 \mathrm{E}- \\
06\end{array}$ & $\begin{array}{c}3.5 \mathrm{E}- \\
07\end{array}$ & $\begin{array}{c}4.1 \mathrm{E}- \\
06\end{array}$ & $1.2 E-06$ & 0.60435 & $\begin{array}{c}7.3 \mathrm{E}- \\
07\end{array}$ & 0.39565 & 0.01121 & 0.0106 & \\
\hline $8: 59$ & 24 & 0.01205 & 0.00143 & 0.01475 & 0.00941 & $7 E-06$ & $\begin{array}{c}\text { 6.4E- } \\
05\end{array}$ & $\begin{array}{c}2.8 \mathrm{E}- \\
05\end{array}$ & 3.5E-05 & 0.50511 & $\begin{array}{c}1.8 \mathrm{E}- \\
05\end{array}$ & 0.49489 & 0.013 & 0.013 & 9 \\
\hline
\end{tabular}

Table D5.7: Kalman Filter for test case $16-3000$

\begin{tabular}{|c|c|c|c|c|c|c|c|c|c|c|c|c|c|c|c|}
\hline time & trip & art3 & art2 & art1 & Average & $\Delta \mathbf{3}$ & $\Delta 2$ & $\Delta 1$ & Var[data]n & $g(k+1)$ & $\theta(k+1)$ & $a(k+1)$ & Prodict & Observed & Boarders \\
\hline $8: 33$ & 17 & 0 & 0 & 0 & 0.025 & 0.0006 & 0.0006 & 0.0006 & 0.0006 & 0.5 & 0 & 0.5 & & 0.025 & \\
\hline $8: 36$ & 18 & 0.00467 & 0.00618 & 0.00437 & 0.00508 & $\begin{array}{c}1.7 \mathrm{E}- \\
07\end{array}$ & $\begin{array}{c}1.2 \mathrm{E}- \\
06\end{array}$ & $\begin{array}{c}4.9 \mathrm{E}- \\
07\end{array}$ & $7 E-07$ & 0.5 & $\begin{array}{c}3.5 \mathrm{E}- \\
07\end{array}$ & 0.5 & 0.01469 & 0.00338 & \\
\hline $8: 39$ & 19 & 0.00743 & 0.00536 & 0.0107 & 0.00783 & $\begin{array}{c}1.6 \mathrm{E}- \\
07\end{array}$ & $\begin{array}{c}6.1 \mathrm{E}- \\
06\end{array}$ & $\begin{array}{c}8.2 \mathrm{E}- \\
06 \\
\end{array}$ & $3.1 E-06$ & 0.52635 & $\begin{array}{c}1.6 \mathrm{E}- \\
06\end{array}$ & 0.47365 & 0.00723 & 0.02005 & \\
\hline $8: 43$ & 20 & 0.00892 & 0.01154 & 0.0126 & 0.01102 & $\begin{array}{c}4.4 \mathrm{E}- \\
06\end{array}$ & $\begin{array}{c}2.7 \mathrm{E}- \\
07\end{array}$ & $\begin{array}{c}2.5 \mathrm{E}- \\
06\end{array}$ & $2.3 \mathrm{E}-06$ & 0.63043 & $\begin{array}{c}1.5 \mathrm{E}- \\
06\end{array}$ & 0.36957 & 0.01535 & 0.01423 & \\
\hline $8: 59$ & 24 & 0.00143 & 0.01475 & 0.0134 & 0.00986 & $\begin{array}{c}7.1 \mathrm{E}- \\
05\end{array}$ & $\begin{array}{c}2.4 \mathrm{E}- \\
05\end{array}$ & $\begin{array}{c}1.3 \mathrm{E}- \\
05\end{array}$ & 4.7E-05 & 0.50764 & $\begin{array}{c}2.4 \mathrm{E}- \\
05\end{array}$ & 0.49236 & 0.014 & 0.017 & 10 \\
\hline
\end{tabular}

Table D5.8: Kalman Filter for test case 21-1200

\begin{tabular}{|c|c|c|c|c|c|c|c|c|c|c|c|c|c|c|c|}
\hline time & trip & art3 & art2 & art1 & Average & $\Delta 3$ & $\Delta 2$ & $\Delta 1$ & Var[data]n & $g(k+1)$ & $\theta(k+1)$ & $\mathbf{a}(\mathbf{k}+1)$ & Predict & Observed & Boarders \\
\hline $8: 33$ & 17 & 0 & 0 & 0 & 0.025 & 0.0006 & 0.0006 & 0.0006 & 0.0006 & 0.5 & 0 & 0.5 & & 0.025 & \\
\hline $8: 36$ & 18 & 0.00685 & 0.01263 & 0.01517 & 0.01155 & $\begin{array}{c}2.2 \mathrm{E}- \\
05\end{array}$ & $\begin{array}{c}1.2 \mathrm{E}- \\
06\end{array}$ & $\begin{array}{c}1.3 \mathrm{E}- \\
05\end{array}$ & $1.2 E-05$ & 0.5 & $\begin{array}{c}5.8 \mathrm{E}- \\
06\end{array}$ & 0.5 & 0.02009 & 0.00761 & \\
\hline $8: 39$ & 19 & 0.01132 & 0.00437 & 0.0015 & 0.00573 & $\begin{array}{c}3.1 \mathrm{E}- \\
05\end{array}$ & $\begin{array}{c}1.9 \mathrm{E}- \\
06\end{array}$ & $\begin{array}{c}1.8 \mathrm{E}- \\
05\end{array}$ & 1.7E-05 & 0.57461 & $\begin{array}{c}9.5 \mathrm{E}- \\
06\end{array}$ & 0.42539 & 0.0041 & 0.00912 & \\
\hline $8: 43$ & 20 & 0.00463 & 0.01354 & 0.00886 & 0.00901 & $\begin{array}{c}1.9 \mathrm{E}- \\
05 \\
\end{array}$ & $\begin{array}{c}2.1 \mathrm{E}- \\
05\end{array}$ & $\begin{array}{c}2.2 \mathrm{E}- \\
08\end{array}$ & 2E-05 & 0.5967 & $\begin{array}{c}1.2 \mathrm{E}- \\
05\end{array}$ & 0.4033 & 0.00897 & 0.01724 & \\
\hline $8: 59$ & 24 & 0.00143 & 0.01029 & 0.00429 & 0.00534 & $\begin{array}{c}1.5 \mathrm{E}- \\
05\end{array}$ & $\begin{array}{c}2.5 \mathrm{E}- \\
05\end{array}$ & $\begin{array}{c}1.1 \mathrm{E}- \\
06\end{array}$ & 2E-05 & 0.61458 & $\begin{array}{c}1.2 \mathrm{E}- \\
05\end{array}$ & 0.38542 & 0.009 & 0.012 & 6 \\
\hline
\end{tabular}


Table D5.9: Kalman Filter for test case 19-1200

\begin{tabular}{|c|c|c|c|c|c|c|c|c|c|c|c|c|c|c|c|}
\hline time & trip & art3 & art2 & $\operatorname{art1}$ & Average & $\Delta \mathbf{3}$ & $\Delta \mathbf{2}$ & $\Delta 1$ & Var[data]n & $g(k+1)$ & $\theta(\mathbf{k}+1)$ & $a(k+1)$ & Predict & Observed & Boarders \\
\hline $8: 33$ & 17 & 0 & 0 & 0 & 0.025 & 0.0006 & 0.0006 & 0.0006 & 0.0006 & 0.5 & 0 & 0.5 & & 0.025 & \\
\hline $8: 36$ & 18 & 0.01517 & 0.00761 & 0.00978 & 0.01085 & $\begin{array}{c}1.9 \mathrm{E}- \\
05\end{array}$ & $\begin{array}{c}1.1 \mathrm{E}- \\
05\end{array}$ & $\begin{array}{c}1.2 \mathrm{E}- \\
06\end{array}$ & $1.5 \mathrm{E}-05$ & 0.5 & $\begin{array}{c}7.3 \mathrm{E}- \\
06\end{array}$ & 0.5 & 0.01739 & 0.00761 & \\
\hline $8: 39$ & 19 & 0.0015 & 0.00912 & 0.01425 & 0.00829 & $\begin{array}{l}4.6 \mathrm{E}- \\
05\end{array}$ & $\begin{array}{c}6.9 \mathrm{E}- \\
07\end{array}$ & $\begin{array}{c}3.6 \mathrm{E}- \\
05\end{array}$ & 2.3E-05 & 0.56753 & $\begin{array}{c}1.3 \mathrm{E}- \\
05\end{array}$ & 0.43247 & 0.01138 & 0.00912 & \\
\hline $8: 43$ & 20 & 0.00886 & 0.01724 & 0.01674 & 0.01428 & $\begin{array}{c}2.9 \mathrm{E}- \\
05\end{array}$ & $\begin{array}{c}8.8 \mathrm{E}- \\
06\end{array}$ & $\begin{array}{c}6.1 \mathrm{E}- \\
06\end{array}$ & $1.9 \mathrm{E}-05$ & 0.6291 & $\begin{array}{c}1.2 \mathrm{E}- \\
05\end{array}$ & 0.3709 & 0.01391 & 0.01279 & \\
\hline $8: 59$ & 24 & 0.00429 & 0.01243 & 0.01423 & 0.01032 & $\begin{array}{c}3.6 \mathrm{E}- \\
05\end{array}$ & $\begin{array}{c}4.5 \mathrm{E}- \\
06\end{array}$ & $\begin{array}{c}1.5 \mathrm{E}- \\
05\end{array}$ & $2 E-05$ & 0.61362 & $\begin{array}{c}1.3 \mathrm{E}- \\
05\end{array}$ & 0.38638 & 0.014 & 0.013 & 8 \\
\hline
\end{tabular}

Table D5.10: Kalman Filter for test case $\mathbf{4 5 - 1 2 0 0}$

\begin{tabular}{|c|c|c|c|c|c|c|c|c|c|c|c|c|c|c|c|}
\hline $\operatorname{tim} \theta$ & trip & art3 & art2 & $\operatorname{art1}$ & Average & $\Delta 3$ & $\Delta 2$ & $\Delta 1$ & Var[data]n & $g(k+1)$ & $\theta(k+1)$ & $a(k+1)$ & Predict & Observed & Boarders \\
\hline $8: 33$ & 17 & 0 & 0 & 0 & 0.025 & 0.0006 & 0.0006 & 0.0006 & 0.0006 & 0.5 & 0 & 0.5 & & 0.025 & \\
\hline $8: 36$ & 18 & 0.00437 & 0.00469 & 0.00618 & 0.00508 & $\begin{array}{c}5.1 \mathrm{E}- \\
07\end{array}$ & $\begin{array}{c}1.5 \mathrm{E}- \\
07\end{array}$ & $\begin{array}{c}1.2 \mathrm{E}- \\
06\end{array}$ & 3.3E-07 & 0.5 & $\begin{array}{c}1.6 \mathrm{E}- \\
07\end{array}$ & 0.5 & 0.01559 & 0.00761 & \\
\hline $8: 39$ & 19 & 0.00536 & 0.00743 & 0.00536 & 0.00605 & $\begin{array}{c}4.8 \mathrm{E}- \\
07\end{array}$ & $\begin{array}{c}1.9 \mathrm{E}- \\
06\end{array}$ & $\begin{array}{l}4.8 \mathrm{E}- \\
07\end{array}$ & $1.2 E-06$ & 0.53226 & $\begin{array}{c}6.4 \mathrm{E}- \\
07\end{array}$ & 0.46774 & 0.00641 & 0.00912 & \\
\hline $8: 43$ & 20 & 0.00808 & 0.00892 & 0.00886 & 0.00862 & $\begin{array}{c}2.9 \mathrm{E}- \\
07\end{array}$ & $\begin{array}{c}9.1 E- \\
08\end{array}$ & $\begin{array}{c}5.9 \mathrm{E}- \\
08\end{array}$ & $1.9 \mathrm{E}-07$ & 0.81132 & $\begin{array}{c}1.6 \mathrm{E}- \\
07\end{array}$ & 0.18868 & 0.00891 & 0.01035 & \\
\hline $8: 59$ & 24 & 0.00143 & 0.01029 & 0.00146 & 0.00439 & $\begin{array}{c}8.8 \mathrm{E}- \\
06\end{array}$ & $\begin{array}{c}3.5 E- \\
05\end{array}$ & $\begin{array}{c}8.6 \mathrm{E}- \\
06\end{array}$ & $2.2 \mathrm{E}-05$ & 0.50179 & $\begin{array}{c}1.1 \mathrm{E}- \\
05\end{array}$ & 0.49821 & 0.006 & 0.013 & 4 \\
\hline
\end{tabular}

Table D5.11: Kalman Filter for test case 39-1200

\begin{tabular}{|c|c|c|c|c|c|c|c|c|c|c|c|c|c|c|c|}
\hline time & trip & art3 & art2 & $\operatorname{art1}$ & Average & $\Delta 3$ & $\Delta 2$ & $\Delta 1$ & Var[data]n & $g(k+1)$ & $\theta(k+1)$ & $a(k+1)$ & Prodict & Observed & Boarders \\
\hline $8: 33$ & 17 & 0 & 0 & 0 & 0.025 & 0.0006 & 0.0006 & 0.0006 & 0.0006 & 0.5 & 0 & 0.5 & & 0.025 & \\
\hline $8: 36$ & 18 & 0.00969 & 0.00624 & 0.00875 & 0.00822 & $\begin{array}{c}2.2 \mathrm{E}- \\
06\end{array}$ & 4E-06 & $\begin{array}{c}2.7 E- \\
07\end{array}$ & $3.1 \mathrm{E}-06$ & 0.5 & $\begin{array}{c}1.5 \mathrm{E}- \\
06\end{array}$ & 0.5 & 0.01687 & 0.00761 & \\
\hline $8: 39$ & 19 & 0.00588 & 0.01506 & 0.01605 & 0.01233 & $\begin{array}{c}4.2 \mathrm{E}- \\
05\end{array}$ & $\begin{array}{c}7.4 \mathrm{E}- \\
06\end{array}$ & $\begin{array}{c}1.4 \mathrm{E}- \\
05\end{array}$ & $2.5 \mathrm{E}-05$ & 0.51511 & $\begin{array}{c}1.3 \mathrm{E}- \\
05\end{array}$ & 0.48489 & 0.01196 & 0.00912 & \\
\hline $8: 43$ & 20 & 0.0202 & 0.01062 & 0.01035 & 0.01372 & $\begin{array}{c}4.2 \mathrm{E}- \\
05\end{array}$ & $\begin{array}{c}9.6 \mathrm{E}- \\
06\end{array}$ & $\begin{array}{c}1.1 \mathrm{E}- \\
05\end{array}$ & $2.6 \mathrm{E}-05$ & 0.5983 & $\begin{array}{c}1.5 \mathrm{E}- \\
05\end{array}$ & 0.4017 & 0.00985 & 0.0134 & \\
\hline $8: 59$ & 24 & 0.00639 & 0.00146 & 0.01339 & 0.00708 & $\begin{array}{l}4.7 \mathrm{E}- \\
07\end{array}$ & $\begin{array}{c}3.2 \mathrm{E}- \\
05\end{array}$ & $4 E-05$ & 1.6E-05 & 0.66256 & $\begin{array}{c}1.1 \mathrm{E}- \\
05\end{array}$ & 0.33744 & 0.013 & 0.009 & 10 \\
\hline
\end{tabular}


Table D5.12: Kalman Filter for test case 38-1200

\begin{tabular}{|c|c|c|c|c|c|c|c|c|c|c|c|c|c|c|c|}
\hline $\operatorname{time}$ & trip & art3 & art2 & art1 & Average & $\mathbf{\Delta} \mathbf{3}$ & $\Delta 2$ & $\Delta \mathbf{1}$ & Var[data]n & $g(k+1)$ & $\theta(k+1)$ & $a(k+1)$ & Predlct & Observed & Boarders \\
\hline $8: 33$ & 17 & 0 & 0 & 0 & 0.025 & 0.0006 & 0.0006 & 0.0006 & 0.0006 & 0.5 & 0 & 0.5 & & 0.025 & \\
\hline $8: 36$ & 18 & 0.00143 & 0.00816 & 0.01356 & 0.00772 & $\begin{array}{c}3.9 \mathrm{E}- \\
05\end{array}$ & 2E-07 & $\begin{array}{c}3.4 \mathrm{E}- \\
05\end{array}$ & 2E-05 & 0.5 & $\begin{array}{c}9.9 \mathrm{E}- \\
06\end{array}$ & 0.5 & 0.01928 & 0,00761 & \\
\hline $8: 39$ & 19 & 0.00446 & 0.00736 & 0.00886 & 0.00689 & $\begin{array}{c}5.9 \mathrm{E}- \\
06\end{array}$ & $\begin{array}{c}2.2 \mathrm{E}- \\
07\end{array}$ & $\begin{array}{c}3.9 \mathrm{E}- \\
06 \\
\end{array}$ & $3.1 \mathrm{E}-06$ & 0.80921 & $\begin{array}{c}2.5 \mathrm{E}- \\
06\end{array}$ & 0.19079 & 0.00862 & 0.00912 & \\
\hline $8: 43$ & 20 & 0.014 & 0.00779 & 0.01132 & 0.01103 & $\begin{array}{c}8.8 \mathrm{E}- \\
06\end{array}$ & $\begin{array}{c}1.1 \mathrm{E}- \\
05\end{array}$ & $\begin{array}{c}8.2 \mathrm{E}- \\
08\end{array}$ & $9.7 E-06$ & 0.55683 & $\begin{array}{c}5.4 \mathrm{E}- \\
06\end{array}$ & 0.44317 & 0.01034 & 0.01204 & \\
\hline 8:59 & 24 & 0.00134 & 0.00939 & 0.00876 & 0.0065 & $\begin{array}{c}2.7 E- \\
05\end{array}$ & $\begin{array}{c}8.4 \mathrm{E}- \\
06\end{array}$ & $\begin{array}{c}5.1 \mathrm{E}- \\
06\end{array}$ & 1.7E-05 & 0.56666 & $\begin{array}{c}9.9 \mathrm{E}- \\
06\end{array}$ & 0.43334 & 0.01 & 0.013 & 7 \\
\hline
\end{tabular}

Table D5.13: Kalman Filter for test case 64-2200

\begin{tabular}{|c|c|c|c|c|c|c|c|c|c|c|c|c|c|c|c|}
\hline time & trip & $\operatorname{art3}$ & art2 & art1 & Average & $\Delta \mathbf{3}$ & $\Delta 2$ & $\Delta 1$ & Var[data]n & $g(k+1)$ & $e(k+1)$ & $a(k+1)$ & Predict & Observed & Boarders \\
\hline $8: 33$ & 17 & 0 & 0 & 0 & 0.025 & 0.0006 & 0.0006 & 0.0006 & 0.0006 & 0.5 & 0 & 0.5 & & 0.025 & \\
\hline $8: 36$ & 18 & 0.00437 & 0.00469 & 0.00618 & 0.00508 & $\begin{array}{c}5.1 \mathrm{E}- \\
07\end{array}$ & $\begin{array}{c}1.5 \mathrm{E}- \\
07\end{array}$ & $\begin{array}{c}1.2 \mathrm{E}- \\
06\end{array}$ & 3.3E-07 & 0.5 & $\begin{array}{c}1.6 \mathrm{E}- \\
07\end{array}$ & 0.5 & 0.01559 & 0.00761 & \\
\hline $8: 39$ & 19 & 0.00536 & 0.00743 & 0.00536 & 0.00605 & $\begin{array}{c}4.8 \mathrm{E}- \\
07 \\
\end{array}$ & $\begin{array}{c}1.9 \mathrm{E}- \\
06\end{array}$ & $\begin{array}{c}4.8 \mathrm{E}- \\
07 \\
\end{array}$ & $1.2 \mathrm{E}-06$ & 0.53226 & $\begin{array}{c}6.4 \mathrm{E}- \\
07\end{array}$ & 0.46774 & 0.00641 & 0.00912 & \\
\hline $8: 43$ & 20 & 0.00808 & 0.00892 & 0.00886 & 0.00862 & $\begin{array}{c}2.9 \mathrm{E}- \\
07\end{array}$ & $\begin{array}{c}9.1 \mathrm{E}- \\
08\end{array}$ & $\begin{array}{c}5.9 \mathrm{E}- \\
08\end{array}$ & $1.9 \mathrm{E}-07$ & 0.81132 & $\begin{array}{c}1.6 \mathrm{E}- \\
07\end{array}$ & 0.18868 & 0.00891 & 0.0108 & \\
\hline $8: 59$ & 24 & 0.00143 & 0.01029 & 0.01048 & 0.0074 & $\begin{array}{c}3.6 \mathrm{E}- \\
05\end{array}$ & $\begin{array}{c}8.4 \mathrm{E}- \\
06\end{array}$ & $\begin{array}{c}9.5 \mathrm{E}- \\
06\end{array}$ & 2.2E-05 & 0.50177 & $\begin{array}{l}\text { 1.1E- } \\
05\end{array}$ & 0.49823 & 0.011 & 0.017 & 8 \\
\hline
\end{tabular}

Table D5.14: Kalman Filter for test case 29-1200

\begin{tabular}{|c|c|c|c|c|c|c|c|c|c|c|c|c|c|c|c|}
\hline time & trip & $\operatorname{art3}$ & art2 & art1 & Average & $\Delta \mathbf{3}$ & $\Delta 2$ & $\Delta 1$ & Var[data]n & $g(k+1)$ & $\theta(k+1)$ & $a(k+1)$ & Predict & Observed & Boarders \\
\hline $8: 33$ & 17 & 0 & 0 & 0 & 0.025 & 0.0006 & 0.0006 & 0.0006 & 0.0006 & 0.5 & 0 & 0.5 & & 0.025 & \\
\hline $8: 36$ & 18 & 0.00437 & 0.00469 & 0.00618 & 0.00508 & $\begin{array}{c}5.1 \mathrm{E}- \\
07\end{array}$ & $\begin{array}{c}1.5 \mathrm{E}- \\
07\end{array}$ & $\begin{array}{c}1.2 \mathrm{E}- \\
06\end{array}$ & 3.3E-07 & 0.5 & $\begin{array}{c}1.6 E- \\
07\end{array}$ & 0.5 & 0.01559 & 0.00326 & \\
\hline $8: 39$ & 19 & 0.00536 & 0.00743 & 0.00536 & 0.00605 & $\begin{array}{c}4.8 \mathrm{E}- \\
07\end{array}$ & $\begin{array}{c}1.9 \mathrm{E}- \\
06\end{array}$ & $\begin{array}{c}4.8 \mathrm{E}- \\
07\end{array}$ & $1.2 \mathrm{E}-06$ & 0.53226 & $\begin{array}{c}6.4 \mathrm{E}- \\
07\end{array}$ & 0.46774 & 0.00437 & 0.00178 & \\
\hline $8: 43$ & 20 & 0.00808 & 0.00892 & 0.00886 & 0.00862 & $\begin{array}{c}2.9 \mathrm{E}- \\
07\end{array}$ & $\begin{array}{c}9.1 \mathrm{E}- \\
08\end{array}$ & $\begin{array}{c}5.9 \mathrm{E}- \\
08\end{array}$ & $1.9 \mathrm{E}-07$ & 0.81132 & $\begin{array}{c}1.6 \mathrm{E}- \\
07\end{array}$ & 0.18868 & 0.00753 & 0.01277 & \\
\hline $8: 59$ & 24 & 0.01729 & 0.01016 & 0.00595 & 0.01113 & $\begin{array}{c}3.8 \mathrm{E}- \\
05\end{array}$ & $\begin{array}{c}9.5 \mathrm{E}- \\
07\end{array}$ & $\begin{array}{c}2.7 E- \\
05\end{array}$ & $1.9 \mathrm{E}-05$ & 0.502 & $\begin{array}{c}9.8 \mathrm{E}- \\
06\end{array}$ & 0.498 & 0.009 & 0.013 & 5 \\
\hline
\end{tabular}


Table D5.15: Kalman Filter for test case 6-3000

\begin{tabular}{|c|c|c|c|c|c|c|c|c|c|c|c|c|c|c|c|}
\hline $\operatorname{tim} \theta$ & trip & art3 & art2 & art1 & Average & $\Delta \mathbf{3}$ & $\Delta 2$ & $\Delta 1$ & Var[data]n & $g(k+1)$ & $\theta(k+1)$ & $a(k+1)$ & Predict & Observed & Boarders \\
\hline $8: 33$ & 17 & 0 & 0 & 0 & 0.025 & 0.0006 & 0.0006 & 0.0006 & 0.0006 & 0.5 & 0 & 0.5 & & 0.025 & \\
\hline $8: 36$ & 18 & 0.00437 & 0.00469 & 0.00618 & 0.00508 & $\begin{array}{c}5.1 \mathrm{E}- \\
07\end{array}$ & $\begin{array}{c}1.5 E- \\
07\end{array}$ & $\begin{array}{c}1.2 \mathrm{E}- \\
06\end{array}$ & 3.3E-07 & 0.5 & $\begin{array}{c}1.6 \mathrm{E}- \\
07\end{array}$ & 0.5 & 0.01559 & 0.00326 & \\
\hline $8: 39$ & 19 & 0.00536 & 0.00743 & 0.00536 & 0.00605 & $\begin{array}{c}4.8 \mathrm{E}- \\
07\end{array}$ & $\begin{array}{c}1.9 \mathrm{E}- \\
06\end{array}$ & $\begin{array}{c}4.8 \mathrm{E}- \\
07\end{array}$ & $1.2 E-06$ & 0.53226 & $\begin{array}{c}6.4 \mathrm{E}- \\
07\end{array}$ & 0.46774 & 0.00437 & 0.00178 & \\
\hline $8: 43$ & 20 & 0.00808 & 0.00892 & 0.00886 & 0.00862 & $\begin{array}{c}2.9 \mathrm{E}- \\
07 \\
\end{array}$ & $\begin{array}{c}9.1 \mathrm{E}- \\
08\end{array}$ & $\begin{array}{c}5.9 \mathrm{E}- \\
08\end{array}$ & $1.9 \mathrm{E}-07$ & 0.81132 & $\begin{array}{c}1.6 \mathrm{E}- \\
07 \\
\end{array}$ & 0.18868 & 0.00753 & 0.0074 & \\
\hline $8: 59$ & 24 & 0.01729 & 0.01016 & 0.00909 & 0.01218 & $\begin{array}{c}2.6 \mathrm{E}- \\
05\end{array}$ & $\begin{array}{c}4.1 \mathrm{E}- \\
06\end{array}$ & $\begin{array}{c}9.5 \mathrm{E}- \\
06\end{array}$ & $1.5 \mathrm{E}-05$ & 0.50258 & $\begin{array}{c}7.6 \mathrm{E}- \\
06\end{array}$ & 0.49742 & 0.008 & 0.008 & 5 \\
\hline
\end{tabular}

Table D5.16: Kalman Filter for test case 56-2200

\begin{tabular}{|c|c|c|c|c|c|c|c|c|c|c|c|c|c|c|c|}
\hline time & trip & art3 & $\operatorname{art2}$ & art1 & Average & $\Delta \mathbf{3}$ & $\Delta \mathbf{2}$ & $\Delta 1$ & Varidatajn & $g(k+1)$ & $\theta(k+1)$ & $a(k+1)$ & Predlct & Observed & Boarders \\
\hline $8: 33$ & 17 & 0 & 0 & 0 & 0.025 & 0.0006 & 0.0006 & 0.0006 & 0.0006 & 0.5 & 0 & 0.5 & & 0.025 & \\
\hline $8: 36$ & 18 & 0.00697 & 0.00952 & 0.00982 & 0.00877 & $\begin{array}{c}3.2 \mathrm{E}- \\
06\end{array}$ & $\begin{array}{c}5.6 \mathrm{E}- \\
07\end{array}$ & $\begin{array}{c}1.1 \mathrm{E}- \\
06\end{array}$ & $1.9 \mathrm{E}-06$ & 0.5 & $\begin{array}{c}9.5 \mathrm{E}- \\
07\end{array}$ & 0.5 & 0.01741 & 0.01099 & \\
\hline $8: 39$ & 19 & 0.00588 & 0.00875 & 0.01257 & 0.00907 & $1 E-05$ & $1 E-07$ & $\begin{array}{c}1.2 \mathrm{E}- \\
05\end{array}$ & 5.1E-06 & 0.54247 & $\begin{array}{c}2.8 \mathrm{E}- \\
06\end{array}$ & 0.45753 & 0.01185 & 0.00446 & \\
\hline $8: 43$ & 20 & 0.00754 & 0.01715 & 0.00895 & 0.01121 & $\begin{array}{c}1.3 \mathrm{E}- \\
05\end{array}$ & $\begin{array}{c}3.5 \mathrm{E}- \\
05\end{array}$ & $\begin{array}{c}5.1 \mathrm{E}- \\
06\end{array}$ & $2.4 \mathrm{E}-05$ & 0.52698 & $\begin{array}{c}1.3 \mathrm{E}- \\
05\end{array}$ & 0.47302 & 0.00683 & 0.00572 & \\
\hline $8: 59$ & 24 & 0.01305 & 0.01636 & 0.01255 & 0.01399 & $\begin{array}{c}8.7 \mathrm{E}- \\
07\end{array}$ & $\begin{array}{c}5.6 \mathrm{E}- \\
06\end{array}$ & $\begin{array}{c}2.1 \mathrm{E}- \\
06\end{array}$ & $3.3 \mathrm{E}-06$ & 0.83155 & $\begin{array}{c}2.7 \mathrm{E}- \\
06\end{array}$ & 0.16845 & 0.011 & 0.009 & 7 \\
\hline
\end{tabular}

Table D5.17: Kalman Filter for test case 8-3000

\begin{tabular}{|c|c|c|c|c|c|c|c|c|c|c|c|c|c|c|c|}
\hline time & trip & art3 & art2 & art1 & Average & $\Delta \mathbf{3}$ & $\Delta \mathbf{2}$ & $\Delta 1$ & Var[data]n & $g(k+1)$ & $\theta(k+1)$ & $a(k+1)$ & Predict & Observed & Boarders \\
\hline $8: 33$ & 17 & 0 & 0 & 0 & 0.025 & 0.0006 & 0.0006 & 0.0006 & 0.0006 & 0.5 & 0 & 0.5 & & 0.025 & \\
\hline $8: 36$ & 18 & 0.00952 & 0.00982 & 0.01099 & 0.01011 & $\begin{array}{c}3.5 \mathrm{E}- \\
07\end{array}$ & $\begin{array}{c}8.4 \mathrm{E}- \\
08\end{array}$ & $\begin{array}{c}7.7 \mathrm{E}- \\
07\end{array}$ & 2.1E-07 & 0.5 & $\begin{array}{c}1.1 \mathrm{E}- \\
07\end{array}$ & 0.5 & 0.01799 & 0.00942 & \\
\hline $8: 39$ & 19 & 0.00875 & 0.01257 & 0.00446 & 0.00859 & $\begin{array}{c}2.5 \mathrm{E}- \\
08\end{array}$ & $\begin{array}{c}1.6 \mathrm{E}- \\
05\end{array}$ & $\begin{array}{c}1.7 \mathrm{E}- \\
05\end{array}$ & 7.9E-06 & 0.50337 & 4E-06 & 0.49663 & 0.00692 & 0.0013 & \\
\hline $8: 43$ & 20 & 0.01715 & 0.00895 & 0.00572 & 0.01061 & $\begin{array}{c}4.3 \mathrm{E}- \\
05\end{array}$ & $\begin{array}{c}2.7 \mathrm{E}- \\
06\end{array}$ & $\begin{array}{c}2.4 \mathrm{E}- \\
05\end{array}$ & 2.3E-05 & 0.54024 & $\begin{array}{c}1.2 \mathrm{E}- \\
05\end{array}$ & 0.45976 & 0.00369 & 0.00923 & \\
\hline $8: 59$ & 24 & 0.01636 & 0.01254 & 0.00869 & 0.01253 & $\begin{array}{c}1.5 \mathrm{E}- \\
05\end{array}$ & $\begin{array}{c}1.4 \mathrm{E}- \\
10\end{array}$ & $\begin{array}{c}1.5 \mathrm{E}- \\
05\end{array}$ & 7.3E-06 & 0.72791 & $\begin{array}{c}5.3 \mathrm{E}- \\
06\end{array}$ & 0.27209 & 0.009 & 0.012 & 6 \\
\hline
\end{tabular}


Table D5.18: Kalman Filter for test case 49-1200

\begin{tabular}{|c|c|c|c|c|c|c|c|c|c|c|c|c|c|c|c|}
\hline time & trip & art3 & art2 & art1 & Average & $\mathbf{\Delta 3}$ & $\Delta 2$ & $\Delta 1$ & Var[data]n & $g(k+1)$ & $e(k+1)$ & $a(k+1)$ & Predict & Observed & Boarders \\
\hline $8: 33$ & 17 & 0 & 0 & 0 & 0.025 & 0.0006 & 0.0006 & 0.0006 & 0.0006 & 0.5 & 0 & 0.5 & & 0.025 & \\
\hline $8: 36$ & 18 & 0.01099 & 0.00942 & 0.0094 & 0.00994 & $\begin{array}{c}1.1 \mathrm{E}- \\
06\end{array}$ & $\begin{array}{c}2.6 E- \\
07\end{array}$ & $\begin{array}{c}2.9 \mathrm{E}- \\
07\end{array}$ & $6.9 \mathrm{E}-07$ & 0.5 & $\begin{array}{c}\text { 3.4E- } \\
07\end{array}$ & 0.5 & 0.0172 & 0.00942 & \\
\hline 8:39 & 19 & 0.01302 & 0.0044 & 0.00587 & 0.00776 & $\begin{array}{c}2.8 \mathrm{E}- \\
05\end{array}$ & $\begin{array}{c}1.1 \mathrm{E}= \\
05\end{array}$ & $\begin{array}{c}3.6 \mathrm{E}- \\
06\end{array}$ & $1.9 \mathrm{E}-05$ & 0.50438 & $\begin{array}{c}9.8 \mathrm{E}- \\
06\end{array}$ & 0.49562 & 0.00763 & 0.0013 & \\
\hline $8: 43$ & 20 & 0.00927 & 0.00535 & 0.00994 & 0.00819 & $\begin{array}{c}1.2 \mathrm{E}- \\
06\end{array}$ & $8 \mathrm{E}-06$ & $\begin{array}{c}3.1 \mathrm{E}- \\
06\end{array}$ & 4.6E-06 & 0.75795 & $\begin{array}{c}3.5 \mathrm{E}- \\
06\end{array}$ & 0.24205 & 0.00785 & 0.00535 & \\
\hline $8: 59$ & 24 & 0.01175 & 0.00802 & 0.0103 & 0.01002 & $3 E-06$ & $4 E-06$ & $\begin{array}{c}7.8 \mathrm{E}- \\
08\end{array}$ & $3.5 E-06$ & 0.66626 & $\begin{array}{c}2.3 \mathrm{E}- \\
06\end{array}$ & 0.33374 & 0.009 & 0.009 & 6 \\
\hline
\end{tabular}

Table D5.19: Kalman Filter for test case 59-2200

\begin{tabular}{|c|c|c|c|c|c|c|c|c|c|c|c|c|c|c|c|}
\hline $\operatorname{tim} \theta$ & trip & art3 & art2 & art1 & Average & $\mathbf{\Delta 3}$ & $\Delta 2$ & $\Delta 1$ & Var[data]n & $g(k+1)$ & $e(k+1)$ & $a(k+1)$ & Predict & Observed & Boarders \\
\hline $8: 33$ & 17 & 0 & 0 & 0 & 0.025 & 0.0006 & 0.0006 & 0.0006 & 0.0006 & 0.5 & 0 & 0.5 & & 0.025 & \\
\hline $8: 36$ & 18 & 0.00942 & 0.00583 & 0.0094 & 0.00821 & $\begin{array}{c}1.5 \mathrm{E}- \\
06\end{array}$ & $\begin{array}{c}5.7 E- \\
06\end{array}$ & $\begin{array}{c}1.4 \mathrm{E}- \\
06\end{array}$ & $3.6 \mathrm{E}-06$ & 0.5 & $\begin{array}{c}1.8 E \\
06\end{array}$ & 0.5 & 0.0172 & 0.00942 & \\
\hline 8:39 & 19 & 0.01302 & 0.00402 & 0.00587 & 0.00763 & $\begin{array}{c}2.9 \mathrm{E}- \\
05\end{array}$ & $\begin{array}{c}1.3 \mathrm{E}- \\
05\end{array}$ & $\begin{array}{c}3.1 \mathrm{E}- \\
06\end{array}$ & $2.1 \mathrm{E}-05$ & 0.52042 & $\begin{array}{c}1.1 \mathrm{E}- \\
05\end{array}$ & 0.47958 & 0.00757 & 0.0013 & \\
\hline $8: 43$ & 20 & 0.00927 & 0.00535 & 0.00994 & 0.00819 & $\begin{array}{c}1.2 \mathrm{E}- \\
06\end{array}$ & $8 \mathrm{E}-06$ & $\begin{array}{c}.1 \mathrm{E}- \\
06 \\
\end{array}$ & 4.6E-06 & 0.77141 & $\begin{array}{c}.6 \mathrm{E}- \\
06 \\
\end{array}$ & 0.22859 & 0.00797 & 0.00896 & \\
\hline 8:59 & 24 & 0.01175 & 0.00802 & 0.0103 & 0.01002 & 3E-06 & $4 E-06$ & $\begin{array}{c}7.8 \mathrm{E}- \\
08 \\
\end{array}$ & 3.5E-06 & 0.66827 & $\begin{array}{c}2.3 \mathrm{E}- \\
06\end{array}$ & 0.33173 & 0.01 & 0.013 & 7 \\
\hline
\end{tabular}

Table D5.20: Kalman Filter for test case 24-1200

\begin{tabular}{|c|c|c|c|c|c|c|c|c|c|c|c|c|c|c|c|}
\hline time & trip & art3 & art2 & art1 & Average & $\Delta \mathbf{3}$ & $\Delta 2$ & $\Delta 1$ & Var[data]n & $g(k+1)$ & $\theta(k+1)$ & $a(k+1)$ & Predict & Observed & Boarders \\
\hline $8: 33$ & 17 & 0 & 0 & 0 & 0.025 & 0.0006 & 0.0006 & 0.0006 & 0.0006 & 0.5 & 0 & 0.5 & & 0.025 & \\
\hline $8: 36$ & 18 & 0.00633 & 0.012 & 0.01109 & 0.00981 & $\begin{array}{c}1.2 \mathrm{E}- \\
05\end{array}$ & $\begin{array}{c}4.8 \mathrm{E}- \\
06\end{array}$ & $\begin{array}{c}1.7 \mathrm{E}- \\
06\end{array}$ & 8.4E-06 & 0.5 & $\begin{array}{c}4.2 \mathrm{E}- \\
06\end{array}$ & 0.5 & 0.01805 & 0.0069 & \\
\hline $8: 39$ & 19 & 0.00515 & 0 & 0.0015 & 0.00222 & $\begin{array}{c}8.6 \mathrm{E}- \\
06\end{array}$ & $\begin{array}{c}4.9 E- \\
06\end{array}$ & $\begin{array}{c}5.1 \mathrm{E}- \\
07\end{array}$ & $6.8 \mathrm{E}-06$ & 0.61911 & $\begin{array}{c}4.2 \mathrm{E}- \\
06\end{array}$ & 0.38089 & 0.00356 & 0.00177 & \\
\hline $8: 43$ & 20 & 0.00635 & 0.0131 & 0.01608 & 0.01184 & $3 E-05$ & $\begin{array}{c}1.6 \mathrm{E}- \\
06\end{array}$ & $\begin{array}{c}1.8 \mathrm{E}- \\
05\end{array}$ & $1.6 \mathrm{E}-05$ & 0.55814 & $\begin{array}{c}8.9 \mathrm{E}- \\
06\end{array}$ & 0.44186 & 0.00976 & 0.02399 & \\
\hline $8: 59$ & 24 & 0.01098 & 0.01318 & 0.00668 & 0.01028 & $\begin{array}{c}4.9 \mathrm{E}- \\
07\end{array}$ & $\begin{array}{c}8.4 \mathrm{E}- \\
06\end{array}$ & $\begin{array}{c}1.3 \mathrm{E}- \\
05\end{array}$ & 4.4E-06 & 0.74975 & $\begin{array}{c}3.3 \mathrm{E}- \\
06\end{array}$ & 0.25025 & 0.011 & 0.007 & 7 \\
\hline
\end{tabular}


$\stackrel{\infty}{\sim}$
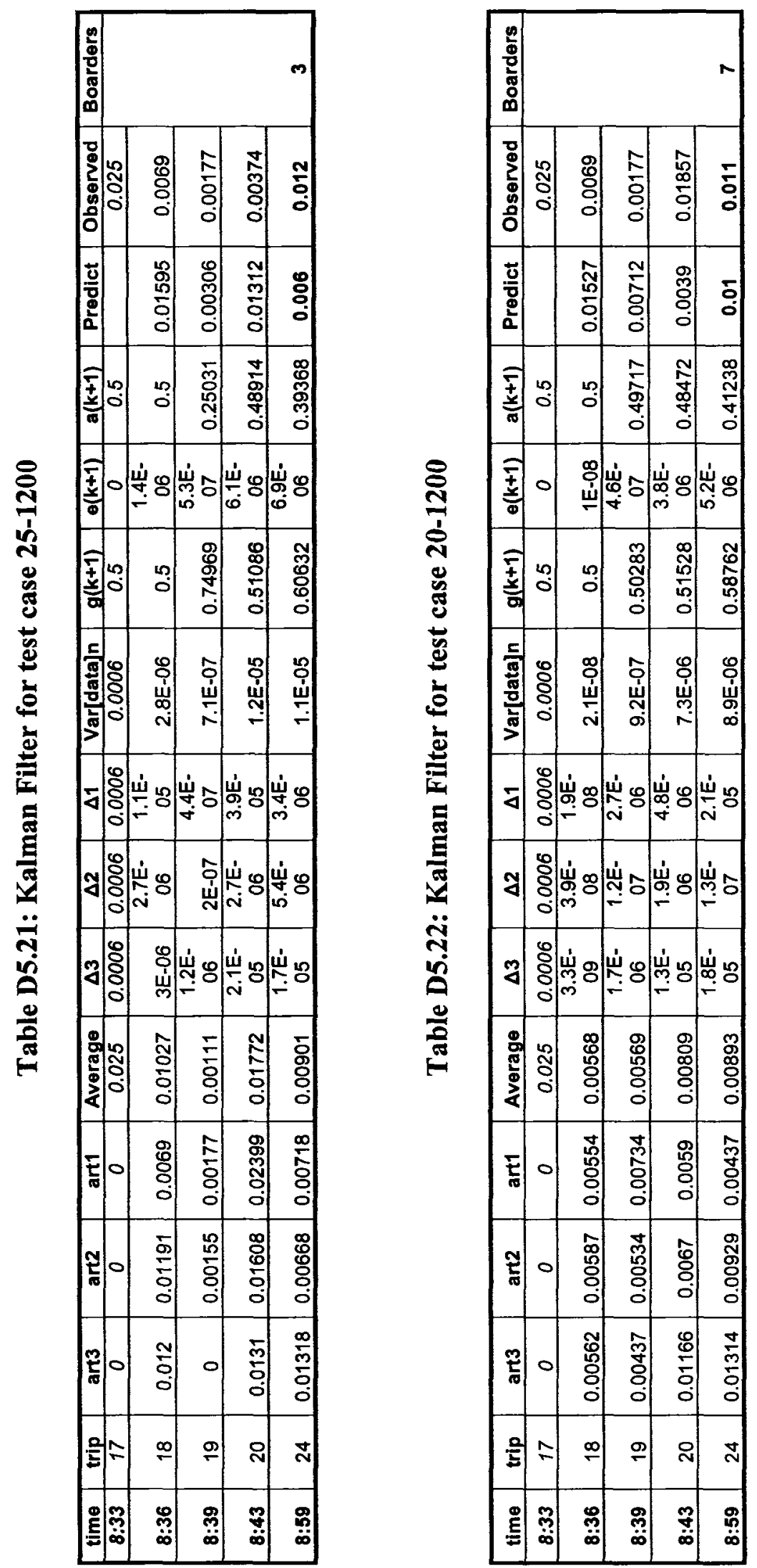
Table D5. 23: Prediction Error of the 3 Predictors

\begin{tabular}{|c|c|c|c|c|c|c|c|c|}
\hline 54 & 2200 & 3 & 5 & 8 & 6 & 0.667 & 1.66667 & 1 \\
\hline 47 & 1200 & 9 & 7 & 10 & 14 & 0.222 & 0.11111 & 0.555556 \\
\hline 48 & 1200 & 11 & 7 & 5 & 2 & 0.364 & 0.54545 & 0.818182 \\
\hline 36 & 1200 & 6 & 7 & 9 & 9 & 0.167 & 0.5 & 0.5 \\
\hline 16 & 3000 & 9 & 7 & 10 & 11 & 0.222 & 0.11111 & 0.222222 \\
\hline 21 & 1200 & 7 & 9 & 6 & 6 & 0.286 & 0.14286 & 0.142857 \\
\hline 19 & 1200 & 7 & 6 & 8 & 5 & 0.143 & 0.14286 & 0.285714 \\
\hline 45 & 1200 & 10 & 6 & 4 & 1 & 0.4 & 0.6 & 0.9 \\
\hline 39 & 1200 & 7 & 7 & 10 & 10 & 0 & 0.42857 & 0.428571 \\
\hline 38 & 1200 & 9 & 7 & 8 & 6 & 0.222 & 0.11111 & 0.333333 \\
\hline 64 & 2200 & 12 & 7 & 8 & 7 & 0.417 & 0.33333 & 0.416667 \\
\hline 29 & 1200 & 7 & 6 & 5 & 4 & 0.143 & 0.28571 & 0.428571 \\
\hline 6 & 3000 & 6 & 8 & 5 & 6 & 0.333 & 0.16667 & 0 \\
\hline 56 & 2200 & 7 & 5 & 7 & 5 & 0.286 & 0 & 0.285714 \\
\hline 8 & 3000 & 8 & 4 & 6 & 11 & 0.5 & 0.25 & 0.375 \\
\hline 49 & 1200 & 6 & 7 & 7 & 5 & 0.167 & 0.16667 & 0.166667 \\
\hline 59 & 2200 & 12 & 7 & 7 & 6 & 0.417 & 0.41667 & 0.5 \\
\hline 24 & 1200 & 8 & 8 & 7 & 5 & 0 & 0.125 & 0.375 \\
\hline 25 & 1200 & 7 & 8 & 3 & 1 & 0.143 & 0.57143 & 0.857143 \\
\hline 20 & 1200 & 8 & 7 & 7 & 8 & 0.125 & 0.125 & 0 \\
\hline
\end{tabular}

Note: d1.d2, d3= Absolute Relative Error the for Developed Model, KF model, and the naïve model, respectively 


\section{Appendix D6}

\section{General}

This Appendix presents the database, the prediction results of the developed model, the Kalman Filter, and the Naive model applied for alighting passenger prediction.

\section{Database for the Developed Model and Kalman Filter Model}

Table D6.1: Database of Alighting Passengers at Mackenzic Station (Route 95)

\begin{tabular}{|c|c|c|c|c|}
\hline 28 & & 9 & 3 & (15: \\
\hline 54 & 2200 & 23 & 27 & 23 \\
\hline 58 & 2200 & 21 & 26 & 23 \\
\hline 33 & 1200 & 25 & 28 & 21 \\
\hline 42 & 1200 & 12 & 13 & 13 \\
\hline 47 & 1200 & 11 & 14 & 13 \\
\hline 62 & 2200 & 21 & 23 & 27 \\
\hline 48 & 1200 & 14 & 17 & 13 \\
\hline 36 & 1200 & 13 & 14 & 10 \\
\hline 16 & 3000 & 17 & 17 & 16 \\
\hline 52 & 2200 & 28 & 28 & 23 \\
\hline 3 & 3000 & 17 & 17 & 17 \\
\hline 2 & 3000 & 17 & 16 & 17 \\
\hline 7 & 3000 & 17 & 16 & 17 \\
\hline 21 & 1200 & 22 & 33 & 25 \\
\hline 28 & 1200 & 22 & 31 & 25 \\
\hline 19 & 1200 & 23 & 33 & 23 \\
\hline 4 & 3000 & 17 & 17 & 15 \\
\hline 11 & 3000 & 17 & 17 & 17 \\
\hline 45 & 1200 & 14 & 16 & 12 \\
\hline 39 & 1200 & 13 & 14 & 11 \\
\hline 5 & 3000 & 17 & 17 & 15 \\
\hline 55 & 2200 & 23 & 31 & 20 \\
\hline 38 & 1200 & 12 & 14 & 12 \\
\hline 13 & 3000 & 17 & 17 & 17 \\
\hline 64 & 2200 & 22 & 21 & 22 \\
\hline 14 & 3000 & 17 & 17 & 16 \\
\hline
\end{tabular}




\begin{tabular}{|c|c|c|c|c|}
\hline 10 & 3000 & 17 & 17 & 16 \\
\hline 29 & 1200 & 25 & 28 & 20 \\
\hline 35 & 1200 & 25 & 27 & 22 \\
\hline 53 & 2200 & 28 & 21 & 26 \\
\hline 17 & 1200 & 25 & 35 & 22 \\
\hline 6 & 3000 & 17 & 17 & 15 \\
\hline 12 & 3000 & 17 & 17 & 17 \\
\hline 18 & 1200 & 29 & 27 & 23 \\
\hline 31 & 1200 & 25 & 28 & 28 \\
\hline 56 & 2200 & 22 & 22 & 20 \\
\hline 8 & 3000 & 17 & 17 & 17 \\
\hline 49 & 1200 & 11 & 14 & 13 \\
\hline 9 & 3000 & 17 & 17 & 17 \\
\hline 59 & 2200 & 27 & 29 & 25 \\
\hline 41 & 1200 & 13 & 17 & 9 \\
\hline 46 & 1200 & 12 & 17 & 12 \\
\hline 57 & 2200 & 24 & 24 & 19 \\
\hline 51 & 2200 & 26 & 29 & 22 \\
\hline 1 & 3000 & 17 & 17 & 16 \\
\hline 22 & 1200 & 27 & 26 & 23 \\
\hline 40 & 1200 & 12 & 12 & 13 \\
\hline 63 & 2200 & 25 & 25 & 22 \\
\hline 50 & 1200 & 12 & 14 & 11 \\
\hline 61 & 2200 & 20 & 30 & 22 \\
\hline 43 & 1200 & 12 & 14 & 11 \\
\hline 24 & 1200 & 23 & 29 & 26 \\
\hline 25 & 1200 & 26 & 29 & 25 \\
\hline 37 & 1200 & 15 & 16 & 13 \\
\hline 65 & 2200 & 20 & 22 & 21 \\
\hline 44 & 1200 & 11 & 14 & 13 \\
\hline 60 & 2200 & 25 & 23 & 23 \\
\hline 20 & 1200 & 26 & 26 & 23 \\
\hline 34 & 1200 & 27 & 26 & 27 \\
\hline 26 & 1200 & 24 & 29 & 26 \\
\hline 23 & 1200 & 25 & 26 & 19 \\
\hline 30 & 1200 & 21 & 27 & 22 \\
\hline 32 & 1200 & 20 & 22 & 23 \\
\hline 27 & 1200 & 19 & 26 & 23 \\
\hline 15 & 3000 & 17 & 17 & 16 \\
\hline
\end{tabular}

Note:

$\mathrm{A} 1, \mathrm{~A} 2$, and $\mathrm{A} 3=$ Alighting Passengers of trip $24^{\text {th }}$, trip $20^{\text {th }}$, and trip $19^{\text {th }}$, respectively. 


\section{Prediction Results of the Developed Model}

The following table shows part of an output returned from Matlab source code for the sake of illustration. All predictions are tabulated in Table D6.22

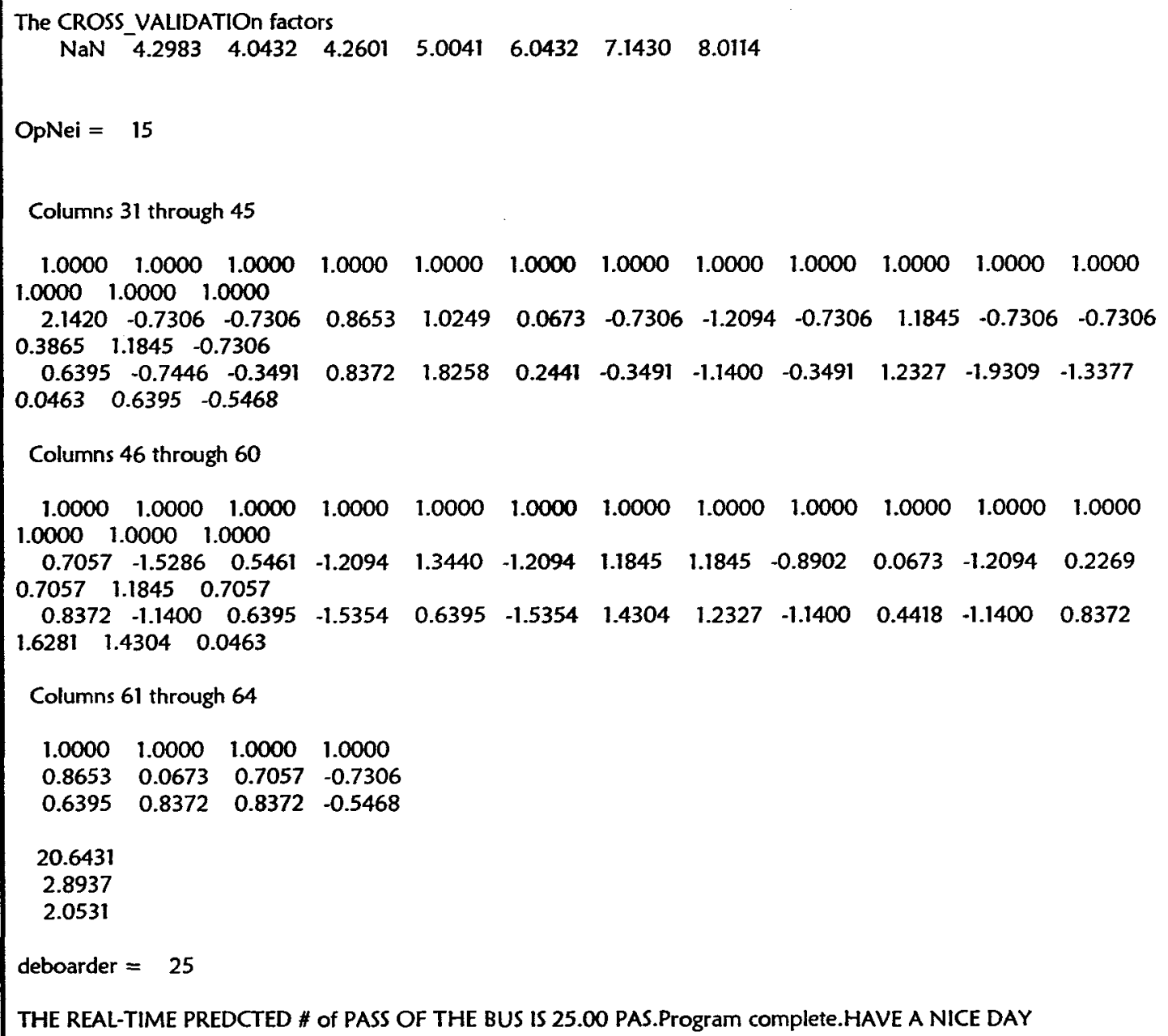

Figure D6.1: An Example of Matlab output for de-boarding Prediction (Case 20-1200) 
Table D6.2: Kalman Filter for test set 54-2200

\begin{tabular}{|c|c|c|c|c|c|c|c|c|c|c|c|c|c|c|}
\hline time & trip & art3 & art2 & art1 & Average & $\Delta 3$ & $\Delta \mathbf{2}$ & $\Delta 1$ & Var[data]n & $g(k+1)$ & $e(k+1)$ & $a(k+1)$ & Predict & Observed \\
\hline $8: 33$ & 17 & 0 & 0 & 0 & 20 & 400 & 400 & 400 & 400 & 0.5 & 0 & 0.5 & & 2 \\
\hline $8: 36$ & 17 & 0 & 0 & 0 & 20 & 400 & 400 & 400 & 400 & 0.5 & 0 & 0.5 & & 2 \\
\hline $8: 39$ & 19 & 23 & 23 & 16 & 20 & 9 & 9 & 16 & 9 & 0.5 & 4.5 & 0.5 & 9 & 23 \\
\hline $8: 43$ & 20 & 22 & 26 & 17 & 21.6667 & 0.1111 & 18.778 & 21.778 & 9.44444 & 0.5962 & 5.63077 & 0.4038 & 19.4228 & 27 \\
\hline $8: 59$ & 24 & 20 & 19 & 17 & 18.6667 & 1.7778 & 0.1111 & 2.7778 & 0.94444 & 0.8744 & 0.82583 & 0.1256 & 18 & 23 \\
\hline
\end{tabular}

Table D6.3: Kalman Filter for test set 47-1200

\begin{tabular}{|c|c|c|c|c|c|c|c|c|c|c|c|c|c|c|}
\hline time & trip & art3 & art2 & art1 & Average & $\Delta 3$ & $\Delta \mathbf{2}$ & $\Delta 1$ & Var[data]n & $g(k+1)$ & $e(k+1)$ & $a(k+1)$ & Predict & Observed \\
\hline $8: 33$ & 17 & 0 & 0 & 0 & 20 & 400 & 400 & 400 & 400 & 0.5 & 0 & 0.5 & & 2 \\
\hline $8: 36$ & 17 & 0 & 0 & 0 & 20 & 400 & 400 & 400 & 400 & 0.5 & 0 & 0.5 & & 2 \\
\hline $8: 39$ & 19 & 23 & 23 & 21 & 20 & 9 & 9 & 1 & 9 & 0.5 & 4.5 & 0.5 & 11.5 & 13 \\
\hline $8: 43$ & 20 & 27 & 26 & 28 & 27 & 0 & 1 & 1 & 0.5 & 0.90909 & 0.45455 & 0.09091 & 26.6364 & 14 \\
\hline $8: 59$ & 24 & 23 & 21 & 25 & 23 & 0 & 4 & 4 & 2 & 0.55102 & 1.10204 & 0.44898 & 20 & 11 \\
\hline
\end{tabular}

Table D6.4: Kalman Filter for test set $\mathbf{4 8 - 1 2 0 0}$

\begin{tabular}{|c|c|c|c|c|c|c|c|c|c|c|c|c|c|c|}
\hline time & trip & art3 & $\operatorname{art2}$ & art1 & Average & $\Delta 3$ & $\Delta 2$ & $\Delta 1$ & Var[data]n & $g(k+1)$ & $e(k+1)$ & $a(k+1)$ & Predict & Observed \\
\hline $8: 33$ & 17 & 0 & 0 & 0 & 20 & 400 & 400 & 400 & 400 & 0.5 & 0 & 0.5 & & 2 \\
\hline $8: 36$ & 17 & 0 & 0 & 0 & 20 & 400 & 400 & 400 & 400 & 0.5 & 0 & 0.5 & & 2 \\
\hline $8: 39$ & 19 & 13 & 13 & 27 & 20 & 49 & 49 & 49 & 49 & 0.5 & 24.5 & 0.5 & 14.5 & 13 \\
\hline $8: 43$ & 20 & 13 & 14 & 23 & 16.6667 & 13.444 & 7.1111 & 40.111 & 10.2778 & 0.77189 & 7.93328 & 0.22811 & 20.7189 & 17 \\
\hline $8: 59$ & 24 & 12 & 11 & 21 & 14.6667 & 7.1111 & 13.444 & 40.111 & 10.2778 & 0.63923 & 6.56991 & 0.36077 & 20 & 14 \\
\hline
\end{tabular}


Table D6.5: Kalman Filter for test set 36-1200

\begin{tabular}{|c|c|c|c|c|c|c|c|c|c|c|c|c|c|c|}
\hline time & trip & art3 & art2 & art1 & Average & $\Delta \mathbf{3}$ & $\Delta 2$ & $\Delta 1$ & Var[data]n & $g(k+1)$ & $\theta(k+1)$ & $a(k+1)$ & Predict & Observed \\
\hline $8: 33$ & 17 & 0 & 0 & 0 & 20 & 400 & 400 & 400 & 400 & 0.5 & 0 & 0.5 & & 2 \\
\hline $8: 36$ & 17 & 0 & 0 & 0 & 20 & 400 & 400 & 400 & 400 & 0.5 & 0 & 0.5 & & 2 \\
\hline $8: 39$ & 19 & 13 & 27 & 13 & 20 & 49 & 49 & 49 & 49 & 0.5 & 24.5 & 0.5 & 7.5 & 10 \\
\hline $8: 43$ & 20 & 14 & 23 & 17 & 18 & 16 & 25 & 1 & 20.5 & 0.68702 & 14.084 & 0.31298 & 14.8092 & 14 \\
\hline $8: 59$ & 24 & 11 & 21 & 14 & 15.3333 & 18.778 & 32.111 & 1.7778 & 25.4444 & 0.60838 & 15.48 & 0.39162 & 14 & 13 \\
\hline
\end{tabular}

Table D6.6: Kalman Filter for test set 16-3000

\begin{tabular}{|c|c|c|c|c|c|c|c|c|c|c|c|c|c|c|}
\hline tlme & trip & art3 & art2 & art1 & Average & $\Delta \mathbf{3}$ & $\Delta \mathbf{2}$ & $\Delta 1$ & Var[data]n & $g(k+1)$ & $e(k+1)$ & $a(k+1)$ & Predict & Observed \\
\hline $8: 33$ & 17 & 0 & 0 & 0 & 20 & 400 & 400 & 400 & 400 & 0.5 & 0 & 0.5 & & 2 \\
\hline $8: 36$ & 17 & 0 & 0 & 0 & 20 & 400 & 400 & 400 & 400 & 0.5 & 0 & 0.5 & & 2 \\
\hline $8: 39$ & 19 & 27 & 13 & 10 & 20 & 49 & 49 & 100 & 49 & 0.5 & 24.5 & 0.5 & 6 & 16 \\
\hline $8: 43$ & 20 & 23 & 17 & 14 & 18 & 25 & 1 & 16 & 13 & 0.74257 & 9.65347 & 0.25743 & 14.5149 & 17 \\
\hline $8: 59$ & 24 & 21 & 14 & 13 & 16 & 25 & 4 & 9 & 14.5 & 0.62487 & 9.06064 & 0.37513 & 15 & 17 \\
\hline
\end{tabular}

Table D6.7: Kalman Filter for test set 21-1200

\begin{tabular}{|c|c|c|c|c|c|c|c|c|c|c|c|c|c|c|}
\hline time & trip & art3 & art2 & art1 & Average & $\Delta 3$ & $\Delta 2$ & $\Delta 1$ & Var[data]n & $g(k+1)$ & $\theta(k+1)$ & $a(k+1)$ & Predict & Observed \\
\hline $8: 33$ & 17 & 0 & 0 & 0 & 20 & 400 & 400 & 400 & 400 & 0.5 & 0 & 0.5 & & 2 \\
\hline $8: 36$ & 17 & 0 & 0 & 0 & 20 & 400 & 400 & 400 & 400 & 0.5 & 0 & 0.5 & & 2 \\
\hline $8: 39$ & 19 & 17 & 17 & 17 & 20 & 9 & 9 & 9 & 9 & 0.5 & 4.5 & 0.5 & 9.5 & 25 \\
\hline $8: 43$ & 20 & 17 & 16 & 16 & 16.3333 & 0.4444 & 0.1111 & 0.1111 & 0.27778 & 0.94505 & 0.26252 & 0.05495 & 16.4945 & 33 \\
\hline $8: 59$ & 24 & 17 & 17 & 17 & 17 & 0 & 0 & 0 & 0 & 1 & 0 & 0 & 17 & 22 \\
\hline
\end{tabular}


Table D6.8: Kalman Filter for test set 19-1200

\begin{tabular}{|c|c|c|c|c|c|c|c|c|c|c|c|c|c|c|}
\hline tíme & trip & art3 & art2 & art1 & Average & $\Delta 3$ & $\Delta 2$ & $\Delta 1$ & Var[data]n & $g(k+1)$ & $e(k+1)$ & $a(k+1)$ & Predict & Observed \\
\hline $8: 33$ & 17 & 0 & 0 & 0 & 20 & 400 & 400 & 400 & 400 & 0.5 & 0 & 0.5 & & 2 \\
\hline $8: 36$ & 17 & 0 & 0 & 0 & 20 & 400 & 400 & 400 & 400 & 0.5 & 0 & 0.5 & & 2 \\
\hline $8: 39$ & 19 & 17 & 25 & 25 & 20 & 9 & 25 & 25 & 17 & 0.5 & 8.5 & 0.5 & 13.5 & 23 \\
\hline $8: 43$ & 20 & 16 & 33 & 31 & 26.6667 & 113.78 & 40.111 & 18.778 & 76.9444 & 0.52617 & 40.486 & 0.47383 & 27.2094 & 33 \\
\hline $8: 59$ & 24 & 17 & 22 & 22 & 20.3333 & 11.111 & 2.7778 & 2.7778 & 6.94444 & 0.87229 & 6.05754 & 0.12771 & 23 & 23 \\
\hline
\end{tabular}

Table D6.9: Kalman Filter for test set 45-1200

\begin{tabular}{|c|c|c|c|c|c|c|c|c|c|c|c|c|c|c|}
\hline time & trip & art3 & art2 & art1 & Average & $\Delta 3$ & $\Delta 2$ & $\Delta 1$ & Var[data]n & $g(k+1)$ & $e(k+1)$ & $a(k+1)$ & Predict & Observed \\
\hline $8: 33$ & 17 & 0 & 0 & 0 & 20 & 400 & 400 & 400 & 400 & 0.5 & 0 & 0.5 & & 2 \\
\hline $8: 36$ & 17 & 0 & 0 & 0 & 20 & 400 & 400 & 400 & 400 & 0.5 & 0 & 0.5 & & 2 \\
\hline $8: 39$ & 19 & 23 & 15 & 17 & 20 & 9 & 25 & 9 & 17 & 0.5 & 8.5 & 0.5 & 9.5 & 12 \\
\hline $8: 43$ & 20 & 33 & 17 & 17 & 22.3333 & 113.78 & 28.444 & 28.444 & 71.1111 & 0.5282 & 37.5607 & 0.4718 & 14.641 & 16 \\
\hline $8: 59$ & 24 & 23 & 17 & 17 & 19 & 16 & 4 & 4 & 10 & 0.82627 & 8.2627 & 0.17373 & 17 & 14 \\
\hline
\end{tabular}

Table D6.10: Kalman Filter for test set 39-1200

\begin{tabular}{|c|c|c|c|c|c|c|c|c|c|c|c|c|c|c|}
\hline time & trip & art3 & art2 & art1 & Average & $\Delta 3$ & $\Delta \mathbf{2}$ & $\Delta 1$ & Var[data]n & $g(k+1)$ & $e(k+1)$ & $a(k+1)$ & Predict & Observed \\
\hline $8: 33$ & 17 & 0 & 0 & 0 & 20 & 400 & 400 & 400 & 400 & 0.5 & 0 & 0.5 & & 2 \\
\hline $8: 36$ & 17 & 0 & 0 & 0 & 20 & 400 & 400 & 400 & 400 & 0.5 & 0 & 0.5 & & 2 \\
\hline $8: 39$ & 19 & 15 & 17 & 12 & 20 & 25 & 9 & 64 & 17 & 0.5 & 8.5 & 0.5 & 7 & 11 \\
\hline $8: 43$ & 20 & 17 & 17 & 16 & 16.6667 & 0.1111 & 0.1111 & 0.4444 & 0.11111 & 0.98726 & 0.1097 & 0.01274 & 15.9363 & 14 \\
\hline $8: 59$ & 24 & 17 & 14 & 14 & 15 & 4 & 1 & 1 & 2.5 & 0.51073 & 1.27684 & 0.48927 & 14 & 13 \\
\hline
\end{tabular}


Table D6.11: Kalman Filter for test set 38-1200

\begin{tabular}{|c|c|c|c|c|c|c|c|c|c|c|c|c|c|c|}
\hline time & trip & $\operatorname{art3}$ & art2 & art1 & Average & $\Delta 3$ & $\Delta \mathbf{2}$ & $\Delta 1$ & Var[data]n & $g(k+1)$ & $e(k+1)$ & $a(k+1)$ & Predict & Observed \\
\hline $8: 33$ & 17 & 0 & 0 & 0 & 20 & 400 & 400 & 400 & 400 & 0.5 & 0 & 0.5 & & 2 \\
\hline $8: 36$ & 17 & 0 & 0 & 0 & 20 & 400 & 400 & 400 & 400 & 0.5 & 0 & 0.5 & & 2 \\
\hline $8: 39$ & 19 & 11 & 15 & 20 & 20 & 81 & 25 & 0 & 53 & 0.5 & 26.5 & 0.5 & 11 & 12 \\
\hline $8: 43$ & 20 & 14 & 17 & 31 & 20.6667 & 44.444 & 13.444 & 106.78 & 28.9444 & 0.65701 & 19.0168 & 0.34299 & 24.4832 & 14 \\
\hline $8: 59$ & 24 & 13 & 17 & 23 & 17.6667 & 21.778 & 0.4444 & 28.444 & 11.1111 & 0.73057 & 8.11742 & 0.26943 & 21 & 12 \\
\hline
\end{tabular}

Table D6.12: Kalman Filter for test set $\mathbf{6 4 - 2 2 0 0}$

\begin{tabular}{|c|c|c|c|c|c|c|c|c|c|c|c|c|c|c|}
\hline time & trip & art3 & art2 & art1 & Average & $\Delta 3$ & $\Delta \mathbf{2}$ & $\Delta 1$ & Var[data]n & $g(k+1)$ & $\theta(k+1)$ & $a(k+1)$ & Predlct & Observed \\
\hline $8: 33$ & 17 & 0 & 0 & 0 & 20 & 400 & 400 & 400 & 400 & 0.5 & 0 & 0.5 & & 2 \\
\hline $8: 36$ & 17 & 0 & 0 & 0 & 20 & 400 & 400 & 400 & 400 & 0.5 & 0 & 0.5 & & 2 \\
\hline $8: 39$ & 19 & 20 & 12 & 17 & 20 & 0 & 64 & 9 & 32 & 0.5 & 16 & 0.5 & 9.5 & 22 \\
\hline $8: 43$ & 20 & 31 & 14 & 17 & 20.6667 & 106.78 & 44.444 & 13.444 & 75.6111 & 0.54784 & 41.4228 & 0.45216 & 19.2608 & 21 \\
\hline $8: 59$ & 24 & 23 & 12 & 17 & 17.3333 & 32.111 & 28.444 & 0.1111 & 30.2778 & 0.7031 & 21.2882 & 0.2969 & 18 & 22 \\
\hline
\end{tabular}

Table D6.13: Kalman Filter for test set 29-1200

\begin{tabular}{|c|c|c|c|c|c|c|c|c|c|c|c|c|c|c|}
\hline time & trip & art3 & art2 & art1 & Average & $\Delta 3$ & $\Delta 2$ & $\Delta 1$ & Var[data]n & $g(k+1)$ & $e(k+1)$ & $a(k+1)$ & Predict & Observed \\
\hline $8: 33$ & 17 & 0 & 0 & 0 & 20 & 400 & 400 & 400 & 400 & 0.5 & 0 & 0.5 & & 2 \\
\hline $8: 36$ & 17 & 0 & 0 & 0 & 20 & 400 & 400 & 400 & 400 & 0.5 & 0 & 0.5 & & 2 \\
\hline $8: 39$ & 19 & 22 & 16 & 16 & 20 & 4 & 16 & 16 & 10 & 0.5 & 5 & 0.5 & 9 & 20 \\
\hline $8: 43$ & 20 & 21 & 17 & 17 & 18.3333 & 7.1111 & 1.7778 & 1.7778 & 4.44444 & 0.68 & 3.02222 & 0.32 & 17.96 & 28 \\
\hline $8: 59$ & 24 & 22 & 17 & 17 & 18.6667 & 11.111 & 2.7778 & 2.7778 & 6.94444 & 0.58936 & 4.09275 & 0.41064 & 22 & 25 \\
\hline
\end{tabular}


Table D6.14: Kalman Filter for test set 6-3000

\begin{tabular}{|c|c|c|c|c|c|c|c|c|c|c|c|c|c|c|}
\hline time & trip & art3 & art2 & art1 & Average & $\Delta 3$ & $\Delta 2$ & $\Delta 1$ & Var[data]n & $g(k+1)$ & $e(k+1)$ & $a(k+1)$ & Predict & Observed \\
\hline $8: 33$ & 17 & 0 & 0 & 0 & 20 & 400 & 400 & 400 & 400 & 0.5 & 0 & 0.5 & & 2 \\
\hline $8: 36$ & 17 & 0 & 0 & 0 & 20 & 400 & 400 & 400 & 400 & 0.5 & 0 & 0.5 & & 2 \\
\hline $8: 39$ & 19 & 22 & 26 & 22 & 20 & 4 & 36 & 4 & 20 & 0.5 & 10 & 0.5 & 12 & 15 \\
\hline $8: 43$ & 20 & 17 & 21 & 35 & 24.3333 & 53.778 & 11.111 & 113.78 & 32.4444 & 0.56677 & 18.3884 & 0.43323 & 26.3353 & 17 \\
\hline $8: 59$ & 24 & 25 & 28 & 25 & 26 & 1 & 4 & 1 & 2.5 & 0.89311 & 2.23277 & 0.10689 & 24 & 17 \\
\hline
\end{tabular}

Table D6.15: Kalman Filter for test set 56-2200

\begin{tabular}{|c|c|c|c|c|c|c|c|c|c|c|c|c|c|c|}
\hline time & trip & $\operatorname{art3}$ & art2 & art1 & Average & $\Delta 3$ & $\Delta 2$ & $\Delta 1$ & Var[data]n & $g(k+1)$ & $\theta(k+1)$ & $a(k+1)$ & Predict & Observed \\
\hline $8: 33$ & 17 & 0 & 0 & 0 & 20 & 400 & 400 & 400 & 400 & 0.5 & 0 & 0.5 & & 2 \\
\hline $8: 36$ & 17 & 0 & 0 & 0 & 20 & 400 & 400 & 400 & 400 & 0.5 & 0 & 0.5 & & 2 \\
\hline $8: 39$ & 19 & 17 & 23 & 28 & 20 & 9 & 9 & 64 & 9 & 0.5 & 4.5 & 0.5 & 15 & 20 \\
\hline $8: 43$ & 20 & 17 & 27 & 28 & 24 & 49 & 9 & 16 & 29 & 0.536 & 15.544 & 0.464 & 24.288 & 22 \\
\hline $8: 59$ & 24 & 17 & 29 & 25 & 23.6667 & 44.444 & 28.444 & 1.7778 & 36.4444 & 0.58789 & 21.4252 & 0.41211 & 24 & 22 \\
\hline
\end{tabular}

Table D6.16: Kalman Filter for test set 8-3000

\begin{tabular}{|c|c|c|c|c|c|c|c|c|c|c|c|c|c|c|}
\hline time & trip & art3 & art2 & art1 & Average & $\Delta \mathbf{3}$ & $\Delta 2$ & $\Delta 1$ & Var[data]n & $g(k+1)$ & $\theta(k+1)$ & $a(k+1)$ & Predict & Observed \\
\hline $8: 33$ & 17 & 0 & 0 & 0 & 20 & 400 & 400 & 400 & 400 & 0.5 & 0 & 0.5 & & 2 \\
\hline $8: 36$ & 17 & 0 & 0 & 0 & 20 & 400 & 400 & 400 & 400 & 0.5 & 0 & 0.5 & & 2 \\
\hline $8: 39$ & 19 & 23 & 28 & 20 & 20 & 9 & 64 & 0 & 36.5 & 0.5 & 18.25 & 0.5 & 11 & 17 \\
\hline $8: 43$ & 20 & 27 & 28 & 22 & 25.6667 & 1.7778 & 5.4444 & 13.444 & 3.61111 & 0.85823 & 3.09918 & 0.14177 & 21.2912 & 17 \\
\hline $8: 59$ & 24 & 29 & 25 & 22 & 25.3333 & 13.444 & 0.1111 & 11.111 & 6.77778 & 0.59304 & 4.01951 & 0.40696 & 20 & 17 \\
\hline
\end{tabular}


Table D6.17: Kalman Filter for test set 49-1200

\begin{tabular}{|c|c|c|c|c|c|c|c|c|c|c|c|c|c|c|}
\hline time & trip & art3 & $\operatorname{art2}$ & art1 & Average & $\Delta \mathbf{3}$ & $\Delta 2$ & $\Delta 1$ & Var[data]n & $g(k+1)$ & $e(k+1)$ & $a(k+1)$ & Predict & Observed \\
\hline $8: 33$ & 17 & 0 & 0 & 0 & 20 & 400 & 400 & 400 & 400 & 0.5 & 0 & 0.5 & & 2 \\
\hline $8: 36$ & 17 & 0 & 0 & 0 & 20 & 400 & 400 & 400 & 400 & 0.5 & 0 & 0.5 & & 2 \\
\hline $8: 39$ & 19 & 20 & 17 & 13 & 20 & 0 & 9 & 49 & 4.5 & 0.5 & 2.25 & 0.5 & 7.5 & 13 \\
\hline $8: 43$ & 20 & 17 & 14 & 17 & 16 & 1 & 4 & 1 & 2.5 & 0.65517 & 1.63793 & 0.34483 & 15.6207 & 14 \\
\hline $8: 59$ & 24 & 25 & 22 & 17 & 21.3333 & 13.444 & 0.4444 & 18.778 & 6.94444 & 0.55275 & 3.83851 & 0.44725 & 16 & 11 \\
\hline
\end{tabular}

Table D6.18: Kalman Filter for test set 59-2200

\begin{tabular}{|c|c|c|c|c|c|c|c|c|c|c|c|c|c|c|}
\hline time & trip & art3 & art2 & art1 & Average & $\Delta 3$ & $\Delta 2$ & $\Delta 1$ & Var[data]n & $g(k+1)$ & $\theta(k+1)$ & $a(k+1)$ & Predict & Observed \\
\hline $8: 33$ & 17 & 0 & 0 & 0 & 20 & 400 & 400 & 400 & 400 & 0.5 & 0 & 0.5 & & 2 \\
\hline $8: 36$ & 17 & 0 & 0 & 0 & 20 & 400 & 400 & 400 & 400 & 0.5 & 0 & 0.5 & & 2 \\
\hline $8: 39$ & 19 & 17 & 13 & 17 & 20 & 9 & 49 & 9 & 29 & 0.5 & 14.5 & 0.5 & 9.5 & 25 \\
\hline $8: 43$ & 20 & 17 & 14 & 17 & 16 & 1 & 4 & 1 & 2.5 & 0.87179 & 2.17949 & 0.12821 & 18.0256 & 29 \\
\hline $8: 59$ & 24 & 17 & 11 & 17 & 15 & 4 & 16 & 4 & 10 & 0.54913 & 5.49133 & 0.45087 & 22 & 27 \\
\hline
\end{tabular}

Table D6.19: Kalman Filter for test set 24-1200

\begin{tabular}{|c|c|c|c|c|c|c|c|c|c|c|c|c|c|c|}
\hline time & trip & art3 & art2 & art1 & Average & $\Delta 3$ & $\Delta 2$ & $\Delta 1$ & Var[data]n & $g(k+1)$ & $e(k+1)$ & $a(k+1)$ & Predlct & Observed \\
\hline $8: 33$ & 17 & 0 & 0 & 0 & 20 & 400 & 400 & 400 & 400 & 0.5 & 0 & 0.5 & & 2 \\
\hline $8: 36$ & 17 & 0 & 0 & 0 & 20 & 400 & 400 & 400 & 400 & 0.5 & 0 & 0.5 & & 2 \\
\hline $8: 39$ & 19 & 11 & 22 & 11 & 20 & 81 & 4 & 81 & 42.5 & 0.5 & 21.25 & 0.5 & 6.5 & 26 \\
\hline $8: 43$ & 20 & 14 & 30 & 14 & 19.3333 & 28.444 & 113.78 & 28.444 & 71.1111 & 0.565 & 40.1775 & 0.435 & 19.2201 & 29 \\
\hline $8: 59$ & 24 & 12 & 20 & 12 & 14.6667 & 7.1111 & 28.444 & 7.1111 & 17.7778 & 0.76526 & 13.6046 & 0.23474 & 16 & 23 \\
\hline
\end{tabular}


Table D6.20: Kalman Filter for test set 25-1200

\begin{tabular}{|c|c|c|c|c|c|c|c|c|c|c|c|c|c|c|}
\hline time & trip & art3 & art2 & art1 & Average & $\Delta 3$ & $\Delta 2$ & $\Delta 1$ & Var[data]n & $g(k+1)$ & $\theta(k+1)$ & $a(k+1)$ & Predict & Observed \\
\hline $8: 33$ & 17 & 0 & 0 & 0 & 20 & 400 & 400 & 400 & 400 & 0.5 & 0 & 0.5 & & 2 \\
\hline $8: 36$ & 17 & 0 & 0 & 0 & 20 & 400 & 400 & 400 & 400 & 0.5 & 0 & 0.5 & & 2 \\
\hline $8: 39$ & 19 & 22 & 11 & 26 & 20 & 4 & 81 & 36 & 42.5 & 0.5 & 21.25 & 0.5 & 14 & 25 \\
\hline $8: 43$ & 20 & 30 & 14 & 29 & 24.3333 & 32.111 & 106.78 & 21.778 & 69.4444 & 0.56635 & 39.3298 & 0.43365 & 27.2654 & 29 \\
\hline $8: 59$ & 24 & 20 & 12 & 23 & 18.3333 & 2.7778 & 40.111 & 21.778 & 21.4444 & 0.73918 & 15.8513 & 0.26082 & 25 & 26 \\
\hline
\end{tabular}

Table D6.21: Kalman Filter for test set 20-1200

\begin{tabular}{|c|c|c|c|c|c|c|c|c|c|c|c|c|c|c|}
\hline time & trip & art3 & art2 & art1 & Average & $\Delta 3$ & $\Delta 2$ & $\Delta 1$ & Var[data]n & $g(k+1)$ & $e(k+1)$ & $a(k+1)$ & Predict & Observed \\
\hline $8: 33$ & 17 & 0 & 0 & 0 & 20 & 400 & 400 & 400 & 400 & 0.5 & 0 & 0.5 & & 2 \\
\hline $8: 36$ & 17 & 0 & 0 & 0 & 20 & 400 & 400 & 400 & 400 & 0.5 & 0 & 0.5 & & 2 \\
\hline $8: 39$ & 19 & 21 & 13 & 23 & 20 & 1 & 49 & 9 & 25 & 0.5 & 12.5 & 0.5 & 12.5 & 23 \\
\hline $8: 43$ & 20 & 23 & 14 & 23 & 20 & 9 & 36 & 9 & 22.5 & 0.6087 & 13.6957 & 0.3913 & 23 & 26 \\
\hline $8: 59$ & 24 & 20 & 11 & 25 & 18.6667 & 1.7778 & 58.778 & 40.111 & 30.2778 & 0.59223 & 17.9313 & 0.40777 & 25 & 26 \\
\hline
\end{tabular}


Table D6.22: Prediction Error of the 3 Predictors for Alighting Passenger Prediction

\begin{tabular}{|r|r|c|c|c|c|c|c|c|}
\hline Dcode & Scode & Actual & $\begin{array}{c}\text { Developed } \\
\text { Model }\end{array}$ & $\begin{array}{c}\text { KF. } \\
\text { Model }\end{array}$ & $\begin{array}{c}\text { Naive } \\
\text { Model }\end{array}$ & $\mathbf{d 1}$ & $\mathbf{d 2}$ & d3 \\
\hline 54 & 2200 & 23 & 25 & 18 & 30 & 0.087 & 0.217 & 0.304348 \\
\hline 47 & 1200 & 11 & 12 & 20 & 13 & 0.091 & 0.818 & 0.181818 \\
\hline 48 & 1200 & 14 & 14 & 20 & 16 & 0 & 0.429 & 0.142857 \\
\hline 36 & 1200 & 13 & 12 & 14 & 12 & 0.077 & 0.077 & 0.076923 \\
\hline 16 & 3000 & 17 & 17 & 15 & 16 & 0 & 0.118 & 0.058824 \\
\hline 21 & 1200 & 22 & 24 & 17 & 35 & 0.091 & 0.227 & 0.590909 \\
\hline 19 & 1200 & 23 & 24 & 23 & 23 & 0.043 & 0 & 0 \\
\hline 45 & 1200 & 14 & 12 & 17 & 16 & 0.143 & 0.214 & 0.142857 \\
\hline 39 & 1200 & 13 & 12 & 14 & 12 & 0.077 & 0.077 & 0.076923 \\
\hline 38 & 1200 & 12 & 12 & 21 & 10 & 0 & 0.75 & 0.166667 \\
\hline 64 & 2200 & 22 & 23 & 18 & 21 & 0.045 & 0.182 & 0.045455 \\
\hline 29 & 1200 & 25 & 23 & 22 & 28 & 0.08 & 0.12 & 0.12 \\
\hline 6 & 3000 & 17 & 17 & 24 & 12 & 0 & 0.412 & 0.294118 \\
\hline 56 & 2200 & 22 & 23 & 24 & 20 & 0.045 & 0.091 & 0.090909 \\
\hline 8 & 3000 & 17 & 17 & 20 & 17 & 0 & 0.176 & 0 \\
\hline 49 & 1200 & 11 & 12 & 16 & 14 & 0.091 & 0.455 & 0.272727 \\
\hline 59 & 2200 & 27 & 24 & 22 & 29 & 0.111 & 0.185 & 0.074074 \\
\hline 24 & 1200 & 23 & 25 & 16 & 25 & 0.087 & 0.304 & 0.086957 \\
\hline 25 & 1200 & 26 & 25 & 25 & 23 & 0.038 & 0.038 & 0.115385 \\
\hline 20 & 1200 & 26 & 25 & 25 & 28 & 0.038 & 0.038 & 0.076923 \\
\hline
\end{tabular}




\section{Appendix D7}

\section{General}

This appendix includes the databases for the two bus routes and the prediction results of the developed model and the reference predictors.

\section{Database for Route 1 and Route 95}

The databases were retrieved from the APC-AVL system embedded on the buses running on the two bus routes.

Table D7.1 Database for route 1 (seconds)

\begin{tabular}{|c|c|c|c|c|}
\hline RCODE & $8: 59$ trip & $8: 47$ trip & 8437 trip & $8: 27$ trip \\
\hline 1 & 877 & 992 & 940 & 818 \\
\hline 2 & 762 & 914 & 900 & 713 \\
\hline 3 & 929 & 903 & 1003 & 789 \\
\hline 4 & 945 & 911 & 1080 & 922 \\
\hline 5 & 810 & 800 & 923 & 850 \\
\hline 6 & 1029 & 917 & 900 & 880 \\
\hline 7 & 1030 & 950 & 946 & 915 \\
\hline 8 & 914 & 968 & 1016 & 726 \\
\hline 9 & 948 & 1074 & 890 & 817 \\
\hline 10 & 934 & 901 & 873 & 814 \\
\hline 11 & 900 & 997 & 956 & 794 \\
\hline 12 & 986 & 1013 & 960 & 846 \\
\hline 13 & 863 & 1062 & 1003 & 951 \\
\hline 14 & 1122 & 846 & 920 & 855 \\
\hline 15 & 905 & 958 & 954 & 933 \\
\hline 16 & 928 & 961 & 932 & 814 \\
\hline 17 & 1017 & 844 & 959 & 858 \\
\hline 18 & 923 & 869 & 1083 & 809 \\
\hline 19 & 909 & 1040 & 919 & 893 \\
\hline 20 & 840 & 926 & 862 & 842 \\
\hline 21 & 945 & 975 & 1001 & 887 \\
\hline 22 & 793 & 947 & 896 & 895 \\
\hline 23 & 984 & 879 & 968 & 852 \\
\hline
\end{tabular}




\begin{tabular}{|c|c|c|c|c|}
\hline 24 & 1070 & 886 & 917 & 912 \\
\hline 25 & 853 & 980 & 1006 & 1000 \\
\hline 26 & 998 & 850 & 1008 & 889 \\
\hline 27 & 1035 & 1012 & 950 & 856 \\
\hline 28 & 768 & 992 & 807 & 913 \\
\hline 29 & 783 & 862 & 975 & 861 \\
\hline 30 & 971 & 914 & 1088 & 789 \\
\hline 31 & 880 & 827 & 1043 & 887 \\
\hline 32 & 982 & 732 & 844 & 857 \\
\hline 33 & 994 & 1031 & 959 & 840 \\
\hline 34 & 984 & 890 & 913 & 936 \\
\hline 35 & 1038 & 969 & 887 & 972 \\
\hline 36 & 980 & 960 & 870 & 825 \\
\hline 37 & 1029 & 940 & 987 & 863 \\
\hline 38 & 805 & 844 & 932 & 807 \\
\hline 39 & 916 & 850 & 970 & 899 \\
\hline 40 & 903 & 903 & 937 & 711 \\
\hline 41 & 767 & 827 & 930 & 918 \\
\hline 42 & 946 & 840 & 994 & 871 \\
\hline 43 & 819 & 1076 & 1021 & 873 \\
\hline 44 & 1050 & 944 & 1127 & 932 \\
\hline 45 & 842 & 824 & 861 & 840 \\
\hline 46 & 967 & 935 & 887 & 849 \\
\hline 47 & 938 & 833 & 1045 & 779 \\
\hline 48 & 831 & 812 & 935 & 754 \\
\hline 49 & 714 & 914 & 859 & 814 \\
\hline 50 & 912 & 1028 & 989 & 874 \\
\hline 51 & 823 & 950 & 1085 & 824 \\
\hline 52 & 975 & 1000 & 1020 & 775 \\
\hline 53 & 965 & 829 & 1116 & 923 \\
\hline 54 & 1076 & 895 & 1004 & 813 \\
\hline 55 & 973 & 914 & 1108 & 856 \\
\hline 56 & 857 & 825 & 939 & 857 \\
\hline 57 & 953 & 815 & 878 & 842 \\
\hline 58 & 823 & 1026 & 949 & 882 \\
\hline 59 & 916 & 939 & 1057 & 841 \\
\hline 60 & 913 & 878 & 880 & 754 \\
\hline 61 & 918 & 1014 & 1014 & 793 \\
\hline 62 & 888 & 960 & 919 & 872 \\
\hline 63 & 1020 & 846 & 1008 & 779 \\
\hline 64 & 742 & 1064 & 881 & 834 \\
\hline 65 & 958 & 966 & 972 & 799 \\
\hline 66 & 1001 & 1077 & 881 & 784 \\
\hline 67 & 986 & 1045 & 927 & 794 \\
\hline 68 & 972 & 874 & 1001 & 832 \\
\hline 69 & 921 & 817 & 941 & 868 \\
\hline 70 & 981 & 932 & 1060 & 850 \\
\hline
\end{tabular}


Table D7.2: Database for route 95

\begin{tabular}{|c|c|c|c|c|c|c|c|c|}
\hline $8: 59$ & $8: 43$ & $8: 40$ & $8: 37$ & B1 & B2 & A1 & A2 & A3 \\
\hline 632 & 611 & 702 & 618 & 6 & 11 & 11 & 13 & 11 \\
\hline 639 & 699 & 594 & 529 & 3 & 22 & 10 & 11 & 12 \\
\hline 571 & 333 & 631 & 553 & 9 & 9 & 9 & 10 & 11 \\
\hline 653 & 465 & 730 & 622 & 6 & 9 & 8 & 7 & 9 \\
\hline 487 & 557 & 563 & 720 & 4 & 16 & 14 & 11 & 10 \\
\hline 667 & 592 & 637 & 567 & 8 & 7 & 11 & 12 & 9 \\
\hline 608 & 484 & 606 & 766 & 8 & 2 & 10 & 10 & 11 \\
\hline 521 & 569 & 528 & 547 & 1 & 3 & 11 & 14 & 8 \\
\hline 542 & 417 & 411 & 687 & 4 & 12 & 10 & 11 & 11 \\
\hline 619 & 520 & 422 & 499 & 6 & 3 & 10 & 12 & 9 \\
\hline 620 & 509 & 562 & 541 & 10 & 17 & 9 & 12 & 11 \\
\hline 554 & 635 & 544 & 579 & 5 & 9 & 12 & 13 & 10 \\
\hline 584 & 486 & 558 & 646 & 7 & 8 & 10 & 14 & 10 \\
\hline 684 & 449 & 488 & 804 & 9 & 10 & 9 & 12 & 11 \\
\hline 583 & 450 & 602 & 635 & 3 & 6 & 12 & 14 & 11 \\
\hline 610 & 567 & 601 & 585 & 1 & 3 & 17 & 23 & 15 \\
\hline 651 & 465 & 536 & 674 & 13 & 12 & 19 & 18 & 15 \\
\hline 656 & 700 & 638 & 510 & 6 & 16 & 15 & 22 & 15 \\
\hline 616 & 597 & 473 & 615 & 11 & 18 & 17 & 17 & 15 \\
\hline 521 & 571 & 513 & 668 & 13 & 9 & 15 & 22 & 17 \\
\hline 519 & 524 & 569 & 596 & 8 & 13 & 18 & 17 & 15 \\
\hline 631 & 571 & 497 & 609 & 9 & 8 & 17 & 17 & 13 \\
\hline 568 & 674 & 671 & 642 & 5 & 16 & 15 & 19 & 17 \\
\hline 639 & 316 & 689 & 665 & 5 & 7 & 17 & 19 & 17 \\
\hline 533 & 669 & 602 & 786 & 2 & 8 & 16 & 19 & 17 \\
\hline 633 & 502 & 489 & 816 & 10 & 13 & 13 & 17 & 15 \\
\hline 642 & 540 & 632 & 692 & 10 & 8 & 15 & 21 & 17 \\
\hline 629 & 628 & 410 & 494 & 11 & 8 & 17 & 19 & 13 \\
\hline 553 & 598 & 549 & 660 & 4 & 1 & 14 & 18 & 15 \\
\hline 589 & 378 & 807 & 570 & 6 & 4 & 17 & 19 & 19 \\
\hline 632 & 389 & 616 & 524 & 8 & 11 & 13 & 15 & 15 \\
\hline 522 & 597 & 507 & 692 & 3 & 4 & 17 & 19 & 14 \\
\hline 570 & 448 & 647 & 671 & 6 & 4 & 18 & 17 & 18 \\
\hline 550 & 376 & 567 & 606 & 7 & 8 & 17 & 18 & 15 \\
\hline 662 & 503 & 658 & 588 & 7 & 4 & 9 & 9 & 7 \\
\hline 701 & 500 & 561 & 540 & 5 & 3 & 10 & 11 & 9 \\
\hline 646 & 423 & 679 & 448 & 11 & 13 & 8 & 9 & 8 \\
\hline 568 & 428 & 656 & 468 & 9 & 1 & 9 & 9 & 7 \\
\hline 620 & 568 & 601 & 512 & 4 & 5 & 8 & 8 & 9 \\
\hline 596 & 348 & 566 & 585 & 9 & 10 & 9 & 11 & 6 \\
\hline 562 & 671 & 604 & 642 & 6 & 5 & 8 & 9 & 9 \\
\hline 627 & 606 & 573 & 647 & 3 & 7 & 8 & 9 & 7 \\
\hline 614 & 512 & 581 & 506 & 12 & 11 & 7 & 9 & 9 \\
\hline 547 & 537 & 634 & 692 & 6 & 8 & 9 & 11 & 8 \\
\hline
\end{tabular}




\begin{tabular}{|c|c|c|c|c|c|c|c|c|}
\hline 571 & 449 & 531 & 666 & 8 & 25 & 8 & 11 & 8 \\
\hline 593 & 435 & 531 & 764 & 1 & 2 & 7 & 9 & 9 \\
\hline 643 & 577 & 586 & 576 & 6 & 14 & 9 & 11 & 9 \\
\hline 541 & 630 & 536 & 624 & 12 & 12 & 7 & 9 & 9 \\
\hline 576 & 667 & 483 & 574 & 8 & 7 & 8 & 9 & 7 \\
\hline 582 & 527 & 646 & 694 & 4 & 3 & 17 & 19 & 15 \\
\hline 661 & 601 & 568 & 729 & 8 & 7 & 19 & 19 & 15 \\
\hline 703 & 735 & 478 & 673 & 5 & 7 & 19 & 14 & 17 \\
\hline 685 & 766 & 559 & 891 & 3 & 6 & 15 & 18 & 15 \\
\hline 657 & 502 & 576 & 609 & 14 & 4 & 15 & 21 & 13 \\
\hline 626 & 763 & 425 & 666 & 8 & 19 & 15 & 15 & 13 \\
\hline 612 & 523 & 492 & 436 & 7 & 4 & 16 & 16 & 13 \\
\hline 527 & 469 & 461 & 572 & 7 & 8 & 14 & 17 & 15 \\
\hline 585 & 596 & 663 & 443 & 12 & 9 & 18 & 19 & 17 \\
\hline 607 & 534 & 576 & 614 & 9 & 13 & 17 & 15 & 15 \\
\hline 620 & 656 & 663 & 498 & 11 & 11 & 13 & 20 & 15 \\
\hline 554 & 605 & 625 & 560 & 3 & 9 & 14 & 15 & 18 \\
\hline 572 & 384 & 619 & 538 & 6 & 3 & 17 & 17 & 15 \\
\hline 638 & 236 & 539 & 610 & 8 & 14 & 15 & 14 & 15 \\
\hline 544 & 593 & 606 & 716 & 9 & 18 & 13 & 15 & 14 \\
\hline 566 & 438 & 494 & 774 & 8 & 3 & 14 & 6 & 5 \\
\hline 623 & 630 & 536 & 666 & 12 & 4 & 16 & 22 & 19 \\
\hline 691 & 698 & 540 & 571 & 6 & 6 & 18 & 26 & 21 \\
\hline 551 & 441 & 600 & 642 & 16 & 8 & 21 & 23 & 17 \\
\hline 642 & 640 & 462 & 551 & 3 & 1 & 18 & 23 & 18 \\
\hline 565 & 555 & 490 & 534 & 17 & 3 & 21 & 23 & 23 \\
\hline 591 & 481 & 600 & 490 & 5 & 7 & 17 & 18 & 19 \\
\hline 576 & 697 & 688 & 442 & 6 & 2 & 21 & 23 & 18 \\
\hline 676 & 488 & 611 & 618 & 6 & 12 & 23 & 22 & 23 \\
\hline 654 & 662 & 556 & 689 & 2 & 7 & 21 & 23 & 18 \\
\hline 494 & 698 & 583 & 645 & 4 & 8 & 11 & 12 & 8 \\
\hline 589 & 330 & 592 & 624 & 8 & 18 & 13 & 13 & 11 \\
\hline 638 & 834 & 721 & 627 & 9 & 2 & 10 & 12 & 10 \\
\hline 524 & 675 & 581 & 571 & 13 & 18 & 11 & 12 & 9 \\
\hline 597 & 644 & 651 & 806 & 7 & 14 & 10 & 10 & 11 \\
\hline 585 & 463 & 581 & 604 & 8 & 6 & 11 & 14 & 8 \\
\hline 635 & 514 & 531 & 489 & 6 & 9 & 10 & 11 & 11 \\
\hline 561 & 402 & 608 & 596 & 15 & 17 & 10 & 12 & 9 \\
\hline 612 & 413 & 659 & 570 & 4 & 9 & 9 & 12 & 11 \\
\hline 580 & 450 & 471 & 613 & 6 & 10 & 12 & 13 & 10 \\
\hline 532 & 598 & 511 & 587 & 9 & 10 & 10 & 14 & 10 \\
\hline 587 & 724 & 573 & 544 & 5 & 6 & 9 & 12 & 11 \\
\hline 583 & 674 & 618 & 458 & 10 & 16 & 12 & 14 & 11 \\
\hline 592 & 490 & 522 & 582 & 9 & 14 & 9 & 12 & 11 \\
\hline 592 & 322 & 541 & 476 & 10 & 19 & 10 & 12 & 9 \\
\hline 522 & 563 & 629 & 616 & 7 & 4 & 22 & 24 & 18 \\
\hline 667 & 466 & 494 & 712 & 12 & 11 & 23 & 23 & 19 \\
\hline
\end{tabular}




\begin{tabular}{|l|l|l|l|l|l|l|l|l|}
\hline 680 & 361 & 658 & 852 & 4 & 12 & 23 & 18 & 22 \\
\hline 601 & 584 & 646 & 675 & 9 & 9 & 19 & 23 & 19 \\
\hline 568 & 597 & 542 & 664 & 14 & 16 & 19 & 26 & 17 \\
\hline 620 & 625 & 632 & 726 & 8 & 11 & 18 & 18 & 17 \\
\hline 586 & 513 & 675 & 666 & 7 & 6 & 20 & 20 & 16 \\
\hline 612 & 498 & 608 & 749 & 1 & 4 & 18 & 22 & 19 \\
\hline 513 & 519 & 645 & 607 & 10 & 6 & 23 & 24 & 21 \\
\hline 699 & 522 & 595 & 637 & 8 & 9 & 21 & 19 & 19 \\
\hline 626 & 561 & 587 & 562 & 2 & 3 & 17 & 25 & 18 \\
\hline
\end{tabular}

For each trip, APC data of the whole year of observations were processed so that the mean and standard deviation can be used. The following tables provided by the OC Transpo are shown for the sake of illustration.

Table D7.3 OC Transpo data for bus trips to Gladstone during 8:30-9:00 AM

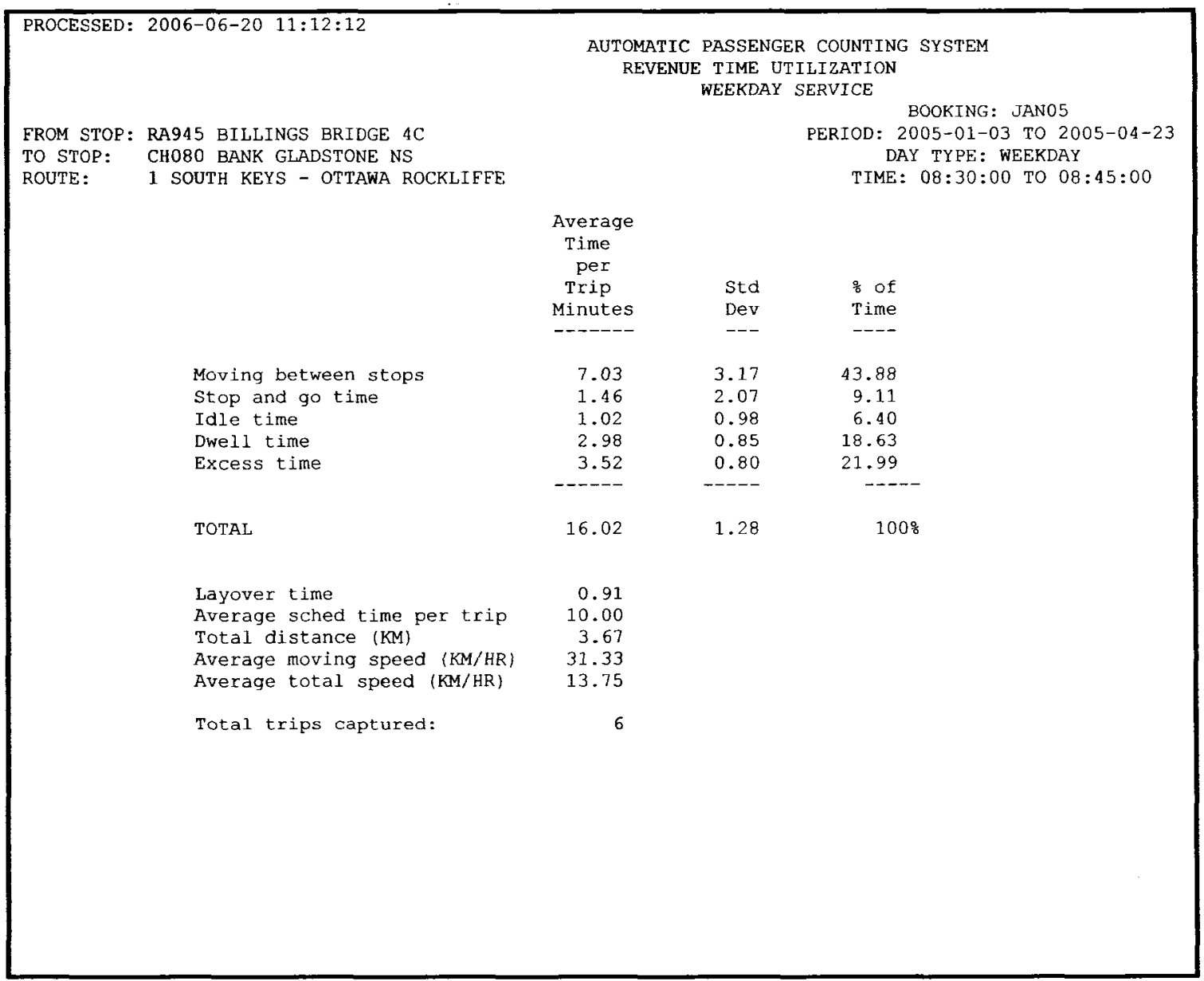


PROCESSED: 2006-06-20 11:24:24

FROM STOP: RA945 BILLINGS BRIDGE 4C

TO STOP: CH080 BANK GLADSTONE NS

ROUTE: 1 Bank - - Rockcliffe
AUTOMATIC PASSENGER COUNTING SYSTEM

REVENUE TIME UTILIZATION

WEEKDAY SERVICE

BOOKING: SEP05

PERIOD: 2005-09-05 TO 2005-12-17

DAY TYPE: WEEKDAY

TIME: $08: 30: 00$ TO $08: 45: 00$

\begin{tabular}{|c|c|c|}
\hline $\begin{array}{c}\text { Average } \\
\text { Time } \\
\text { per }\end{array}$ & & \\
\hline Trip & Std & $\frac{a}{8}$ of \\
\hline Minutes & Dev & Time \\
\hline
\end{tabular}

Moving between stops

Stop and go time

Idle time

Dwell time

Excess time

TOTAL

-------

1.02

65.78

10.52

0.22

2.45

2.80

0.00

0.43

0.79

0.69

0.00

1.36

15.35

17.51

15.99

1.66

$100 \%$

\section{Layover time}

Average sched time per trip

Total distance (KM)

Average moving speed (KM/HR)

Average total speed (KM/HR)

Total trips captured:

PROCESSED: 2006-06-20 $11: 12: 32$

FROM STOP: RA945 BILIINGS BRIDGE 4C

TO STOP: CHO8O BANK GLADSTONE NS

ROUTE: 1 SOUTH KEYS - OTTAWA ROCKLIFFE

$$
0.00
$$

10.00

3.67

20.94

13.77

4

AUTOMATIC PASSENGER COUNTING SYSTEM REVENUE TIME UTILIZATION WEEKDAY SERVICE

BOOKING: JAN05

2005-01-03 TO 2005-04-23

DAY TYPE: WEEKDAY

TIME : 08:45:00 TO 09:00:00

Average

Time

per

Trip

Minutes

Std $\quad$ s of

Moving between stops

Stop and go time

Idle time

Dwell time

Excess time

TOTAL

10.21

0.29

0.45

2.06

2.39

66.28

$1.21 \quad 1.91$

$0.60 \quad 2.94$

$0.54 \quad 13.37$

$\begin{array}{ll}0.54 & 13.37 \\ 0.86 & 15.49\end{array}$

15.41

(1)

1.56

1008

Layover time

1.56

7.20

10.00

10.00
3.67

21.56

14.29

17 


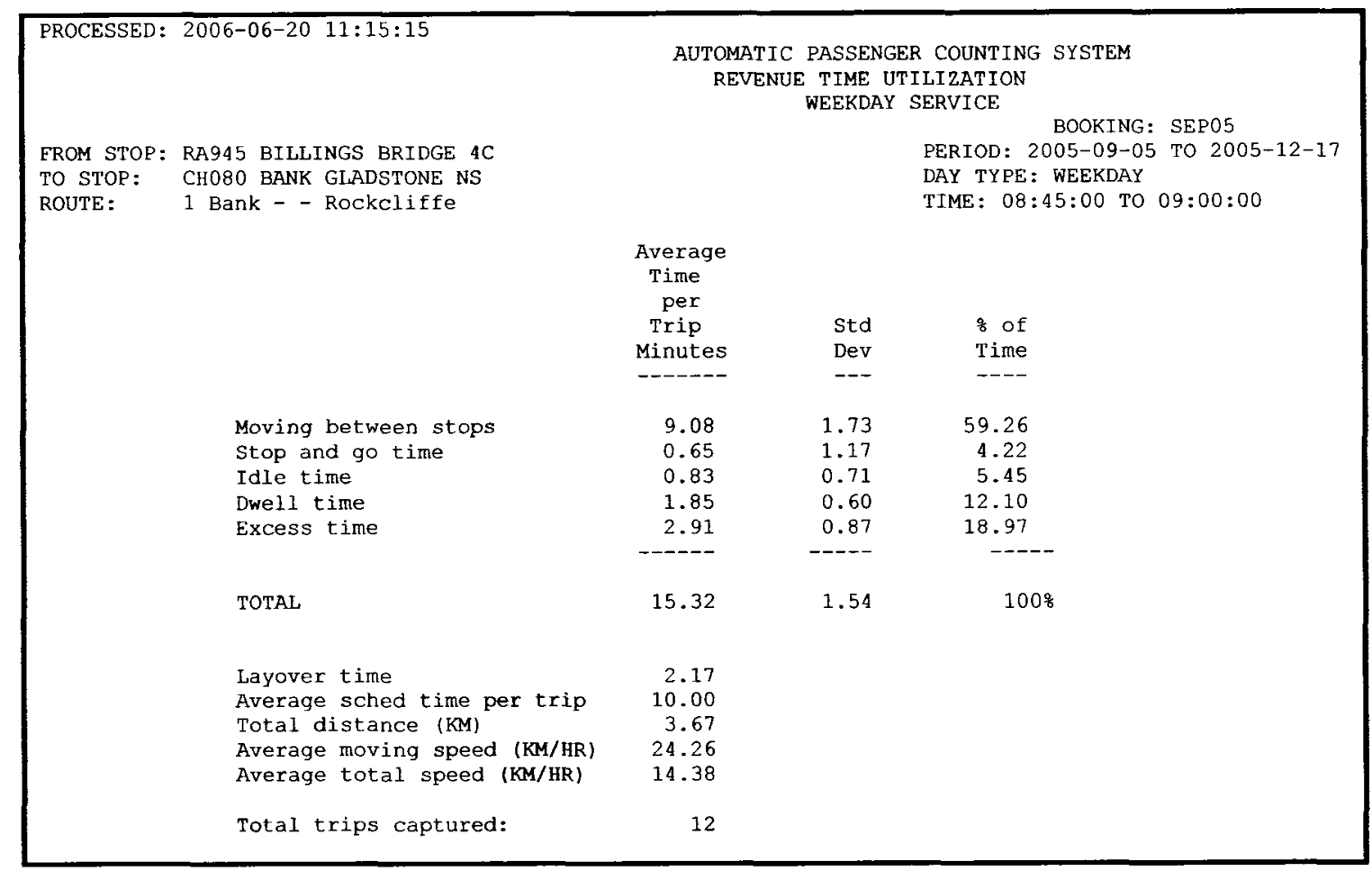

Table D7.4 OC Transpo data for bus trips to Mackenzie during 8:00 -10:00 AM

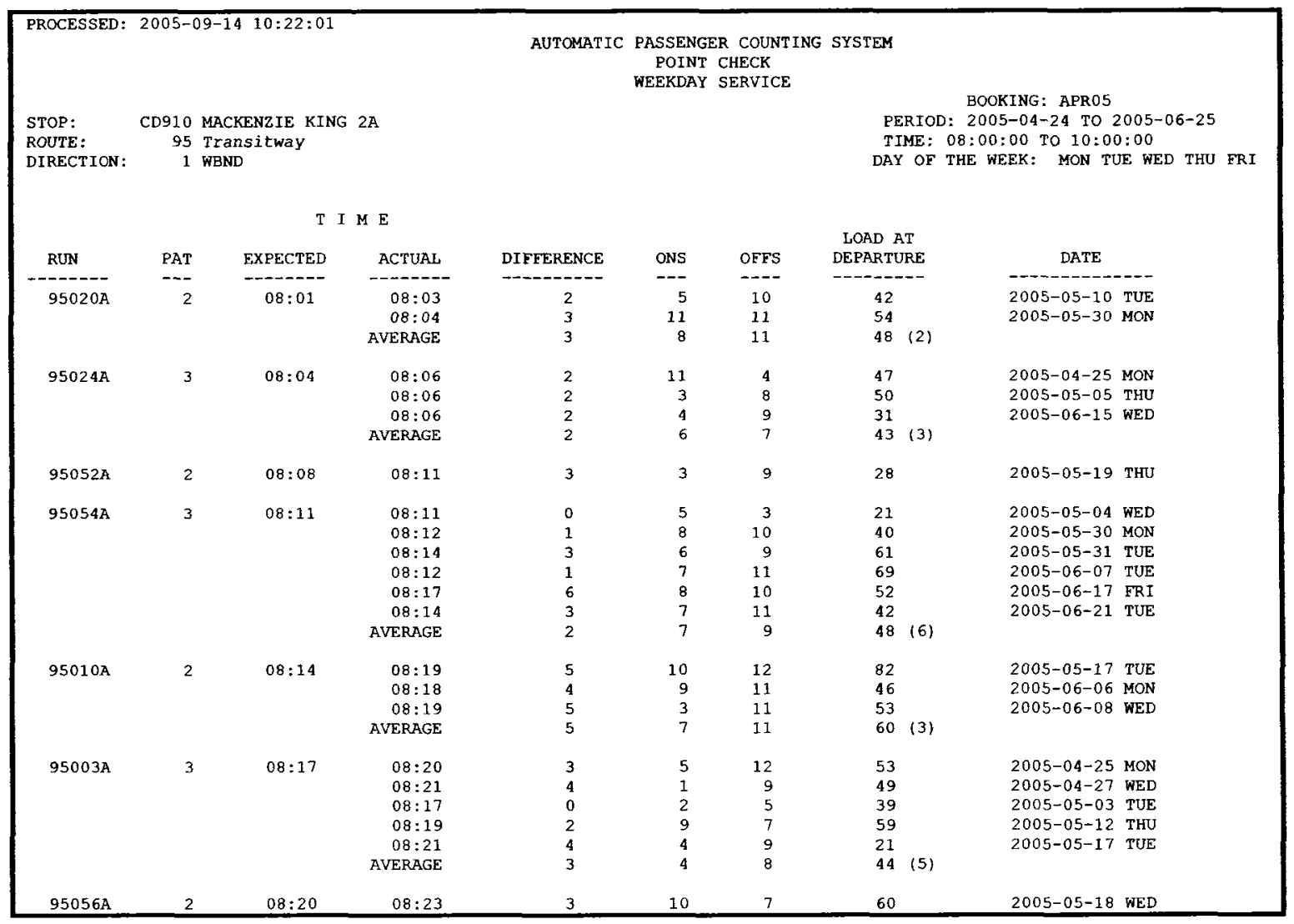




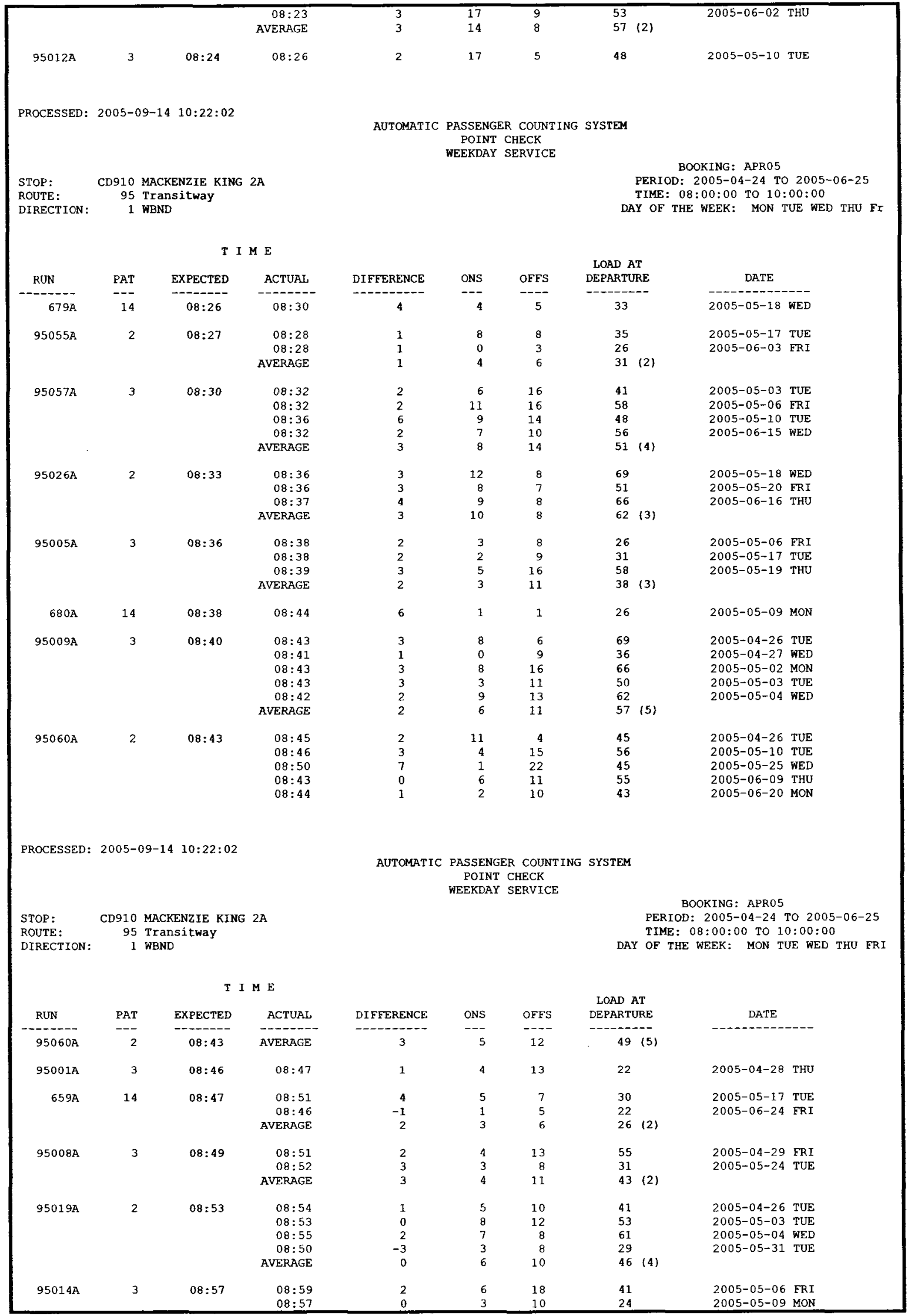




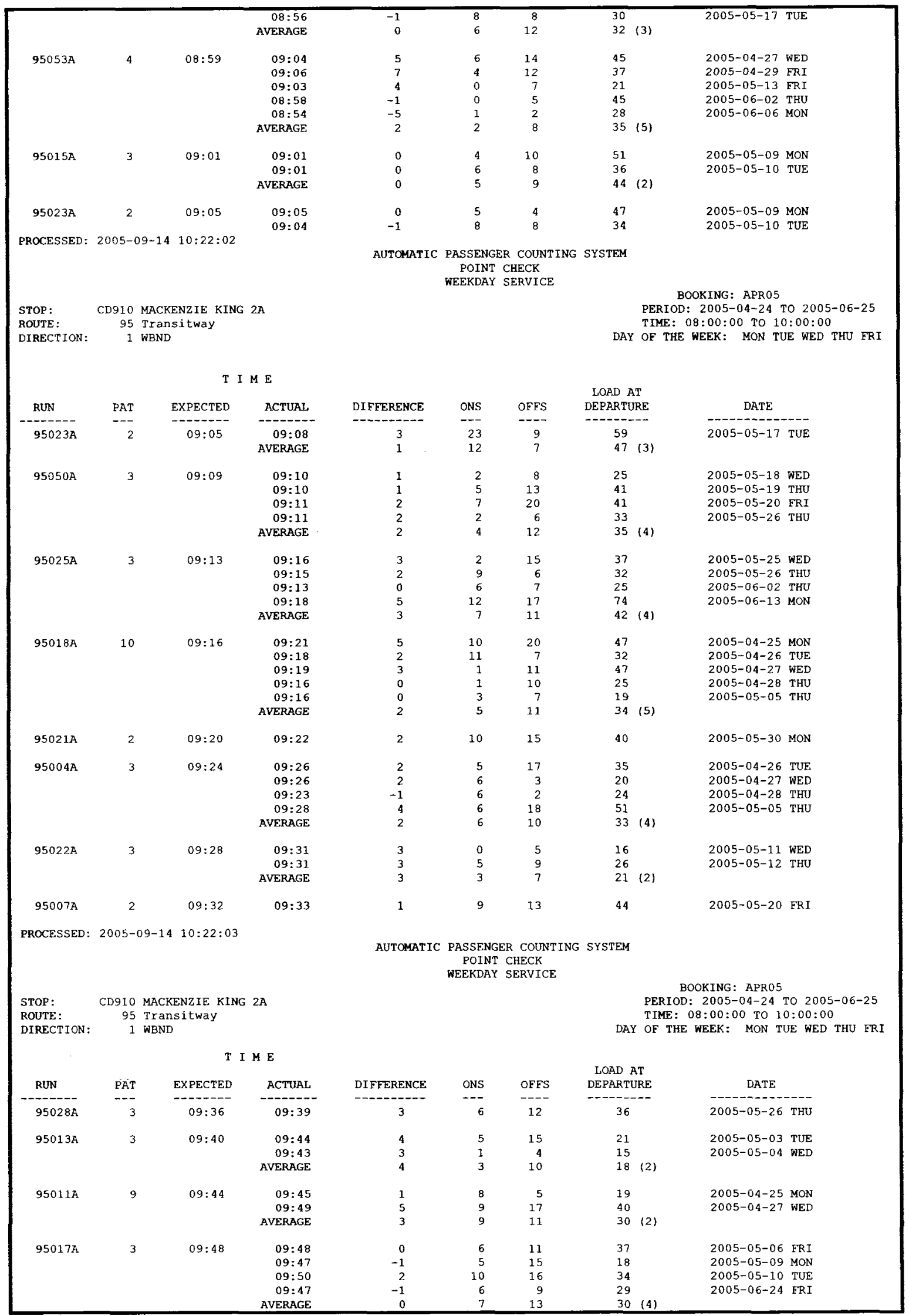




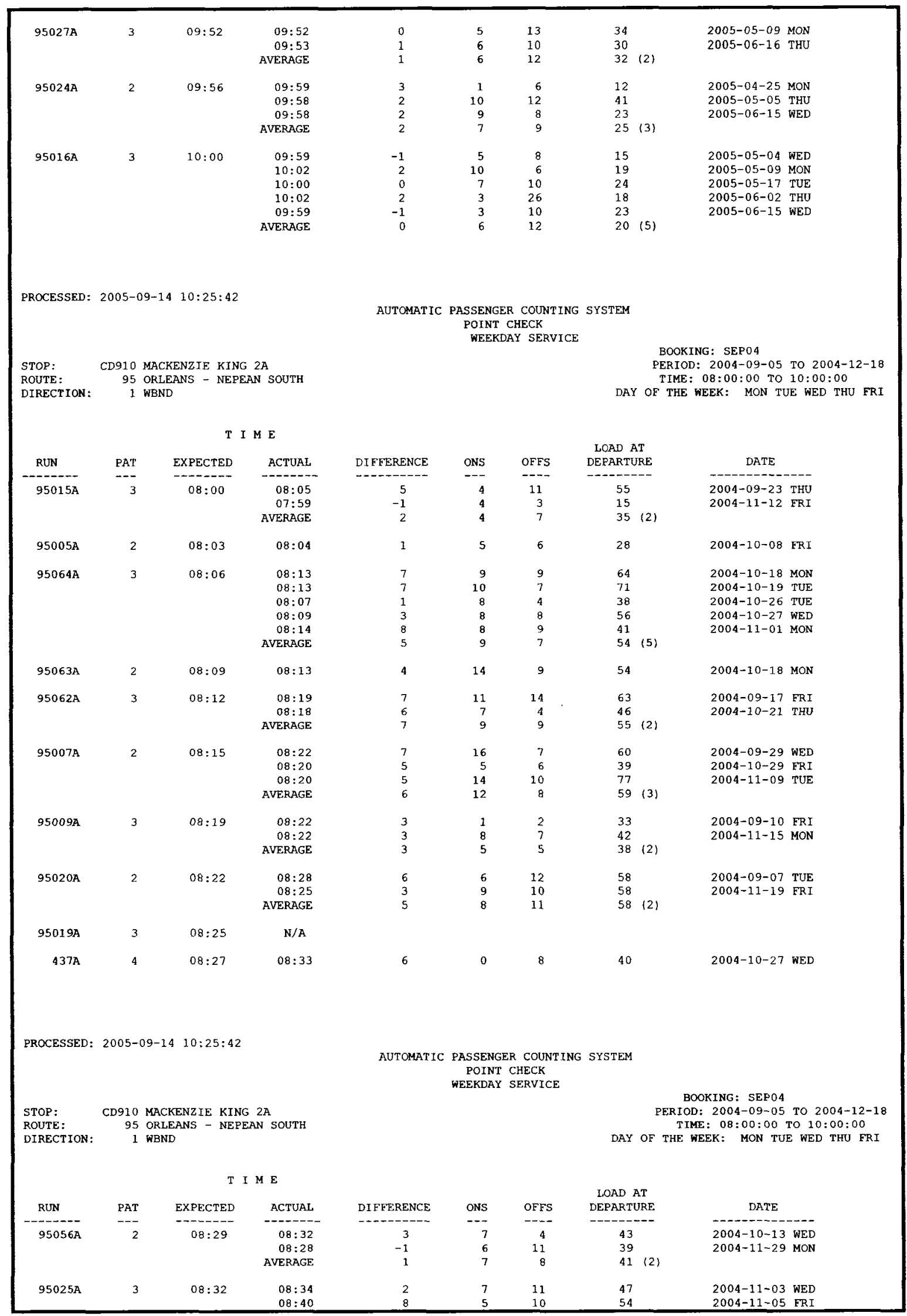




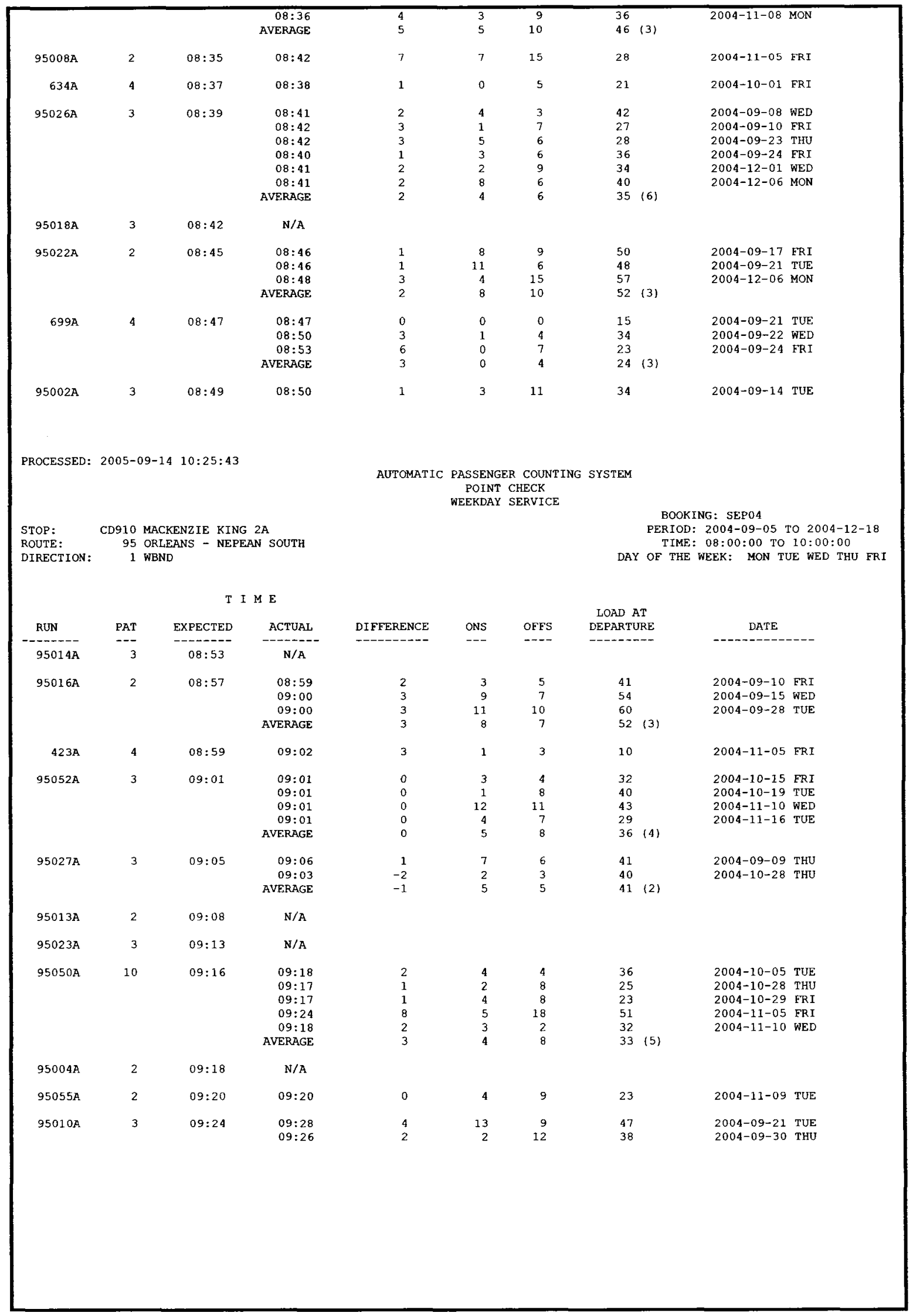




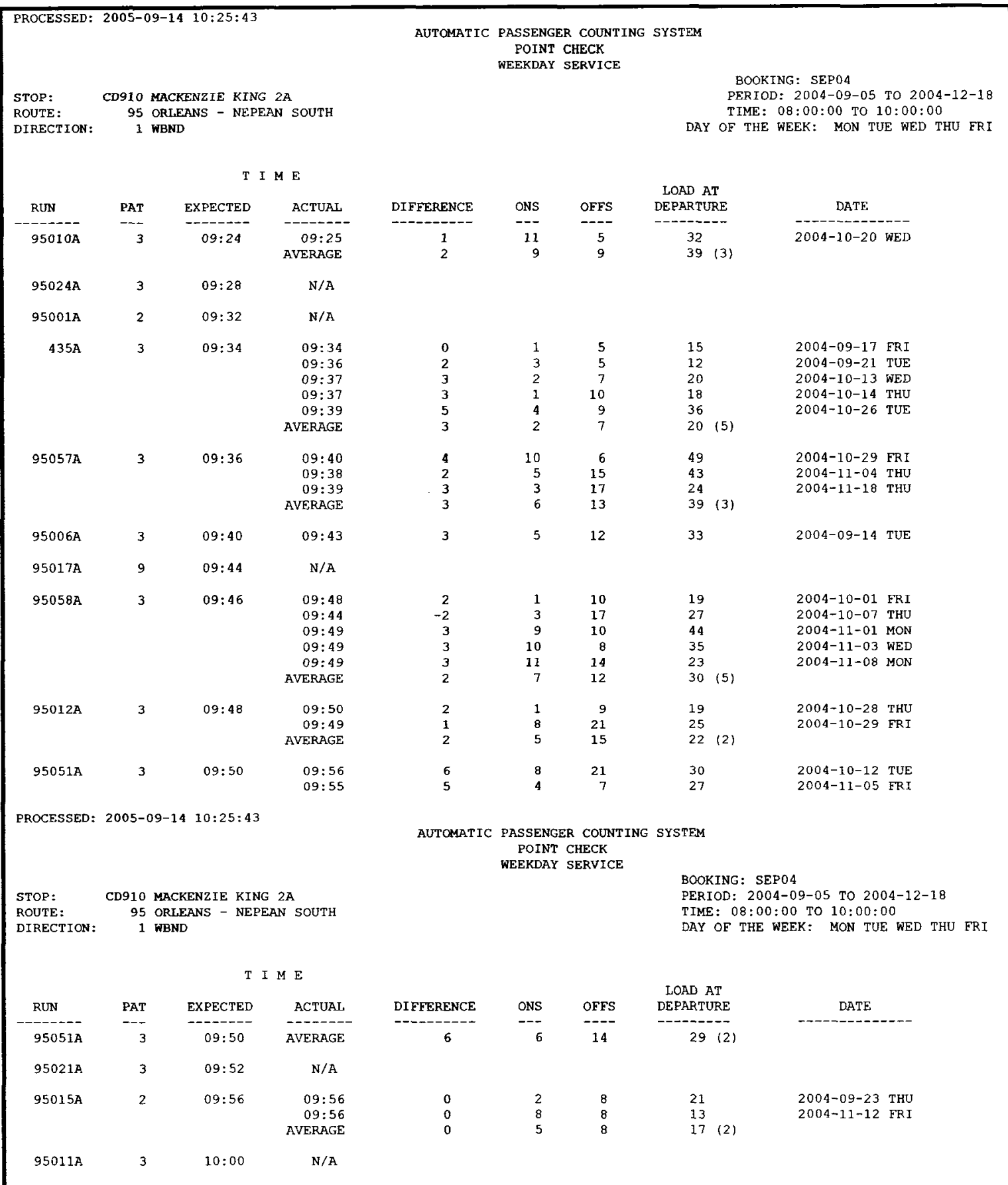




\section{Running Time Prediction Results of the Developed Model :Route 1}

The running time predictions obtained from the developed model are tabulated in Table D7.19 of this appendix. For illustration, the following table depicts the output of the model applied for test case $\mathrm{RCODE}=18$.

Table D7.5: An Example of Developed Model's Output

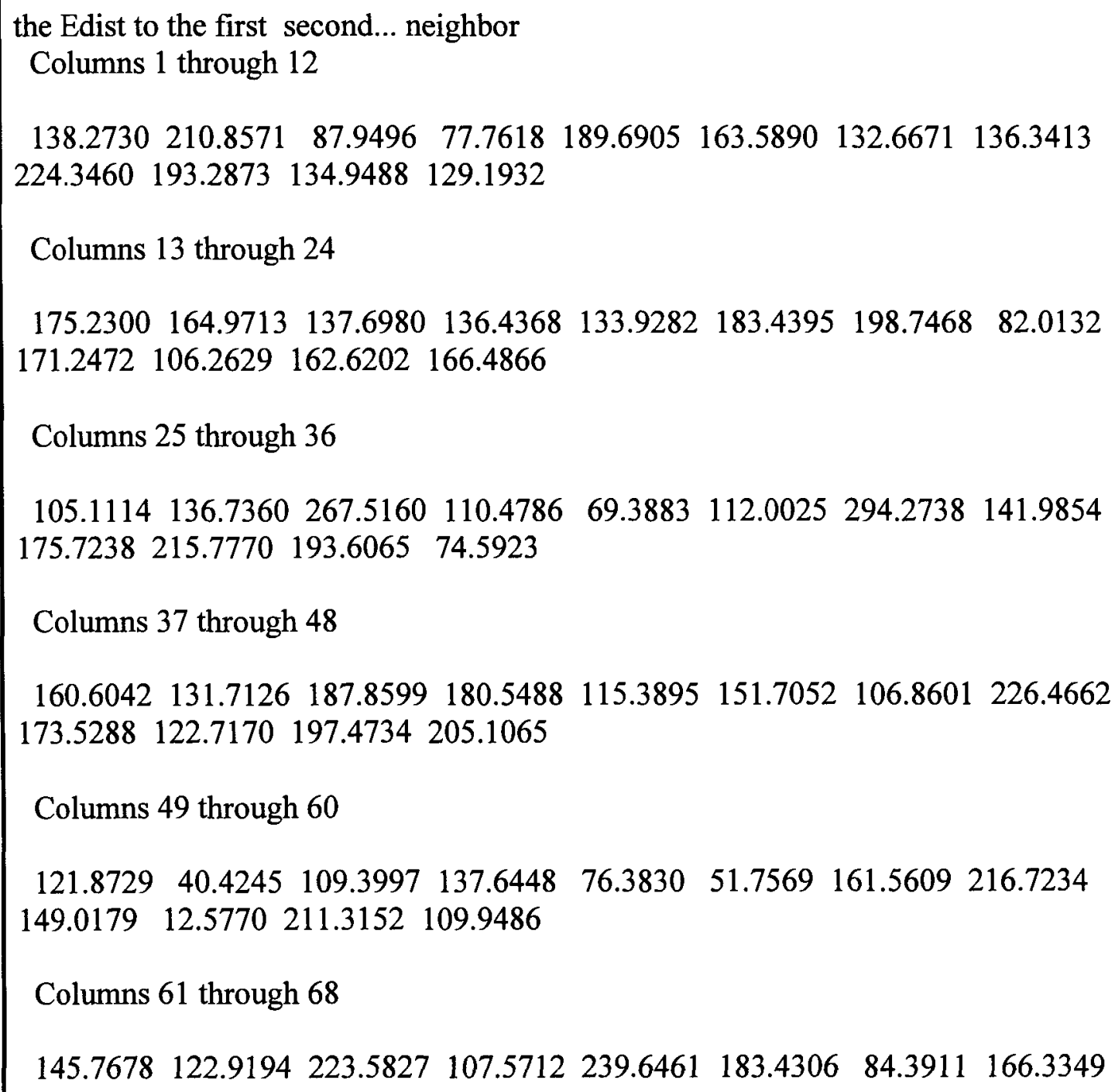




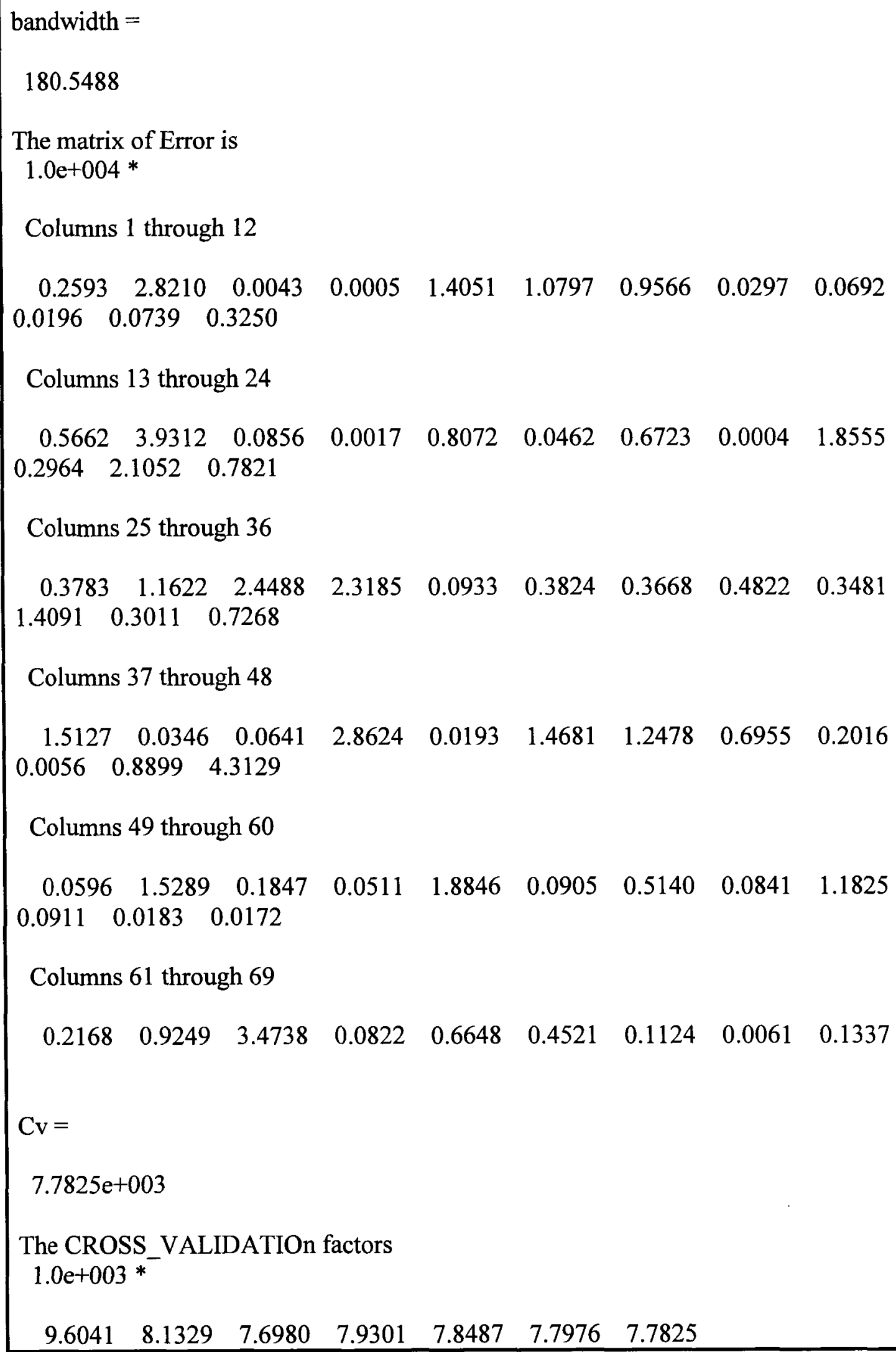


OpNei $=$

21

Beta $=$

$1.0 \mathrm{e}+003^{*}$

1.3238

$-0.0000$

$-0.0002$

$-0.0002$

THE PREDCTED BUS RUNNING TIME IS 948.96 SECONDS.Program complete ans $=$

948.9596

Note: Only the most important part of the output is printed out. 


\section{Running Time Prediction Results of the KF Predictor: Route 1}

The predictions of Kalman Filter predictors are tabulated in Tables D7.6-D7.20. 
in
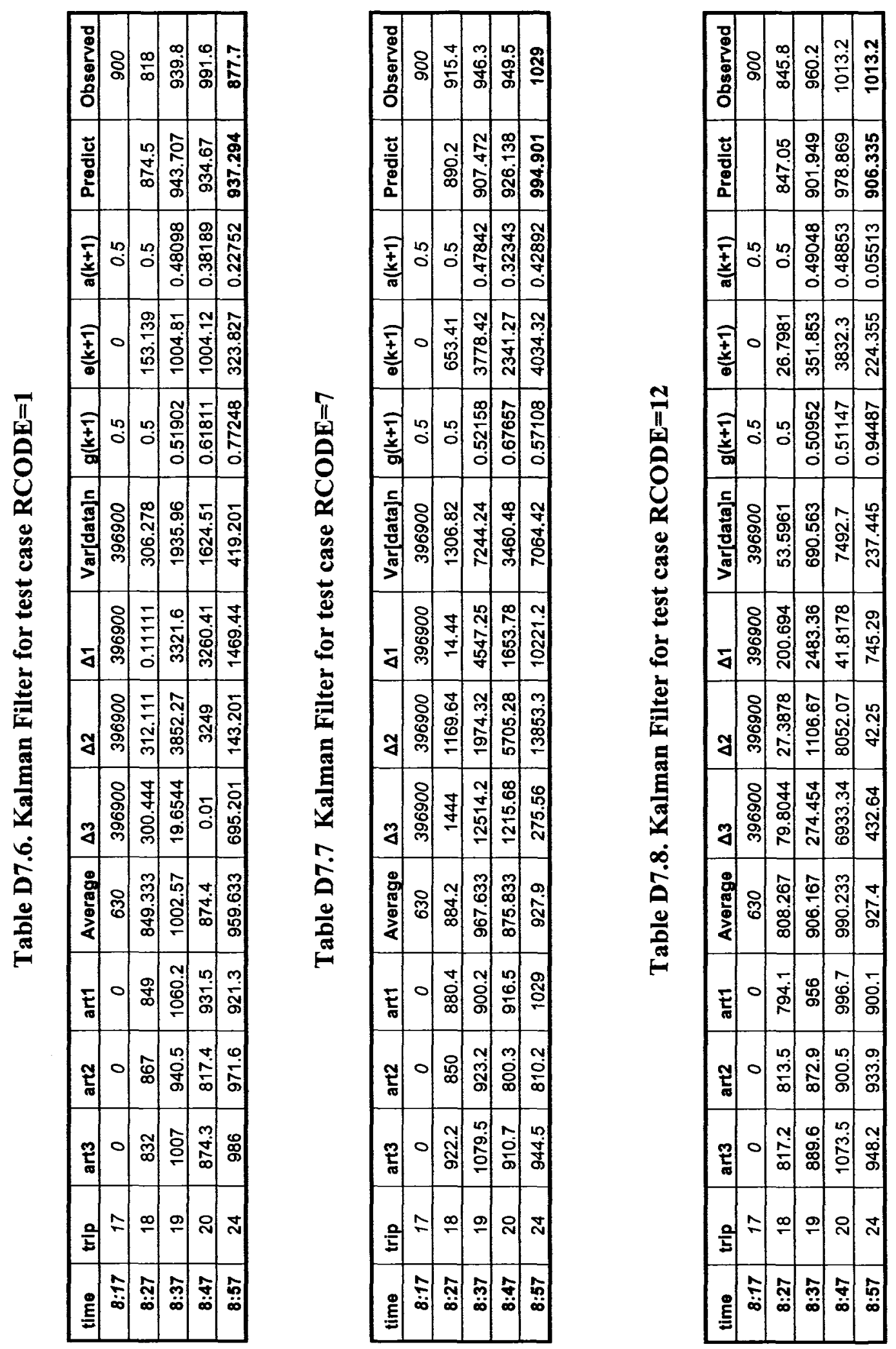
Table D7.9: Kalman Filter for test case RCODE=17

\begin{tabular}{|c|c|c|c|c|c|c|c|c|c|c|c|c|c|c|}
\hline time & trip & art3 & art2 & art1 & Average & $\Delta 3$ & $\Delta 2$ & $\Delta 1$ & Var[data]n & $g(k+1)$ & $\theta(k+1)$ & $a(k+1)$ & Predict & Observed \\
\hline $8: 17$ & 17 & 0 & 0 & 0 & 630 & 396900 & 396900 & 396900 & 396900 & 0.5 & 0 & 0.5 & & 900 \\
\hline $8: 27$ & 18 & 854.6 & 932.9 & 814 & 867.167 & 157.921 & 4320.87 & 2826.69 & 2239.4 & 0.5 & 1119.7 & 0.5 & 857 & 931 \\
\hline $8: 37$ & 19 & 919.7 & 953.7 & 931.8 & 935.067 & 236.134 & 347.201 & 10.6711 & 291.668 & 0.82874 & 241.716 & 0.17126 & 931.663 & 959 \\
\hline $8: 47$ & 20 & 845 & 948 & 960.6 & 917.867 & 5309.55 & 908.018 & 1826.14 & 3108.78 & 0.51871 & 1612.56 & 0.48129 & 959.83 & 844 \\
\hline $8: 57$ & 24 & 1121.9 & 904.8 & 928.2 & 984.967 & 18750.7 & 6426.69 & 3222.45 & 12588.7 & 0.5301 & 6673.23 & 0.4699 & 888.634 & 1017 \\
\hline
\end{tabular}

Table D7.10: Kalman Filter for test case $\mathrm{RCODE}=18$

\begin{tabular}{|c|c|c|c|c|c|c|c|c|c|c|c|c|c|c|}
\hline time & trip & art3 & art2 & art1 & Average & $\Delta 3$ & $\Delta \mathbf{2}$ & $\Delta 1$ & Var[data]n & $g(k+1)$ & $e(k+1)$ & $a(k+1)$ & Predict & Observed \\
\hline $8: 17$ & 17 & 0 & 0 & 0 & 630 & 396900 & 396900 & 396900 & 396900 & 0.5 & 0 & 0.5 & & 900 \\
\hline $8: 27$ & 18 & 932.9 & 814 & 857.5 & 868.133 & 4194.72 & 2930.42 & 113.068 & 3562.57 & 0.5 & 1781.28 & 0.5 & 878.75 & 808.6 \\
\hline $8: 37$ & 19 & 953.7 & 931.8 & 959.1 & 948.2 & 30.25 & 268.96 & 118.81 & 149.605 & 0.92809 & 138.847 & 0.07191 & 948.278 & 1083.1 \\
\hline $8: 47$ & 20 & 958.2 & 960.6 & 844 & 920.933 & 1388.8 & 1573.44 & 5918.74 & 1481.12 & 0.52239 & 773.72 & 0.47761 & 958.197 & 868.9 \\
\hline $8: 57$ & 24 & 904.8 & 928.2 & 1017.4 & 950.133 & 2055.11 & 481.071 & 4524.8 & 1268.09 & 0.61688 & 782.259 & 0.38312 & 960.507 & 923 \\
\hline
\end{tabular}

Table D7.11. Kalman Filter for test case $\mathbf{R C O D E}=\mathbf{2 3}$

\begin{tabular}{|c|c|c|c|c|c|c|c|c|c|c|c|c|c|c|}
\hline time & trip & art3 & art2 & art1 & Average & $\Delta 3$ & $\Delta \mathbf{2}$ & $\Delta 1$ & Var[data]n & $g(k+1)$ & $\theta(k+1)$ & $a(k+1)$ & Predict & Observed \\
\hline $8: 17$ & 17 & 0 & 0 & 0 & 630 & 396900 & 396900 & 396900 & 396900 & 0.5 & 0 & 0.5 & & 900 \\
\hline $8: 27$ & 18 & 841.5 & 886.7 & 895.3 & 874.5 & 1089 & 148.84 & 432.64 & 618.92 & 0.5 & 309.46 & 0.5 & 897.65 & 852.2 \\
\hline $8: 37$ & 19 & 861.7 & 1001.2 & 895.8 & 919.567 & 3348.55 & 6664 & 564.854 & 5006.28 & 0.51499 & 2578.18 & 0.48501 & 874.654 & 967.9 \\
\hline $8: 47$ & 20 & 926.1 & 974.9 & 946.6 & 949.2 & 533.61 & 660.49 & 6.76 & 597.05 & 0.84173 & 502.553 & 0.15827 & 949.971 & 878.9 \\
\hline $8: 57$ & 24 & 839.6 & 945.1 & 792.5 & 859.067 & 378.951 & 7401.73 & 4431.12 & 3890.34 & 0.53034 & 2063.19 & 0.46966 & 833.079 & 984.4 \\
\hline
\end{tabular}


in
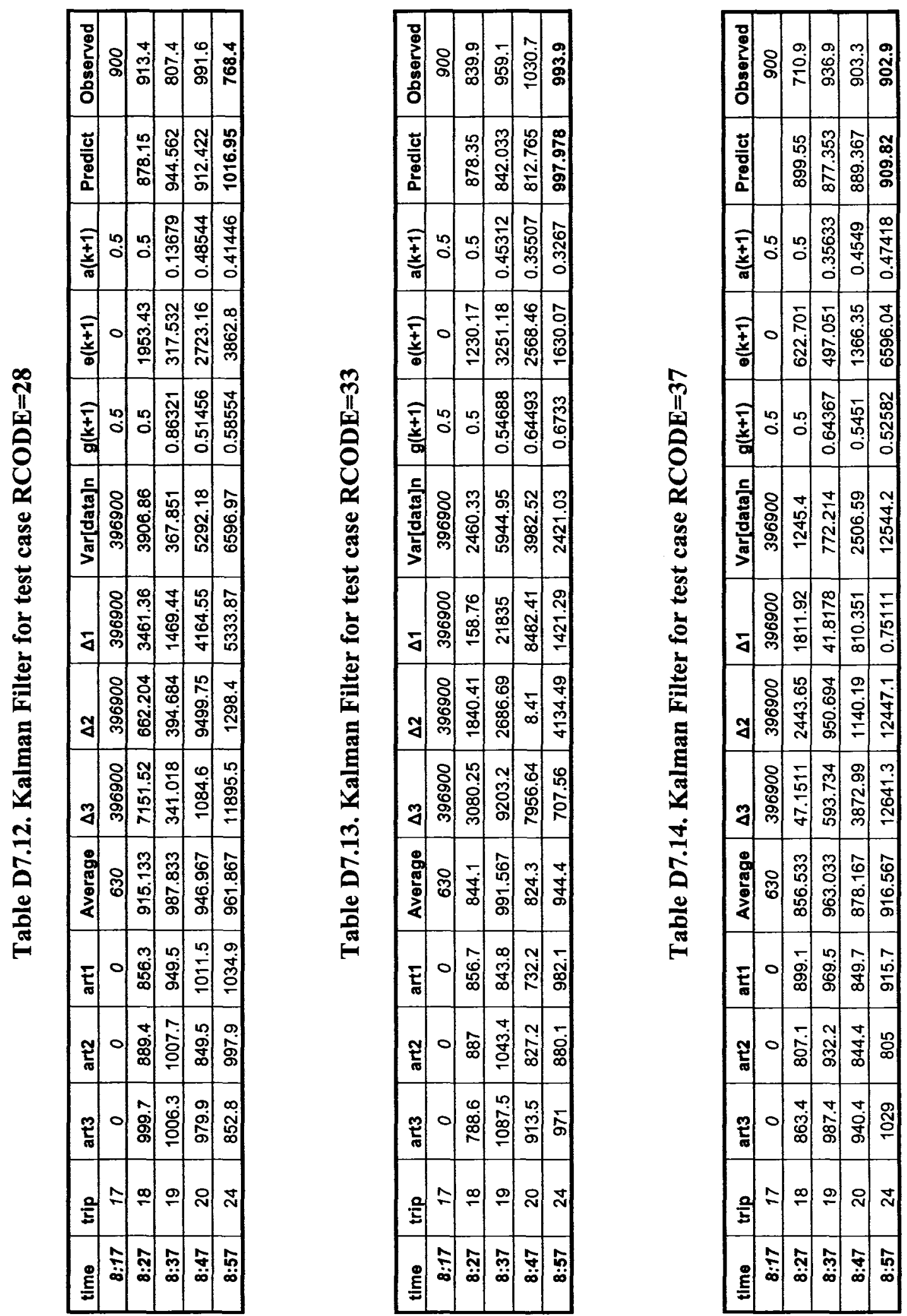
\&
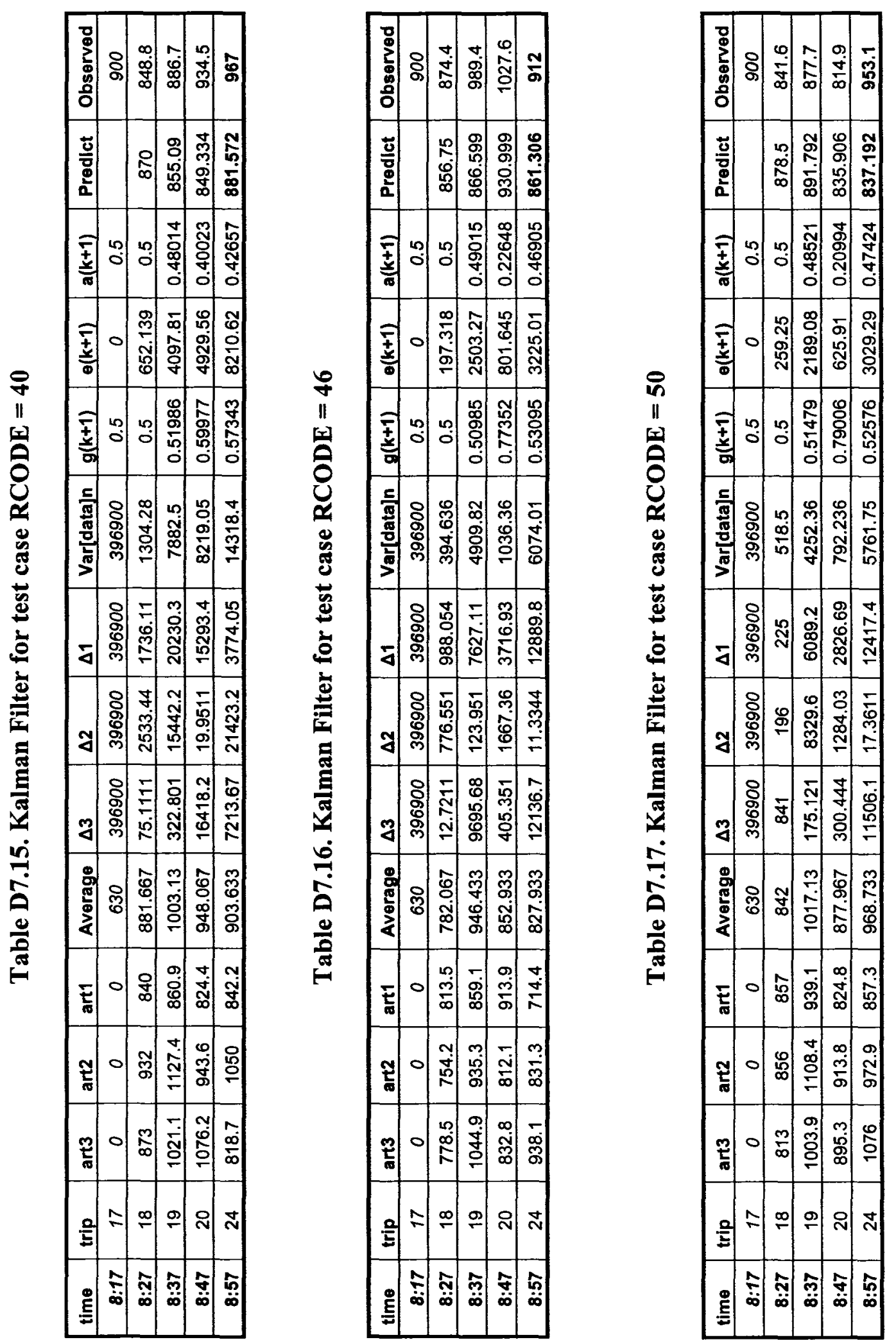

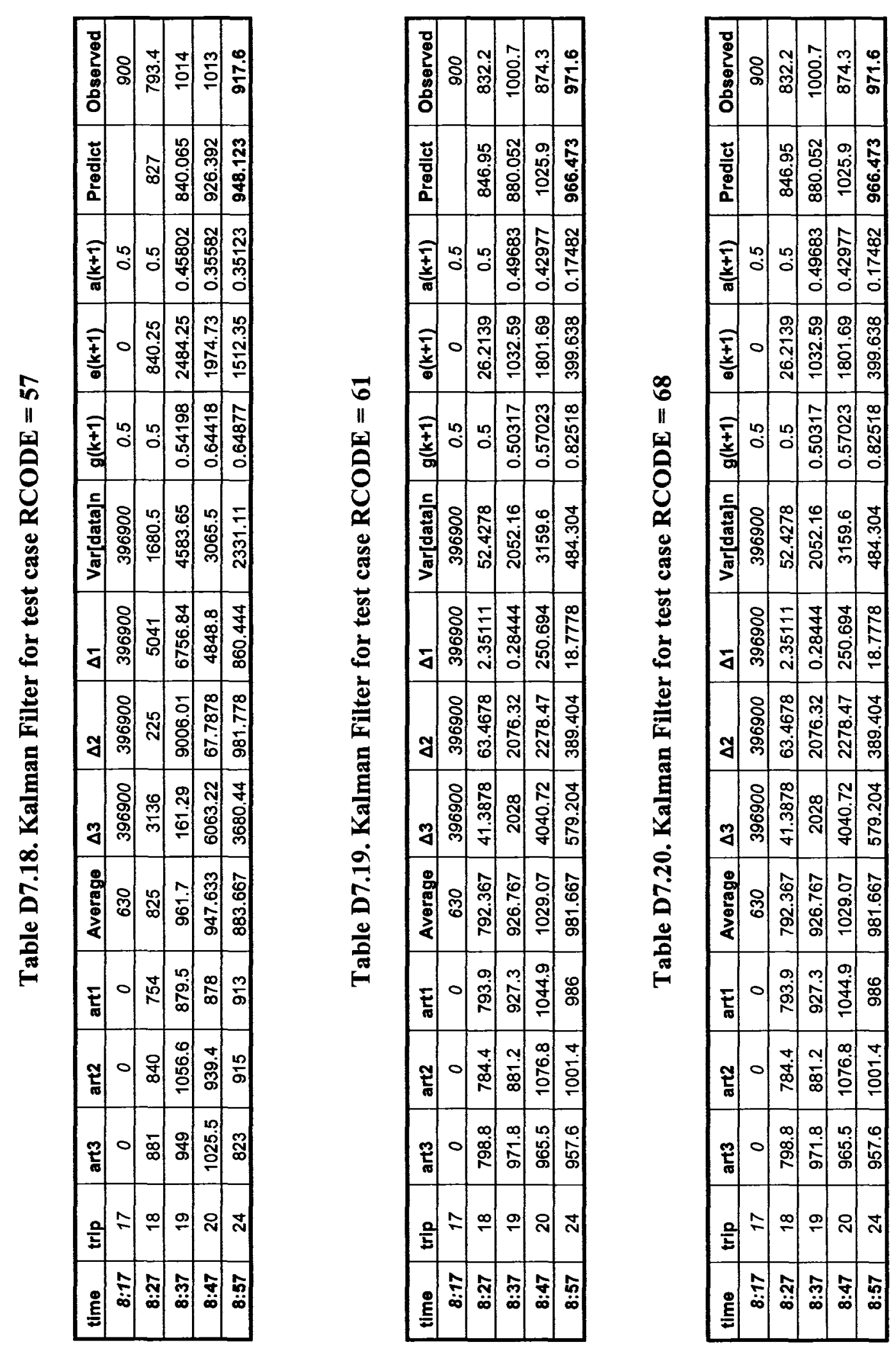
Table D7.21: Prediction Error of the Predictors

\begin{tabular}{|c|c|c|c|c|c|c|c|c|c|c|}
\hline Rcode & Actual & $\begin{array}{c}\text { Developed } \\
\text { Model }\end{array}$ & $\begin{array}{c}\text { KF } \\
\text { Model }\end{array}$ & $\begin{array}{c}\text { Naive } \\
\text { Model }\end{array}$ & $\begin{array}{c}\text { Devlp. } \\
\text { Error }\end{array}$ & $\begin{array}{c}\text { KF } \\
\text { Error }\end{array}$ & $\begin{array}{c}\text { Naive } \\
\text { Error }\end{array}$ & d1 & d2 & d3 \\
\hline 1 & 877.1 & 946 & 984 & 1117.82 & -68.90 & -106.90 & -240.72 & 0.0786 & 0.1130 & 0.2446 \\
\hline 7 & 1029.8 & 962.11 & 994.9 & 1066.05 & 67.69 & 34.90 & -36.25 & 0.0657 & 0.0363 & 0.0364 \\
\hline 12 & 985.5 & 923.07 & 906.33 & 915 & 62.43 & 79.17 & 70.50 & 0.0633 & 0.0858 & 0.0778 \\
\hline 17 & 1017.4 & 924.92 & 888.63 & 815.53 & 92.48 & 128.77 & 201.87 & 0.0909 & 0.1392 & 0.2272 \\
\hline 18 & 923.1 & 948.96 & 960.5 & 1047.42 & -25.86 & -37.40 & -124.32 & 0.0280 & 0.0394 & 0.1294 \\
\hline 23 & 984.4 & 935.03 & 833.08 & 735.82 & 49.37 & 151.32 & 248.58 & 0.0502 & 0.1618 & 0.2984 \\
\hline 28 & 768.4 & 858.62 & 1016.95 & 1014.54 & -88.19 & -248.55 & -246.14 & 0.1148 & 0.2902 & 0.2420 \\
\hline 33 & 993.9 & 911.56 & 997.97 & 1382.48 & 82.34 & -4.07 & -388.58 & 0.0828 & 0.0045 & 0.3894 \\
\hline 37 & 1029 & 950.59 & 967.12 & 959.79 & 78.41 & 61.88 & 69.21 & 0.0762 & 0.0651 & 0.0716 \\
\hline 40 & 902.9 & 828.25 & 909.82 & 973.46 & 74.65 & -6.92 & -70.56 & 0.0827 & 0.0084 & 0.0776 \\
\hline 46 & 967 & 896.47 & 881.57 & 954.68 & 70.53 & 85.43 & 12.32 & 0.0729 & 0.0953 & 0.0140 \\
\hline 50 & 912 & 908.77 & 861.31 & 803.28 & 3.23 & 50.69 & 108.72 & 0.0035 & 0.0558 & 0.1262 \\
\hline 57 & 953.1 & 899.4 & 837.19 & 847 & 53.70 & 115.91 & 106.10 & 0.0563 & 0.1289 & 0.1267 \\
\hline 61 & 917.6 & 903.53 & 948.12 & 1054.21 & 14.07 & -30.52 & -136.61 & 0.0153 & 0.0338 & 0.1441 \\
\hline 68 & 971.6 & 953.53 & 966.47 & 825.02 & 18.07 & 5.13 & 146.58 & 0.0186 & 0.0054 & 0.1517 \\
\hline
\end{tabular}

Note: $\mathrm{d} 1, \mathrm{~d} 2, \mathrm{~d} 3$ : The absolute relative error (\%) of the Proposed Model, the Kalman Filter and the Naïve predictor 


\section{Running Time Prediction Results of the Developed Model: Route 95}

The running time prediction results of the developed model are tabulated in Table D7.36 of this appendix.

\section{Running Time Prediction Results of the KF Model: Route 95}

Tables from D7.22-D7.35 present the running time prediction results of KF model applied to route 95 .

\section{Boarding Passenger Prediction Results of the Developed Model:} Route 95

The boarding passenger prediction results of the developed model are tabulated in Table D7.51 in this appendix.

\section{Boarding Passenger Prediction Results of the KF Model: Route 95}

Tables from D7.37-D7.50 show the predictions results of the KF model applied for boarding passenger predictions.

\section{Alighting Passenger Prediction Results of the Developed Model: Route 95}

The alighting passenger prediction results of the developed model are tabulated in Table D7.65 of this appendix. 
10. Alighting Passenger Prediction Results of the KF Model: Route 95

Tables from D7.52 -D7.64 show the prediction results of the KF model applied for alighting passenger predictions. 
吕
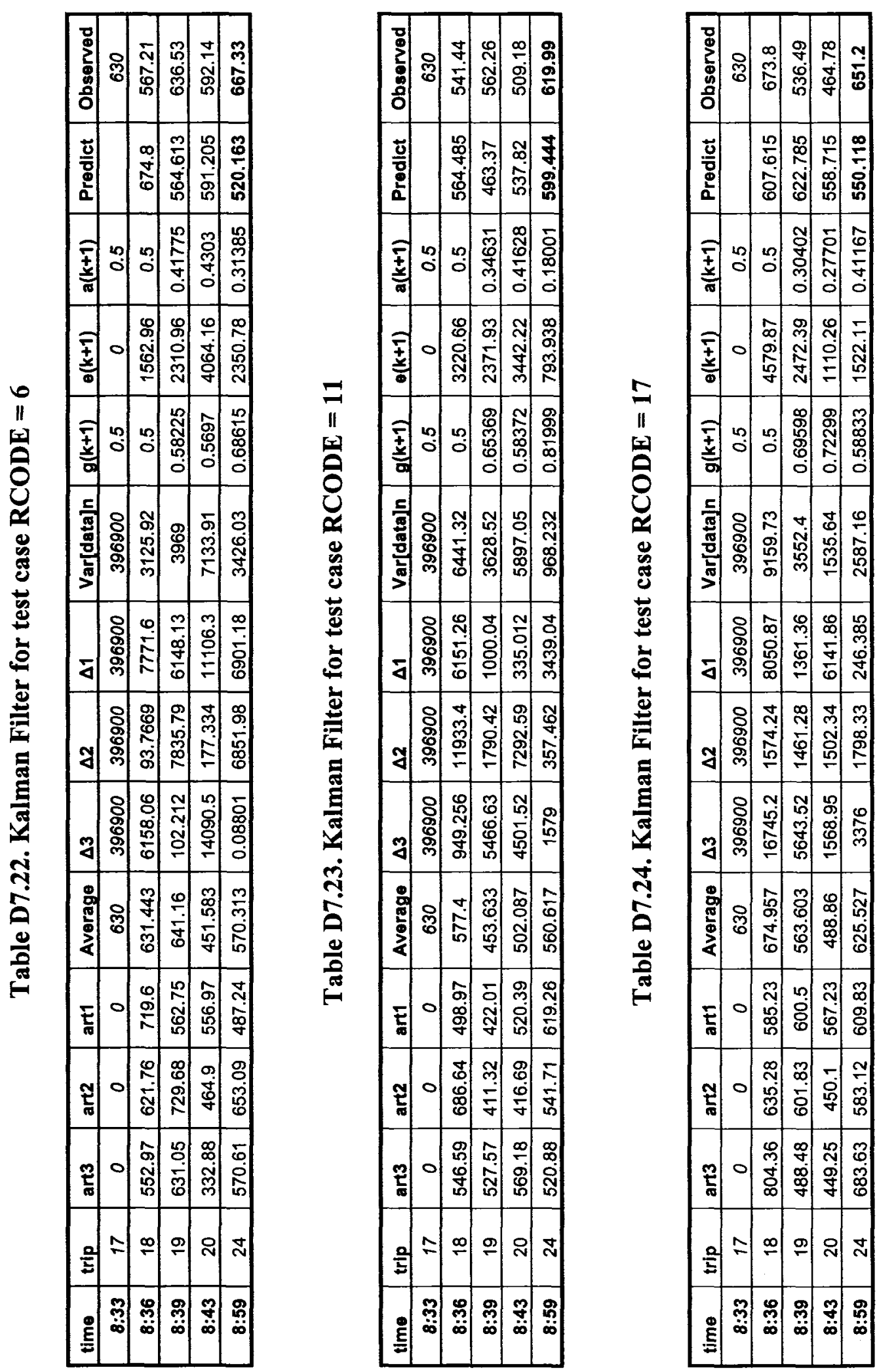
遂
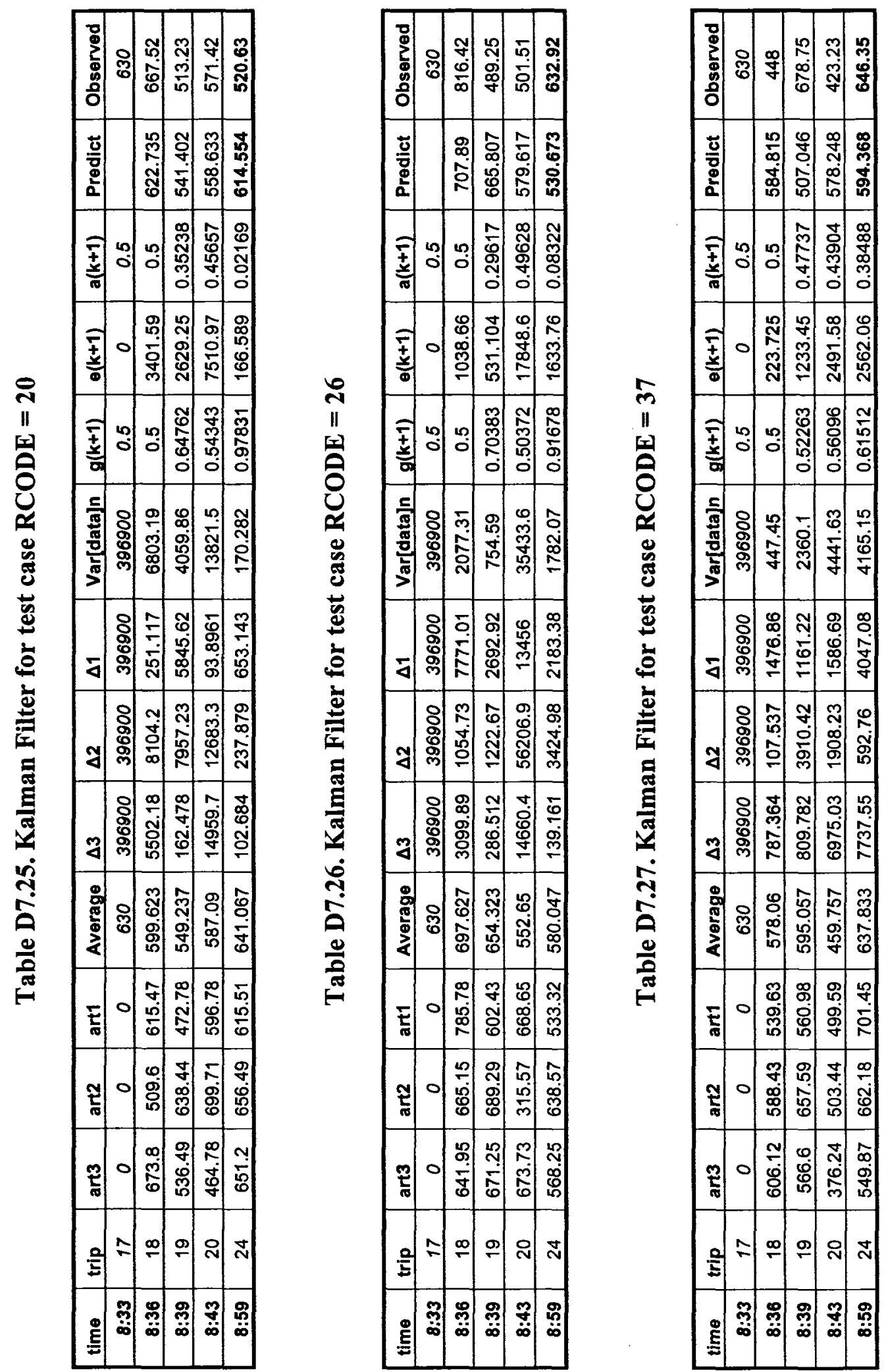
চ
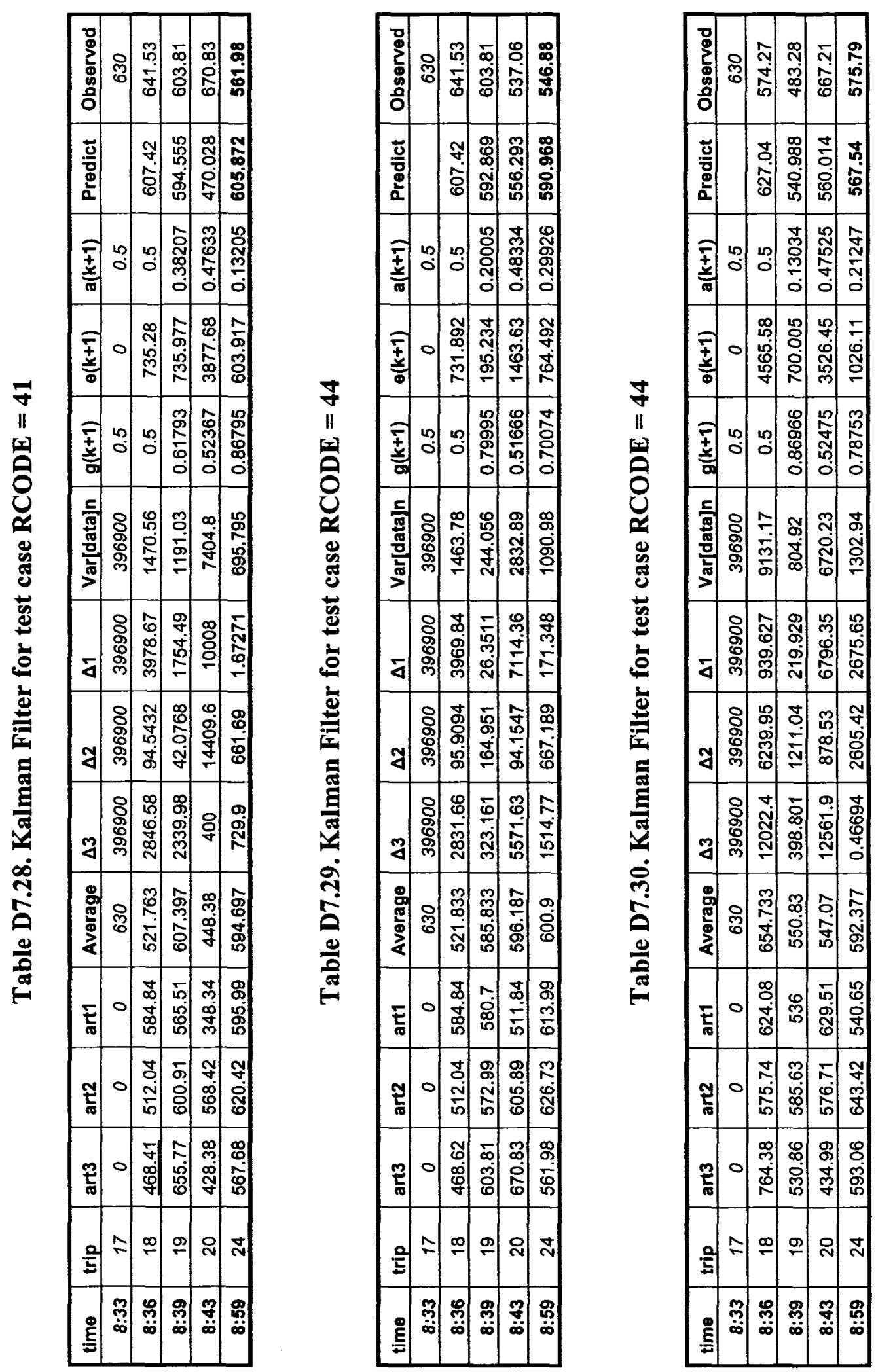
Table D7.31. Kalman Filter for test case RCODE $=49$

\begin{tabular}{|c|c|c|c|c|c|c|c|c|c|c|c|c|c|c|}
\hline time & trip & art3 & art2 & art1 & Average & $\Delta 3$ & $\Delta \mathbf{2}$ & $\Delta 1$ & Var[data]n & $g(k+1)$ & $\theta(k+1)$ & $a(k+1)$ & Predict & Observed \\
\hline $8: 33$ & 17 & 0 & 0 & 0 & 630 & 396900 & 396900 & 396900 & 396900 & 0.5 & 0 & 0.5 & & 630 \\
\hline $8: 36$ & 18 & 614.36 & 498.37 & 560.15 & 557.627 & 3218.67 & 3511.35 & 6.36721 & 3365.01 & 0.5 & 1682.51 & 0.5 & 595.07 & 538.09 \\
\hline $8: 39$ & 19 & 576.35 & 662.93 & 624.75 & 621.343 & 2024.4 & 1729.45 & 11.6054 & 1876.93 & 0.65475 & 1228.91 & 0.345254 & 594.83 & 619.16 \\
\hline $8: 43$ & 20 & 534.33 & 655.71 & 605.24 & 598.427 & 4108.38 & 3281.38 & 46.4215 & 3694.88 & 0.57129 & 2110.86 & 0.428707 & 611.20 & 384.35 \\
\hline $8: 59$ & 24 & 606.93 & 620.4 & 553.62 & 593.65 & 176.358 & 715.563 & 1602.4 & 445.96 & 0.85148 & 379.728 & 0.148516 & 528.48 & 572.17 \\
\hline
\end{tabular}

Table D7.32. Kalman Filter for test case RCODE $=\mathbf{6 2}$

\begin{tabular}{|c|c|c|c|c|c|c|c|c|c|c|c|c|c|c|}
\hline $\operatorname{tim} \theta$ & trip & art3 & $\operatorname{art2}$ & art1 & Average & $\Delta 3$ & $\Delta 2$ & $\Delta 1$ & Var[data]n & $g(k+1)$ & $e(k+1)$ & $a(k+1)$ & Predict & Observed \\
\hline $8: 33$ & 17 & 0 & 0 & 0 & 630 & 396900 & 396900 & 396900 & 396900 & 0.5 & 0 & 0.5 & & 630 \\
\hline $8: 36$ & 18 & 550.66 & 534.16 & 489.59 & 524.803 & 668.567 & 87.5472 & 1239.98 & 378.057 & 0.5 & 189.029 & 0.5 & 559.80 & 441.73 \\
\hline $8: 39$ & 19 & 462.31 & 490.41 & 599.8 & 517.507 & 3046.67 & 734.229 & 6772.19 & 1890.45 & 0.52381 & 990.232 & 0.476192 & 524.53 & 687.54 \\
\hline $8: 43$ & 20 & 640.15 & 555.21 & 480.67 & 558.677 & 6637.9 & 12.0178 & 6085.04 & 3324.96 & 0.5648 & 1877.95 & 0.435196 & 570.70 & 696.77 \\
\hline $8: 59$ & 24 & 641.84 & 564.99 & 591.14 & 599.323 & 1807.67 & 1178.78 & 66.9669 & 1493.22 & 0.69303 & 1034.85 & 0.30697 & 623.57 & 575.55 \\
\hline
\end{tabular}

Table D7.33. Kalman Filter for test case $\mathrm{RCODE}=\mathbf{8 0}$

\begin{tabular}{|c|c|c|c|c|c|c|c|c|c|c|c|c|c|c|}
\hline time & trip & art3 & art 2 & art1 & Average & $\Delta 3$ & $\Delta \mathbf{2}$ & $\Delta 1$ & Var[data]n & $g(k+1)$ & $\theta(k+1)$ & $a(k+1)$ & Predict & Observed \\
\hline $8: 33$ & 17 & 0 & 0 & 0 & 630 & 396900 & 396900 & 396900 & 396900 & 0.5 & 0 & 0.5 & & 630 \\
\hline $8: 36$ & 18 & 626.64 & 571.11 & 805.78 & 667.843 & 1697.71 & 9357.34 & 19026.5 & 5527.53 & 0.5 & 2763.76 & 0.5 & 717.89 & 603.65 \\
\hline $8: 39$ & 19 & 721.25 & 581.13 & 650.93 & 651.103 & 4920.55 & 4896.27 & 0.03004 & 4908.41 & 0.60984 & 2993.36 & 0.390158 & 632.48 & 580.66 \\
\hline $8: 43$ & 20 & 834.31 & 674.97 & 644.36 & 717.88 & 13555.9 & 1841.27 & 5405.19 & 7698.61 & 0.58138 & 4475.84 & 0.418617 & 617.69 & 462.64 \\
\hline $8: 59$ & 24 & 637.95 & 524.02 & 596.9 & 586.29 & 2668.76 & 3877.55 & 112.572 & 3273.15 & 0.70304 & 2301.15 & 0.296962 & 557.03 & 585.08 \\
\hline
\end{tabular}


is

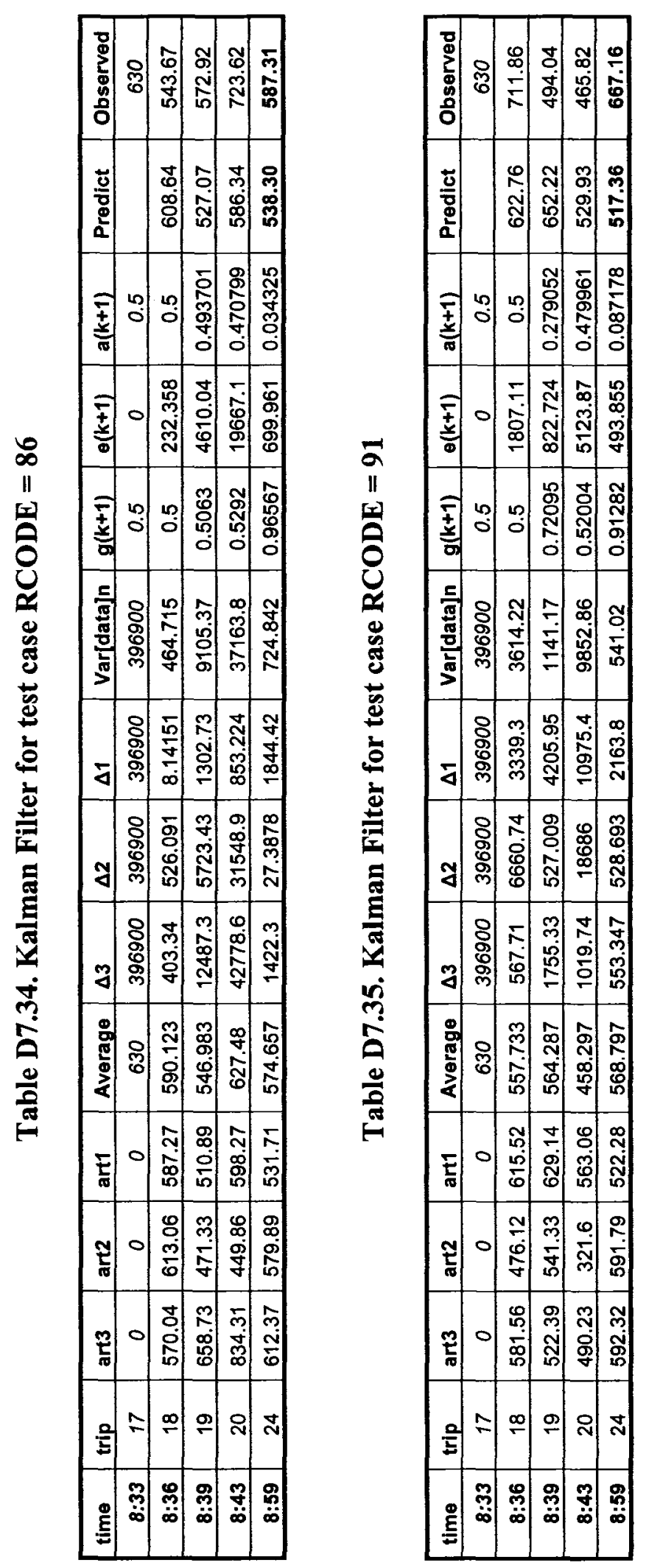


Table D7.36: Prediction Error of the Predictors

\begin{tabular}{|c|c|c|c|c|c|c|c|}
\hline RCODE & $\begin{array}{c}\text { Actual } \\
\text { Running } \\
\text { slme }\end{array}$ & $\begin{array}{l}\text { Developed } \\
\text { Modtel }\end{array}$ & MF & $\begin{array}{l}\text { Naive } \\
\text { Modol. }\end{array}$ & c1 & di 6 & d3 \\
\hline 6 & 667 & 600.44 & 520.16 & 518.0104 & 0.100247 & 0.220546 & 0.223767 \\
\hline 11 & 620 & 597.99 & 599.44 & 605.9335 & 0.035495 & 0.033156 & 0.022683 \\
\hline 17 & 651 & 590.09 & 550.12 & 499.6859 & 0.093843 & 0.155222 & 0.232669 \\
\hline 20 & 521 & 594.16 & 614.55 & 589.355 & 0.141228 & 0.180392 & 0.131999 \\
\hline 26 & 633 & 610.78 & 530.67 & 400.0117 & 0.034981 & 0.161553 & 0.367991 \\
\hline 37 & 646 & 607.15 & 594.37 & 594.2325 & 0.060654 & 0.080426 & 0.080639 \\
\hline 41 & 562 & 595.24 & 605.87 & 1147.79 & 0.059166 & 0.078081 & 1.04237 \\
\hline 44 & 547 & 598.06 & 590.97 & 644.2334 & 0.093582 & 0.080617 & 0.178012 \\
\hline 49 & 576 & 600.62 & 567.54 & 573.0316 & 0.043107 & 0.014343 & 0.004806 \\
\hline 62 & 572 & 606.61 & 528.48 & 351.5722 & 0.06018 & 0.076369 & 0.385553 \\
\hline 72 & 576 & 615.58 & 623.57 & 856.9015 & 0.069557 & 0.083439 & 0.488848 \\
\hline 80 & 585 & 593.81 & 557.03 & 428.5672 & 0.014912 & 0.047951 & 0.267513 \\
\hline 86 & 587 & 606.63 & 538.3 & 643.1271 & 0.032888 & 0.083455 & 0.095031 \\
\hline 91 & 667 & 587.2 & 517.36 & 432.0833 & 0.119848 & 0.224531 & 0.352352 \\
\hline 96 & 586 & 604.26 & 574.94 & 508.2925 & 0.030391 & 0.019606 & 0.133254 \\
\hline
\end{tabular}

Note: d1, d2, d3: The absolute relative error (\%) of the Developed Model, the Kalman Filter and the Naïve predictors, respectively 
Table D7.37. Kalman Filter for test case RCODE $=6$

\begin{tabular}{|c|c|c|c|c|c|c|c|c|c|c|c|c|}
\hline time & trip & art3 & art2 & art1 & Average & Var[data]n & $g(k+1)$ & $\theta(k+1)$ & $a(k+1)$ & Predicted & Observed & Boarders \\
\hline $8: 33$ & 17 & 0 & 0 & 0 & 0.025 & 0.000625 & 0.5 & 0 & 0.5 & & 0.025 & \\
\hline $8: 36$ & 18 & 0 & 0 & 0 & 1.025 & 1.050625 & 0.5 & 0.525313 & 0.5 & 0.0125 & 0 & \\
\hline $8: 39$ & 19 & 0.011776 & 0.005482 & 0.010662 & 0.009307 & $1.04 E-05$ & 0.99998 & $1.04 \mathrm{E}-05$ & $1.97 E-05$ & 0.010662 & 0.018852 & \\
\hline $8: 43$ & 20 & 0.027036 & 0.019355 & 0.028726 & 0.025039 & 1.81E-05 & 0.611056 & 1.11E-05 & 0.388944 & 0.024886 & 0.011821 & \\
\hline $8: 59$ & 24 & 0.015773 & 0.009187 & 0.008209 & 0.011056 & $1.29 \mathrm{E}-05$ & 0.650571 & 8.37E-06 & 0.349429 & 0.009472 & 0.011988 & 6 \\
\hline
\end{tabular}

Table D7.38. Kalman Filter for test case RCODE $=11$

\begin{tabular}{|c|c|c|c|c|c|c|c|c|c|c|c|c|}
\hline time & trip & art3 & $\operatorname{art2}$ & art1 & Average & $\operatorname{Var}[$ data]n & $g(k+1)$ & $\theta(k+1)$ & $a(k+1)$ & Predicted & Observed & Boarders \\
\hline $8: 33$ & 17 & 0 & 0 & 0 & 0.025 & 0.000625 & 0.5 & 0 & 0.5 & & 0.025 & \\
\hline $8: 36$ & 18 & 0 & 0 & 0 & 1.025 & 1.050625 & 0.5 & 0.525313 & 0.5 & 0.0125 & 0 & \\
\hline $8: 39$ & 19 & 0.003791 & 0.003791 & 0.009478 & 0.005687 & $3.59 \mathrm{E}-06$ & 0.999993 & $3.59 \mathrm{E}-06$ & $6.84 \mathrm{E}-06$ & 0.009478 & 0.010671 & \\
\hline $8: 43$ & 20 & 0.005271 & 0.028798 & 0.005765 & 0.013278 & 0.000152 & 0.505824 & 7.71E-05 & 0.494176 & 0.008189 & 0.033386 & \\
\hline $8: 59$ & 24 & 0.00192 & 0.007384 & 0.009689 & 0.006331 & $1.03 E-05$ & 0.894747 & $9.2 \mathrm{E}-06$ & 0.105253 & 0.012183 & 0.016129 & 7 \\
\hline
\end{tabular}

Table D7.39. Kalman Filter for test case RCODE $=17$

\begin{tabular}{|c|c|c|c|c|c|c|c|c|c|c|c|c|}
\hline time & trip & art3 & art2 & art1 & Average & Var[data]n & $g(k+1)$ & $e(k+1)$ & $a(k+1)$ & Predict & Observed & Boarders \\
\hline $8: 33$ & 17 & 0 & 0 & 0 & 0.025 & 0.000625 & 0.5 & 0 & 0.5 & & 0.025 & \\
\hline $8: 36$ & 18 & 0 & 0 & 0 & 1.025 & 1.050625 & 0.5 & 0.525313 & 0.5 & 0.0125 & 0 & \\
\hline $8: 39$ & 19 & 0.005376 & 0.011631 & 0.004996 & 0.007334 & $1.11 \mathrm{E}-05$ & 0.999979 & 1.11E-05 & $2.12 \mathrm{E}-05$ & 0.004996 & 0.020503 & \\
\hline $8: 43$ & 20 & 0.022259 & 0.01333 & 0.005289 & 0.013626 & $3.73 E-05$ & 0.564989 & $2.11 \mathrm{E}-05$ & 0.435011 & 0.011907 & 0.025819 & \\
\hline $8: 59$ & 24 & 0.013165 & 0.005145 & 0.00164 & 0.00665 & 2.24E- 05 & 0.660199 & $1.48 \mathrm{E}-05$ & 0.339801 & 0.009856 & 0.019963 & 6 \\
\hline
\end{tabular}


Table D7.40. Kalman Filter for test case RCODE $=\mathbf{2 0}$

\begin{tabular}{|c|c|c|c|c|c|c|c|c|c|c|c|c|}
\hline time & trip & $\operatorname{art3}$ & art2 & art1 & Average & Var[data]n & $g(k+1)$ & $e(k+1)$ & $a(k+1)$ & Predict & Observed & Boarders \\
\hline $8: 33$ & 17 & 0 & 0 & 0 & 0.025 & 0.000625 & 0.5 & 0 & 0.5 & & 0.025 & \\
\hline $8: 36$ & 18 & 0 & 0 & 0 & 1.025 & 1.050625 & 0.5 & 0.525313 & 0.5 & 0.0125 & 0 & \\
\hline $8: 39$ & 19 & 0.020503 & 0.014097 & 0.021151 & 0.018584 & $1.19 \mathrm{E}-05$ & 0.999977 & 1.19E-05 & 2.27E-05 & 0.021151 & 0.021433 & \\
\hline $8: 43$ & 20 & 0.025819 & 0.022867 & 0.030161 & 0.026282 & $5.94 \mathrm{E}-06$ & 0.750292 & 4.46E-06 & 0.249708 & 0.027982 & 0.01575 & \\
\hline $8: 59$ & 24 & 0.019963 & 0.009139 & 0.017871 & 0.015658 & $3.05 \mathrm{E}-05$ & 0.534033 & 1.63E-05 & 0.465967 & 0.016883 & 0.02497 & 10 \\
\hline
\end{tabular}

Table D7.41. Kalman Filter for test case $\mathrm{RCODE}=\mathbf{2 6}$

\begin{tabular}{|c|c|c|c|c|c|c|c|c|c|c|c|c|}
\hline time & trip & art3 & art2 & art1 & Avorage & Var[data]n & $g(k+1)$ & $e(k+1)$ & $a(k+1)$ & Predlat & Observed & Boarders \\
\hline $8: 33$ & 17 & 0 & 0 & 0 & 0.025 & 0.000625 & 0.5 & 0 & 0.5 & & 0.025 & \\
\hline $8: 36$ & 18 & 0 & 0 & 0 & 1.025 & 1.050625 & 0.5 & 0.525313 & 0.5 & 0.0125 & 0 & \\
\hline $8: 39$ & 19 & 0.005959 & 0.007254 & 0.011619 & 0.008277 & $3.21 \mathrm{E}-06$ & 0.999994 & $3.21 \mathrm{E}-06$ & $6.11 \mathrm{E}-06$ & 0.011619 & 0.016351 & \\
\hline $8: 43$ & 20 & 0.023748 & 0.022182 & 0.011964 & 0.019298 & $1.41 \mathrm{E}-05$ & 0.551251 & 7.75E-06 & 0.448749 & 0.013933 & 0.025921 & \\
\hline $8: 59$ & 24 & 0.008799 & 0.00783 & 0.00375 & 0.006793 & 2.55E-06 & 0.801586 & 2.04E-06 & 0.198414 & 0.008149 & 0.0158 & 5 \\
\hline
\end{tabular}

Table D7.42. Kalman Filter for test case $\mathrm{RCODE}=37$

\begin{tabular}{|c|c|c|c|c|c|c|c|c|c|c|c|c|}
\hline time & trip & art3 & art2 & art1 & Average & Var[data]n & $g(k+1)$ & $\theta(k+1)$ & a(k+1) & Predict & Observed & Boarders \\
\hline $8: 33$ & 17 & 0 & 0 & 0 & 0.025 & 0.000625 & 0.5 & 0 & 0.5 & & 0.025 \\
\hline $8: 36$ & 18 & 0 & 0 & 0 & 1.025 & 1.050625 & 0.5 & 0.525313 & 0.5 & 0.0125 & 0 \\
\hline $8: 39$ & 19 & 0.012354 & 0.010645 & 0.010696 & 0.011232 & $8.02 \mathrm{E}-07$ & 0.999998 & $8.02 \mathrm{E}-07$ & $1.53 \mathrm{E}-06$ & 0.010696 & 0.007366 \\
\hline $8: 43$ & 20 & 0.021263 & 0.007945 & 0.006005 & 0.011738 & $5.26 \mathrm{E}-05$ & 0.503788 & $2.65 \mathrm{E}-05$ & 0.496212 & 0.00668 & 0.030716 \\
\hline $8: 59$ & 24 & 0.01273 & 0.010571 & 0.007128 & 0.010143 & $3.44 \mathrm{E}-06$ & 0.896926 & $3.08 \mathrm{E}-06$ & 0.103074 & $\mathbf{0 . 0 0 9 5 5 9}$ & $\mathbf{0 . 0 1 7 0 1 9}$ & $\mathbf{6}$ \\
\hline
\end{tabular}


Table D7.43. Kalman Filter for test case $\mathrm{RCODE}=\mathbf{4 1}$

\begin{tabular}{|c|c|c|c|c|c|c|c|c|c|c|c|c|}
\hline time & trip & art3 & art2 & art1 & Average & Var[data]n & $g(k+1)$ & $\theta(k+1)$ & $a(k+1)$ & Predict & Observed & Boarders \\
\hline 8:33 & 17 & 0 & 0 & 0 & 0.025 & 0.000625 & 0.5 & 0 & 0.5 & & 0.025 & \\
\hline $8: 36$ & 18 & 0 & 0 & 0 & 1.025 & 1.050625 & 0.5 & 0.525313 & 0.5 & 0.0125 & 0 & \\
\hline $8: 39$ & 19 & 0.012199 & 0.006657 & 0.012378 & 0.010411 & 8.65E-06 & 0.999984 & 8.65E-06 & 1.65E-05 & 0.012378 & 0.008281 & \\
\hline $8: 43$ & 20 & 0.002334 & 0.008796 & 0.028708 & 0.013279 & $6.99 \mathrm{E}-05$ & 0.529109 & 3.7E-05 & 0.470891 & 0.019089 & 0.007453 & \\
\hline $8: 59$ & 24 & 0.015854 & 0.006447 & 0.015101 & 0.012467 & 2.39E-05 & 0.718421 & 1.71E-05 & 0.281579 & 0.012947 & 0.010676 & 8 \\
\hline
\end{tabular}

Table D7.44. Kalman Filter for test case $\mathrm{RCODE}=\mathbf{4 4}$

\begin{tabular}{|c|c|c|c|c|c|c|c|c|c|c|c|c|}
\hline time & trip & art3 & art2 & art1 & Average & Var[data]n & $g(k+1)$ & $\theta(k+1)$ & $a(k+1)$ & Predict & Observed & Boarders \\
\hline $8: 33$ & 17 & 0 & 0 & 0 & 0.025 & 0.000625 & 0.5 & 0 & 0.5 & & 0.025 & \\
\hline $8: 36$ & 18 & 0 & 0 & 0 & 1.025 & 1.050625 & 0.5 & 0.525313 & 0.5 & 0.0125 & 0 & \\
\hline $8: 39$ & 19 & 0.008281 & 0.006981 & 0.017221 & 0.010827 & $1.06 \mathrm{E}-05$ & 0.99998 & $1.06 \mathrm{E}-05$ & $2.03 E-05$ & 0.01722 & 0.014186 & \\
\hline $8: 43$ & 20 & 0.007453 & 0.011553 & 0.021491 & 0.013499 & $2.02 E-05$ & 0.604362 & 1.22E-05 & 0.395638 & 0.018601 & 0.014896 & \\
\hline $8: 59$ & 24 & 0.010676 & 0.004787 & 0.019544 & 0.011669 & 2.42E-05 & 0.600668 & $1.45 \mathrm{E}-05$ & 0.3993332 & 0.017688 & 0.010971 & 11 \\
\hline
\end{tabular}

Table D7.45. Kalman Filter for test case $\mathrm{RCODE}=49$

\begin{tabular}{|c|c|c|c|c|c|c|c|c|c|c|c|c|}
\hline time & trip & $\operatorname{art3}$ & art2 & art1 & Average & Var[data]n & $g(k+1)$ & $\theta(k+1)$ & $a(k+1)$ & Prodict & Observed & Boarders \\
\hline $8: 33$ & 17 & 0 & 0 & 0 & 0.025 & 0.000625 & 0.5 & 0 & 0.5 & & 0.025 & \\
\hline $8: 36$ & 18 & 0 & 0 & 0 & 1.025 & 1.050625 & 0.5 & 0.525313 & 0.5 & 0.0125 & 0 & \\
\hline $8: 39$ & 19 & 0.005651 & 0.011953 & 0.020522 & 0.012709 & 2.52E-05 & 0.999952 & 2.52E-05 & 4.79E-05 & 0.020521 & 0.006208 & \\
\hline $8: 43$ & 20 & 0.004598 & 0.024276 & 0.019062 & 0.015979 & $9.92 \mathrm{E}-05$ & 0.556339 & $5.52 \mathrm{E}-05$ & 0.443661 & 0.013359 & 0.010491 & \\
\hline $8: 59$ & 24 & 0.001686 & 0.009325 & 0.022195 & 0.011069 & 4.55E-05 & 0.688638 & $3.14 \mathrm{E}-05$ & 0.311362 & 0.018551 & 0.013894 & 11 \\
\hline
\end{tabular}


Table D7.46. Kalman Filter for test case $\mathrm{RCODE}=\mathbf{6 2}$

\begin{tabular}{|c|c|c|c|c|c|c|c|c|c|c|c|c|}
\hline $\operatorname{tim} \theta$ & trip & $\operatorname{art3}$ & art2 & art1 & Average & Var[data]n & $g(k+1)$ & $\theta(k+1)$ & $a(k+1)$ & Predict & Observed & Boarders \\
\hline $8: 33$ & 17 & 0 & 0 & 0 & 0.025 & 0.000625 & 0.5 & 0 & 0.5 & & 0.025 & \\
\hline $8: 36$ & 18 & 0 & 0 & 0 & 1.025 & 1.050625 & 0.5 & 0.525313 & 0.5 & 0.0125 & 0 & \\
\hline $8: 39$ & 19 & 0.01388 & 0.016593 & 0.009604 & 0.013359 & $5.36 \mathrm{E}-06$ & 0.99999 & 5.36E-06 & $1.02 E-05$ & 0.009604 & 0.012921 & \\
\hline $8: 43$ & 20 & 0.024329 & 0.016775 & 0.01487 & 0.018658 & 1.79E-05 & 0.565307 & $1.01 \mathrm{E}-05$ & 0.434693 & 0.014023 & 0.007805 & \\
\hline $8: 59$ & 24 & 0.014829 & 0.01773 & 0.005419 & 0.012659 & $1.52 E-05$ & 0.624555 & $9.5 \mathrm{E}-06$ & 0.375445 & 0.006315 & 0.010486 & 4 \\
\hline
\end{tabular}

Table D7.47. Kalman Filter for test case RCODE $=72$

\begin{tabular}{|c|c|c|c|c|c|c|c|c|c|c|c|c|}
\hline time & trip & art3 & art2 & art1 & Average & Var[data]n & $g(k+1)$ & $e(k+1)$ & $a(k+1)$ & Prodict & Observed & Boarders \\
\hline 8:33 & 17 & 0 & 0 & 0 & 0.025 & 0.000625 & 0.5 & 0 & 0.5 & & 0.025 & \\
\hline $8: 36$ & 18 & 0 & 0 & 0 & 1.025 & 1.050625 & 0.5 & 0.525313 & 0.5 & 0.0125 & 0 & \\
\hline $8: 39$ & 19 & 0.012978 & 0.010196 & 0.01167 & 0.011615 & $1.94 \mathrm{E}-06$ & 0.999996 & $1.94 \mathrm{E}-06$ & $3.69 \mathrm{E}-06$ & 0.01167 & 0.005818 & \\
\hline $8: 43$ & 20 & 0.001562 & 0.005403 & 0.014563 & 0.007176 & $1.73 E-05$ & 0.526468 & $9.12 E-06$ & 0.473542 & 0.010422 & 0.00287 & \\
\hline $8: 59$ & 24 & 0.004674 & 0.030089 & 0.008458 & 0.014407 & 0.00017 & 0.513042 & $8.74 E-05$ & 0.486958 & 0.005737 & 0.010425 & 4 \\
\hline
\end{tabular}

Table D7.48. Kalman Filter for test case $\mathrm{RCODE}=\mathbf{8 6}$

\begin{tabular}{|c|c|c|c|c|c|c|c|c|c|c|c|c|}
\hline time & trip & art3 & art2 & art1 & Average & Var[data]n & $g(k+1)$ & $\theta(k+1)$ & $a(k+1)$ & Predict & Observed & Boarders \\
\hline $8: 33$ & 17 & 0 & 0 & 0 & 0.025 & 0.000625 & 0.5 & 0 & 0.5 & & 0.025 & \\
\hline $8: 36$ & 18 & 0 & 0 & 0 & 1.025 & 1.050625 & 0.5 & 0.525313 & 0.5 & 0.0125 & 0 & \\
\hline $8: 39$ & 19 & 0.008319 & 0.024091 & 0.018435 & 0.016948 & $6.27 \mathrm{E}-05$ & 0.999881 & $6.27 \mathrm{E}-05$ & 0.000119 & 0.018433 & 0.013777 & \\
\hline $8: 43$ & 20 & 0.002397 & 0.026668 & 0.021727 & 0.016931 & 0.000153 & 0.58506 & $8.95 \mathrm{E}-05$ & 0.41494 & 0.018428 & 0.012969 & \\
\hline $8: 59$ & 24 & 0.014108 & 0.024808 & 0.011727 & 0.016881 & 3.53E-05 & 0.779663 & 2.75E-05 & 0.220337 & 0.012001 & 0.013673 & 7 \\
\hline
\end{tabular}


Table D7.349. Kalman Filter for test case RCODE = 91

\begin{tabular}{|c|c|c|c|c|c|c|c|c|c|c|c|c|}
\hline time & trip & art3 & art2 & art1 & Average & Var[data]n & $g(k+1)$ & $e(k+1)$ & $a(k+1)$ & Predict & Observed & Boarders \\
\hline $8: 33$ & 17 & 0 & 0 & 0 & 0.025 & 0.000625 & 0.5 & 0 & 0.5 & & 0.025 & \\
\hline $8: 36$ & 18 & 0 & 0 & 0 & 1.025 & 1.050625 & 0.5 & 0.525313 & 0.5 & 0.0125 & 0 & \\
\hline $8: 39$ & 19 & 0.010626 & 0.016973 & 0.015659 & 0.01442 & $1.05 E-05$ & 0.99998 & $1.05 \mathrm{E}-05$ & $1.99 \mathrm{E}-05$ & 0.015659 & 0.006982 & \\
\hline $8: 43$ & 20 & 0.021813 & 0.022229 & 0.016715 & 0.020252 & 3.17E-06 & 0.811191 & $2.57 \mathrm{E}-06$ & 0.188809 & 0.014877 & 0.008292 & \\
\hline $8: 59$ & 24 & 0.006532 & 0.010347 & 0.016926 & 0.011268 & $1.16 \mathrm{E}-05$ & 0.549753 & $6.4 \mathrm{E}-06$ & 0.450247 & 0.013039 & 0.008513 & 8 \\
\hline
\end{tabular}

Table D7.50. Kalman Filter for test case RCODE $=96$

\begin{tabular}{|c|c|c|c|c|c|c|c|c|c|c|c|c|}
\hline time & trip & art3 & art2 & art1 & Average & Var[data]n & $g(k+1)$ & $\theta(k+1)$ & $a(k+1)$ & Predict & Observed & Boarders \\
\hline $8: 33$ & 17 & 0 & 0 & 0 & 0.025 & 0.000625 & 0.5 & 0 & 0.5 & & 0.025 & \\
\hline $8: 36$ & 18 & 0 & 0 & 0 & 1.025 & 1.050625 & 0.5 & 0.525313 & 0.5 & 0.0125 & 0 & \\
\hline $8: 39$ & 19 & 0.010626 & 0.022168 & 0.007947 & 0.01358 & 4.12E-05 & 0.999922 & $4.12 E-05$ & $7.85 \mathrm{E}-05$ & 0.007947 & 0.018217 & \\
\hline $8: 43$ & 20 & 0.028558 & 0.059079 & 0.007104 & 0.03158 & 0.000383 & 0.525559 & 0.000201 & 0.474441 & 0.012377 & 0.023614 & \\
\hline $8: 59$ & 24 & 0.015194 & 0.016898 & 0.013403 & 0.015165 & 1.5E-06 & 0.992643 & 1.49E-06 & 0.007357 & 0.013478 & 0.017987 & 8 \\
\hline
\end{tabular}


Table D7.51: Boarding Passenger Prediction Results of the 3 Predictors

\begin{tabular}{|c|c|c|c|c|c|c|c|}
\hline Reple & $\begin{array}{c}\text { Actual } \\
\text { Boarding } \\
\text { Passengers }\end{array}$ & $\begin{array}{c}\text { Beveloped } \\
\text { Model }\end{array}$ & hodel & $\begin{array}{l}\text { Naive } \\
\text { Model }\end{array}$ & (x) & $y^{2}+2=$ & $f^{2}+\cos ^{2} x^{2}$ \\
\hline 6 & 8 & 7 & 6 & 2 & 0.1250 & 0.2500 & 0.7500 \\
\hline 11 & 10 & 9 & 7 & 34 & 0.1000 & 0.3000 & 2.4000 \\
\hline 17 & 13 & 8 & 6 & 4 & 0.3846 & 0.5385 & 0.6923 \\
\hline 20 & 13 & 7 & 10 & 6 & 0.4615 & 0.2308 & 0.5385 \\
\hline 26 & 10 & 8 & 5 & 3 & 0.2000 & 0.5000 & 0.7000 \\
\hline 37 & 11 & 8 & 6 & 21 & 0.2727 & 0.4545 & 0.9091 \\
\hline 41 & 6 & 7 & 8 & 5 & 0.1667 & 0.3333 & 0.1667 \\
\hline 44 & 6 & 7 & 11 & 9 & 0.1667 & 0.8333 & 0.5000 \\
\hline 49 & 8 & 7 & 11 & 7 & 0.1250 & 0.3750 & 0.1250 \\
\hline 62 & 6 & 7 & 4 & 1 & 0.1667 & 0.3333 & 0.8333 \\
\hline 72 & 6 & 6 & 4 & 1 & 0.0000 & 0.3333 & 0.8333 \\
\hline 80 & 8 & 7 & 10 & 3 & 0.1250 & 0.2500 & 0.6250 \\
\hline 86 & 5 & 7 & 7 & 5 & 0.4000 & 0.4000 & 0.0000 \\
\hline 91 & 12 & 8 & 8 & 19 & 0.3333 & 0.3333 & 0.5833 \\
\hline 96 & 7 & 7 & 8 & 2 & 0.0000 & 0.1429 & 0.7143 \\
\hline
\end{tabular}

Note: d1, d2, d3: The absolute relative error (\%) of the Developed Model, the Kalman Filter and the Nainve predictors, respectively 
Table D7.52. Kalman Filter for test case RCODE $=1$

\begin{tabular}{|c|c|c|c|c|c|c|c|c|c|c|c|}
\hline time & trip & art3 & art2 & art1 & Average & Varidata]n & $g(k+1)$ & $\theta(k+1)$ & $a(k+1)$ & Predict & Observed \\
\hline $8: 33$ & 17 & 0 & 0 & 0 & 0.025 & 0.000625 & 0.5 & 0 & 0.5 & & 0.025 \\
\hline $8: 36$ & 18 & 0 & 0 & 0 & 0.25 & 0.0625 & 0.5 & 0.03125 & 0.5 & 0.0125 & 9 \\
\hline $8: 39$ & 19 & 15 & 17 & 15 & 15.66667 & 1.111111 & 0.506934 & 0.56326 & 0.493066 & 12.0416 & 15 \\
\hline $8: 43$ & 20 & 17 & 19 & 15 & 17 & 2 & 0.561717 & 1.123434 & 0.438283 & 15 & 20 \\
\hline $8: 59$ & 24 & 14 & 18 & 17 & 16.33333 & 4.111111 & 0.560105 & 2.302652 & 0.439895 & 18 & 13 \\
\hline
\end{tabular}

Table D7.53. Kalman Filter for test case RCODE $=6$

\begin{tabular}{|c|c|c|c|c|c|c|c|c|c|c|c|}
\hline time & trip & art3 & art2 & art1 & Average & Var[data]n & $g(k+1)$ & $e(k+1)$ & $a(k+1)$ & Predict & Observed \\
\hline $8: 33$ & 17 & 0 & 0 & 0 & 0.025 & 0.000625 & 0.5 & 0 & 0.5 & & 0.025 \\
\hline $8: 36$ & 18 & 0 & 0 & 0 & 0.25 & 0.0625 & 0.5 & 0.03125 & 0.5 & 0.0125 & 9 \\
\hline $8: 39$ & 19 & 11 & 9 & 10 & 10 & 1 & 0.507692 & 0.507692 & 0.492308 & 9.507692 & 9 \\
\hline $8: 43$ & 20 & 10 & 7 & 11 & 9.333333 & 2.944444 & 0.539685 & 1.589072 & 0.460315 & 10.07937 & 12 \\
\hline $8: 59$ & 24 & 9 & 8 & 14 & 10.33333 & 3.611111 & 0.590172 & 2.131178 & 0.409828 & 13 & 11 \\
\hline
\end{tabular}

Table D7.54. Kalman Filter for test case $\mathrm{RCODE}=11$

\begin{tabular}{|c|c|c|c|c|c|c|c|c|c|c|c|}
\hline time & trip & art3 & art2 & art1 & Average & Var[data]n & $g(k+1)$ & $\theta(k+1)$ & $a(k+1)$ & Predict & Observed \\
\hline $8: 33$ & 17 & 0 & 0 & 0 & 0.025 & 0.000625 & 0.5 & 0 & 0.5 & & 0.025 \\
\hline $8: 36$ & 18 & 0 & 0 & 0 & 0.25 & 0.0625 & 0.5 & 0.03125 & 0.5 & 0.0125 & 9 \\
\hline $8: 39$ & 19 & 8 & 11 & 9 & 9.333333 & 2.277778 & 0.503407 & 1.146648 & 0.496593 & 9 & 11 \\
\hline $8: 43$ & 20 & 14 & 11 & 12 & 12.33333 & 2.277778 & 0.600544 & 1.367906 & 0.399456 & 11.60054 & 12 \\
\hline $8: 59$ & 24 & 11 & 10 & 10 & 10.33333 & 0.277778 & 0.855584 & 0.237662 & 0.144416 & 11 & 9 \\
\hline
\end{tabular}


Table D7.55. Kalman Filter for test case $\mathrm{RCODE}=14$

\begin{tabular}{|r|c|c|c|c|c|c|c|c|c|c|c|}
\hline time & trip & art3 & art2 & art1 & Average & Var[data]n & $\mathbf{g}(\mathbf{k}+1)$ & $\mathbf{e}(\mathbf{k}+1)$ & $\mathbf{a}(\mathbf{k}+1)$ & Predict & Observed \\
\hline $8: 33$ & 17 & 0 & 0 & 0 & 0.025 & 0.000625 & 0.5 & 0 & 0.5 & & 0.025 \\
\hline $8: 36$ & 18 & 0 & 0 & 0 & 0.25 & 0.0625 & 0.5 & 0.03125 & 0.5 & 0.0125 & 9 \\
\hline $8: 39$ & 19 & 11 & 10 & 10 & 10.33333 & 0.277778 & 0.526627 & 0.146285 & 0.473373 & 9.526627 & 11 \\
\hline $8: 43$ & 20 & 12 & 13 & 14 & 13 & 0.5 & 0.563808 & 0.281904 & 0.436192 & 12.69143 & 12 \\
\hline $8: 59$ & 24 & 9 & 12 & 10 & 10.33333 & 2.277778 & 0.529138 & 1.205258 & 0.470862 & 11 & 9 \\
\hline
\end{tabular}

Table D7.56. Kalman Filter for test case $\mathrm{RCODE}=17$

\begin{tabular}{|c|c|c|c|c|c|c|c|c|c|c|c|}
\hline time & trip & art3 & art2 & art1 & Average & Var[data]n & $g(k+1)$ & $e(k+1)$ & $a(k+1)$ & Predict & Observed \\
\hline $8: 33$ & 17 & 0 & 0 & 0 & 0.025 & 0.000625 & 0.5 & 0 & 0.5 & & 0.025 \\
\hline $8: 36$ & 18 & 0 & 0 & 0 & 0.25 & 0.0625 & 0.5 & 0.03125 & 0.5 & 0.0125 & 8 \\
\hline $8: 39$ & 19 & 11 & 11 & 15 & 12.33333 & 1.777778 & 0.504356 & 0.896633 & 0.495644 & 11.53049 & 15 \\
\hline $8: 43$ & 20 & 12 & 14 & 23 & 16.33333 & 12.11111 & 0.517848 & 6.271712 & 0.482152 & 19.14278 & 18 \\
\hline $8: 59$ & 24 & 9 & 12 & 17 & 12.66667 & 6.944444 & 0.655544 & 4.552387 & 0.344456 & 17 & 19 \\
\hline
\end{tabular}

Table D7.57. Kalman Filter for test case $\mathrm{RCODE}=\mathbf{2 3}$

\begin{tabular}{|c|c|c|c|c|c|c|c|c|c|c|c|}
\hline time & trip & art3 & art2 & art1 & Average & Var[data]n & $\mathbf{g}(\mathbf{k + 1})$ & $\boldsymbol{\theta}(\mathbf{k + 1})$ & $\mathbf{a}(\mathbf{k + 1})$ & Predict & Observed \\
\hline $\mathbf{8 : 3 3}$ & 17 & 0 & 0 & 0 & 0.025 & 0.000625 & 0.5 & 0 & 0.5 & 0.025 \\
\hline $\mathbf{8 : 3 6}$ & 18 & 0 & 0 & 0 & 0.25 & 0.0625 & 0.5 & 0.03125 & 0.5 & 0.0125 & $\mathbf{8}$ \\
\hline $\mathbf{8 : 3 9}$ & 19 & 15 & 15 & 15 & 15 & 0 & 1 & 0 & 0 & 15 & 17 \\
\hline $\mathbf{8 : 4 3}$ & 20 & 18 & 22 & 17 & 19 & 5 & 0.5 & 2.5 & 0.5 & 17 & 22 \\
\hline $\mathbf{8 : 5 9}$ & 24 & 19 & 15 & 17 & 17 & 4 & 0.619048 & 2.47619 & 0.380952 & 19 & 15 \\
\hline
\end{tabular}


Table D7.58. Kalman Filter for test case $\mathbf{R C O D E}=\mathbf{2 6}$

\begin{tabular}{|r|c|c|c|c|c|c|c|c|c|c|c|}
\hline tlme & trip & art3 & art2 & art1 & Average & Var[data]n & $\mathbf{g}(\mathbf{k}+1)$ & $\boldsymbol{\theta}(\mathbf{k}+1)$ & $\mathbf{a}(\mathbf{k}+1)$ & Predict & Observed \\
\hline $\mathbf{8 : 3 3}$ & 17 & 0 & 0 & 0 & 0.025 & 0.000625 & 0.5 & 0 & 0.5 & & 0.025 \\
\hline $\mathbf{8 : 3 6}$ & 18 & 0 & 0 & 0 & 0.25 & 0.0625 & 0.5 & 0.03125 & 0.5 & 0.0125 & 8 \\
\hline $\mathbf{8 : 3 9}$ & 19 & 17 & 17 & 17 & 17 & 0 & 1 & 0 & 0 & 17 & 17 \\
\hline $\mathbf{8 : 4 3}$ & 20 & 19 & 19 & 18 & 18.66667 & 0.111111 & 0.5 & 0.055556 & 0.5 & 17.5 & 15 \\
\hline $8: 59$ & 24 & 15 & 17 & 16 & 16 & 1 & 0.513514 & 0.513514 & 0.486486 & 15 & 13 \\
\hline
\end{tabular}

Table D7.59. Kalman Filter for test case $\mathbf{R C O D E}=\mathbf{3 0}$

\begin{tabular}{|c|c|c|c|c|c|c|c|c|c|c|c|}
\hline time & trip & art3 & art2 & art1 & Average & Var[data]n & $g(k+1)$ & $e(k+1)$ & $a(k+1)$ & Predict & Observed \\
\hline $8: 33$ & 17 & 0 & 0 & 0 & 0.025 & 0.000625 & 0.5 & 0 & 0.5 & & 0.025 \\
\hline $8: 36$ & 18 & 0 & 0 & 0 & 0.25 & 0.0625 & 0.5 & 0.03125 & 0.5 & 0.0125 & 8 \\
\hline $8: 39$ & 19 & 17 & 13 & 15 & 15 & 4 & 0.501946 & 2.007782 & 0.498054 & 11.51362 & 19 \\
\hline $8: 43$ & 20 & 21 & 19 & 18 & 19.33333 & 1.444444 & 0.705015 & 1.018355 & 0.294985 & 18.29498 & 19 \\
\hline $8: 59$ & 24 & 15 & 17 & 14 & 15.33333 & 1.444444 & 0.630316 & 0.910457 & 0.369684 & 16 & 17 \\
\hline
\end{tabular}

Table D7.60. Kalman Filter for test case RCODE $=37$

\begin{tabular}{|r|c|c|c|c|c|c|c|c|c|c|c|}
\hline time & trip & art3 & art2 & art1 & Average & Var[data]n & $\mathbf{g}(\mathbf{k}+1)$ & $\mathbf{e}(\mathbf{k}+1)$ & $\mathbf{a}(\mathbf{k}+\mathbf{1})$ & Predict & Observed \\
\hline $\mathbf{8 : 3 3}$ & 17 & 0 & 0 & 0 & 0.025 & 0.000625 & 0.5 & 0 & 0.5 & & 0.025 \\
\hline $\mathbf{8 : 3 6}$ & 18 & 0 & 0 & 0 & 0.25 & 0.0625 & 0.5 & 0.03125 & 0.5 & 0.0125 & 8 \\
\hline $\mathbf{8 : 3 9}$ & 19 & 15 & 7 & 9 & 10.33333 & 16.44444 & 0.500475 & 8.230027 & 0.499525 & 8.500475 & 8 \\
\hline $\mathbf{8 : 4 3}$ & 20 & 18 & 9 & 11 & 12.66667 & 20.94444 & 0.582105 & 12.19187 & 0.417895 & 9.746315 & 9 \\
\hline $\mathbf{8 : 5 9}$ & 24 & 17 & 9 & 10 & 12 & 17 & 0.63197 & 10.74349 & 0.36803 & 10 & $\mathbf{8}$ \\
\hline
\end{tabular}


Table D7.61. Kalman Filter for test case $\mathrm{RCODE}=\mathbf{4 1}$

\begin{tabular}{|c|c|c|c|c|c|c|c|c|c|c|c|}
\hline time & trip & art3 & art2 & art1 & Average & Var[data]n & $\mathbf{g}(\mathbf{k}+1)$ & $\boldsymbol{\theta}(\mathbf{k}+1)$ & $\mathbf{a}(\mathbf{k}+1)$ & Predict & Observed \\
\hline $8: 33$ & 17 & 0 & 0 & 0 & 0.025 & 0.000625 & 0.5 & 0 & 0.5 & 0.025 \\
\hline $8: 36$ & 18 & 0 & 0 & 0 & 0.25 & 0.0625 & 0.5 & 0.03125 & 0.5 & 0.0125 & 8 \\
\hline $8: 39$ & 19 & 7 & 9 & 6 & 7.333333 & 1.444444 & 0.505351 & 0.729951 & 0.494649 & 6.989298 & 9 \\
\hline $8: 43$ & 20 & 9 & 8 & 11 & 9.333333 & 0.944444 & 0.639365 & 0.603845 & 0.360635 & 10.27873 & 9 \\
\hline $8: 59$ & 24 & 9 & 8 & 9 & 8.666667 & 0.277778 & 0.760413 & 0.211226 & 0.239587 & 9 & 8 \\
\hline
\end{tabular}

Table D7.62. Kalman Filter for test case RCODE $=44$

\begin{tabular}{|c|c|c|c|c|c|c|c|c|c|c|c|}
\hline time & trip & art3 & art2 & art1 & Average & Var[data]n & $g(k+1)$ & $e(k+1)$ & $a(k+1)$ & Predict & Observed \\
\hline $8: 33$ & 17 & 0 & 0 & 0 & 0.025 & 0.000625 & 0.5 & 0 & 0.5 & & 0.025 \\
\hline $8: 36$ & 18 & 0 & 0 & 0 & 0.25 & 0.0625 & 0.5 & 0.03125 & 0.5 & 0.0125 & 9 \\
\hline $8: 39$ & 19 & 9 & 9 & 9 & 9 & 0 & 1 & 0 & 0 & 9 & 7 \\
\hline $8: 43$ & 20 & 9 & 11 & 9 & 9.666667 & 1.111111 & 0.5 & 0.555556 & 0.5 & 8 & 9 \\
\hline $8: 59$ & 24 & 7 & 9 & 7 & 7.666667 & 1.111111 & 0.6 & 0.666667 & 0.4 & 9 & 8 \\
\hline
\end{tabular}

Table D7.63. Kalman Filter for test case RCODE $=\mathbf{5 5}$

\begin{tabular}{|c|c|c|c|c|c|c|c|c|c|c|c|}
\hline time & trip & art3 & art2 & art1 & Average & Var[data]n & $g(k+1)$ & $e(k+1)$ & $a(k+1)$ & Predict & Observed \\
\hline $8: 33$ & 17 & 0 & 0 & 0 & 0.025 & 0.000625 & 0.5 & 0 & 0.5 & & 0.025 \\
\hline $8: 36$ & 18 & 0 & 0 & 0 & 0.25 & 0.0625 & 0.5 & 0.03125 & 0.5 & 0.0125 & 9 \\
\hline $8: 39$ & 19 & 17 & 15 & 13 & 15 & 2 & 0.503876 & 1.007752 & 0.496124 & 11.0155 & 13 \\
\hline $8: 43$ & 20 & 14 & 18 & 21 & 17.66667 & 6.777778 & 0.534599 & 3.623393 & 0.465401 & 17.27679 & 15 \\
\hline $8: 59$ & 24 & 19 & 15 & 15 & 16.33333 & 4.444444 & 0.644793 & 2.865749 & 0.355207 & 15 & 15 \\
\hline
\end{tabular}


Table D7.64. Kalman Filter for test case $\mathrm{RCODE}=\mathbf{5 9}$

\begin{tabular}{|c|c|c|c|c|c|c|c|c|c|c|c|}
\hline time & trip & art3 & art2 & art1 & Average & Var[data]n & $\mathbf{g}(\mathbf{k}+1)$ & $\mathbf{e}(\mathbf{k}+1)$ & $\mathbf{a}(\mathbf{k}+1)$ & Predict & Observed \\
\hline $\mathbf{8 : 3 3}$ & 17 & 0 & 0 & 0 & 0.025 & 0.000625 & 0.5 & 0 & 0.5 & 0.025 \\
\hline $\mathbf{8 : 3 6}$ & 18 & 0 & 0 & 0 & 0.25 & 0.0625 & 0.5 & 0.03125 & 0.5 & 0.0125 & 9 \\
\hline $\mathbf{8 : 3 9}$ & 19 & 13 & 15 & 17 & 15 & 2 & 0.503876 & 1.007752 & 0.496124 & 13.03101 & 15 \\
\hline $\mathbf{8 : 4 3}$ & 20 & 16 & 17 & 19 & 17.33333 & 0.944444 & 0.673952 & 0.63651 & 0.326048 & 17.69581 & 15 \\
\hline $\mathbf{8 : 5 9}$ & 24 & 16 & 14 & 18 & 16 & 2 & 0.568641 & 1.137282 & 0.431359 & $\mathbf{1 7}$ & $\mathbf{1 7}$ \\
\hline
\end{tabular}

Table D7.65: Boarding Passenger Prediction Results of the 3 Predictors

\begin{tabular}{|c|c|c|c|c|c|c|c|}
\hline mapole & $\begin{array}{l}\text { Actual } \\
\text { number of } \\
\text { De-boarders }\end{array}$ & $\begin{array}{l}\text { Doveloped } \\
\text { Model }\end{array}$ & $\begin{array}{l}\text { kr } \\
\text { Moolel }\end{array}$ & $\begin{array}{l}\text { Naves } \\
\text { nores }\end{array}$ & an: & (2) & $d 8$ \\
\hline 1 & 11 & 11 & 18 & 17 & 0.0000 & 0.3846 & 0.5455 \\
\hline 6 & 11 & 10 & 13 & 15 & 0.0909 & 0.0833 & 0.6667 \\
\hline 11 & 9 & 11 & 10 & 10 & 0.2222 & 0.1667 & 0.0909 \\
\hline 14 & 9 & 11 & 11 & 9 & 0.2222 & 0.1667 & 0.1818 \\
\hline 17 & 19 & 16 & 17 & 13 & 0.1579 & 0.0556 & 0.1333 \\
\hline 20 & 15 & 16 & 19 & 22 & 0.0667 & 0.1364 & 0.2941 \\
\hline 23 & 15 & 17 & 18 & 19 & 0.1333 & 0.0526 & 0.1176 \\
\hline 26 & 13 & 16 & 15 & 13 & 0.2308 & 0.1176 & 0.1333 \\
\hline 30 & 17 & 16 & 16 & 15 & 0.0588 & 0.1579 & 0.2105 \\
\hline 37 & 8 & 8 & 10 & 8 & 0.0000 & 0.1111 & 0.0000 \\
\hline 41 & 8 & 8 & 9 & 7 & 0.0000 & 0.0000 & 0.2222 \\
\hline 44 & 9 & 9 & 9 & 9 & 0.0000 & 0.1818 & 0.1250 \\
\hline 49 & 8 & 8 & 8 & 7 & 0.0000 & 0.1111 & 0.0000 \\
\hline 55 & 15 & 15 & 15 & 11 & 0.0000 & 0.0000 & 0.1538 \\
\hline 59 & 17 & 16 & 17 & 14 & 0.0588 & 0.1333 & 0.0667 \\
\hline
\end{tabular}

Note: $\mathrm{d} 1, \mathrm{~d} 2, \mathrm{~d} 3$ : The absolute relative error (\%) of the Developed Model, the Kalman Filter and the Naïve predictors, respectively 


\section{Appendix D8}

\section{General}

This appendix covers the mathematical concepts of the Tukey test used for the purpose of evaluating the models' performance. As discussed in chapter 6, section 6.4.3.6 and section 6.4.1, MAPE is the most suitable criterion to be compared so that the performance of the models can be ranked.

\section{Tukey Test Procedure}

The results presented in chapter 6 were obtained by following the steps suggested by Netter et al., (1985).

Step 1: Statistical hypothesis

Ho: MAPE $_{\mathrm{N}} \mathrm{MAPE}_{\mathrm{K}}=\mathrm{MAPE}_{\mathrm{P}}$

$\boldsymbol{H a}: \mathrm{MAPE}_{\mathrm{N}} \geq \mathrm{MAPE}_{\mathrm{K}} \geq \mathrm{MAPE}_{\mathrm{K}}$ or MAPE $\mathrm{K} \geq \mathrm{MAPE}_{\mathrm{P}} \geq \mathrm{MAPE}_{\mathrm{P}}$

Where $\mathrm{N}=$ Naïve model, $\mathrm{K}=$ Kalman Filter Model, $\mathrm{P}=$ Developed model

Let $\quad i=$ The number of models being compared; $i=3$

$j=$ the number of elements of the sample test

Select level of significance $\alpha=0.05$, then select suitable $Q_{\alpha, i, i . j-1)}$ from the studentized range distribution.

Step 2: Determine range $\mathrm{w}=Q_{\alpha} \sqrt{M S E / j}$ where MSE $=$ mean square error within group. 
Step 3: List the sample's MAPE in the increasing order and make pairwise comparisons. If the difference is smaller than the predetermined range, the null hypothesis cannot be rejected, or there is no statically significant difference between the pairwise models at $\alpha=0.05$. Such a difference is underlined. 


\section{Appendix D9}

\section{General}

This appendix covers the predicted arrival times of 15 full trips at three selected stops. These are Holmwood, Gladstone, and Rideau stops.

\section{Prediction Results}

The results are tabulated in Tables D9.1-D9.45. The left hand side part of the tables shows the predicted running times between stops and dwell times at the stops by applying developed running time and dwell time prediction modules, respectively. The right hand side part of the tables presents the predicted arrival time obtained from the general equations 3.4 and 3.5 (chapter 3). The updated predictions followed the procedure presented in chapter 3 , section 3.3.4.

The following legends are used in Tables D9.1 to D9.45

T6-R1-54: Running time from stop 1 to stop 54, trip 6;

T6-R54-3: Running time from stop 54 to stop 3, trip 6

Boar: Number of boarding Passenger at a stop

Ali: Number of alighting passenger at a stop

Dwell: Dwell time

Arv.Holwood: Arival time at Holmwood stop 
(number): The number showing the times the prediction is updated.

Error: Prediction Error, the difference between the predicted arrival time at last stop to that of schedule time.

\%. Relative Prediction Error

Numbers in the colored boxes are the predicted values

Numbers in non-colored boxes are the actual values 
Table D9.1- D9.15. Real-time Bus Arrival Prediction at Stops: Holmwood, Gladstone, and Rideau. Route 1-Direction: Downtown 8:59 a.m. bus trips. The Developed Model

Table D9.1

\begin{tabular}{|c|c|c|c|c|c|c|c|c|c|c|c|c|c|}
\hline dcode & Update & $t 6-R 1-54$ & boar54 & all.54 & dwell 54 & $T 6-R 54-3(1)$ & boar3 & al/3 & dwell3 & $T 6 . R 3-53(1)$ & $\begin{array}{l}\text { Arv. } \\
\text { Holmwood }\end{array}$ & $\begin{array}{l}\text { Arv } \\
\text { Gladstone }\end{array}$ & $\begin{array}{l}\text { Arv. } \\
\text { RIdeau }\end{array}$ \\
\hline \multirow[t]{7}{*}{17} & 1st & 556.61 & 11 & & 34.5 & 368.75 & 7 & 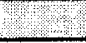 & 24.5 & 603.94 & 556.61 & 959.86 & 1588.30 \\
\hline & & 630.5 & 13 & & 39.5 & & 10 & & 32 & & & & \\
\hline & & & & & & $T 6-R 54-3(2)$ & & & & T6-R3-53(2) & & & \\
\hline & 2nd & & & & & 354.55 & C. & 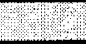 & 7 & 583.95 & & 1024.55 & 1615.50 \\
\hline & & & & & & & & & & T6-R3-53(3) & & & \\
\hline & last & & & & & & & & & 585.62 & & & 1255.62 \\
\hline & & & & & & & & & & 569.5 & 630.5 & 670 & 1239.50 \\
\hline
\end{tabular}

Table D9.2

\begin{tabular}{|c|c|c|c|c|c|c|c|c|c|c|c|c|c|}
\hline dcode & Update & $t 6-R 1-54$ & boar 54 & ali. 54 & $d w e l / 54$ & $T 6-R 54-3(1)$ & boar3 & all3 & dwe//3 & $T 6-R 3-53(1)$ & $\begin{array}{l}\text { Arv. } \\
\text { Holmwood }\end{array}$ & $\begin{array}{l}\text { Arv. } \\
\text { Gladstone }\end{array}$ & Arv. Rideau \\
\hline 12 & 1st & 513.47 & 2 & प्र? & 12 & 457.22 & 2.7 & 3.1. & 12 & 605.42 & 513.47 & 982.69 & 1600.11 \\
\hline & & 604.5 & 2 & & 12 & & 3 & & 14.5 & & & & \\
\hline & & & & & & $T 6 . R 54-3(2)$ & & & & $T 6 \cdot R 3-53(2)$ & & & \\
\hline & 2nd & & & & & 407.63 & 4 & 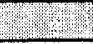 & 17 & 585.36 & & 1024.13 & 1626.49 \\
\hline & & & & & & 397.5 & 3 & & 14.5 & T6-R3-53(3) & & & \\
\hline & last & & & & & & & & & 614,92 & & & 1643.42 \\
\hline & & & & & & & & & & 673 & 604.5 & 1014 & 1701.50 \\
\hline
\end{tabular}




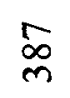

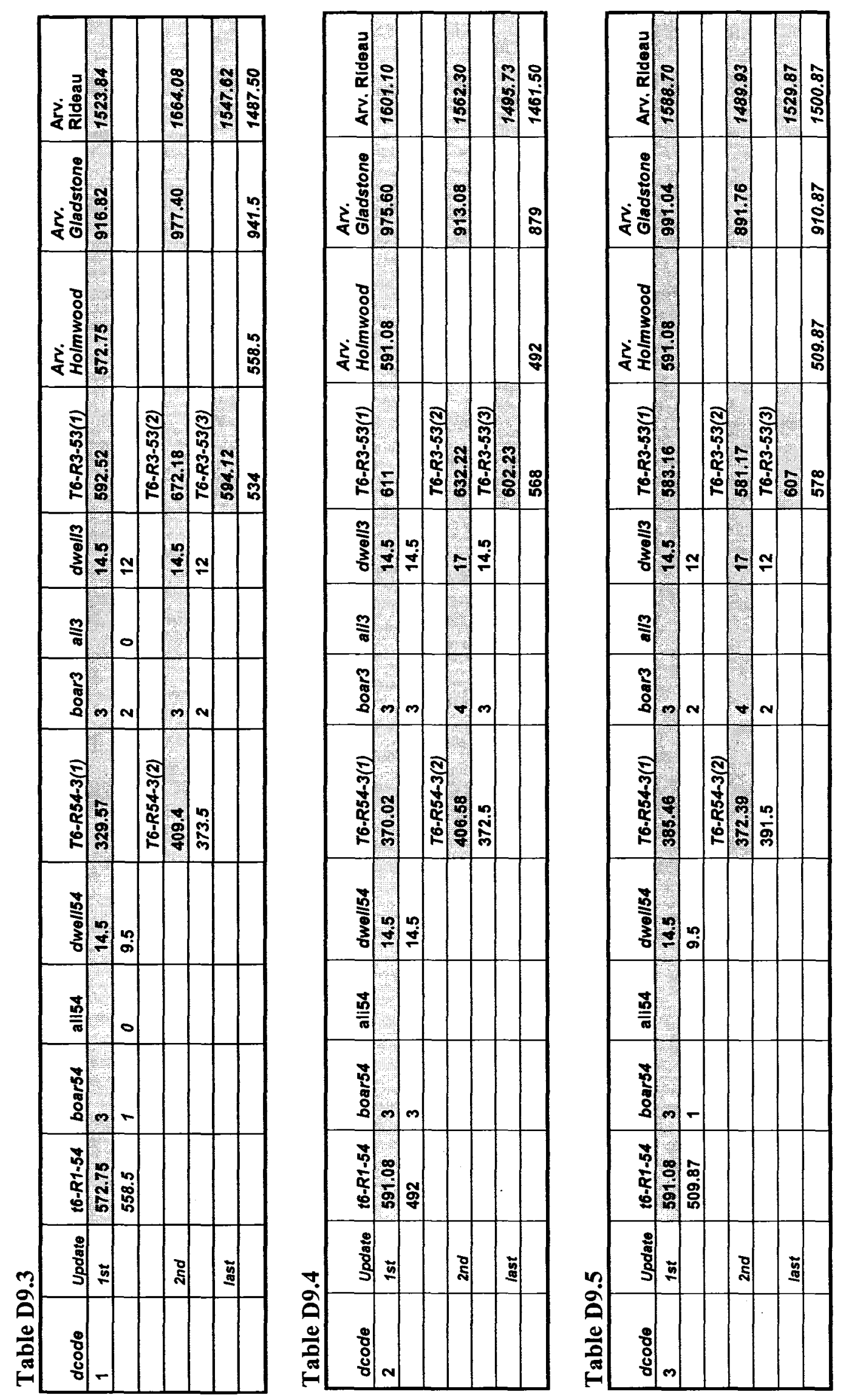


$\infty$
$\infty$
$m$
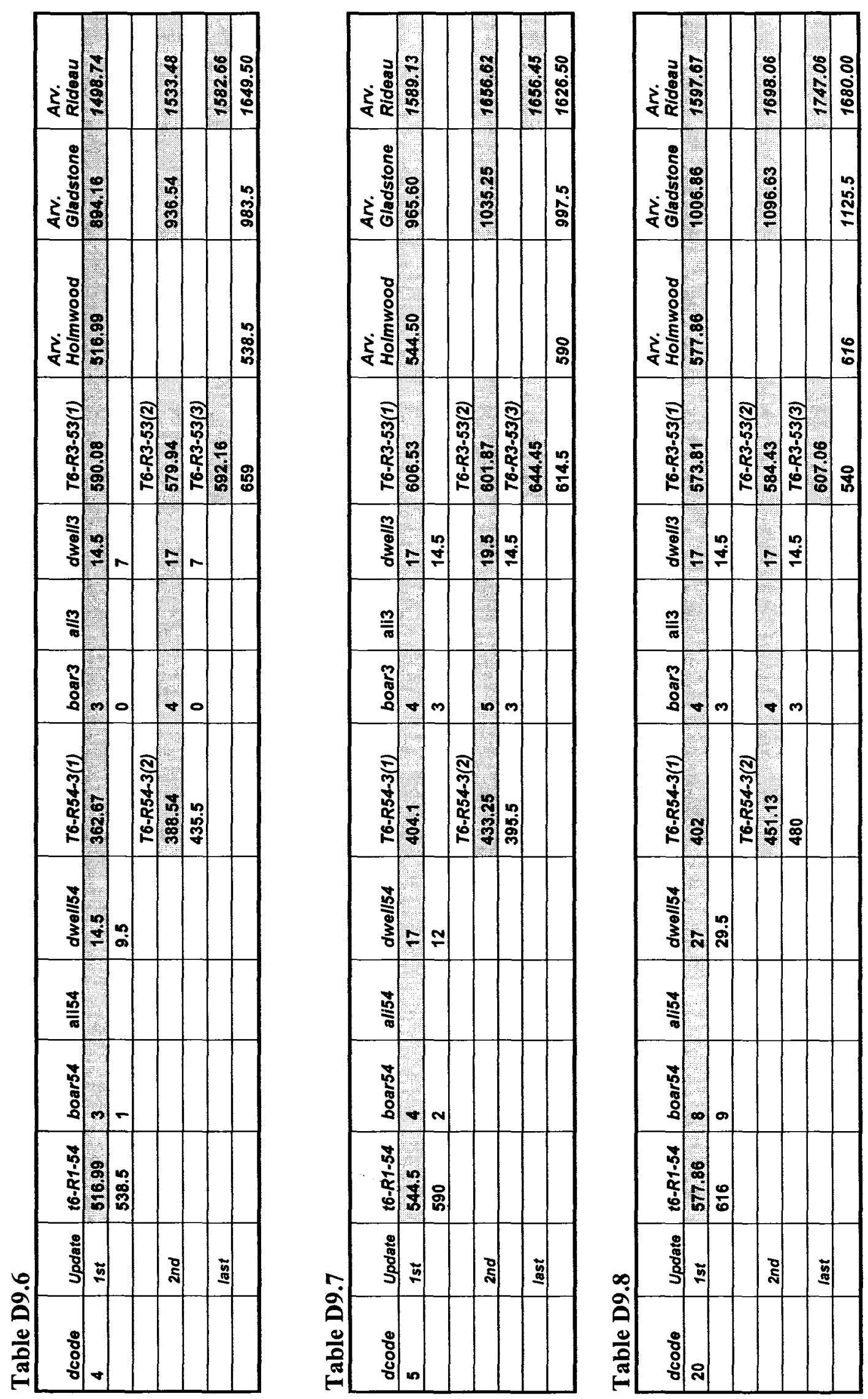
ळ
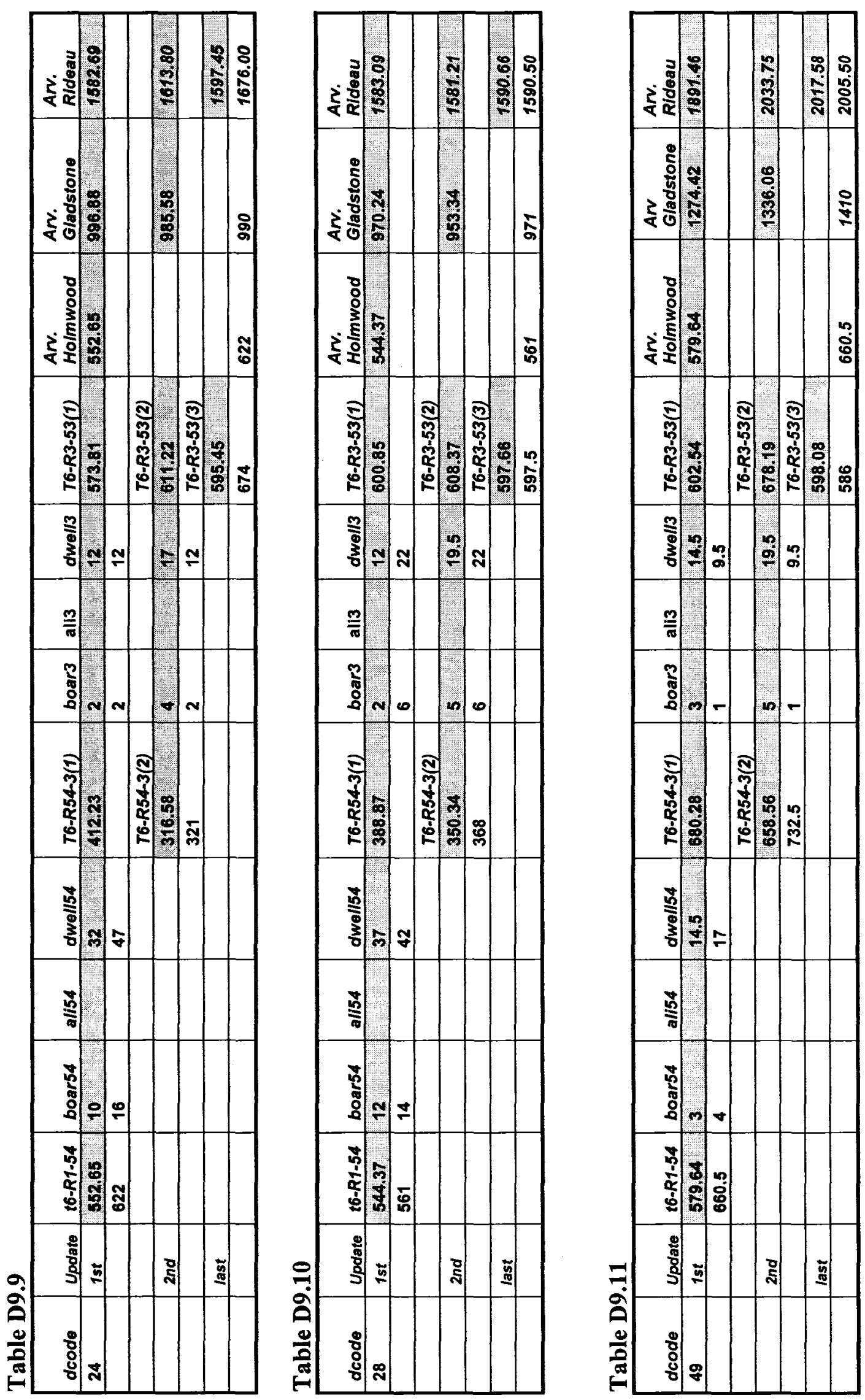
雍

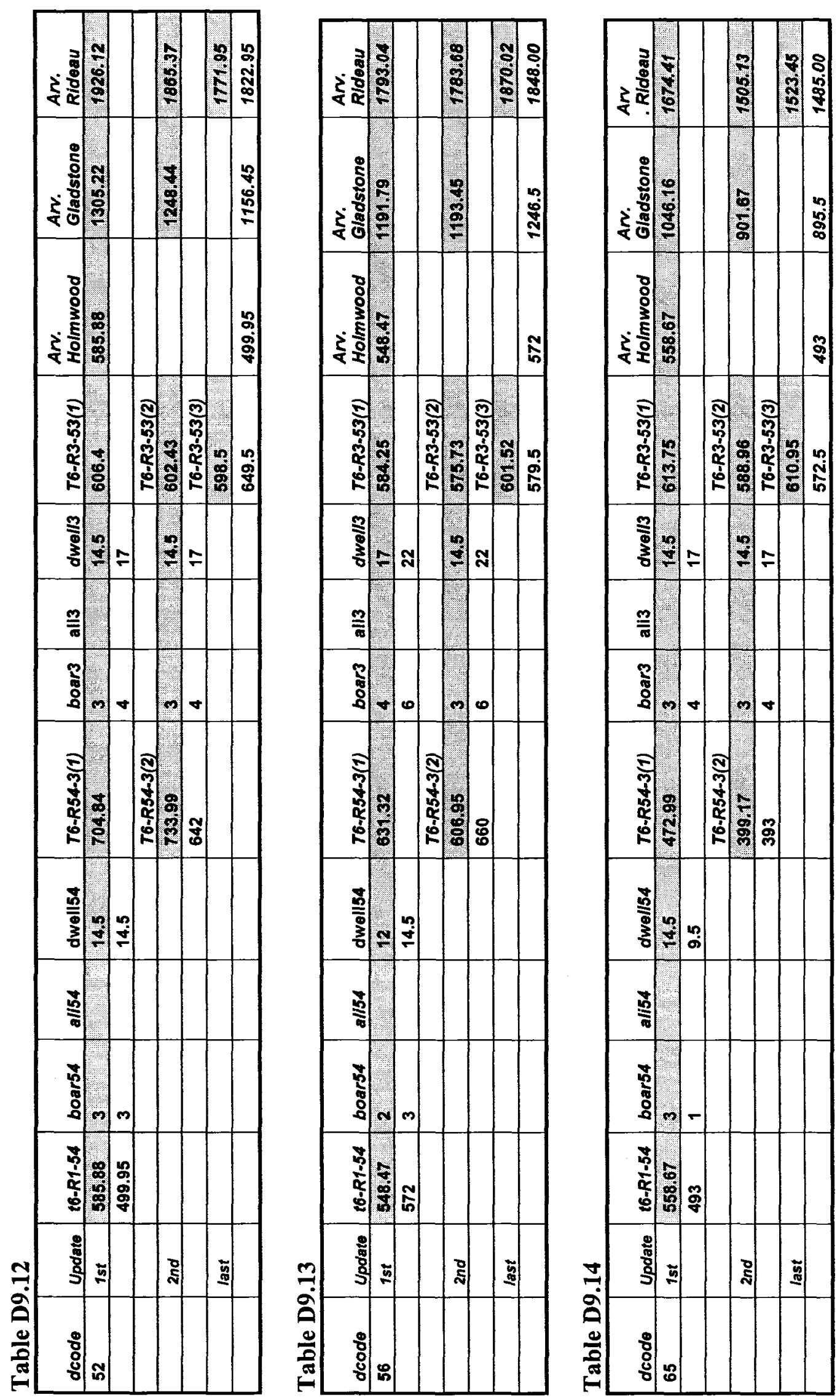


$\bar{\sigma}$

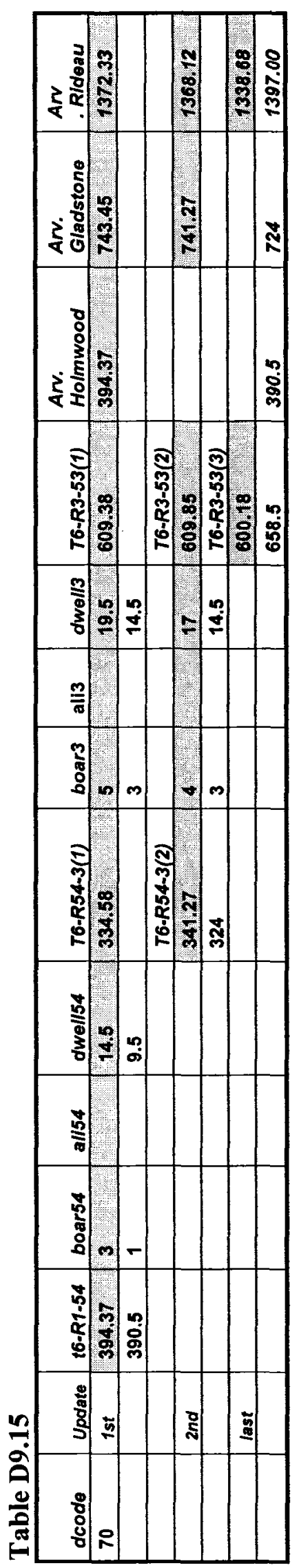


Table D9.16 -D9.30. Real-time Bus Arrival Prediction at Stops: Holmwood, Gladstone, and Rideau. Route 1-Direction: Downtown 8:59 a.m. bus trips. Kalman Filter-based model

Table D9.16

\begin{tabular}{|c|c|c|c|c|c|c|c|c|c|c|c|c|c|}
\hline dcode & t6-R1-54 & boar54 & ali.54 & dwell 54 & T6-R54-3(1) & boar3 & $a / / 3$ & dwell3 & T6-R3-53(1) & $\begin{array}{l}\text { Arv. } \\
\text { Holmwood }\end{array}$ & $\begin{array}{l}\text { Arv. } \\
\text { Gladstone }\end{array}$ & $\begin{array}{l}\text { Arv. } \\
\text { Rideau }\end{array}$ & Update \\
\hline 17 & 508.01 & 11 & 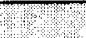 & 34.5 & 412.6 & 7 & 20 & 24.5 & 547.52 & 508.01 & 955.11 & 1527.13 & $1 \mathrm{st}$ \\
\hline & 630.5 & 13 & & 39.5 & & 10 & & 32 & & & & & \\
\hline & & & & & T6-R54-3(2) & & & & T6.R3-53(2) & & & & \\
\hline & & & & & 406.79 & $w^{2}$ & 20 & 7 & 543.44 & & 1076.79 & 1627,23 & $2 s t$ \\
\hline & & & & & 342.5 & & & & T6-R3-53(3) & & & & \\
\hline & & & & & & & & & 544.06 & & & 1556.56 & last \\
\hline & & & & & & & & & 569.5 & 630.5 & 1012.5 & 1582.00 & \\
\hline
\end{tabular}

Table D9.17

\begin{tabular}{|c|c|c|c|c|c|c|c|c|c|c|c|c|c|}
\hline dcode & t6-R1-54 & $\begin{array}{l}\text { boar } \\
54\end{array}$ & ali. 54 & $d w e / / 54$ & T6-R54-3(1) & boar3 & ali3 & dwel/3 & T6-R3-53(1) & $\begin{array}{l}\text { Arv. } \\
\text { Holmwood }\end{array}$ & $\begin{array}{l}\text { Arv. } \\
\text { Gladstone }\end{array}$ & $\begin{array}{l}\text { Arv. } \\
\text { Rideau }\end{array}$ & Updated \\
\hline 12 & 480.39 & 2 & $\sqrt{1.04}$ & 12 & 367.15 & 2 & Pry & 12 & 574.08 & 480.39 & 859.54 & 1415.62 & $1 s t$ \\
\hline & & & & & T6-R54-3(2) & & & & $T 6-R 3-53(2)$ & & & & \\
\hline & & & & & 393.51 & 4 & ren & 17 & 577.56 & & 1010.01 & 1604.57 & $2 s t$ \\
\hline & & & & & & 3 & & 14.5 & T6-R3-53(3) & & & & \\
\hline & & & & & & & & & 885.39 & & & 121,33 & last \\
\hline
\end{tabular}


ồ

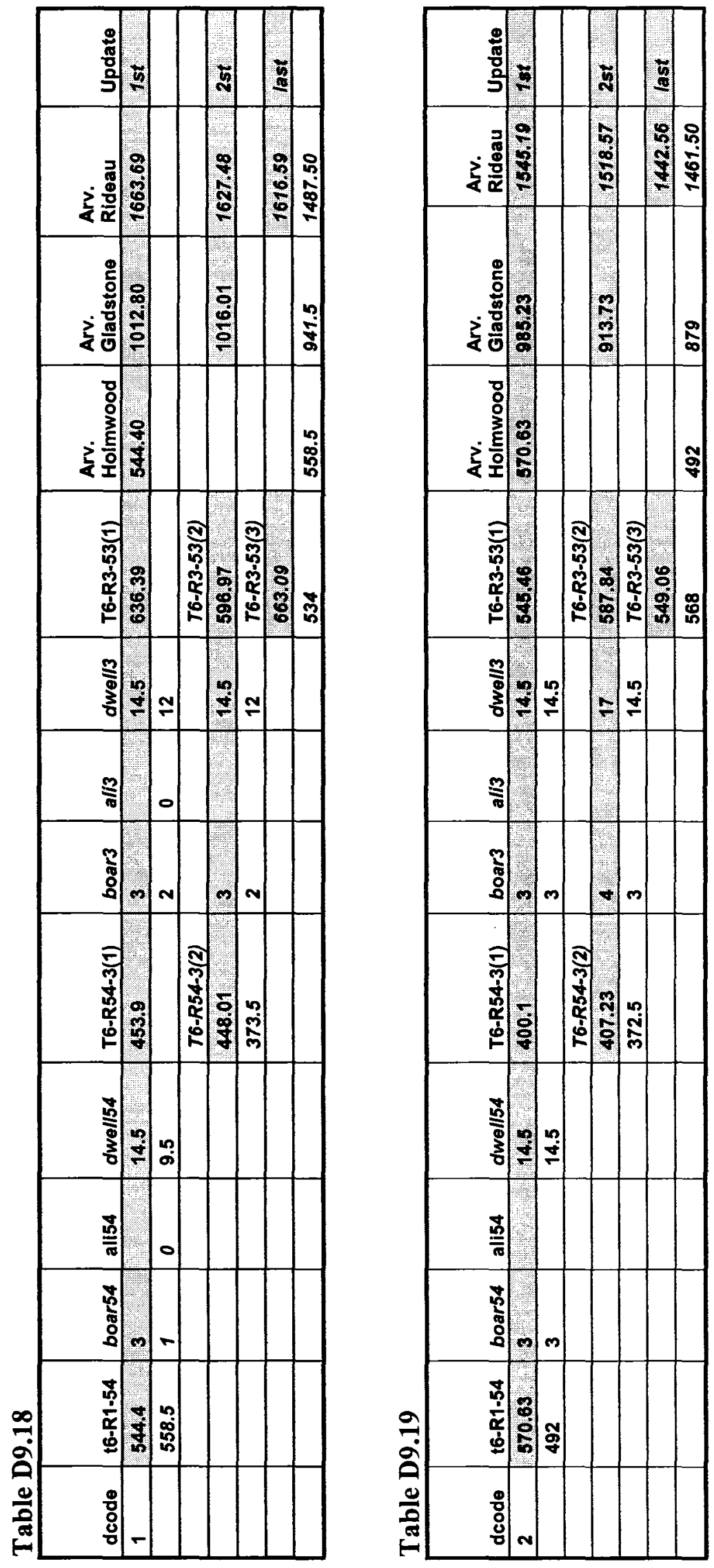


市
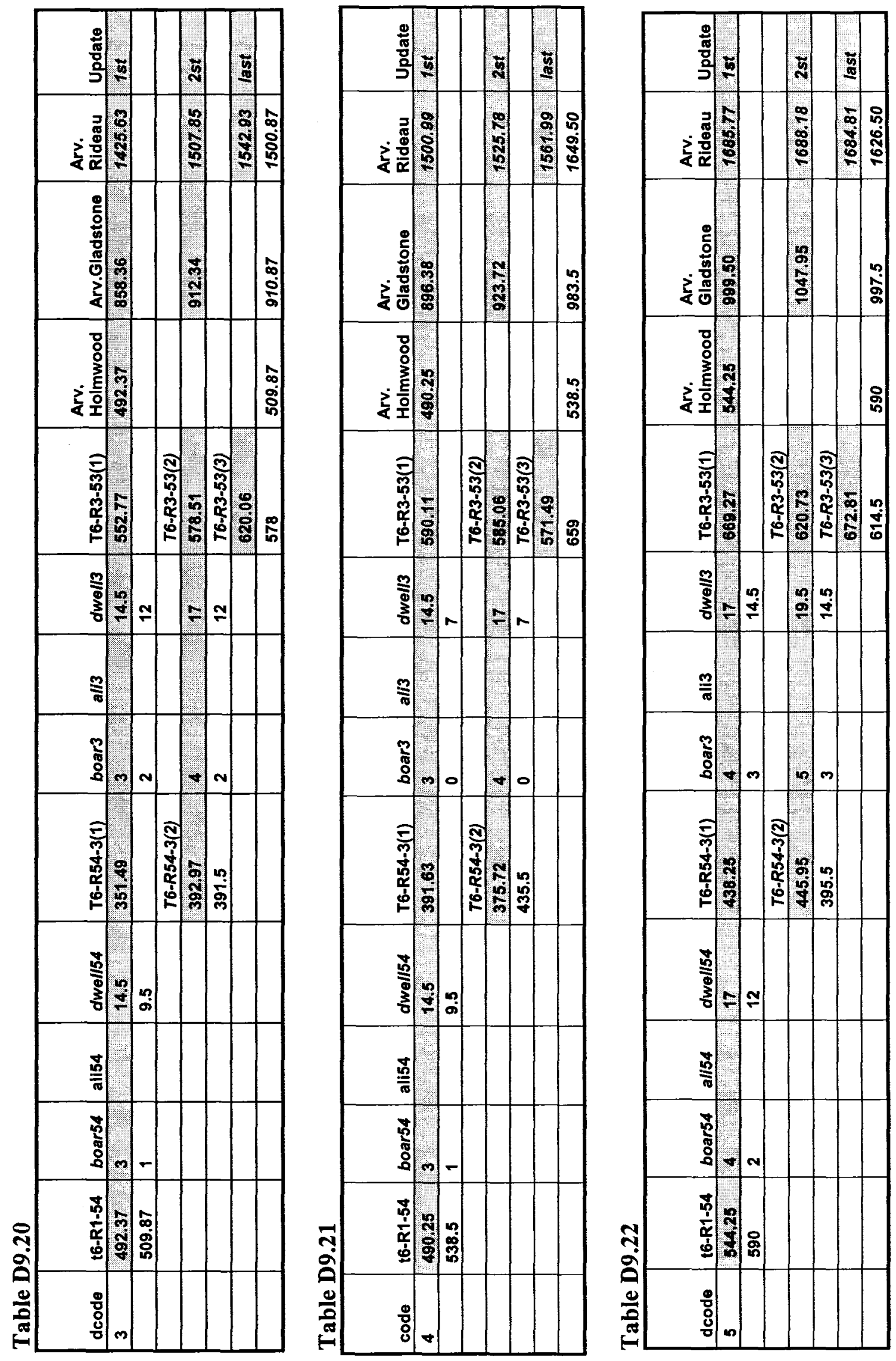
$\stackrel{n}{n}$
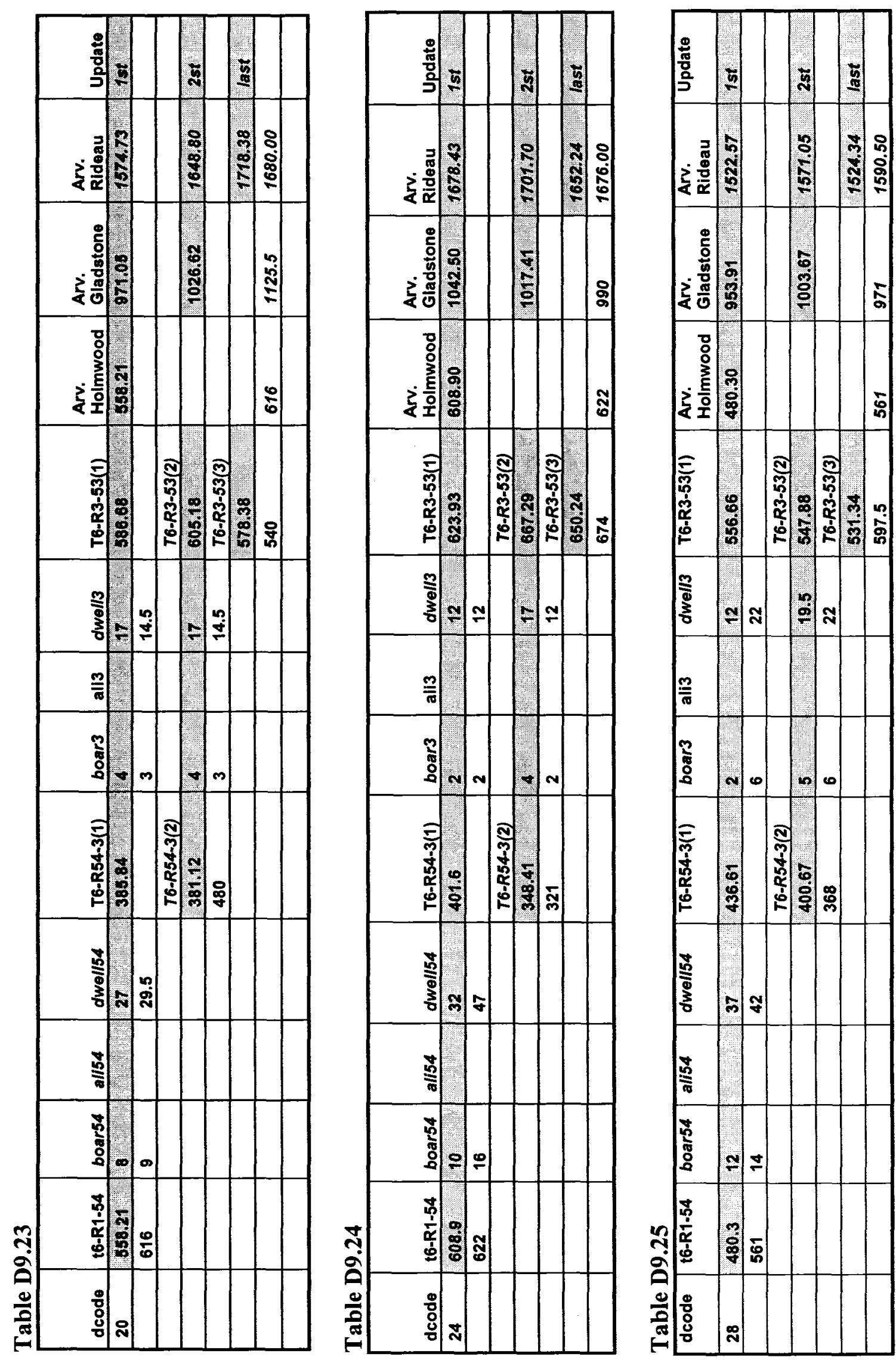

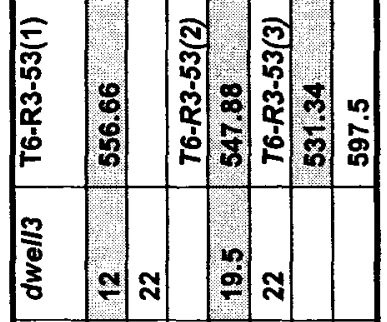

$\frac{m}{\frac{0}{0}}$

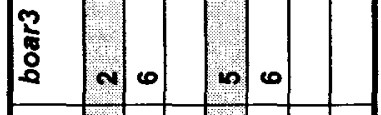

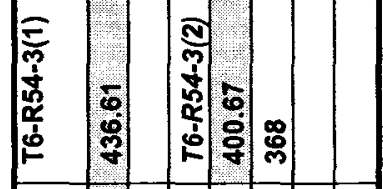

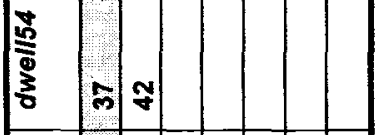
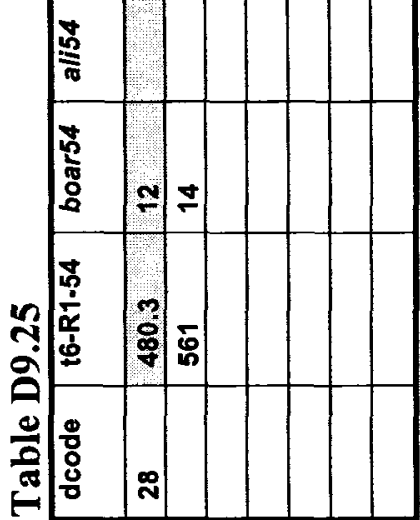
용
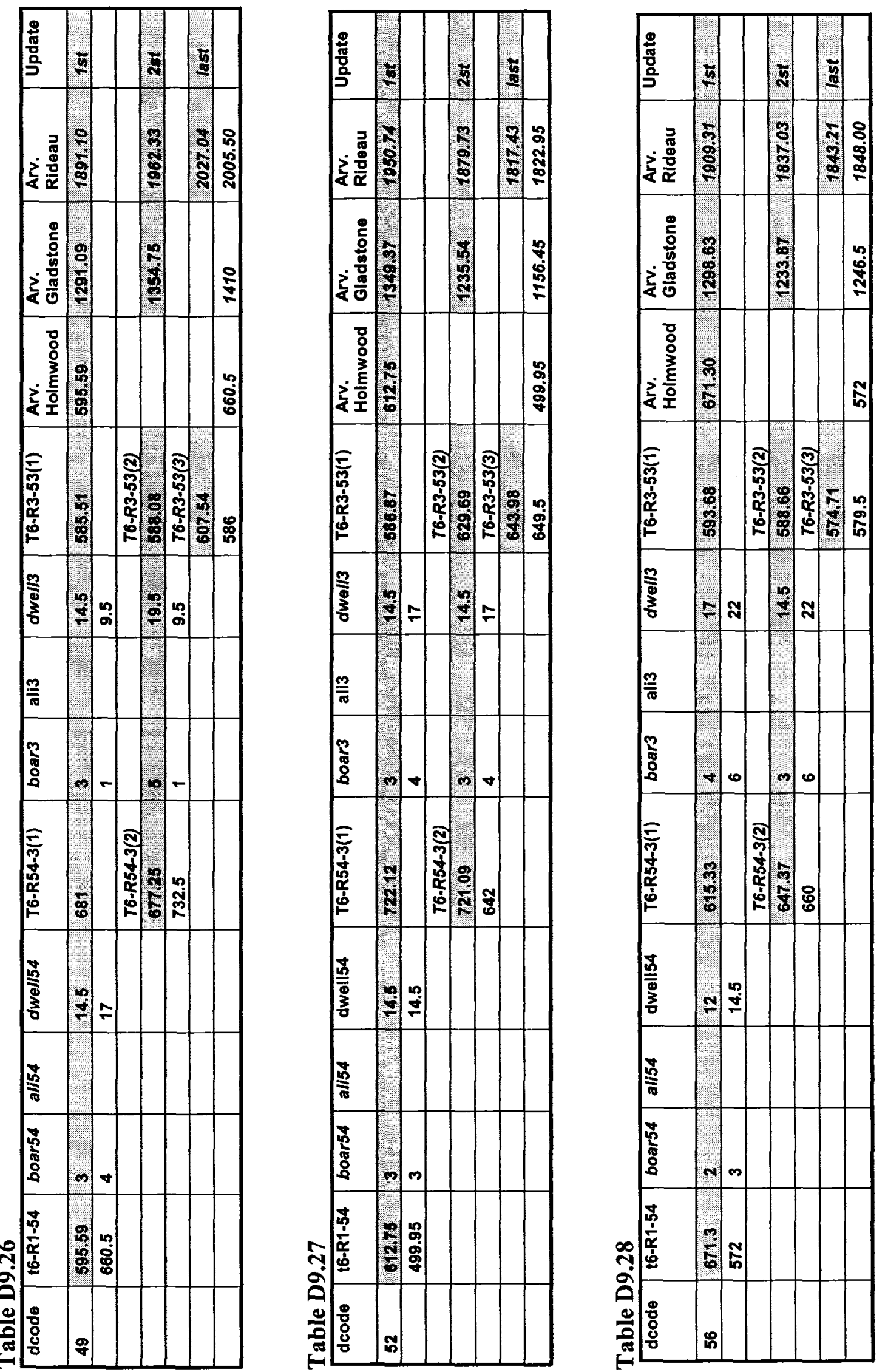
ล̀
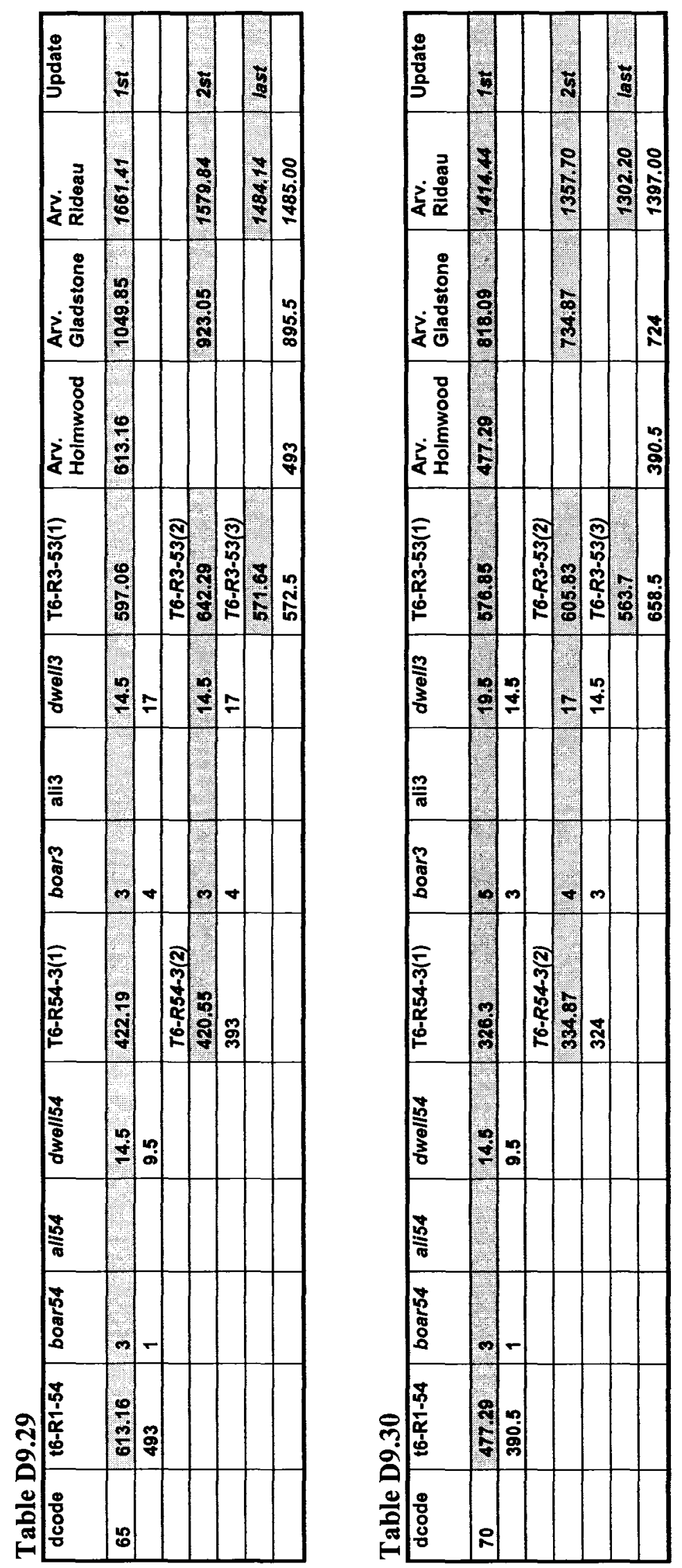
Table D9.31 -D9.45. Real-time Bus Arrival Prediction at Stops: Holmwood, Gladstone, and Rideau. Route 1-Direction: Downtown 8:59 a.m. bus trips. The Naïve model

Table D9.31

\begin{tabular}{|c|c|c|c|c|c|c|c|c|c|c|c|c|c|}
\hline dcode & t6-R1-54 & boar54 & ali.54 & dwell 54 & T6-R54-3(1) & boar3 & ali3 & dwell3 & $T 6-R 3-53(1)$ & $\begin{array}{l}\text { Arv. } \\
\text { Holmwood }\end{array}$ & $\begin{array}{l}\text { Arv. } \\
\text { Gladstone }\end{array}$ & $\begin{array}{l}\text { Arv. } \\
\text { Rideau }\end{array}$ & Update \\
\hline \multirow[t]{7}{*}{17} & 465.59 & 11 & (1) & 34.5 & 328.23 & 7 & 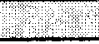 & 24.5 & 519.84 & 465.59 & 828.32 & 1372.66 & $1 s t$ \\
\hline & 630.5 & 13 & & 39.5 & & 10 & & 32 & & & & & \\
\hline & & & & & $T 6-R 54-3(2)$ & & & & $T 6-R 3-53(2)$ & & & & \\
\hline & & & & & 439.5 & 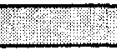 & $7=0.8$ & tur & 460.5 & & 1109.50 & 1577.00 & $2 s t$ \\
\hline & & & & & 342.5 & & & & $T 6-R 3-53(3)$ & & & & \\
\hline & & & & & & & & & 620 & & & 1632.50 & last \\
\hline & & & & & & & & & 569.5 & 630.5 & 1012.5 & 1582.00 & \\
\hline
\end{tabular}

Table D9.32

\begin{tabular}{|c|c|c|c|c|c|c|c|c|c|c|c|c|c|}
\hline dcode & t6-R1-54 & $\begin{array}{l}\text { boar } \\
54\end{array}$ & ali. 54 & dwell54 & T6-R54-3(1) & boar3 & al/3 & $d w e / / 3$ & T6-R3-53(1) & $\begin{array}{l}\text { Arv. } \\
\text { Holmwood }\end{array}$ & $\begin{array}{l}\text { Arv. } \\
\text { Gladstone }\end{array}$ & $\begin{array}{l}\text { Arv. } \\
\text { Rideau }\end{array}$ & Updated \\
\hline \multirow[t]{7}{*}{12} & 436.09 & 2 & 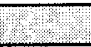 & 12 & 353.7 & 2 & 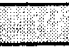 & 12 & 558.89 & 436.09 & 801.78 & 1372.68 & $1 \mathrm{st}$ \\
\hline & 604.5 & 2 & & 12 & & 3 & & 14.5 & & & & & \\
\hline & & & & & $T 6-R 54-3(2)$ & & & & $T 6-R 3-53(2)$ & & & & \\
\hline & & & & & 350.94 & 4 & 02 & 17 & 529.48 & & 967.44 & 1513.93 & $2 s t$ \\
\hline & & & & & 397.5 & 3 & & 14.5 & $T 6-R 3-53(3)$ & & & & \\
\hline & & & & & & & & & 550.56 & & & 1579.06 & last \\
\hline & & & & & & & & & 673 & 604.5 & 1014 & 1701.50 & \\
\hline
\end{tabular}


๙
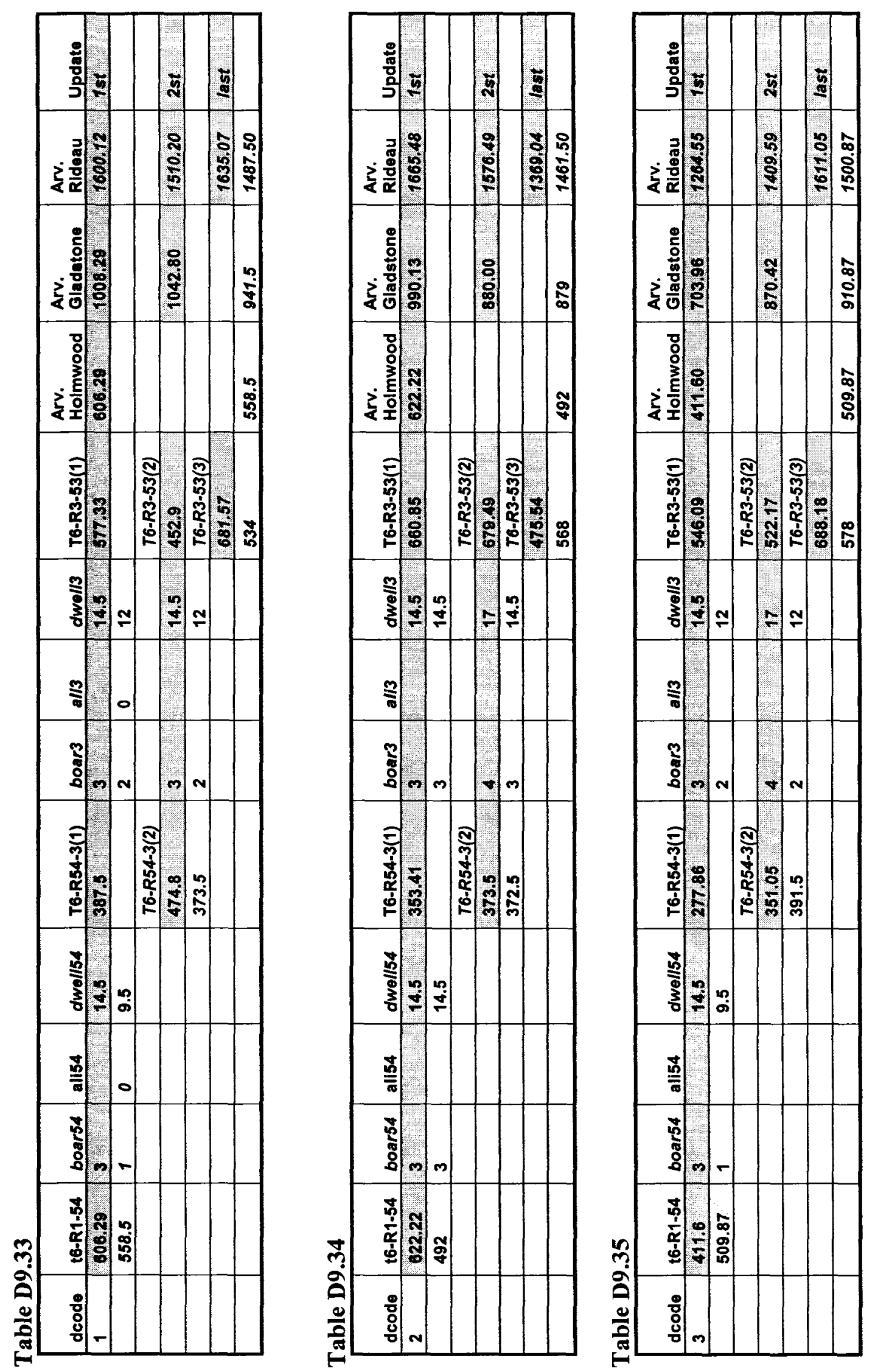
ఫ

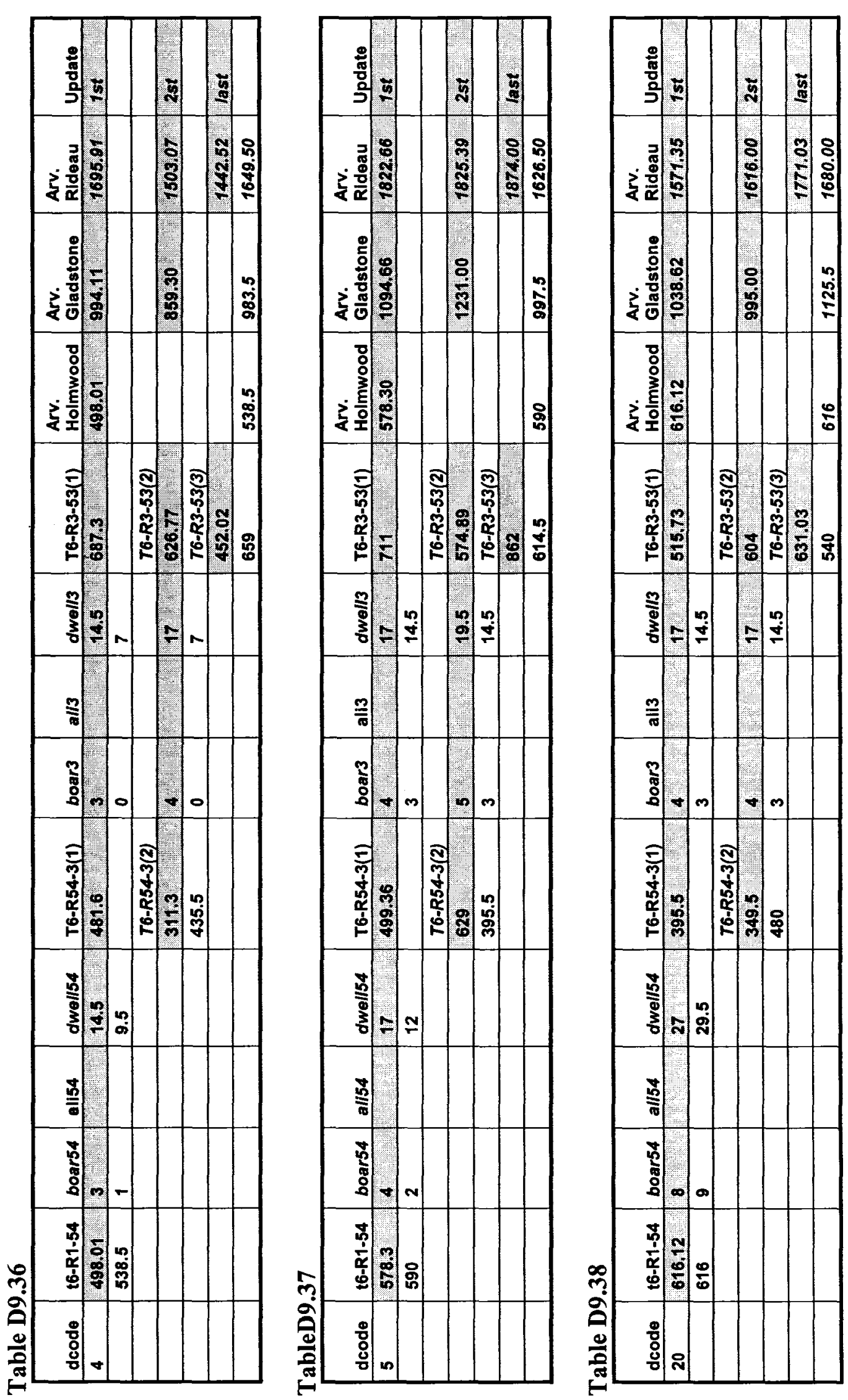


$\overline{8}$
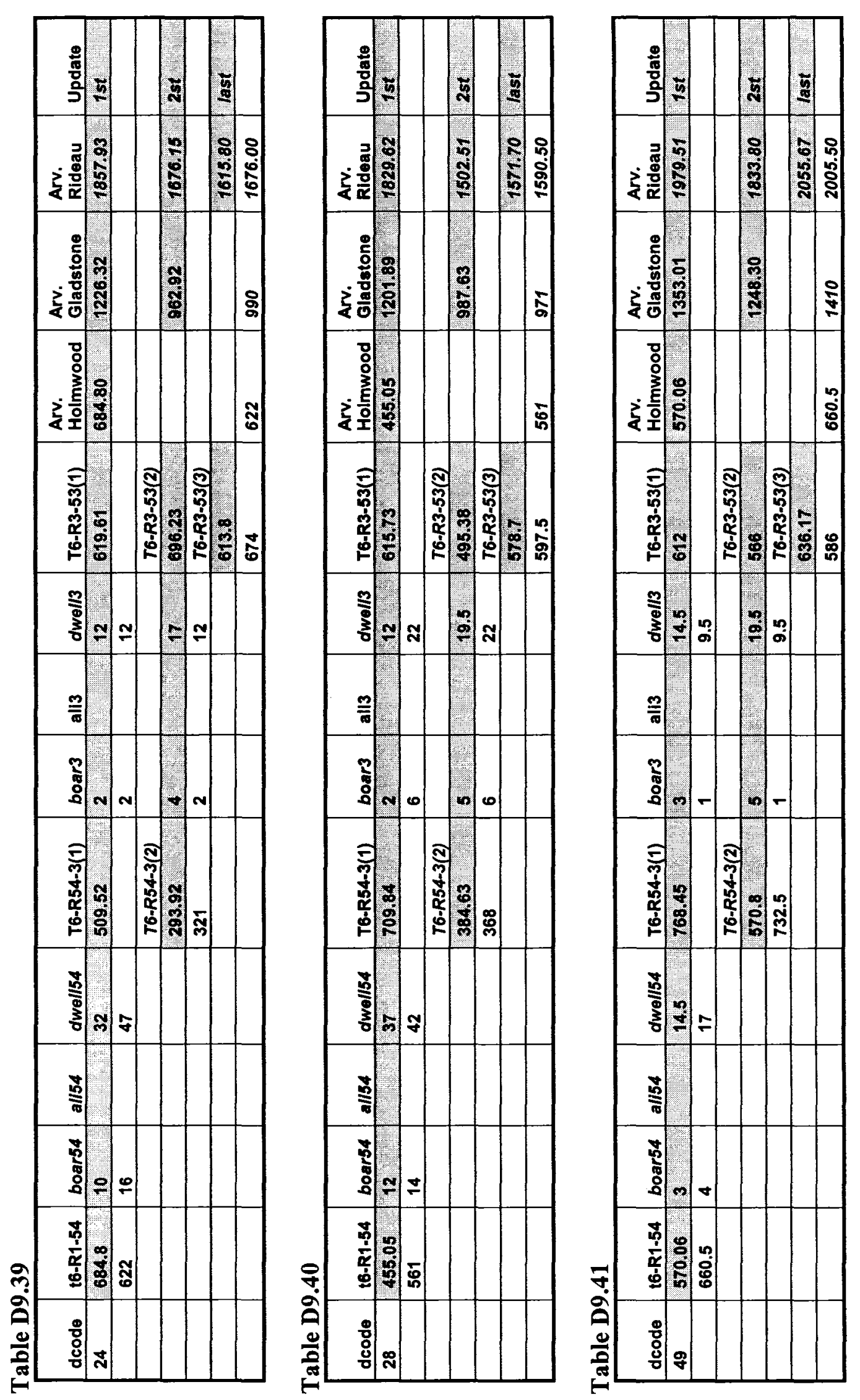
宇
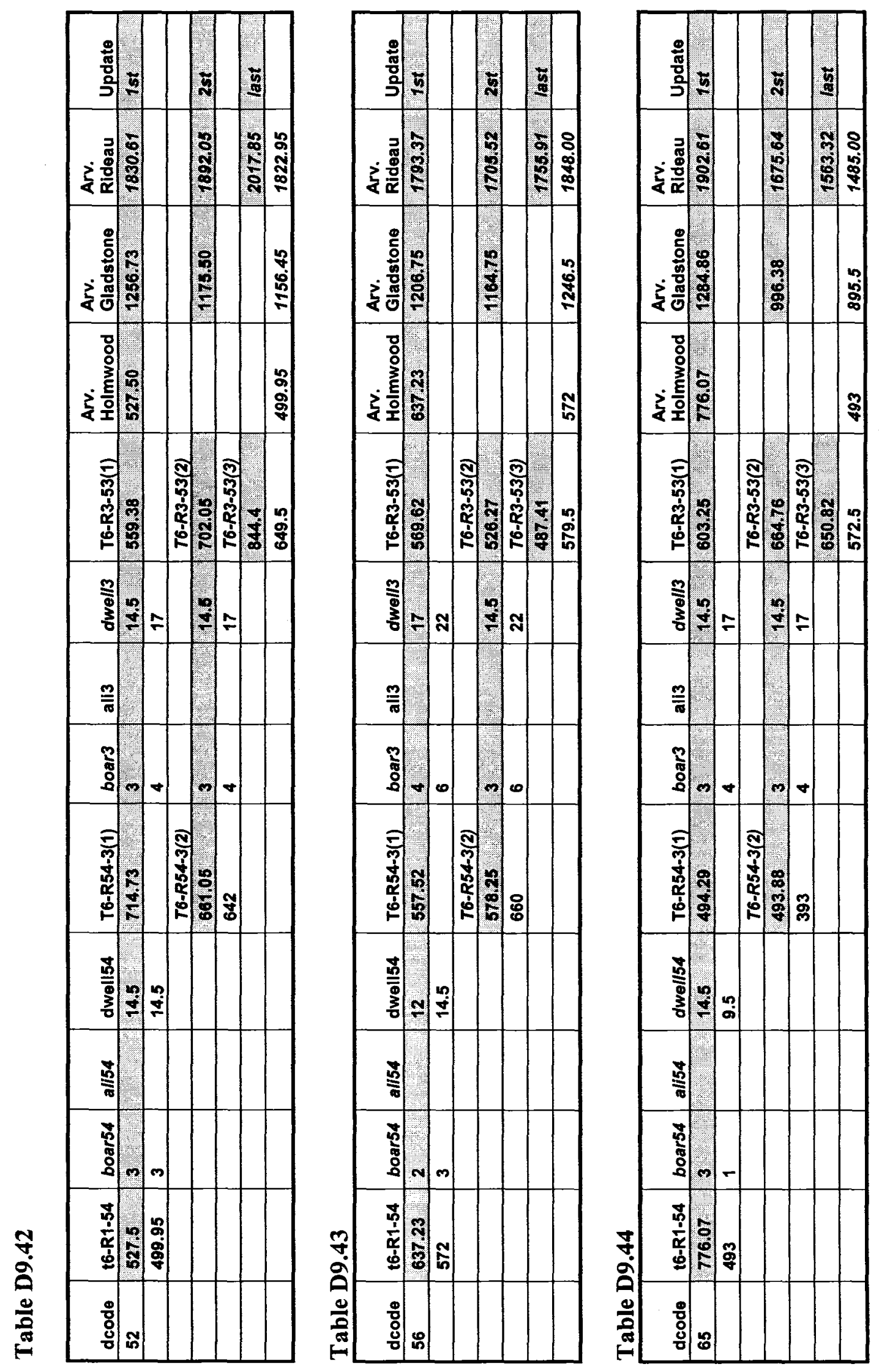
$\stackrel{m}{q}$

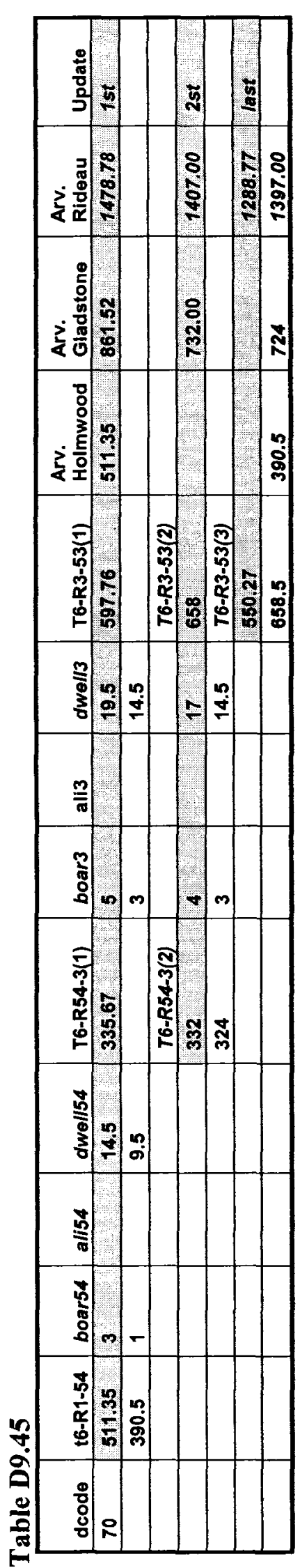

